Defense Threat Reduction Agency 8725 John J. Kingman Road, MS

6201 Fort Belvoir, VA 22060-6201

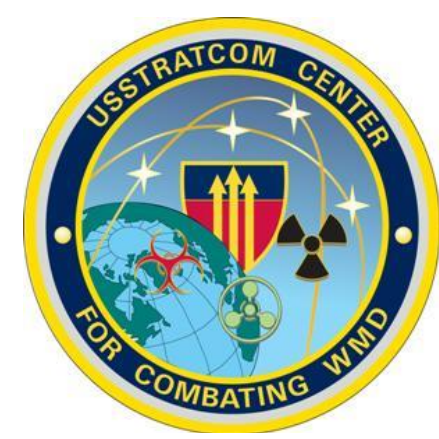

DTRA-TR-16-71






\section{DESTRUCTION NOTICE:}

Destroy this report when it is no longer needed.

Do not return to sender.

PLEASE NOTIFY THE DEFENSE THREAT REDUCTION AGENCY, ATTN: DTRIAC/ J9STT, 8725 JOHN J. KINGMAN ROAD, MS-6201, FT BELVOIR, VA 22060-6201, IF YOUR ADDRESS IS INCORRECT, IF YOU WISH IT DELETED FROM THE DISTRIBUTION LIST, OR IF THE ADDRESSEE IS NO LONGER EMPLOYED BY YOUR ORGANIZATION. 


\section{REPORT DOCUMENTATION PAGE}

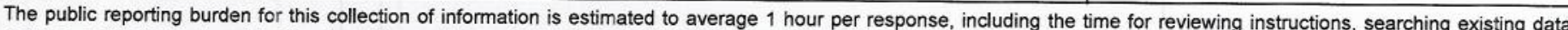

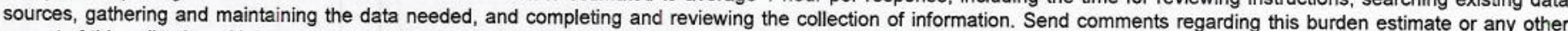

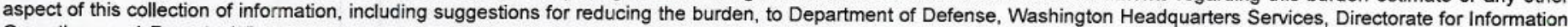

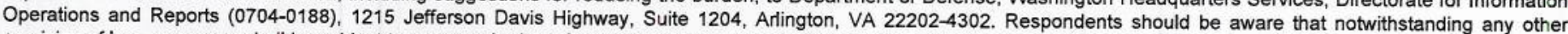
provision of law, no person shall be subject to any penalty for failing to comply with a collection of information if it does not display a currently valid OMB control number. PLEASE DO NOT RETURN YOUR FORM TO THE ABOVE ADDRESS.

\begin{tabular}{|l|l|l}
\hline $\begin{array}{l}\text { 1. REPORT DATE (DD-MM-YYYY) } \\
00-06-2016\end{array}$ & $\begin{array}{l}\text { 2. REPORT TYPE } \\
\text { Final Report }\end{array}$ & $\begin{array}{l}\text { 3. DATES COVERED (From - To) } \\
\text { Feb 2012 - Dec 2105 }\end{array}$
\end{tabular}

\section{TITLE AND SUBTITLE}

Luminescence materials as nanoparticle thermal sensors

5a. CONTRACT NUMBER

HDTRA1-10-1-0007

5b. GRANT NUMBER

5c. PROGRAM ELEMENT NUMBER

6. $A U T H O R(S)$

5d. PROJECT NUMBER

Yukihara, E. G., Talghader, J. J., Jacobsohn, L. G., Ballato, J.

\section{5e. TASK NUMBER}

5f. WORK UNIT NUMBER

\section{PERFORMING ORGANIZATION NAME(S) AND ADDRESS(ES)}

Physics Department, Oklahoma State University, 145 Physical Sciences II, Stillwater, OK 74078

Department of Electrical and Computer Engineering, University of Minnesota,

9. SPONSORING/MONITORING AGENCY NAME(S) AND ADDRESS(ES)

Defense Threat Reduction Agency

8725 John J. Kingman Road, STOP 6201

Fort Belvoir, VA 22060-6201

8. PERFORMING ORGANIZATION REPORT NUMBER

10. SPONSOR/MONITOR'S ACRONYM(S)

DTRA

11. SPONSOR/MONITOR'S REPORT NUMBER(S)

DTRA-TR-16-71

\section{DISTRIBUTION/AVAILABILITY STATEMENT}

Distribution Statement A. Approved for public release; distribution is unlimited.

\section{SUPPLEMENTARY NOTES}

\section{ABSTRACT}

The objective of this project was to create and study novel materials for temperature sensing in support of detonation studies using thermoluminescence (TL) technique. Commercial materials were investigated and new TL materials were developed. The best candidate materials, specifically LiF, Li2B4O7, MgB4O7 and CaSO4, were tested under closed and open chamber detonation experiments in collaboration with the Naval Surface Warface Center and DTRA. A multiparametric analysis procedure was also developed to recover the temperatures from the TL data. The results

15. SUBJECT TERMS

particle sensors; thermoluminescence; agent defeat tests; thermometry;

16. SECURITY CLASSIFICATION OF:

a. REPORT

Unclass

\begin{tabular}{|l|l|}
\hline b. ABSTRACT & c. THIS PAGE \\
Unclass & Unclass \\
\end{tabular}

17. LIMITATION OF ABSTRACT

SAR
18. NUMBER 19 a. NAME OF RESPONSIBLE PERSON

OF

PAGES

213 Allen Dalton

19b. TELEPHONE NUMBER (Include area code) 703-767-3054 


\section{UNIT CONVERSION TABLE}

U.S. customary units to and from international units of measurement ${ }^{*}$

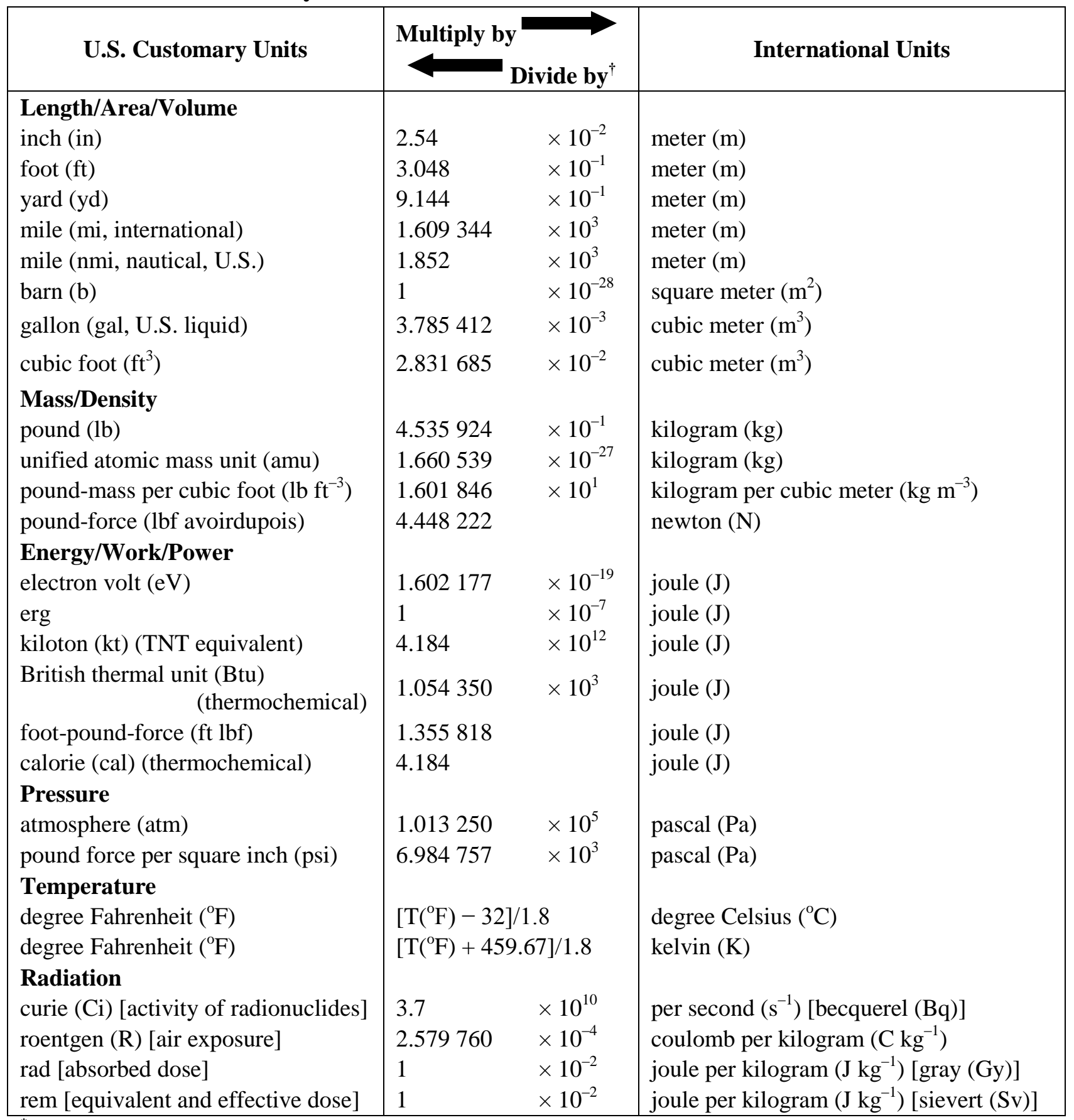

"Specific details regarding the implementation of SI units may be viewed at http://www.bipm.org/en/si/.

${ }^{\dagger}$ Multiply the U.S. customary unit by the factor to get the international unit. Divide the international unit by the factor to get the U.S. customary unit. 
Final Technical Report

\title{
LUMINESCENCE MATERIALS AS NANOPARTICLE THERMAL SENSORS
}

\author{
Contract number HDTRA1-10-0007
}

\section{Prepared for}

DEFENSE THREAT REDUCTION AGENCY

For the period

18 February 2010 - 15 December 2015

\section{Submitted by}

Eduardo G. Yukihara, Principal Investigator, Oklahoma State University (OSU)

Joseph J. Talghader, Co-Investigator, University of Minnesota (UMN)

John Ballato, Co-Investigator, Clemson University (CU)

Luiz G. Jacobsohn, Co-Investigator, Clemson University (CU)

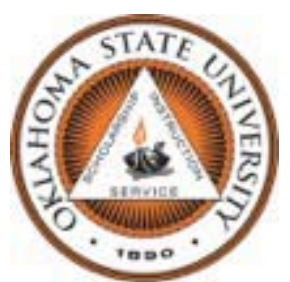

OKLAHOMA STATE UNIVERSITY

Physics Department

145 Physical Sciences II

Stillwater OK 74078

(405) 744-5051

eduardo.yukihara@okstate.edu

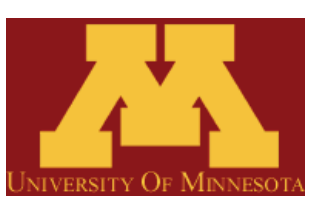

UNIVERSITY OF MINNESOTA

Department of Electrical and

Computer Engineering

Minneapolis, MN 55455

joey@ece.umn.edu

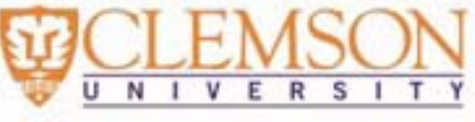

CLEMSON UNIVERSITY

Department of Materials Science and Engineering, and Center for Optical Materials Science and Engineering Technologies (COMSET)

Clemson, South Carolina 29634 


\section{CONTENTS}

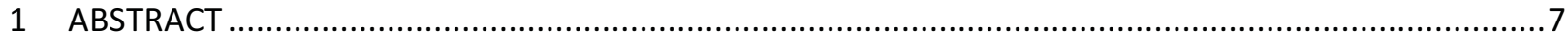

2 OBJECTIVES

3 APPROACH

3.1 Temperature sensing using thermoluminescence .............................................................12

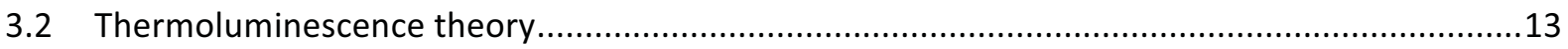

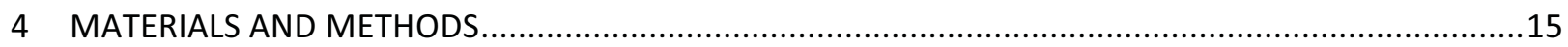

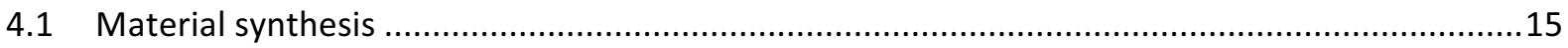

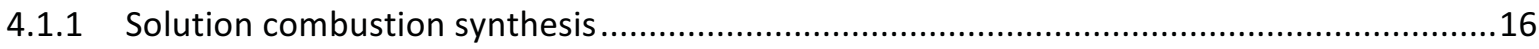

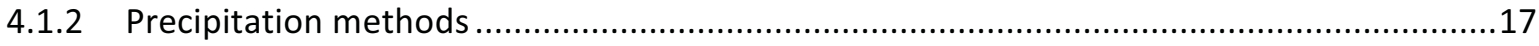

4.1.3 Solution precipitation of core/shell nanoparticles ......................................................18

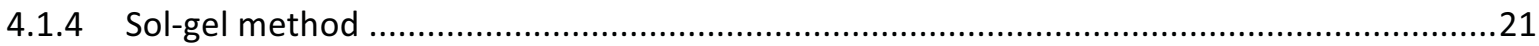

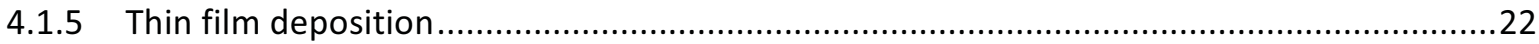

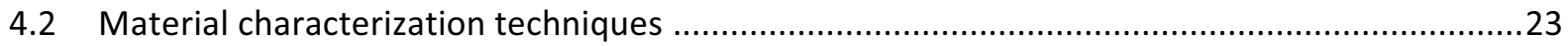

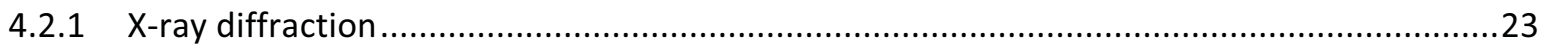

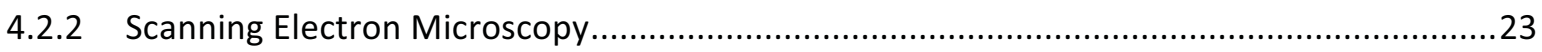

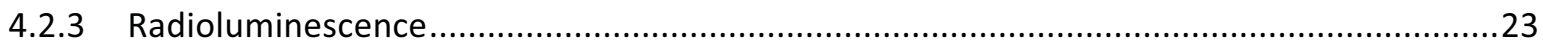

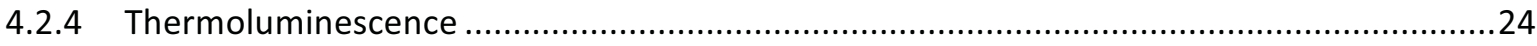

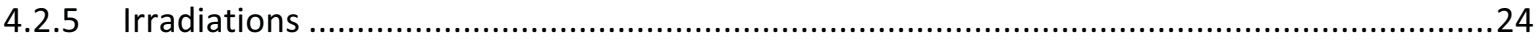

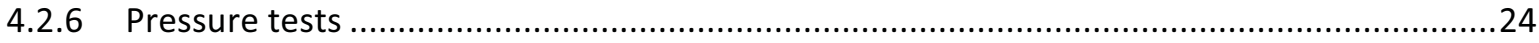

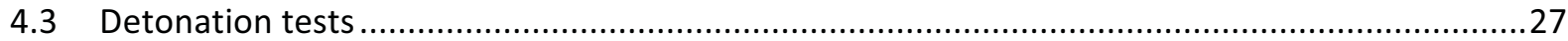

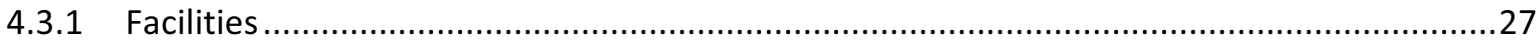

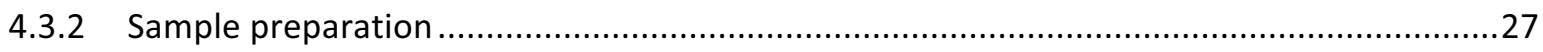

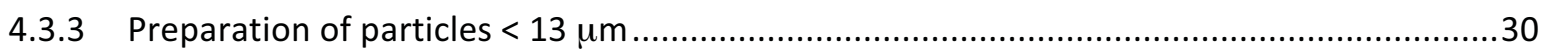

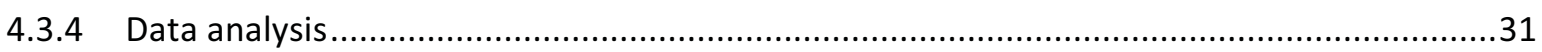

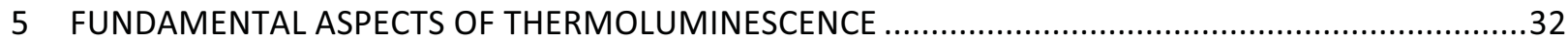

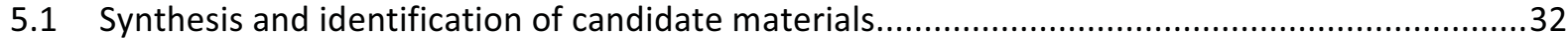

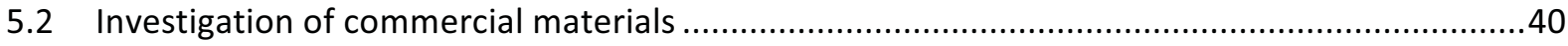

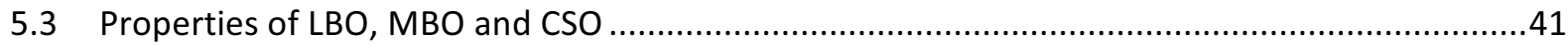

5.3.1 Crystal structure and morphology ..................................................................................

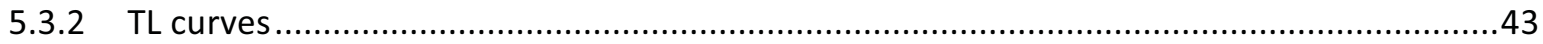

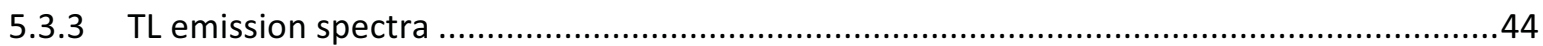

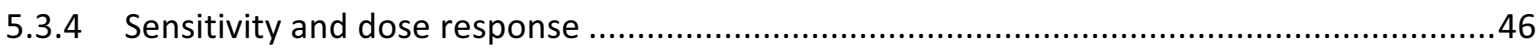




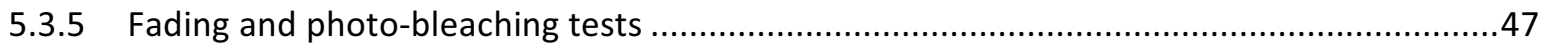

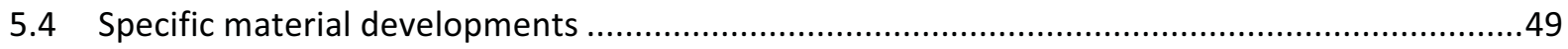

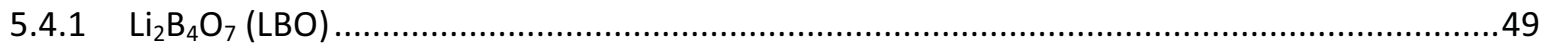

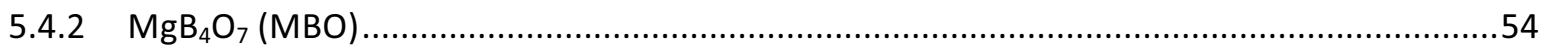

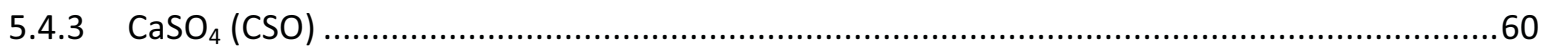

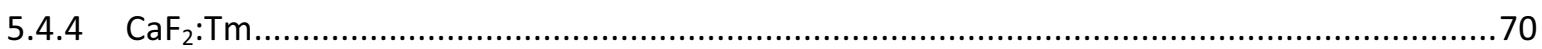

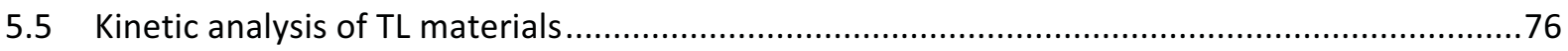

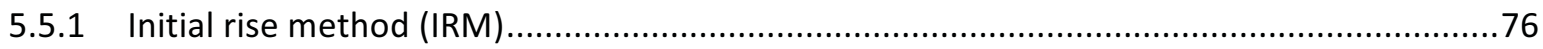

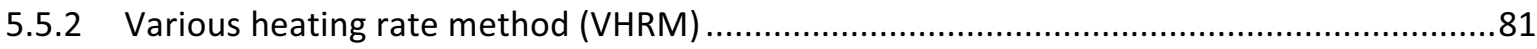

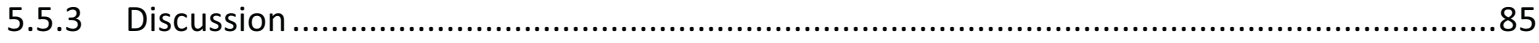

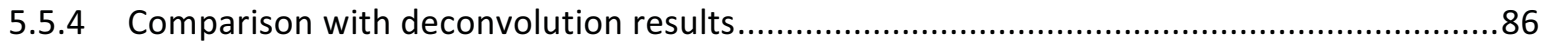

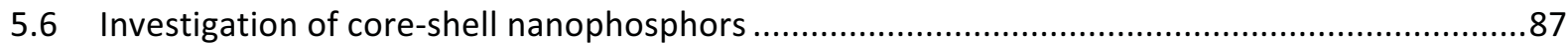

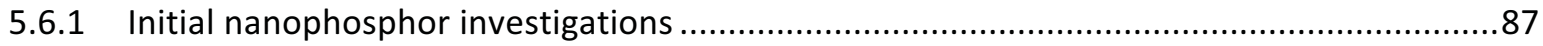

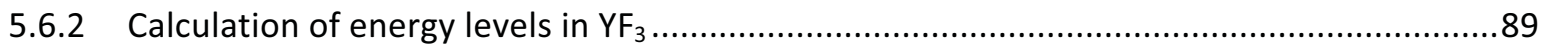

5.6.3 Characterization of nanophosphors produced at Clemson University...............................90

5.6.4 Computer simulation of luminescence of idealized particles..............................................94

6 DEVELOPMENT OF MULTIPARAMETRIC TEMPERATURE RECONSTRUCTION ..................................96

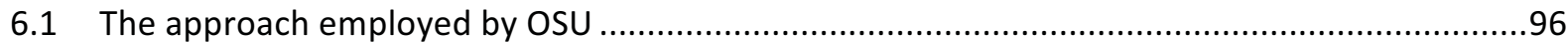

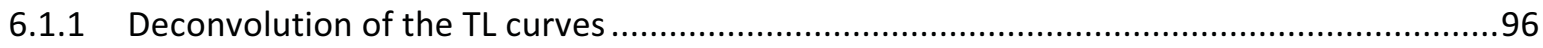

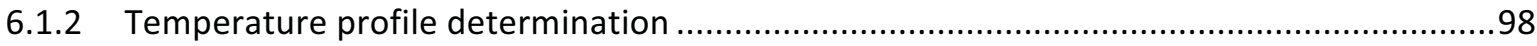

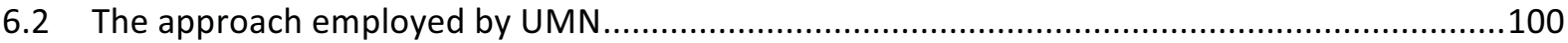

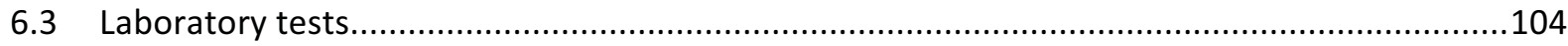

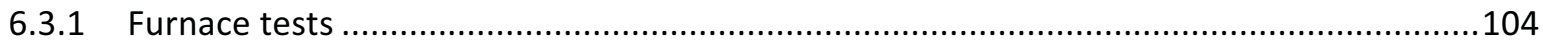

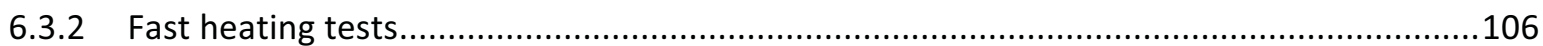

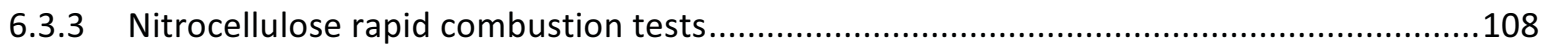

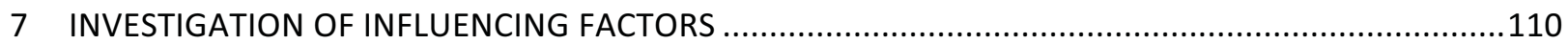

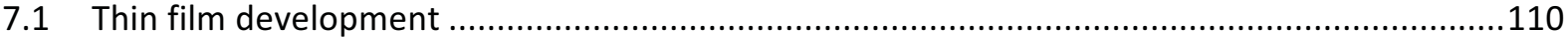

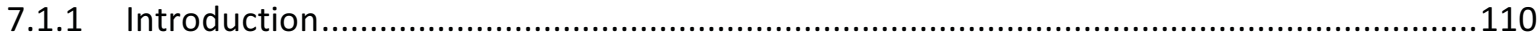

7.1.2 Deposition process and measurements ….............................................................110

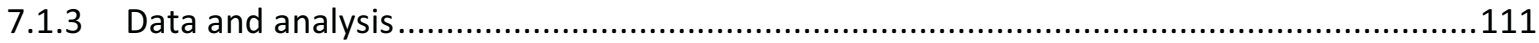

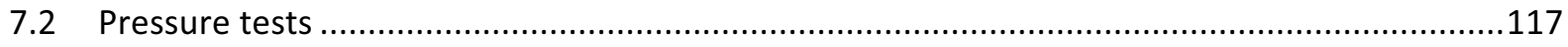

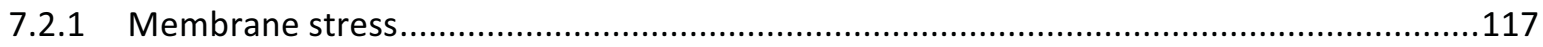

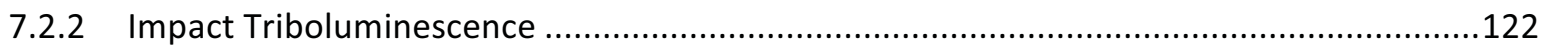




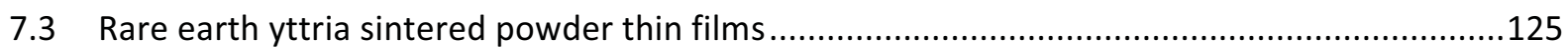

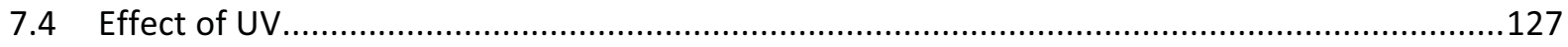

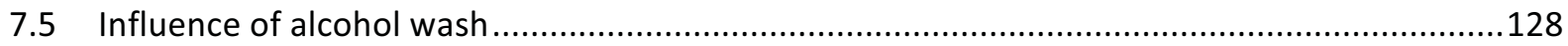

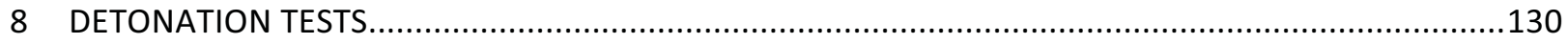

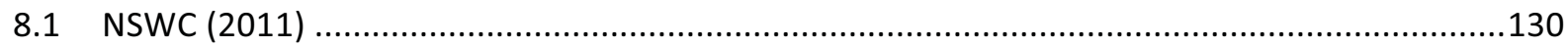

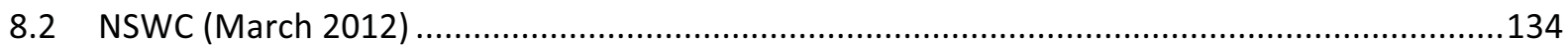

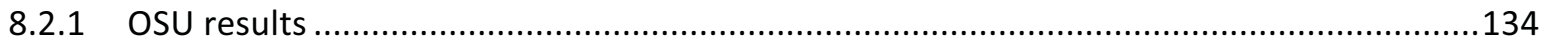

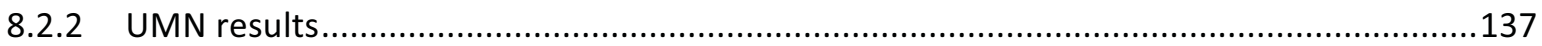

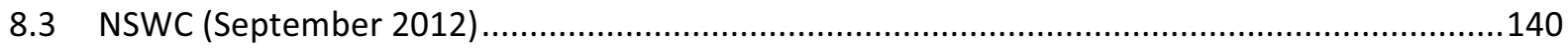

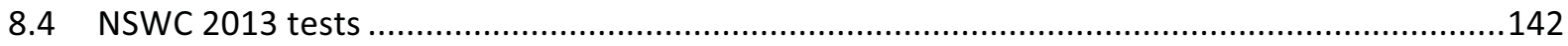

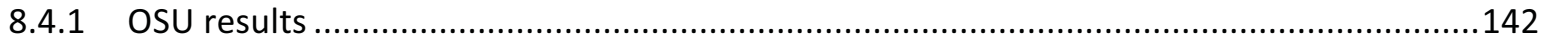

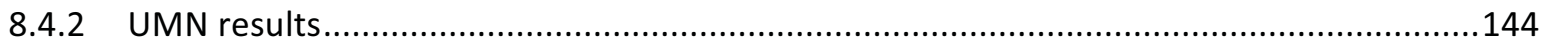

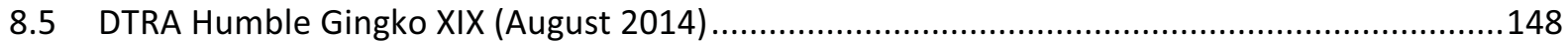

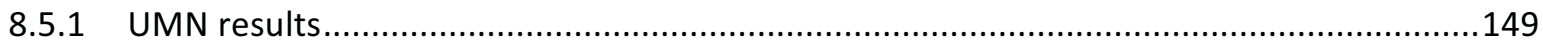

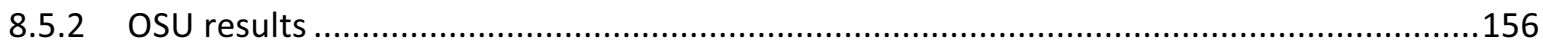

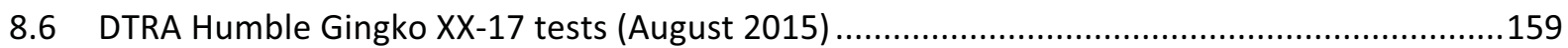

8.7 Shock-tube tests at University of Illinois Urbana-Champaign (Jan. 2013)...............................162

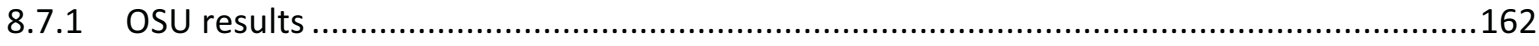

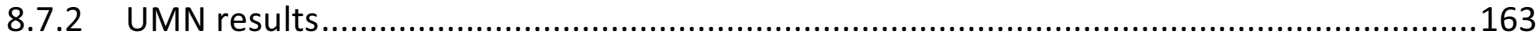

8.8 Shock Tube tests at University of Illinois - Urbana Champaign (Oct. 2014)............................166

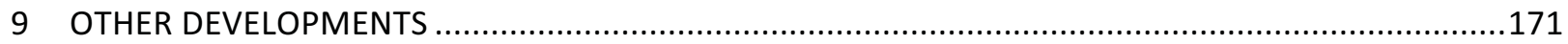

9.1 Development of low thermal emission heaters for high temperature TL analysis .................171

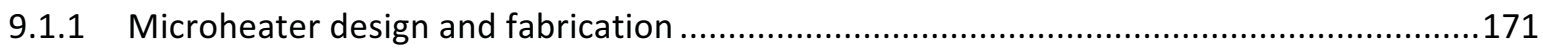

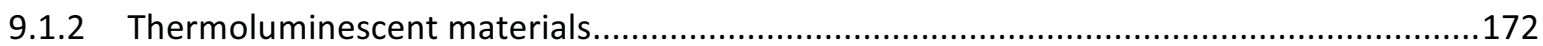

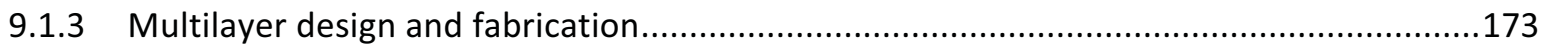

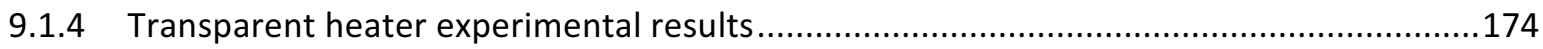

9.1.5 Multilayer method experimental results....................................................................176

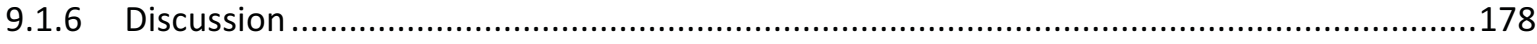

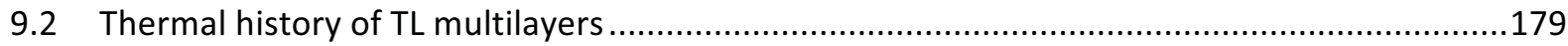

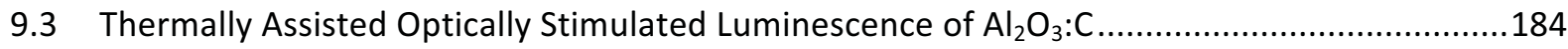

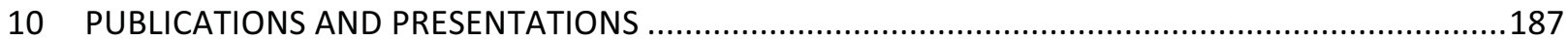

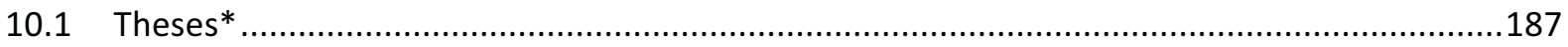

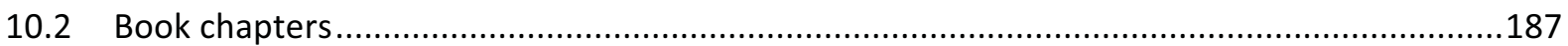




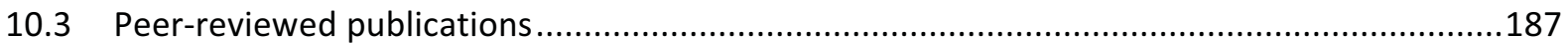

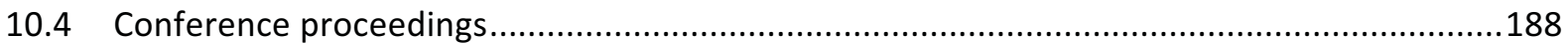

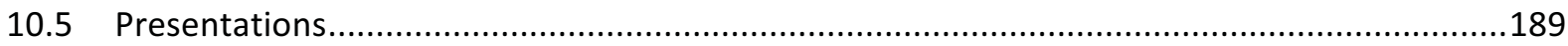

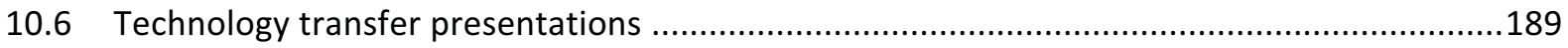

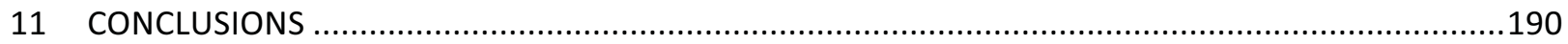

APPENDIX A - ENERGY DISTRIBUTION DETERMINATION ALGORITHM

APPENDX B - TEMPERATURE DETERMINATION ALGORITHM 


\section{ABSTRACT}

This report presents the result of a fundamental research program to create and study novel luminescence particles (phosphors) capable of sensing and retaining the time-temperature information to which they were exposed, therefore acting as nano- and microsized thermosensors. The thermometric property is the latent thermoluminescence (TL) signal associated with electron/hole pairs trapped at defect energy levels, which are differently affected by the environmental temperature.

The Defense Threat Reduction Agency (DTRA) mission of combating WMD includes the research and development of Agent Defeat Weapons (ADW) capable of destroying chemical and biological agent facilities and stockpiles with minimum collateral damage, particularly avoiding the dispersion of viable agents to the environment. High-temperature produced by slow-burning incendiary materials is one of the kill mechanisms investigated to neutralize different types of agents, including dry spores, vegetative cells, viruses, toxins and chemical agents. One of the obstacles on advancing the research on new types of energetic materials and mechanisms of biological agent neutralization, however, is the inability of current technology to measure the entire time-temperature profile of very small particles in extreme conditions.

The TL technique investigated in this study has the potential to record the entire temperature history during an agent-defeat test (ADT). Traditional contact (thermocouples, thermistors, etc.) and noncontact temperature measurement methods (spectroscopy, pyrometry) are not capable of determining the temperature experienced by a particle during an ADT. Other techniques under development, such as fluorescence nanoparticle probes [1] are also not applicable, because they rely on real-time changes in the fluorescence as a function of temperature, and therefore cannot be used in extreme environments (inside blast and fireball, or in dark, smoky blast cloud and dust plume).

The objective of this project was to create and study novel luminescence particles capable of sensing and retaining the time-temperature information to which they were exposed in their thermoluminescence (TL) curves, therefore acting as nano- to microsized thermosensors.

Specific aims of the present project were:

(a) To understand the fundamental aspects of the TL mechanism in luminescent particles, learning how to engineer the TL properties during synthesis to develop suitable thermosensors, and create core-shell TL nanostructures capable of distinguishing fast radiative heating from slower convective processes;

(b) To develop and test a multiparametric procedure to extract the critical portions of the temperature profile including heating, maximum temperature, and cooling and to differentiate between fast radiative and slower convection effects based on the TL from single-material and core-shell nanoparticles;

(c) To understand how complicating factors such as pressure and ultraviolet radiation during the explosive process will impact the TL and thermometric properties of the phosphors and, if necessary, how to correct for them.

To achieve these objectives, we investigated commercial candidate materials for use as temperature sensors, and performed a systematic study to synthesize and develop new materials by looking at various host/dopant combinations [2]. The study also included the development of TL core/shell nanophosphors. 
By investigating different host/dopant combinations we improved our understanding of the TL properties necessary for temperature sensing (e.g. multiplicity of TL peaks over a wide range of temperatures, emission in the UV region, light insensitivity, minimum fading, etc.), and developed models to obtain these required properties by suitable choice of host and dopant.

From this study, we found that specific host materials (e.g., $\mathrm{LiF} \mathrm{Li}_{2} \mathrm{~B}_{4} \mathrm{O}_{7}, \mathrm{MgB}_{4} \mathrm{O}_{7}, \mathrm{CaSO}_{4}$ ) are less light sensitive and can be used as the basis for development of temperature sensors [3, 4]. With suitable dopants these materials can be made to emit in the UV region (e.g. by $\mathrm{Cu}^{+}$or $\mathrm{Ce}^{3+}$ doping), and $\mathrm{TL}$ peaks in a wide range of temperatures from $<100{ }^{\circ} \mathrm{C}$ up to $>600{ }^{\circ} \mathrm{C}$ (when using $1{ }^{\circ} \mathrm{C} / \mathrm{s}$ heating rate) can be introduced (e.g. using various lanthanides) $[5,6]$. The wide range of TL peaks that can be obtained have been demonstrated using $\mathrm{CaSO}_{4}$. We also demonstrate how the synthesis conditions (e.g. fuel type and annealing temperature) can be used to improve the luminescence properties $[7,8]$.

Commercial materials identified (e.g. LiF) and new materials developed (e.g. $\mathrm{Li}_{2} \mathrm{~B}_{4} \mathrm{O}_{7}, \mathrm{MgB}_{4} \mathrm{O}_{7}, \mathrm{CaSO}_{4}$ ) were characterized for the structural and thermoluminescence properties [4, 9]. This provided the basic information to develop a multiparametric algorithm to extract the temperature profile from the $\mathrm{TL}$ curve data obtained from the particles. The algorithm from Oklahoma State University (OSU) is based on the deconvolution of the TL from the samples to obtain a TL model, which can then be used together with experimental TL curves to estimate the temperature experience by the particles [10]. The algorithm from the University of Minnesota (UMN) is based on the use of multiple points in the TL curve and a TL model for the materials to the same effect [9]. The results from both algorithms, developed completely independently, were comparable.

The materials mentioned above were also tested in laboratory (furnace and a temperature chamber) and showed perfect agreement with thermocouple data, except when particle aggregation prevented some of the grains from being heated uniformly [10]. Tests in close-chamber experiments showed agreement with thermocouple data for various materials, while highlighting the need to develop materials with a wider range of temperature applicability [11]. Materials with a narrow range of TL peaks have a narrow range of applicability. Therefore, it is desirable to have materials with multiple TL peaks distributed over a wide range of temperatures. Finally, open chamber tests performed in collaboration with DTRA revealed the complexity of the temperature environment, with different particles resulting in different temperatures, probably because of the various possible paths during the detonation event.

The project required the development of thin TL films to investigate the effect of pressure [12], but we did not observed any effect up to MPa pressures. Furthermore, because the samples are prepared with very high doses of ionizing radiation ( $k G y)$, no effect of UV light in inducing or depleting the TL peaks was noticed during experiments. The influence of particle size was investigated either by simulation of the response of core-shell nanophosphors, as well as experimentally by the use of TL materials with multilayers [13]. Materials for core-shell nanophosphors were also developed [14], but the properties were not sufficient for temperature sensing applications.

Finally, new heaters with low thermal emission were also developed as part of this project with the objective of providing a method to measure TL peaks at high temperature, which has the potential to increase the range of applicability of the TL materials [15].

As is clear from this abstract, the project generated several publications, in addition to conference proceedings, presentations and student's theses.

Overall the project successfully demonstrated TL particles as thermal sensors and developed the fundamental materials science and data analysis procedures to apply it. Successful deployment of the 
technique in realistic conditions depends now on a close collaboration between researchers with expertise on the TL technique and TL materials, and researchers interested in employing the technique. The major obstacle seems to be not scientific or technical, but the need to bridge the gap between the two branches of investigation to identify and solve some of the practical issues related with sample collection and handling, choice of best materials for application, and strategies to obtain useful conclusions from the information provided by the particles, since the novelty of the technique also implies that there are no reference or "gold-standard" to compare the technique to in real applications.

\section{ACKNOWLEDGEMENTS}

This project would not be successful without the support, suggestions, encouragement and collaboration from various individuals, including: Suhithi Peiris, Douglas Dalton and Howard Ross from Defense Threat Reduction Agency; James Lightstone, Joel Carney, Amber Daniels, Demitrios Stamatis, Christopher Milby, Jillian Horn and Forrest Svingala from the Naval Surface Warfare Center; Nick Glumac and Shuyue Guo from the University of Illinois Urbana-Champaign; Dr. Hergen Eilers from University of Washington; Don Littrell from Air Force Research Laboratory; Liping Huang from RPI, and William Lewis from University of Dayton Research Institute; Charles Hunt from Physics Department, Oklahoma State University. 


\section{OBJECTIVES}

The objective of this project was to create and study novel luminescence particles capable of sensing and retaining the time-temperature information to which they were exposed in their thermoluminescence (TL) curves, therefore acting as nano-to microsized thermosensors.

Specific aims of the present project were:

(a) To understand the fundamental aspects of the TL mechanism in luminescent particles, learning how to engineer the TL properties during synthesis to develop suitable thermosensors, and create core-shell TL nanostructures capable of distinguishing fast radiative heating from slower convective processes;

(b) To develop and test a multiparametric procedure to extract the critical portions of the temperature profile including heating, maximum temperature, and cooling and to differentiate between fast radiative and slower convection effects based on the TL from single-material and core-shell nanoparticles;

(c) To understand how complicating factors such as pressure and ultraviolet radiation during the explosive process will impact the TL and thermometric properties of the luminescent particles and, if necessary, how to correct for them.

Ceramic particles are extremely sensitive to temperature and could be used to probe the thermal history during an Agent Defeat Test (ADT).

We identified three main challenges that must be faced if luminescent particle sensors are to become practical: (i) one must develop sets of TL materials with properties specific to a given agent defeat procedure; (ii) one must extract not only the maximum temperature, but the entire thermal history experienced by the particle from the various TL signals; and (iii) one must eliminate or compensate for the luminescence effects of other environmental parameters (e.g. pressure, UV light) in the data.

This research addressed these challenges by combining materials science and engineering, investigations on thermal history extraction, and materials testing (Fig. 2). We investigated new TL materials and nanophosphors with defects and characteristics suitable for thermal sensing, strategies to extract the entire thermal history experienced by the particles based on the multiple particles or multiple TL peaks, and tested the materials in extreme environments, such as fast heating and cooling rates simulating the profiles experienced during explosions ( $\mu \mathrm{s}$ to $\sim 10^{2} \mathrm{~s}$ time-temperature profiles) and extreme pressures (MPa).

This research program aimed at developing the fundamental knowledge to create new TL luminescent particles with engineered properties for use as thermosensors, with impact on the understanding and development of this new class of materials, as well as to practical aspects in temperature measurements in ADTs. Ultimately, the temperature measurements will assist in the development of more effective agent defeat weapons.

The knowledge gained during the project is also useful for thermosensor application in other industrial and non-defense applications, such as process optimization of industrial manufacturing, mapping of temperature in turbines, etc. The project also benefits other areas not related to temperature measurements. Defects are responsible not only for the thermometric properties of the luminescence materials, but also for their performance in radiation detection, lighting, imaging, and dosimetry, where they are used for illicit radioactive material detection, medical imaging, dosimetry, and quality control in radiotherapy. Therefore, trapping center identification in a variety of matrices and the 
understanding of the underlying luminescence mechanism provides not only the scientific basis for new material development for thermosensors, but also for applications.

This project was in support of the Basic and Applied Sciences Directorate and the DTRA Research Topic Area C - Nano-sized Thermosensor Materials, in support of DTRA initiative C (Thrust 1 ) aimed at advancing the state of the science and body of knowledge for nano-sized thermosensor materials.

This project was a multi-disciplinary effort combining physical sciences, engineering, and material sciences towards the understanding the fundamental aspects of TL nanophosphors for use as thermosensors in ADTs. The team consisted of Dr. Eduardo G. Yukihara (PI, Physics Department, Oklahoma State University - OSU), Dr. Joseph J. Talghader (Co-I, Department of Electrical and Computer Engineering, University of Minnesota - UMN), Dr. Luiz G. Jacobsohn, and Dr. John Ballato (Co-l's, Department of Materials Science and Engineering, and Center for Optical Materials Science and Engineering Technologies, Clemson University - CU), in additional to graduate students in all three institutions. Clemson University was funded only for the first three years of project and Dr. John Ballato worked as an internal consultant at Clemson University at no cost to the project.

The TL material development was based on research on commercial TL materials and development of novel TL materials using syntheses methods such as solution combustion synthesis (SCS) and solution precipitation (SP), which offer new opportunities to easily produce a variety of nanophosphors. In the SCS method the material is produced by the self-sustained combustion of a mixture of metal nitrate and fuel (e.g., urea, glycine) initiated at relatively low temperatures $\left(\sim 500^{\circ} \mathrm{C}\right)$ in relatively short periods of time ( $\sim$ s) [16-19]. In the SP method, metal nitrate solutions are used as precursors and heated under continuous stirring to induce precipitation of metal hydroxide nanoparticles. Further calcination yields the production of oxides. As a result, a variety of materials can be easily produced at a low cost and without the need for high temperature furnaces. These synthesis methods are well-established in the literature and have been used to produce myriad different materials [20]. In particular, they have been used to produce phosphors doped with rare earth impurities with enhanced luminescence properties [21].

Core-shell nanoparticles have also been produced and demonstrate to have unique properties such as tunable energy transfer between lanthanides in different shells [22]. The concept of core-shell nanoparticle to alter the heat transfer mechanism in nanophosphors is novel and we explored its potential for distinguishing between different heat conduction mechanisms present in an ADT. 


\section{APPROACH}

\subsection{Temperature sensing using thermoluminescence}

The temperature measurement technique investigated in this project is based on thermoluminescence $(\mathrm{TL})$, a phenomenon observed in crystalline insulator and semiconductors of sizes from macro to the nanoscale [23], and which can be used to record the time-temperature profile experienced by a nanoor micro-particle used as a surrogate for bio-agent stimulants (see Fig. 1a). The concept has been presented by Talghader and colleagues $[3,24]$ and is briefly described here.

$\mathrm{TL}$ is the transient light emitted by material upon heating, caused by the existence of charges trapped at defects in insulating or semiconductor materials [25]. When a crystal is exposed to UV or ionizing radiation, free electrons are created and captured by energy levels introduced by defects in the crystal (traps) (Fig. 1a). If the material is heated during an explosion or in laboratory, some of these trapped charges will be released at a rate that depends on the temperature, time, and properties of the trapping center: the energy difference $E$ between the defect energy level and the conduction band, and the attempt-to-escape frequency s. In laboratory, the trapped charge population can be determined recording the TL curve, which is the curve of TL intensity versus temperature. The TL curve is composed of several peaks, each corresponding to a different trapping center (Fig. 1b), i.e., with a different $E$ and $s$ values, with an intensity that depends on the trapping population.

The resultant TL curve recorded in laboratory is affected by the temperature history of the particle. This is illustrated in Fig. $1 \mathrm{~b}$, where a TL curve before and after heating the material to $180{ }^{\circ} \mathrm{C}$ is recorded. One can see that the pre-heating decreases the intensity of one of the peaks to $40 \%$ of its original value, without affecting the other peak. Fig. 1c demonstrates how different TL peaks are affected by the temperature.

Therefore, using materials with multiple TL peaks (i.e., multiple types of defects) and appropriate algorithms, one can determine the temperature history of the particle by seeing how the different TL peaks are affected. On that basis, the particle acts as "passive" thermosensor, registering the temperatures experienced by the particle.

(a)
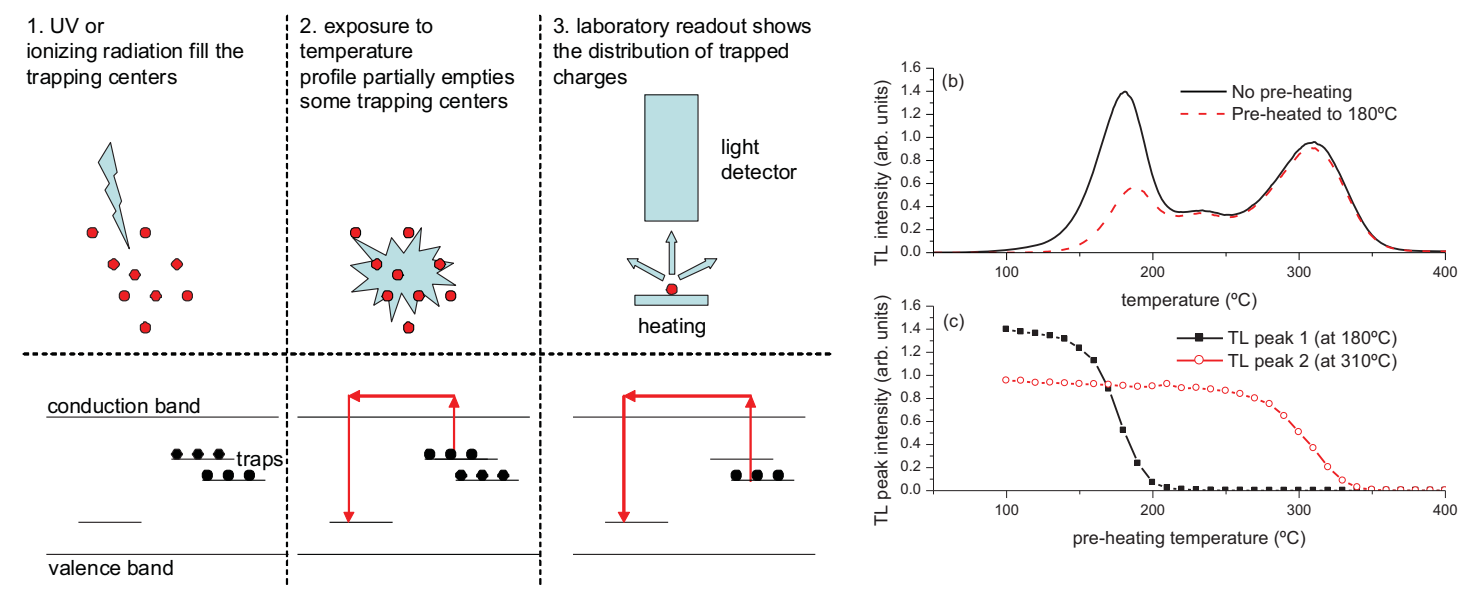

Fig. 1. (a) Illustration of TL particles can be used as thermosensors: trapping centers are initially filled using UV or ionizing radiation; then, the TL particles are exposed to a temperature profile; readout of the resultant TL curve in laboratory allows the determination of the thermal history; (b) example of TL curve obtained after filling the trapping centers 
$\left(\mathrm{Mg}_{2} \mathrm{SiO}_{4}: \mathrm{Tb}, \mathrm{Co}\right)$, as well as the curve after pre-heating to $180{ }^{\circ} \mathrm{C} ;(\mathrm{c})$ decrease in intensity of the two prominent TL peaks as a function of the maximum temperature reached in a given heating scheme.

The concept implementation is depicted in Fig. 2.

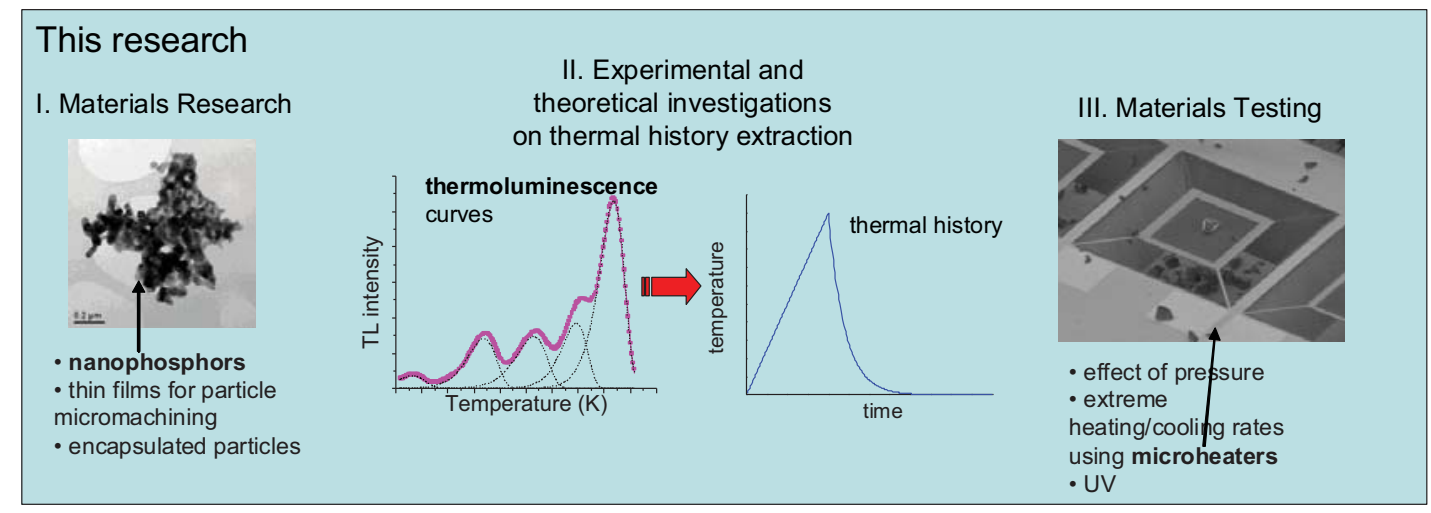

Concept implementation

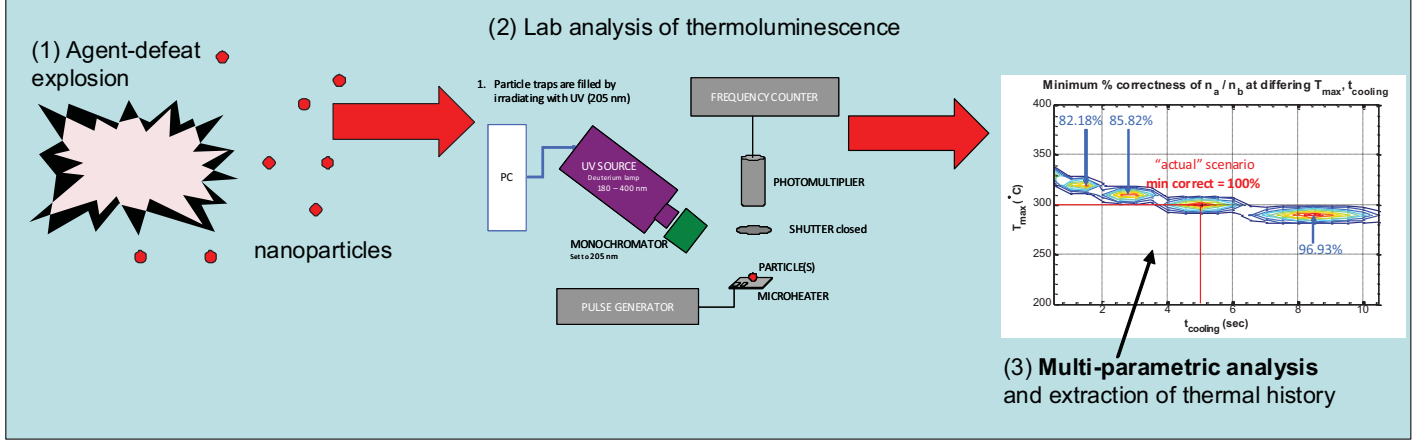

Fig. 2. Conceptual diagram of the primary thrust of the proposed luminescent particle research, including development of luminescent materials, determination of algorithms to extract thermal history, and experimental testing of both materials and algorithms under temperature profiles typical of agent defeat events.

\subsection{Thermoluminescence theory}

As other thermally stimulated processes, the rate of which the electrons escape from the traps is given by Arrhenius equation. In the case of TL, the detrapping rate depends on the number of trapped charges $n$, on the trap depth $E$, on the "attempt-to-escape" frequency $s$, and on the temperature $T$ according to [25]:

$$
\frac{d n}{d t}=-n s e^{-E / k T}
$$

If the crystal is submitted to a thermal profile $T(t)$, the number of trapped charges is given by: 


$$
n(t)=n_{0} \exp \left[-s \int_{0}^{t} e^{-\frac{E}{k T(t)}} d t\right]
$$

Assuming that all trapped charges recombined immediately, the TL curve is then given by the sum of the detrapping rates of all available trapping centers:

$$
I(t)=\sum_{i}\left|\frac{d n_{i}}{d t}\right|=\sum_{i} n_{0, i} s_{i} e^{-\frac{E_{i}}{k T}} \exp \left[-s_{i} \int_{0}^{t} e^{-\frac{E_{i}}{k T(t)}} d t\right]
$$

where $n_{0, i}, s_{i}, E_{i}$ are the initial trap population, attempt-to-escape frequency, and activation energy of the $i$-th trapping center, respectively.

The advantage of the TL technique is that the population in each trap can be determined accurately by the light intensity of each TL peak. By comparing the TL intensities of a grain "not exposed" and of a grain "exposed" to an explosion, associated with knowledge on the trapping parameters for each trap, one can apply an algorithm to extract the time-temperature profile experienced by the particle, i.e., determine what kind of time-temperature profile would result in the observed changes in the TL curve.

Another advantage of the proposed TL technique is precision, achieved by the possibility of regenerating the signal after the detonation. After recovering the thermosensor, one can measure the resultant TL related to the trap population that was partially emptied by "exposure" to the explosion. Then, one can again fill the traps of the thermosensor with UV light or ionizing radiation to measure the TL intensity of the same grain that would be obtained with all the traps filled, i.e., the TL of the grain if it was "not exposed" to the explosion. By comparing these two TL curves, one can determine to which extend the trapping centers were affected by the explosion, and therefore infer the temperature profile experienced by the grain. This "self-calibration" allows a very precise characterization of the trap depletion, irrespective of the grain size, TL sensitivity, or other parameters that may affect the overall luminescence output of the thermosensor.
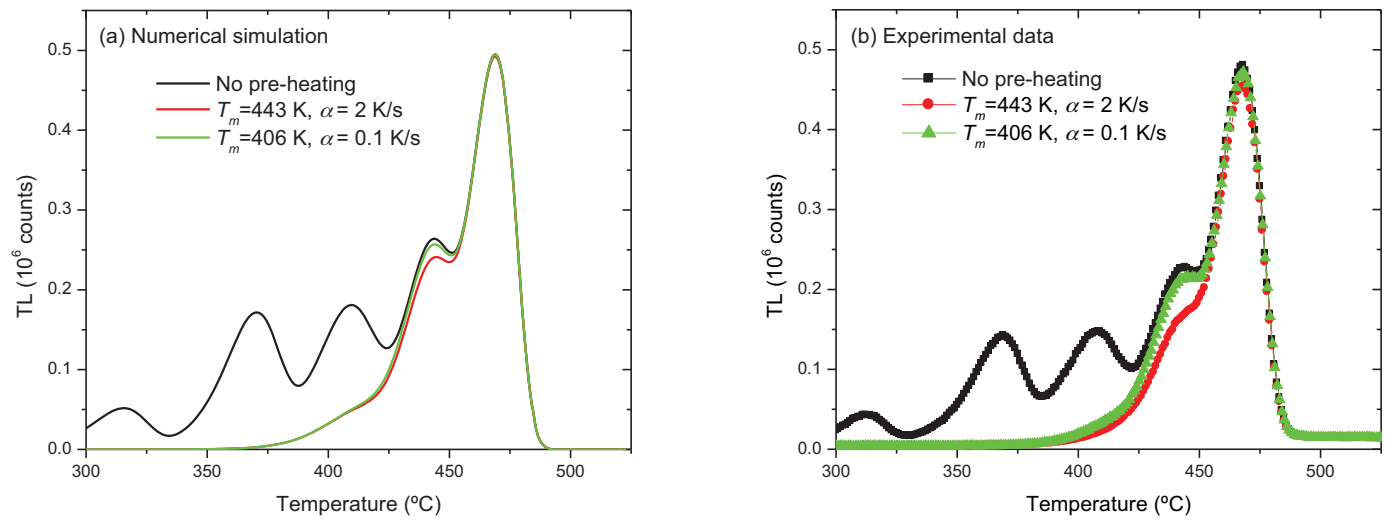

Fig. 3. Example of the effect of a "pre-heating" on the TL based on: (a) a numerical model for LiF, and (b) actual experimental data on LiF. The three curves represent LiF subjected to three different time-temperature profiles: "no preheating", a pre-heating up to a maximum temperature of $443 \mathrm{~K}$ and heating rate of $2 \mathrm{~K} / \mathrm{s}$, and a pre-heating up to a maximum temperature of $406 \mathrm{~K}$ and heating rate of $0.1 \mathrm{~K} / \mathrm{s}$. The later two heating regimes were designed to affect the third TL peak in the same way, but one can clearly see that peak 4 can "register" the different heating regimes. 


\section{MATERIALS AND METHODS}

\subsection{Material synthesis}

A very large number of host materials and dopant combinations were synthesized in this project using solution combustion synthesis (SCS) $[16,18,26]$, solid-state reaction, sol-gel, and solution precipitation methods [27]. Table 1 provides an overview of the wide range of materials, synthesized to identify appropriate materials for temperature sensing.

From these, three materials were identified as further developed for temperature sensing at OSU: $\mathrm{Li}_{2} \mathrm{~B}_{4} \mathrm{O}_{7}: \mathrm{Cu}, \mathrm{Ag}(\mathrm{LBO}), \mathrm{MgB}_{4} \mathrm{O}_{7}: \mathrm{Dy}$, $\mathrm{Li}(\mathrm{MBO})$ and $\mathrm{CaSO}_{4}: \mathrm{Ce}$, Tb (CSO) (Table 2).

Table 1. Examples of materials synthesized in this project by Solution Combustion Synthesis (SCS), Solid State Reaction (SSR), Precipitation (P), Solution Precipitation (SP), and sol-gel methods. Dopants in parenthesis indicate co-doped samples.

\begin{tabular}{|c|c|c|}
\hline Formula & Method & Dopants investigated \\
\hline $\mathrm{Al}_{2} \mathrm{O}_{3}$ & SCS & $\begin{array}{l}\mathrm{Ce}, \mathrm{Pr}, \mathrm{Nd}, \mathrm{Sm}, \mathrm{Eu}, \mathrm{Gd}, \mathrm{Tb}, \mathrm{Dy}, \mathrm{Ho}, \mathrm{Er}, \mathrm{Tm}, \mathrm{Yb}, \mathrm{Li}, \mathrm{Zn}, \mathrm{Zr}, \mathrm{Si}, \mathrm{Ag}, \mathrm{Fe}, \mathrm{Mn}, \mathrm{Ni}, \mathrm{Cu} \text {, } \\
(\mathrm{Zr}, \mathrm{Si}),(\mathrm{Zr}, \mathrm{P}),(\mathrm{Zr}, \mathrm{Tb}), \mathrm{Li}\end{array}$ \\
\hline $\mathrm{BaB}_{2} \mathrm{O}_{4}$ & SSR & Dy, $(D y, L i)$ \\
\hline $\mathrm{BaB}_{4} \mathrm{O}_{7}$ & SSR & Dy \\
\hline $\mathrm{BaB}_{8} \mathrm{O}_{13}$ & SSR & $\mathrm{Ce}$ \\
\hline $\mathrm{BaF}_{2}$ & SP & $\mathrm{Ce}$ \\
\hline $\mathrm{Ca}_{3} \mathrm{~B}_{2} \mathrm{O}_{6}$ & SSR & Dy \\
\hline $\mathrm{CaF}$ & SP & Eu (core + up to 3 shells) \\
\hline $\mathrm{CaAl}_{2} \mathrm{O}_{4}$ & SCS & $\mathrm{Ce}, \mathrm{Eu}, \mathrm{Li}$ \\
\hline $\mathrm{CaAl}_{12} \mathrm{O}_{19}$ & SCS & $\mathrm{Ce}, \mathrm{Eu}, \mathrm{Li}$ \\
\hline $\mathrm{CaO}$ & SCS & $\mathrm{Ce}, \mathrm{Pr}, \mathrm{Nd}, \mathrm{Sm}, \mathrm{Eu}, \mathrm{Gd}, \mathrm{Tb}, \mathrm{Dy}, \mathrm{Ho}, \mathrm{Er}, \mathrm{Tm}, \mathrm{Yb}, \mathrm{Li}$ \\
\hline $\mathrm{CaSO}_{4}$ & $\mathrm{P}$ & $\begin{array}{l}\mathrm{Dy}, \mathrm{Ce}, \mathrm{Tm}, \mathrm{Eu}, \mathrm{Y}, \mathrm{Tb},(\mathrm{Ce}, \mathrm{Na}),(\mathrm{Ce}, \mathrm{Sm}),(\mathrm{Ce}, \mathrm{Dy}),(\mathrm{Ce}, \mathrm{Nd}),(\mathrm{Ce}, \mathrm{Pr}),(\mathrm{Ce}, \mathrm{Er}) \text {, } \\
(\mathrm{Ce}, \mathrm{Ag}),(\mathrm{Eu}, \mathrm{Sm}),(\mathrm{Eu}, \mathrm{Tb}),(\mathrm{Eu}, \mathrm{Dy}),(\mathrm{Eu}, \mathrm{Ag}),(\mathrm{Dy}, \mathrm{P}),(\mathrm{Dy}, \mathrm{Ag})\end{array}$ \\
\hline $\mathrm{Gd}_{2} \mathrm{O}_{3}$ & SP & $\mathrm{Eu}$ \\
\hline $\operatorname{LaMgB}_{5} \mathrm{O}_{10}$ & SCS & $\mathrm{Ce}, \mathrm{Pr}, \mathrm{Nd}, \mathrm{Sm}, \mathrm{Eu}, \mathrm{Gd}, \mathrm{Tb}, \mathrm{Dy}, \mathrm{Ho}, \mathrm{Er}, \mathrm{Tm}, \mathrm{Yb}, \mathrm{Li}$ \\
\hline $\mathrm{LiAlO}_{2}$ & SCS & $\mathrm{Ce}$ \\
\hline $\mathrm{Li}_{2} \mathrm{~B}_{4} \mathrm{O}_{7}$ & SCS & $\mathrm{Dy}, \mathrm{Ce}, \mathrm{Mn}, \mathrm{Cu}, \mathrm{Ag}, \mathrm{Ni}, \mathrm{Cr},(\mathrm{Cu}, \mathrm{Ag}),(\mathrm{Cu}, \mathrm{P}),(\mathrm{Cu}, \mathrm{Dy}),(\mathrm{Cu}, \mathrm{Ti}),(\mathrm{Cu}, \mathrm{In}),(\mathrm{Cu}, \mathrm{Co})$ \\
\hline $\mathrm{LiCaBO}_{3}$ & SSR & Dy, Tm \\
\hline $\mathrm{Li}_{4} \mathrm{Sr}\left(\mathrm{BO}_{3}\right)_{3}$ & SSR & $\mathrm{Ce}, \mathrm{Tm}, \mathrm{Dy}, \mathrm{Tb},(\mathrm{Ce}, \mathrm{Eu}),(\mathrm{Ce}, \mathrm{Yb}),(\mathrm{Ce}, \mathrm{Tb}),(\mathrm{Ce}, \mathrm{Cu})$ \\
\hline $\mathrm{LiSrBO}_{3}$ & SSR & Tm, Ce, Tb, Dy, (Ce, Tm), (Ce,Tb), (Ce, Dy), (Ce,Eu),(Ce,Ag), (Dy, Ag), (Tm, Ag) \\
\hline $\mathrm{MgAl}_{2} \mathrm{O}_{4}$ & SCS & $\mathrm{Ce}, \mathrm{Pr}, \mathrm{Nd}, \mathrm{Sm}, \mathrm{Eu}, \mathrm{Gd}, \mathrm{Tb}, \mathrm{Dy}, \mathrm{Ho}, \mathrm{Er}, \mathrm{Tm}, \mathrm{Yb}$ \\
\hline $\mathrm{MgB}_{4} \mathrm{O}_{7}$ & SCS & $\begin{array}{l}\mathrm{Ce} ;(\mathrm{Ce}, \mathrm{K}),(\mathrm{Ce}, \mathrm{Li}),(\mathrm{Ce}, \mathrm{Na}),(\mathrm{Ce}, \mathrm{Nd}),(\mathrm{Ce}, \mathrm{Dy}, \mathrm{Li}),(\mathrm{Ce}, \mathrm{Nd}, \mathrm{Li}),(\mathrm{Ce}, \mathrm{Pr}, \mathrm{Li}),(\mathrm{Ce}, \mathrm{Tm}, \mathrm{Li}), \\
\mathrm{Dy},(\mathrm{Dy}, \mathrm{K}),(\mathrm{Dy}, \mathrm{Li}),(\mathrm{Dy}, \mathrm{Na}), \mathrm{Er},(\mathrm{Er}, \mathrm{Li}), \mathrm{Eu},(\mathrm{Eu}, \mathrm{Li}),(\mathrm{Eu}, \mathrm{K}),(\mathrm{Eu}, \mathrm{Na}), \mathrm{Gd},(\mathrm{Gd}, \mathrm{Li}), \mathrm{Ho}, \\
(\mathrm{Ho}, \mathrm{Li}), \mathrm{K}, \mathrm{Li}, \mathrm{Mn}, \mathrm{Na}, \mathrm{Nd},(\mathrm{Nd}, \mathrm{K}),(\mathrm{Nd}, \mathrm{Li}),(\mathrm{Nd}, \mathrm{Na}), \mathrm{Pr},(\mathrm{Pr}, \mathrm{K}),(\mathrm{Pr}, \mathrm{Li}),(\mathrm{Pr}, \mathrm{Na}), \mathrm{Sm} \text {, } \\
(\mathrm{Sm}, \mathrm{K}),(\mathrm{Sm}, \mathrm{Li}),(\mathrm{Sm}, \mathrm{Na}), \mathrm{Tb},(\mathrm{Tb}, \mathrm{K}),(\mathrm{Tb}, \mathrm{Li}),(\mathrm{Tb}, \mathrm{Na}),(\mathrm{Tb}, \mathrm{Dy}, \mathrm{Li}),(\mathrm{Tb}, \mathrm{Na}, \mathrm{Li}), \\
(\mathrm{Tb}, \mathrm{Pr}, \mathrm{Li}),(\mathrm{Tb}, \mathrm{Tm}, \mathrm{Li}), \mathrm{Tm},(\mathrm{Tm}, \mathrm{K}),(\mathrm{Tm}, \mathrm{Li}),(\mathrm{Tm}, \mathrm{Na}), \mathrm{Yb},(\mathrm{Yb}, \mathrm{Li})\end{array}$ \\
\hline $\mathrm{MgB}_{4} \mathrm{O}_{7}$ & SCS & Dy, $($ Dy, Li) \\
\hline $\mathrm{Mg}_{3} \mathrm{~B}_{2} \mathrm{O}_{6}$ & SCS & $(\mathrm{Dy}, \mathrm{Li}),(\mathrm{Ce}, \mathrm{Li}),(\mathrm{Tm}, \mathrm{Li}),(\mathrm{Eu}, \mathrm{Li}),(\mathrm{Gd}, \mathrm{Li})$ \\
\hline $\mathrm{MgSO}_{4}$ & $\mathrm{SP}$ & $\mathrm{Tm}$ \\
\hline $\mathrm{MgO}$ & SCS & $\begin{array}{l}\mathrm{Ce}, \mathrm{Pr}, \mathrm{Nd}, \mathrm{Sm}, \mathrm{Eu}, \mathrm{Gd}, \mathrm{Tb}, \mathrm{Dy}, \mathrm{Ho}, \mathrm{Er}, \mathrm{Tm}, \mathrm{Yb}, \mathrm{Li}, \mathrm{Al},(\mathrm{Ce}, \mathrm{Gd}),(\mathrm{Nd}, \mathrm{Gd}),(\mathrm{Ce}, \mathrm{Ca}) \text {, } \\
(\mathrm{Ce}, \mathrm{La}),(\mathrm{Ce}, \mathrm{Dy}),(\mathrm{Ce}, \mathrm{Eu}),(\mathrm{Ce}, \mathrm{Er}),(\mathrm{Ce}, \mathrm{Tm}),(\mathrm{Ce}, \mathrm{Yb}), \mathrm{La}, \mathrm{Ca}, \mathrm{Na}, \mathrm{Ba}, \mathrm{K}, \mathrm{Al}, \mathrm{Fe} \text {, } \\
\mathrm{Cr}, \mathrm{Mn}, \mathrm{P}, \mathrm{Si}, \mathrm{Co}, \mathrm{Zn}, \mathrm{Zr}, \mathrm{In},(\mathrm{In}, \mathrm{Gd}),(\mathrm{In}, \mathrm{Nd}), \mathrm{Ti}, \mathrm{Cu}, \mathrm{Ag},(\mathrm{Cu}, \mathrm{Ag}), \mathrm{Ni},(\mathrm{Ce}, \mathrm{Gd}),\end{array}$ \\
\hline
\end{tabular}




\begin{tabular}{|c|c|c|}
\hline & & $\begin{array}{l}(\mathrm{Nd}, \mathrm{Gd}),(\mathrm{Nd}, \mathrm{Ho}),(\mathrm{Nd}, \mathrm{Tm}),(\mathrm{Ce}, \mathrm{Ca}),(\mathrm{Fe}, \mathrm{Mn}),(\mathrm{Nd}, \mathrm{Ho}),(\mathrm{Nd}, \mathrm{Dy}),(\mathrm{Nd}, \mathrm{Gd}),(\mathrm{Nd} \text {, } \\
\mathrm{Tm}),(\mathrm{Nd}, \mathrm{Ho}),(\mathrm{Nd}, \mathrm{Er})\end{array}$ \\
\hline $\mathrm{PbF}_{2}$ & SP & $\mathrm{Er}, \mathrm{Yb}$ \\
\hline $\mathrm{SiO}_{2}$ & sol-gel & $\begin{array}{l}\mathrm{Ce},(\mathrm{Ce}, \mathrm{Li}), \mathrm{Dy},(\mathrm{Dy}, \mathrm{Li}), \mathrm{Eu},(\mathrm{Eu}, \mathrm{Li}), \mathrm{Gd},(\mathrm{Gd}, \mathrm{Li}), \mathrm{Ho},(\mathrm{Ho}, \mathrm{Li}), \mathrm{Li}, \mathrm{Pr},(\mathrm{Pr}, \mathrm{Li}), \mathrm{Sm} \text {, } \\
(\mathrm{Sm}, \mathrm{Li}), \mathrm{Tb},(\mathrm{Tb}, \mathrm{Li})\end{array}$ \\
\hline $\mathrm{Sr}_{2} \mathrm{MgB}_{2} \mathrm{O}_{6}$ & SSR & Dy \\
\hline $\mathrm{Y}_{2} \mathrm{O}_{3}$ & SCS & $\begin{array}{l}\text { Undoped, } \mathrm{Ce}, \mathrm{Pr}, \mathrm{Nd}, \mathrm{Sm}, \mathrm{Eu}, \mathrm{Gd}, \mathrm{Tb}, \mathrm{Dy}, \mathrm{Ho}, \mathrm{Er}, \mathrm{Tm}, \mathrm{Yb},(\mathrm{Ce}, \mathrm{Dy}),(\mathrm{Ce}, \mathrm{Sm}) \text {, } \\
(\mathrm{Ce}, \mathrm{Tm}),(\mathrm{Eu}, \mathrm{Dy}),(\mathrm{Eu}, \mathrm{Sm}),(\mathrm{Eu}, \mathrm{Tm}), \mathrm{Gd}, \mathrm{Ho}, \mathrm{Nd}, \mathrm{Pr}, \mathrm{Sm}, \mathrm{Tb},(\mathrm{Tb}, \mathrm{Dy}),(\mathrm{Tb}, \mathrm{Eu}) \text {, } \\
(\mathrm{Tb}, \mathrm{Sm}),(\mathrm{Tb}, \mathrm{Tm}), \mathrm{Yb}\end{array}$ \\
\hline $\mathrm{Y}_{3} \mathrm{Al}_{5} \mathrm{O}_{12}$ & SCS & $\begin{array}{l}\mathrm{Ce}, \mathrm{Pr}, \mathrm{Nd}, \mathrm{Sm}, \mathrm{Eu}, \mathrm{Gd}, \mathrm{Tb}, \mathrm{Dy}, \mathrm{Ho}, \mathrm{Er}, \mathrm{Tm}, \mathrm{Yb},(\mathrm{Ce}, \mathrm{Sm}),(\mathrm{Ce}, \mathrm{Eu}),(\mathrm{Ce}, \mathrm{Yb}) \\
(\mathrm{Ce}, \mathrm{Eu}, \mathrm{Yb}),(\mathrm{Pr}, \mathrm{Eu}),(\mathrm{Pr}, \mathrm{Yb}),(\mathrm{Pr}, \mathrm{Eu}, \mathrm{Yb}),(\mathrm{Tb}, \mathrm{Eu}),(\mathrm{Tb}, \mathrm{Sm}),(\mathrm{Tb}, \mathrm{Yb}),(\mathrm{Tb}, \mathrm{Eu}, \mathrm{Yb})\end{array}$ \\
\hline $\mathrm{YAlO}_{3}$ & SCS & $\mathrm{Ce},(\mathrm{Ce}, \mathrm{Sm}),(\mathrm{Ce}, \mathrm{Tb}), \mathrm{Sm}, \mathrm{Tb}, \mathrm{Tm}$ \\
\hline $\mathrm{ZrO}_{2}$ & SCS & $\mathrm{Ce}, \mathrm{Pr}, \mathrm{Nd}, \mathrm{Sm}, \mathrm{Eu}, \mathrm{Gd}, \mathrm{Tb}, \mathrm{Dy}, \mathrm{Ho}, \mathrm{Er}, \mathrm{Tm}, \mathrm{Yb}$ \\
\hline
\end{tabular}

Table 2. New TL materials synthesized at OSU with good properties for temperature sensing.

\begin{tabular}{|c|c|c|c|c|}
\hline Label & Material & Reagents & Preparation Method & Annealing \\
\hline LBO & $\mathrm{Li}_{2} \mathrm{~B}_{4} \mathrm{O}_{7}: \mathrm{Cu}, \mathrm{Ag}$ & $\begin{array}{l}0.4919 \mathrm{~g} \mathrm{LiNO}_{3}(99 \%, \text { Alfa- } \\
\text { Aesar), } 0.8653 \mathrm{~g} \mathrm{H}_{3} \mathrm{BO}_{3}(99.5 \% \text {, } \\
\text { Alfa Aesar) and } 0.2919 \mathrm{~g} \\
\text { glycine ( } 99 \% \text {, Alfa Aesar), } \\
35 \text { mol DI water }\end{array}$ & $\begin{array}{l}\text { Solution Combustion } \\
\text { Synthesis }\end{array}$ & $875^{\circ} \mathrm{C}$ for $1 \mathrm{~h}$ \\
\hline MBO & $\mathrm{MgB}_{4} \mathrm{O}_{7}: \mathrm{Dy}, \mathrm{Li}$ & $\begin{array}{l}0.1612 \text { g MgO }(98 \% \text {, Alfa } \\
\text { Aesar), } 1.0186 \mathrm{~g} \mathrm{H}_{3} \mathrm{BO}_{3}(99.5 \% \text {, } \\
\text { Alfa Aesar), } 0.3988 \mathrm{~g} \text { urea } \\
(99 \% \text {, alfa Aesar) }\end{array}$ & $\begin{array}{l}\text { Solution Combustion } \\
\text { Synthesis }\end{array}$ & $\begin{array}{l}900^{\circ} \mathrm{C} \text { for } 2 \mathrm{~h} \\
\left(5^{\circ} \mathrm{C} / \mathrm{min},\right. \\
\text { cooled inside } \\
\text { oven) }\end{array}$ \\
\hline CSO & $\mathrm{CaSO}_{4}: \mathrm{Ce}, \mathrm{Tb}$ & $\begin{array}{l}2.00 \mathrm{~g} \mathrm{CaCO}_{3} ; 4 \mathrm{ml} \mathrm{H} \mathrm{SO}_{4} \\
0.0173 \mathrm{~g} \mathrm{Ce}\left(\mathrm{NO}_{3}\right)_{3} \cdot 6 \mathrm{H}_{2} \mathrm{O} \\
0.01379 \mathrm{~g} \mathrm{~Tb}\left(\mathrm{NO}_{3}\right)_{3} \cdot x \mathrm{H}_{2} \mathrm{O}\end{array}$ & $\begin{array}{l}\text { Ion exchange, dried at } 170^{\circ} \mathrm{C} \\
\text { for } 3 \mathrm{~h} \text { and } 350^{\circ} \mathrm{C} \text { for } 3 \mathrm{~h}\end{array}$ & $\begin{array}{l}700^{\circ} \mathrm{C} \text { for } 2 \mathrm{~h} \\
\left(5^{\circ} \mathrm{C} / \mathrm{min},\right. \\
\text { cooled inside } \\
\text { oven }) .\end{array}$ \\
\hline
\end{tabular}

\subsubsection{Solution combustion synthesis}

In the solution combustion synthesis method the material is produced by self-sustained combustion of a mixture of metal nitrate and fuel (e.g., urea, glycine) initiated at relatively low temperatures $\left(\sim 500{ }^{\circ} \mathrm{C}\right)$ in relatively short periods of time ( $\sim$ seconds). Fig. 4 shows an example of the reaction involved in the synthesis of $\mathrm{MgO}$ doped with a lanthanide, accompanied by photos of the actual reaction that takes place in the beaker. After synthesis, the samples are annealed in a furnace to remove organic residues and improve the crystal structure. 


$$
\mathrm{Mg}\left(\mathrm{NO}_{3}\right)_{2} \cdot 6 \mathrm{H}_{2} \mathrm{O}+\left(\mathrm{NH}_{2}\right)_{2} \mathrm{C}=\mathrm{O}+\operatorname{Ln}\left(\mathrm{NO}_{3}\right)_{3} \rightarrow \mathrm{MgO}: \mathrm{Ln}_{(\mathrm{s})}+\mathrm{NH}_{3}(\mathrm{~g})+\mathrm{CO}_{2(\mathrm{~g})}+\mathrm{H}_{2} \mathrm{O}(\mathrm{g})
$$
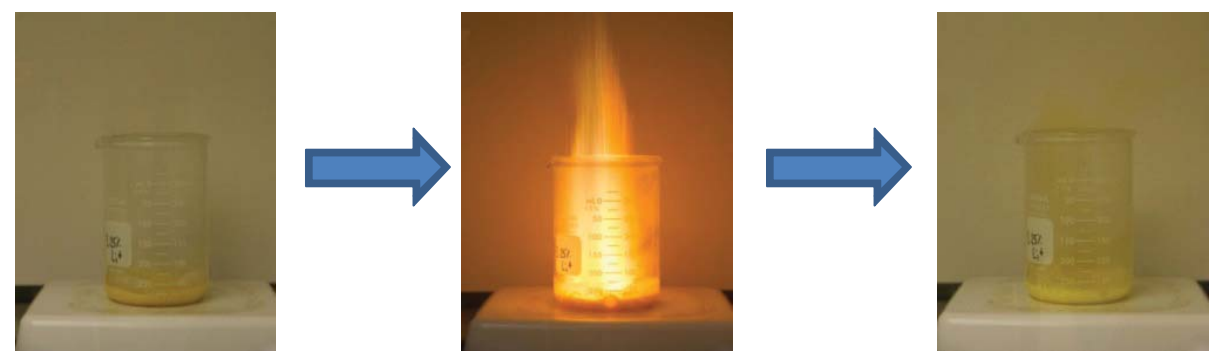

Fig. 4. Example of synthesis of MgO doped with a lanthanide (Ln) using the Solution Combustion Synthesis technique.

$\mathrm{Li}_{2} \mathrm{~B}_{4} \mathrm{O}_{7}: \mathrm{Cu}, \mathrm{Ag}(\mathrm{LBO})$ was synthesized by dissolving $\mathrm{LiNO}_{3}, \mathrm{H}_{3} \mathrm{BO}_{3}$, glycine and corresponding dopants in deionized water in a beaker and drying it on a hot plate at $300{ }^{\circ} \mathrm{C}$ for $30 \mathrm{~min}$. Once dried, the temperature was increased to $500{ }^{\circ} \mathrm{C}$ where the reaction took place, resulting in a slightly brown powder, which was crushed using agate mortar and pestle. The obtained material was then annealed in a furnace preheated to $875^{\circ} \mathrm{C}$ for $1 \mathrm{~h}$. After annealing, the samples were removed from the furnace and allowed to cool to room temperature outside the furnace. The sample was then crushed again using agate mortar and pestle.

$\mathrm{MgB}_{4} \mathrm{O}_{7}$ :Dy, Li (MBO) was synthesized by dissolving $\mathrm{Mg}\left(\mathrm{NO}_{3}\right)_{2} \cdot 6 \mathrm{H}_{2} \mathrm{O}, \mathrm{H}_{3} \mathrm{BO}_{3}$, urea and corresponding dopants in deionized water in a beaker and drying it on a hot plate at $300^{\circ} \mathrm{C}$ for $30 \mathrm{~min}$. Once dried, the temperature was increased to $500{ }^{\circ} \mathrm{C}$ until combustion took place. Once the reaction was exhausted, the remaining powder was allowed to cool to room temperature, collected, ground using an agate mortar and pestle, then annealed at $900{ }^{\circ} \mathrm{C}$ for $2 \mathrm{~h}$ (temperature ramped at $5{ }^{\circ} \mathrm{C} / \mathrm{min}$ from room temperature, cooled inside the oven).

\subsubsection{Precipitation methods}

$\mathrm{CaSO}_{4}$ with different dopants was prepared using the precipitation technique described by Lakshamanan [28].

$\mathrm{CaSO}_{4}: \mathrm{Ce}, \mathrm{Tb}(\mathrm{CSO})$ was prepared mixing $\mathrm{CaCO}_{3}$ and suitable amount of dopant in $50 \mathrm{ml}$ of deionized water in a $400 \mathrm{ml}$ beaker. In a $150 \mathrm{ml}$ beaker, the sulfuric acid was diluted in $30 \mathrm{ml}$ of deionized water. The mixture containing $\mathrm{CaCO}_{3}$ and dopant was placed on a hot plate at room temperature under vigorous stirring for $2 \mathrm{~min}$. The solution of sulfuric acid was poured in a separatory funnel place above the beaker. The valve was adjusted to allow the acid solution to drop into the $\mathrm{CaCO}_{3}$ solution slowly. The magnetic stirrer was kept while the solution of sulfuric acid was dropped. The product was dried in a furnace at $170{ }^{\circ} \mathrm{C}$ for $3 \mathrm{~h}$. After water evaporation, the hot plate temperature was raised to $350{ }^{\circ} \mathrm{C}$ for the evaporation of unreacted sulfuric acid and kept in this temperature for $3 \mathrm{~h}$. Sample was annealed in alumina crucible at $700{ }^{\circ} \mathrm{C}$ for $2 \mathrm{~h}$ (temperature ramped at $5^{\circ} \mathrm{C} / \mathrm{min}$ from room temperature, cooled inside oven). The powder obtained was crushed again in agate mortar.

CSO:Ce, $\mathrm{Pr}$ were also synthesized through the following procedure: $4.003 \mathrm{~g}$ of $\mathrm{CaCO}_{3}, 2 \mathrm{ml}$ of $\mathrm{Ce}\left(\mathrm{NO}_{3}\right)_{3} \cdot 6 \mathrm{H}_{2} \mathrm{O}(0.04 \mathrm{M})$ and $2 \mathrm{ml}$ of $\operatorname{Pr}\left(\mathrm{NO}_{3}\right)_{3} \cdot \mathrm{XH}_{2} \mathrm{O}(0.04 \mathrm{M})$ were mixed with $30 \mathrm{ml}$ of deionized water. The solution was stirred at room temperature for $20 \mathrm{~min}$. In a separate beaker containing $52 \mathrm{ml}$ of water, $8 \mathrm{ml}$ of sulfuric acid was added with a pipet slowly. The diluted sulfuric acid was dropped slowly into the $\mathrm{CaCO}_{3}$ solution using a separatory funnel. After the separatory funnel was empty, the solution was heated on hot plate at $170{ }^{\circ} \mathrm{C}$ for $30 \mathrm{~min}$. After the solution was cooled to room temperature, it 
was poured into several test tubes. Test tubes were settled for $4 \mathrm{~h}$ to let the precipitates separate from the liquid. The liquid was pipetted off from the top of precipitates. Test tubes were rinsed off with deionized water into the beaker. The solution was then heated at $200{ }^{\circ} \mathrm{C}$ until it completely dried. The product was annealed in box furnace at $700{ }^{\circ} \mathrm{C}$ for $2 \mathrm{~h}$. Each batch yielded about $5 \mathrm{~g}$ of CSO. The total amount of material for test is about $20 \mathrm{~g}$. Reproducibility, dark fading and light sensitivity were tested on all batches.

\subsubsection{Solution precipitation of core/shell nanoparticles}

The solution precipitation method was used to the preparation of core-shell nanoparticles $[22,29]$. Metal nitrate solutions are used as precursors and heated under continuous stirring to induce precipitation of metal hydroxide nanoparticles. Further calcination yields the production of oxides. As a result, a variety of materials can be easily produced at a low cost and without the need for high temperature furnaces. This method can be used to synthesize core-shell nanoparticles with unique properties such as tunable energy transfer between lanthanides in different nanoparticle shells [22]. It can also be important if the concept of core-shell nanoparticle is used to alter the heat transfer mechanism in nanophosphors, as originally proposed for distinguishing between different heat conduction mechanisms present in an ADT.

The solution precipitation method was carried out as follows:

- $\quad \mathrm{A} \mathrm{NH}_{4} \mathrm{~F}$ solution in ethanol/water at $75^{\circ} \mathrm{C}$ with ADDP (ammonium di-noctadecyldithiophosphate) ligand is prepared;

- $\quad \mathrm{A} \mathrm{NH}_{4} \mathrm{~F}$ and host and dopant metal nitrates solution is prepared in water;

- These solutions are combined drop-wise and well stirred to form the core;

- Additional $\mathrm{NH}_{4} \mathrm{~F}$ and host metal nitrate solution is added and stirred to form a shell;

- This step is repeated to add more shells;

- Per design, only the core is RE-doped;

- Centrifuging, washing, and drying are carried out to extract NPs in the form of powders.

This process is illustrated in Fig. 5. 

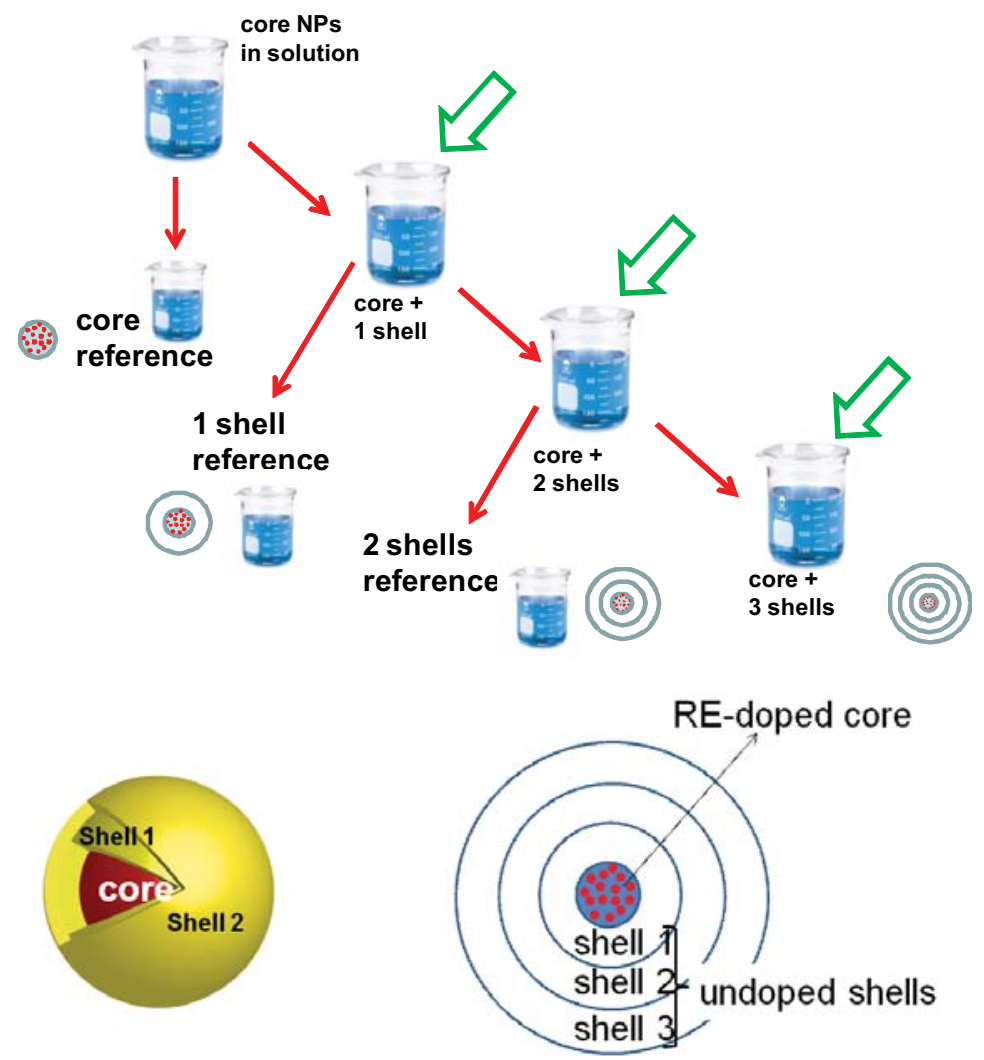

Fig. 5. Scheme for the production of core-shell nanoparticles at CU.

Clemson University synthesized a variety of materials with different dopants, as indicated in Table 3. Strong emphasis was placed in fluorides, because of the excellent properties of LiF and because this complements the synthesis capabilities from OSU. These materials were doped and co-doped with rare earths combined with structural characterization ( $x$-ray diffraction and electron microscopy), as well as luminescence and TL measurements.

Table 3. Samples synthesized at Clemson University by solution precipitation.

\begin{tabular}{|c|c|}
\hline Host & Samples \\
\hline $\mathrm{CaF}_{2}$ & $\begin{array}{l}\mathrm{CaF} 2 \mathrm{Eu}(\mathrm{KBS}-19-53) \\
\mathrm{CaF}_{2}: \mathrm{Eu}(\mathrm{KBS}-19-48) \text { core / up to } 3 \text { shells }\end{array}$ \\
\hline $\mathrm{BaF}_{2}$ & BaF2:Ce (KBS-19-39) \\
\hline $\mathrm{PbF}_{2}$ & $\mathrm{PbF} 2: \mathrm{Er}, \mathrm{Yb}$ \\
\hline $\mathrm{LaF}_{3}$ & $\begin{array}{l}\text { LaF3:Eu (KBS-19-50) } \\
\text { LaF3:Ce (KBS-19-51) various shells }\end{array}$ \\
\hline $\mathrm{Gd}_{2} \mathrm{O}_{3}$ & $\begin{array}{l}\text { Gd2O3:Eu (SR-22-65) } 1000^{\circ} \mathrm{C} \\
\text { Gd2O3:Eu (SR-22-65) } 1700^{\circ} \mathrm{C}\end{array}$ \\
\hline $\mathbf{Y F}_{3}$ & Undoped, $\mathrm{YF}_{3}: \mathrm{Eu}, \mathrm{YF}_{3}: \mathrm{Ce}, \mathrm{YF}_{3}: \mathrm{Tb} \mathrm{YF}_{3}: \mathrm{Ce}, \mathrm{Tm}, \mathrm{YF} \mathrm{F}_{3}: \mathrm{Pr}, \mathrm{Tm}, \mathrm{YF}_{3}: \mathrm{Tb}, \mathrm{Sm}, \mathrm{YF}$ : $: \mathrm{Pr}, \mathrm{Sm}$ \\
\hline $\mathrm{MgF}_{2}$ & Undoped, $\mathrm{MgF}_{2}: \mathrm{Tb}, \mathrm{MgF}_{2}: \mathrm{Eu}, \mathrm{MgF}_{2}: \mathrm{Ce}$ \\
\hline
\end{tabular}


The main preparation steps are exemplified below for $\mathrm{MgF}_{2}$ and $\mathrm{YF}_{3}$. Other samples did not result in significant TL intensities.

$\mathrm{MgF}_{2}$ : A solution of ammonium fluoride was created by dissolving $0.184 \mathrm{~mol}$ of $\mathrm{NH}_{4} \mathrm{~F}$ in $400 \mathrm{ml}$ of methanol. The solution was stirred at $250 \mathrm{rpm}$ until completely dissolved. A second solution was created containing magnesium chloride and, if applicable, rare earth ions. For the undoped control sample $26.6 \mathrm{mmol}$ of $\mathrm{MgCl}_{2} \cdot 6 \mathrm{H}_{2} \mathrm{O}$ was dissolved in $80 \mathrm{ml}$ of methanol. For the rare earth-doped samples $26.3 \mathrm{mmol}$ of $\mathrm{MgCl}_{2} \cdot 6 \mathrm{H}_{2} \mathrm{O}$ and $0.266 \mathrm{mmol}$ of a $\mathrm{RE}\left(\mathrm{NO}_{3}\right)_{3} \cdot 6 \mathrm{H}_{2} \mathrm{O}$, where RE is either $\mathrm{Tb}$, Eu, or $\mathrm{Ce}$, was dissolved in $80 \mathrm{ml}$ of methanol. The solution was stirred at $250 \mathrm{rpm}$ until completely dissolved. The magnesium chloride solution was slowly added to the ammonium fluoride solution, the solution was stirred at $250 \mathrm{rpm}$ for $1.5 \mathrm{~h}$, and then centrifuged at $3000 \mathrm{rpm}$ for $6 \mathrm{~min}$ to recover the nanoparticles. The supernatant was replaced with fresh methanol, redispersed, and centrifuged again. After the supernatant was removed again, the nanoparticles were placed in a vacuum chamber to dry and, once dried, they were ball milled. XRD measurements showed the precipitate did not correspond to $\mathrm{MgF}_{2}$ and calcination in air at $500{ }^{\circ} \mathrm{C}$ for one hour was used to convert the precipitate into $\mathrm{MgF}_{2}$. Fig. 6 summarizes the XRD results, whereas Fig. 7 illustrates the $\mathrm{MgF}_{2}$ nanoparticles obtained this way.

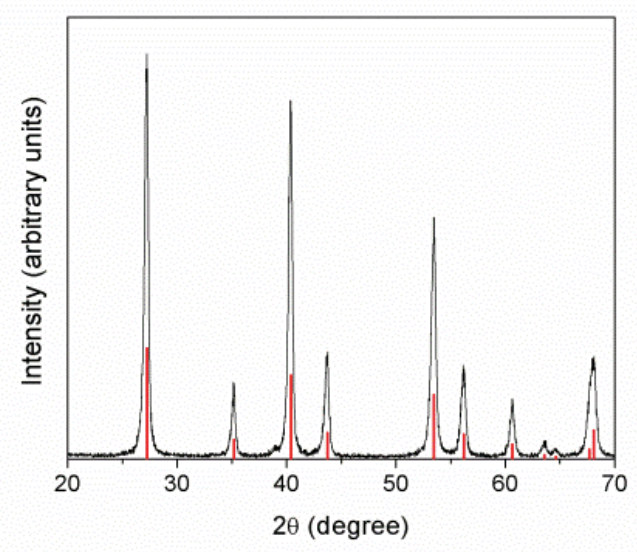

Fig. 6. X-ray diffraction (XRD) data from calcined samples, with $\mathrm{MgF}_{2}$ powder diffraction file (PDF) $41-1443$ represented as vertical lines, confirming the formation of crystalline phase-pure tetragonal $\mathrm{MgF}_{2}$.

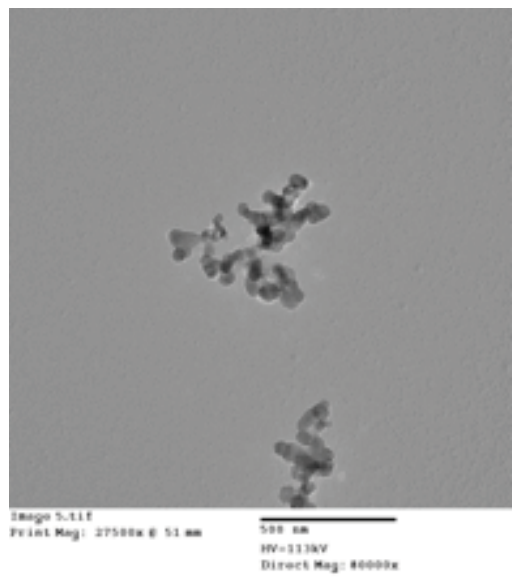

Fig. 7. Transmission electron microscopy (TEM) image of $\mathrm{MgF}_{2}$ nanoparticles after calcination. 
$\mathrm{YF}_{3}$ : For the formation of $\mathrm{YF}_{3}, \mathrm{Y}\left(\mathrm{NO}_{3}\right)_{3} \cdot 6 \mathrm{H}_{2} \mathrm{O}$ and the respective dopant precursor (a rare earth nitrate hydrate) were dissolved in methanol. A separate solution of $\mathrm{NH}_{4} \mathrm{~F}$ in methanol was added to the nitrate solution, followed by $1.5 \mathrm{~h}$ of stirring at about $400 \mathrm{rpm}$. The final solution was cleaned by centrifuging at $3000 \mathrm{rpm}$ several times, $6 \mathrm{~min}$ at a time. After each cycle, methanol was added to the tubes and the precipitates were forced back into solution using a Vortex spinner. The resulting material was allowed to dry under vacuum for at least $48 \mathrm{~h}$. Ball milling using two milling balls and the Vortex spinner for about $90 \mathrm{~s}$ was used to bring the dried material into powder. As in the case of $\mathrm{MgF}_{2}$, calcination was used to transform the as-precipitated material into orthorhombic $\mathrm{YF}_{3}$. This transformation is demonstrated by XRD measurements shown in Fig. 8, whereas Fig. 9 presents a typical TEM image of the nanoparticles after calcination.

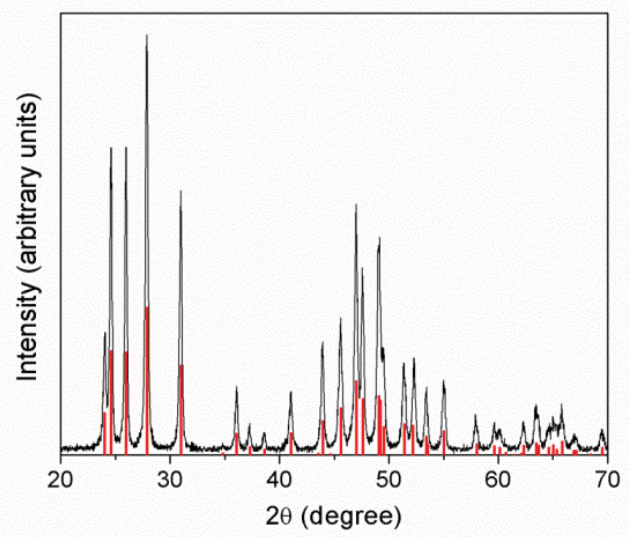

Fig. 8. XRD data of calcined samples, with $\mathrm{YF}_{3}$ powder diffraction file (PDF) 32-1431 represented as vertical lines, confirming the formation of crystalline phase-pure orthorhombic $\mathrm{YF}_{3}$.

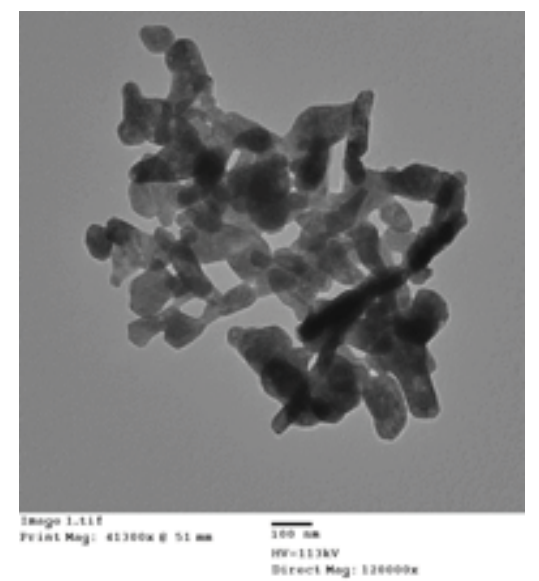

Fig. 9. Transmission electron microscopy image of $\mathrm{YF}_{3}$ nanoparticles after calcination.

\subsubsection{Sol-gel method}

In the alkoxide-based sol-gel method and nitrate-modified alkoxide-based sol-gel method the precursors are silicon alkoxide and metal nitrates, respectively. The silicon alkoxide (e.g. tetraethyl orthosilicate - TEOS) is reactive toward nucleophilic reagents such as water [30]. The hydrolysis reaction occurs with the formation of $\mathrm{Si}-\mathrm{OH}$ hydroxo groups, which readily undergo condensation with the formation of Si-O-Si bridges. The condensation product has the aspect of a gel. The gelation occurs also 
with the incorporation of the metal nitrates when they are present in the initial solution either as a dopant or major component. The final product is obtained at further heating, during which the reaction between individual components takes place as a solid-state reaction.

\subsubsection{Thin film deposition}

We developed a thin film material, $\mathrm{Y}_{2} \mathrm{O}_{3}$ :Tb that could be easily deposited using standard microfabrication techniques without any specialized crystal growth methods. This was part of our preparations for pressure testing, but since this material was so successful, we wanted to more fully characterize the behavior of $\mathrm{Y}_{2} \mathrm{O}_{3}: \mathrm{Tb}$ and perform other materials studies to see if other dopants of $\mathrm{Y}_{2} \mathrm{O}_{3}$ and some other common microfabrication materials would luminesce as strongly.

In examining other dopants of yttrium oxide, we started with lanthanides because of earlier studies on crystalline materials performed by Dorenbos [31], who was able to derive the trap levels of all the lanthanides in the band gap of yttrium aluminum garnet (YAG) by only knowing the trap levels of a few lanthanides. Using the data from the $\mathrm{Y}_{2} \mathrm{O}_{3}$ : Tb we tried to pick out lanthanides that had deeper trap levels. The relative position of the trap levels of lanthanides was not heavily dependent on the host material, so we decided to continue to primarily use $\mathrm{Y}_{2} \mathrm{O}_{3}$ as a host, particularly since prior work in other groups used YAG host material, which cannot be reproduced well using standard microfabrication deposition. However, we also attempted to deposit a few other types of thin films. Unfortunately, most materials showed little luminescence at low temperatures. Exceptions were $\mathrm{ZrO}_{2}: \mathrm{Tb}$ and any codopants used with $\mathrm{Y}_{2} \mathrm{O}_{3}: \mathrm{Tb}$ (although Ce used as a co-dopant seemed to degrade the luminescence). An overview of the lanthanides we tried in $\mathrm{Y}_{2} \mathrm{O}_{3}$ is listed in Table 4.

The main features about the $\mathrm{Y}_{2} \mathrm{O}_{3}$ :Tb that we found to be important were its large bandgap and its ease of deposition in polycrystalline form. The films were all deposited by electron beam evaporation except for $\mathrm{CaF}_{2}$ :Dy, which was deposited by thermal evaporation. Thermal evaporation was used because the pellet sources are more difficult to get a constant low deposition rate in the e-beam evaporator. All sources were in a powder form then compressed into pellets. For $\mathrm{Y}_{2} \mathrm{O}_{3}: \mathrm{Tb}$ and all the other lanthanides listed in Table 4 except for $\mathrm{Dyl}_{3}$ and $\mathrm{DyF}_{3}$ the oxide form of the lanthanide was used in a powder mixture with the host material. For example $\mathrm{Tb}_{4} \mathrm{O}_{7}$ powder was mixed with $\mathrm{Y}_{2} \mathrm{O}_{3}$ powder. Terbium oxide is known to decompose when evaporated. That is why we chose $\mathrm{Dyl}_{3}$ and $\mathrm{DyF}_{3}$. Both these substances are known to decompose when evaporated. We were hoping for the Dy to disassociate from the molecule during deposition and then become active in the host material after annealing. All of the before mentioned materials were annealed at $1100{ }^{\circ} \mathrm{C}$ and $900{ }^{\circ} \mathrm{C}$ for $30 \mathrm{~s}$ and $120 \mathrm{~s}$ respectively before the TL curves were measured. This was done to increase the crystallinity of the films and activate the dopants.

Table 4. Summary of attempted TL materials by thin film deposition.

\begin{tabular}{|c|c|c|c|c|}
\hline Material & Crystallinity & $\begin{array}{l}\mathrm{TL} \\
\text { response }\end{array}$ & $\begin{array}{l}\text { Peak } \\
\text { location(s) }\end{array}$ & $\begin{array}{l}\text { UV } \\
\text { sensitive }\end{array}$ \\
\hline $\mathrm{Y}_{2} \mathrm{O}_{3}:(\mathrm{Tb}) ;(\mathrm{Tb}, \mathrm{Er}) ;(\mathrm{Tb}, \mathrm{Yb}) ;(\mathrm{Tb}, \mathrm{Eu})$ & polycrystalline & strong & $130^{\circ} \mathrm{C}, 250^{\circ} \mathrm{C}$ & yes \\
\hline $\mathrm{Y}_{2} \mathrm{O}_{3}: \mathrm{Tb}, \mathrm{Ce}$ & polycrystalline & weak & $130^{\circ} \mathrm{C}$ & yes \\
\hline $\begin{array}{l}\mathrm{Y}_{2} \mathrm{O}_{3}:(\mathrm{Ce}) ;(\mathrm{Ce}, \mathrm{Yb}) ;(\mathrm{Ce}, \mathrm{Er}) ;(\mathrm{Pr}) ; \\
\left.(\mathrm{Nd}) ;(\mathrm{Dy}) ;(\mathrm{Er}) ;(\mathrm{Dyl})_{3}\right) ;\left(\mathrm{DyF}_{3}\right)\end{array}$ & polycrystalline & none & $\mathrm{n} / \mathrm{a}$ & $\mathrm{n} / \mathrm{a}$ \\
\hline $\mathrm{ZrO}_{2}$ & polycrystalline & none & $\mathrm{n} / \mathrm{a}$ & $\mathrm{n} / \mathrm{a}$ \\
\hline $\mathrm{ZrO}_{2}: \mathrm{Tb}$ & polycrystalline & weak & $110^{\circ} \mathrm{C}$ & yes \\
\hline
\end{tabular}




\begin{tabular}{|lllll|}
\hline $\mathrm{CeO}_{2}:() ;(\mathrm{Er}) ;(\mathrm{Tb})$ & polycrystalline & none & $\mathrm{n} / \mathrm{a}$ & $\mathrm{n} / \mathrm{a}$ \\
\hline $\mathrm{MgO}^{\text {ame);(Tb) }}$ & $\begin{array}{l}\text { amorphous/ } \\
\text { polycrystalline }\end{array}$ & none & $\mathrm{n} / \mathrm{a}$ & $\mathrm{n} / \mathrm{a}$ \\
\hline $\mathrm{CaF}_{2}:$ Dy (TLD-200) & $\begin{array}{l}\text { amorphous/ } \\
\text { polycrystalline }\end{array}$ & none & $\mathrm{n} / \mathrm{a}$ & $\mathrm{n} / \mathrm{a}$ \\
\hline LiF:Mg,Ti (TLD-100) & amorphous & none & $\mathrm{n} / \mathrm{a}$ & $\mathrm{n} / \mathrm{a}$ \\
\hline $\mathrm{CaO}$ & amorphous & none & $\mathrm{n} / \mathrm{a}$ & $\mathrm{n} / \mathrm{a}$ \\
\hline YAG:Ce & amorphous & none & $\mathrm{n} / \mathrm{a}$ & $\mathrm{n} / \mathrm{a}$ \\
\hline
\end{tabular}

\subsection{Material characterization techniques}

\subsubsection{X-ray diffraction}

The crystal structure from the samples synthesized was characterized using a Bruker Advance D8 diffractometer (model D8-25-ADVANCE, Bruker AXS GmbH) with CuKa radiation filter by a $0.02 \mathrm{~mm} \mathrm{Ni}$ foil to suppress the $\mathrm{Kb}$ line (scanning $2 \theta$ in 0.02 degrees step size and $0.5 \mathrm{~s}$ step time).

\subsubsection{Scanning Electron Microscopy}

The morphology of samples was determined by using FEI Quanta 600 field-emission Scanning Electron Microscope (SEM) with magnification around 5000, acceleration voltage of $20 \mathrm{kV}$. The SEM samples were prepared by suspending the particles in ethanol by ultrasonification and drying a drop of the suspension on an aluminum stub and then coating with gold palladium.

\subsubsection{Radioluminescence}

$\mathrm{RL}$ measurements were carried out exciting the sample with X-rays (40 kV Magnum ${ }^{\mathrm{TM}} \mathrm{X}$-ray tube, Ag transmission target, Moxtek Inc.), delivering a dose rate of approximately $150 \mathrm{mGy} / \mathrm{s}$. The RL emission from the sample was collected using a f/2 fused silica lens coupled to an optical fiber ( $1 \mathrm{~mm}$ core diameter, transmission between $200-1100 \mathrm{~nm}$ ), connected to an Ocean Optics USB-2000 fiber spectrometer (Ocean Optics, Inc., Dunedin, FL, USA). The spectrometer has an order-sorting detection filter to eliminate the second order interference light and a resolution of $\sim 7.2 \mathrm{~nm}$. The RL spectra were measured using approximately $10 \mathrm{mg}$ of powder placed in stainless steel cups. The spectra were not corrected for the response of the entire system, but the relative sensitivity of the instrument can be found in Ref. [32]

The system described above was automated to characterize the radioluminescence (RL) properties from the nanophosphors. The system is based on a sample handler system constructed using a rotary step-motor (Zaber Technologies Inc.), as shown in Fig. 10. The system has three optical ports on which other instruments can be mounted. This instrument allows the automated characterization of the RL from 24 samples with minimum user input. The other two optical ports can be used for other types of luminescence characterization (e.g., optically stimulated luminescence or photoluminescence). 


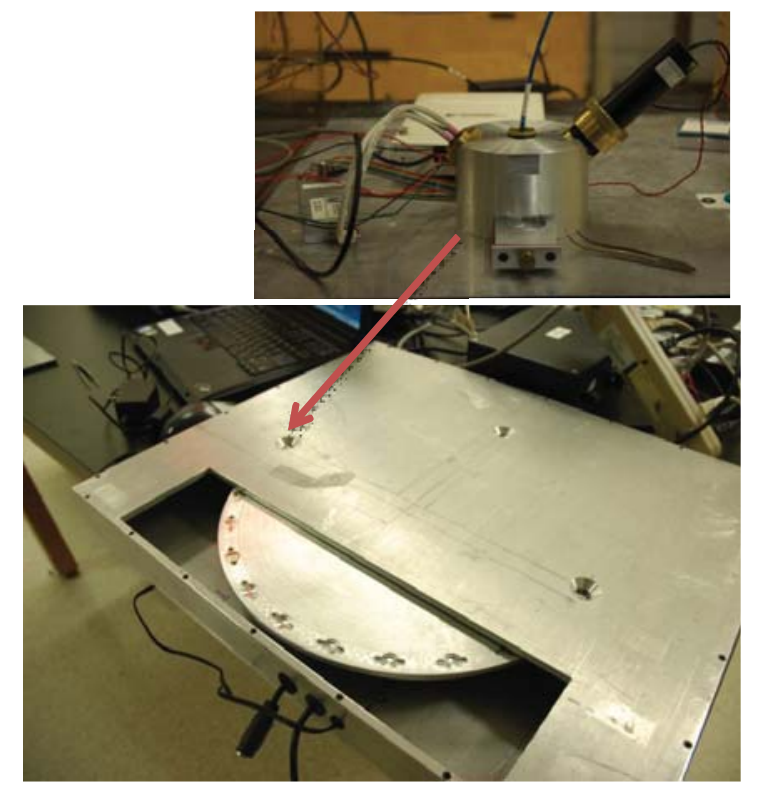

Fig. 10. Characterization apparatus constructed for the characterization of nanophosphors.

\subsubsection{Thermoluminescence}

TL measurements were carried out using a Ris $\varnothing$ TL/OSL-DA-15 reader under $\mathrm{N}_{2}$ atmosphere using a heating rate of $5 \mathrm{~K} / \mathrm{s}$. The TL was detected using a photomultiplier tube (PMT) (model 9235QB, Electron Tubes Inc.). Aliquots of $10 \mathrm{mg}$ of sample in stainless steel cups were used for the readouts. The samples were irradiated with beta rays from a ${ }^{90} \mathrm{Sr} /{ }^{90} \mathrm{Y}$ source. Different optical filters were used in front of the PMT to select either visible emission (Schott BG-39 glass filters, transmission between 330 and $620 \mathrm{~nm}$, $6 \mathrm{~mm}$ total thickness, Schott AG, Mainz, Germany) or UV emission (Hoya U-340 filters, transmission between $290-390 \mathrm{~nm}, 7.5 \mathrm{~mm}$ total thickness, Hoya Corporation), depending on the material.

TL emission spectra were measured replacing the PMT with the optical fiber system described for the $\mathrm{RL}$ system. The data reported in this work are the average of multiple spectra obtained around the main TL peak. Again, the spectra were not corrected for the relative detection efficiency of the entire optical system.

\subsubsection{Irradiations}

A variety of radiation sources were used. At OSU we used either ${ }^{90} \mathrm{Sr} /{ }^{90} \mathrm{Y}$ beta source delivering a dose rate of $\sim 100 \mathrm{mGy} / \mathrm{s}$ or X-rays from a $40 \mathrm{kV}$ X-ray tube (40 kV Magnum ${ }^{\mathrm{TM}} \mathrm{X}$-ray tube, $\mathrm{Ag}$ transmission target, Moxtek Inc.). For irradiation of the materials prior to detonation tests we ${ }^{137} \mathrm{Cs}$ gamma source with higher dose rate, including a Gammacell ${ }^{\circledR} 1000$ Elite.

At the University of Minnesota, we use two X-ray sources: an X-RAD 320 operated at $320 \mathrm{kV}$ and $12.5 \mathrm{~mA}$ with a $2 \mathrm{~mm}$ Al filter, and an RS 2000 operated at $160 \mathrm{kV}$ at $25 \mathrm{~mA}$ with $0.3 \mathrm{~mm}$ copper filter. The gamma source was a JLS Mark $\mathrm{I}-25$ hot cell using a ${ }^{137} \mathrm{Cs}$ isotope as a source.

\subsubsection{Pressure tests}

Ideal methods for measuring temperature and pressure would completely separate their effects, that is, the temperature testing would induce no extraneous pressures, and the pressure testing would involve no elevated temperatures. From the beginning of the program, our temperature testing has 
used microheaters, which apply no pressure at all during heating, and we designed our pressure testing actuators with the same philosophy.

The current design is a micromechanical electrostatic membrane actuator (see Fig. 11 and Fig. 12). In function, the actuator has two electrodes, one of which has a TL film deposited on it. As voltage is applied across the actuator electrodes, the electrode with the TL film deflects, which applies a biaxial stress to the film. The biaxial stress is identical to a biaxial pressure (both are measured in $\mathrm{kPa}, \mathrm{MPa}$, or GPa). We do not expect major differences in effects caused by biaxially applied or uniform threedimensional pressures (except for the geometric Poisson factors) because the films are polycrystalline and should have no overall preference for crystal orientation. Note also that the actuation can be applied on millisecond time scales (dictated by the mechanical time constant of the membrane), making this a good simulation of explosive pressures.

The devices have a large enough area that the luminescence is easily measureable. They have a voltage-controlled adjustable electrode spacing and thus a variable maximum applied force, and they contain structural materials that do not tend to display $\mathrm{TL}$, to avoid confounding background signals when the entire device is heated on a hotplate.

To meet the size and adjustability requirements, the actuator device is fabricated in two pieces, dubbed the "top" and "bottom" dies. The top die comprises the flexible membrane, made of a lowstress silicon nitride applied to a bare silicon wafer using low-pressure chemical vapor deposition (LPCVD) and etch released from the silicon by potassium hydroxide. This nitride is electrically insulating, provides a stalwart barrier against diffusion, is mechanically robust even at thicknesses of $250 \mathrm{~nm}$ or less, and can withstand the high-temperature anneals needed to re-crystallize and "activate" many deposited TL thin films. The side of the membrane covering its silicon frame receives a $50 \mathrm{~nm}$ metal film, usually gold with a thin chrome adhesion layer, to form the actuator's top electrode. The other side, which will face upward in use, hosts the TL film to be studied.

The other electrode is hosted by the bottom die. A Bosch polymer-assisted plasma etch is used on a patterned silicon wafer to define a grid of square pillars, with heights adjustable from several tens to hundreds of $\mu \mathrm{m}$, spaced such that four will support a membrane die placed between them with the pillars contacting only the membrane's silicon frame at the center each side and with half of the pillar top left uncovered. Silicon oxide is then thermally grown on the entire wafer as an electrical insulation layer. Finally, a thick (300 $\mathrm{nm}$ ) chrome/gold layer is deposited as the electrical layer, with the pillars isolated from the surrounding areas by metal-free "moats" defined by lift-off resist. When the metallized surface of the top membrane die is placed in contact with the base pillars, the pillar tops conduct electrically to the membrane metal, allowing it to be energized without having to directly probe or wirebond the downward-facing top electrode; the bottom electrode is one continuous piece and can be connected to at any convenient point. 


\section{TL active layer \\ LPCVD silicon nitride released membrane \\ evaporated $\mathrm{Cr}+\mathrm{Au}$ electrode}

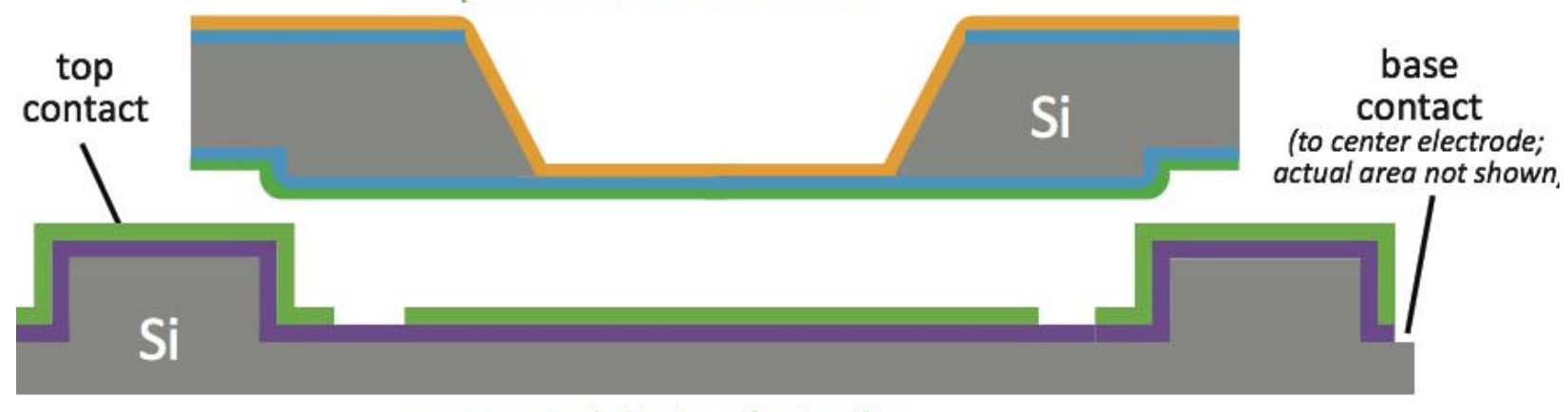

evaporated $\mathrm{Cr}+\mathrm{Au}$ electrode

\section{$\mathrm{SiO}_{2}$ electrical isolation}

Fig. 11. A side-view schematic (not to scale) of the membrane actuator and its materials. The indentations at the edges of the top membrane die are receptors for the bottom die's support pillars, which are Bosch etched into the silicon before nitride deposition; these receptors are meant to provide mechanical stability and further adjustability to the electrode spacing height, but as performance has been found to be satisfactory without them, they are now considered optional and often omitted to save fabrication time and cost.

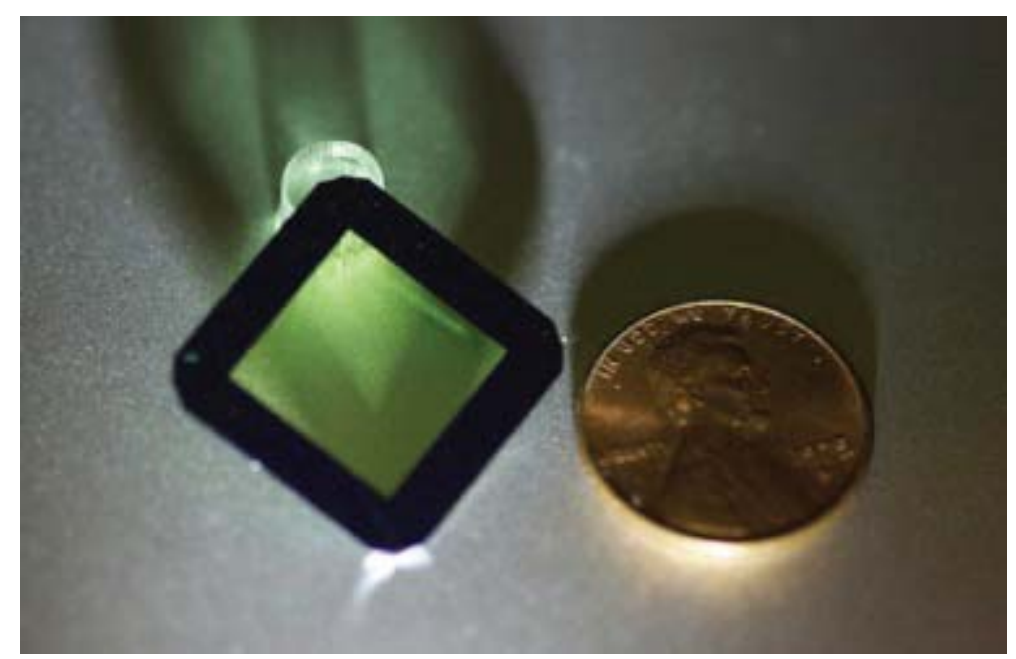

Fig. 12. A silicon nitride membrane die shares a portrait with a penny. This example is $\mathbf{1 2 . 5} \mathbf{~ m m}$ on a side. For actuator use, the side facing up in this photo would receive a conductive metal coating - usually gold, with a chrome adhesion layer - and the TL film of interest would be deposited on the reverse; neither has been done for this photo.

A Zygo Mark GPI XPS laser interferometer is used to profile the membrane surface during actuation. With electrode heights in the $300 \mu \mathrm{m}$ range, we have achieved maximum deflections of nearly a micron at $380 \mathrm{~V}$, from which we calculate a maximum stress of over $100 \mathrm{MPa}$. Note that this is not the average stress, which is much smaller, but merely the stress at the edges where the membrane bending strain is at a maximum. This is towards the high end of achievable electrode spacings with this process architecture, so we expect to rapidly reach much greater deflection and stresses - since the electrostatic force will scale with the inverse square of distance - as we complete the fabrication of devices with smaller spacings. 


\subsection{Detonation tests}

\subsubsection{Facilities}

Test of the TL particles were performed at different facilities, including closed-chamber detonations at the Naval Surface Warfare Center (NSWC) in collaboration with Dr. James Lightstone and his team. In August 2014 and 2015, Merlin Mah and Joseph Talghader traveled to Kirtland AFB to work with the DTRA Albuquerque (including Howard Ross, Kiran Shah, and Carl Denton) and several ARA personnel to test three types of microparticles in the Humble Gingko test chamber. Shock-tube experiments were also performed at the University of Illinois Urbana-Champaign (UIUC) in collaboration with Dr. Nick Glumac. Table 5 provides an overview of the tests performed and materials tested.

Table 5. Detonation and shock-tube tests performed by OSU and UMN.

\begin{tabular}{|c|c|c|}
\hline Test & Materials investigated & General conditions \\
\hline NSWC (2011) & LiF:Mg,Ti & Closed chamber \\
\hline NSWC (Mar 2012) & $\begin{array}{l}\mathrm{Li}_{2} \mathrm{~B}_{4} \mathrm{O}_{7}: \mathrm{Cu}, \mathrm{Ag}, \\
\mathrm{MgB}_{4} \mathrm{O}_{7}: \mathrm{Dy}, \mathrm{Li}, \mathrm{LiF}: \mathrm{Mg}, \mathrm{Ti}, \\
\mathrm{CaF}_{2}: \mathrm{Dy}\end{array}$ & Closed chamber \\
\hline NSWC (Sep 2012) & $\begin{array}{l}\mathrm{Li}_{2} \mathrm{~B}_{4} \mathrm{O}_{7}: \mathrm{Cu}, \mathrm{Ag}, \\
\mathrm{MgB}_{4} \mathrm{O}_{7}: \mathrm{Dy}, \mathrm{Li}, \\
\mathrm{CaSO}_{4}: \mathrm{Ce}, \mathrm{Tb}, \mathrm{LiF}: \mathrm{Mg}, \mathrm{Ti},\end{array}$ & $\begin{array}{l}\text { Closed chamber, plastic bonded explosive (PBX) asnd } \\
\text { aluminized plastic bonded explosive (A1PBX), } 10 \text { shots }\end{array}$ \\
\hline NSWC (2013) & $\begin{array}{l}\mathrm{Li}_{2} \mathrm{~B}_{4} \mathrm{O}_{7}: \mathrm{Cu}, \mathrm{Ag}, \\
\mathrm{MgB}_{4} \mathrm{O}_{7}: \mathrm{Dy}, \mathrm{Li}, \\
\mathrm{CaSO}_{4}: \mathrm{Ce}, \mathrm{Tb}, \mathrm{LiF}: \mathrm{Mg}, \mathrm{Ti},\end{array}$ & Closed chamber, HPBX and LPBX \\
\hline $\begin{array}{l}\text { DTRA Humble } \\
\text { Gingko XIX (2014) }\end{array}$ & $\begin{array}{l}\mathrm{MgB}_{4} \mathrm{O}_{7}: \mathrm{Dy}, \mathrm{Li} \\
\mathrm{CaSO}_{4}: \mathrm{Ce}, \mathrm{Tb}\end{array}$ & Open chamber \\
\hline $\begin{array}{l}\text { DTRA Humble } \\
\text { Gingko XIX (2015) }\end{array}$ & $\mathrm{CaSO}_{4}: \mathrm{Ce}, \mathrm{Pr}$ & Open chamber, 1 shot \\
\hline UIUC (2013) & $\begin{array}{l}\mathrm{Li}_{2} \mathrm{~B}_{4} \mathrm{O}_{7}: \mathrm{Cu}, \mathrm{Ag} \\
\mathrm{MgB}_{4} \mathrm{O}_{7}: \mathrm{Dy}, \mathrm{Li} \\
\mathrm{CaSO}_{4}: \mathrm{Ce}, \mathrm{Tb}\end{array}$ & Shock-tube \\
\hline UIUC (2014) & $\begin{array}{l}\mathrm{Li}_{2} \mathrm{~B}_{4} \mathrm{O}_{7}: \mathrm{Cu}, \mathrm{Ag} \\
\mathrm{MgB}_{4} \mathrm{O}_{7}: \mathrm{Dy}, \mathrm{Li} \\
\mathrm{CaSO}_{4}: \mathrm{Ce}, \mathrm{Tb}\end{array}$ & Shock-tube \\
\hline
\end{tabular}

\subsubsection{Sample preparation}

Prior to the tests, the materials were irradiated with doses of the order of hundreds of Gys ( 100-240 Gy) packaged into vials. The vials were then sealed in light-tight envelopes.

When particle size reduction was needed, the materials were ball-milled about $3.5 \mathrm{~g}$ at a time for $30 \mathrm{~min}$ at $400 \mathrm{rpm}$ using a Planetary Micro Mill (Pulverisette 7, Fritsch GmbH). TL reproducibility, dose 
response, and fading data were collected to verify the properties of the material matched those of material used for previous tests. The results are shown in Fig. 13. Ball milling reduced the brightness of the material in comparison to non-milled material, but less than $50 \%$. The sensitivity should still be sufficient for detonation tests.

Test of the particles were performed for overall sensitivity, dark fading and light sensitivity, as exemplified in Fig. 14.

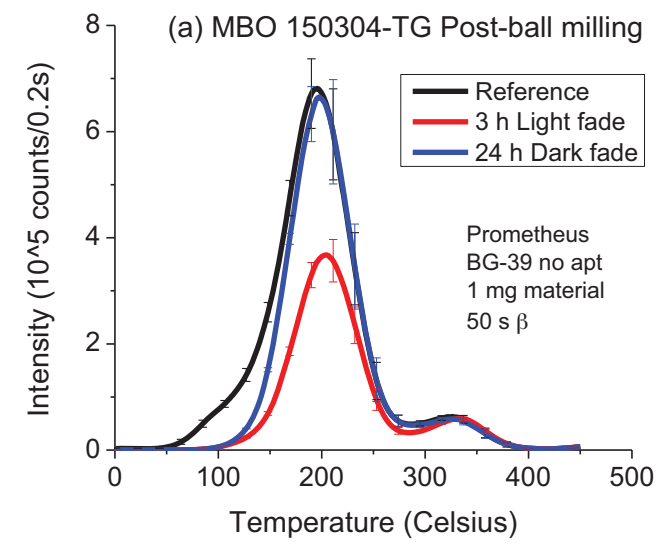

Fig. 13. Fading data for MBO. The data match the behavior of previous material used. 

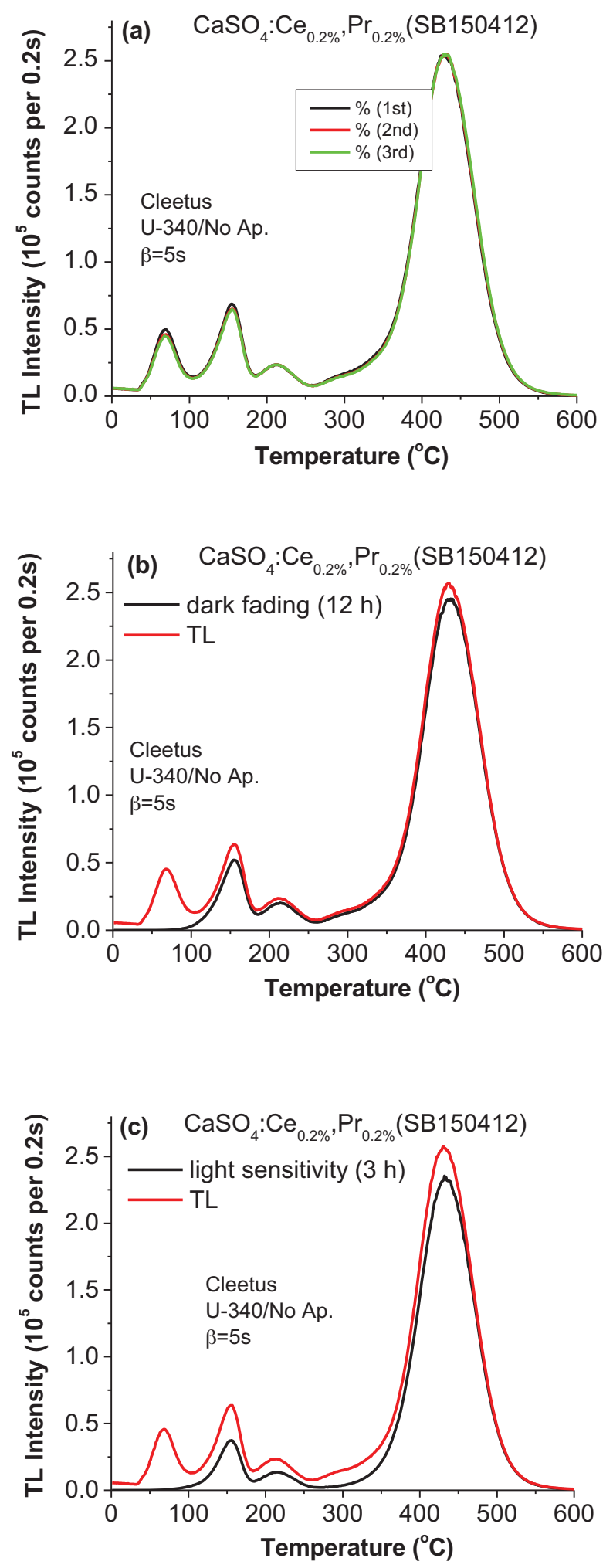

Fig. 14. Testing TL properties of CSO:Ce,Pr: (a) reproducibility; (b) dark fading; and (c) light sensitivity $\left(5^{\circ} \mathrm{C} / \mathrm{s}\right.$ heating rate).TL properties are acceptable for use of particles in tests. 


\subsubsection{Preparation of particles $<13 \mu \mathrm{m}$}

Materials were wet sieved to get the percentage of the particles $<13 \mu \mathrm{m}$ by mass. We compared TL of the material before and after sieving to show that the wet sieving process did not destroy the material. SEM images were also collected as a verification of the size. For MBO, dry sieving passed none of the material so wet sieving was used. In the wet sieving, $100 \mathrm{mg}$ of material was sonicated to break up aggregates in $200 \mathrm{~mL}$ of ethanol for $25 \mathrm{~min}$. The material was then poured into a pre-wetted sieve. The liquid that passed was collected in weigh trays. The ethanol was allowed to evaporate. The trays were weighed again to find the mass of the particles that passed. For MBO, $89.8 \%$ of the material passed through the sieve. In Fig. 15, the TL curves of the sieved and non-sieved material are shown as well as material that was not ball milled. As one can see, neither ball milling nor wet sieving destroyed MBO. SEM is shown in and confirms that most of the particles are $<13 \mu \mathrm{m}$.

For CSO, dry sieving also did not pass any measurable material. We used the same wet sieving method as we used for MBO with the following modifications. $100 \mathrm{mg}$ of CSO was mixed into $250 \mathrm{~mL}$ of ethanol. The sonication was $45 \mathrm{~min}$ followed by $10 \mathrm{~min}$ of stirring. This was done since CSO was still appearing to aggregate in the ethanol after the initial sonication. For CSO, 95\% of the material passed through the sieve. TL for sieved and non-sieved material are shown in Fig. 15b. As one can see, CSO was not destroyed passing through the sieve. SEM data shows that most particles have one dimension $<13$ $\mu \mathrm{m}$ (Fig. 16).
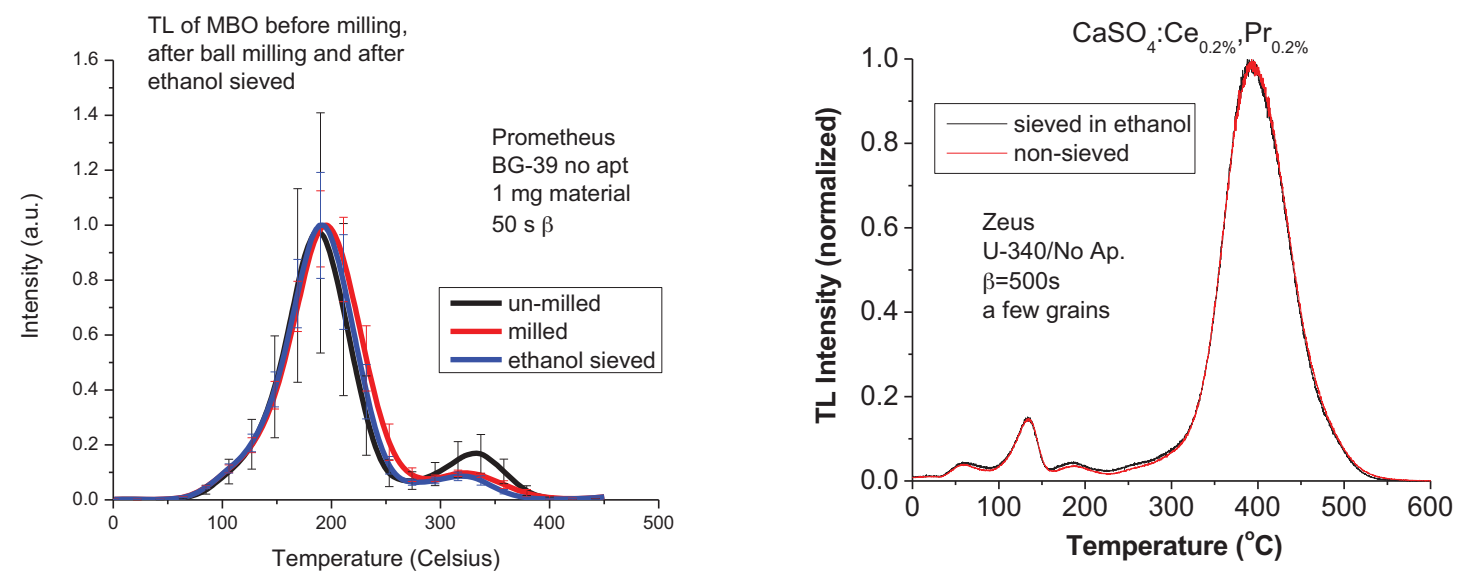

Fig. 15. Normalized comparison of the prepared (a) MBO and (b) CSO compared with not milled and sieved material. Milling and ethanol sieving do not change the TL properties of the material. 


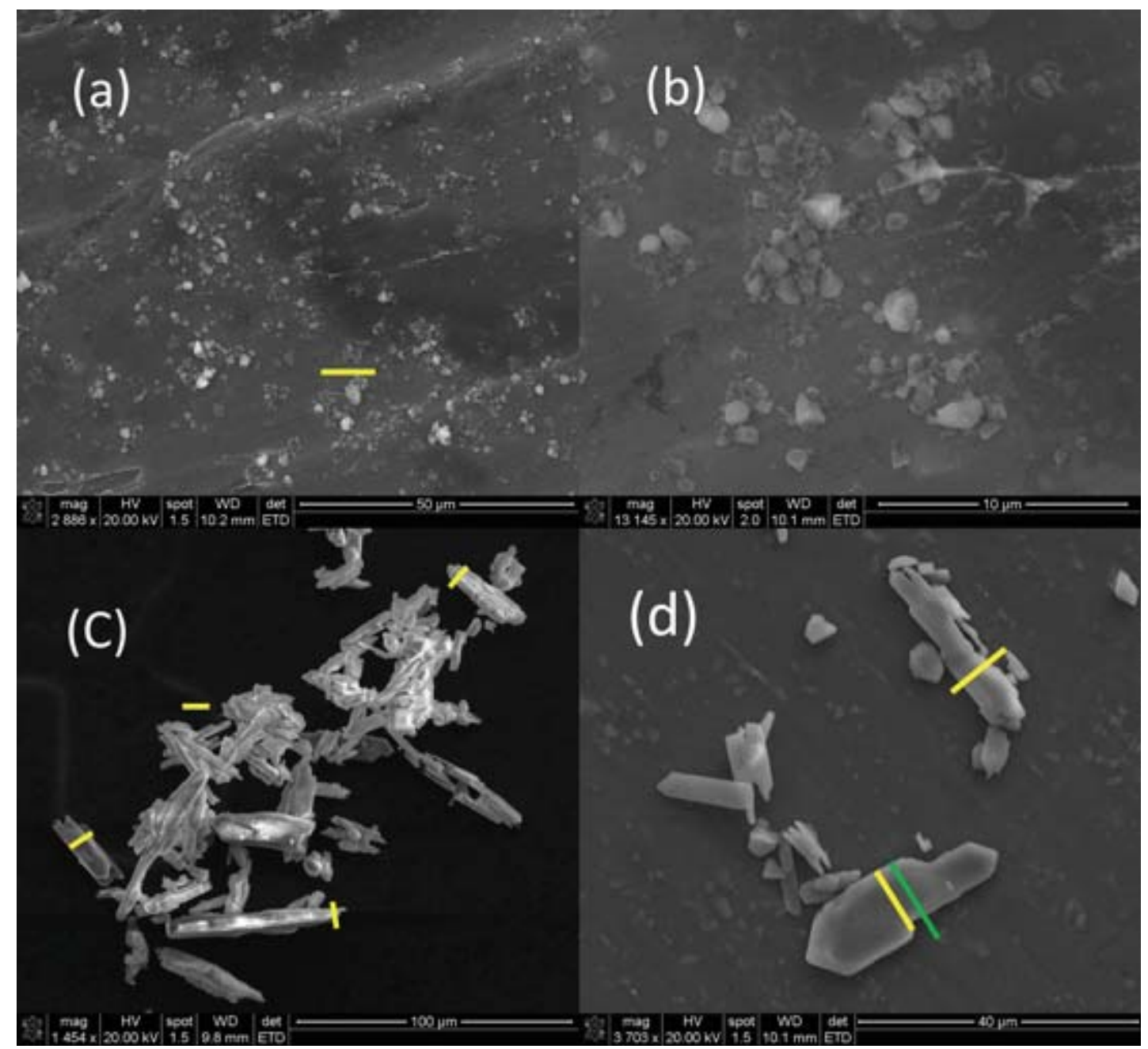

Fig. 16. SEM for MBO, (a) and (b), as well as for CSO, (c) and (d), are shown. Scaled lines have been added as a help to the reader. The yellow lines are approximately $10 \mu \mathrm{m}$ and green lines are approximately $13 \mu \mathrm{m}$. They were added using the scale in an image processing software. As can be seen, most of the particles imaged have at least one cross section $<10 \mu \mathrm{m}$.

\subsubsection{Data analysis}

Data analysis was for the temperature reconstructions were carried out using user-written codes in Mathematica (Wolfram Research Inc.) or MATLAB (MathWorks Inc.). The detailed codes are presented in the Appendices. 


\section{FUNDAMENTAL ASPECTS OF THERMOLUMINESCENCE}

\subsection{Synthesis and identification of candidate materials}

In this project we undertook a large and systematic synthesis of new luminescence materials doped with lanthanides, transition metals, and alkali metals to identify potential materials for temperature sensing [2]. A list of materials synthesized is presented in Table 1 along with dopants investigated. In addition, for the most promising materials the influence of other synthesis parameters (e.g. fuel, annealing temperature, etc.) was also investigated and will be described in more detail later in this report. The crystalline structure of the materials was confirmed by XRD (Fig. 17) and the luminescence properties were characterized by radioluminescence and TL.

In addition to what already published $[2,4-6,32,33]$, we present here some examples of the results.
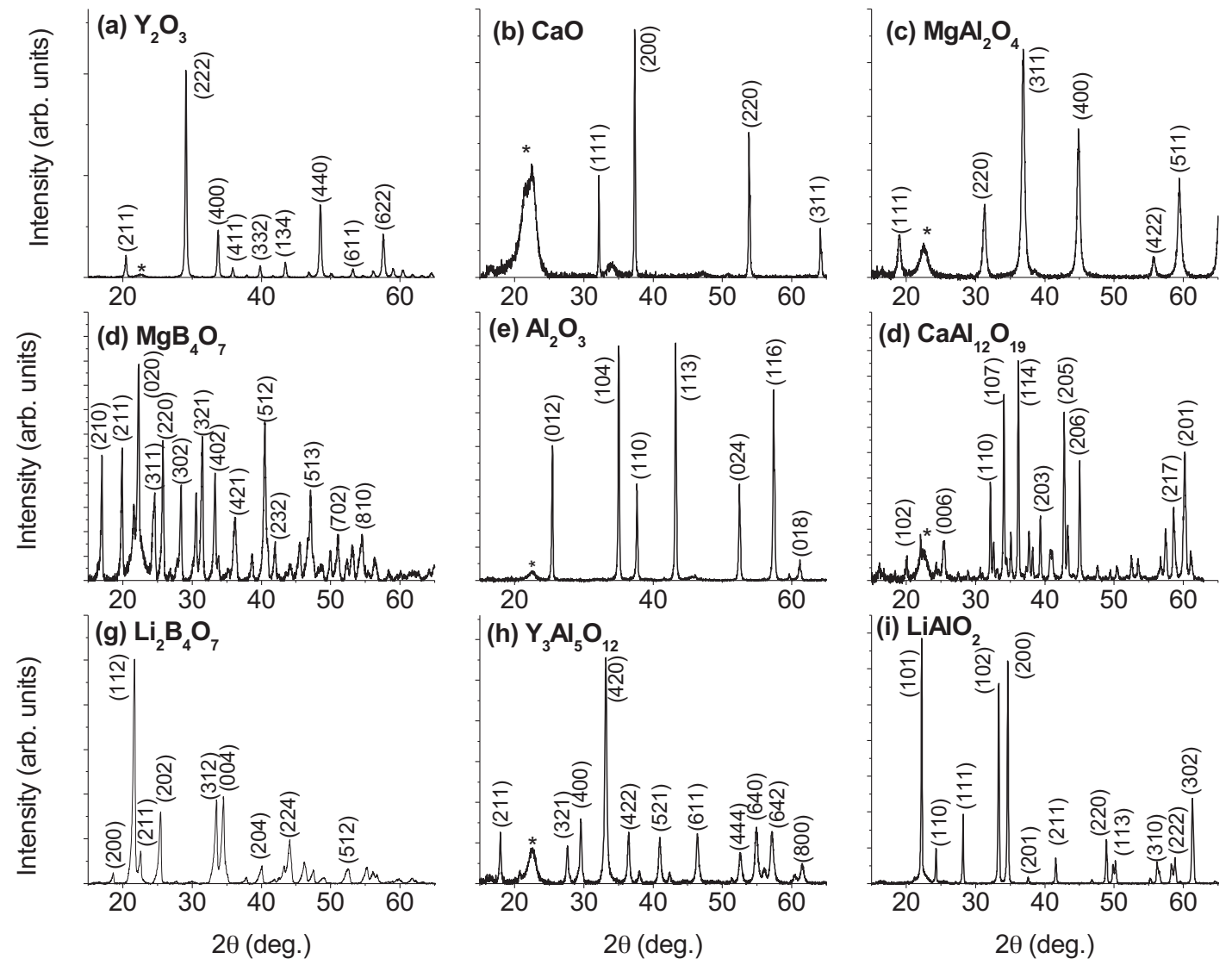

Fig. 17. XRD patterns of samples produced by SCS, accompanied by information on annealing temperature and duration, powder diffraction card number, crystal system and space group: (a) $\mathrm{Y}_{2} \mathrm{O}_{3}\left(1100{ }^{\circ} \mathrm{C}\right.$ for $\left.2 \mathrm{~h}, 01-083-0927, \mathrm{cubic}, \mathrm{la}-3\right)$, (b) $\mathrm{CaO}$ (900 ${ }^{\circ} \mathrm{C}$ for 2h, 01-077-2376, cubic, $\mathrm{Fm}-3 \mathrm{~m}$ ), (c) $\mathrm{MgAl}_{2} \mathrm{O}_{4}\left(900{ }^{\circ} \mathrm{C}\right.$ for 2h, 01-071-6329, cubic, $\left.\mathrm{Fd}-3 \mathrm{~m}\right),(\mathrm{d}) \mathrm{MgB}_{4} \mathrm{O}_{7}\left(900{ }^{\circ} \mathrm{C}\right.$ for 2h, 00-017-0927, orthorhombic, $P b c a)$, (e) $\mathrm{Al}_{2} \mathrm{O}_{3}\left(900^{\circ} \mathrm{C}\right.$ for $2 \mathrm{~h}, 00-042-1468$, rhombohedral, $\left.R-3 \mathrm{C}\right),(\mathrm{f}) \mathrm{CaAl}_{12} \mathrm{O}_{19}\left(1200{ }^{\circ} \mathrm{C}\right.$ for 4h, 00-038-0470, hexagonal, $P 63 / \mathrm{mmc})$, (g) $\mathrm{LiB}_{4} \mathrm{O}_{7}\left(860^{\circ} \mathrm{C}\right.$ for $40 \mathrm{~min}, 01-084-2191$, tetragonal, $\left.141 \mathrm{~cd}\right),(\mathrm{h}) \mathrm{Y}_{3} \mathrm{Al}_{5} \mathrm{O}_{12}\left(900{ }^{\circ} \mathrm{C}\right.$ 
for $2 \mathrm{~h}, 01-071-1853$, cubic, Ia-3d), (i) $\mathrm{LiAlO}_{2}\left(120{ }^{\circ} \mathrm{C}\right.$ for $4 \mathrm{~h}, 00-038-1464$, tetragonal, $\mathrm{P4212}$ ). Miller indices are presented only for the most intense peaks. The asterisk (*) indicates an artifact introduced by the sample holder.

Fig. 18 shows the RL for three different host materials with the most intense signals. The data demonstrate the possibility of introducing luminescence centers by appropriate Ln doping, although not all host materials showed large RL signals. Characteristic emission lines of several $\mathrm{Ln}^{3+}$ can be seen.

TL materials with intensity comparable to commercial TL material (LiF:Mg,Ti) were obtained in this project. Fig. 19 shows the TL curves for the most intense TL materials, all measured in the same conditions. Comparing the intensities, it can be seen that $\mathrm{MgO}: \mathrm{Sm}, \mathrm{Li}$ and several $\mathrm{MgB}_{4} \mathrm{O}_{7}$ present $\mathrm{TL}$ intensity similar to LiF:Mg,Ti. Moreover, the dopants change the structure of the TL peak, indicating that different trapping centers are formed.

One of the biggest challenges was to identify light insensitive host materials or TL peaks, which is required for the thermal sensors to be used in explosive events. Testing of the materials developed indicate that most are light sensitive, i.e., light can either increase or decrease the intensity of TL peaks.
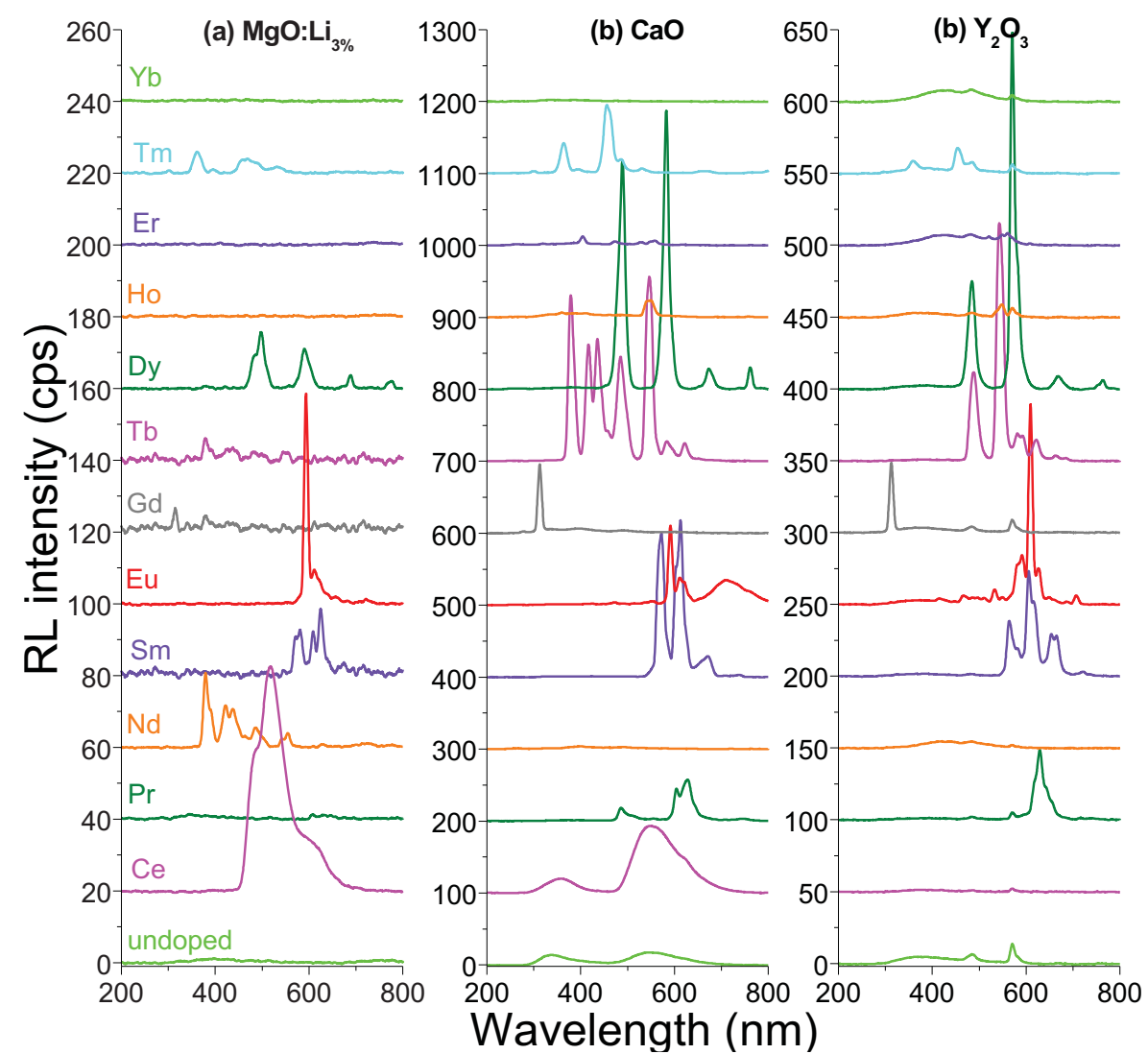

Fig. 18. RL emission spectra of undoped and lanthanide-doped (a) $\mathrm{MgO}$, (b) $\mathrm{CaO}$ and (c) $\mathrm{Y}_{2} \mathrm{O}_{3}$ synthesized by solution combustion synthesis. 


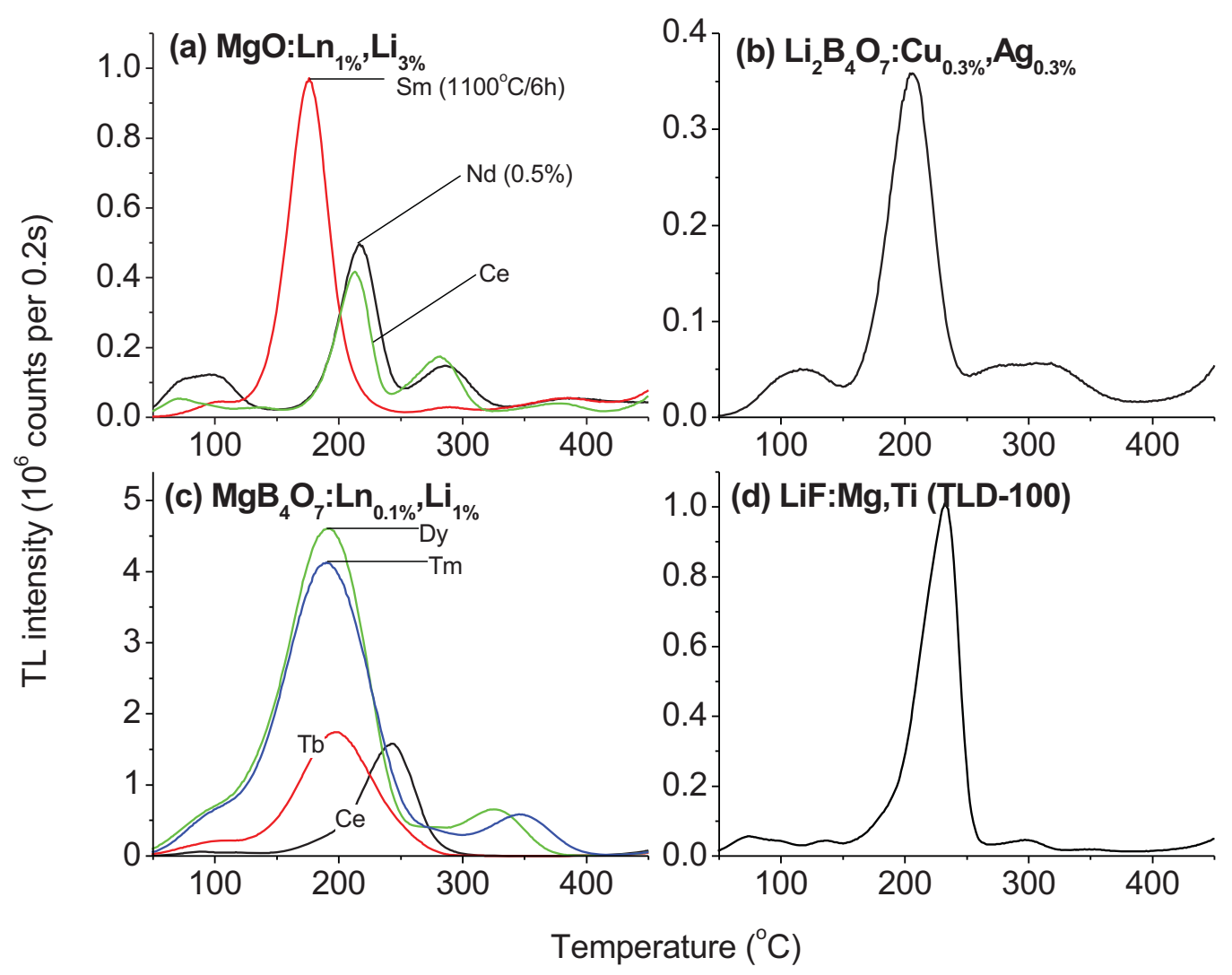

Fig. 19. TL curves for different lanthanide(Ln)-doped compounds synthesized by SCS: (a) MgO; (b) $\mathrm{Li}_{2} \mathrm{~B}_{4} \mathrm{O}_{7}$ and (c) $\mathrm{MgB}_{4} \mathrm{O}_{7}$. Panel (d) shows the TL from commercial LiF:Mg,Ti (TLD-100) for comparison.

Fig. 20 shows an example of $\mathrm{RL}$ spectra of $\mathrm{SiO}_{2}$ undoped or with various dopants. In this study, $\mathrm{Li}^{+}$was investigated as a co-dopant to elucidate the role of charge-compensation during the doping process. The results in this figure lead to the following conclusions:

- The lanthanides are acting effectively as luminescence centers in $\mathrm{SiO}_{2}$, as demonstrated by the characteristic emission lines of $\mathrm{Tb}^{3+}, \mathrm{Dy}^{3+}$, and $\mathrm{Gd}^{3+}$, or the band emission from $\mathrm{Ce}^{3+}$. This enables us to control the emission wavelength of the nanophosphors, although it remains to be demonstrated that these luminescence centers are also acting as recombination centers during the TL process.

- In $\mathrm{SiO}_{2}, \mathrm{Li}^{+}$as a co-dopant increases the $\mathrm{RL}$ intensity in most cases by at least an order of magnitude. One possible explanation is that $\mathrm{Si}^{4+}$ is more easily replaced by a complex defect consisting of $\mathrm{Ln}^{3+}$ with a nearby $\mathrm{Li}^{+}$ion, therefore preserving local charge neutrality. However, this enhancement does not seem to work with $\mathrm{Ce}^{3+}$ in this material, as co-doping with $\mathrm{Li}$ and $\mathrm{Ce}$ resulted in a decrease in $\mathrm{RL}$ intensity.

The corresponding $\mathrm{TL}$ curves for the same samples are presented in Fig. 21. The data shows that in $\mathrm{SiO}_{2}$ the TL curve is dominated by a peak around $100^{\circ} \mathrm{C}$, regardless of the dopant. In most cases, $\mathrm{Li}^{+}$doping 
increased the intensity of the TL curve, although again this was not the case for the Ce-doped sample. The fact that the TL curve does not depend much on the doping used as a luminescence center opens the possibility of using co-dopants to control the other trapping centers responsible for the TL peaks. This hypothesis remains to be demonstrated.
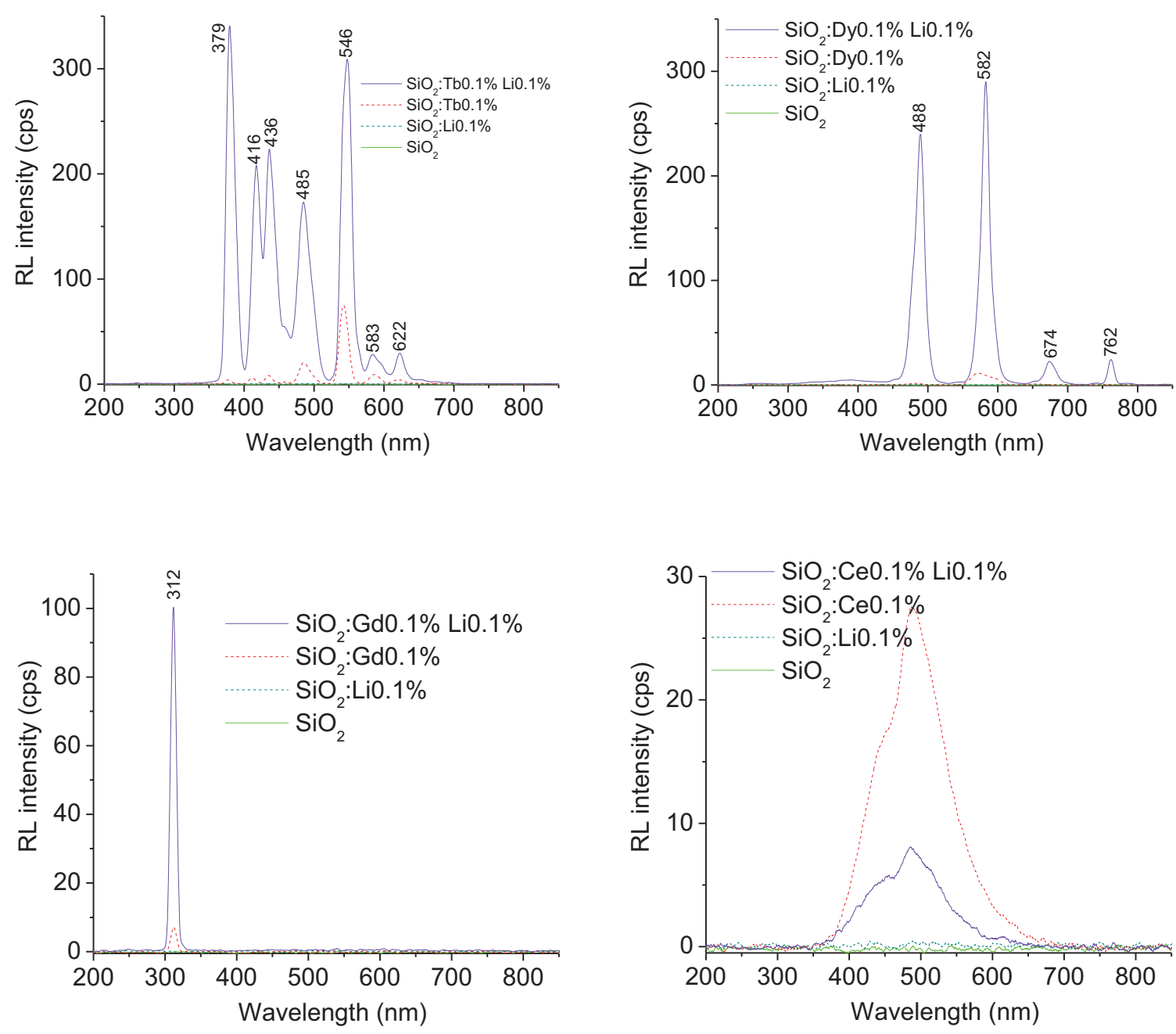

Fig. 20. Radioluminescence (RL) curves of various samples of $\mathrm{SiO}_{2}$ undoped or doped with $\mathrm{Li}^{+}$or different lanthanides, or both, synthesized at OSU using the sol-gel method. 

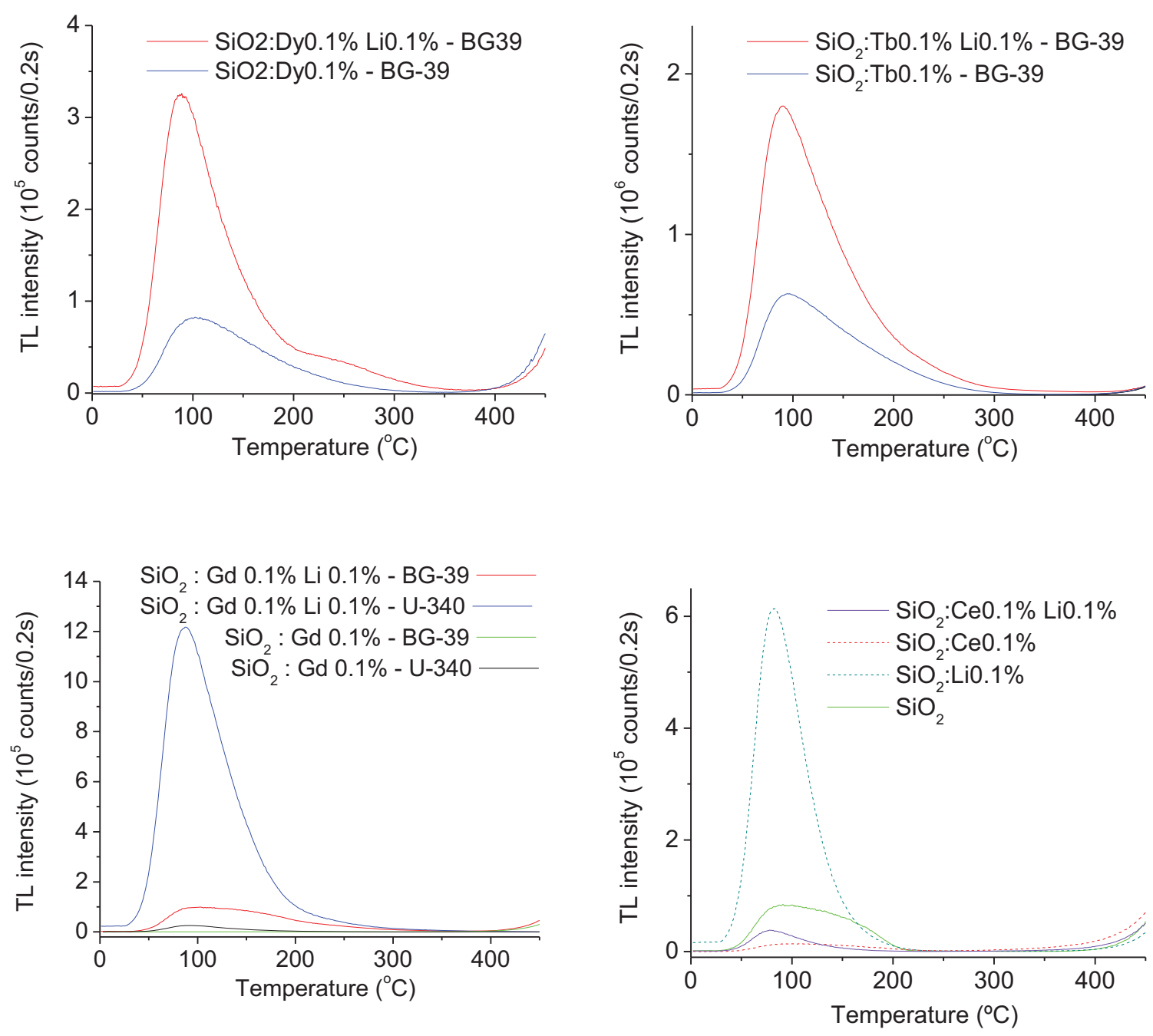

Fig. 21. TL curves of various samples of $\mathrm{SiO}_{2}$ undoped or doped with $\mathrm{Li}^{+}$or different lanthanides, or both, synthesized at OSU using the sol-gel method.

Fig. 22 and Fig. 23 shows a few examples of the RL and TL data obtained for $\mathrm{CaO}$ doped with various dopants. Although this material is hygroscopic and unlikely to be used as a thermal sensor in an actual application, it was investigated here to gain further insight on the relationship between dopants and the luminescence properties in different materials.

The RL data in Fig. 22 shows that there are relatively strong luminescence centers even in the undoped $\mathrm{CaO}$. Doping this material can alter the emission spectrum, but the luminescence bands characteristic of the undoped samples are still observable in most cases. In this material, $\mathrm{Li}^{+}$doping has not been effective in improving the RL intensity, showing that it does not seem to work as a charge compensator to improve the incorporation of luminescence centers.

The TL curves of $\mathrm{CaO}$ shown in Fig. 23 demonstrate that the TL intensity of the undoped samples is in some cases comparable to the TL intensity of the doped samples. Although doping has changed the shape of the TL curves and the relative intensity of the TL peaks, it has not increased the TL intensity as much as observed for example with $\mathrm{SiO}_{2}$. 

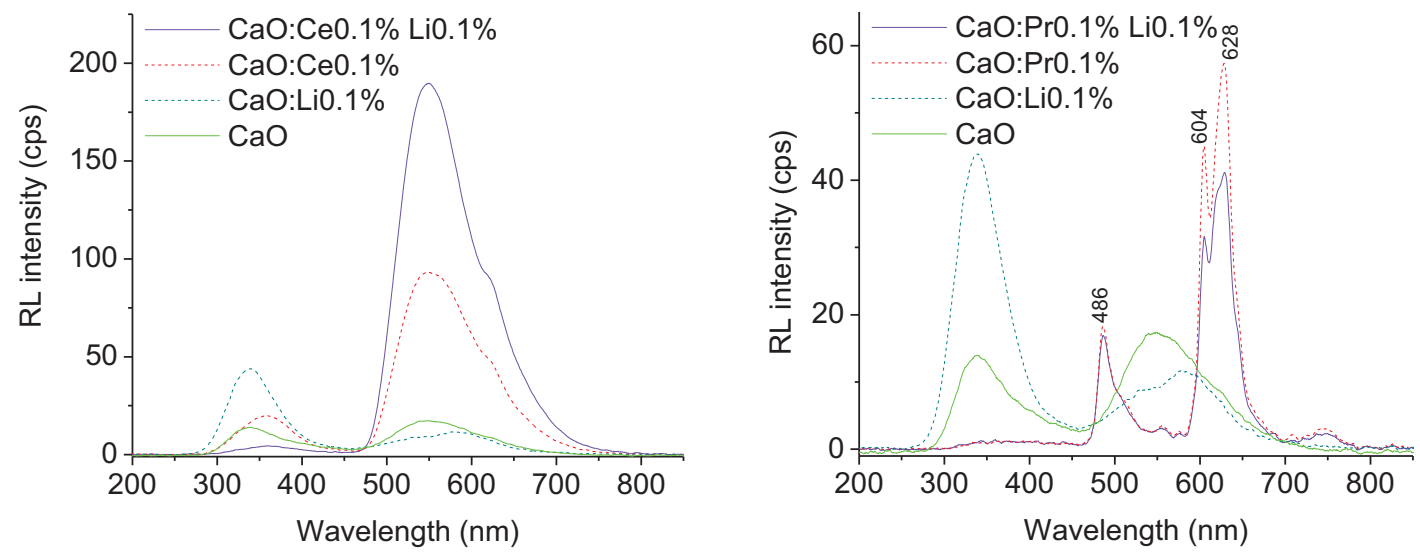

Fig. 22. $\mathrm{RL}$ emission spectrum of $\mathrm{CaO}$ undoped or doped with various lanthanides, synthesized at OSU using the solution combustion synthesis method.
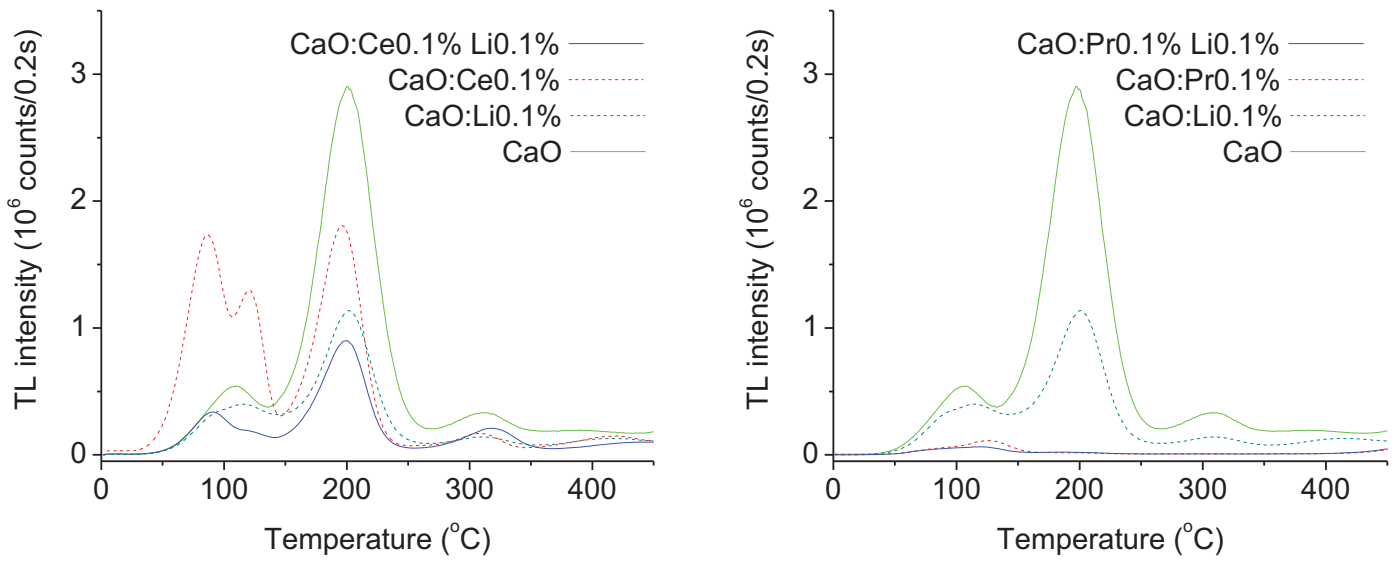

Fig. 23. TL curves of $\mathrm{CaO}$ undoped or doped with various lanthanides, synthesized at OSU using the solution combustion synthesis method.

Finally, Fig. 24 and Fig. 25 show the RL and $\mathrm{TL}$ data for $\mathrm{Y}_{2} \mathrm{O}_{3}$. In this case, the data demonstrates that the dopants are acting as luminescence centers. However, the TL emission in most cases has been weak, with the exception of $\mathrm{Y}_{2} \mathrm{O}_{3}:$ Tb. This sample shows a relatively well-defined TL peak of high intensity in a temperature range which, according to our previous simulations, is of interest for thermometry applications. 

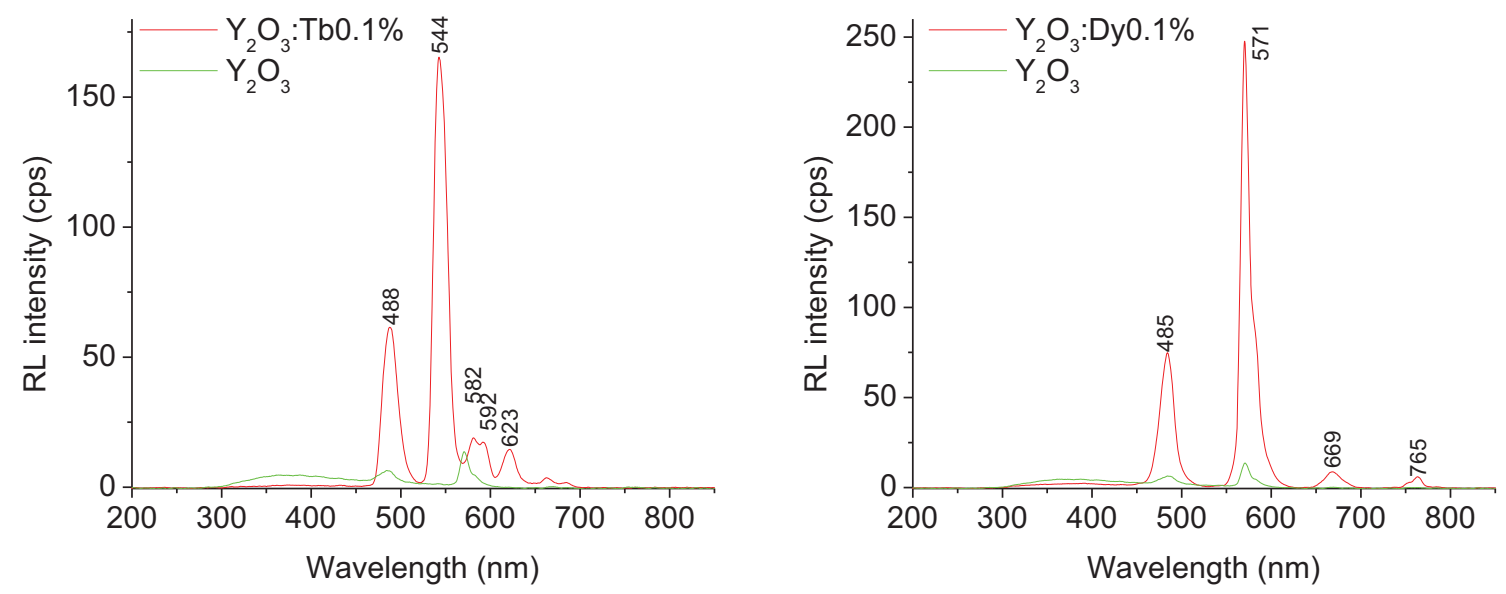

Fig. 24. RL emission spectrum of $\mathrm{Y}_{2} \mathrm{O}_{3}$ undoped or doped with various lanthanides, synthesized at OSU using the solution combustion synthesis method.
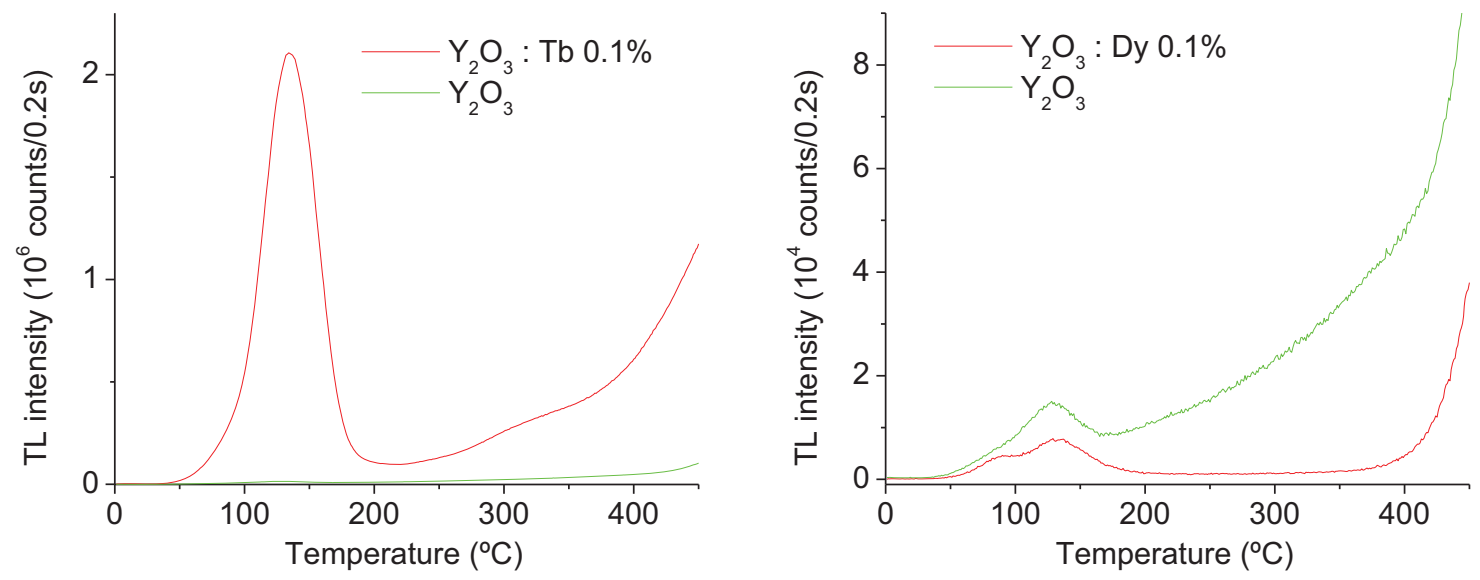

Fig. 25. TL curves of $\mathrm{Y}_{2} \mathrm{O}_{3}$ undoped or doped with various lanthanides, synthesized at OSU using the solution combustion synthesis method.

Fig. 26 shows the XRD and TL of $\mathrm{LiSr}_{4}\left(\mathrm{BO}_{3}\right)_{3}$ prepared with different dopants. All TL curves showed similar TL structure, with the main peak at $\sim 20{ }^{\circ} \mathrm{C}$ and sometimes strong $\mathrm{TL}$ intensity (e.g. with $\mathrm{Ce}, \mathrm{Tb}$ dopants). This material did not show significant fading, but the main TL peak decreased $\sim 20-40 \%$ with $3 \mathrm{~h}$ of room light exposure. The secondary $\mathrm{TL}$ peak at $100^{\circ} \mathrm{C}$ could be of interest for measurement of low temperatures, provided the exposures and readout are made quickly enough to avoid the inevitable thermal fading.

Fig. 27 shows the XRD and $\mathrm{TL}$ of $\mathrm{LiSr}_{4} \mathrm{BO}_{3}$. In this case a wider variety of TL curves was observed with the type of dopant. The main TL peak of the Ce-doped sample is at $300{ }^{\circ} \mathrm{C}$, which can be of interest for temperature sensing, although the intensity is not so high. This TL peak is promising, because it did not show evidence of fading or light sensitivity.

Fig. 28 shows the XRD and TL results for $\mathrm{Mg}_{3} \mathrm{~B}_{2} \mathrm{O}_{6}$. Though the TL intensity is high, the main TL peak at $\sim 180{ }^{\circ} \mathrm{C}$ showed significant decrease ( $\left.50 \%\right)$ with $3 \mathrm{~h}$ of room light exposure. 
Finally, Fig. 29 shows the results for $\mathrm{MgSO}_{4}: \mathrm{Tm}$. This material is extremely interesting for temperature sensing because of the presence of two peaks at relatively high temperatures $\left(260^{\circ} \mathrm{C}\right.$ and $\left.360{ }^{\circ} \mathrm{C}\right)$ with high intensity. These peaks did not seem to be affected by $3 \mathrm{~h}$ of room light exposure.

These results exemplify the type of investigations that were carried out to identify materials with appropriate characteristics for temperature sensing. Future tests focused on the most promising materials, but some of the materials identified here can be developed here in the future for temperature sensing applications.

(a) XRD

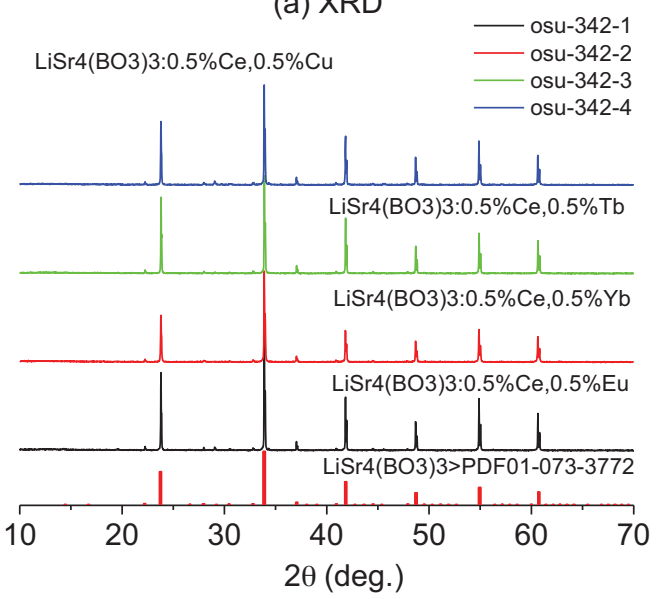

(b) TL

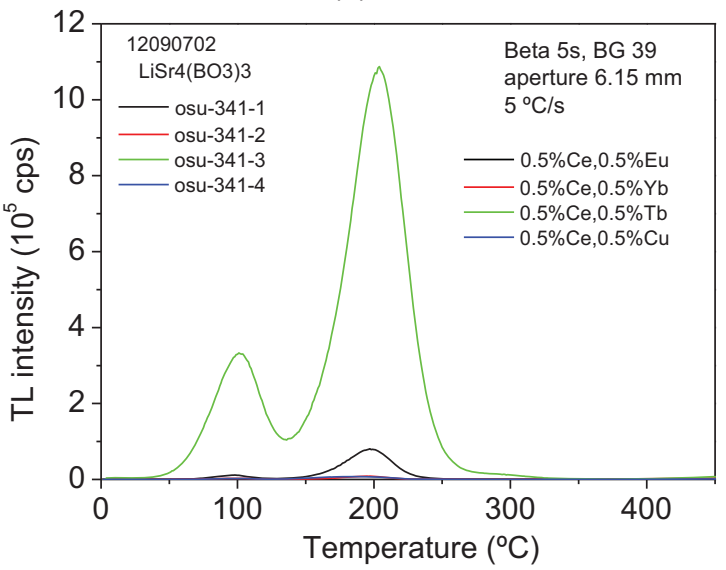

Fig. 26. (a) XRD and (b) TL of $\mathrm{LiSr}_{4}\left(\mathrm{BO}_{3}\right)_{3}$ prepared with different dopants.

(a) XRD

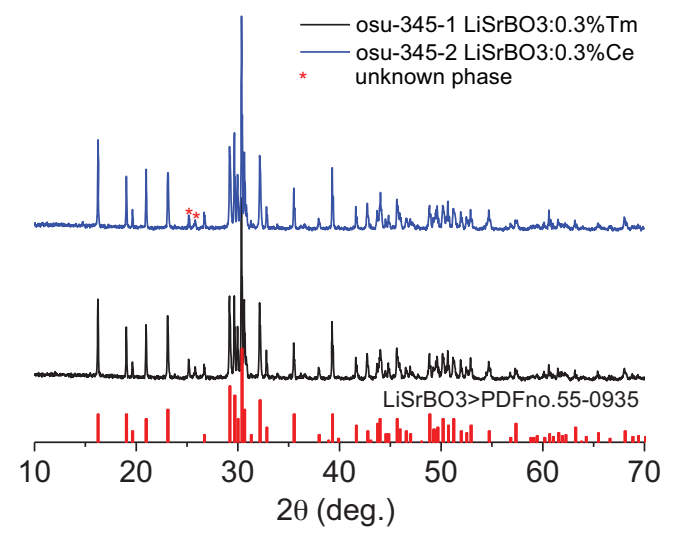

(b) $\mathrm{TL}$

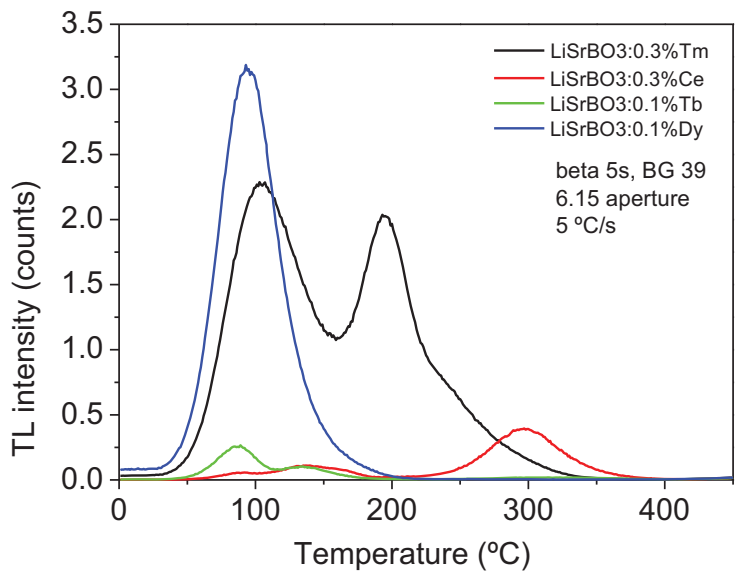

Fig. 27. (a) XRD and (b) $\mathrm{TL}$ of $\mathrm{LiSrBO}_{3}$ prepared with different dopants. 
(a) XRD

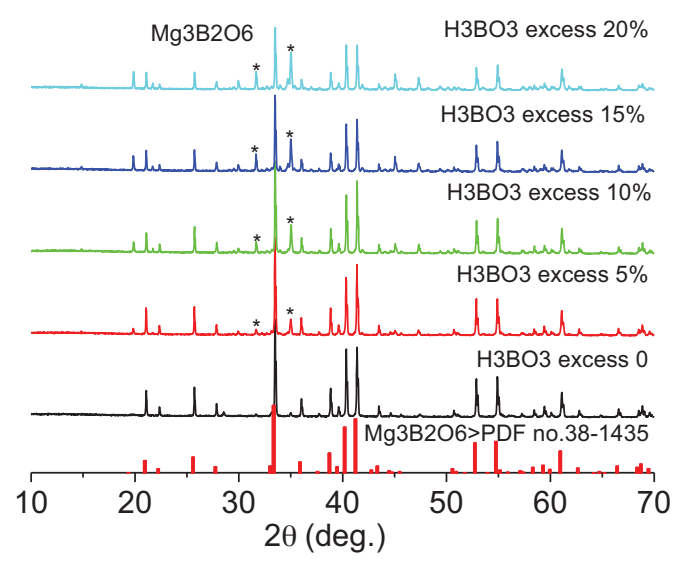

(b) TL

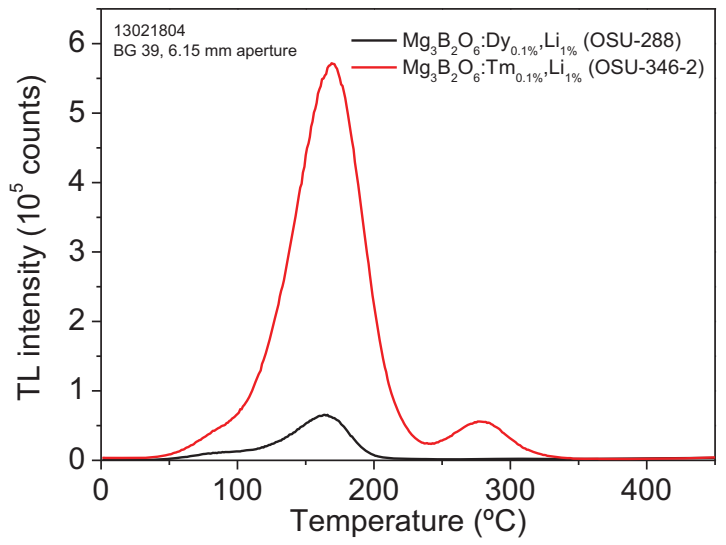

Fig. 28. (a) XRD and (b) TL of $\mathrm{Mg}_{3} \mathrm{~B}_{2} \mathrm{O}_{6}$ prepared with different dopants.

(a) fast cooling

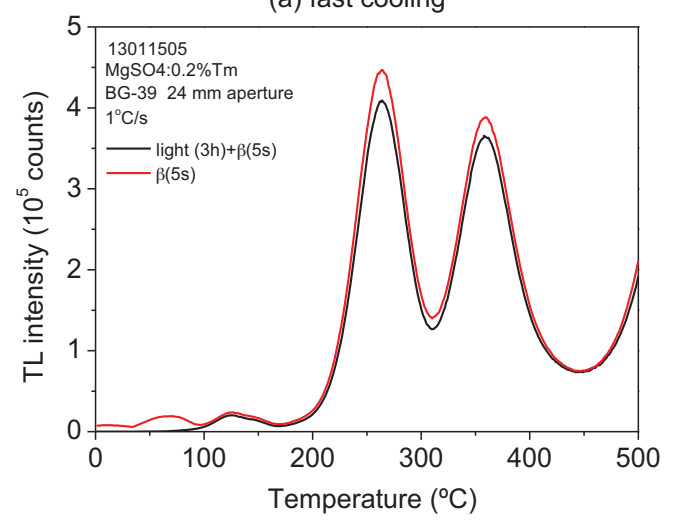

(b) slow cooling

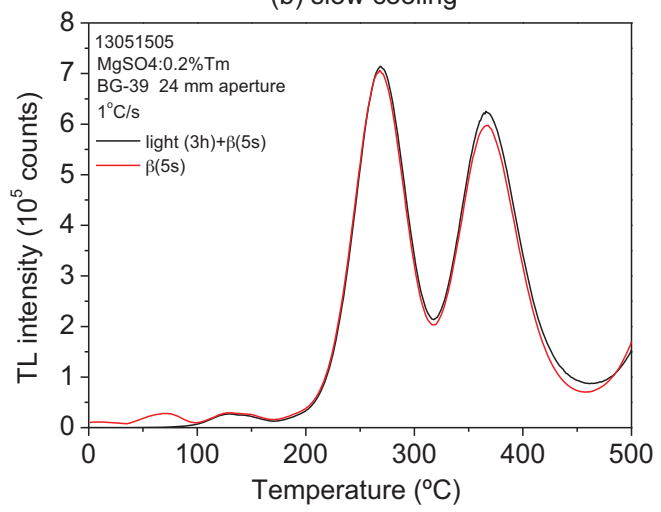

Fig. 29. $\mathrm{TL}$ of $\mathrm{MgSO}_{4}: \mathrm{Tm}$ synthesized with (a) fast cooling and (b) slow cooling, showing the effect of $3 \mathrm{~h}$ of room light exposure on the TL peaks.

\subsection{Investigation of commercial materials}

In addition to materials developed at OSU, we started several collaborations with groups who produce commercially materials that show potential for temperature sensing, including the Institute of Nuclear Physics (INP, Krakow, Poland), the Instituto de Pesquisas Energéticas e Nucleares (IPEN-CNEN, Brazil), and the Korea Atomic Energy Research Institute (KAERI, Korea).

The materials investigated are:

LiF:Mg,Cu,P (INP). LiF:Mg,Cu,P is a well-known TL material [34], but it has recently been reported that the use of high doses of radiation (>5 kGy) can induce new high temperature peaks in this material [35]. Since these high temperature peaks are of interest for temperature sensing, we established a collaboration with Dr. Barbara Obryk from the INP to further characterize this material for temperature sensing applications. LiF:Mg,Cu,P was provided by Dr. Obryk and irradiated with doses of $5 \mathrm{kGy}, 50 \mathrm{kGy}$ and 500 kGy using a gamma cell (courtesy Dr. Helen Khoury, Nuclear Energy Department, UFPE, Brazil). Then, the materials were characterized to determine the TL curve, reproducibility, TL emission spectrum, and effect of temperature. No change in TL was observed as a result of light exposure, indicating that the material could be used for temperature sensing. The TL curve changed considerably 
with the dose. The TL after irradiation with $500 \mathrm{kGy}$ is interesting because of the presence of a dominant TL peak at $\sim 410{ }^{\circ} \mathrm{C}$. The only problem with this material is the impossibility of regenerating the signal for comparison with the TL after exposure to a temperature profile. Because of that, it is difficult to determine in practice how much the TL peak at $\sim 410^{\circ} \mathrm{C}$ is affected by the temperature.

LiF:Mg,CU,Si (KAERI). Because of the observed light insensitivity of LiF:Mg,Ti and LiF:Mg,Cu,P, we decided to test samples of LiF:Mg,Cu,Si produced by KAERI also. The TL curve is dominated by a TL peak at $\sim 270{ }^{\circ} \mathrm{C}$. The presence of a single peak is not necessarily interesting for temperature sensing, but it can be used in the future if a TL with this thermal stability is required. Attempts to characterize the light sensitivity were hampered by the sensitivity loss with repeated readout. Irradiation with high doses introduces a high temperature $\left(\sim 320^{\circ} \mathrm{C}\right) \mathrm{TL}$ peak in the material. The dose response is strongly sublinear, probably because of the sensitivity loss observed due to repeated readout.

$\mathrm{CaSO}_{4}:$ Dy (IPEN). CaSO 4 :Dy chips from IPEN showed a wide range of TL peaks and linear-supralinear dose response. The material seems suitable for temperature sensing, with small light sensitivity and small fading. This can ben an alternative commercial material for temperature sensing, but in this study we have focused on $\mathrm{CaSO}_{4}$ developed at OSU because of the possibility to control the trapping centers during the synthesis process.

\subsection{Properties of LBO, MBO and CSO}

From this initial investigation, a series of materials were identified for further development and testing, the main ones being $\mathrm{Li}_{2} \mathrm{~B}_{4} \mathrm{O}_{7}: \mathrm{Cu}, \mathrm{Ag}(\mathrm{LBO}), \mathrm{MgB}_{4} \mathrm{O}_{7}: \mathrm{Dy}, \mathrm{Li}(\mathrm{MBO})$ and $\mathrm{CaSO}_{4}: \mathrm{Ce}, \mathrm{Tb}(\mathrm{CSO})$. The development of these materials represent a breakthrough in this project, because the materials identified in the literature or previously obtained in this project were light sensitive and, therefore, not suitable for temperature sensing (except for commercial LiF:Mg, Ti).

\subsubsection{Crystal structure and morphology}

The XRD curves of LBO, MBO and CSO (Fig. 30) confirm the crystalline structure of the materials. SEM images of the grains (Fig. 31) provide an idea of the grain sizes that can be obtained by sieving. Smaller grain sizes can be obtained by ball-milling, but aggregation is always a problem for small particles. 

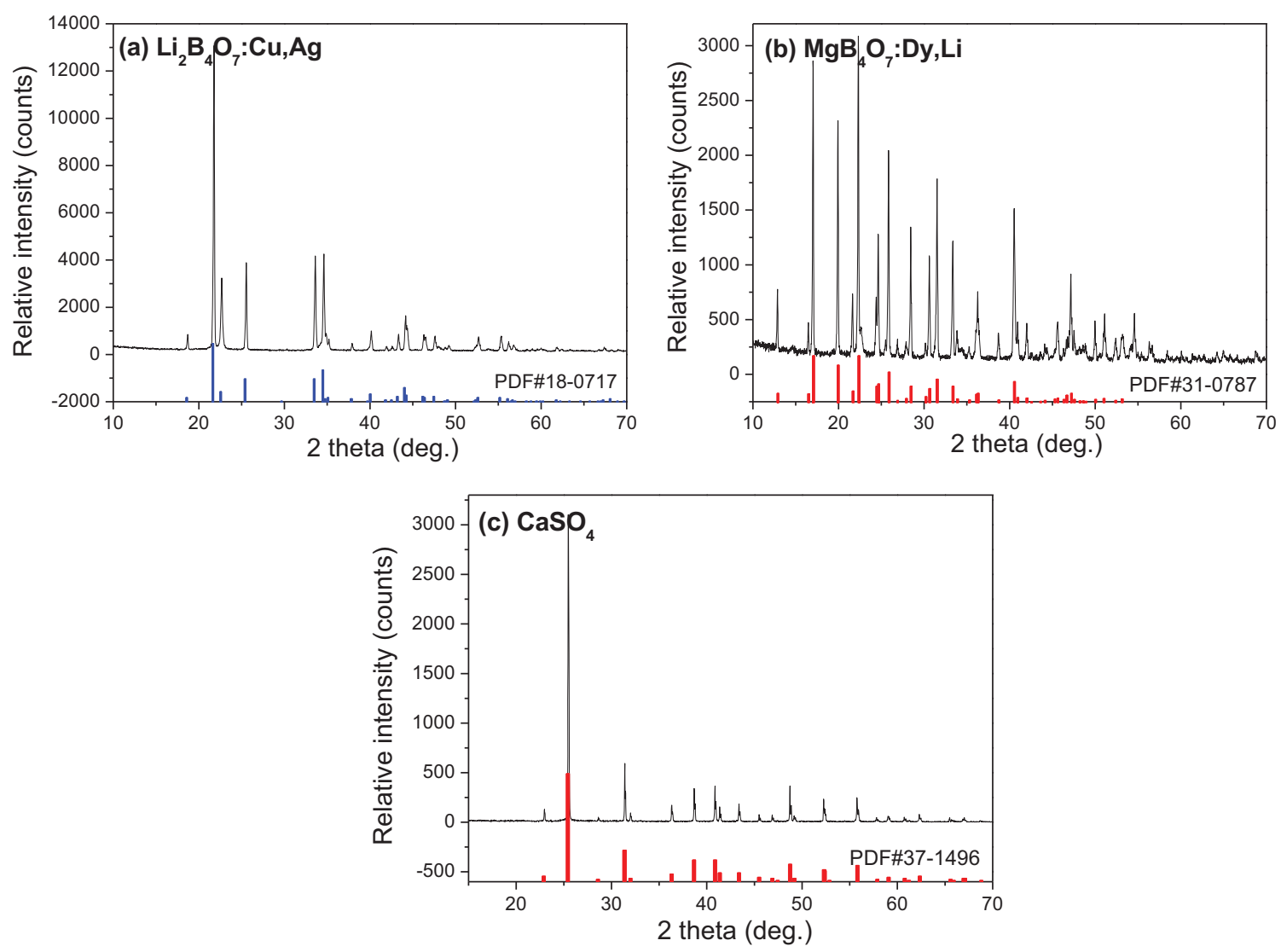

Fig. 30. XRD patterns of LBO, MBO and CSO.

(a)

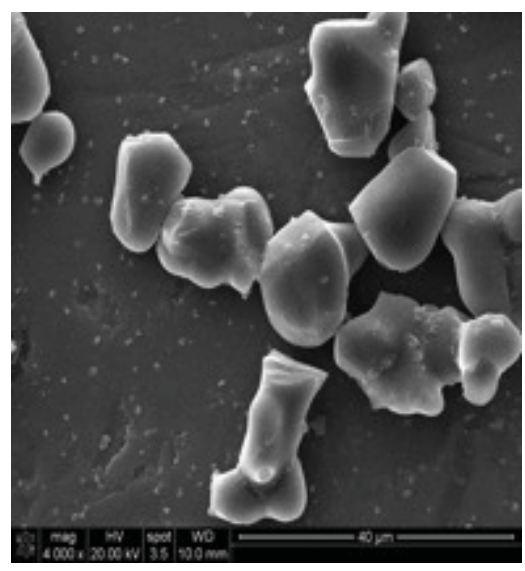

(b)

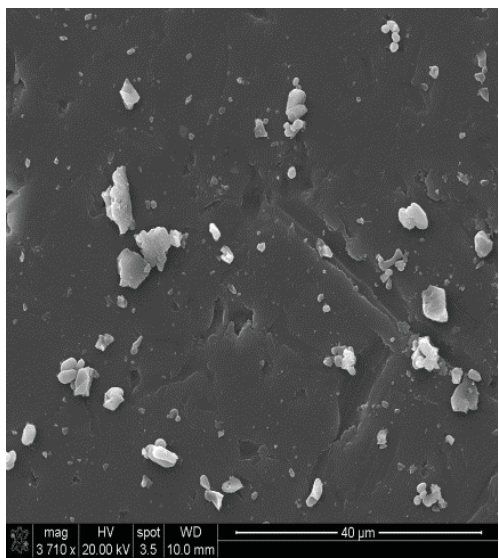

(c)

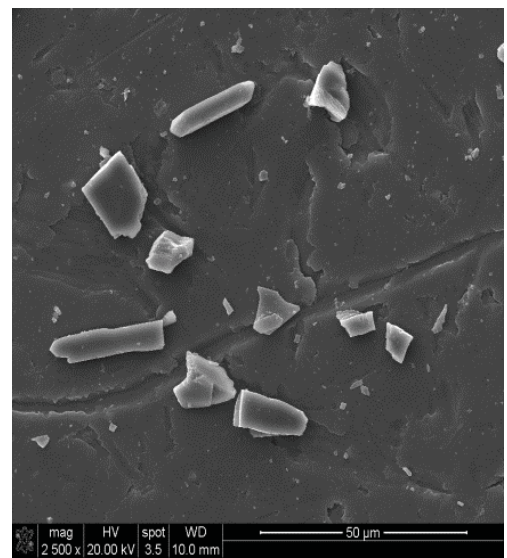

Fig. 31. SEM from (a) LBO sieved with 10-20 $\mu \mathrm{m}$ mesh size, (b) MBO sieved with 20-32 $\mu \mathrm{m}$ mesh size and (c) CSO sieved with 29-32 $\mu \mathrm{m}$ mesh size. 


\subsubsection{TL curves}

Fig. 32 shows the TL curves of LBO, MBO and CSO after repeated readout and irradiation cycles, demonstrating the material's repeatability.
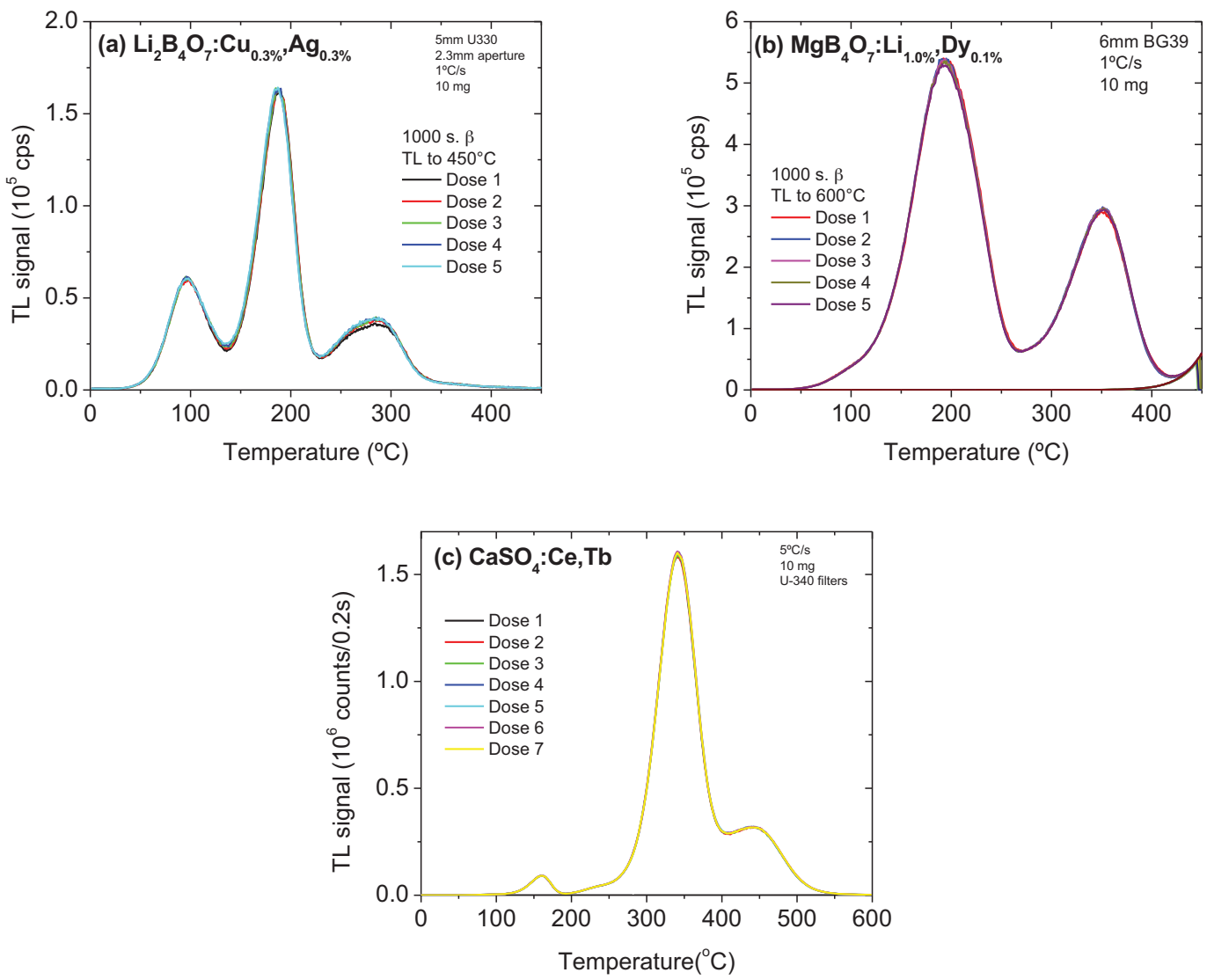

Fig. 32. Repeatability of TL curves of (a) LBO, (b) MBO, and (c) CSO, obtained using repeated irradiation and TL readout. Irradiations were performed using a beta source delivering a dose rate of $\sim 0.1 \mathrm{~Gy} / \mathrm{s}$. The data demonstrates the repeatability of the TL readouts. CSO was read using UV transmitting filters, which removes the blackbody radiation and allows TL measurements up to $600^{\circ} \mathrm{C}$.

Fig. 32 also demonstrates that we achieved the goal of developing materials with TL peaks in a wide range of temperatures. Since each TL peak is sensitive to a certain temperature range, the presence of several TL peaks means that the materials can be chosen to cover a wide range of measurement temperatures. The TL peaks of $\mathrm{LBO}$ and $\mathrm{MBO}$ are probably more suitable for temperature measurements in the $200-400{ }^{\circ} \mathrm{C}$ range, whereas CSO has a TL peak at $450{ }^{\circ} \mathrm{C}$, which can probably extend the applicability of this material beyond $500^{\circ} \mathrm{C}$.

TL data for different grains of LBO (Fig. 33) show that it is possible to obtain powder in which each grain has similar TL properties. The homogeneity of the samples prepared may vary with the type of sample and synthesis conditions. Fig. 33a shows the TL curves for different grains, which of course will vary because of the mass of each grain. When normalized to the maximum intensity (Fig. 33a), the shape of the curves is the same, indicating the good homogeneity. 

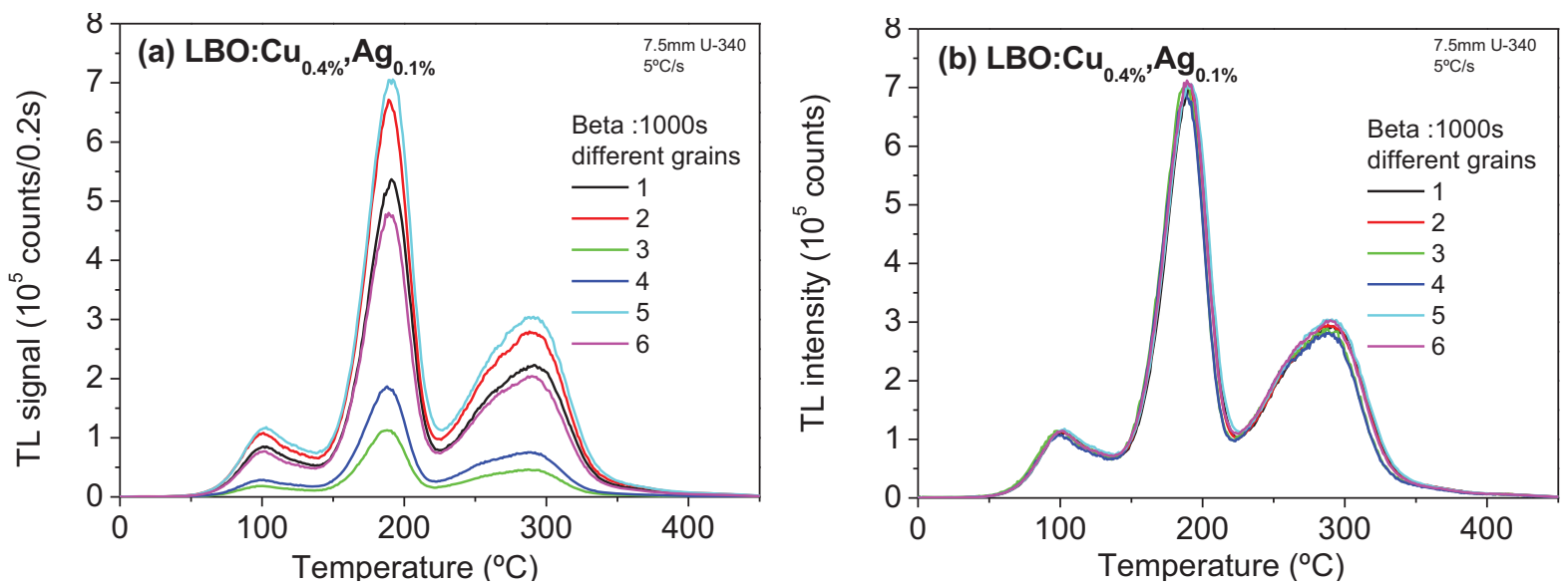

Fig. 33. TL curves for different grains of $\mathrm{LBO}: \mathrm{Cu}_{0.4 \%}, \mathrm{Ag}_{0.1 \%}$ (a) before normalization and (b) after normalization to the peak intensity.

\subsubsection{TL emission spectra}

An important TL property is the related emission wavelength, because it determines the optimum optical filters to read the TL from each sample.

Samples emitting in the UV are also interesting for temperature measurement applications, because UV emission allows one to measure the TL at high temperatures using optical filters to cut the blackbody radiation from the heaters. Black-body radiation makes the readout of $\mathrm{TL}>450^{\circ} \mathrm{C}$ in the visible range difficult, so samples that emit in the UV are preferred to measure TL peaks at high temperature.

Fig. 34 shows the TL emission spectra of LBO, MBO and CSO. The emission from LBO peaks at $350 \mathrm{~nm}$ and is attributed to the $\mathrm{Cu}^{+}$ion. The emission from $\mathrm{MBO}$ shows the characteristic emission lines from $\mathrm{Dy}^{3+}$. Emission from CSO consists of a combination of $\mathrm{Ce}^{3+}$ emission band and $\mathrm{Tb}^{3+}$ emission lines. 
(a)

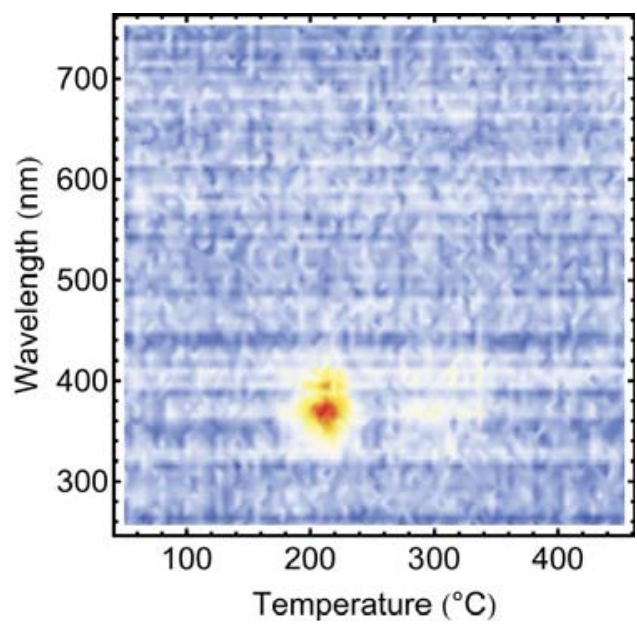

(b)

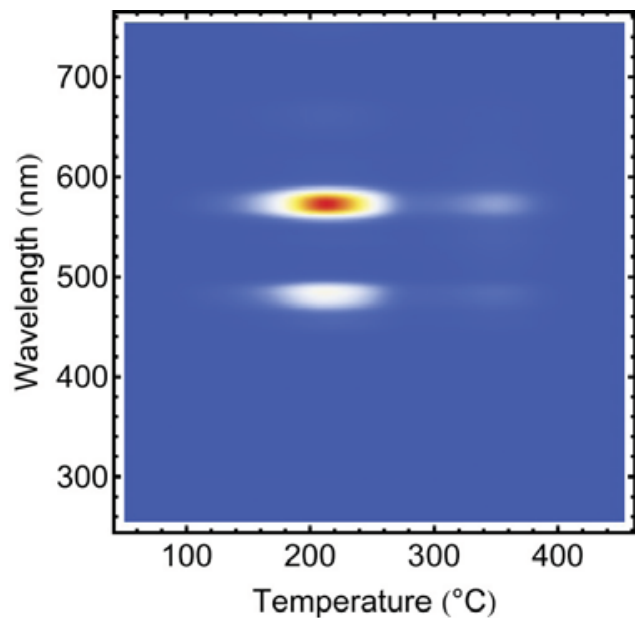

(c)

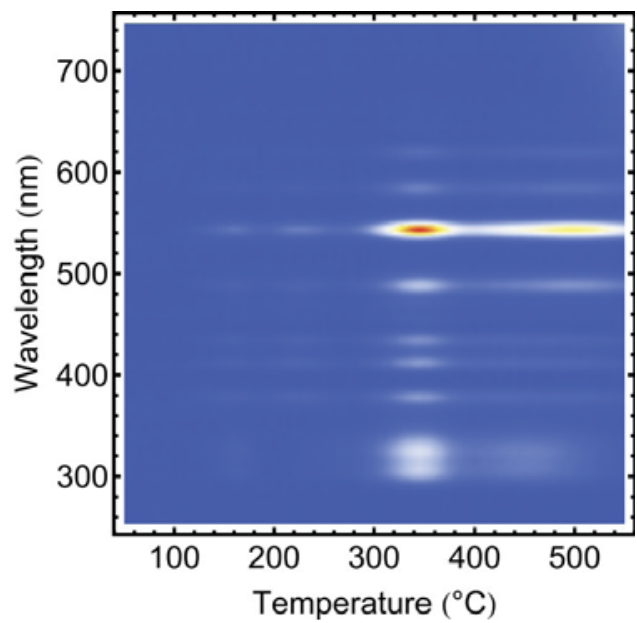

Fig. 34. TL emission spectra of (a) LBO:Cu,Ag, (b) MBO:Dy, Li and (c) CaSO:Ce,Tb. The graph shows that $L B O$ and MBO emit in very separate spectral regions because of the dopants used. Therefore, it is possible to perform detonation tests using both materials and use optical filters to isolate the TL from only one of these materials at a time. 
It is also important to note that the different emissions for observed LBO and MBO observed in Fig. 34 can be important for temperature measurement applications. Since it is possible to separate the emissions using optical filters, we can perform detonation tests using both LBO and MBO simultaneously (e.g., placed at two different distances). We can then use optical filters to isolate the signal from LBO or MBO separately, obtaining twice the amount of data in a single detonation test.

This concept can possibly be extended to other materials. Tests may be performed in which several materials are used in a single detonation test.

We investigated the optimum filters to separate the emission from these samples and arrived at the configuration presented in Table 6 . We tested these configurations with a 50/50 mixture of LBO and $\mathrm{MBO}$ and confirmed that, using these filters, it is possible to block most of the emission from one of the materials, whereas the luminescence from the other material is detected.

Table 6. Optical filters used to separate the emission from $\mathrm{L}_{2} \mathrm{~B}_{4} \mathrm{O}_{7}: \mathrm{Cu}, \mathrm{Ag}$ and $\mathrm{MgB}_{4} \mathrm{O}_{7}: \mathrm{Li}, \mathrm{Dy}$.

\begin{tabular}{|ll|}
\hline Material & Recommended optical filter \\
\hline $\mathrm{L}_{2} \mathrm{~B}_{4} \mathrm{O}_{7}: \mathrm{Cu}, \mathrm{Ag}$ & $4 \mathrm{~mm} \mathrm{U}-330$ (Hoya Corporation) \\
\hline $\mathrm{MgB}_{4} \mathrm{O}_{7}: \mathrm{Li}, \mathrm{Dy}$ & $4 \mathrm{~mm} \mathrm{BG}-39+3 \mathrm{~mm} \mathrm{GG}-435$ (Schott AG) \\
\hline
\end{tabular}

\subsubsection{Sensitivity and dose response}

Fig. 35 compares the TL signal from LBO, MBO and CSO and LiF:Mg,Ti to different doses (same mass). One can see that TL signal from MBO is higher than commercial LiF:Mg,Ti, whereas the TL signal from LBO is lower than LiF:Mg,Ti.

The advantage of LBO and MBO over LiF:Mg, Ti is that the signal does not show saturation, whereas the TL from LiF:Mg,Ti is already saturated at a dose of $400 \mathrm{~Gy}$. This means that, if necessary, it is still possible to increase the TL signal from LBO and MBO by increasing the dose. Moreover, LBO and MBO are more reproducible for repeated irradiation with high doses than LiF:Mg,Ti.

One needs, however, to pay attention to the dose delivered to the samples. Fig. 36 shows that the TL curves of these materials vary with dose, so it is important to compare the curves at the same dose levels. 


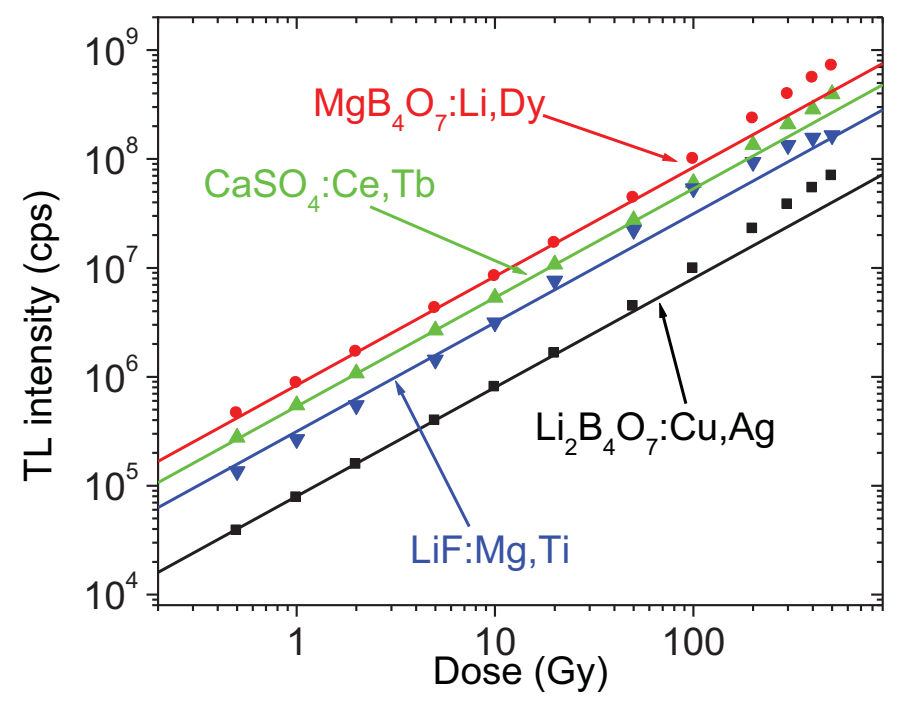

Fig. 35. Dose response for LBO, MBO, CSO and LiF:Mg,Ti (TLD-100), defined as the maximum intensity of the TL curve. For LBO and CSO we used Hoya U-340 filters, whereas for MBO and LiF we used Schott BG-39 filters. The data was obtained using $10 \mathrm{mg}$ of material and a heating rate of $1{ }^{\circ} \mathrm{C} / \mathrm{s}$.
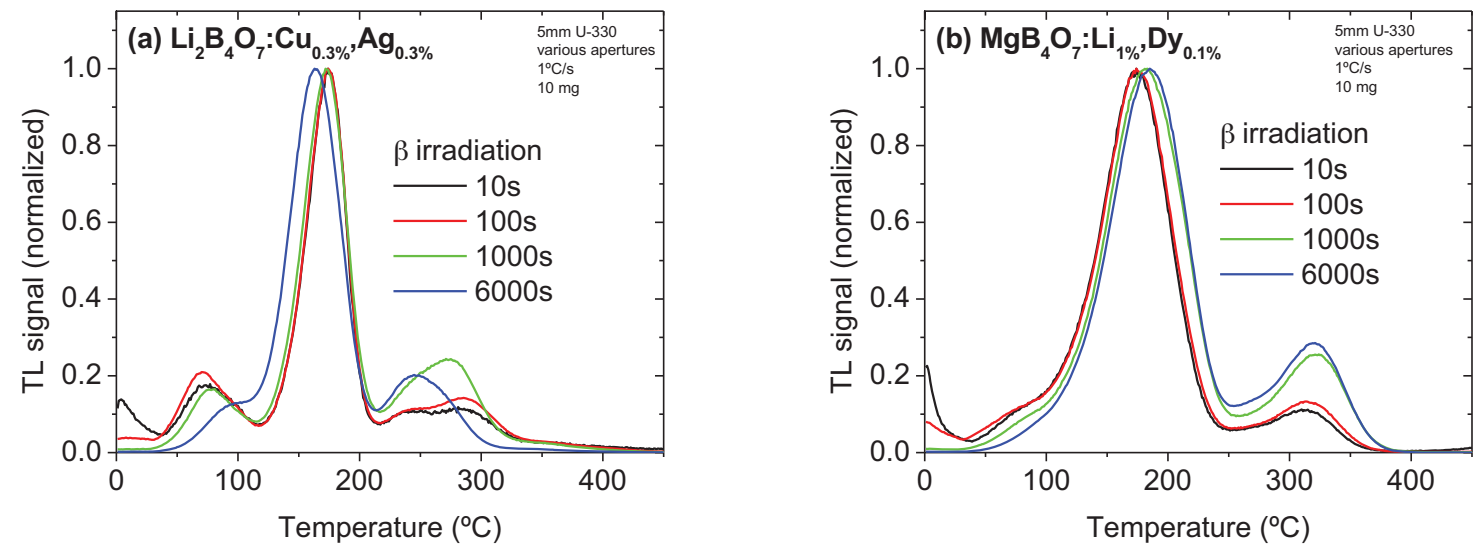

Fig. 36. TL curves of $L B O$ and MBO irradiated with different doses of beta radiation. The irradiations were carried out using a beta source delivering approximately $0.1 \mathrm{~Gy} / \mathrm{s}$.

\subsubsection{Fading and photo-bleaching tests}

The following tests were performed to identify problems with fading (thermal stimulation of trapped charges at room temperature) or photo-bleaching (optical stimulation of trapped charges) in the TL signal:

- $\quad$ ading tests: The samples were irradiated and kept in the dark for $24 \mathrm{~h}$. After that, the TL was recorded. Then, the sample was again irradiated and the TL recorded. We compared the TL obtained immediately after irradiation with TL after $24 \mathrm{~h}$ of storage in the dark. 
- Photo-bleaching tests: The samples were irradiated and exposed to $3 \mathrm{~h}$ of room light. After that, the TL was recorded. Then, the sample was again irradiated and the TL recorded. We compared the TL obtained immediately after irradiation with TL after $3 \mathrm{~h}$ of light exposure

The fading results are shown in Fig. 37, which demonstrates that these samples show little fading. The differences observed for temperature $<150{ }^{\circ} \mathrm{C}$ are associated with the expected decay of $\mathrm{TL}$ peaks unstable at room temperature (shallow traps).

Fig. 38 shows the degree of photo-bleaching observed for the various samples. In the case of LBO and CSO, the TL curves decayed only $10 \%$ with $3 \mathrm{~h}$ of light exposure. In the case of MBO, the decay was $\sim 40 \%$ over the same period of time. Although this may be a problem, we should mention that we did not observe any problem in samples that were used in detonation tests. So, the amount of photobleaching that is tolerable depends on the experimental conditions during the explosive tests, which has not been clearly specified. For now, the best is to minimize light exposure as much as possible.
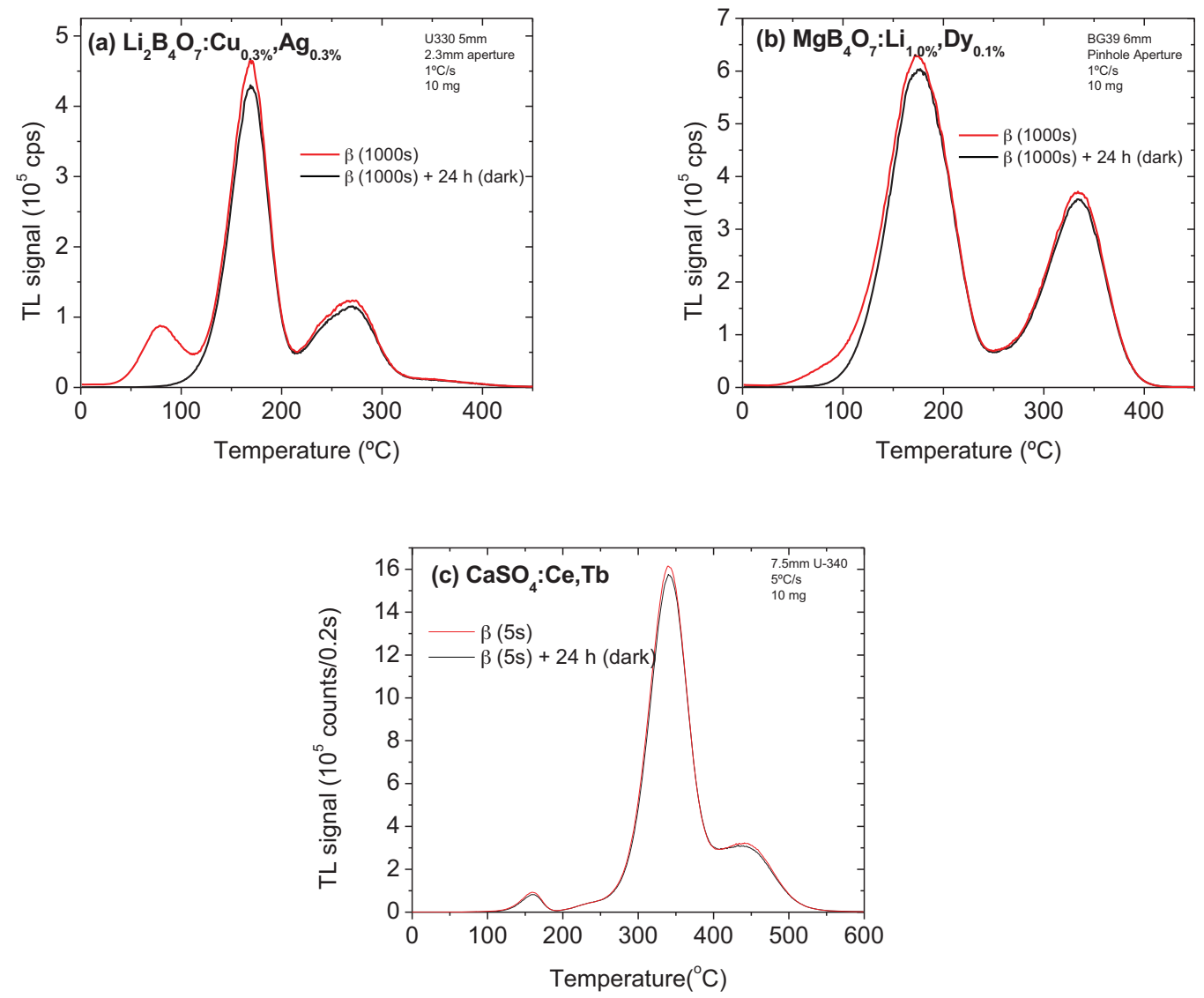

Fig. 37. TL signal of (a) LBO, (b) MBO and (c) CSO immediately after irradiation or after irradiation and $24 \mathrm{~h}$ of storage in the dark. 

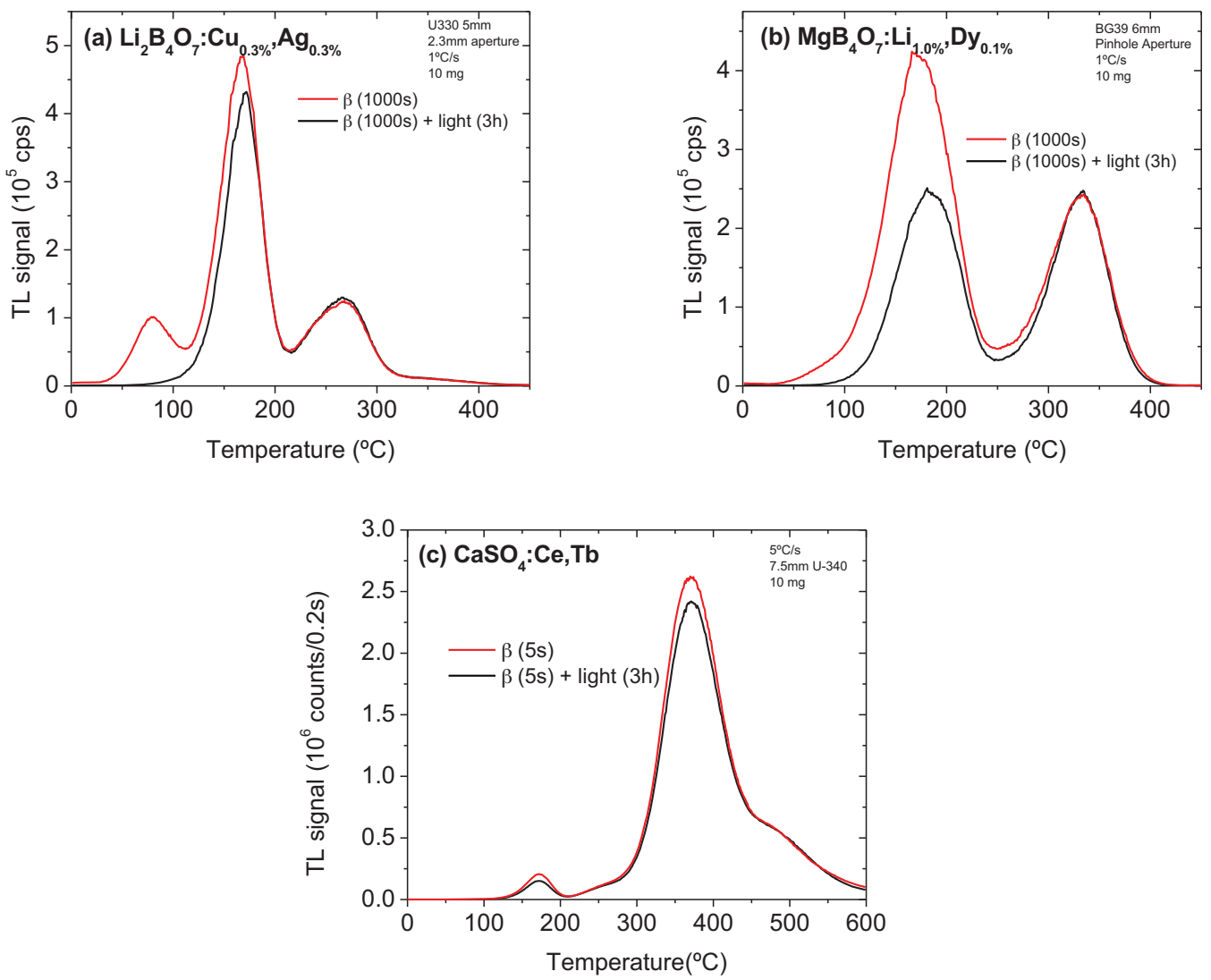

Fig. 38. TL signal of (a) LBO, (b) MBO and (c) CSO immediately after irradiation or after irradiation and $3 \mathrm{~h}$ of light exposure.

\subsection{Specific material developments}

For the most promising materials, additional investigations were carried out to optimize them for temperature sensing.

\subsection{1 $\mathrm{Li}_{2} \mathrm{~B}_{4} \mathrm{O}_{7}(\mathrm{LBO})$}

Effect of co-dopant type and concentration on $\mathrm{Li}_{2} \mathrm{~B}_{4} \mathrm{O}_{7}$

Fig. 39 shows the $\mathrm{TL}$ curves of $\mathrm{Li}_{2} \mathrm{~B}_{4} \mathrm{O}_{7}$ doped with copper and various types and concentration of codopants (In, Dy, Ti or Ag). The data indicates that there is some variability in the TL curve obtained, but they all have very similar characteristics, the main difference being the relative intensity of the TL peaks. The most intense TL were obtained with $\mathrm{Cu}$ and $\mathrm{Ag}$ doping. 

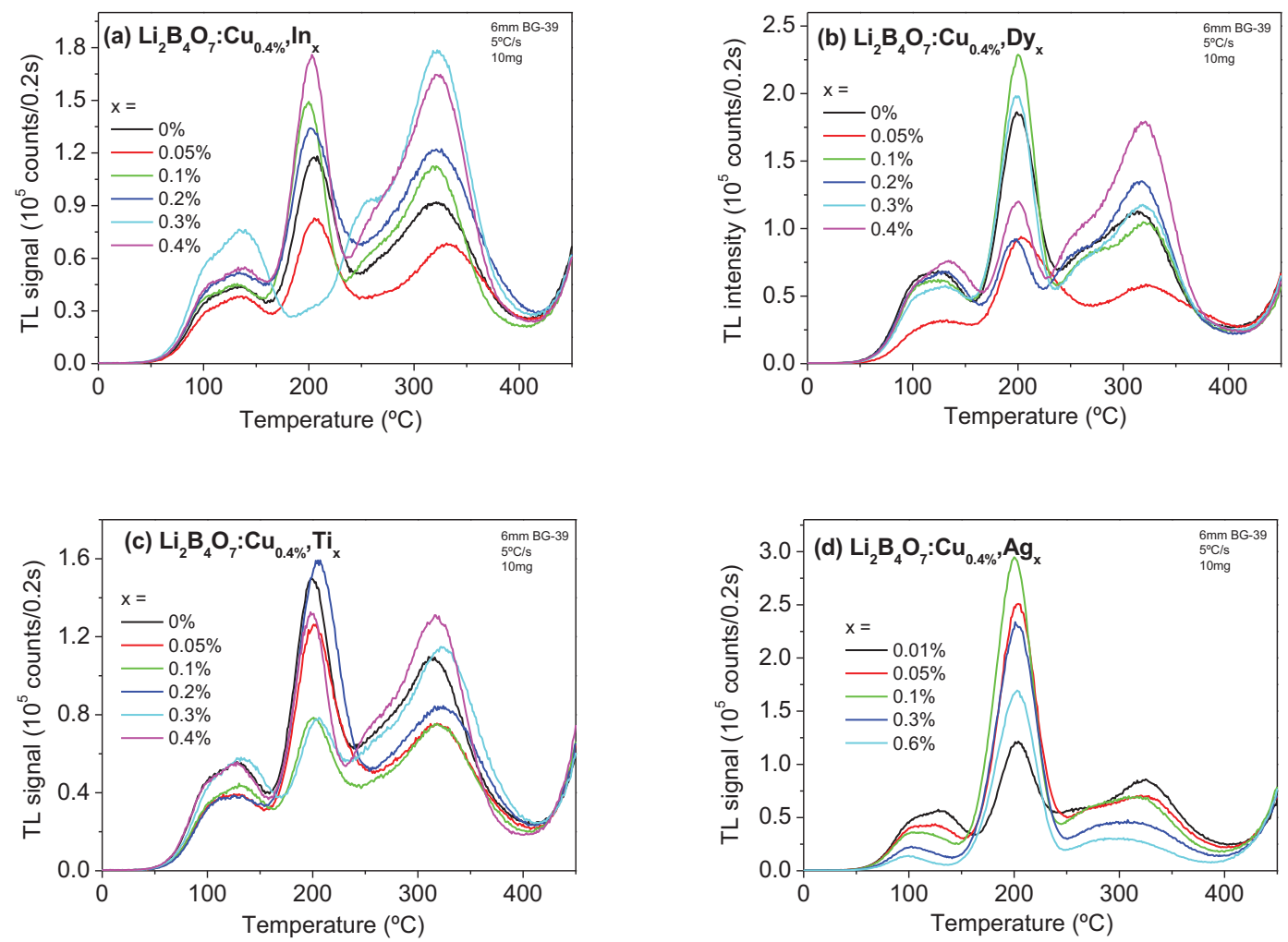

Fig. 39. TL curves of $\mathrm{Li}_{2} \mathrm{~B}_{4} \mathrm{O}_{7}: \mathrm{Cu}$ co-doped with different concentrations of (a) In, (b) Dy, (c) Ti, or (d) Ag.

To check the reproducibility of the synthesis and the effect of $\mathrm{Ag}$ concentration, Fig. 40 shows the TL curves for four different syntheses (combustions) for four Ag doping levels, from $0.01 \%$ to $0.3 \%$. One can observe that the effect of $\mathrm{Ag}$ is to increase the intensity of the $200{ }^{\circ} \mathrm{C}$ peak and improve the reproducibility of the synthesis process. The exact role of $\mathrm{Ag}$ co-doping is not understood.
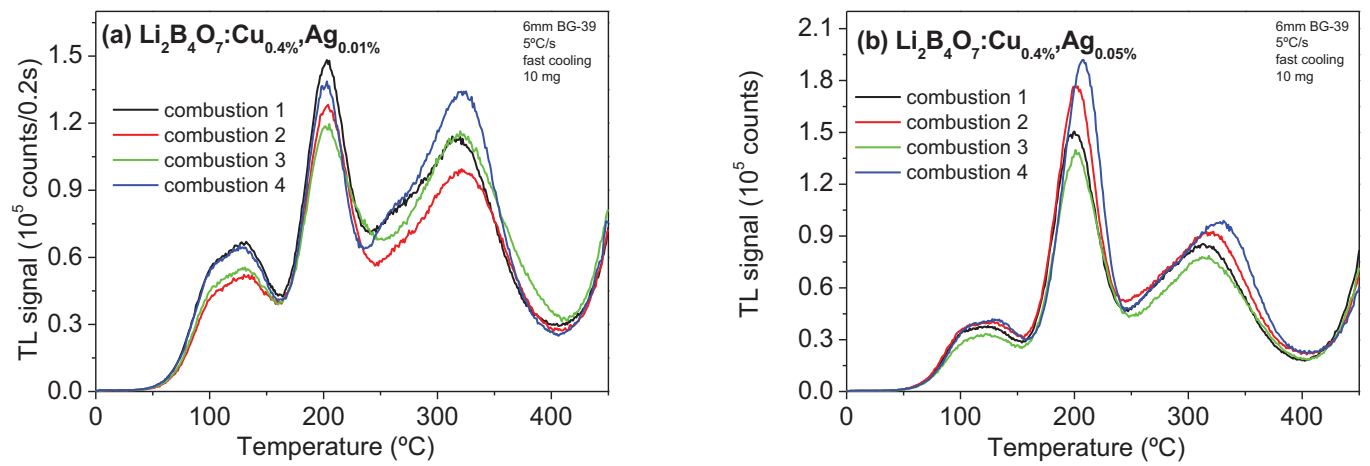

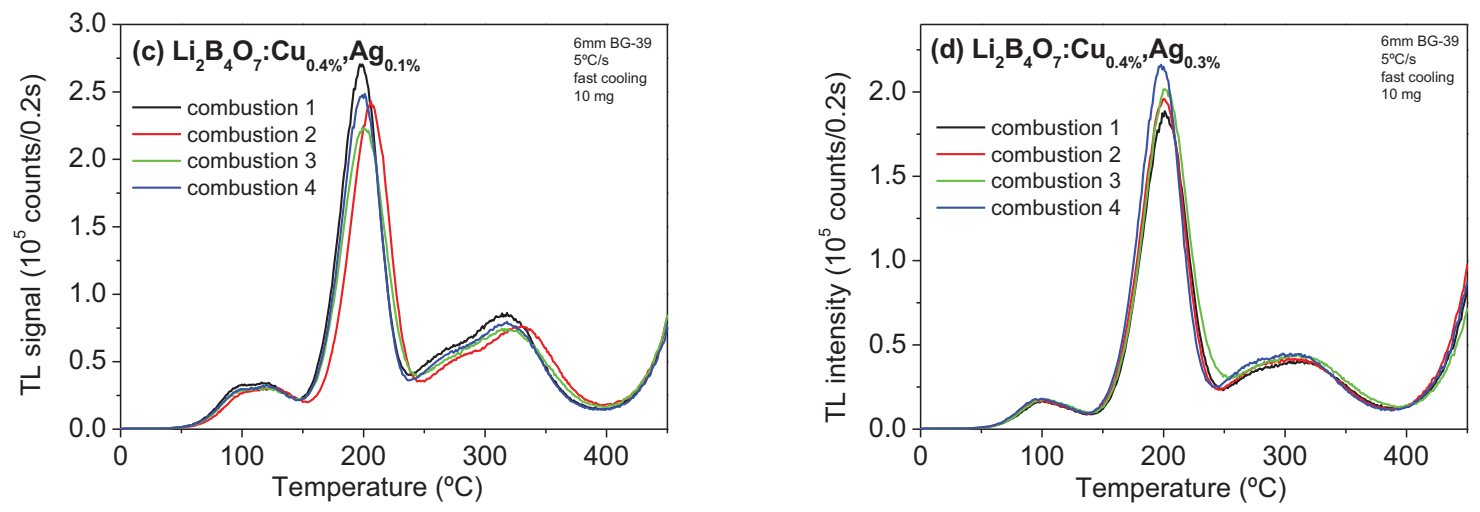

Fig. 40. TL curves of LBO doped with $\mathrm{Cu}(0.4 \%)$ and various concentrations of $\mathrm{Ag}$ : (a) $0.01 \%$, (b) $0.05 \%$, (c) $0.1 \%$, and (d) $0.3 \%)$. Each sample was synthesized 4 times.

\section{Effect of type of fuel on LBO}

Fig. 41 investigates the effect of different fuels used in the preparation of LBO in the final crystal structure. Although we observed that the degree of crystallinity was very different after combustion, after annealing all samples showed similar crystal structures. The conclusion is that fuel does not seem to affect the crystalline structure of the samples.

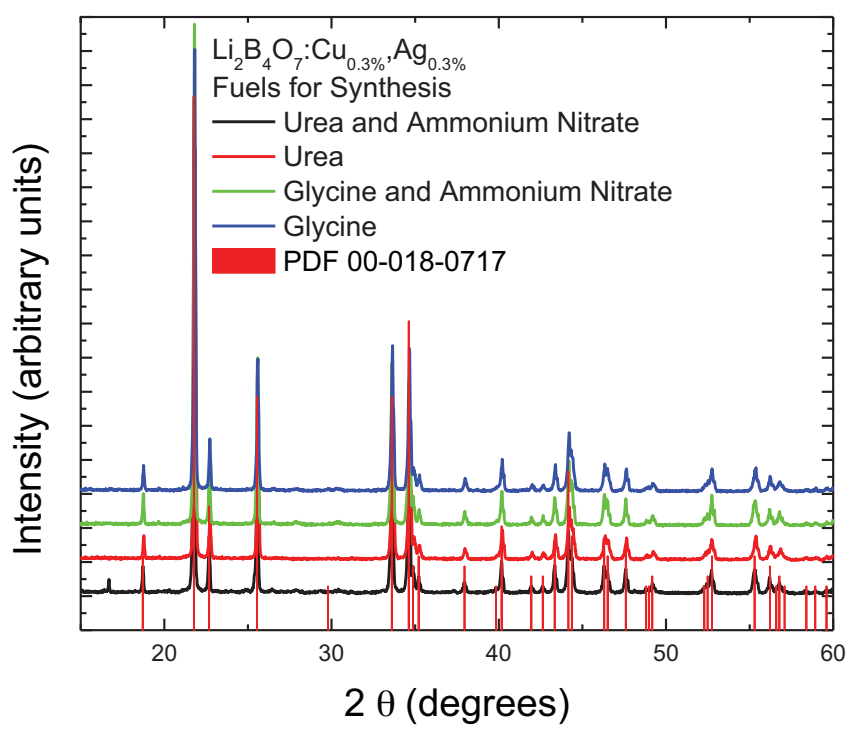

Fig. 41. XRD of LBO synthesized with different fuel combinations after annealing $\left(860^{\circ} \mathrm{C} / 1 \mathrm{~h}\right.$ in preheated furnace, fast cooling). This XRD data indicates that glycine/glycine and ammonium nitrate result in a more complete reaction and more crystalline material following synthesis, however post annealing no difference is seen.

On the other hand, the fuel may have an influence on the relative intensity of the shallow traps and on the degree of photo-bleaching, as previously observed in another oxide prepared by solution combustion synthesis [36], but the variations are too small to allow a definite conclusion. This observation is based on Fig. 42, which compares the photo-bleaching for samples prepared with different fuel types. Based on these results we decided to use glycine in the synthesis of LBO. 

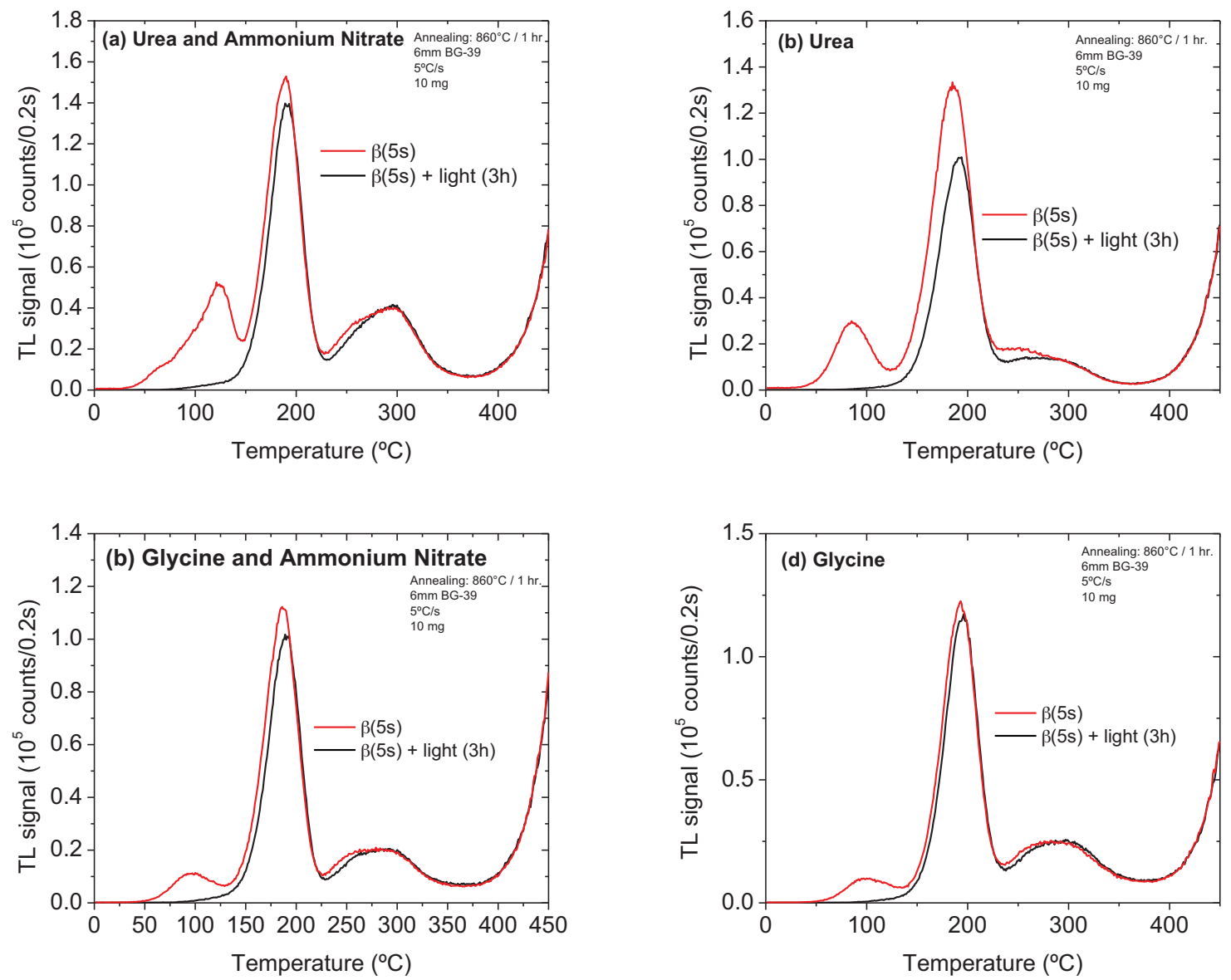

Fig. 42. Photo-bleaching tests of $\mathrm{LBO}\left(\mathrm{Li}_{2} \mathrm{~B}_{4} \mathrm{O}_{7}: \mathrm{Cu}_{0.3 \%}, \mathrm{Ag}_{0.3 \%}\right)$ synthesized with four fuel combinations.

\section{Effect of annealing temperature and duration}

Fig. 43a shows the effect of annealing temperature ( $80 \mathrm{~min}$ annealing) on the TL curves of LBO. For this annealing time, the maximum intensity is achieved around $840^{\circ} \mathrm{C}$. Fig. $43 \mathrm{~b}$ shows the effect of annealing time and various temperatures on the TL of LBO. The effect of annealing on photo-bleaching is shown in Fig. 44. 

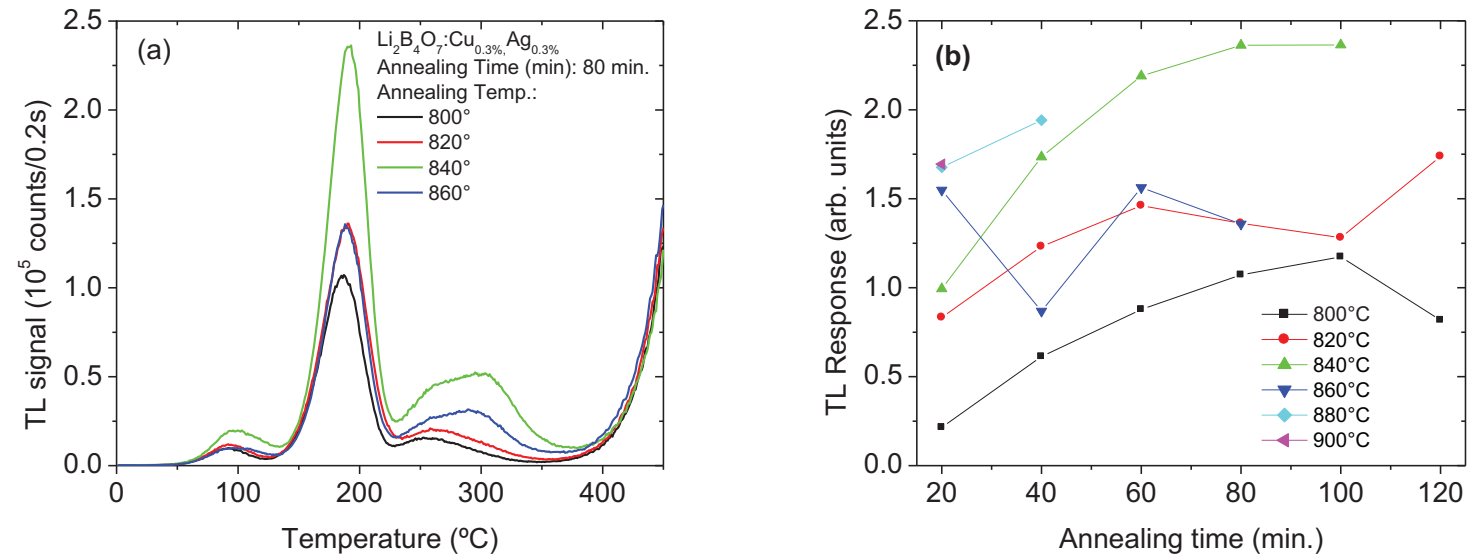

Fig. 43. (a) TL response of LBO for various annealing treatments and (b) representative TL plots for different annealing temperatures showing the effect of annealing on TL response. The TL response was defined as the maximum TL intensity.
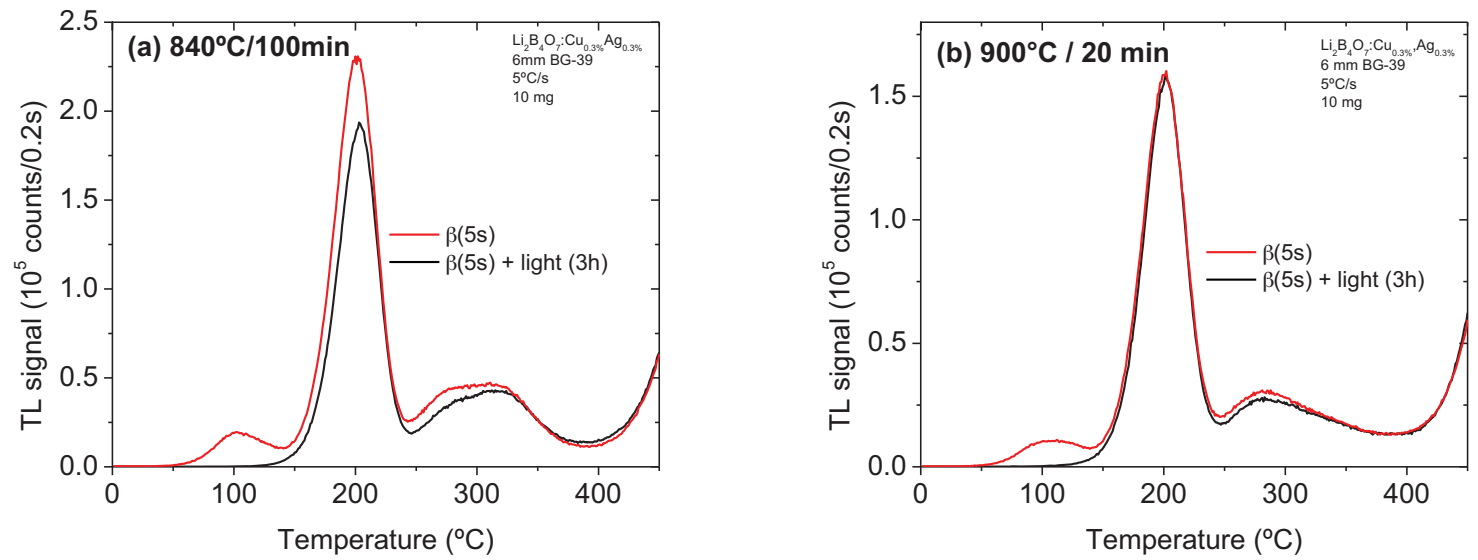

Fig. 44. Photo-bleaching tests of LBO (a) annealed at $840^{\circ} \mathrm{C}$ for $100 \mathrm{~min}$ and (b) annealed at $900^{\circ} \mathrm{C}$ for 20 min showing the effect of annealing.

\section{Effect of $L i$ source on the $T L$ of $\angle B O$ and $\mathrm{Li}_{2} \mathrm{~B}_{4} \mathrm{O}_{7}: \mathrm{Cu}$}

We tested the influence of the $\mathrm{Li}$ source in the synthesis of $\angle B O$ and $\mathrm{Li}_{2} \mathrm{~B}_{4} \mathrm{O}_{7}: \mathrm{Cu}$. We synthesized one sample using $\mathrm{Li}_{2} \mathrm{CO}_{3}$ dissolved in nitric acid and another using $\mathrm{LiNO}_{3}$ as the $\mathrm{Li}$ source. The TL curves for both samples are presented in Fig. 45, showing the difference in TL peak shape. Similar differences were observed for samples doped only with $\mathrm{Cu}\left(\mathrm{Li}_{2} \mathrm{~B}_{4} \mathrm{O}_{7}: \mathrm{Cu}\right)$, as shown in Fig. 46. We do not have a clear explanation for these differences, but they may be related to impurities found in the different reagents and/or impurities incorporated in the final material, e.g., C versus $\mathrm{N}$. In any case, these data are evidence of the variability that can be observed due to the synthesis method and reagents used. 

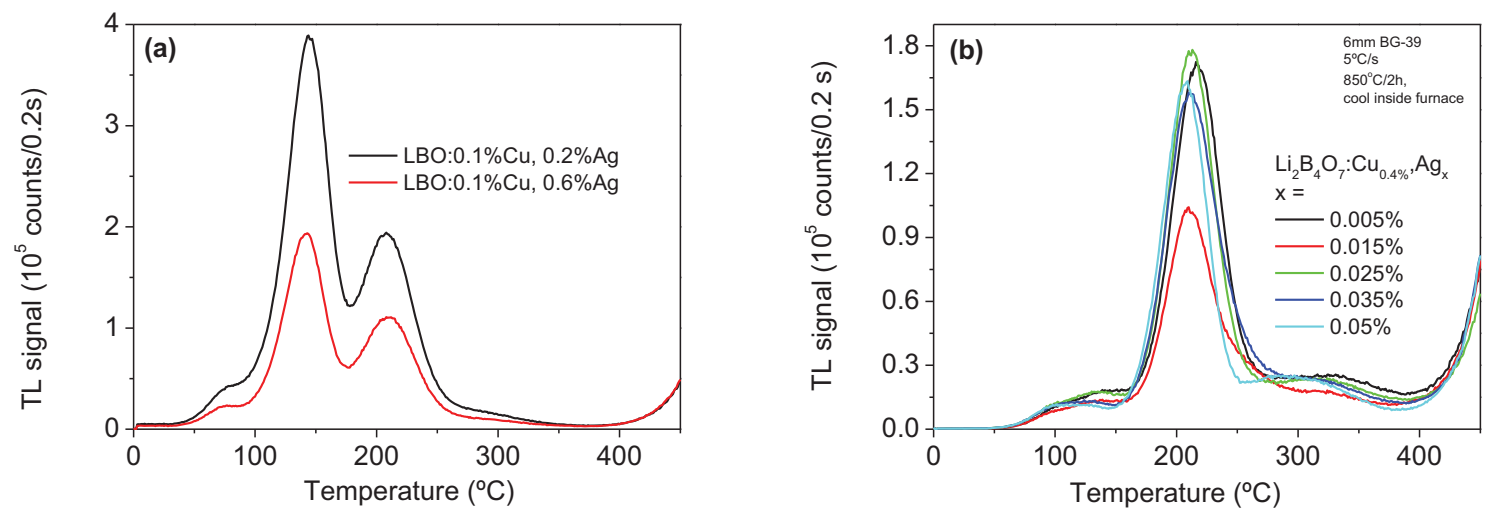

Fig. 45. TL curves of $\mathrm{LBO}$ obtained using (a) $\mathrm{Li}_{2} \mathrm{CO}_{3}$ dissolved in nitric acid or (b) $\mathrm{LiNO}_{3}$ as the $\mathrm{Li}$ source.
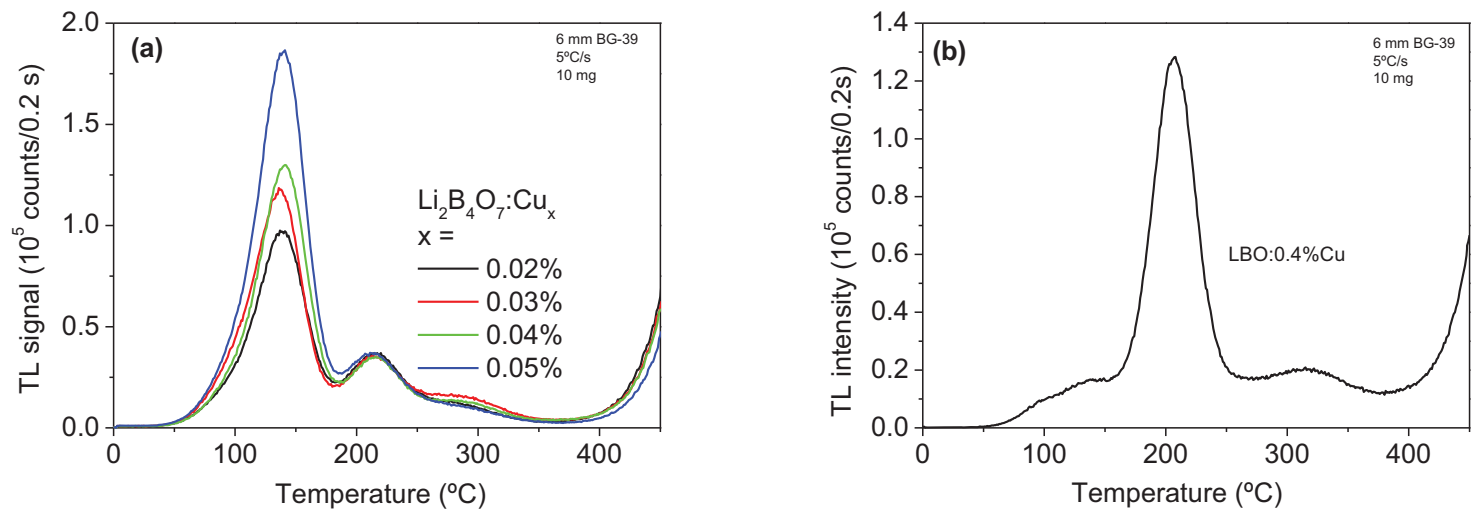

Fig. 46. TL curves of $\mathrm{Li}_{2} \mathrm{~B}_{4} \mathrm{O}_{7}: \mathrm{Cu}$ obtained using (a) $\mathrm{Li}_{2} \mathrm{CO}_{3}$ dissolved in nitric acid or (b) $\mathrm{LiNO}_{3}$ as the $\mathrm{Li}$ source.

\section{Effect of annealing cooling regime on $L B O$}

The effect of cooling after annealing on the TL properties of LBO can be observed by comparing the data obtained with fast cooling (Fig. 40d) and slow cooling (Fig. 45b). We observed that if LBO is cooled inside the furnace, the high temperature TL peak is not as prominent as when the cooling is fast outside the furnace.

\subsection{2 $\mathrm{MgB}_{4} \mathrm{O}_{7}(\mathrm{MBO})$}

We carried out a systematic investigation of MBO doped with lanthanides to develop a model for the $\mathrm{TL}$ in this material that could assist in better engineering the trapping centers associated with the TL peaks [6].

Various samples of MBO doped with different lanthanides were synthesized by Solution Combustion Synthesis (SCS) and characterized using XRD, radioluminescence (RL), and TL. As an example, Fig. 47 shows the TL emission spectra of samples doped with different lanthanides (Ln). The data presented in this figure suggest that there are two types of recombination centers active in this material: recombination centers for electrons $(\mathrm{Ce}, \mathrm{Mn})$ and recombination centers for holes $(\mathrm{Sm}, \mathrm{Gd}, \mathrm{Tb}$, Dy, and $\mathrm{Tm}$ ). Furthermore, specific trapping centers are associated with these recombination centers (Fig. 48). 
These and other results led to the proposed TL mechanism for electron and hole traps (Fig. 49). The mechanism proposed suggests the appropriate dopants for specific trapping centers, therefore providing a more guided approach to the development of TL materials.

(a) Ce

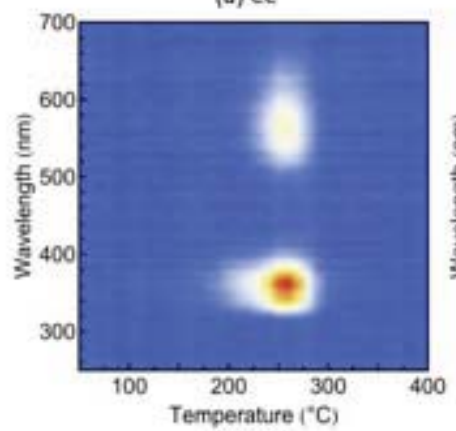

(d) $\mathrm{Sm}$

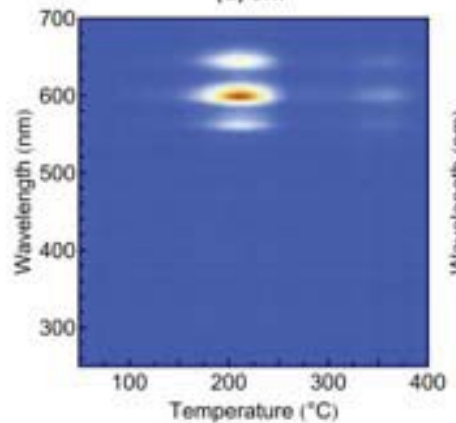

(g) Tb

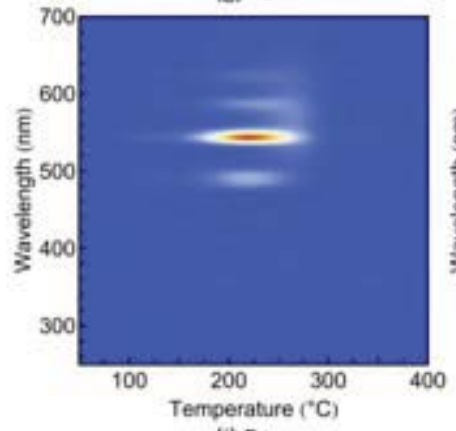

(j) Er

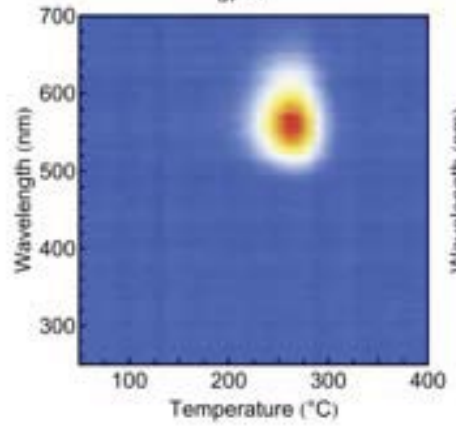

(b) $\operatorname{Pr}$

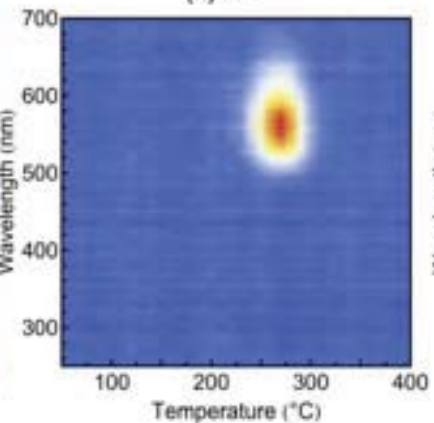

(e) Eu

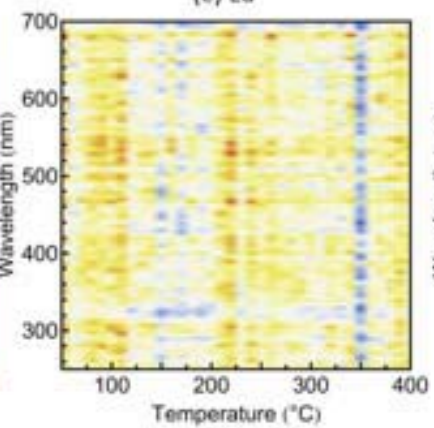

(h) Dy

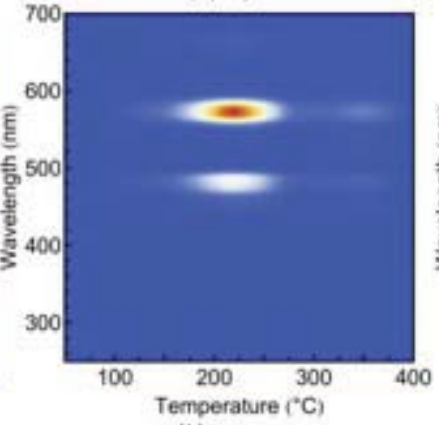

(k) Tm

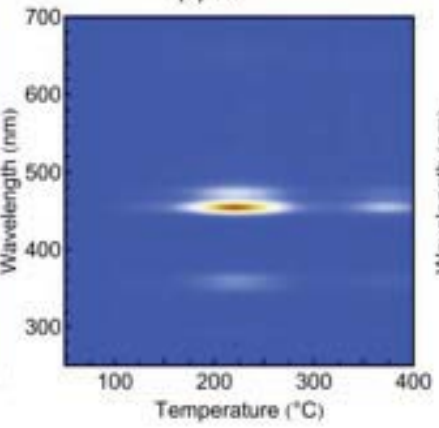

(c) Nd

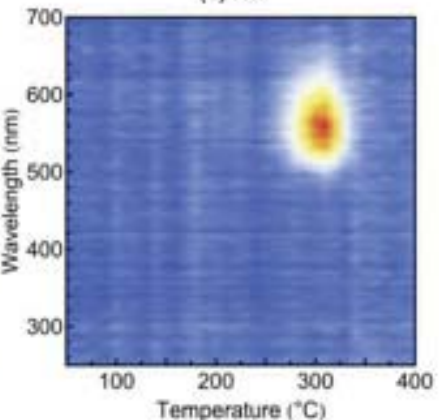

(f) Gd

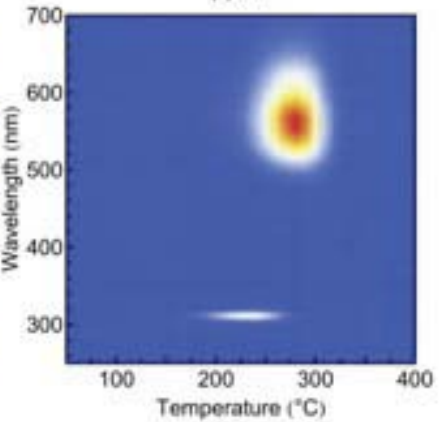

(i) $\mathrm{Ho}$

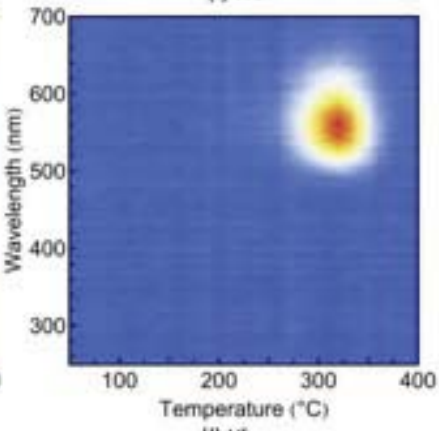

(I) $\mathrm{Yb}$

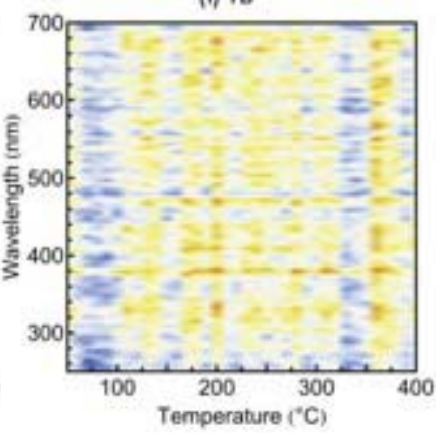

Fig. 47. TL emission spectra of $\mathrm{MgB}_{4} \mathrm{O}_{7}$ doped with different lanthanides. 


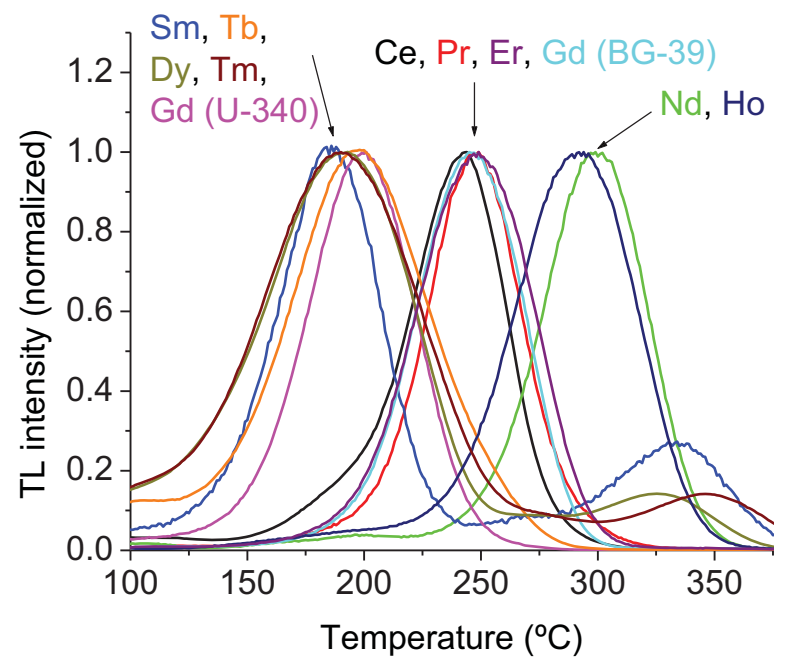

Fig. 48. Trapping centers associated with specific dopants. Based on interpretation of the data, the TL peaks at $180{ }^{\circ} \mathrm{C}$ and $350{ }^{\circ} \mathrm{C}$ are likely associated with hole traps, whereas the ones at $250{ }^{\circ} \mathrm{C}$ and $300^{\circ} \mathrm{C}$ are likely associated with electron traps.

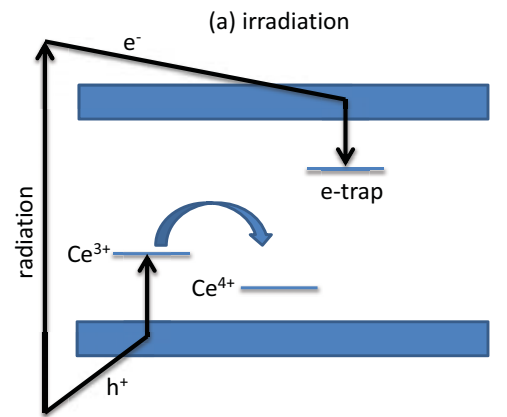

(c) irradiation

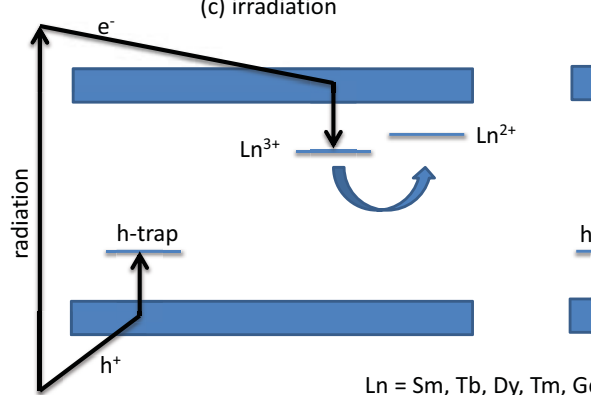

$\mathrm{Ln}=\mathrm{Sm}, \mathrm{Tb}, \mathrm{Dy}, \mathrm{Tm}, \mathrm{Gd}$ (b) readout

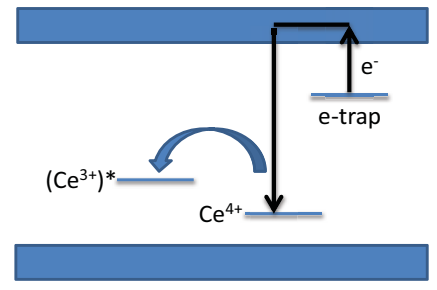

(d) readout

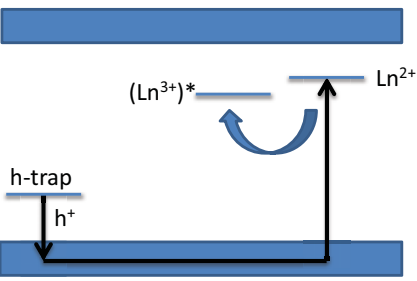

Fig. 49. Proposed TL mechanism for $\mathrm{MgB}_{4} \mathrm{O}_{7}$ for electron traps during (a) irradiation and (b) readout, and for hole traps during (c) irradiation and (d) readout, as well as associated luminescence centers.

\section{Transition metal doping}

We tested if specific transition metals could be used to introduce additional trapping centers in MBO and extend the temperature range of applicability of this material.

Transition metals (TM) with ionic radii close to that of magnesium and having a greater electron affinity than magnesium were selected (Table 7). MBO samples were synthesized by SCS with various transition 
metals each singly and co-doped with Ce, Gd, or Li (Table 8). The samples were then analyzed using TL and RL.

Table 7. Radii and electron affinity of dopants and $\mathrm{Mg}$.

\begin{tabular}{|ccc|}
\hline Ion & $\begin{array}{c}\text { Ionic Radius } \\
(\AA)\end{array}$ & $\begin{array}{c}\text { Electron Affinity } \\
\text { (Pauling) }\end{array}$ \\
\hline $\mathbf{M g}$ & 0.72 & 1.31 \\
\hline $\mathbf{C r}$ & 0.62 & 1.66 \\
\hline $\mathrm{Mn}$ & 0.67 & 1.55 \\
\hline $\mathrm{Co}$ & 0.65 & 1.88 \\
\hline $\mathbf{N i}$ & 0.69 & 1.91 \\
\hline $\mathrm{Cu}$ & 0.73 & 1.9 \\
\hline $\mathbf{Z n}$ & 0.74 & 1.65 \\
\hline
\end{tabular}

Table 8. Samples prepared in this study and dopant concentrations. $\mathrm{TM}=\mathrm{Cr}, \mathrm{Mn}, \mathrm{Co}, \mathrm{Ni}, \mathrm{Cu}$ or $\mathrm{Zn}$.

\begin{tabular}{|c|c|c|}
\hline & Concentrations & Compared with \\
\hline Singly Doped & $\mathrm{MgB}_{4} \mathrm{O}_{7}: \mathrm{TM}_{1.0 \%}$ & \\
\hline \multirow{3}{*}{ Doubly Doped } & $\mathrm{MgB}_{4} \mathrm{O}_{7}: \mathrm{Ce}_{1.0 \%}, \mathrm{TM}_{1.0 \%}$ & $\mathrm{MgB}_{4} \mathrm{O}_{7}: \mathrm{Ce}_{1.0 \%}$ \\
\hline & $\mathrm{MgB}_{4} \mathrm{O}_{7}: \mathrm{Gd}_{1.0 \%}, \mathrm{TM}_{1.0 \%}$ & $\mathrm{MgB}_{4} \mathrm{O}_{7}: \mathrm{Gd}_{1.0 \%}$ \\
\hline & $\mathrm{MgB}_{4} \mathrm{O}_{7}: \mathrm{Li}_{1.0 \%}, \mathrm{TM}_{1.0 \%}$ & $\mathrm{MgB}_{4} \mathrm{O}_{7}: \mathrm{Li}_{1.0 \%}$ \\
\hline
\end{tabular}

$\mathrm{RL}$ measurements revealed that the transition metals introduced no new luminescence centers with the exception of $\mathrm{Mn}$, which we previously confirmed as acting as an efficient luminescence center with emission at $\sim 550 \mathrm{~nm}$ [6]. Fig. 50 shows the TL for the materials of at least moderate brightness.

Overall, the only dopant combination that may warrant more investigation is (Gd,Co). MBO:Gd,Co showed a high temperature peak around $400{ }^{\circ} \mathrm{C}$, but the signal was not as intense as other materials already developed in our laboratory. 

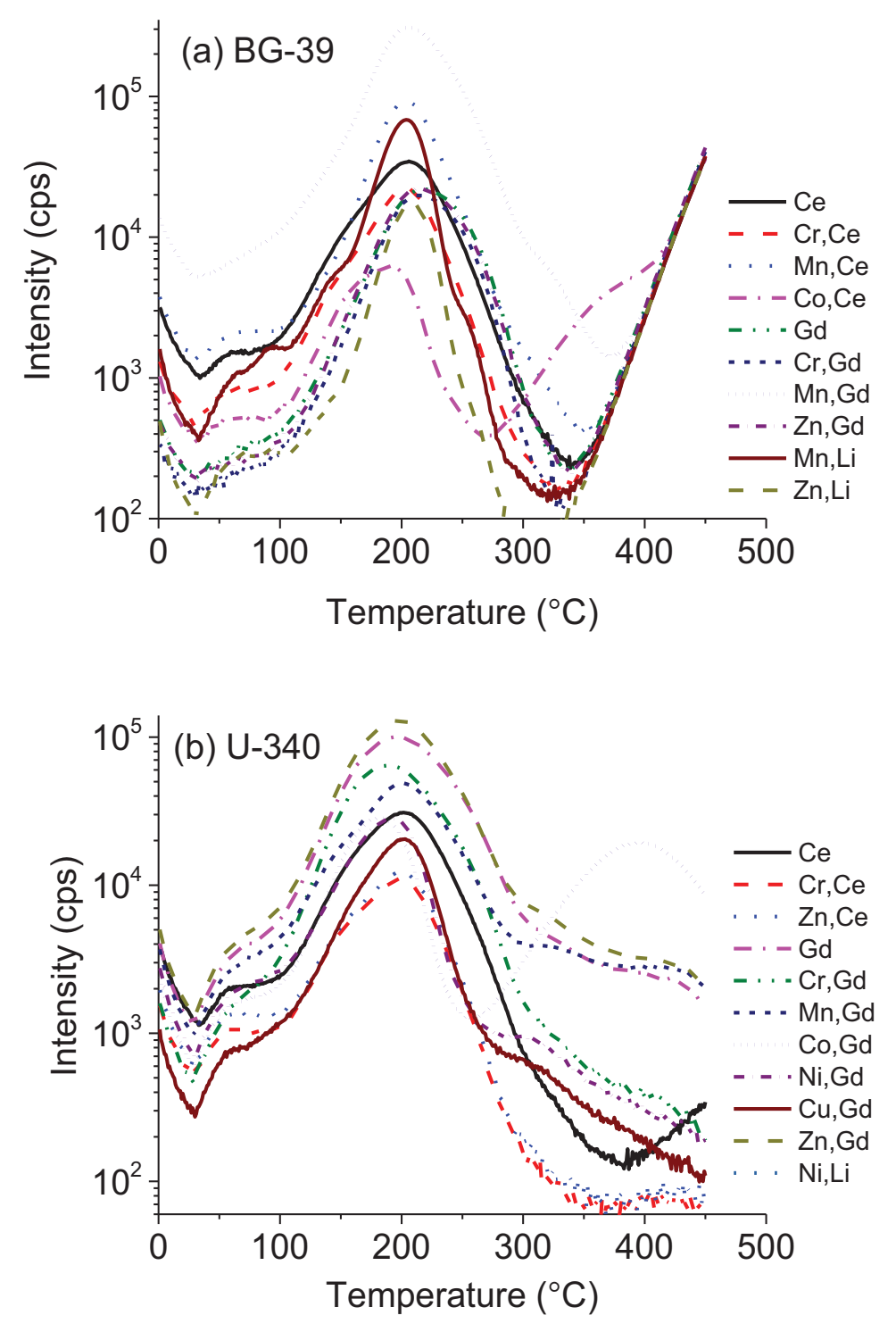

Fig. 50. TL curves of MBO with various dopants, measured using (a) visible transmitting filters (Schott BG-39) or (b) UV transmitting filters (Hoya U-340). The legends denote the dopants for each particular sample.

\section{Sensitization}

It has been observed that MBO's high temperature TL peak increases in intensity with multiple irradiations and TL readouts, which may result in inaccurate temperature measurements. Therefore, we tried to understand the cause for this increase in the intensity of the high temperature TL peak (sensitization).

Based on previous data, it appeared that either the dose of radiation or the atmosphere during heating could be related to the high temperature peak sensitization. To investigate that, multiple $10 \mathrm{mg}$ aliquots were taken from the same synthesis batch of $\mathrm{MBO}$ and pre-irradiated with various doses between $0 \mathrm{~Gy}$ and $1500 \mathrm{~Gy}$. A TL readout was then performed on all samples, except the control, to empty all TL traps. This cycle was run with an air atmosphere for half of the samples and a nitrogen 
atmosphere for the other half. Next, all samples were irradiated with $100 \mathrm{~Gy}$, run through a TL cycle $\left(\mathrm{N}_{2}\right.$ atmosphere), irradiated a second time with $100 \mathrm{~Gy}$, and run through a second identical TL cycle. Data for the two irradiations of 100 Gy were compared to identify any additional sensitization of the samples.

The data in Fig. 51 show that the sensitization does depend on the atmosphere during readout or on previous irradiation. The data seem to indicate only a dependence on the material being heated and cooled. This is likely due to trap modification in the heating and cooling process.

The next step is to test our hypothesis by comparing samples that we heat and fast cool with samples that are slow cooled after heating.
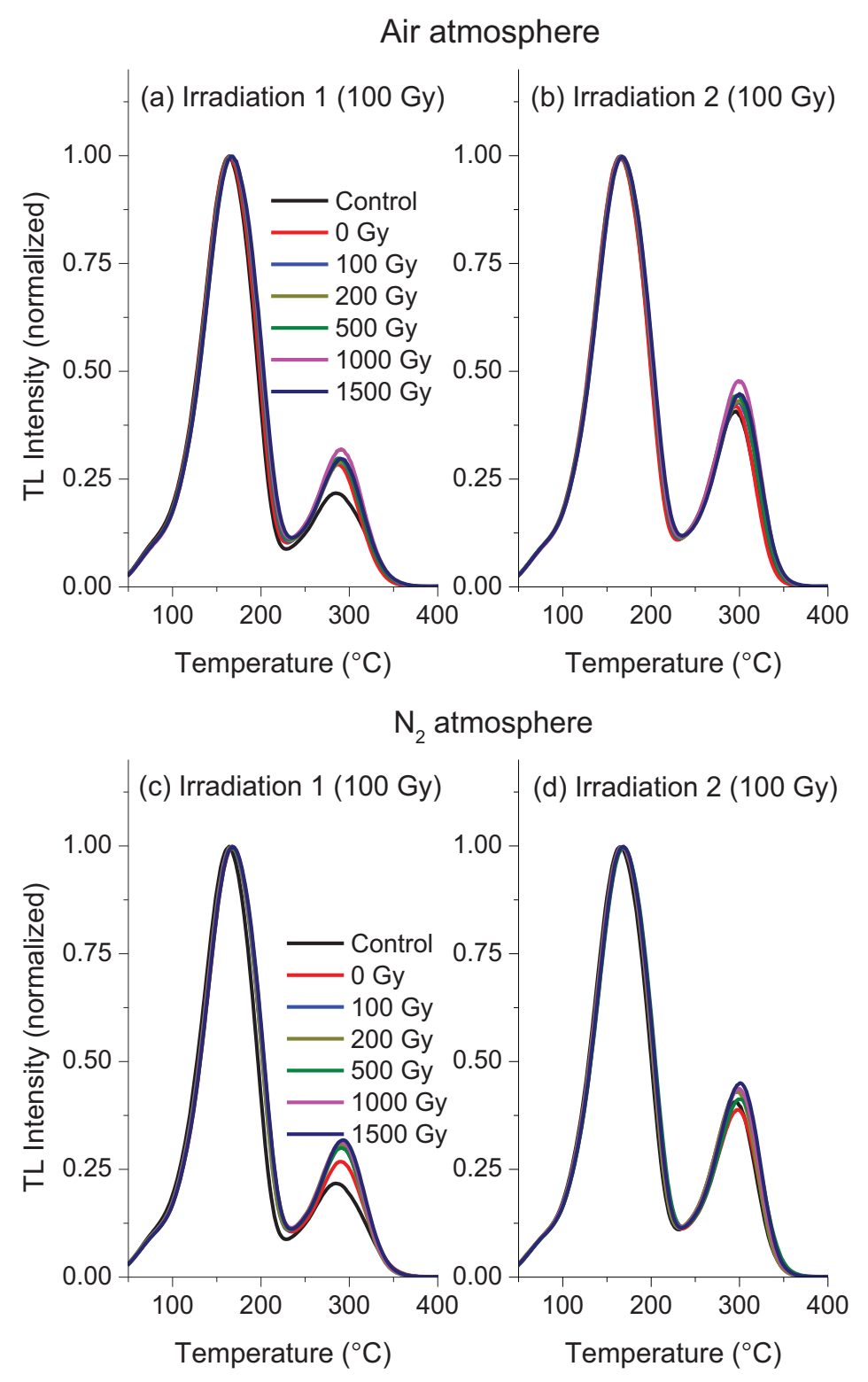

Fig. 51. Sensitization of $\mathrm{MBO}$ as a function of pre-sensitization dose after thermal cleaning in (a) air or in (b) $\mathrm{N}_{2}$ atmosphere. 


\subsection{3 $\mathrm{CaSO}_{4}(\mathrm{CSO})$}

We identified $\mathrm{CaSO}_{4}: \mathrm{Ln}$ as one of the most promising materials for temperature sensing, because of our ability to introduce different TL peaks using selected co-dopants, and because of the presence of high temperature TL peaks. CSO also seems to be of interest for temperature sensing application, because of the potential biotoxicity of other fluoride-based compounds.

In previous tests we have used $\mathrm{CSO}: \mathrm{Ce}, \mathrm{Tb}$ for temperature sensing because of its applicability to temperatures higher than LBO:Ce,Li and MBO:Dy, Li $[10,11]$. We have also tested the influence of single dopants in the TL curve. Here we investigate the effect of co-dopants to introduce TL peaks at even higher temperatures.

\section{Basic characterization}

CSO has been identified as a material of interest for temperature sensing for several reasons. First of all, the TL from this material is light insensitive. Second, there are TL peaks at high temperature that could be used to extend the measurement range of the thermal sensors. Third, the TL seems to vary considerably depending on the dopants. Therefore, in this study we looked into the variation in TL curves for Ce- and Tb-doped CSO samples.

Fig. 52 show the RL emission spectrum from CSO:Ce,Tb. One can see both $\mathrm{Ce}^{3+}$ emission bands and $\mathrm{Tb}^{3+}$ emission lines. Because of these luminescence centers, we investigated the TL using detection in the $290-390 \mathrm{~nm}$ range (using Hoya U-340 filters) or in the visible range (using Schott BG-39 filters).

Fig. 53 compares the TL curves for various dopants and different optical filters used in the TL readout. The range of TL curves that can be observed depends on the dopants and optical filters. For Ce-doped samples, the $\mathrm{TL}$ is dominated by a peak at $\sim 450{ }^{\circ} \mathrm{C}$. For Tb-doped samples, the peak at $250{ }^{\circ} \mathrm{C}$ dominates. For co-doped samples an intermediate peak at $\sim 350{ }^{\circ} \mathrm{C}$ is observed.

These results demonstrates the possibility of introducing TL peaks at high temperatures by appropriate doping of CSO, with the potential of extending the range of temperature sensing towards higher temperatures. 


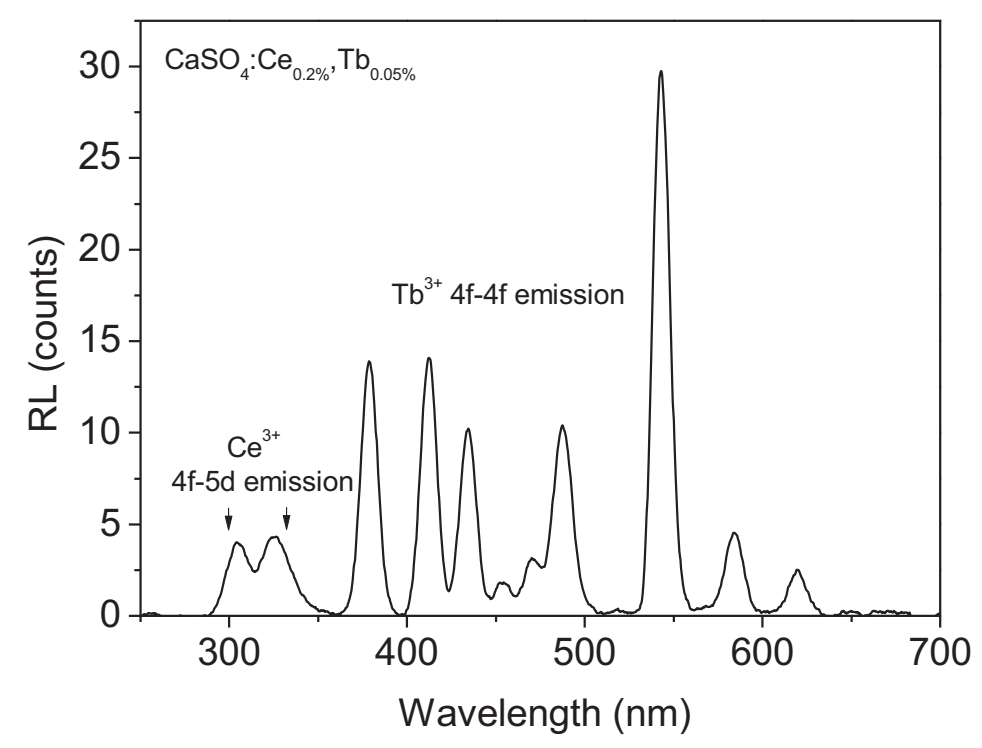

Fig. 52. RL emission spectra of $\mathrm{CaSO}_{4}: \mathrm{Ce}, \mathrm{Tb}$ produced at $\mathrm{OSU}$.

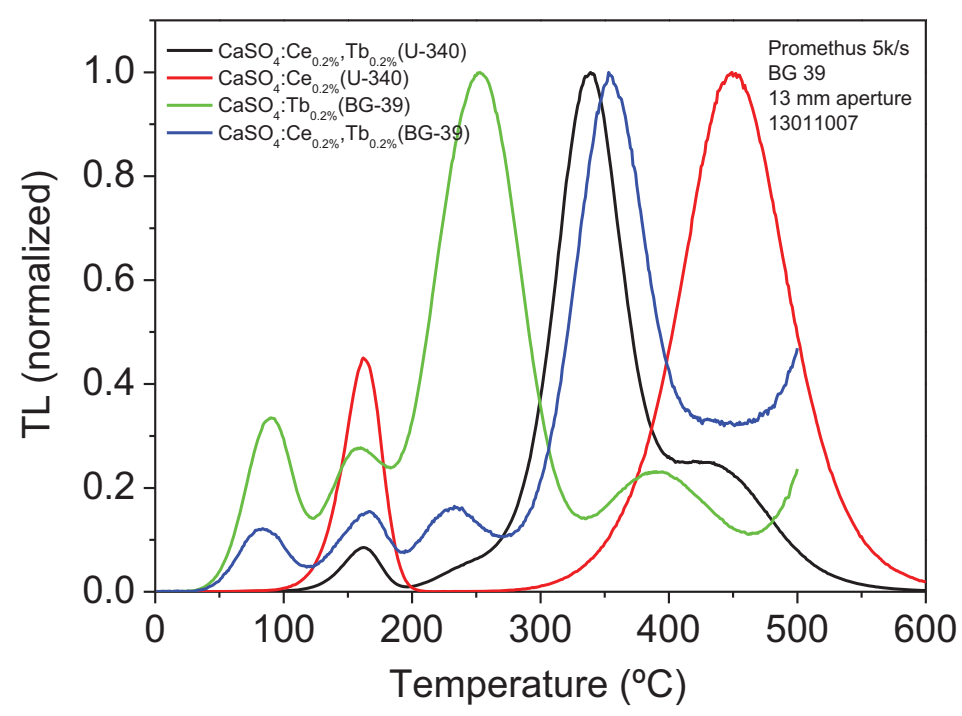

Fig. 53. TL of $\mathrm{CaSO}_{4}$ with various dopants produced at OSU. The TL peak structure changes considerably with the type of dopant, allowing us to fine-tune the temperature of the peak. 
(a) BG-39 filter

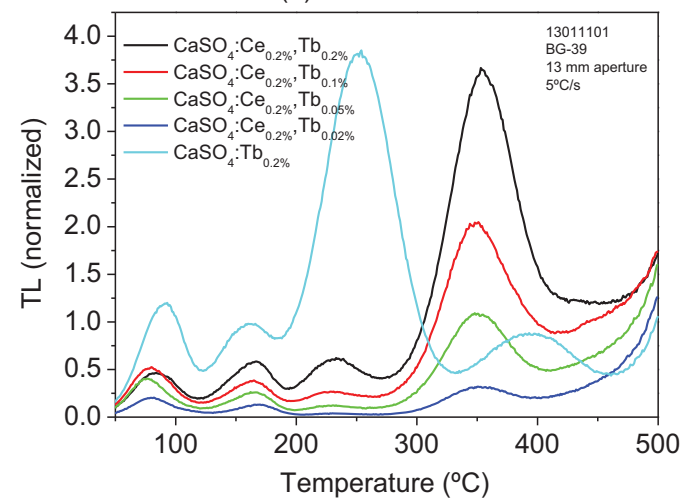

(b) U-340 filter

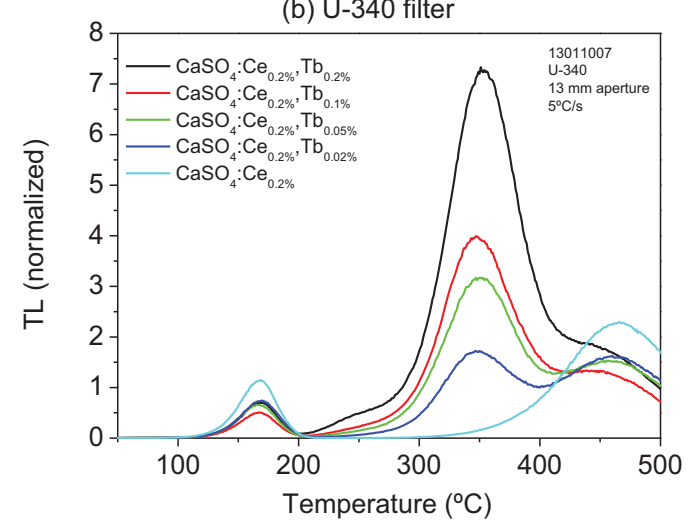

Fig. 54. TL curves of $\mathrm{CaSO}_{4}$ produced at OSU with various dopant concentrations. The TL was measured with either (a) BG-39 filters (monitors $\mathrm{Tb}^{3+}$ emission) or (b) $\mathrm{U}-340$ filters (monitors $\mathrm{Ce}^{3+}$ emission).

\section{Effect of dopants}

We investigate CSO doped with different lanthanides of major interest for luminescence ( $\mathrm{Ce}, \mathrm{Pr}, \mathrm{Nd}$, $\mathrm{Sm}, \mathrm{Eu}, \mathrm{Gd}, \mathrm{Tb}, \mathrm{Dy}, \mathrm{Ho}, \mathrm{Er}, \mathrm{Tm}, \mathrm{Yb}$ ) and co-dopants (Ce,Tb). The resultant RL emission, $\mathrm{TL}$ curves, and TL emission spectra were characterized.

As an example of the measurements carried out, Fig. 55a shows the $\mathrm{RL}$ emission from $\mathrm{CaSO}_{4}: \mathrm{Eu}$, indicating $\mathrm{Eu}^{2+}$ emission at $380 \mathrm{~nm}$ and weaker $\mathrm{Eu}^{3+}$ emission around $\sim 600 \mathrm{~nm}$. Fig. $55 \mathrm{~b}$ shows that the main TL peak of this sample is at $180^{\circ} \mathrm{C}$, with a higher temperature peak around $\sim 250{ }^{\circ} \mathrm{C}$. Finally, Fig. $55 \mathrm{C}$ shows that the TL peak at $180^{\circ} \mathrm{C}$ is associated with $\mathrm{Eu}^{2+}$ emission, whereas the secondary peak at $250{ }^{\circ} \mathrm{C}$ is associated with $\mathrm{Eu}^{3+}$ emission.

(a)

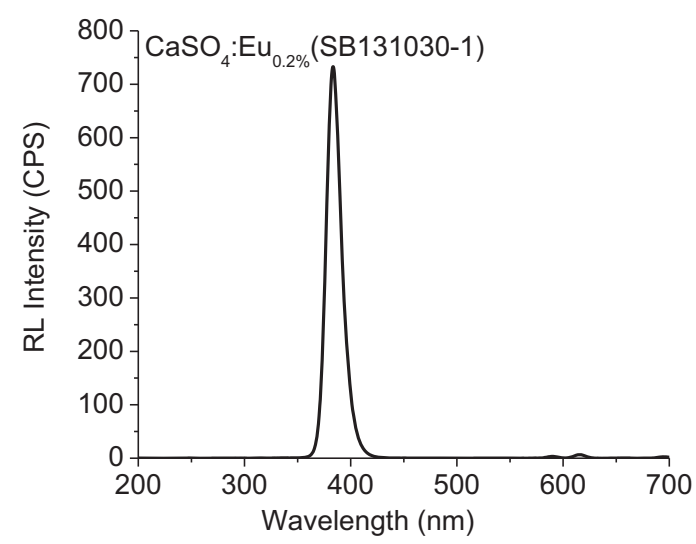

(b)

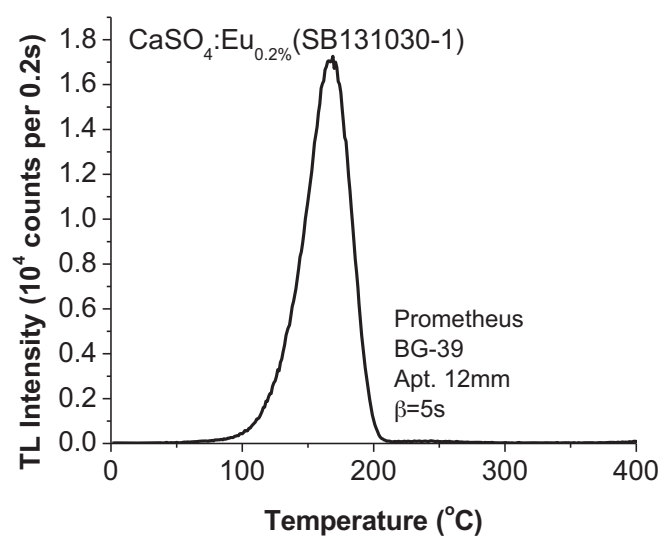


(c)

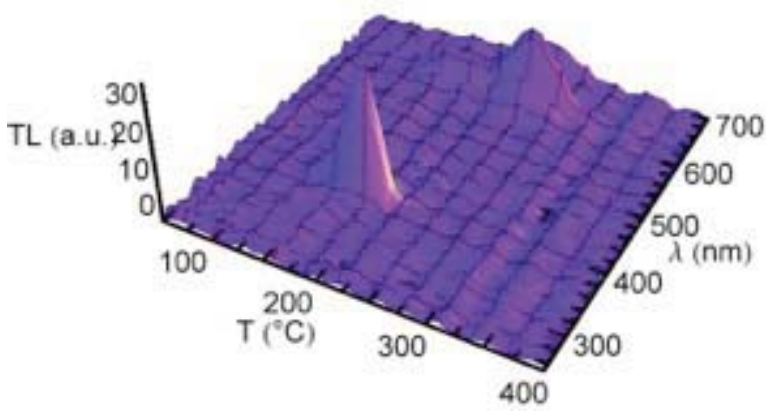

Fig. 55. (a) RL spectrum of $\mathrm{CaSO}_{4}: \mathrm{Eu}$; (b) $\mathrm{TL}$ curve of $\mathrm{CaSO}_{4}: \mathrm{Eu}\left(5^{\circ} \mathrm{C} / \mathrm{s}, 0.5 \mathrm{~Gy}\right)$; and (c) TL emission spectrum of $\mathrm{CaSO}_{4}: \mathrm{Eu}$ $\left(10^{\circ} \mathrm{C} / \mathrm{s}, 100 \mathrm{~Gy}\right)$.

The undoped CSO sample (Fig. 56) shows a weak TL emission at $150{ }^{\circ} \mathrm{C}$ emission associated with unidentified luminescence centers due to intrinsic defects or contaminants.

On the other hand, when the samples are doped with lanthanides a variety of luminescence centers are introduced. Fig. 57 presents the TL spectra of all CSO:Ln samples synthesized in this study. From these results, we can identify nine types of luminescence centers participating in the TL process of $\mathrm{CaSO}_{4}$ : $\mathrm{Ce}^{3+}, \mathrm{Pr}^{3+}, \mathrm{Sm}^{3+}, \mathrm{Eu}^{3+}, \mathrm{Eu}^{2+}, \mathrm{Gd}^{3+}, \mathrm{Tb}^{3+}, \mathrm{Dy}^{3+}$ and $\mathrm{Tm}^{3+}$.

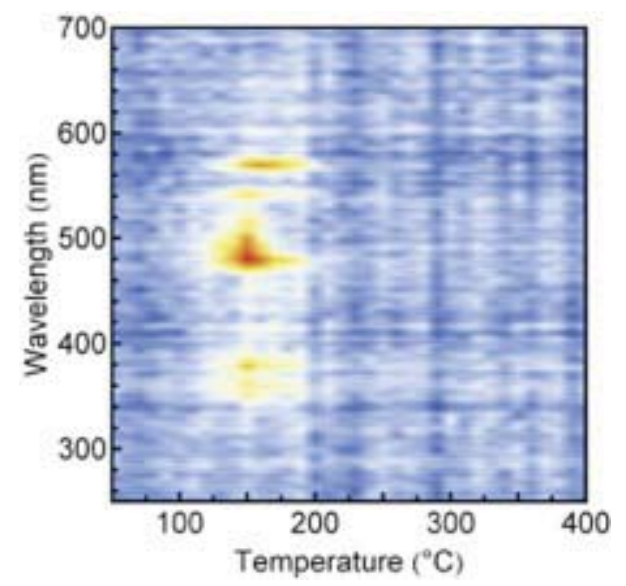

Fig. 56. TL emission spectrum of undoped $\operatorname{CSO}\left(10^{\circ} \mathrm{C} / \mathrm{s}, 100 \mathrm{~Gy}\right)$. The weak emissions observed could be due to contaminants. 
(a) $\mathrm{Ce}$

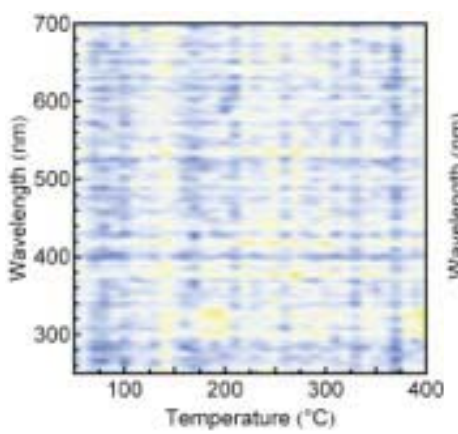

(d) $\mathrm{Sm}$

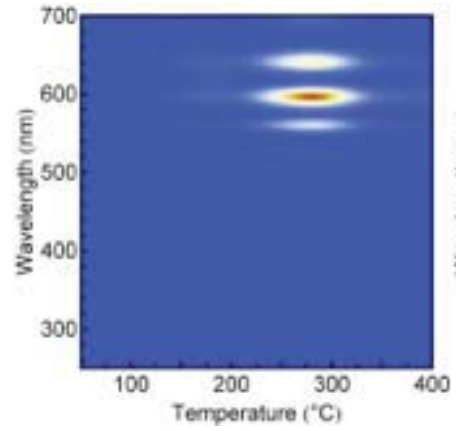

(g) $\mathrm{Tb}$

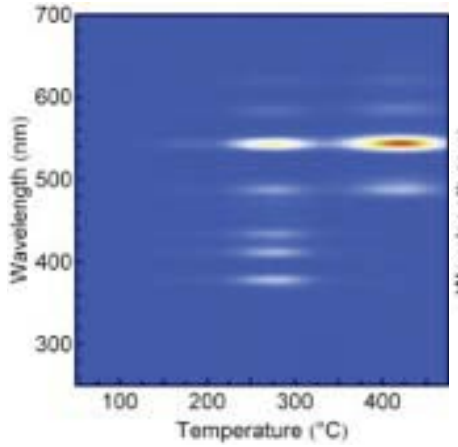

(j) $\mathrm{Er}$

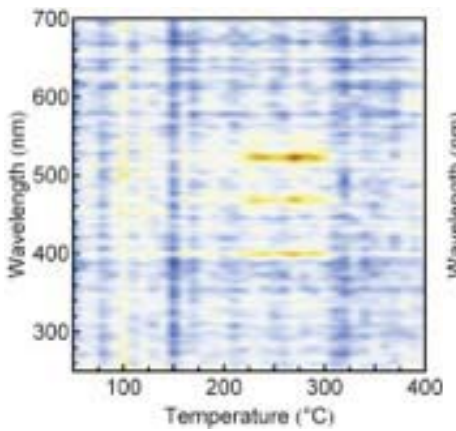

(b) $\operatorname{Pr}$

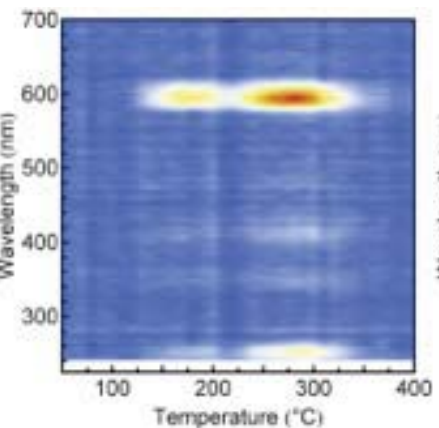

(e) Eu

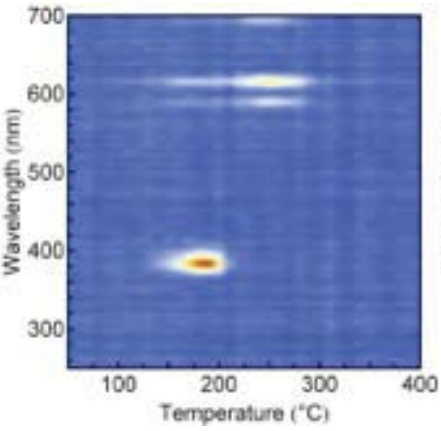

(h) Dy

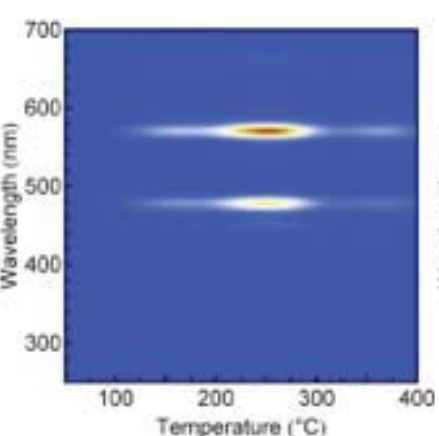

(k) $\mathrm{Tm}$

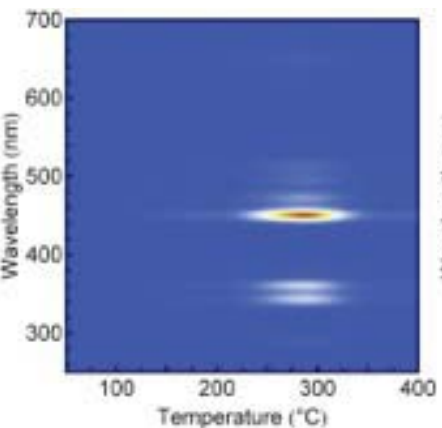

(c) $\mathrm{Nd}$

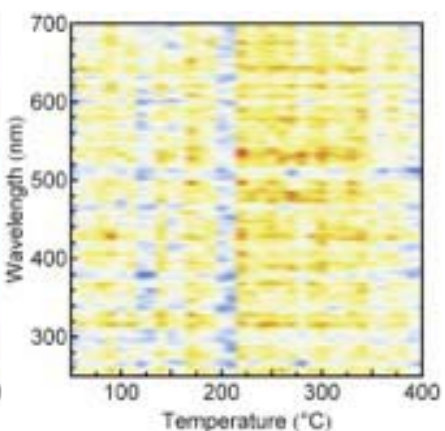

(f) $\mathrm{Gd}$

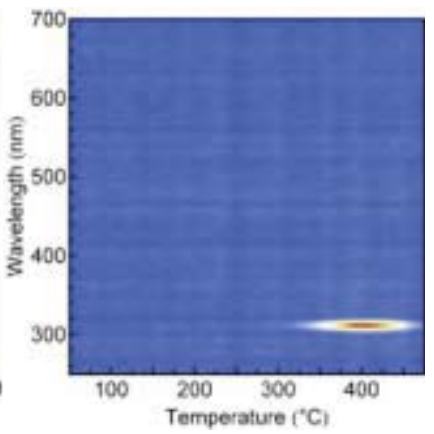

(i) $\mathrm{Ho}$

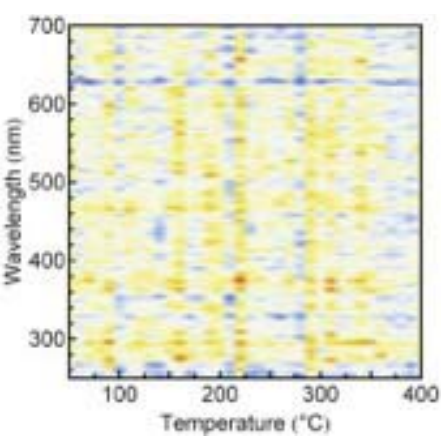

(I) $\mathrm{Yb}$

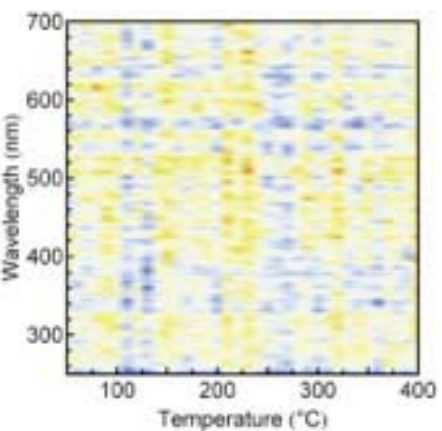

Fig. 57. TL emission spectra of $\mathrm{CaSO}_{4}: \operatorname{Ln}\left(10^{\circ} \mathrm{C} / \mathrm{s}, 100 \mathrm{~Gy}\right)$. 
If we plot the normalized TL curves for various samples, there seems to be approximately five main TL peaks (Fig. 58), resulting in TL emission over a wide temperature range, from $50{ }^{\circ} \mathrm{C}$ up to $600{ }^{\circ} \mathrm{C}$. Fig. 59 shows a comparison of the maximum intensity of TL curves of this host material when doped with different lanthanides can be compared.

The results seem to confirm CSO:Ce,Tb as the most promising material for temperature sensing, since the samples obtained possessed TL peaks over a wide temperature range and exhibits the high temperature peak associated with Ce-doped samples (see Fig. 60). The data obtained provides guidance for controlling the luminescence properties of CSO, but a complete model such as the one obtained for MBO [6] is not straightforward in this case.

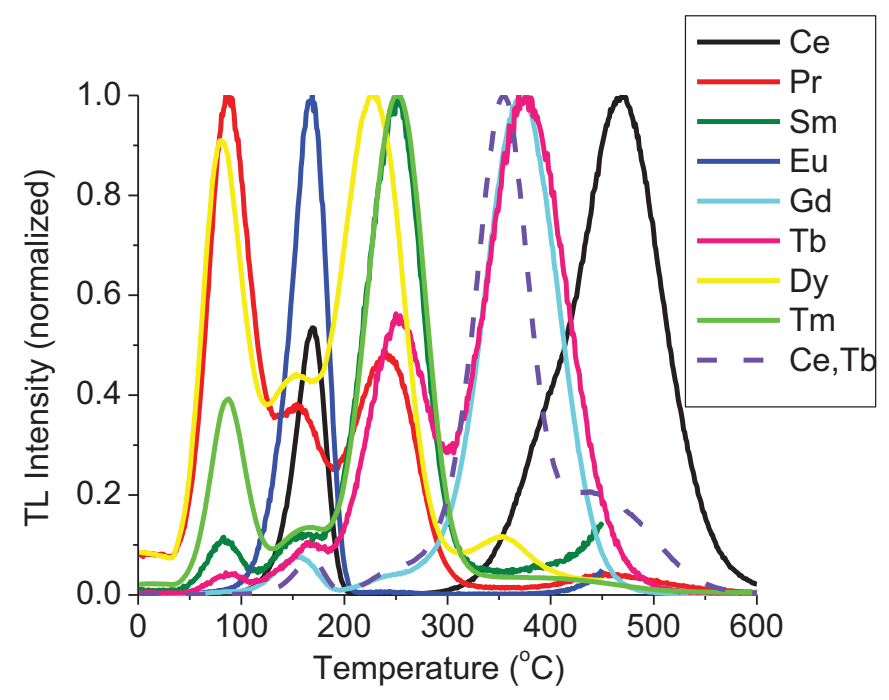

Fig. 58. Normalized TL curves for $\mathrm{CaSO}_{4}$ :Ln showing 5 main TL peaks.

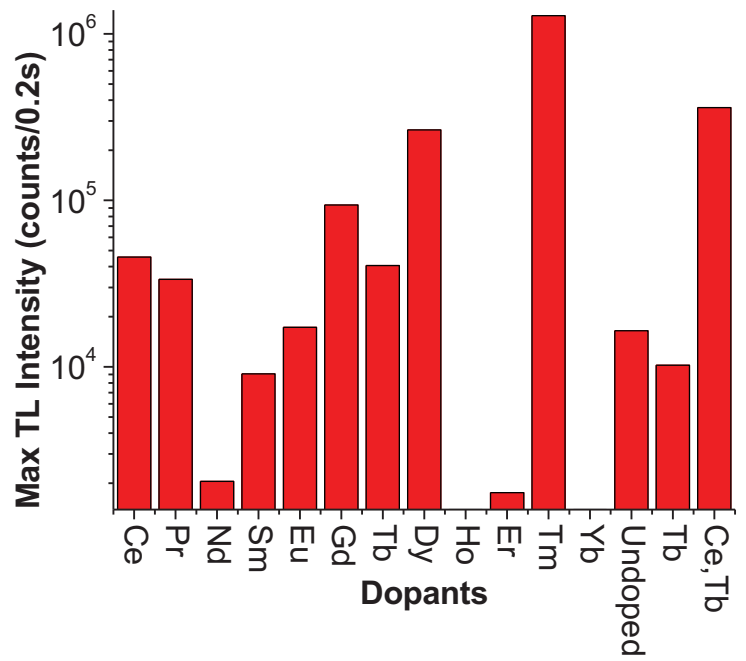

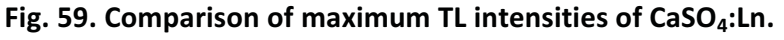




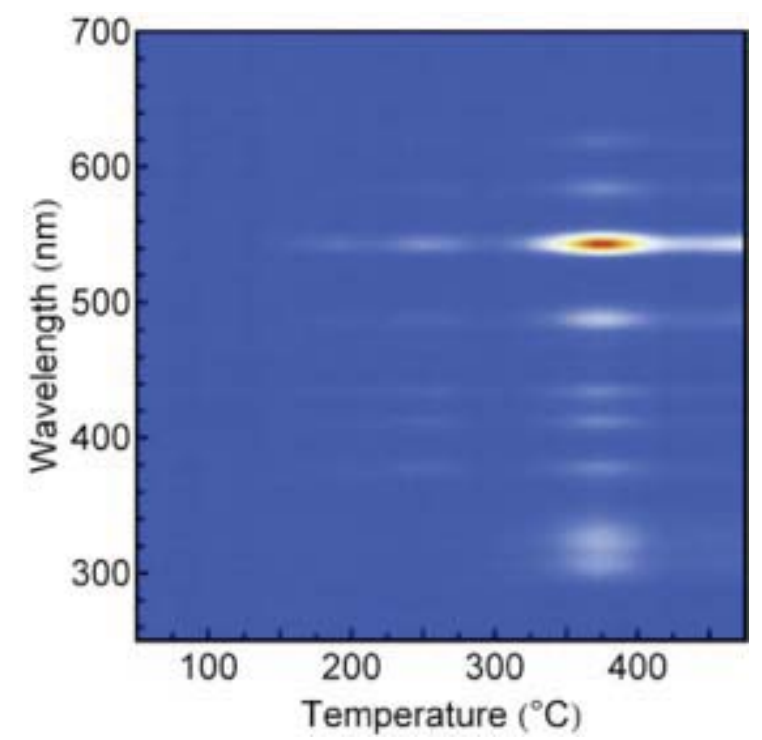

Fig. 60. TL emission spectra of $\mathrm{CaSO}_{4}: \mathrm{Ce}, \mathrm{Tb}\left(10^{\circ} \mathrm{C} / \mathrm{s}, 100 \mathrm{~Gy}\right)$

\section{Effect of co-dopants}

CSO was codoped with Ce because that leads to UV emission and a TL peak in the high temperature range $\left(\sim 500^{\circ} \mathrm{C}\right)$, combined other co-dopants $(\mathrm{Pr}, \mathrm{Gd}, \mathrm{Tb}$, and $\mathrm{Tm})$ to obtain as many $\mathrm{TL}$ peaks as possible in a broad range of temperature. This selection was based on 11 lanthanides investigated previously.

By codoping/triple-doping lanthanides with $\mathrm{Ce}$, we could produce multiple TL peaks at higher temperatures with more intensity comparing to the singly doped samples. Fig. 61 to Fig. 65 show how codoping and triple doping of CSO affect the TL properties of the material.

Fig. 61a shows that the TL intensity of CSO co-doped with Ce and Pr is higher than the TL intensity of CSO singly doped with Ce or Pr. On the other hand, the position of the high temperature peak of the co-doped sample seems to have a $20^{\circ} \mathrm{C}$ shift to the lower temperature comparing to the singly doped samples (Fig. 61b). The results also show that the co-doped sample combines the TL peaks from the singly doped samples. Similar observations can be made for Ce and Tb co-doping (Fig. 62), and Ce and Gd co-doping (Fig. 63).

In the case of Ce and Tm co-doping, however, the co-doped samples show lower intensity than the sample singly-doped with Tm (Fig. 64). Similarly observation can be made for CSO triple-doped with Ce and $\mathrm{Tm}$ and Tb (Fig. 65). 

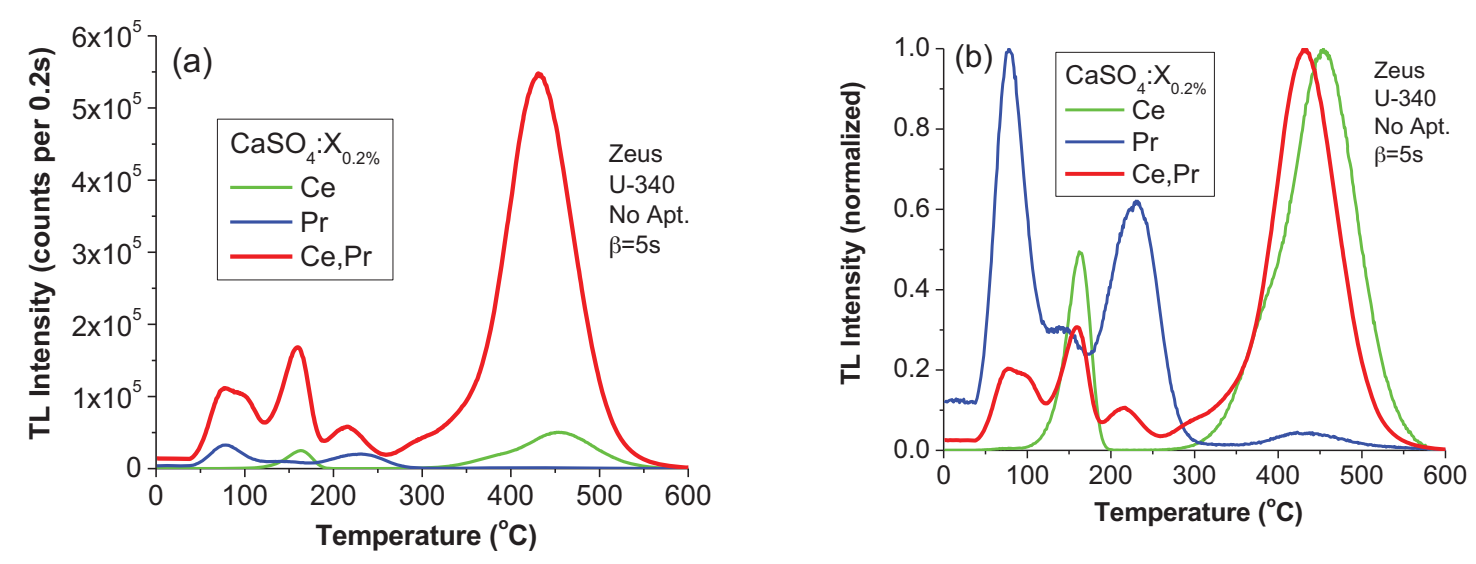

Fig. 61. TL curves of CSO singly doped with Ce or Pr, or co-doped with Ce and Pr: (a) absolute intensity comparison; (b) normalized curves.
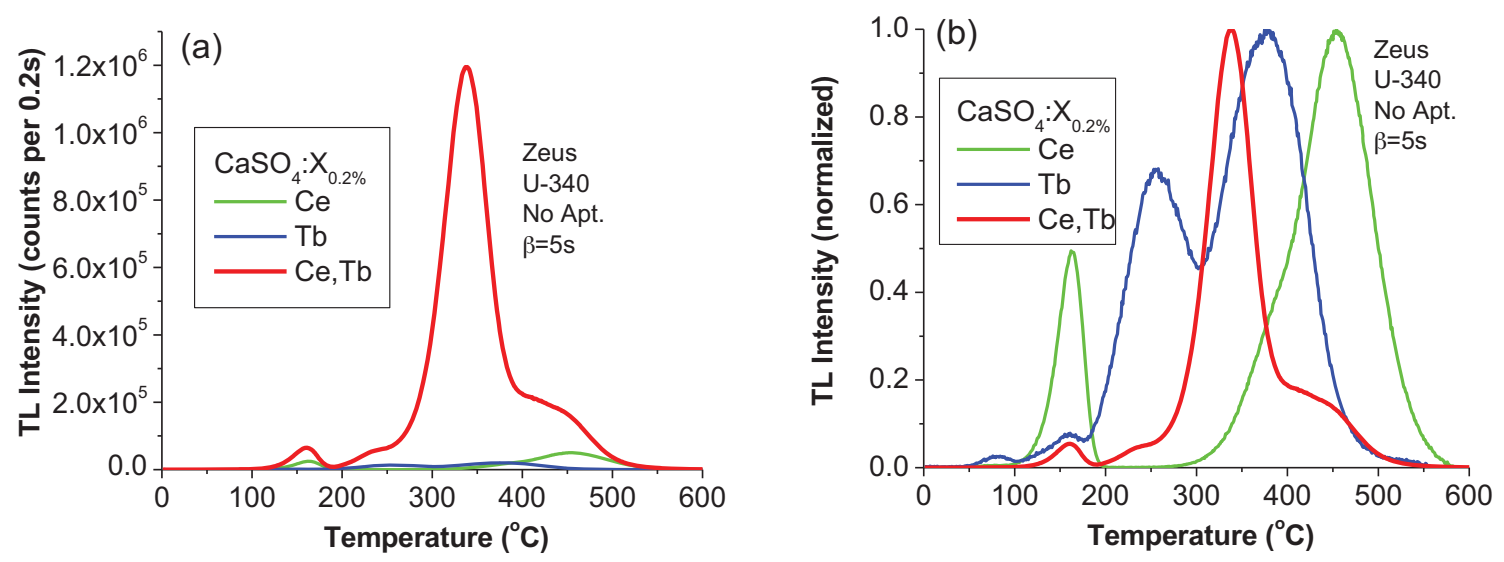

Fig. 62. TL curves of CSO singly doped with Ce or Tb, or co-doped with Ce and Tb: (a) absolute intensity comparison; (b) normalized curves.
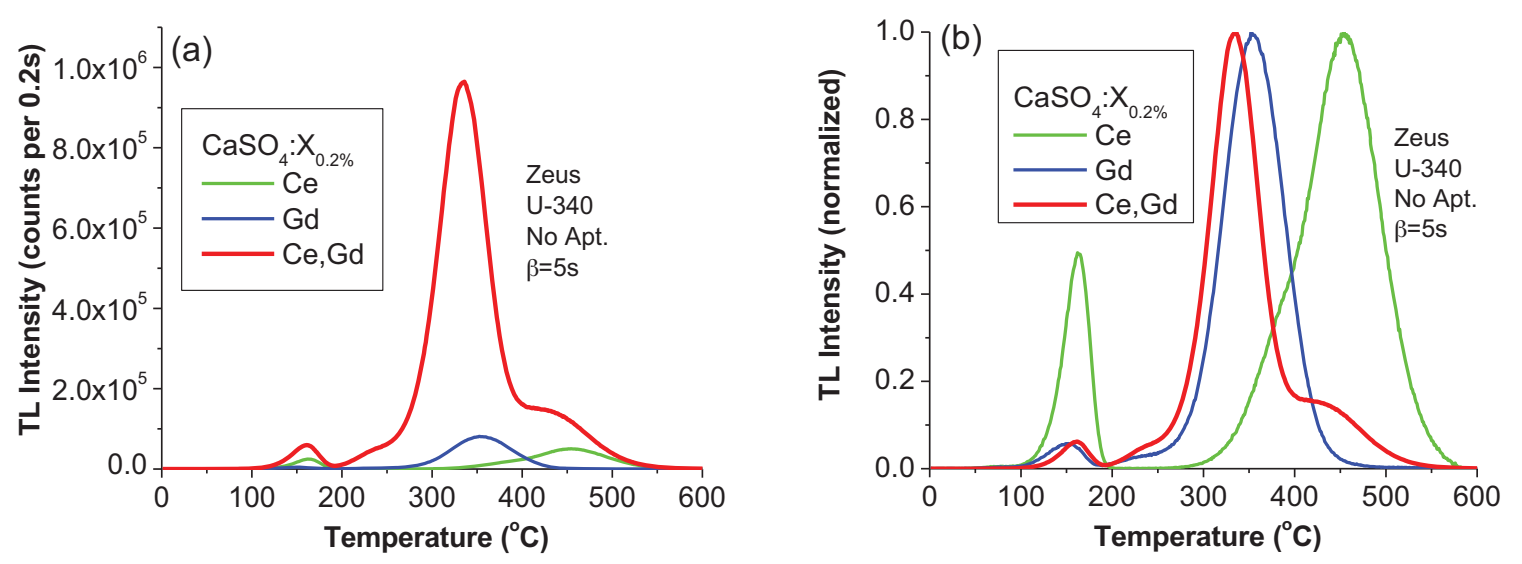

Fig. 63. TL curves of CSO singly doped with Ce or Gd, or co-doped with Ce and Gd: (a) absolute intensity comparison; (b) normalized curves. 

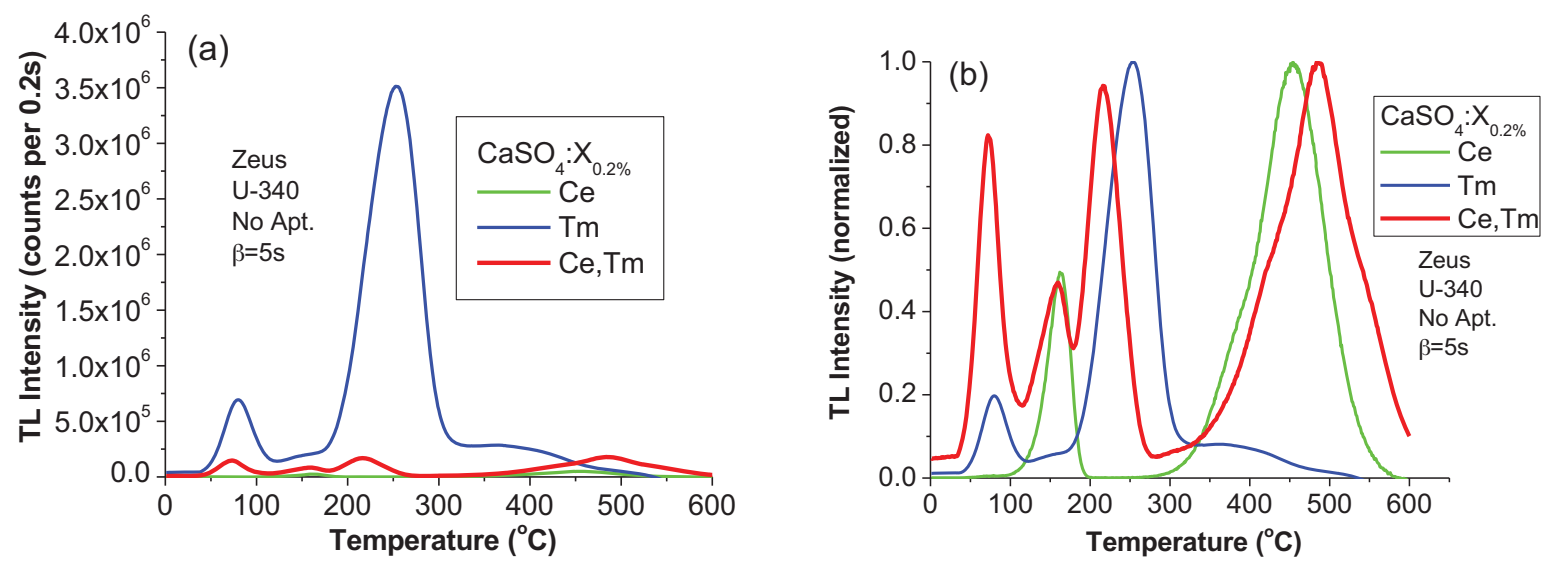

Fig. 64. TL curves of CSO singly doped with Ce or Tm, or co-doped with Ce and Tm: (a) absolute intensity comparison; (b) normalized curves.
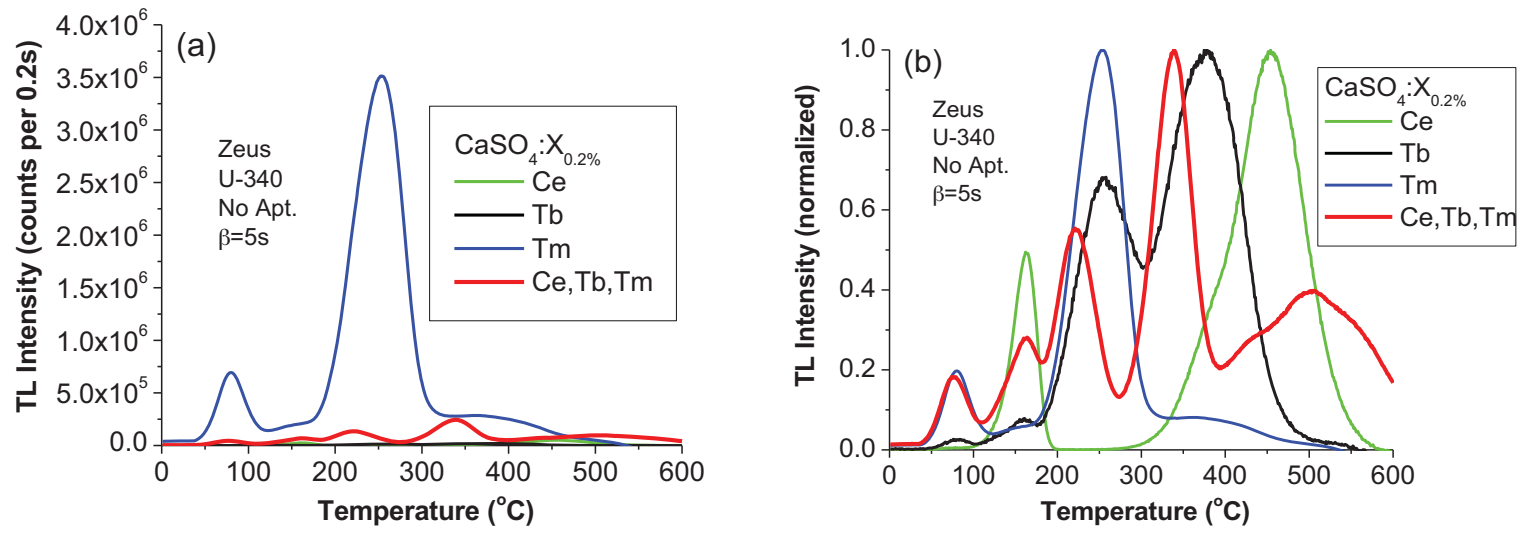

Fig. 65. TL curves of CSO singly doped with Ce or Tm or Tb, or triply-doped with Ce and Tb and Tm: (a) absolute intensity comparison; (b) normalized curves.

Based on the results presented we can conclude that:

(a) In samples co-doped with Tm, the TL intensity has been reduced, but the TL peak positions were shifted to higher temperature relative to singly-doped samples (Fig. 64 and Fig. 65).

(b) In other co-doped samples, the TL intensity of co-doped sample has been increased and the TL peak positions were shifted towards lower temperature relative to singly-doped samples. (e.g., CSO:Ce,Pr)

(c) The multiplicity of TL peaks was observed in all co/triply doped samples.

Finally, Fig. 66 compares the maximum TL intensity of all co/triply doped CSO. The results showed that $\mathrm{CSO}: \mathrm{Ce}, \mathrm{Gd}$ has the highest TL intensity. $\mathrm{CSO}: \mathrm{Ce}, \mathrm{Tm}$ is not as bright, but has the highest temperature peak. CSO:Ce,Pr shows both multiple TL peaks as well as having the peak in higher temperature range.

The results above indicate that $\mathrm{CSO}: \mathrm{Ce}, \mathrm{Pr}$ may be used in place of $\mathrm{CSO}: \mathrm{Ce}, \mathrm{Tb}$ in future detonation tests. 


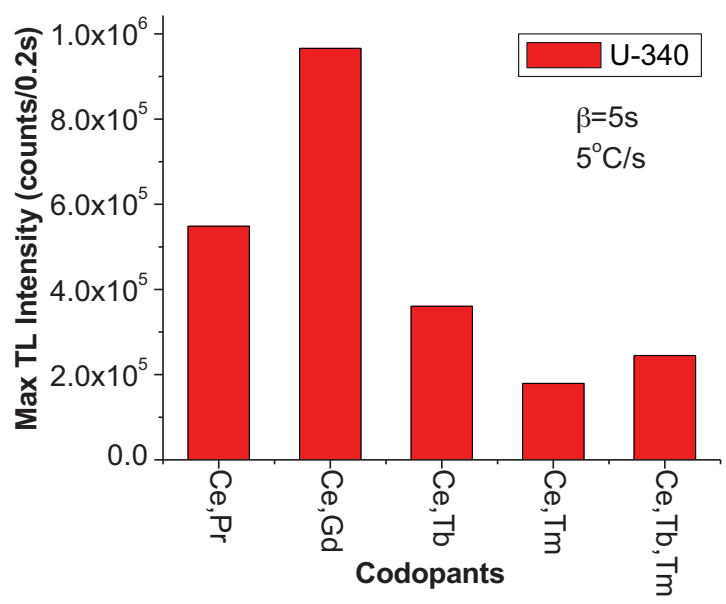

Fig. 66. Comparison of the maximum TL intensity of co/triply doped CSO.

\section{Optimization of acid concentration}

The material synthesis was optimized by investigating the amount of sulfuric acid used in the synthesis that resulted in maximal $\mathrm{TL}$ intensity. $\mathrm{CaSO}_{4}: \mathrm{Ce}$, Tb samples were synthesized by co-precipitation as described earlier (Section 4.1.1). Three different amounts of sulfuric acid were investigated. The first amount was chosen from the correct stoichiometric balance of the reaction, $\mathrm{CaCO}_{3}+\mathrm{H}_{2} \mathrm{SO}_{4} \rightarrow \mathrm{CaSO}_{4}+$ $\mathrm{H}_{2} \mathrm{O}+\mathrm{CO}_{2}$. The second amount (145\% of stoichiometric amount) was chosen to achieve a pH-neutral solution at the end of the reaction. The final amount was the same as Doull et al. [4], 360\% of the stoichiometric amount. Any excess acid was removed by evaporation and calcination of the precipitate.

Fig. 67 shows the TL results from the respective sulfuric acid synthesis amounts. The synthesis using $360 \%$ of the stoichiometric balance of sulfuric acid had the greatest TL intensity and, as a result, was the amount used through the duration of this study. 


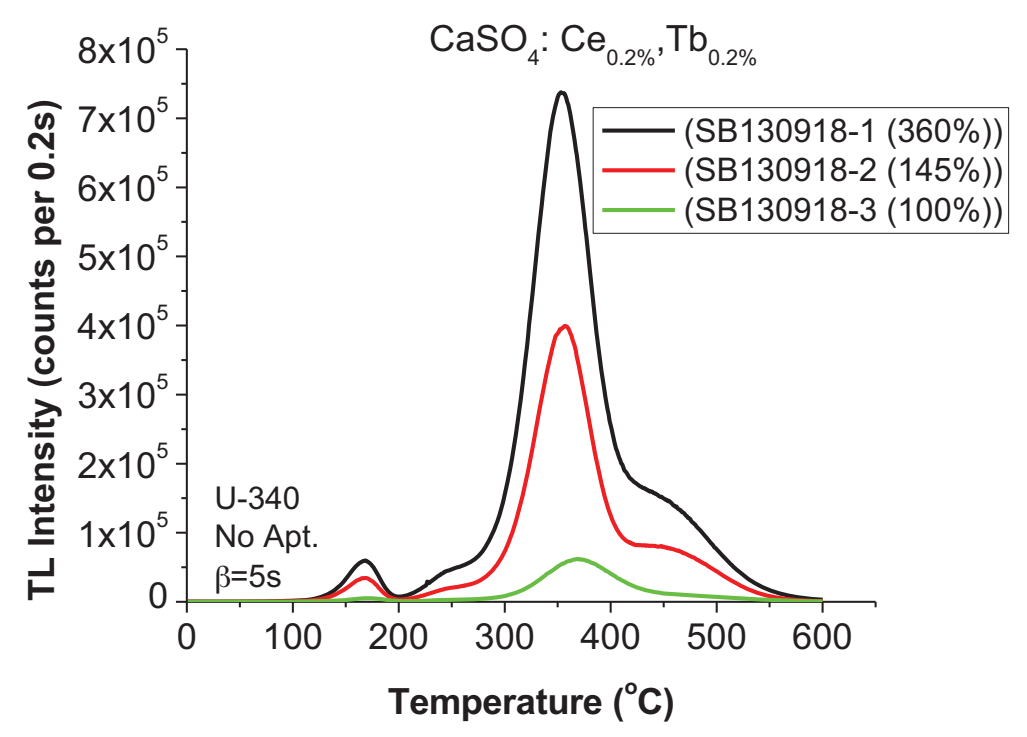

Fig. 67. TL curves of $\mathrm{CaSO}_{4}: \mathrm{Ce}_{0.2 \%}, \mathrm{~Tb}_{0.2 \%}$ with different amount of sulfuric acid as a percent of stoichiometrically balanced amount $\left(5^{\circ} \mathrm{C} / \mathrm{s}\right)$.

\subsection{4 $\mathrm{CaF}_{2}: \mathrm{Tm}$}

To meet DTRA needs of extending the range of applicability of TL materials to higher temperatures, we investigated $\mathrm{CaF}_{2}: \mathrm{Tm}$ (TLD-300, Harshaw, Inc). TLD-300 is promising because it has a high melting temperature $1418{ }^{\circ} \mathrm{C}$, is inert, and has UV emission, which is technically relevant for measurement of high temperature TL. There is essentially no data in the high temperature region of TLD-300 in the literature. Preliminary testing, however, showed TL peaks from $110^{\circ} \mathrm{C}$ up to $625^{\circ} \mathrm{C}$, indicating a large applicable temperature sensing range.

Characterization of TLD-300 was conducted to determine feasibility of the material as a temperature sensor. We investigated the TL curve response to dose, repeatability of TL signal, light sensitivity, TL comparison with current materials, step-annealing, and preliminary modeling (deconvolution).

\section{Dose response}

The curves of $\mathrm{CaF}_{2}: \mathrm{Tm}$ at different doses (Fig. 68a) shows no change in TL curve shape for doses between $0.1 \mathrm{~Gy}$ and $1 \mathrm{~Gy}$, but the relative intensity of the TL peaks at $110^{\circ} \mathrm{C}$ and $300^{\circ} \mathrm{C}$ increased with dose. Higher temperature peaks also became more prominent.

\section{Repeatability}

Since a sample may be irradiated and heated numerous times during analysis it is important that no changes to the TL curve structure occur or else it would hinder ability to recover temperature history. TLD-300 chips were annealed in a muffle furnace for 1 hour at $400{ }^{\circ} \mathrm{C}$ to clean residual signal. Then, the chips were given a beta dose of $100 \mathrm{~Gy}$ and read to $700{ }^{\circ} \mathrm{C}$ at $5{ }^{\circ} \mathrm{C} / \mathrm{s}$. This process was repeated 10 times. The results shown in Fig. $68 \mathrm{~b}$ demonstrate little variability between successive runs, indicating that TLD-300 behaves in a predictable manner. 


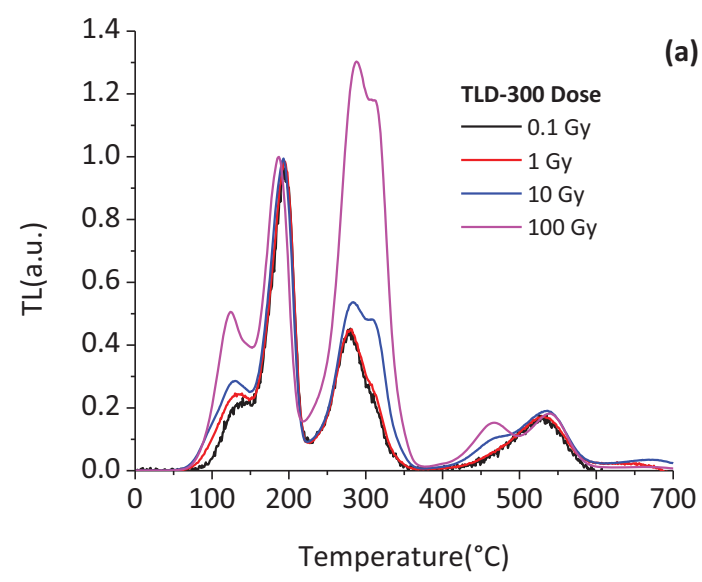

(a)

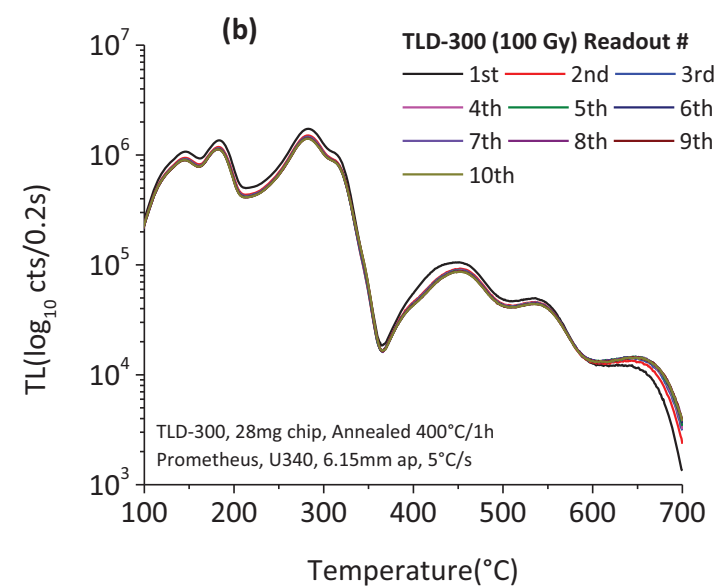

Fig. 68. (a) Normalized TL curves of TLD-300 subject to radiation doses from 0.1 - $100 \mathrm{~Gy}$, and (b) TLD-300 following repeated $\mathrm{TL}$ readouts to $700^{\circ} \mathrm{C}$ at $5^{\circ} \mathrm{C} / \mathrm{s}$ with $100 \mathrm{~Gy}$ beta ray dose.

\section{Light sensitivity}

The effect of light on the TL signal of $\mathrm{CaF}_{2}: \mathrm{Tm}$ TLD-300 was investigated using TLD-300 chips beta irradiated with $100 \mathrm{~Gy}$. The TL was then measured following $3 \mathrm{~h}$ of room light exposure or $3 \mathrm{~h}$ in the dark using the same samples. A reference TL was also measured immediately following irradiation.

Fig. 69a shows that TL curves after $3 \mathrm{~h}$ of light exposure or $3 \mathrm{~h}$ in the dark are identical, indicating that any reduction in TL intensity with respect compared to reference curve is due to room temperature fading. Fig. $69 \mathrm{~b}$ shows the TL from TLD-300 chips irradiated and pre-heated at $400^{\circ} \mathrm{C}(2 \mathrm{~h})$ with and without $3 \mathrm{~h}$ light exposure, to check for the presence of charge phototransfer from deeper to shallower traps. Phototransfer could confuse the interpretation of the results. Reference TL curves were also obtained for normalization. Fig. 69b shows no noticeable phototransfer. The small shifts in TL peak position are likely due to variations in the chip position in the TL reader cups.
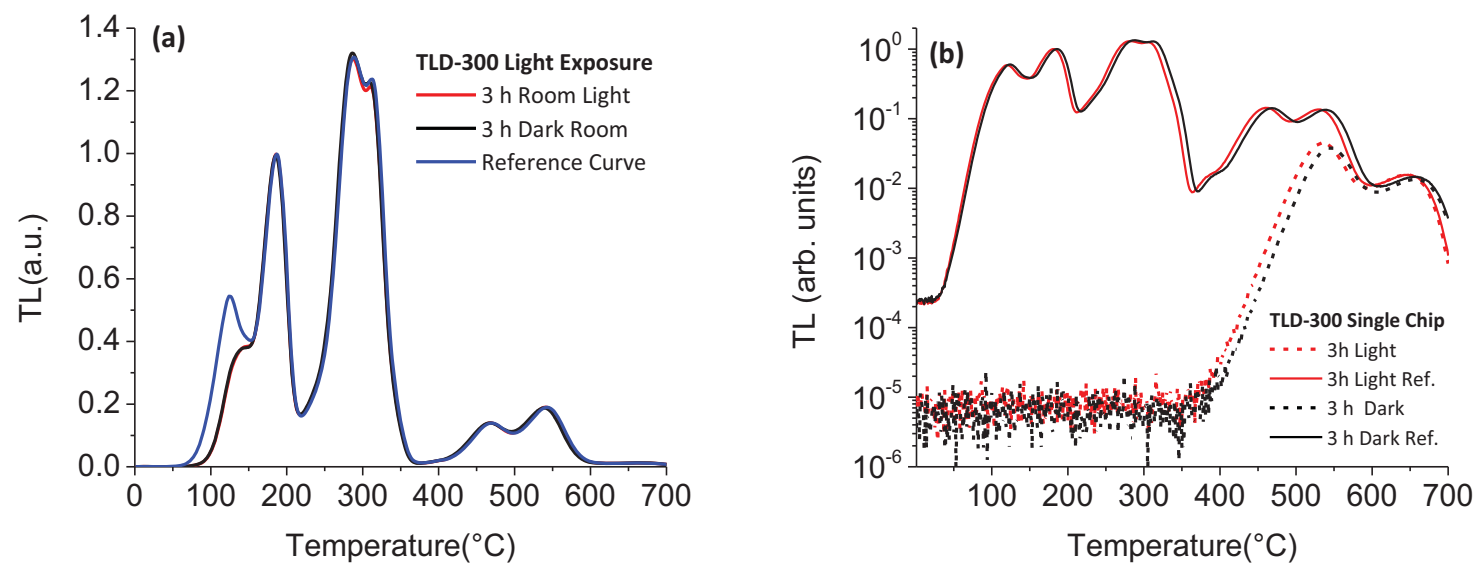

Fig. 69. TL curves of (a) TLD-300 chips given a dose of 100 Gy then exposed to light or dark for $3 \mathrm{~h}$. (b) Thermally cleaned irradiated TLD-300 $\left(400^{\circ} \mathrm{C} / 2 \mathrm{~h}\right)$ with and without room light exposure, showing no evidence of phototransfer compared to the chips kept in the dark. 
The TL intensity of $\mathrm{CaF}_{2}: \mathrm{Tm}$ TLD-300 was compared with $\mathrm{LBO}, \mathrm{MBO}$ and CSO samples previous developed in this study [4]. Chips of TLD-300 were ground using mortar and pestle and grains were sieved with sizes between 75-125 $\mu \mathrm{m}$. Aliquots of MBO, LBO, CSO, and TLD-300 were given a dose of $0.5 \mathrm{~Gy}$ in the Ris $\varnothing$ reader and the TL was measured. Hoya U-340 filters (UV transmission) were used for all TL readouts with the exception of $\mathrm{MBO}$, for which we used a Schott BG-39 filter (visible transmission). We can see in Fig. 70 that TLD-300 is has the strongest TL signal above $600^{\circ} \mathrm{C}$ and a clear peak at $\sim 650{ }^{\circ} \mathrm{C}$.

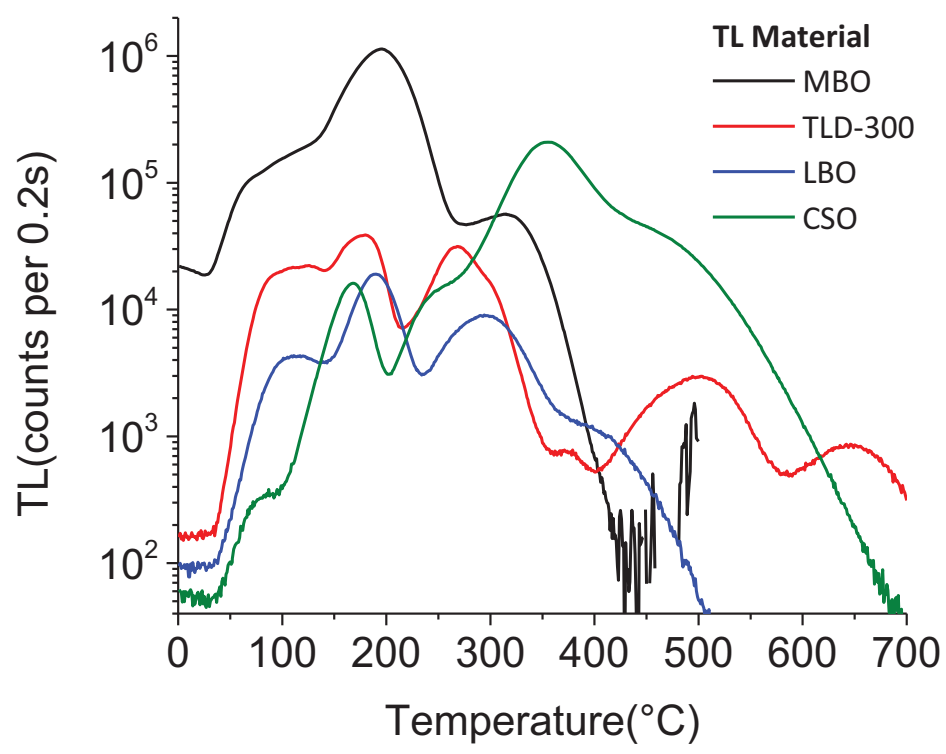

Fig. 70. TL curves of MBO, TLD-300, LBO, and CSO following $0.5 \mathrm{~Gy}$ beta irradiation. TLD-300 has the highest TL temperature peak of all materials investigated, at $650^{\circ} \mathrm{C}$ when read at $5^{\circ} \mathrm{C} / \mathrm{s}$.

\section{Step-annealing}

Step annealing was performed using a single TLD-300 chip ( $28 \mathrm{mg}$ of material) to demonstrate the successive depletion of TL peaks with increasing temperatures. The sequential depletion of the TL curve with increasing annealing temperature is an important property for temperature sensing. The data may also yield more information on the kinetics of the TL process of individual TL peaks.

Step-annealing consists of giving a known dose to a sample and then annealing to a set temperature, $T_{\text {stop }}$, at a linear heating rate of $1^{\circ} \mathrm{C} / \mathrm{s}$. Following the annealing, a TL curve is recorded to measure the annealing effect on the TL signal. The annealing temperature $\left(T_{\text {stop }}\right)$ was varied from $100{ }^{\circ} \mathrm{C}$ to $700{ }^{\circ} \mathrm{C}$ using $20^{\circ} \mathrm{C}$ increments.

Fig. 71 shows the result of the step-annealing procedure of TLD-300, demonstrating the sequential depletion of the TL peaks. 


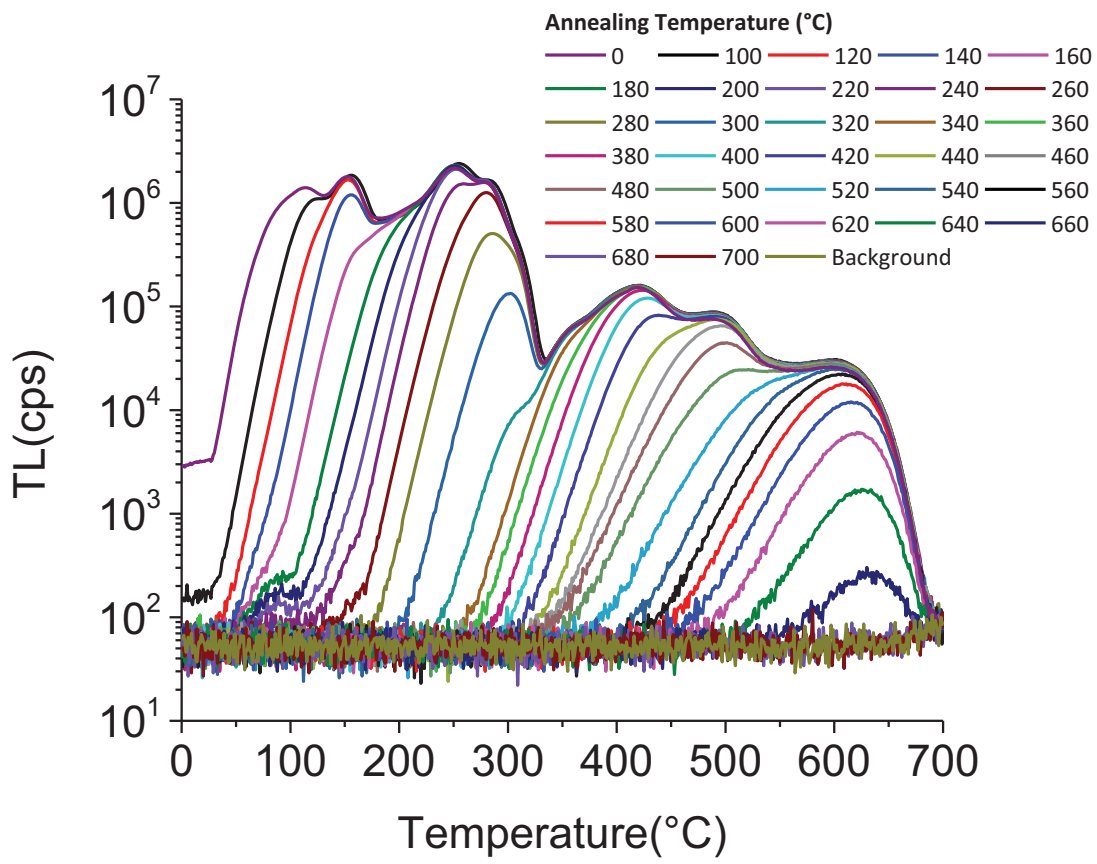

Fig. 71. TL curves of $\mathrm{CaF}_{2}: \mathrm{Tm}$ after irradiation with fixed dose and subsequent heating to increasing temperatures.

\section{Modeling}

To determine a model for $\mathrm{CaF}_{2}: \mathrm{Tm}$ TLD-300, a single TL curve of TLD-300 chip ( $28 \mathrm{mg}$ ) was obtained for $100 \mathrm{~Gy}$. Using the deconvolution method outlined in Yukihara et al. [10] the TL curve was fitted assuming a fixed frequency factor of $10^{14} \mathrm{~s}^{-1}$ and 30 first order TL peaks. Using the distribution of activation energies obtained from the deconvolution, a TL model was created to estimate the effect of constant temperature for a time exposure of $1 \mathrm{~ms}, 1 \mathrm{~s}$, and $2 \mathrm{~h}$ on the residual TL curve.

From the results shown in Fig. 72 , we can see that TLD-300 has the potential to measure temperatures up to $1000^{\circ} \mathrm{C}$ for $1 \mathrm{~ms}$ heating times.
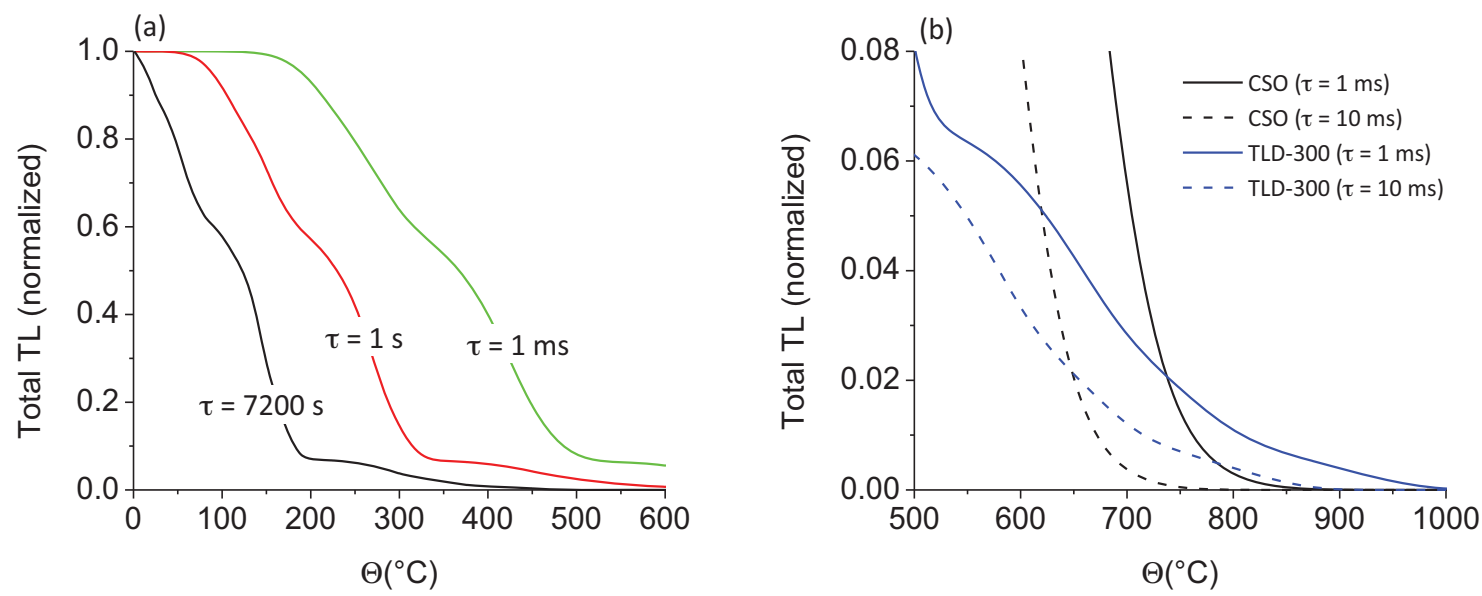

Fig. 72. (a) Residual TL for TLD-300 as a function of heating temperature for $1 \mathrm{~ms}, 1 \mathrm{~s}$, and $7200 \mathrm{~s}$. (b) Residual TL as a function of heating for $1 \mathrm{~ms}$ and $10 \mathrm{~ms}$ for CSO and TLD-300 for higher temperatures. 
Characterization of TLD-300's light fading and light contamination demonstrated the materials ability to survive handling without loss or contamination of TL curve. Step-annealing of TLD-300 showed sequential depletion with increases of annealing temperature, and revealed the presence of high temperature peaks, vital for extending temperature sensing ranges. Preliminary modeling of trapping energies also demonstrated the ability of TLD-300 to measure temperatures of $900{ }^{\circ} \mathrm{C}-1000^{\circ} \mathrm{C}$ for heating in the ms timescale.

\section{Synthesis attempts}

TLD-300 is no longer commercially produced. Therefore, we attempted to synthesize $\mathrm{CaF}_{2}: \mathrm{Tm}$ by sintering $[37,38]$. The sintering process consists in mixing the host material, $\mathrm{CaF}_{2} 99.95 \%$ purity, with the dopants in solid form, $\mathrm{Tm}_{2} \mathrm{O}_{3} 99.99 \%$ purity, pressing the solid precursors into a pellet, and heating the pellet at a temperature around $85 \%$ of the melting temperature of the host material for some amount of time to diffuse the dopants into the host material.

In this study the samples were made to yield approximately $1 \mathrm{~g}$ of $\mathrm{CaF}_{2}: \mathrm{Tm} 0.6 \%$. The molar concentration for Tm is based on the literature [39]. The solid precursors were mixed together and pressed into pellets in steel molds under 12000 psi of pressure for $5 \mathrm{~min}$, resulting in a pellet of about $10 \mathrm{~mm}$ diameter and $1 \mathrm{~mm}$ height. A soak temperature of $1100^{\circ} \mathrm{C}$ was used for all the samples, but the duration of the soaking and atmosphere varied.

A study was conducted to determine the soak time required to produce materials similar to TLD-300. The pellets were placed into alumina crucibles in a furnace. The temperature was initially ramped to $600^{\circ} \mathrm{C}$ over $1 \mathrm{~h}$ and soaked at that temperature for $30 \mathrm{~min}$. They were then heated to $900^{\circ} \mathrm{C}$ over $30 \mathrm{~min}$ and soaked for $1 \mathrm{~h}$. Finally, they were ramped to $1100^{\circ} \mathrm{C}$ over $30 \mathrm{~min}$ and soaked for various periods $(1 \mathrm{~h}-30 \mathrm{~h})$. They were allowed to cool slowly back to room temperature inside the furnace. Two samples were produced for each soak time tested.

The same soak time study was conducted in $\mathrm{N}_{2}$ atmosphere to avoid the potential formation $\mathrm{CaO}$ in oxygen atmosphere. The preparation of the pellets for this study was the same as the soak time study, but the pellets were placed into a platinum crucible rather than alumina. The crucible was placed into a standing tube furnace with atmosphere control. The sample was ramped to $250^{\circ} \mathrm{C}$ over $1 \mathrm{~h}$ and soaked for $21 \mathrm{~h}$ in vacuum ( $10 \mu$ Torr). It was then backfilled with high purity $\mathrm{N}_{2}$ ( $\sim-10$ psi relative to atm). The temperature was ramped to $1100{ }^{\circ} \mathrm{C}$ over $6 \mathrm{~h}$ and allowed to soak for varying amounts of time, after which the sample was allowed to cool naturally to room temperature. Finally, the sample pellets were powdered using a ceramic mortar and pestle.

TL and RL of the produced materials were measured and compared with TLD-300 (Fig. 73 and Fig. 74). The materials produced were unfortunately not as bright as TLD-300 and presented TL curves different than TLD-300 (Fig. 73). The RL of the materials were in general more intense than that of TLD-300, indicating the possibility that the materials synthesized are lacking trapping centers.

XRD were also obtained for the sintered materials. In all XRD collected (e.g., Fig. 75), $\operatorname{Tm}_{2} \mathrm{O}_{3}$ was still observed in small amounts, but no $\mathrm{CaO}$ was detected in the XRD data.

The conclusion is that sintering does not produce $\mathrm{CaF}_{2}$ with sufficient quality for temperature measurements and alternate synthesis methods must be explored. 

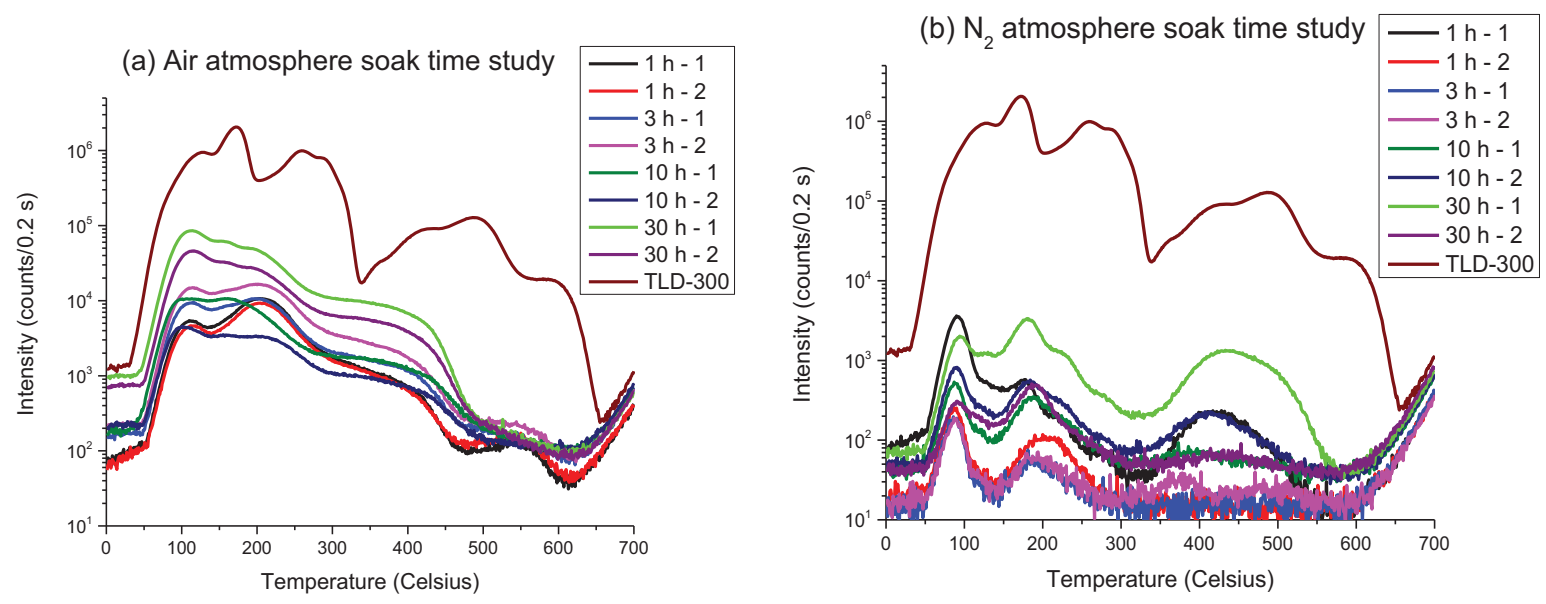

Fig. 73. TL curves of $\mathrm{CaF}_{2}$ prepared by sintering in (a) air atmosphere and (b) $\mathrm{N}_{2}$ atmosphere. The materials produced showed weak luminescence in comparison with TLD-300. (Heating rate of $5^{\circ} \mathrm{C} / \mathrm{s}$ ).

(a) $\mathrm{RL}$ of air atm sintered $\mathrm{CaF}_{2}$

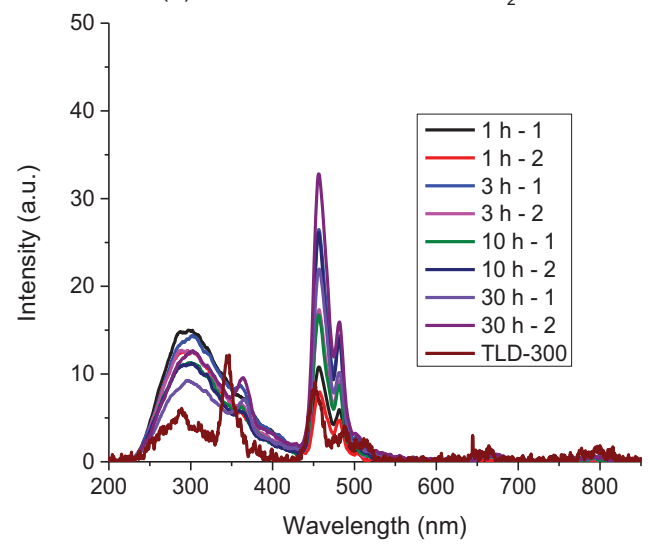

(b) $\mathrm{RL}$ of nitrogen atm sintered $\mathrm{CaF}_{2}$

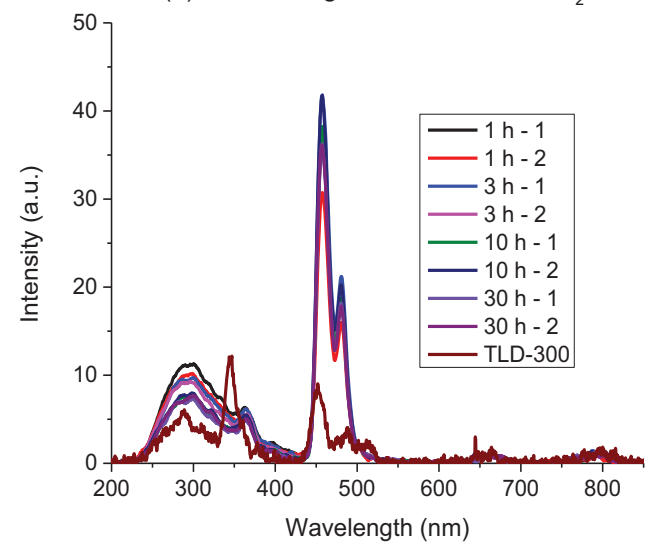

Fig. 74. Radioluminescence of $\mathrm{CaF}_{2}$ prepared by sintering using (a) air atmosphere and (b) $\mathbf{N}_{2}$ atmosphere. The strong RL signal in the synthesized samples indicate that the lack of TL intensity may be related to the deficiency in trapping centers.

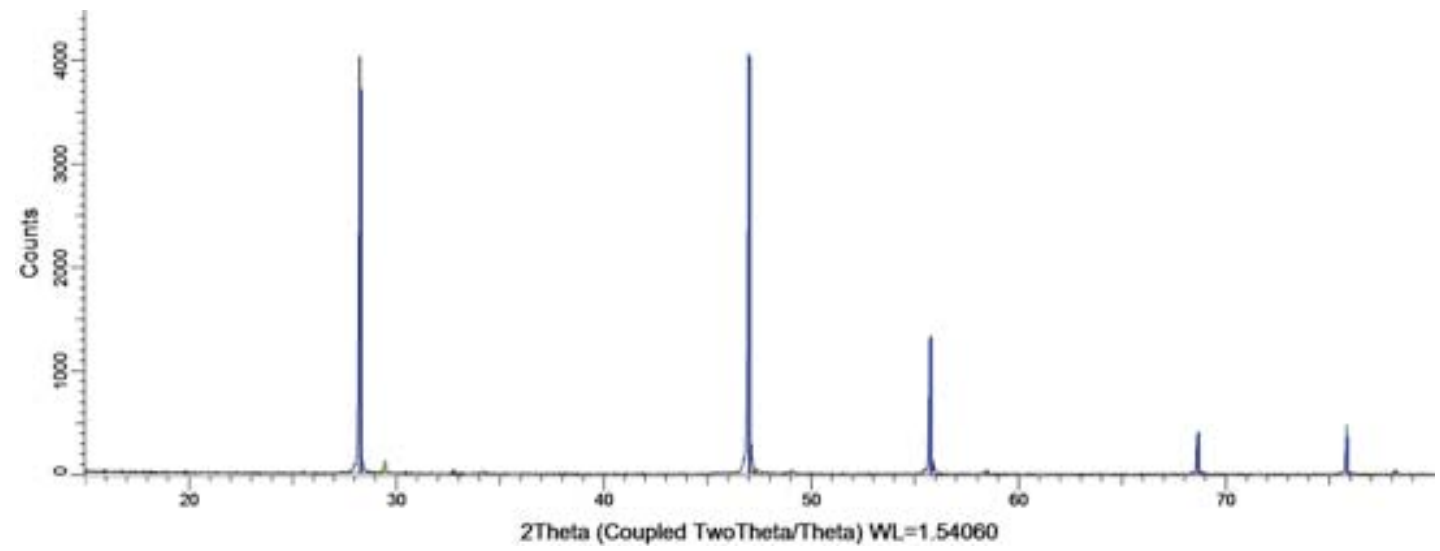

Fig. 75. Typical XRD collected for the sintered materials, here for the sample soaked for $30 \mathrm{~h}$ in a nitrogen atmosphere. Analysis shows a $99.45 \%$ concentration of $\mathrm{CaF}_{2}$ (blue lines) and $0.55 \% \mathrm{Tm}_{2} \mathrm{O}_{3}$ (green lines). The presence of detectable amounts of $\mathrm{Tm}_{2} \mathrm{O}_{3}$ is evidence that $\mathrm{Tm}$ is not entering the $\mathrm{CaF}_{2}$ lattice. 


\subsection{Kinetic analysis of TL materials}

The temperature reconstruction algorithms rely on a model for underlying TL process in the various materials. In the past we have used the assumption that: (a) the materials can be described by a superposition of trapping centers characterized by a distribution of activation energies; and (b) that the frequency factor that best describes the behavior of the material under a variety of conditions and preheating temperatures was $s=10^{14} \mathrm{~s}^{-1}$.

To test the validity of these assumptions, we are carrying out analysis of all TL materials used in this study using traditional TL analysis techniques, such as the initial rise method [40] and the various heating rate method $[41,42]$. This research is still in progress as a part of Ph. D. student at Oklahoma State University, and the results presented here are preliminary.

\subsubsection{Initial rise method (IRM)}

The IRM has long been used to determine the activation energy $E$ of single TL peaks because $E$ can be obtained independently of the kinetic order. The method is based on the fact that, in the initial part of the TL curve, the trap occupancy remains approximately constant, the effect of which is an approximately exponential increase in TL intensity for the low temperature region of a TL peak approximated by [40]:

$$
I(t) \propto n_{0} s \exp (-E / k T) .
$$

A linear fit of $\ln I(t)$ vs $1 / T$ yields a slope of $-E / k$, allowing for the determination of the activation energy, $E$. Knowledge of the exact peak location, $T_{m}$, for a single and isolated $1^{\text {st }}$ order TL peak the frequency factor is calculated as

$$
s=\beta E / k T_{m}^{2} \cdot \exp \left(E / k T_{m}\right),
$$

where $\beta$ is the heating rate $(\mathrm{K} / \mathrm{s})$, and $k$ is the Boltzmann constant.

For the case of multiple TL peaks, step-annealing is used to isolate the initial rise portion of TL peaks and to aid in kinetic order determination [43]. Step-annealing (or $T_{m}-T_{\text {stop }}$ method) works by heating an irradiated TL material to a temperature below the maximum readout temperature $T_{\text {stop, }}$, cooling to room temperature, then heating to the maximum readout temperature. The process is repeated increasing $T_{\text {stop }}$ every step. The result is a sequential depletion of the low temperature TL structures to aid in isolation of the initial rise region of higher temperature TL peaks. Plots of the first TL maximum $T_{\mathrm{m}}$ with increasing $T_{\text {stop }}$ and plots of activation energy with increasing $T_{\text {stop }}$ should result in a staircase structure for isolated $1^{\text {st }}$ order TL peaks, whereas more complex TL structures or kinetics result in different structures [43].

Step-annealing data was collected for three OSU materials; $\mathrm{Li}_{2} \mathrm{~B}_{4} \mathrm{O}_{7}: \mathrm{Cu}_{0.4 \%}, \mathrm{Ag}_{0.1 \%}$ (LBO), $\mathrm{MgB}_{4} \mathrm{O}_{7}: \mathrm{Dy}_{0.1 \%}, \mathrm{Li}_{1 \%}(\mathrm{MBO}), \mathrm{CaSO}_{4}: \mathrm{Ce}_{0.2 \%}, \mathrm{~Tb}_{0.2 \%}$ (CSO) for $T_{\text {stop }}$ in the range of $50-450{ }^{\circ} \mathrm{C}$ for $\mathrm{MBO}$ and LBO, and $50-600{ }^{\circ} \mathrm{C}$ for $\mathrm{CSO}$ in $5{ }^{\circ} \mathrm{C}$ increments. Linear fitting of the initial rise section was conducted up to $15 \%$ of the TL intensity at a readout temperature of $T_{m}$. Data was not considered for fitting if the intensity for a given $T_{\mathrm{m}}-T_{\text {stop }}$ curve was found to be less than the average plus three times the standard deviation of the background (first 50 data points in the TL curve). An example of data used for analysis and the associated fits is shown in Fig. 76 . Aliquots for TL collection were small $(<1 \mathrm{mg})$ to ensure good thermal contact. Frequency factors were calculated for each material using the activation energy and peak position for each $T_{\text {stop }}$, but the $s$ value is only an estimate, since $E$ and $T_{m}$ do not necessarily 
correspond to the same TL peak in case of a superposition of TL peaks. Analysis was conducted for all $T_{\mathrm{m}}-T_{\text {stop }} \mathrm{TL}$ curves where sufficient data points existed for linear fitting.

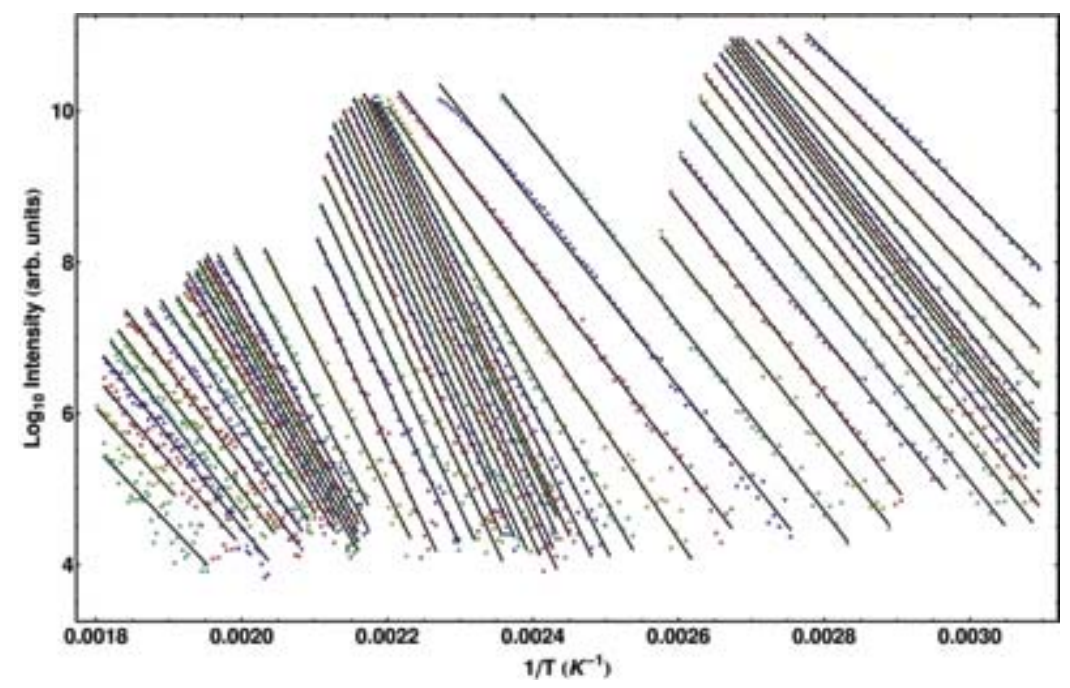

Fig. 76. Initial rise data obtained from LBO (<1 mg sample) step annealing curves $\left(\mathrm{T}_{\text {stop }}, 95-345^{\circ} \mathrm{C}\right)$ using a $1^{\circ} \mathrm{C} / \mathrm{s}$ heating rate. Linear fits were calculated to $15 \%$ of max TL peak intensity as determined from $T_{m}-T_{\text {stop }}$ analysis.

$\angle i_{2} B_{4} O_{7}: C U, A g(L B O)$

A typical set of initial rise TL curves for LBO is found in Fig. 77a. IRM curves from LBO show the presence of five distinct TL peaks confirmed by the $T_{m}-T_{\text {stop }}$ results (Fig. 77b). The plateau regions for TL peaks 2 and 3 indicate $1^{\text {st }}$ order behavior, whereas peaks 4 and 5 are described by either a distribution of peaks or non- $1^{\text {st }}$ order kinetics. Activation energies obtained using the IRM are plotted as a function of $T_{\text {stop }}$ along with $T_{\mathrm{m}}$ data in Fig. $77 \mathrm{c}$ and along with estimated frequency factors in Fig. $77 \mathrm{~d}$. It should be noted that the plateau regions from the $T_{m}-T_{\text {stop }}$ results approximately match the plateau regions in the activation energy data, indicating that the activation energies obtained in the plateau may describe the correspond $T_{m}$ plateau. Regions of constant activation energy values (and corresponding frequency factors) are highlighted by the shaded regions and labeled in Fig. $77 \mathrm{~d}$ and are used for the calculation of the results shown in Fig. 77. 

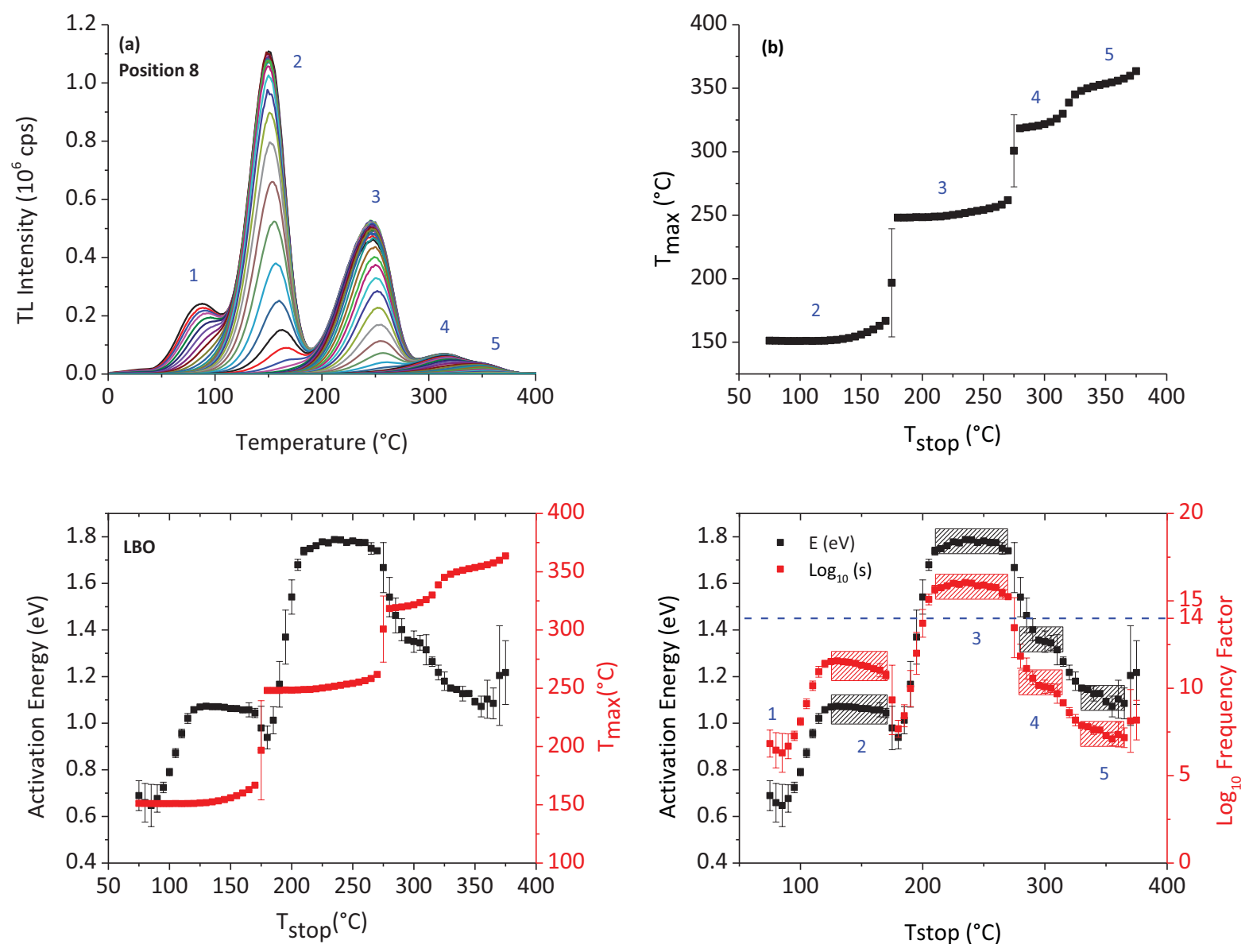

Fig. 77. LBO $T_{\mathrm{m}}-T_{\text {stop }}$ TL curves are shown in plot (a) and with peak positions in plot (b). Activation energies obtained from initial rise analysis are plotted with $T_{m}$ data in plot (c) alongside the corresponding peak positions. Plot (d) shows estimated frequency factors with associated activation energies. Data for plots $b$-d represent averages and errors from three aliquots.

Table 9. Results obtained from LBO initial rise and step-annealing analysis. Results were obtained from three aliquots of $<1 \mathrm{mg}$ of material. Frequency factors $\left(\log _{10} \mathrm{~s}\right)$ are estimates, as analysis does not indicate the initial rise analysis was of individual TL peaks. Peaks $1-5$ correspond to the shaded regions of Fig. $77 d$.

\begin{tabular}{|ccccc|}
\hline Peak & Activation Energy $(\mathrm{eV})$ & $\log _{10} \mathbf{S}$ & $\mathbf{T}_{\max }\left({ }^{\circ} \mathbf{C}\right)$ & $\mathbf{T}_{\text {stop }}$ Range $\left({ }^{\circ} \mathbf{C}\right)$ \\
\hline $\mathbf{1}$ & - & - & - & - \\
\hline $\mathbf{2}$ & $1.06 \pm 0.01$ & $11.37 \pm 0.20$ & $157 \pm 5$ & $125-170$ \\
\hline $\mathbf{3}$ & $1.77 \pm 0.02$ & $15.84 \pm 0.17$ & $253 \pm 4$ & $210-270$ \\
\hline $\mathbf{4}$ & $1.38 \pm 0.09$ & $11.34 \pm 0.97$ & $322 \pm 4$ & $280-315$ \\
\hline $\mathbf{5}$ & $1.11 \pm 0.03$ & $7.61 \pm 0.22$ & $353 \pm 3$ & $330-365$ \\
\hline
\end{tabular}

$\mathrm{MgB}_{4} \mathrm{O}_{7}: \mathrm{Dy}, \mathrm{Li}(\mathrm{MBO})$

A typical set of initial rise (IR) TL curves for MBO is shown in Fig. 78a. IRM data for MBO show the presence of low intensity shoulder that precedes the $150{ }^{\circ} \mathrm{C}$ peak and a complex $\mathrm{TL}$ structure between readout temperatures of $200-300^{\circ} \mathrm{C}$, which complicates the IRM analysis. The $T_{\mathrm{m}}-T_{\text {stop }}$ results (Fig. 78b) confirm the presence of two distinct TL peaks illustrated by the plateau regions, but the subsequent rises in $T_{m}-T_{\text {stop }}$ indicate either non-first-order kinetics, a distribution of closely spaced TL 
peaks, or both [43]. Activation energies obtained using the IRM are plotted as a function of $T_{\text {stop }}$ along with $T_{\mathrm{m}}$ data in Fig. 78c and along with estimated frequency factors in Fig. 78d. It should be noted that the plateau regions from the $T_{\mathrm{m}}-T_{\text {stop }}$ results do not match the plateau regions in the activation energy data, indicating that, although the peak position does not change, the initial rise region does not belong to that peak. Regions of constant activation energy values (and corresponding frequency factors) are highlighted by the shaded regions and labeled in Fig. 78d and are used for the calculation of the results shown in Table 10.
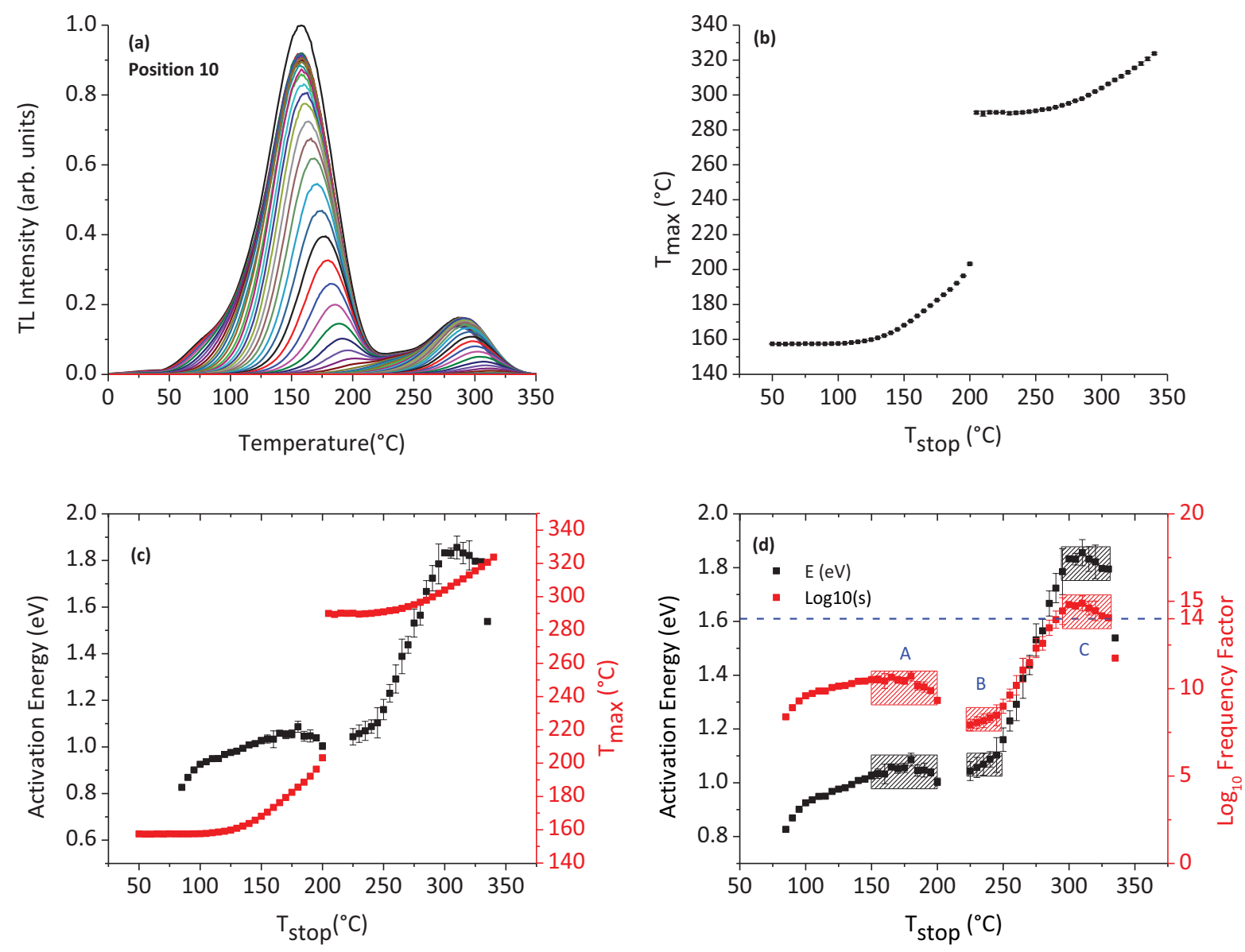

Fig. 78. MBO $T_{\mathrm{m}}-T_{\text {stop }}$ TL curves are shown in plot (a) and with peak positions in plot (b). Activation energies obtained from initial rise analysis are plotted with Tm data in plot (c) alongside the corresponding peak positions. Plot (d) shows estimated frequency factors with associated activation energies. Data for plots $b$-d represent averages and errors from three aliquots.

Table 10. Results obtained following MBO initial rise and step-annealing analysis. Results were obtained from three aliquots of $<1 \mathrm{mg}$ of material. Frequency factors $\left(\log _{10} s\right)$ are estimates, as analysis does not indicate the initial rise analysis was of individual TL peaks. Peaks A - C correspond to the shaded regions of Fig. 78d.

\begin{tabular}{|ccccc|}
\hline Peak & Activation Energy $(\mathrm{eV})$ & $\log _{10} \mathbf{S}$ & $\mathbf{T}_{\max }\left({ }^{\circ} \mathrm{C}\right)$ & $\mathbf{T}_{\text {stop }}$ Range $\left({ }^{\circ} \mathbf{C}\right)$ \\
\hline A & $1.04 \pm 0.02$ & $10.46 \pm 1.36$ & $183 \pm 11$ & $150-200$ \\
\hline B & $1.07 \pm 0.02$ & $8.38 \pm 1.66$ & $290 \pm 0$ & $225-245$ \\
\hline C & $1.82 \pm 0.02$ & $14.7 \pm 1.42$ & $310 \pm 6$ & $295-330$ \\
\hline
\end{tabular}


$\mathrm{CaSO}_{4}: \mathrm{Ce}_{0.2 \%} \mathrm{~Tb}_{0.2 \%}(\mathrm{CSO})$

A typical set of initial rise (IR) TL curves for CSO is found in Fig. 79a. IRM data for CSO show the presence of two distinct TL peaks with a third peak like structure at a readout temperature of $\sim 400{ }^{\circ} \mathrm{C}$. Results from $T_{\mathrm{m}}-T_{\text {stop }}$ analysis are found in Fig. 79b and confirmed the two peaks as well as the presence of a more complex higher temperature structure. Activation energies obtained using the IRM are plotted as a function of $T_{\text {stop }}$ along with $T_{\mathrm{m}}$ data in Fig. $79 \mathrm{c}$ and along with estimated frequency factors in Fig. $79 \mathrm{~d}$. It should be noted that the plateau regions from the $T_{m}-T_{\text {stop }}$ results fail to match the plateau regions in the activation energy data. Furthermore the inset in Fig. 79d show small staircase like behavior indicating closely spaced TL peaks. Regions of constant activation energy values (and corresponding frequency factors) are highlighted by the shaded regions and labeled in Fig. 79d and are used for the calculation of the results shown in Table 11.
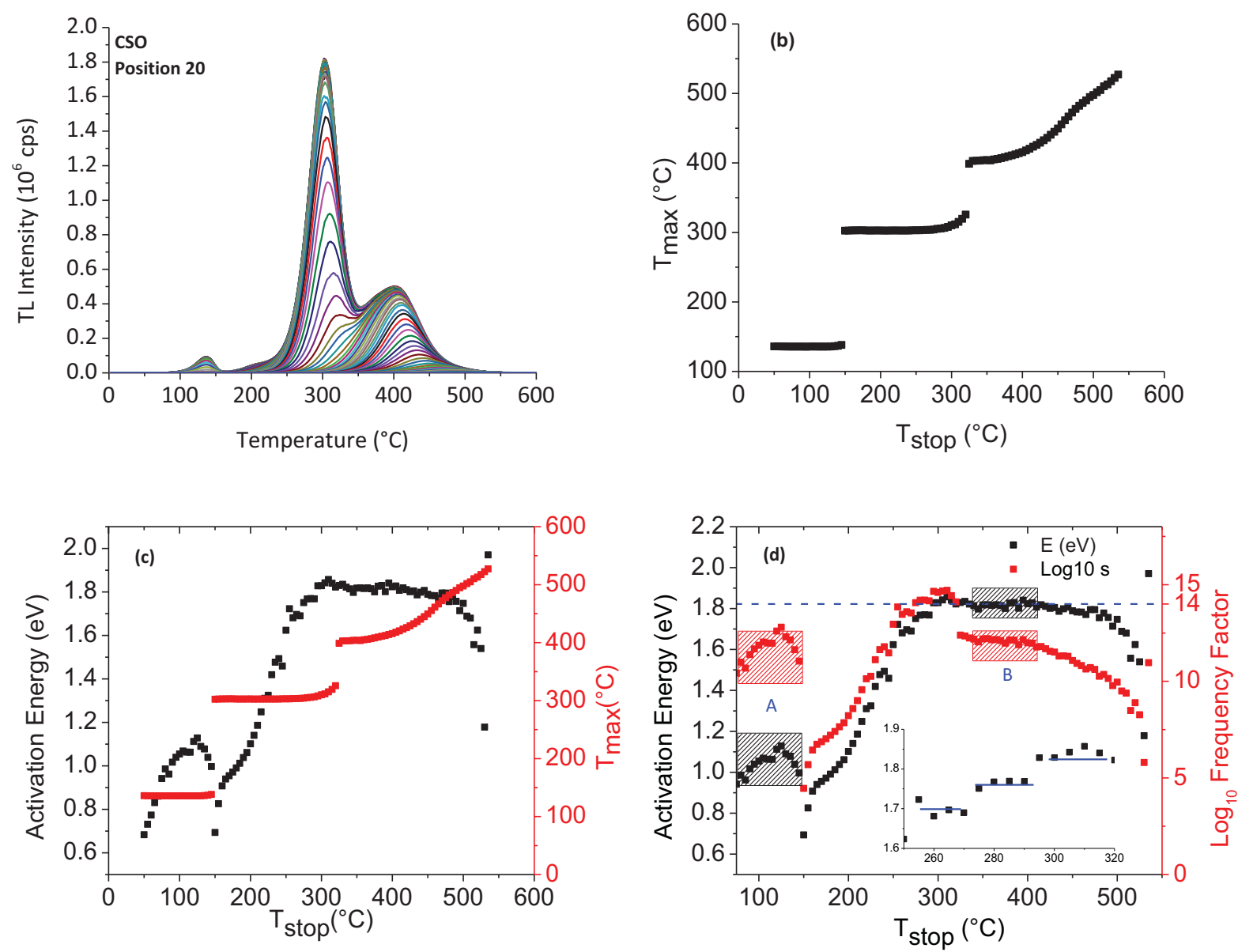

Fig. 79. CSO $T_{\mathrm{m}}-T_{\text {stop }}$ TL curves are shown in plot (a) and with peak positions in plot (b). Activation energies obtained from initial rise analysis are plotted with Tm data in plot (c) alongside the corresponding peak positions. Plot (d) shows estimated frequency factors with associated activation energies. The inset in plot(c) shows small regions of constant activation energy values indicating a cluster of TL peaks. Data for plots b-d represent averages and errors from three aliquots.

Table 11. Results obtained from CSO initial rise and step-annealing analysis. Results were obtained from three aliquots of $<1 \mathrm{mg}$ of material. Frequency factors $\left(\log _{10} \mathrm{~s}\right)$ are estimates, as analysis does not indicate the initial rise analysis was of individual TL peaks. Peaks A and B correspond to the shaded regions of Fig. 79d.
Peak
Activation Energy (eV)
$\log _{10} s$
$\mathrm{T}_{\max }\left({ }^{\circ} \mathrm{C}\right)$
$\mathrm{T}_{\text {stop }}$ Range $\left({ }^{\circ} \mathrm{C}\right)$ 


\begin{tabular}{|ccccc|}
\hline A & $1.05 \pm 0.05$ & $12.10 \pm 3.19$ & $136 \pm 1$ & $75-145$ \\
\hline B & $1.82 \pm 0.01$ & $12.10 \pm 0.56$ & $410 \pm 6$ & $340-410$ \\
\hline
\end{tabular}

LBO results are promising as regions of constant peak position align well with regions of constant activation energy with regards to $T_{\text {stop }}$, but more work must be done to determine the reliability of the results. $\mathrm{MBO}$ and CSO results indicate that, although the TL curves show distinct features, the underlying peak structure is more complex and not easily resolved by the IRM. A deconvolution method informed by experimental results may be preferred for these two materials.

\subsubsection{Various heating rate method (VHRM)}

The VHRM is derived from Eq. (5-2) restated below as:

$$
\beta=s \cdot\left(k T_{m}^{2} / E\right) \exp \left(-E / k T_{m}\right) .
$$

By making use of different linear heating rates, $\beta_{i}$, a plot of $\ln \left(T_{m}^{2} / \beta_{i}\right) v s 1 / T_{m}$ yields a straight line with a slope of $-E / k$ with a $y$-intercept of $\ln (s k / E)$ allowing for simultaneous calculation of both trap parameters [41]. TL curves were obtained for LiF:Mg,Ti (TLD-100) and three OSU materials $\mathrm{Li}_{2} \mathrm{~B}_{4} \mathrm{O}_{7}: \mathrm{Cu}_{0.4 \%}, \mathrm{Ag}_{0.1 \%}(\mathrm{LBO}), \mathrm{MgB}_{4} \mathrm{O}_{7}: \mathrm{Dy}_{0.1 \%}, \mathrm{Li}_{1 \%}(\mathrm{MBO}), \mathrm{CaSO}_{4}: \mathrm{Ce}_{0.2 \%}, \mathrm{~Tb}_{0.2 \%}$ (CSO) for heating rates from $0.1-10^{\circ} \mathrm{C} / \mathrm{s}$.

LiF:Mg,Ti (TLD-100)

Three different aliquots of TLD-100 were used for the varying heating rate method to study the effect of mass on the obtained trap parameters. The samples are characterized as a TLD-100 chip ( $23 \mathrm{mg}$ ) and two smaller samples; a crushed chip $(5.6 \mathrm{mg}$ ) and powder (2 mg). Typical TLD-100 TL curves obtained from different heating rates are presented in Fig. 80a. Following TL collection peak positions were obtained and plots of $\ln \left(T_{m}^{2} / \beta_{i}\right)$ vs $1 / T_{m}$ were fit, Fig. $80 \mathrm{~b}$. The VHRM results are compared with those from literature in Table 12. Activation energies for the smaller mass samples are comparable to those from literature, whereas the TLD-100 chip results did not agree due to poor heating, as illustrated in Fig. 80 b by the deviation from linear (black data points).
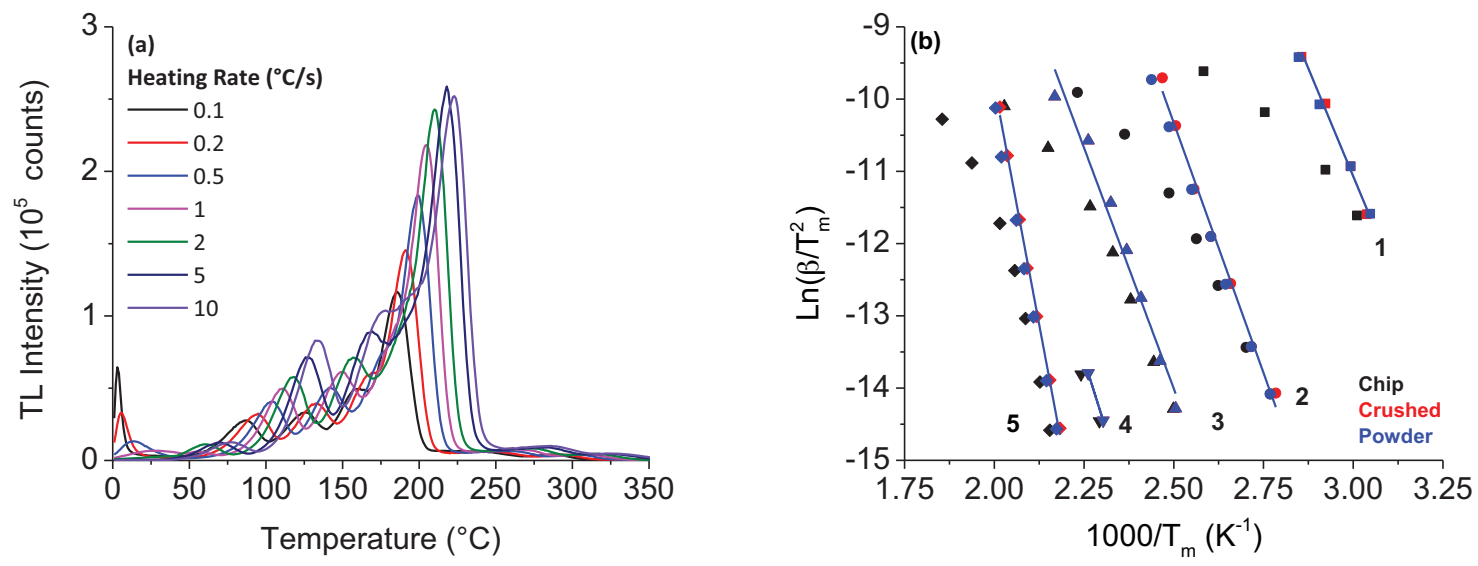

Fig. 80. Plot (a) shows TL curves obtained from a TLD-100 sample (crushed chip) for heating rates between $0.1-10^{\circ} \mathrm{C} / \mathrm{s}$. Plot (b) shows the results for three TLD-100 samples in different forms chip, crushed chip and powder. The larger mass of the chip introduces a thermal lag, which is seen in the deviation from linear in the black data points in plot (b). Numbers indicate the peak number. 
Table 12. TLD-100 kinetic parameters obtained using the VHRM. Peak positions provided were obtained using the $1^{\circ} \mathrm{C} / \mathrm{s}$ heating rate; if unavailable an "*" denotes a calculated peak temperature using Eq. (5-2). Results obtained from Ref. [44] were from a different analysis method (Glow Curve Deconvolution, GCD).

\begin{tabular}{|c|c|c|c|c|c|c|}
\hline Peak & Parameter & $\begin{array}{l}\text { Chip } \\
\text { (23 mg) }\end{array}$ & $\begin{array}{l}\text { Crushed Chip } \\
\text { (2 mg) }\end{array}$ & $\begin{array}{l}\text { Powder } \\
\text { (5.6 mg) }\end{array}$ & Ref. [44] & Ref. [45] \\
\hline \multirow[t]{3}{*}{1} & $E(e V)$ & 0.39 & 1.03 & 0.92 & - & - \\
\hline & $\log _{10} S$ & 4.67 & 14.83 & 13.16 & - & - \\
\hline & $\mathrm{T}_{\mathrm{m}}\left({ }^{\circ} \mathrm{C}\right)$ & 59 & 56 & 55 & - & - \\
\hline \multirow[t]{3}{*}{2} & $E(e V)$ & 0.68 & 1.19 & 1.14 & 1.26 & $1.11 \pm 0.02$ \\
\hline & $\log _{10} S$ & 7.42 & 14.68 & 13.88 & 15.23 & 13.48 \\
\hline & $\mathrm{T}_{\mathrm{m}}\left({ }^{\circ} \mathrm{C}\right)$ & 117 & 111 & 111 & $118^{*}$ & $112^{*}$ \\
\hline \multirow[t]{3}{*}{3} & $E(e V)$ & 0.78 & 1.15 & 1.15 & 1.33 & $1.27 \pm 0.03$ \\
\hline & $\log _{10} S$ & 7.67 & 12.72 & 12.51 & 14.81 & 13.95 \\
\hline & $\mathrm{T}_{\mathrm{m}}\left({ }^{\circ} \mathrm{C}\right)$ & 156 & 149 & 149 & $149 *$ & $152 *$ \\
\hline \multirow[t]{3}{*}{4} & $E(e V)$ & 1.08 & 1.36 & 1.36 & 1.62 & $1.6 \pm 0.07$ \\
\hline & $\log _{10} S$ & 10.37 & 13.68 & 13.68 & 16.88 & 16.48 \\
\hline & $\mathrm{T}_{\mathrm{m}}\left({ }^{\circ} \mathrm{C}\right)$ & $196 *$ & $190 *$ & $190 *$ & $182^{*}$ & $186^{*}$ \\
\hline \multirow[t]{3}{*}{5} & $\mathrm{E}(\mathrm{eV})$ & 1.23 & 2.28 & 2.21 & 2.12 & $2.06 \pm 0.11$ \\
\hline & $\log _{10} S$ & 11.40 & 23.16 & 22.32 & 21.18 & 20.3 \\
\hline & $\mathrm{T}_{\mathrm{m}}\left({ }^{\circ} \mathrm{C}\right)$ & 213 & 205 & 207 & $209 *$ & $214^{*}$ \\
\hline
\end{tabular}

$\mathrm{Li}_{2} \mathrm{~B}_{4} \mathrm{O}_{7}: \mathrm{Cu}_{0.4 \%} \mathrm{Ag} \mathrm{g}_{0.1 \%}$ (LBO)

A total of four aliquots of LBO were prepared with three aliquots of $1 \mathrm{mg}$, and one $10 \mathrm{mg}$ aliquot. Fig. 81a shows the average TL obtained from the $1 \mathrm{mg}$ LBO samples for various heating rates, with numbers to denote the approximate location of the TL peaks. The data used for linear fitting is displayed in Fig. $81 \mathrm{~b}$ and for the $10 \mathrm{mg}$ sample the thermal lag is apparent. The results from the fits from Fig. $81 \mathrm{~b}$ are available in Table 13 and moderate agreement for peaks 3 and 4 however the results are much different for all other peaks most likely due to thermal lag.
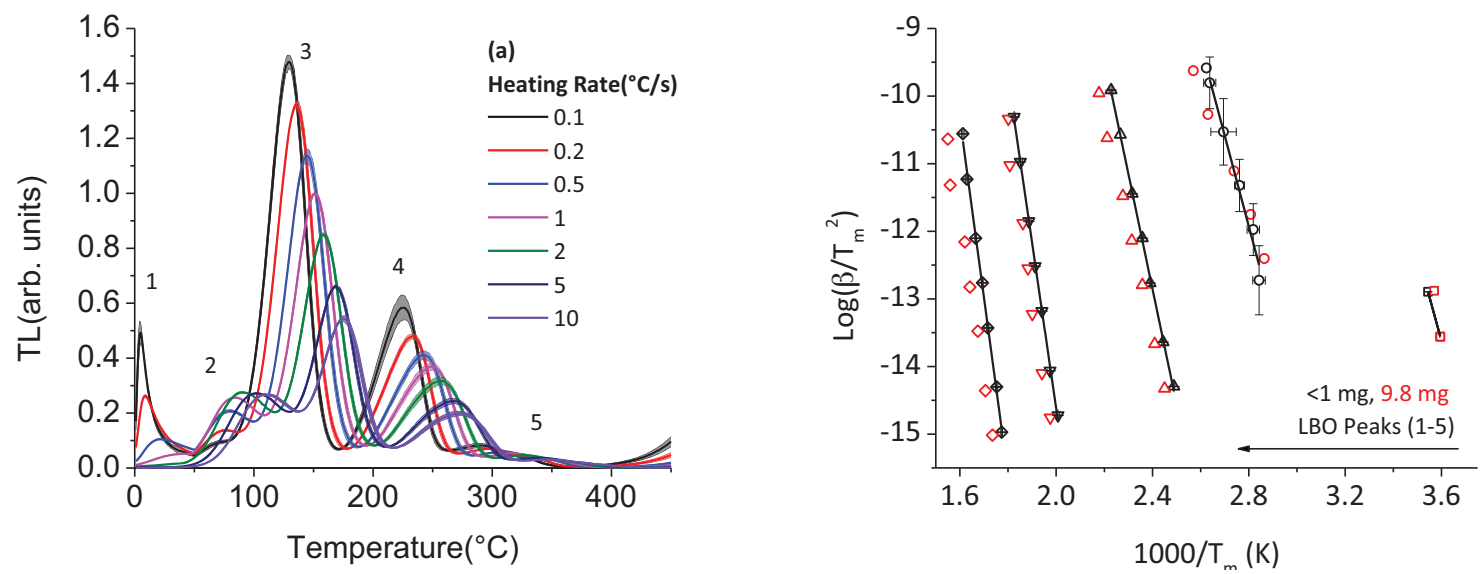

Fig. 81. Plot (a) shows the average TL curve obtained for $<1 \mathrm{mg}$ of LBO using various heating rates. The shaded region indicates one standard deviation. Plot (b) shows the data used for fitting from the $1 \mathrm{mg}$ and $10 \mathrm{mg}$ samples. 
Table 13. LBO kinetic parameters obtained using the VHRM. Peak positions provided were obtained using the $1^{\circ} \mathrm{C} / \mathrm{s}$ heating rate; if unavailable an "*” denotes a calculated peak temperature using Eq. (5-2). Results for the $<1 \mathrm{mg}$ samples are the averages of three samples, whereas the $10 \mathrm{mg}$ results are from a single sample.

\begin{tabular}{|cccc|}
\hline Peak & Parameter & $<1 \mathrm{mg}$ & $10 \mathrm{mg}$ \\
\hline $\mathbf{1}$ & $E(\mathrm{eV})$ & $1.12 \pm 0.0$ & 2.27 \\
& $\log _{10} S$ & $18.58 \pm 0.0$ & 39.83 \\
& $T_{\mathrm{m}}\left({ }^{\circ} \mathrm{C}\right)$ & $18^{*}$ & $11^{*}$ \\
\hline $\mathbf{2}$ & $E(\mathrm{eV})$ & $1.13 \pm 0.06$ & 0.78 \\
& $\log _{10} S$ & $15.37 \pm 0.64$ & 9.95 \\
& $T_{\mathrm{m}}\left({ }^{\circ} \mathrm{C}\right)$ & $84.33 \pm 1.53$ & 83 \\
\hline $\mathbf{3}$ & $E(\mathrm{eV})$ & $1.46 \pm 0.01$ & 1.36 \\
& $\log _{10} S$ & $16.33 \pm 0.17$ & 14.87 \\
& $T_{\mathrm{m}}\left({ }^{\circ} \mathrm{C}\right)$ & $151 \pm 1$ & 159 \\
\hline \multirow{4}{*}{$\mathbf{E}$} & $E(\mathrm{eV})$ & $2.10 \pm 0.03$ & 2.12 \\
& $\log _{10} S$ & $19.27 \pm 0.28$ & 19.05 \\
& $T_{\mathrm{m}}\left({ }^{\circ} \mathrm{C}\right)$ & $249.67 \pm 1.53$ & 258 \\
\hline $\mathbf{5}$ & $E(\mathrm{eV})$ & $2.27 \pm 0.03$ & 1.93 \\
& $\log _{10} S$ & $18.29 \pm 0.25$ & 14.76 \\
& $T_{\mathrm{m}}\left({ }^{\circ} \mathrm{C}\right)$ & $317.33 \pm 1.15$ & 336 \\
\hline
\end{tabular}

$\mathrm{MgB}_{4} \mathrm{O}_{7}: D y_{0.1 \%} L i_{1 \%}(\mathrm{MBO})$

A total of five aliquots of $\mathrm{MBO}$ were prepared with three aliquots of $1 \mathrm{mg}$, and one aliquot each for $2 \mathrm{mg}$ and $10 \mathrm{mg}$ sample sizes. Fig. 82a shows the average TL obtained from the $1 \mathrm{mg} \mathrm{MBO}$ samples for various heating rates. It is observed that for the two higher heating rates of $5{ }^{\circ} \mathrm{C}$ and $10{ }^{\circ} \mathrm{C}$ the standard deviation between the samples is larger than for lower heating rates, indicating inconsistent heating of the samples between readouts. The data used for linear fitting is displayed in Fig. 82b, showing clearly thermal lag for the $10 \mathrm{mg}$ sample. The results from the fits from Fig. $82 \mathrm{~b}$ are available in Table 14 and show relatively good agreement between the $1 \mathrm{mg}$ and $2 \mathrm{mg}$ samples, whereas the $10 \mathrm{mg}$ sample results are poor for the higher temperature TL peak.
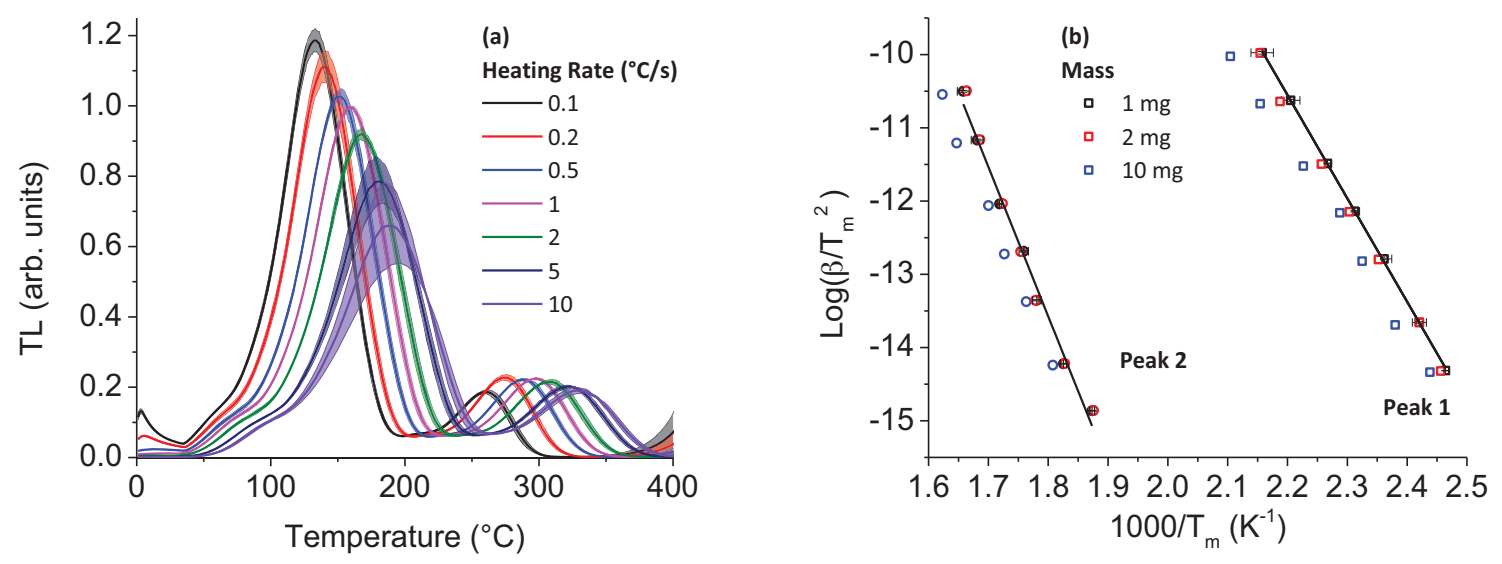

Fig. 82. Plot(a) shows the average TL curve obtained for $1 \mathrm{mg}$ of $\mathrm{MBO}$ using various heating rates. The shaded region indicates one standard deviation. Plot(b) shows the data used for fitting from the $1 \mathrm{mg}, 2 \mathrm{mg}$ and $10 \mathrm{mg}$ samples. 
Table 14. MBO kinetic parameters obtained using the VHRM. Peak positions provided were obtained using the $1^{\circ} \mathrm{C} / \mathrm{s}$ heating rate. A single sample was used to obtain the $2 \mathrm{mg}$ and $10 \mathrm{mg}$ results, whereas the average and standard deviation of three samples are presented for $1 \mathrm{mg}$.

\begin{tabular}{|ccccc|}
\hline Peak & Parameter & $\mathbf{1 ~} \mathbf{~ m g}$ & $\mathbf{2} \mathbf{~ m g}$ & $\mathbf{1 0} \mathbf{~ m g}$ \\
\hline $\mathbf{1}$ & $\mathrm{E}(\mathrm{eV})$ & $1.21 \pm 0.05$ & 1.19 & 1.21 \\
& $\log _{10} s$ & $13.29 \pm 0.53$ & 12.72 & 11.69 \\
& $\mathrm{~T}_{\mathrm{m}}\left({ }^{\circ} \mathrm{C}\right)$ & $159 \pm 2$ & 161 & 164 \\
\hline $\mathbf{2}$ & $\mathrm{E}(\mathrm{eV})$ & $1.75 \pm 0.05$ & 1.80 & 1.51 \\
& $\log _{10} s$ & $14.52 \pm 0.40$ & 14.68 & 11.90 \\
& $\mathrm{~T}_{\mathrm{m}}\left({ }^{\circ} \mathrm{C}\right)$ & $295 \pm 3$ & 297 & 306 \\
\hline
\end{tabular}

$\mathrm{CaSO}_{4}: \mathrm{Ce}_{0.2 \%} \mathrm{~Tb}_{0.2 \%}(\mathrm{CSO})$

TL curves were obtained for three small aliquots ( $<1 \mathrm{mg}$ ) of CSO and one typical aliquot size (10 mg). The TL curves obtained for the $<1 \mathrm{mg}$ aliquot are shown as averages in Fig. 83a with approximate peak positions denoted by numbers $1-3$. The corresponding data used for fitting can be found in Fig. 83b which shows a strong linear trend for the $<1 \mathrm{mg}$ samples, whereas the $10 \mathrm{mg}$ sample suffers from thermal lag as illustrated by the deviation from linear and shown by the differences in obtained kinetic parameters in Table 15.
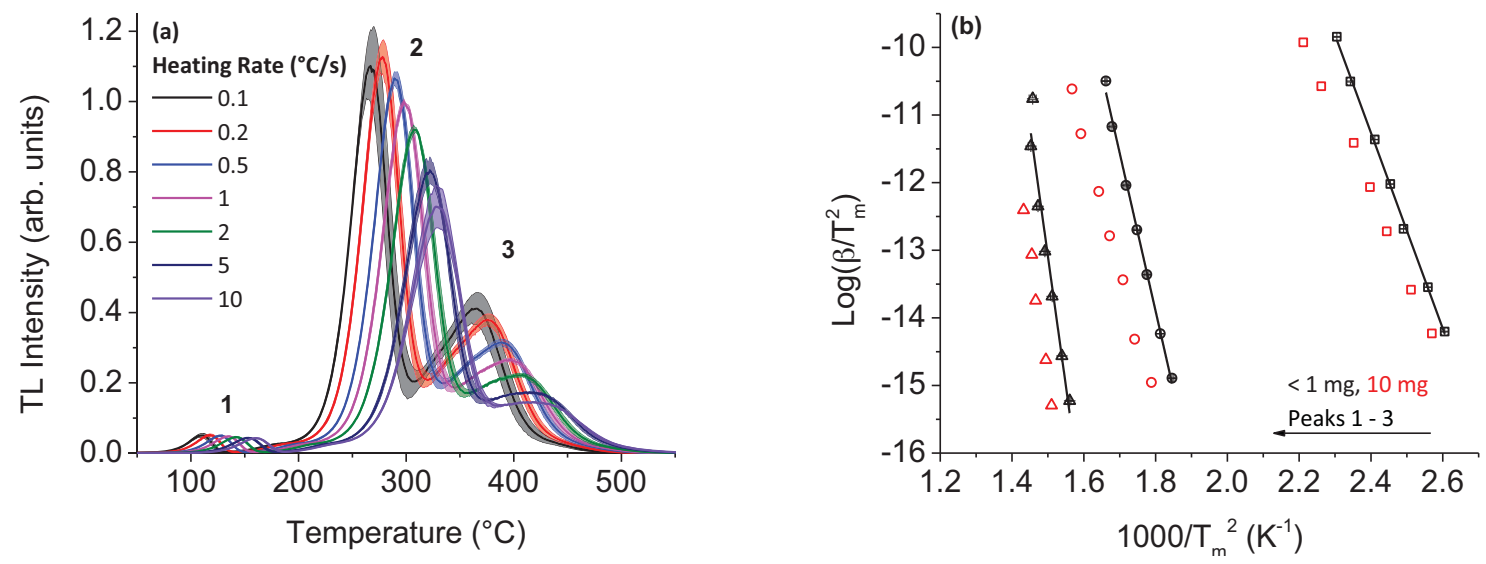

Fig. 83. Plot (a) shows the average TL curve obtained for $<1 \mathrm{mg}$ of CSO using various heating rates. The shaded region indicates one standard deviation. Plot (b) shows the data used for fitting from the $1 \mathrm{mg}$ and $10 \mathrm{mg}$ samples.

Table 15. CSO kinetic parameters obtained from the various heating rate method. Peak positions provided were obtained using the $1^{\circ} \mathrm{C} / \mathrm{s}$ heating rate. Results for the $<1 \mathrm{mg}$ samples are the averages of three samples, whereas the $10 \mathrm{mg}$ results are from a single sample.

\begin{tabular}{|cccc|}
\hline Peak & Parameter & $<1 \mathrm{mg}$ & $10 \mathrm{mg}$ \\
\hline $\mathbf{1}$ & $E(\mathrm{eV})$ & $1.24 \pm 0.03$ & 1.04 \\
& $\log _{10} S$ & $14.4 \pm 0.4$ & 11.46 \\
& $T_{\mathrm{m}}\left({ }^{\circ} \mathrm{C}\right)$ & $134.3 \pm 0.6$ & 144 \\
\hline $\mathbf{2}$ & $E(\mathrm{eV})$ & $2.01 \pm 0.03$ & 1.69 \\
& $\log _{10} \mathrm{~S}$ & $16.7 \pm 0.3$ & 13.04 \\
& $T_{\mathrm{m}}\left({ }^{\circ} \mathrm{C}\right)$ & $299 \pm 3$ & 325 \\
\hline $\mathbf{3}$ & $E(\mathrm{eV})$ & $3.28 \pm 0.21$ & 3.21 \\
\hline
\end{tabular}




\begin{tabular}{|ccc|}
\hline $\log _{10} S$ & $24.7 \pm 0.7$ & 22.40 \\
$T_{\mathrm{m}}\left({ }^{\circ} \mathrm{C}\right)$ & $397.3 \pm 1.1$ & 414 \\
\hline
\end{tabular}

\subsubsection{Discussion}

Kinetic parameters were obtained using the VHRM for three OSU materials and one commercial material for various sample weights. Results indicated that smaller mass samples ( $\leq 1 \mathrm{mg}$ ) performed better and were comparable to available results obtained from literature.

Finally the parameters obtained from the varying heating rate method found in Table 12 - Table 15 were used to simulate $1^{\text {st }}$ order TL curves using the Randall Wilkins model for a $1^{\circ} \mathrm{C} / \mathrm{s}$ heating rate. Peak intensities were normalized to the experimental TL curve intensity and are therefore not indicative of the trap carrier population and thus are for illustration only. The simulation results are found in Fig. 84 and show that for the OSU materials the TL curves obtained by the VHRM do not adequately explain the full TL curve indicating a more complex TL structure then that obtained by this method. This indicates that a deconvolution approach, informed by but not limited to traditional methods, maybe best suited for model development.
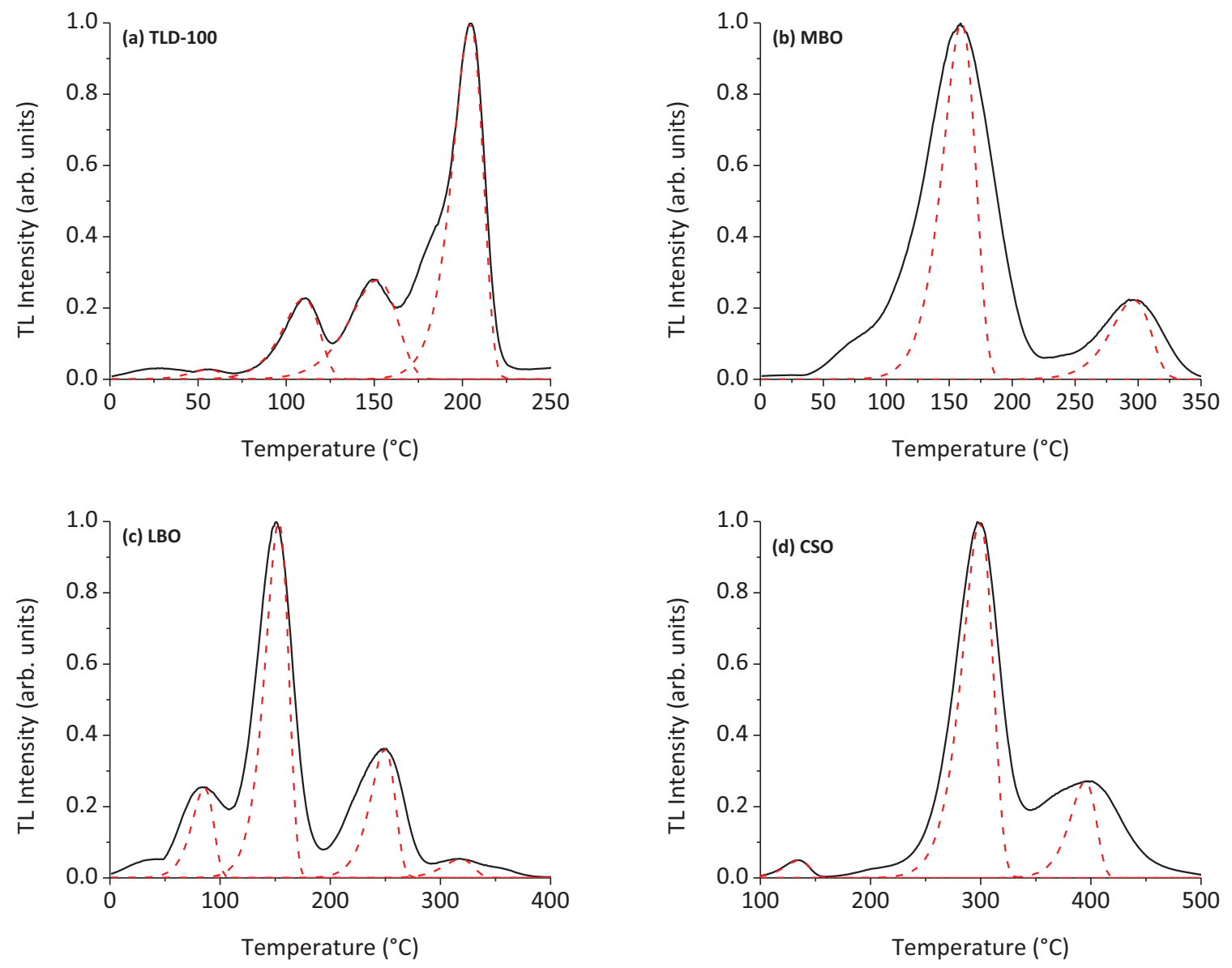

Fig. 84. Comparison between simualted TL curves and expiremential TL curves obtained for (a) TLD-100, (b) MBO, (c) LBO and (d) CSO using a $1^{\circ} \mathrm{C} / \mathrm{s}$ heating rate. Dashed red curves are simulated $1^{\text {st }}$ order TL peaks obtained using the kinetic parameters from the VHRM. Solid black curves are experimental TL data. 


\subsubsection{Comparison with deconvolution results}

A formal analysis of the trap parameters obtained from the IRM and VHRM has not been conducted and is the subject of future investigation. Nevertheless, we present a comparison of activation energies obtained using the IRM and VHRM with those obtained using deconvolution [10].

TL curves obtained during the VHRM for the $1^{\circ} \mathrm{C} / \mathrm{s}$ heating rate were used to calculate TL models using a fixed frequency factor $s=10^{14} \mathrm{~s}^{-1}$. Three aliquots per material were used for model generation and the resulting distributions are presented in Fig. 85.
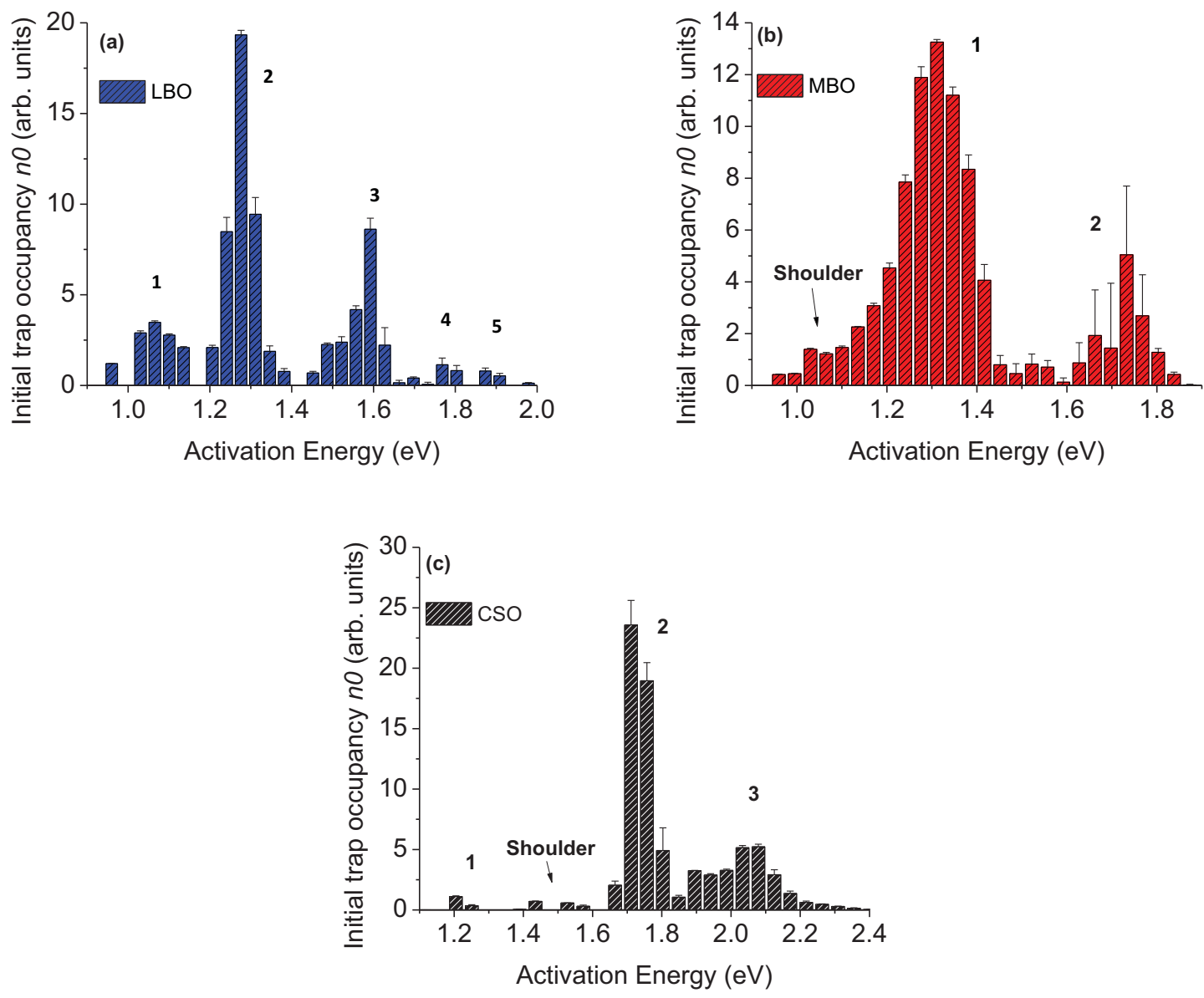

Fig. 85. Activation energy distributions obtained from OSU material TL curves using a $1{ }^{\circ} \mathrm{C} / \mathrm{s}$ heating rate and a fixed frequency factor of $s=10^{14} \mathrm{~s}^{-1}$. Numbers are included in each plot to signify the approximate location of each TL peak. Plot (b) an indicator to show where the low temperature TL shoulder occurs.

The VHRM resulted in a better resolution of parameters with regards to individual TL peaks, the results of which are provided in Table 14 - Table 15. VHR analysis of MBO returned activation energies of $1.21 \mathrm{eV}$ and $1.74 \mathrm{eV}$ for peaks 1 and 2 respectively. These values are close to those obtained through deconvolution, which is expected, as the corresponding frequency factors from VHR are within an order of magnitude the fixed value used in the deconvolution. Activation energy values obtained by the VHRM for the low temperature peaks of LBO $(1.12 \mathrm{eV})$ and CSO $(1.24 \mathrm{eV})$ are in good agreement with deconvolution method (Fig. 85a,c), but the higher temperature peaks differ by more than $0.1 \mathrm{eV}$. A comparison of the frequency factors obtained through the VHR method to the fixed value of $10^{14} \mathrm{~s}^{-1}$ shows a difference of multiple orders of magnitude. 
The deconvolution method works by fixing the frequency factor and choosing a distribution of activation energies that span the readout temperatures for a given TL curve. For a $1^{\text {st }}$ order TL peak (fixed $\mathrm{s}, 1^{\circ} \mathrm{C} / \mathrm{s}$ ) the relationship between the activation energy and the peak position $\left(T_{m}\right)$ is given by Eq. (5-2). Therefore by fixing $s$, there is a direct loss of precision in the activation energy if the real frequency factor deviates from the fixed value. This relationship is also responsible for the distribution resembling the TL curve structure. The IRM and VHRM results suggests a need to move to a more realistic TL model, but the complexity of the TL curves from OSU material also suggest that such a model cannot be easily achieved by traditional methods (IRM, VHRM, etc.) alone. Future model development of OSU materials should focus on combining deconvolution with physical analysis.

\subsection{Investigation of core-shell nanophosphors}

Several different materials were synthesized at Clemson University (CU) as part of this project. Their luminescence properties were characterized at OSU and the main results are reported here.

\subsubsection{Initial nanophosphor investigations}

Fig. 86 shows examples of RL data obtained from samples synthesized at CU using the solution precipitation method. The $\mathrm{RL}$ data again indicates that the trivalent dopants are acting as luminescence centers, although in the case of $\mathrm{CaF}_{2}$ :Eu luminescence from $\mathrm{Eu}^{2+}$ at $\sim 430 \mathrm{~nm}$ is also observed, in addition to the $\mathrm{Eu}^{3+}$ emission lines.

The TL data for the same samples, shown in Fig. 87, indicates that the TL peaks for these compounds tend to be concentrated in the high-temperature region of the TL curve. This is relevant because it covers a different temperature range from the samples investigated before. The other samples produced at CU did not resulted in high TL intensity in comparison to the background signal. 

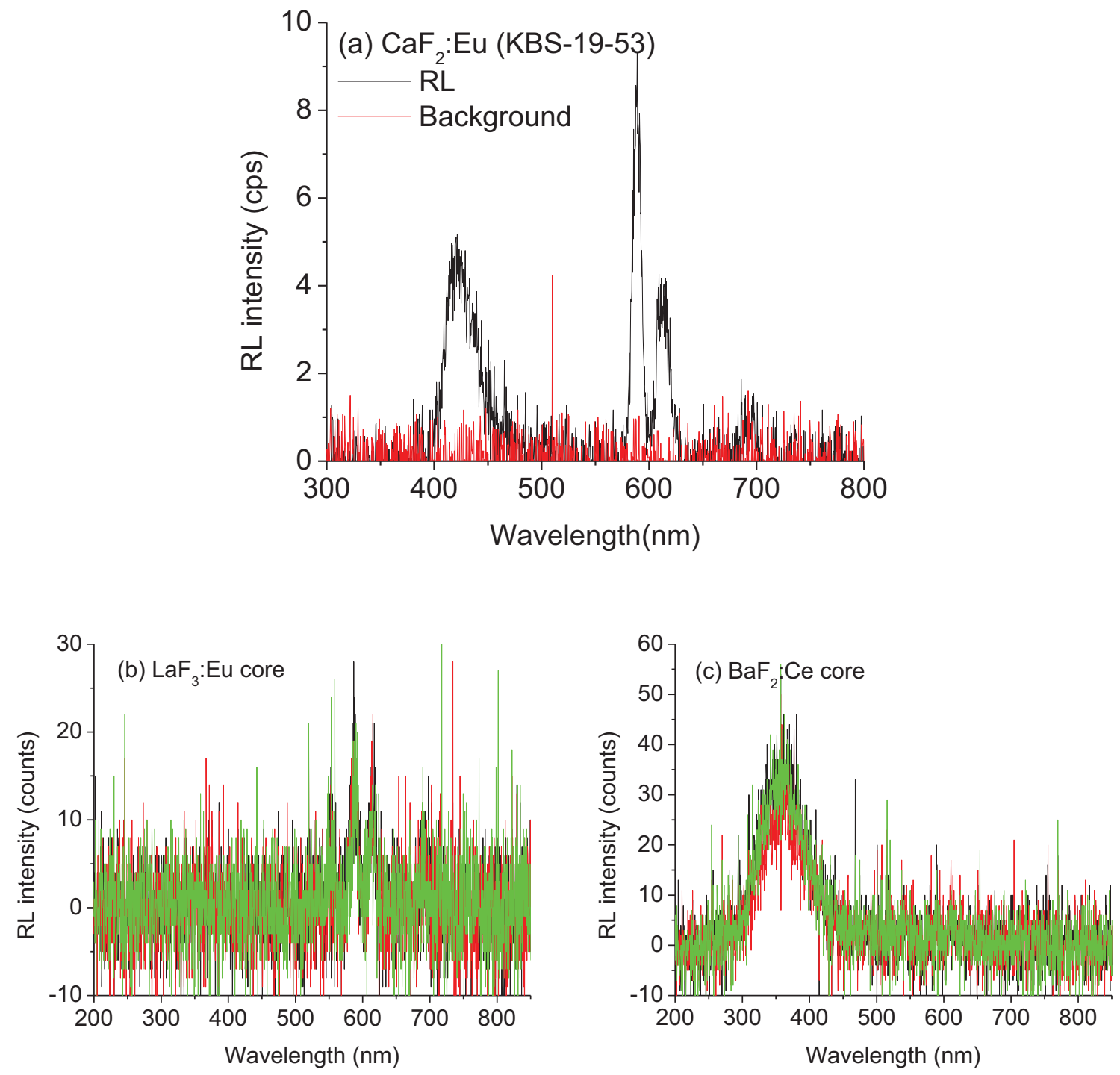

Fig. 86. Radioluminescence $(\mathrm{RL})$ emission spectrum of $\mathrm{CaF}_{2}: \mathrm{Eu}, \mathrm{LaF}_{3}: \mathrm{Eu}$ and $\mathrm{BaF}_{2}: \mathrm{Ce}$ synthesized at $\mathrm{CU}$ by the solution precipitation method. The data shows the characteristic emissions from $\mathrm{Eu}^{3+}$ and $\mathrm{Ce}^{3+}$, indicating that the dopants are acting as luminescence centers. 

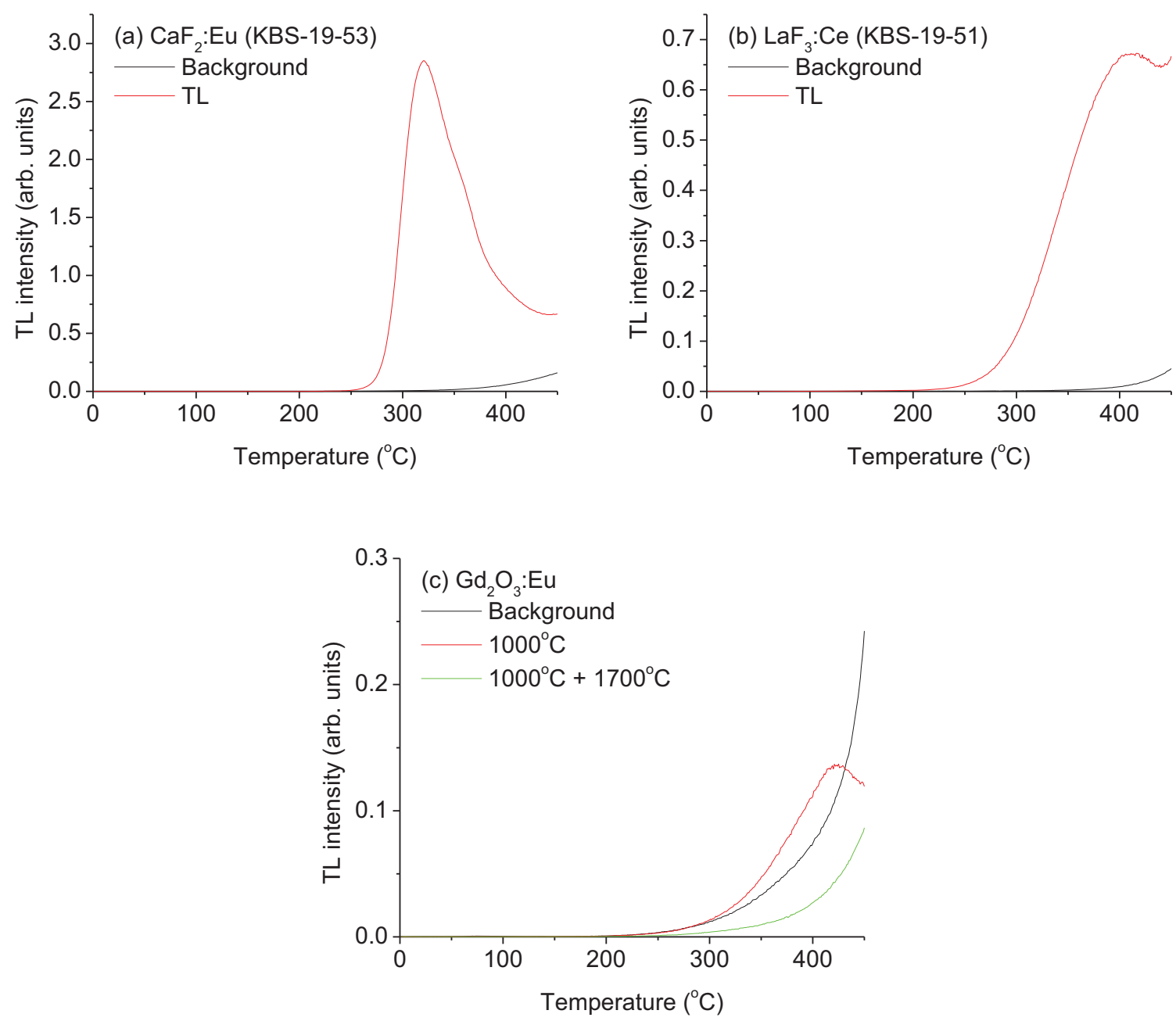

Fig. 87. TL curves of various samples synthesized at CU by the solution precipitation method. The data show that the TL emission from the samples produced so far at CU are concentrated in the high-temperature region of the TL curve.

The results obtained demonstrate the initial steps in the investigation to understand and control the luminescence process in the nanophosphors, particularly the TL process. Because of the intrinsically complex nature of the TL process, which requires the presence of luminescence and trapping centers, this investigation requires the efficient synthesis and characterization of a large number of samples.

Our results demonstrated the possibility of introducing the appropriate luminescence centers in various host materials.

\subsubsection{Calculation of energy levels in $\mathrm{YF}_{3}$}

To address the difficulty in introducing simultaneously trapping center and recombination centers in nanophosphors, we investigated the possibility of using Dorenbos model to guide the choice of dopants to be used [31, 46-49]

Based on Dorenbos model, we calculated the energy levels of lanthanides in this host materials, as we have done before for YAG [5]. The energy diagram obtained (Fig. 88) indicates that Sm, Eu, Yb and Tm 
are the most promising dopants to act as trapping centers. In addition, $\mathrm{Ce}, \mathrm{Tb}$ and $\mathrm{Pr}$ were used as codopants to introduce potential luminescence centers.

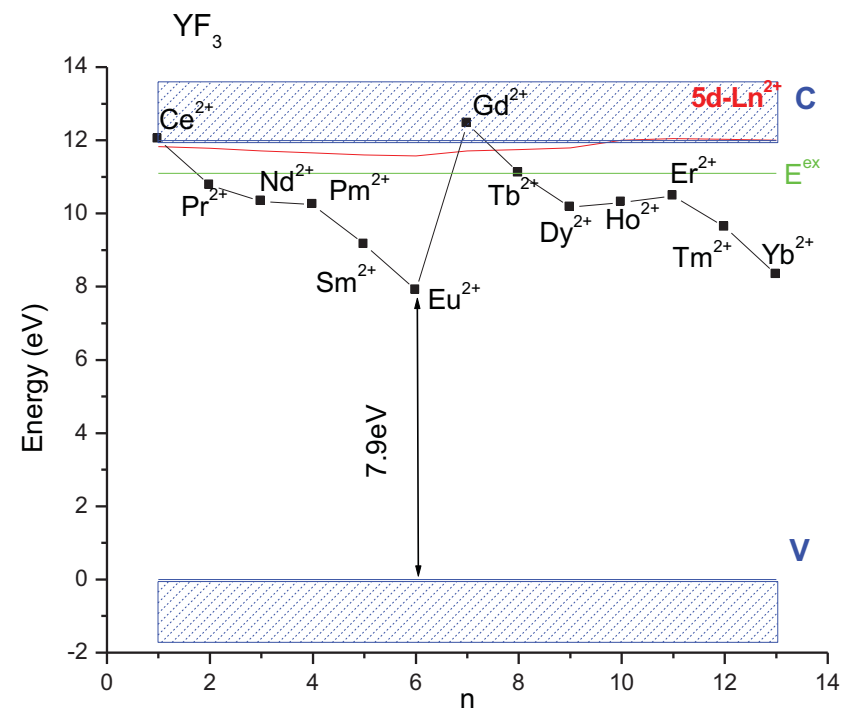

Fig. 88. $\mathrm{YF}_{3}$ band diagram calculated as in $\mathrm{YAG}$ using data from the literature: band gap $11.988 \mathrm{eV}, \mathrm{Eu}^{2+} 7.9 \mathrm{eV}^{2}$ and $\mathrm{E}^{\mathrm{ex}}=$ $11.1 \mathrm{eV}$ [see 5 and references therein].

\subsubsection{Characterization of nanophosphors produced at Clemson University}

We characterized the $R L$ and $T L$ of the samples produced in Clemson to test for the incorporation of the luminescence centers and the introduction of new trapping centers. We also tested the photobleaching ( $3 \mathrm{~h}$ of room light exposure) on the TL of irradiated samples.

Fig. 89 and Fig. 90 present the RL signal from representative $\mathrm{YF}_{3}$ and $\mathrm{MgF}_{2}$ samples, showing that the emission is characteristic of the trivalent or divalent lanthanides introduced as dopants.

Fig. 91 shows the TL and photo-bleaching tests for $\mathrm{YF}_{3}$, indicating that the TL intensity is relatively weak compared to samples investigated previously and are significantly different before and after light exposure (Fig. 91b). This difference can be caused by both fading (thermal stimulation of trapped charges at room temperature) and photo-bleaching (optical stimulation of trapped charges). Similar data are presented for $\mathrm{MgF}_{2}$ in Fig. 92.

Based on the diagram presented in Fig. 88, Clemson University prepared several samples with codopants. The TL curves from some of these samples are shown in Fig. 93. In general, the TL signal was improved when compared to the singly-doped materials, in some cases presenting well-defined peaks, but it was again observed that the TL curves before and after light exposure were significantly different (Fig. 94). This prevents the use of these materials as temperature sensors.

Because of the difficulties in the synthesis of nanophosphors with adequate properties for temperature sensing, as well as estimations showing that materials produced by solution combustion synthesis have adequate time response for the initial tests to be performed (see Section 5.6.4), investigations on nanophosphors were not further pursued in Option Years 1 and 2 of this project. 

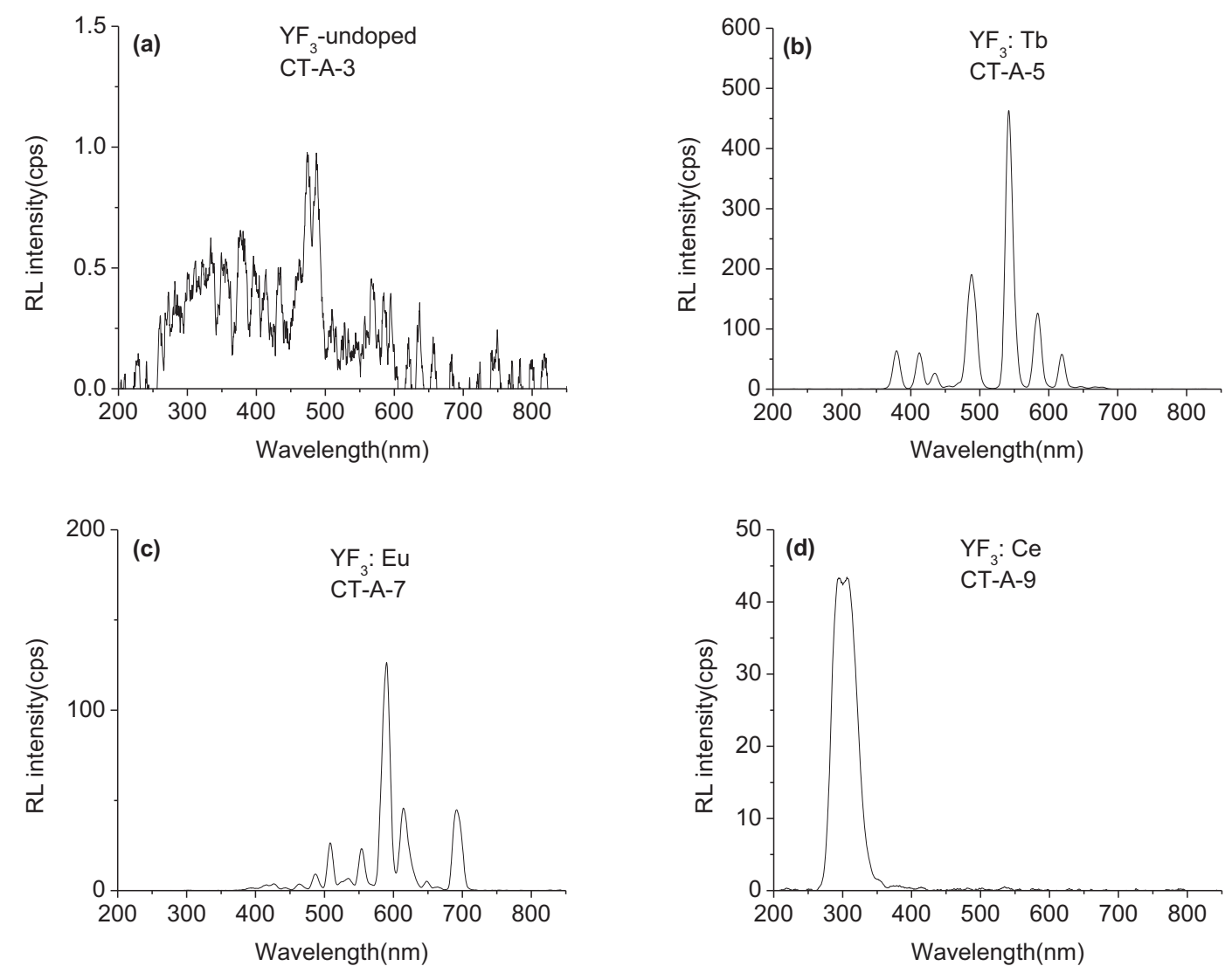

Fig. 89. RL signal from $\mathrm{YF}_{3}$ doped with several lanthanides. The data show that the lanthanides are successfully acting as recombination center in these materials. 

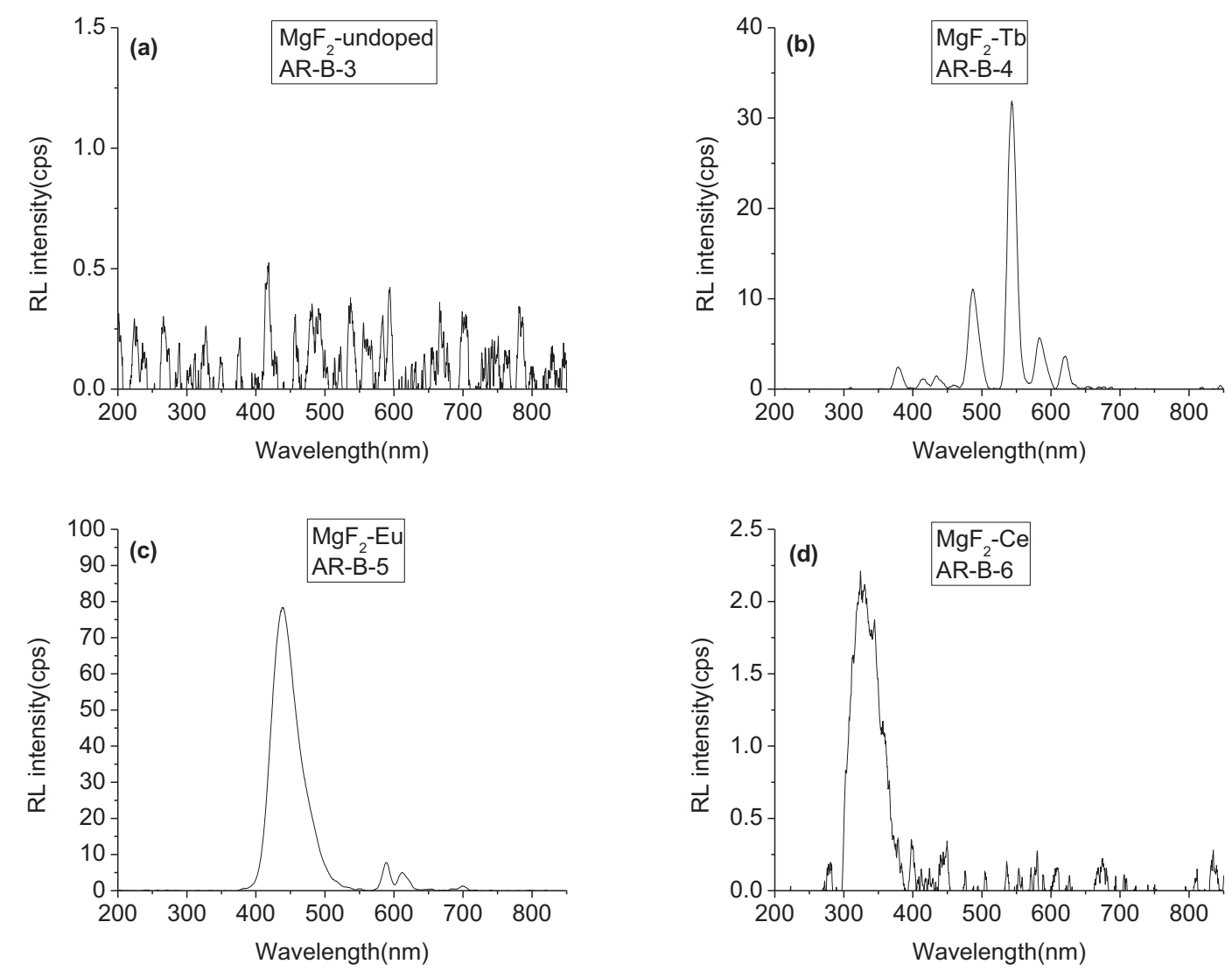

Fig. 90. $\mathrm{RL}$ signal from $\mathrm{MgF}_{2}$ doped with several lanthanides. The data shows that the lanthanides are successfully acting as recombination center in these materials.
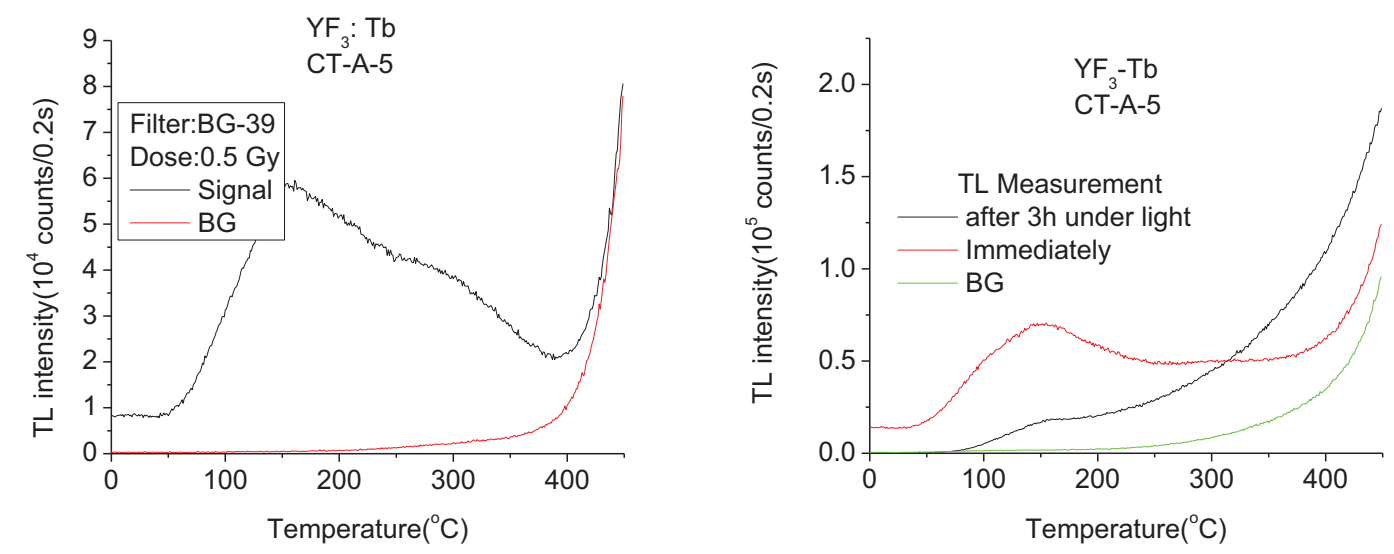

Fig. 91. (a) TL from some of the best samples of $\mathrm{YF}_{3}$ and (b) photo-bleaching test for the same sample. The data show that the $\mathrm{TL}$ is in general weak compared to other samples presented before and, in general, the curves were significantly different immediately after irradiation or after irradiation and $3 \mathrm{~h}$ of light exposure. 

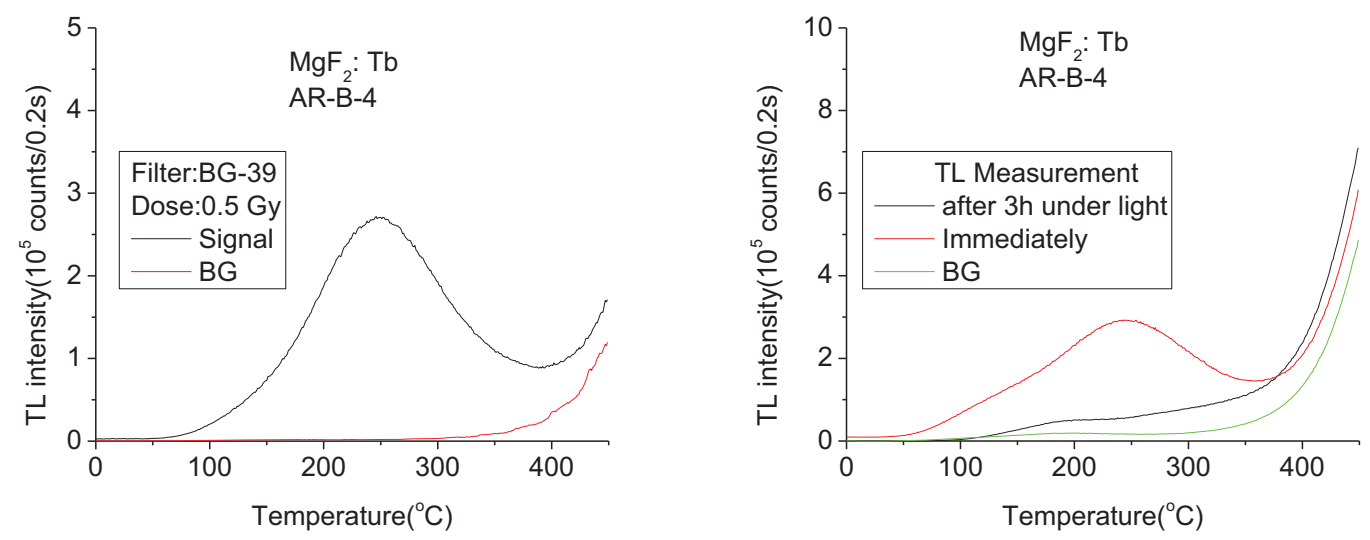

Fig. 92. (a) TL from some of the best samples of $\mathrm{MgF}_{2}$ and (b) photo-bleaching test for the same sample. Again, the curves were significantly different immediately after irradiation or after irradiation and $\mathbf{3} \mathrm{h}$ of light exposure.
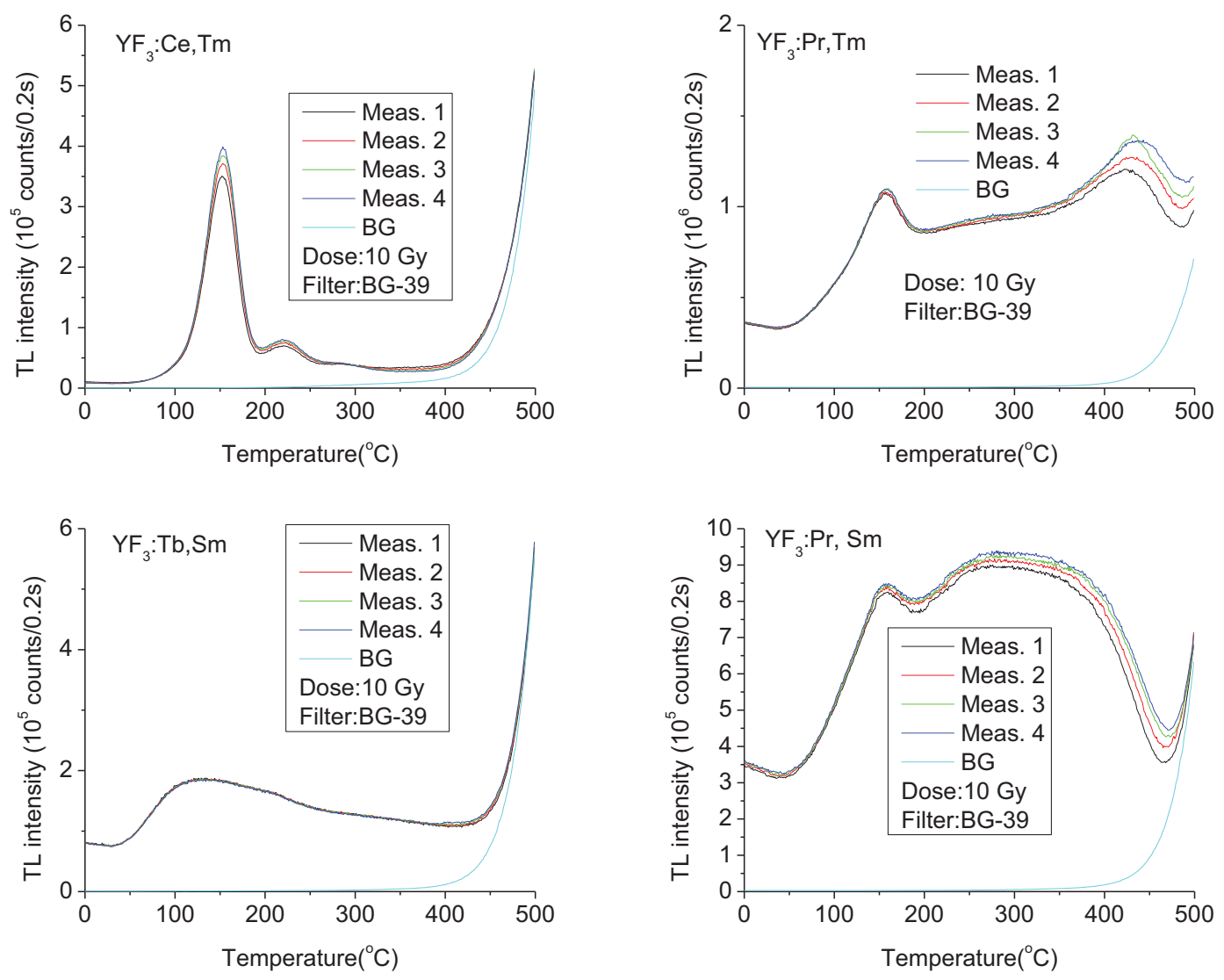

Fig. 93. TL from some samples co-doped with both a recombination center ( $\mathrm{Ce}, \mathrm{Pr}, \mathrm{Tb}, \mathrm{Pr}$ ) and a trapping center ( $\mathrm{Tm}$, Sm) after repeated irradiation with $10 \mathrm{~Gy}$ and TL readout. The dose given (10 Gy) here was significantly higher than the dose given in previous experiments ( $0.5 \mathrm{~Gy})$ because the samples were not very sensitive to ionizing radiation. 

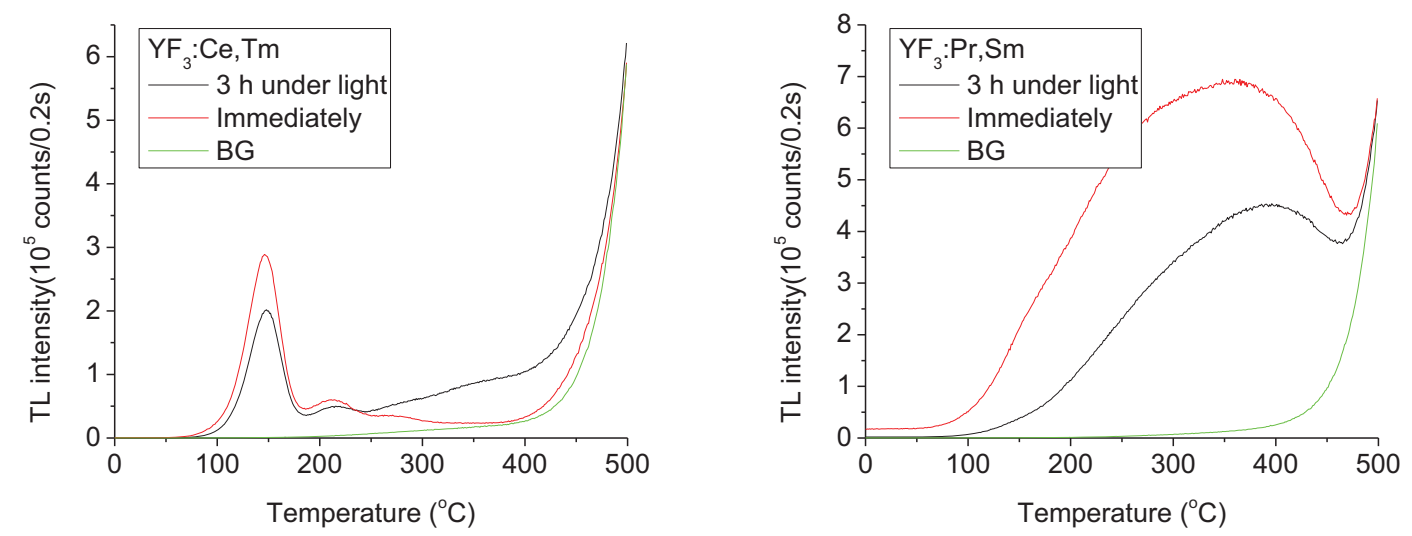

Fig. 94. Photo-bleaching tests for (a) $\mathrm{YF}_{3}: \mathrm{Ce}, \mathrm{Tm}$ and $(\mathrm{b}) \mathrm{YF}_{3}: \mathrm{Pr}, \mathrm{Sm}$. The data show that the samples are significantly different before and after light exposure (after irradiation with the same dose).

\subsubsection{Computer simulation of luminescence of idealized particles}

We investigate the possibility of using core-shell nanoparticles to discriminate between different heating profiles. The concept, illustrated in Fig. 95, is that a core-shell structure with the right properties could result in the shell responding to the fast thermal profile, whereas the core would correspond to the time-average of the temperature profile.

This scheme was tested using computer simulation of an idealized particle subjected to a $1 \mathrm{~ms}$ pulse, as shown in Fig. 96. First of all, it was concluded that the core-shell structure would have to be on the micrometer scale to respond differently to the thermal profile. Fig. 97 shows the temperature profile as a function of depth of the particle for different sizes of particles. For the particle with $200 \mu \mathrm{m}$ core, we also show the temperature profiles for different times.

The data in Fig. 97 show that, whereas the temperature of the shell can increase significantly during the fast thermal profile, the temperature of the core varies slowly and corresponds to a time-average of the temperature profile. Of course these simple calculations have been carried out with a simplified system just to demonstrate the principle.

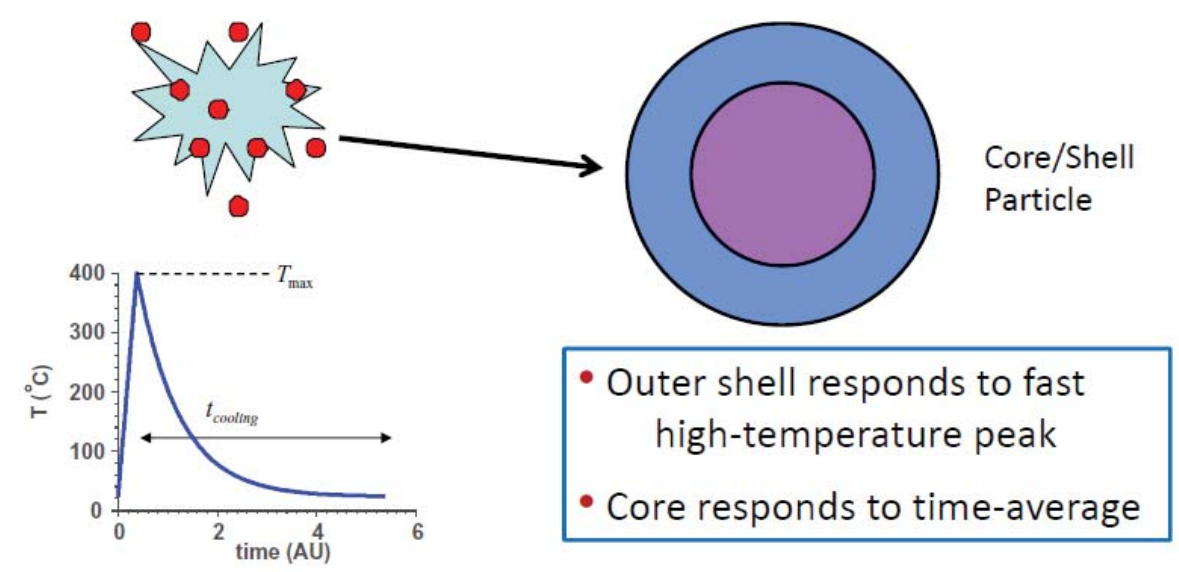

Fig. 95. Illustration of the concept of using a core-shell particle to discriminate between the fast radiative and slow convective thermal profiles. 


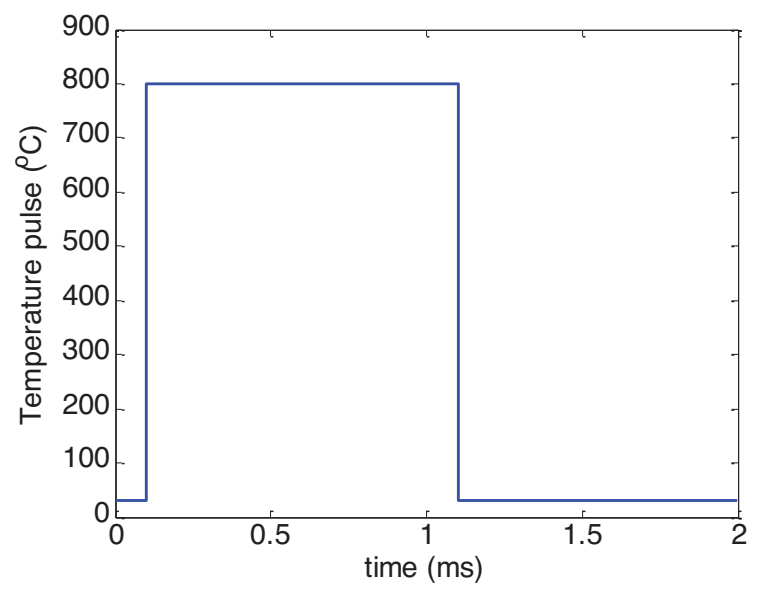

Fig. 96. Thermal pulse used in the simulations of the effect of heating on a core-shell particle.

$\square 90 \mu \mathrm{m}$ Core with $10 \mu \mathrm{m}$ shell

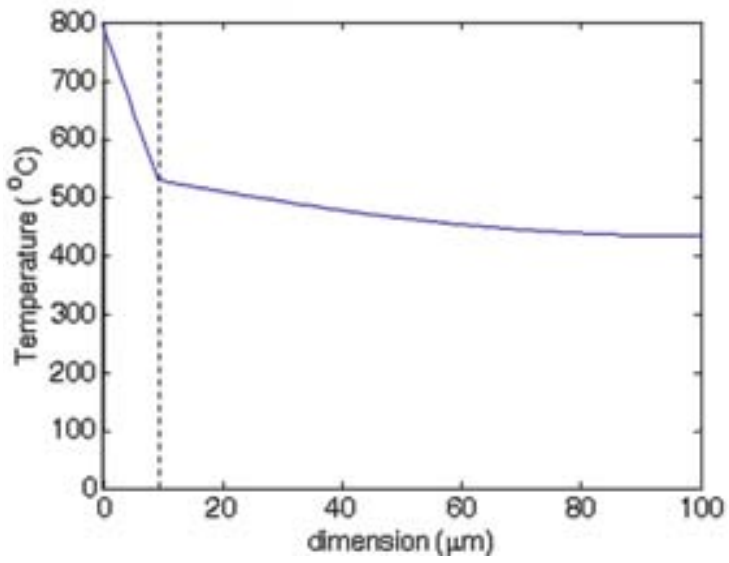

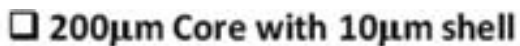

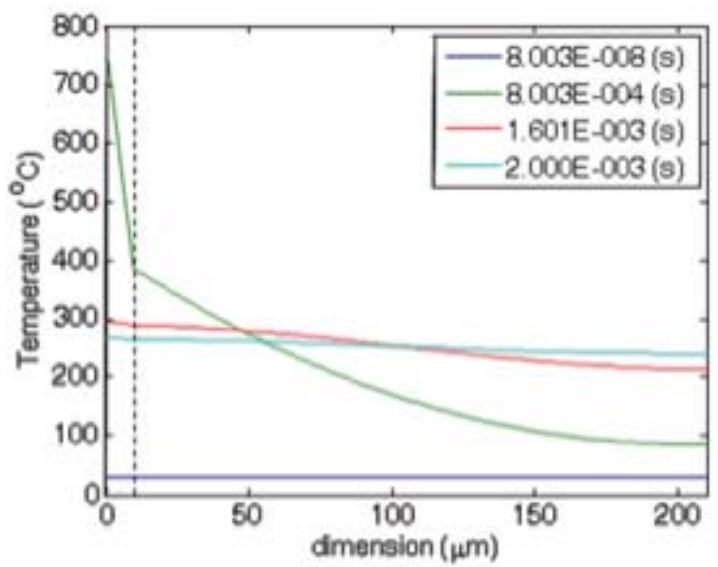

Fig. 97. Temperature profiles for an idealized core-shell particle of $90 \mu \mathrm{m}$ or $200 \mu \mathrm{m}$ core and $10 \mu \mathrm{m}$ shell subjected to a $1 \mathrm{~ms}$ thermal pulse. The figure shows that, depending on the thermal properties and relative sizes of the core-shell structure, the core may be less susceptible to the fast increase in temperature experienced by the surface of the particle, acting as an averaging sensor. 


\section{DEVELOPMENT OF MULTIPARAMETRIC TEMPERATURE RECONSTRUCTION}

Algorithms were tested independently at UMN and at OSU to verify the consistency of the results and also to take advantage of the equipment available at each institution. Below we describe briefly the fundamentals of the algorithms investigated in each institution.

\subsection{The approach employed by OSU}

The algorithm developed at OSU makes use of a TL model for the materials developed, which then allows one to calculate the expected TL curve for any heating profile. These curves are then compared to the experimental curves for exposed particles to determine the time-temperature profile to which they were exposed.

\subsubsection{Deconvolution of the TL curves}

The model assumes first-order kinetic model of $\operatorname{TL}[25,50]$, i.e., negligible re-trapping of the thermally stimulated charges. In the first-order model, the rate of change in the trapped charge population $n$ for a trapping center characterized by activation energy $E$ and frequency factor $s$ is given by:

$$
\frac{d n}{d t}=-n s e^{-E / k T}
$$

By integration, and assuming prompt recombination, the TL intensity is given by:

$$
I(T) \propto n_{0} s e^{-E / k T} \exp \left(-\frac{s}{\beta} \int_{T_{0}}^{T} e^{-E / k \theta} d \theta\right)
$$

where $n_{0}$ is the initial occupancy of the trapping center, $\beta$ is the heating rate, and $k$ is the Boltzmann constant [25].

To obtain data to develop the TL model, we recorded the TL curves after the sample had been irradiated with a fixed dose and heated with a known profile. The procedure consisted in heating to $T_{\max }$ at $1{ }^{\circ} \mathrm{C} / \mathrm{s}$, holding (soak) at $T_{\mathrm{ph}}$ for $5 \mathrm{~min}$, and cooling it back to room temperature. This cycle was performed automatically using the Ris $\varnothing \mathrm{TL}$ reader. The long soak time $(5 \mathrm{~min}$ ) was chosen to guarantee that the trap depopulation during heating and cooling can be considered negligible. This sequence was repeated several times for increasing values of $T_{\max }$ in $10^{\circ} \mathrm{C}$ steps.

The entire set of TL curves $y_{i, T p h}$ was then fitted simultaneously using a superposition of $N$ individual TL peaks with activation energies $E_{1}, E_{2}, \ldots, E_{N}$ and fixed frequency factor $s$, where the values have been chosen to cover the temperature range of the TL curve, as illustrated in Fig. 98. 

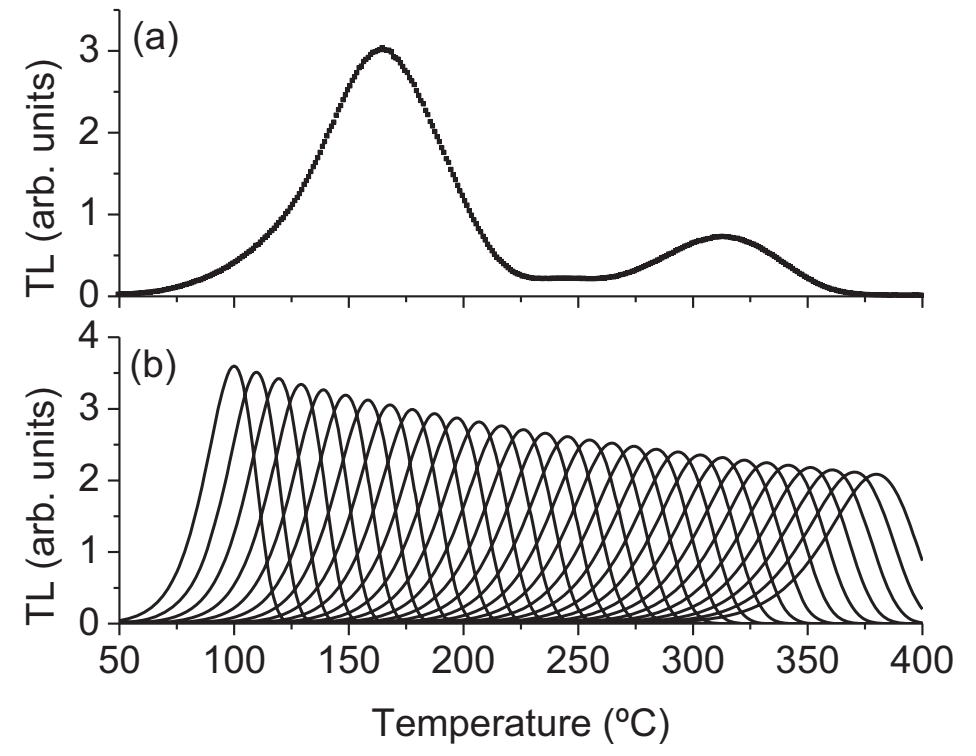

Fig. 98. (a) Experimental TL curve for MBO after pre-heating to $T p h=50$ ㅇ $\mathrm{C}$ for $\mathbf{5} \mathrm{min}$; and (b) first-order TL peaks used to simultaneously fit the set of TL curves obtained for various Tph.

In matrix form, the problem can be written as:

$$
\mathbf{y}=\mathbf{X a}
$$

where $\mathbf{y}$ is a column-vector containing the intensity of all TL curves, $\mathbf{a}$ is the column-vector containing the parameters that describe the population of the trapping centers $n_{0}$ for each trapping center, and $\mathbf{X}$ is the project matrix. To be more explicit, Eq. (6-6) can be written as:

$$
\left(\begin{array}{c}
y_{1, T p h 1} \\
y_{2, T p h 1} \\
\ldots \\
y_{n, T p h 1} \\
y_{1, T p h 2} \\
y_{2, T p h 2} \\
\ldots \\
y_{n, T p h 2} \\
y_{1, T p h M} \\
y_{2, T p h M} \\
\ldots \\
y_{n, T p h M}
\end{array}\right)=\left(\begin{array}{cccc}
f\left(E_{1}, T_{1}\right) \phi\left(E_{1}, T p h_{1}\right) & f\left(E_{2}, T_{1}\right) \phi\left(E_{2}, T p h_{1}\right) & \ldots & f\left(E_{N}, T_{1}\right) \phi\left(E_{1}, T p h_{2}\right) \\
f\left(E_{1}, T_{2}\right) \phi\left(E_{1}, T p h_{1}\right) & f\left(E_{2}, T_{2}\right) \phi\left(E_{2}, T p h_{1}\right) & \ldots & f\left(E_{N}, T_{1}\right) \phi\left(E_{1}, T p h_{2}\right) \\
\ldots & \ldots & \ldots & \ldots \\
f\left(E_{1}, T_{n}\right) \phi\left(E_{1}, T p h_{1}\right) & f\left(E_{2}, T_{n}\right) \phi\left(E_{2}, T p h_{1}\right) & \ldots & f\left(E_{N}, T_{1}\right) \phi\left(E_{1}, T p h_{2}\right) \\
f\left(E_{1}, T_{1}\right) \phi\left(E_{1}, T p h_{2}\right) & f\left(E_{2}, T_{1}\right) \phi\left(E_{2}, T p h_{2}\right) & \ldots & f\left(E_{N}, T_{1}\right) \phi\left(E_{1}, T p h_{2}\right) \\
f\left(E_{1}, T_{2}\right) \phi\left(E_{1}, T p h_{2}\right) & f\left(E_{2}, T_{2}\right) \phi\left(E_{2}, T p h_{2}\right) & \ldots & f\left(E_{N}, T_{2}\right) \phi\left(E_{1}, T p h_{2}\right) \\
\ldots & \ldots & \ldots & \ldots \\
f\left(E_{1}, T_{n}\right) \phi\left(E_{1}, T p h_{2}\right) & f\left(E_{2}, T_{1}\right) \phi\left(E_{2}, T p h_{2}\right) & \ldots & f\left(E_{N}, T_{1}\right) \phi\left(E_{1}, T p h_{2}\right) \\
\ldots & \ldots & \ldots & \ldots \\
f\left(E_{1}, T_{1}\right) \phi\left(E_{1}, T p h_{M}\right) & f\left(E_{2}, T_{1}\right) \phi\left(E_{2}, T p h_{M}\right) & \ldots & f\left(E_{N}, T_{1}\right) \phi\left(E_{1}, T p h_{M}\right) \\
f\left(E_{1}, T_{2}\right) \phi\left(E_{1}, T p h_{M}\right) & f\left(E_{2}, T_{2}\right) \phi\left(E_{2}, T p h_{M}\right) & \ldots & f\left(E_{N}, T_{2}\right) \phi\left(E_{1}, T p h_{M}\right) \\
\ldots & \ldots & \ldots & \ldots \\
f\left(E_{1}, T_{n}\right) \phi\left(E_{1}, T p h_{M}\right) & f\left(E_{2}, T_{1}\right) \phi\left(E_{2}, T p h_{M}\right) & \ldots & f\left(E_{N}, T_{1}\right) \phi\left(E_{1}, T p h_{M}\right)
\end{array}\right) \cdot\left(\begin{array}{c}
n_{0,1} \\
n_{0,2} \\
n_{0, N}
\end{array}\right)
$$


where $f\left(E_{i}, T_{j}\right)$ is the TL intensity corresponding to an activation energy $E_{i}$, temperature $T_{j}$ and unitary trap occupancy, and $\phi\left(E_{i}, T p h_{k}\right)$ is the occupancy rate of a trapping center with activation energy $E_{\mathrm{i}}$ subjected to the pre-heating with maximum temperature $T_{p h}$. In the first-order model, this is simply obtaining by integrating Eq. (6-1) for a constant temperature $T$ and soak time $\tau$, which results in:

$$
\phi(E, T p h)=\exp \left(-s e^{-E / k T p h} \tau\right),
$$

Therefore, the project matrix $\mathbf{X}$ takes into account the fact that some of the TL curves were obtained after the sample has been heated to a temperature Tph for a duration $\tau$. The parameter vector a represents the original trapping population before the sample was pre-heated; this parameter is common to all TL curves and can be found by solving the least-squares problem for Eq. (6-3). This was accomplished using the non-negative least square algorithm given by Lawson and Hanson [51]. The solution a was used as the input in the analysis of the heated samples.

Fig. 99 shows an example of the activation energy distribution obtained with the formalism above for three materials developed at OSU: LBO, MBO and CSO. A complete algorithm is presented in the Appendices and more detailed results are presented in Ref. [10].

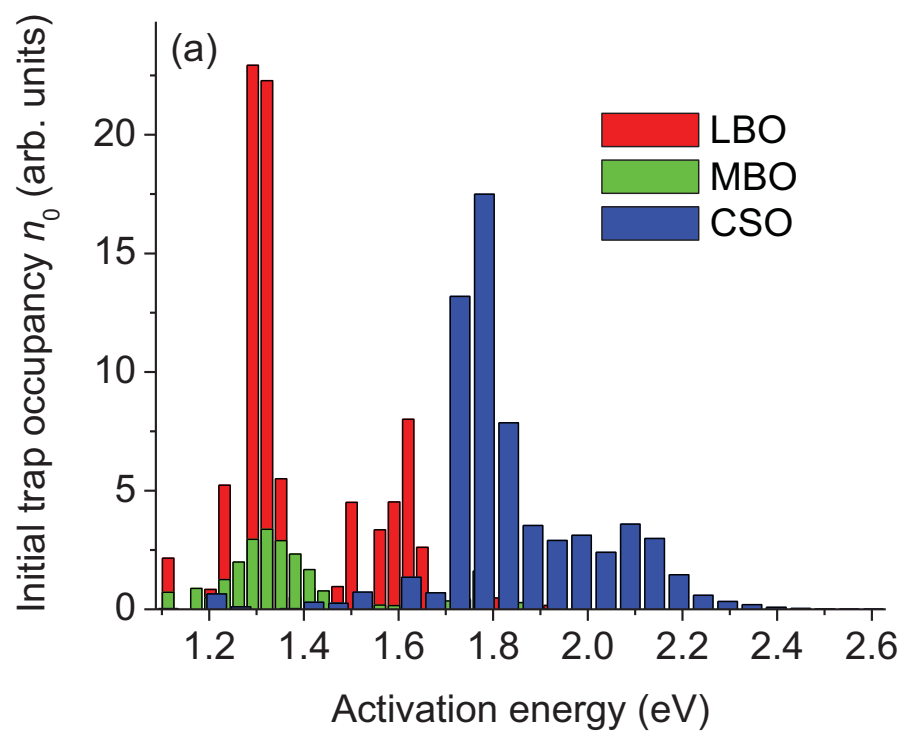

Fig. 99. Initial trap occupancy versus activation energy for LBO, MBO and CSO obtained by fitting simultaneously the TL curves from these materials obtained after irradiation and pre-heating to different temperatures for 5 min. A fixed frequency factor $s=10^{14} \mathrm{~s}^{-1}$ was used in these fittings.

\subsubsection{Temperature profile determination}

To determine the temperature profile to which the sample was heated, we used the solution a obtained in the previous section and calculated the TL curves that would be expected had the sample been heated to a temperature $T$ for a duration $\tau$. The resultant TL curve $y$ was compared to the experimental TL curve $y_{\text {exp }}$, obtained for the heated sample, using a parameter given by: 


$$
\eta=\frac{n}{\sqrt{\sum_{i}\left(y_{i}-y_{i, \exp }\right)^{2}}},
$$

where the values $y_{i}$ and $y_{i, \text { exp }}$ are after both TL curves have been normalized to the maximum intensity. Therefore, variations in absolute intensity of the TL curves are not taken into account, only the relative intensity of the TL peaks is considered. In this definition, a large $\eta$ value indicates good agreement between the shape of the TL curves, whereas a small $\eta$ value indicates poor agreement between them. The value $\eta$ was calculated for various $T$ and $\tau$ combinations, generating a matrix that maps the solutions for which there is good agreement between the predicted and experimental TL curves.

The contour plots in Fig. 100 shows examples of values of $\phi$ versus $T$ and $\log _{10}(\tau)$ for four LBO aliquots heated to different temperatures in the furnace for $2 \mathrm{~h}$. The lighter (yellow) regions indicate a high value of $\phi$, calculated using Eq. (6-6), and therefore indicating a good agreement between the experimental TL curve and the TL curves calculated based on the model in Fig. 99 for a given preheating temperature $T$ and duration $\tau$.

Fig. 101 shows a comparison between the experimental curve (data points) and the calculated TL curves (lines) for LBO heated in the furnace set at $100{ }^{\circ} \mathrm{C}$. The figure shows the TL curve for the best solution $\left(91^{\circ} \mathrm{C}\right.$ ) obtained for a heating of $\tau=7200 \mathrm{~s}$. The inset shows the value of $\eta$ along the $\tau=7200 \mathrm{~s}$ line as a function of the heating temperature $\Theta$, indicating that the best agreement is observed for $\Theta=$ $91{ }^{\circ} \mathrm{C}$ for this aliquot. The figure also shows the TL curves expected for $\pm 10{ }^{\circ} \mathrm{C}$ heating from the best solution, indicating the high sensitivity of the TL process to small temperature variations in this case.

The complete algorithm is presented in the Appendices.
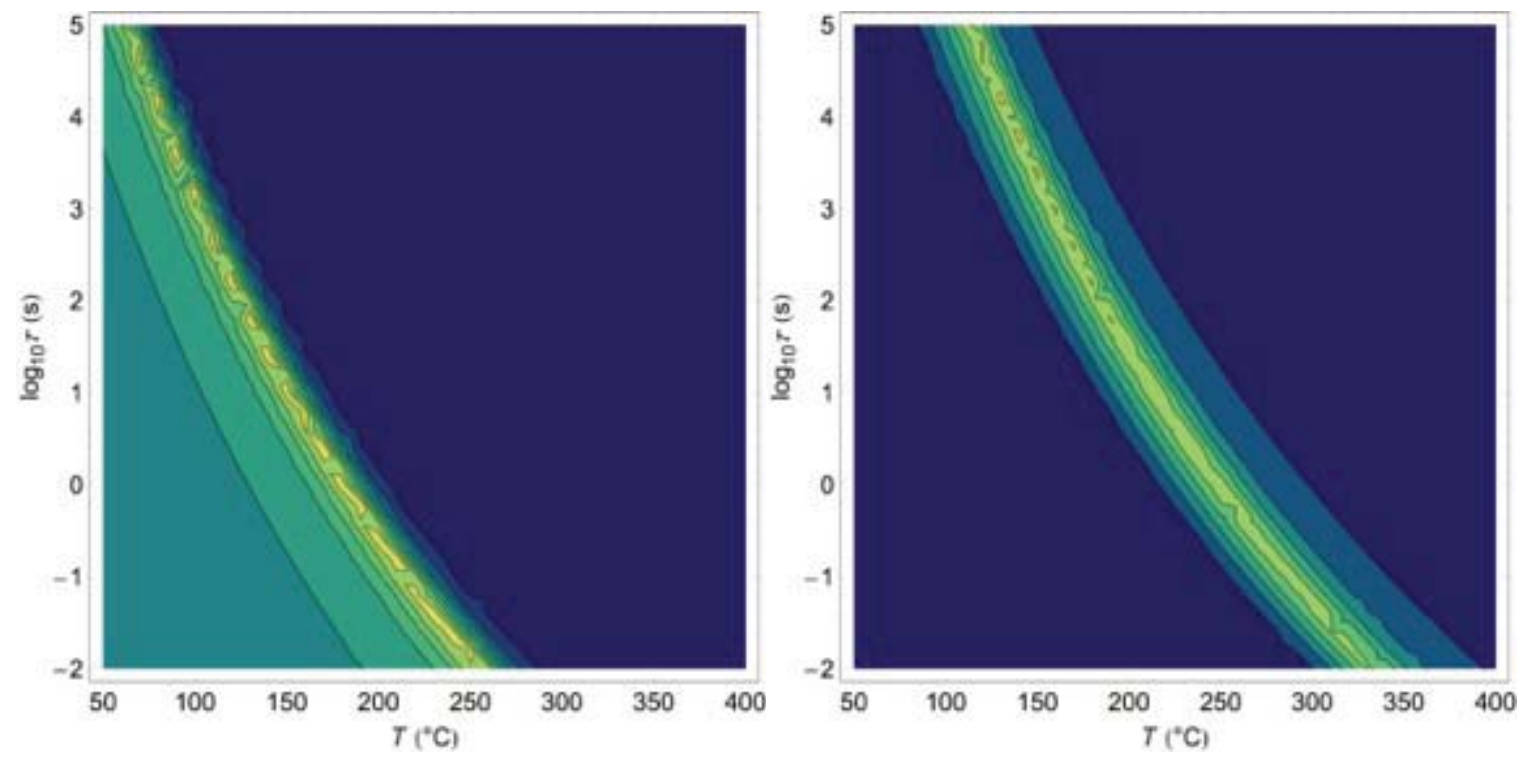

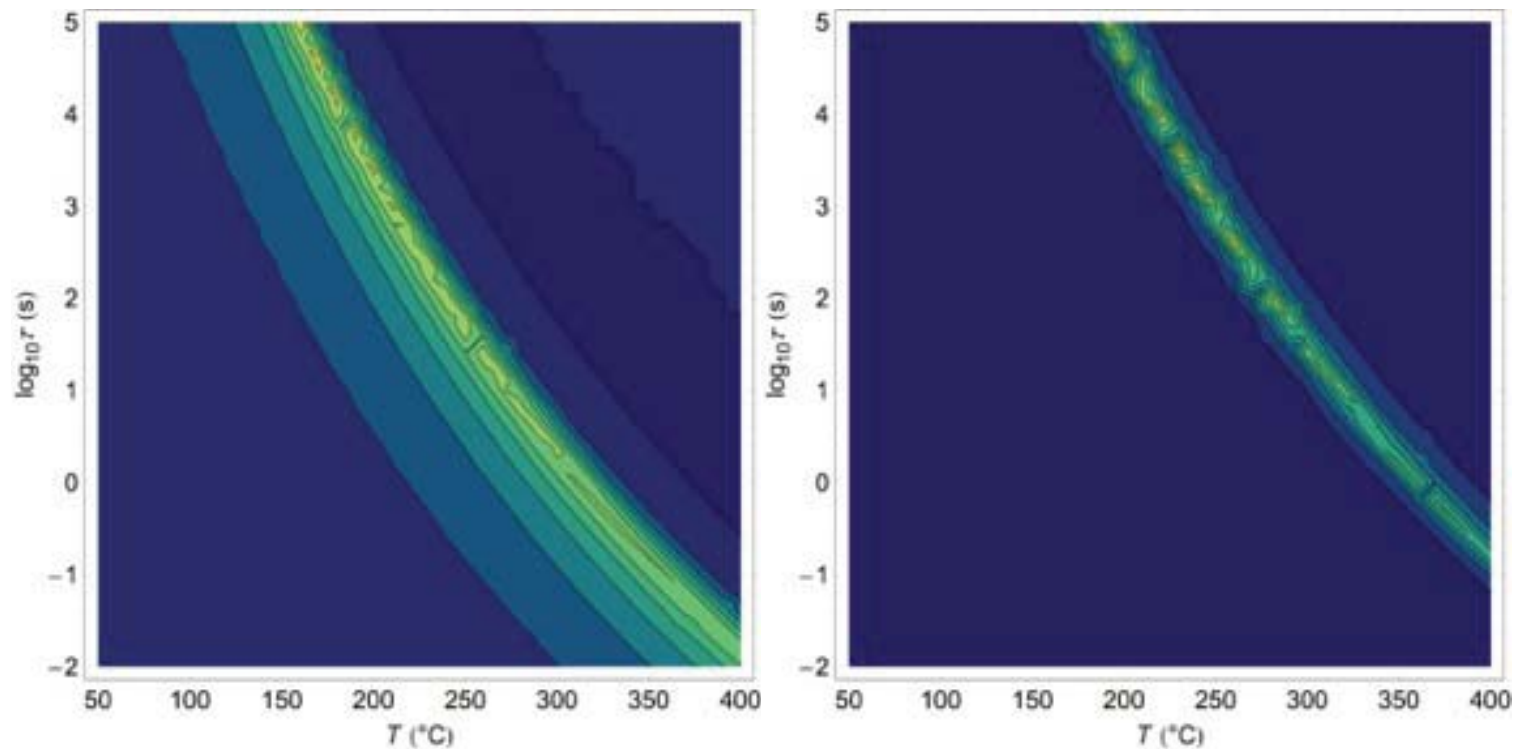

Fig. 100. Examples of contour plots of $\phi$ versus temperature $T$ for duration $\tau$. $\phi$ is calculated by Eq. (6-6) and expresses the comparison between the experimental TL curves of LBO samples irradiated and heated in furnace to (a) $100{ }^{\circ} \mathrm{C},(\mathrm{b}) 150{ }^{\circ} \mathrm{C}$, (c) $200^{\circ} \mathrm{C}$ and (d) $250^{\circ} \mathrm{C}$, with the TL curves after irradiation and pre-heating to temperature $T$ for duration $\tau$ calculated using the models in Fig. 99.

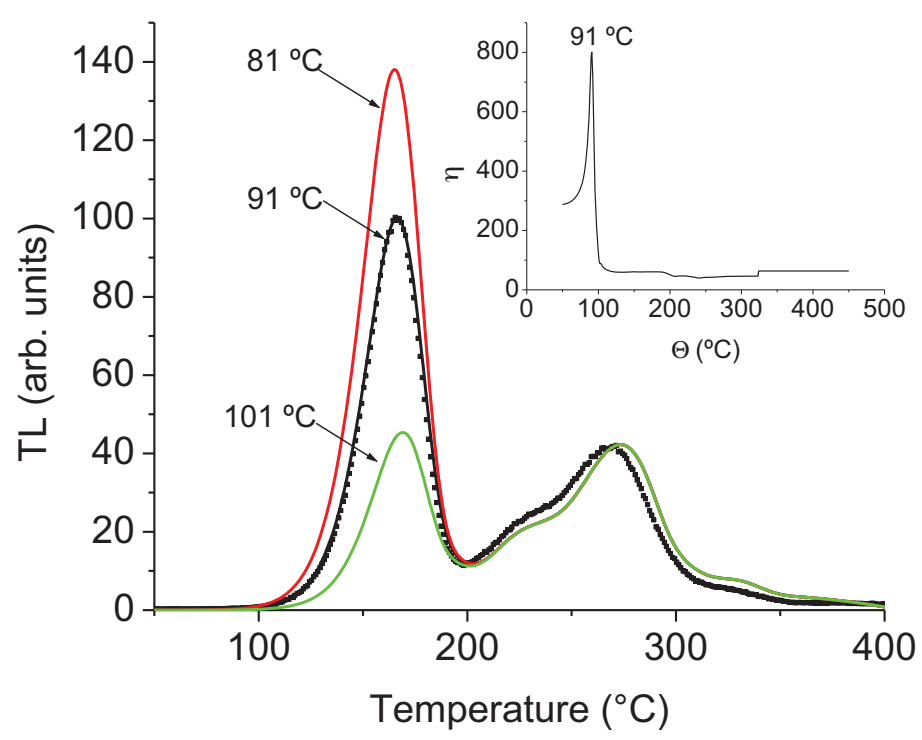

Fig. 101. Comparison of TL curve for LBO heated in the furnace set to $100 \mathrm{\circ}$ for $\mathbf{2} \mathrm{h}$ (points) with the TL curves calculated based on the model shown in Fig. 99 for $81{ }^{\circ} \mathrm{C}, 91^{\circ} \mathrm{C}$ or $101{ }^{\circ} \mathrm{C}$ and heating time $\tau=7200 \mathrm{~s}$. The inset shows the value of $\eta$ as a function of temperature for a fixed heating time $\tau=7200 \mathrm{~s}$.

\subsection{The approach employed by UMN}

When a TL material is subjected to a temperature excursion such as that induced in a detonation fireball, its carrier traps will be depopulated to a degree dependent on the trap's energy, depth, frequency factor, and the heating profile experienced, assuming first order kinetics. The simplest model under which this is quantified is the Randall-Wilkins model, which assumes that carriers will not be re- 
trapped once freed [25]. TLD-100 is generally held to be well represented by this model [52], although the question of whether the actual order of certain peaks can vary with irradiation dosage has been the subject of some dispute $[53,54]$. Under first-order kinetics, the population of an electron trap after experiencing temperature profile $T(t)$ is expressed by

$$
n(t)=n_{0} \exp \left(-\int_{0}^{t} s e^{-\left(\frac{E}{k T(\tau)}\right)} d \tau\right)
$$

where the defining trap parameters are the depth $E$, frequency factor $s$ in $\mathrm{s}^{-1}$, and initial trap population $n_{0}$ in volume density [50]. (We have also replaced the variable of integration $t$ with $\tau$ to avoid confusion with the upper limit of integration, as convention dictates.) If the electron concentration once $T(t)$ has run its course is taken to be $n^{\prime}$, the TL intensity observed during a subsequent linear temperature ramp $T^{\prime}\left(t^{\prime}\right)$ is then

$$
I\left(t^{\prime}\right)=C \frac{d n}{d t^{\prime}}=C n_{0}^{\prime} \exp \left(-\int_{0}^{t \prime} s e^{-\frac{E}{k T^{\prime}(\tau \prime)}} d \tau^{\prime}\right) s e^{-\frac{E}{k T^{\prime}\left(t^{\prime}\right)}}
$$

Unfortunately, Eq. (6-8) cannot be solved analytically for general $T(t)$, but the problem can be simplified to a form that can be handled numerically, if a functional form is set for $T(t)$ which is defined by a small number of unknown parameters. In this case, we assumed that the temperature profile experienced by the particles took the form of an instantaneous jump from ambient temperature to a maximum temperature $T_{\max }$, followed by an exponential decrease back to the original ambient. The time at which the temperature descends to $1{ }^{\circ} \mathrm{C}$ above the ambient is taken as the cooling time $t_{\text {cooling. }}$. Thermocouple measurements of the detonations bear this functional form out as a fairly good approximation (Fig. 102), taking into account that the conventional sensor dwarfs the microparticles in size and will respond much more slowly to rapid changes in temperature.

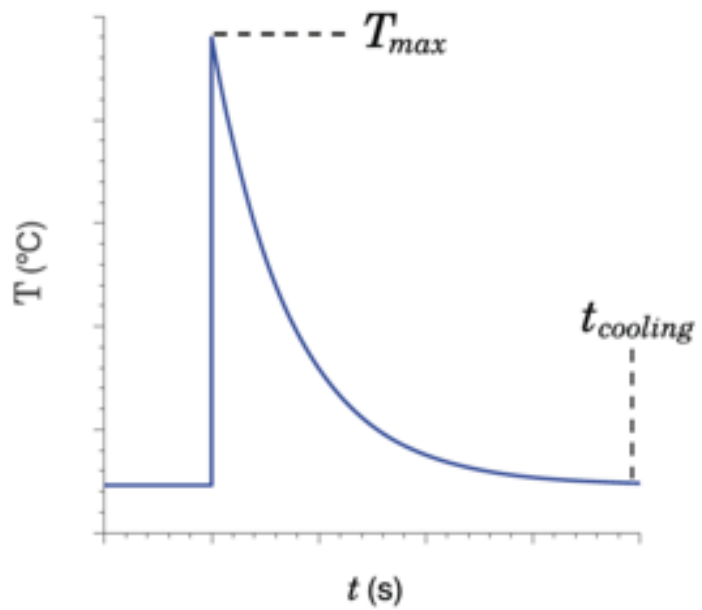

(a)

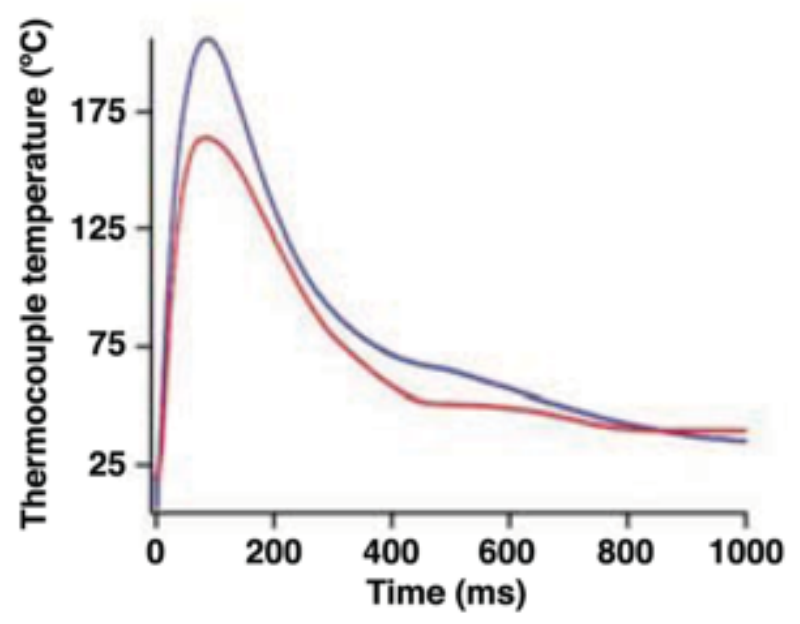

(b)

Fig. 102. Assumed functional form of the temperature profile inside the postdetonation environment, and thermocouple readings of two such detonations.

Intuitively, the transition of a single trap species from its initially filled state to a certain partiallydepleted population cannot be unique: one would expect that there are multiple thermal profiles which would produce the same final carrier density. Indeed, simulations of the two-dimensional space 
defined by $T_{\max }$ and $t_{\text {cooling }}$ demonstrate that a one-to-one curve of parameter pairs exists along which the degree of depopulation is the same.

Since carrier densities are conventionally expressed per cubic centimeter and thus stretch over a wide range of large values, it is convenient to define the "correctness" value of the population resulting from a single possible heating scenario $n$ with respect to that of the actual sought heating scenario $n_{\text {actual }}$ as the complement of the percent error:

$$
\text { correctness }=\left\{\begin{array}{cc}
0 & \text { if } \frac{n_{\text {actual }}-n}{n_{\text {actual }}} \geq 1 \\
1-\frac{n_{\text {actual }}-n}{n_{\text {actual }}} & \text { otherwise }
\end{array}\right.
$$

The existence of an entire set of "correctness" 100\% scenarios, as seen in Fig. 103, implies that a single TL trap species cannot be used to select any heating scenario defined by more than a single parameter. A second trap species, however, will be depopulated at a different rate in response to the same heating profile, and its curve (in this two-dimensional case) of fully-correct scenarios will thus take a different course. The only point which the two curves are certain to both include is the heating scenario which produces the degrees of depopulation actually observed in each trap. Therefore, two TL trap species can be used to recover two thermal history parameters, provided that the trap characteristics are sufficiently different to provide easily distinguishable curves.

Fig. 104 illustrates this process for two heating parameters. This concept logically scales to recovering a general $n$ thermal history parameters using $n$ distinct trap species, or $n+1$ species if a trap sufficiently deep to be unaffected by the heating is used as a normalization intensity reference.

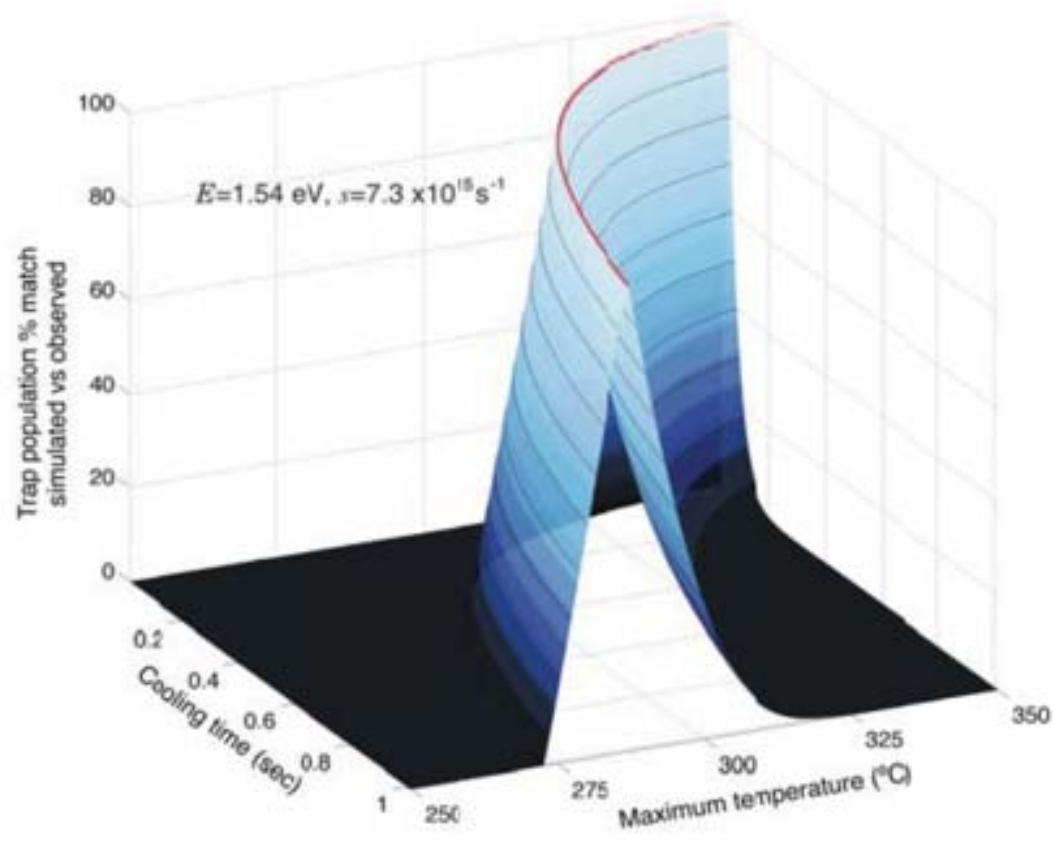

Fig. 103. Simulation of a single TL trap species with trap parameters taken, for illustration purposes, from McKeever's study of $\beta$-irradiated TLD-100 [52]. The vertical axis represents the correctness, Eq. (6-9), of the trap's post-heating population as compared to that produced by the "actual" scenario, which in experimental use would be that observed from the TL curve of a sample. The thermal profile is assumed to be in the form described earlier, and the two parameters thereof form the horizontal plane axes; in this example, the actual scenario is set as $T_{\max }=300{ }^{\circ} \mathrm{C}$ and $t_{\text {cooling }}=0.5 \mathrm{~s}$. All heating scenarios along the red line at the top of the curve give a TL curve identical to that of this scenario, and thus a correctness of $100 \%$. 


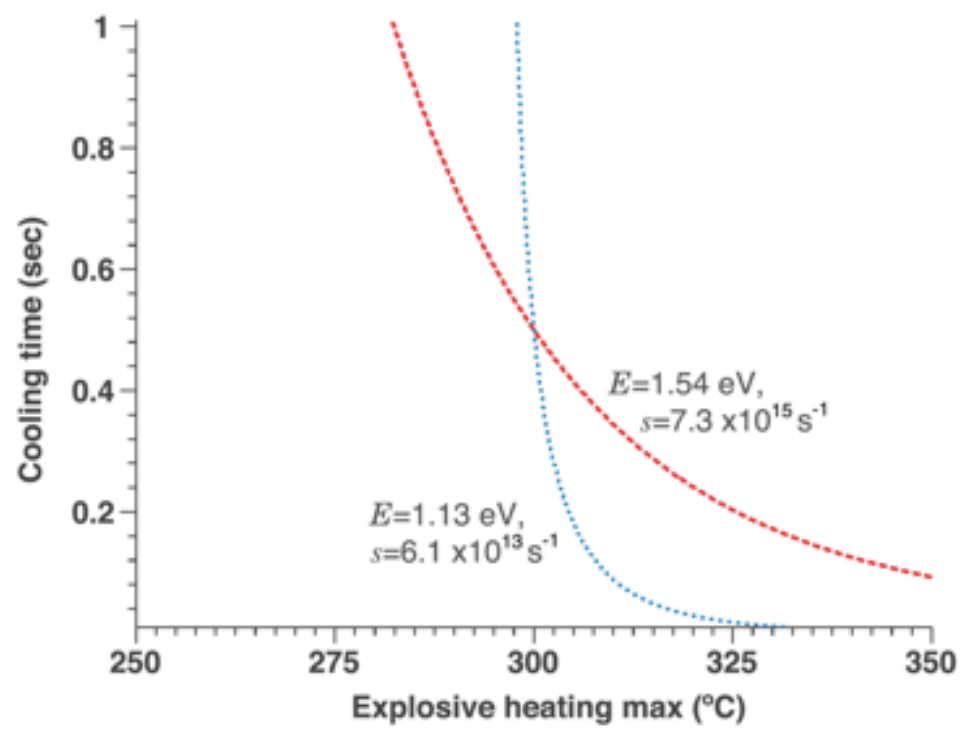

Fig. 104. Line of scenarios yielding a correctness of $100 \%$ previously seen in Fig. 4 intersected with its equivalent from a second trap species (with parameters again drawn from [52]). The intersection point does indeed lie at the correct scenario.

To recover the parameters of a sample's thermal history, we must find the point shared amongst the correctness curves of every trap species present in the sample. The most straightforward and robust numerical approach is simply to simulate the depopulation effects and resulting TL curve of a speculative temperature profile for the detonation or other thermal event, compare the result to the observed TL curve data, and adjust the speculative profile for a closer match. If a correct general form is assumed for the unknown temperature profile, and given sufficient iterations, this procedure will eventually converge on the intersection point of the correctness curves and thus the temperature profile most likely to have produced the observed TL response.

To reconstruct the thermal history profile experienced by the detonation-exposed TLD-100 particles, such an algorithm was implemented in MATLAB. A set of the trap parameters $E$, $s$, and no representing our particular TLD-100 samples and excitation methods was obtained by manually fitting five first-order TL traps such that their summed TL curves closely resembled that observed from a control sample, as shown in Fig. 105a; the parameter values are listed in Table I. These virtual particles were then used to simulate the response of TLD-100 to different heating scenarios.

For each search iteration, an initial range of temperature profile parameters $T$ max and $t$ cooling is evenly subdivided and paired; this is conveniently visualized as forming a uniform grid in the two-dimensional solution space of ( $\left.T_{\max }, t_{\text {cooling}}\right)$ pairs, as shown in Fig. 105b. At each point of this search grid, the values were shifted by a randomized amount (not exceeding the grid subdivision size) and the resulting explosive heating scenario applied via numerical evaluation of Eqs. (6-7) and (6-8). The resulting simulated TL curve was evaluated at five temperatures corresponding roughly to the five component intensity peaks of the model. (No scenario could be found to explain the aberrant shape of the lowest temperature peak.)

To facilitate comparison with the experimental intensity, which will vary with the sample size and the proportion of inactive debris, both simulated and experimental TL curves are normalized to a stable intensity peak. The heating profiles whose simulated intensity ratios came closest to those of the experimental data are subsequently expanded into new, more focused search grids, and the process repeated until the desired level of accuracy in the scenario parameters is reached. 


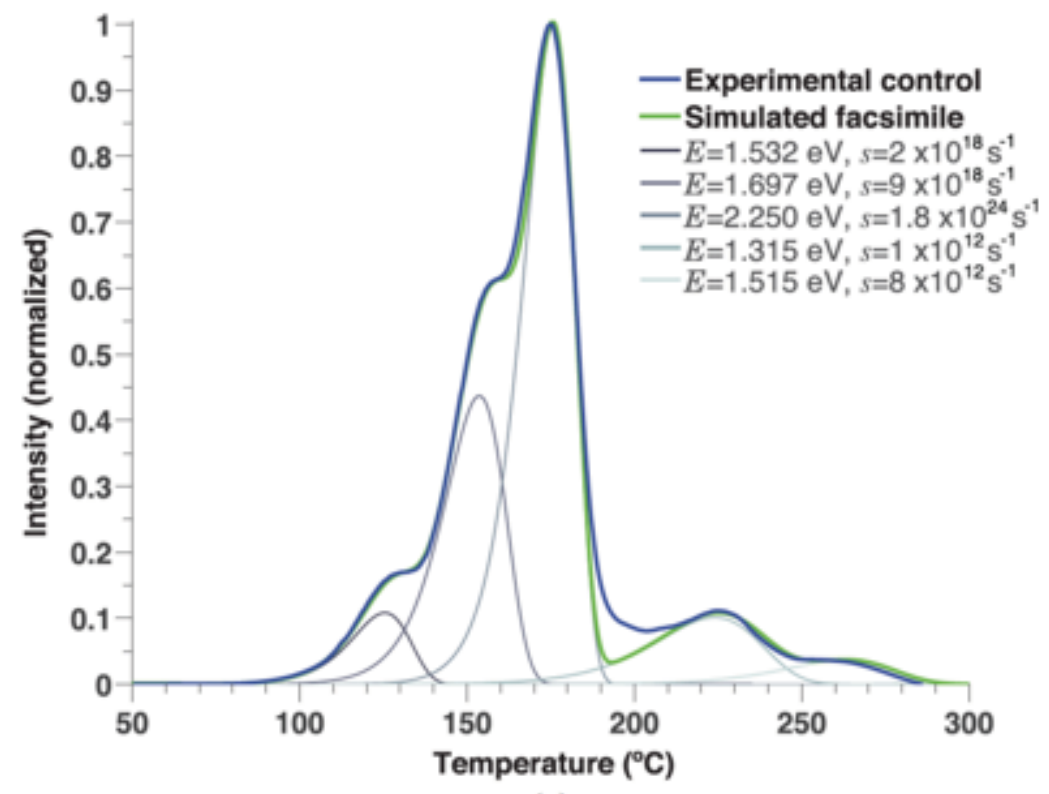

(a)

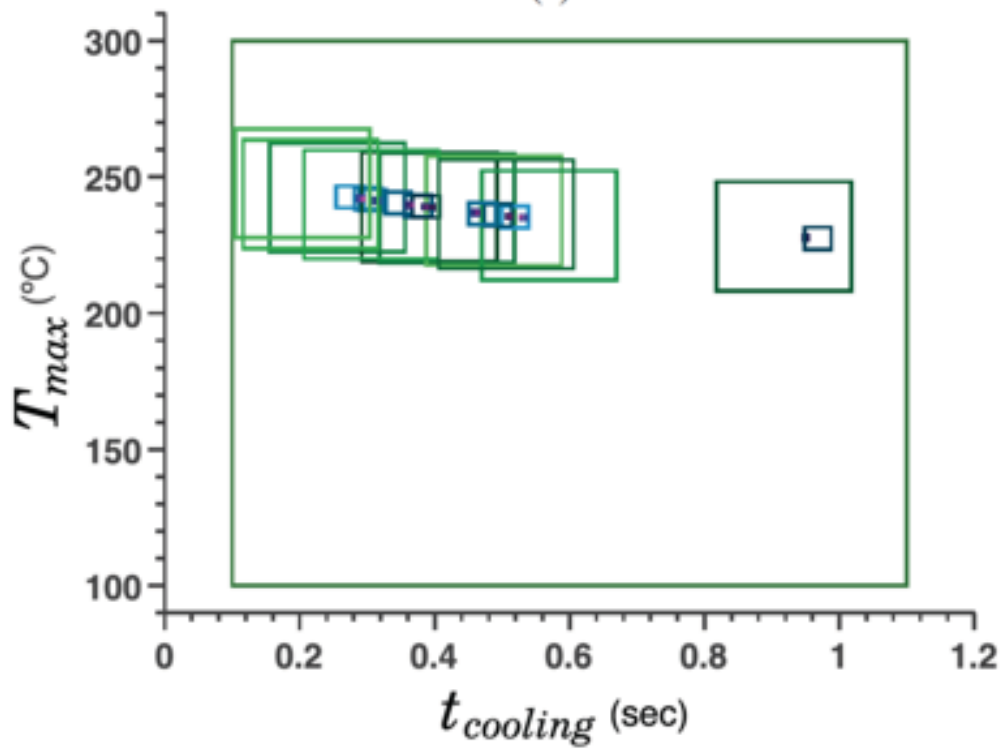

(b)

Fig. 105. (a) Observed TL curve of a control sample (unexposed to detonation) of TLD-100 compared to its first-order model. (b) Activity over several cycles of the iterative randomized search algorithm. The areas searched in each iteration are delineated by square outlines, which rapidly dwindle from legibility at this scale.

\subsection{Laboratory tests}

\subsubsection{Furnace tests}

Tests on the materials developed at OSU (LBO, MBO and CSO) were performed in laboratory to evaluate their performance as temperature sensors [10].

LBO, MBO and CSO samples were heated for $2 \mathrm{~h}$ to different temperatures $\left(100{ }^{\circ} \mathrm{C}, 150{ }^{\circ} \mathrm{C}, 200{ }^{\circ} \mathrm{C}\right.$ and $250^{\circ} \mathrm{C}$ ) inside a muffle furnace (Omegalux model LMF-3550). For CSO, we added two additional temperatures, $300^{\circ} \mathrm{C}$ and $350{ }^{\circ} \mathrm{C}$, because of the material's ability to register higher temperatures. 
Three aliquots in stainless steel cups were placed in an alumina crucible, covered by aluminum foil to protect them from light, and placed in the pre-heated furnace. After $2 \mathrm{~h}$, the alumina crucibles were removed and placed on a metal block to quickly cool them back to room temperature. Three other aliquots in stainless steel cups were used as controls.

The results in Table 16 seemed to indicate an under-response of the TL sensors. However, upon further examination of the furnace heating profile we found out that the front panel temperature indicator of the furnaces were not reliably indicating the temperature reached at the sample position. As an example, Fig. 106 shows the temperature profiles measured with a thermocouple for the furnaces when the temperature was set to $100{ }^{\circ} \mathrm{C}$ and $250{ }^{\circ} \mathrm{C}$. As one can see, even after the furnace indicator shows that the temperature has been reached, the temperature at the sample position is significantly lower.

Therefore, the data shown in Table 16 is reliably indicating the temperature the samples experienced, helping reveal problems with the furnace indicator.

Table 16. Temperature measurements using LBO, MBO and CSO for samples heated to a fixed temperature for $2 \mathrm{~h}$.

\begin{tabular}{|c|c|c|c|}
\hline Furnace setting & $\begin{array}{l}\text { LBO } \\
\left({ }^{\circ} \mathrm{C}\right)\end{array}$ & $\begin{array}{l}\mathrm{MBO} \\
\left({ }^{\circ} \mathrm{C}\right)\end{array}$ & $\begin{array}{l}\mathrm{CSO} \\
\left({ }^{\circ} \mathrm{C}\right)\end{array}$ \\
\hline \multirow[t]{3}{*}{$100^{\circ} \mathrm{C}$} & 86 & 95 & 188 \\
\hline & 85 & 92 & 110 \\
\hline & 86 & 95 & 205 \\
\hline \multirow[t]{3}{*}{$150^{\circ} \mathrm{C}$} & 138 & 144 & 136 \\
\hline & 133 & 142 & 139 \\
\hline & 136 & 143 & 133 \\
\hline \multirow[t]{3}{*}{$200^{\circ} \mathrm{C}$} & 187 & 168 & 206 \\
\hline & 179 & 175 & 197 \\
\hline & 178 & 172 & 182 \\
\hline \multirow[t]{3}{*}{$250^{\circ} \mathrm{C}$} & 222 & 229 & 232 \\
\hline & 222 & 224 & 231 \\
\hline & 226 & 228 & 231 \\
\hline \multirow[t]{3}{*}{$300^{\circ} \mathrm{C}$} & - & - & 337 \\
\hline & & & 280 \\
\hline & & & 276 \\
\hline \multirow[t]{3}{*}{$350^{\circ} \mathrm{C}$} & - & - & 327 \\
\hline & & & 324 \\
\hline & & & 332 \\
\hline
\end{tabular}



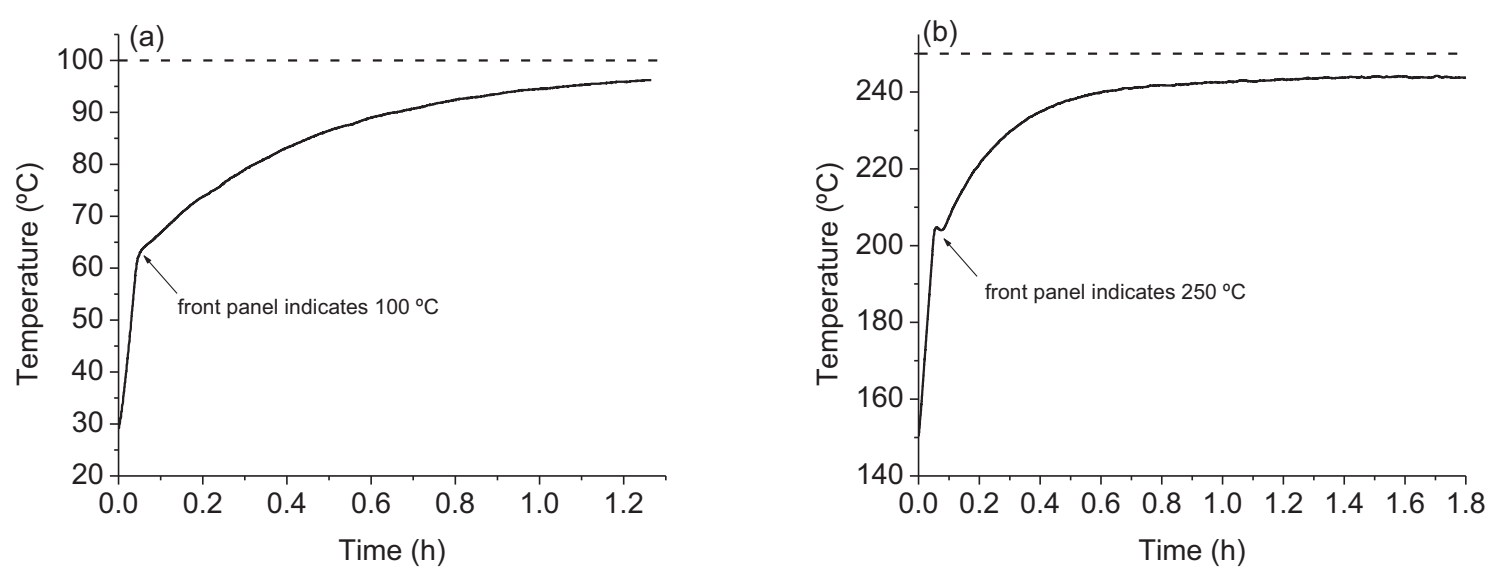

Fig. 106. Thermocouple measurements at the sample position inside the furnace. The arrows indicate the time when the furnace indicator shows that the setpoint was reached.

\subsubsection{Fast heating tests}

LBO, MBO and CSO were tested in laboratory at OSU for a heating profile faster than the one achievable using the furnaces [10].

The samples were heated using a custom-built temperature chamber (Fig. 107) which consists in an insulated 1/8-inch thick stainless steel cylinder of 18 inches outside diameter by 36 inches length, heated by four band heaters (Watlow, $220 \mathrm{~V} 562 \mathrm{~W}$ each half-band). The temperatures insider the chamber were measured using K-type thermocouple probes with the tip approximately at the symmetry axis of the cylinder positioned at three points along the length of the cylinder: one at the top, one at the middle, and one at the bottom. The middle thermocouple is connected to a temperature controller (model CN4800, Omega), which controls the current to the band heaters through a silicon-controlled rectifier (Eurotherm model 831). The other thermocouples were measured using a multimeter (Omega model HHM31). Approximately $50 \mathrm{mg}$ of powder was dropped from the top of the temperature chamber and a fraction of it was collected at the bottom in a beaker. It took approximately 1-2 s for the samples to reach the beaker. The collected powder was then transferred to stainless steel cups. In the case of LBO, the powder was collected using stainless steel cups placed at the bottom of the beaker because very little powder was obtained. Again, three aliquots in stainless steel cups were used as controls.

The $L B O$ and $M B O$ results are shown in Table 17, in comparison with the three thermocouple measurements. The results show agreement between the temperatures obtained using the TL particles, which were obtained assuming a $1 \mathrm{~s}$ heating period, and the thermocouple temperatures. 


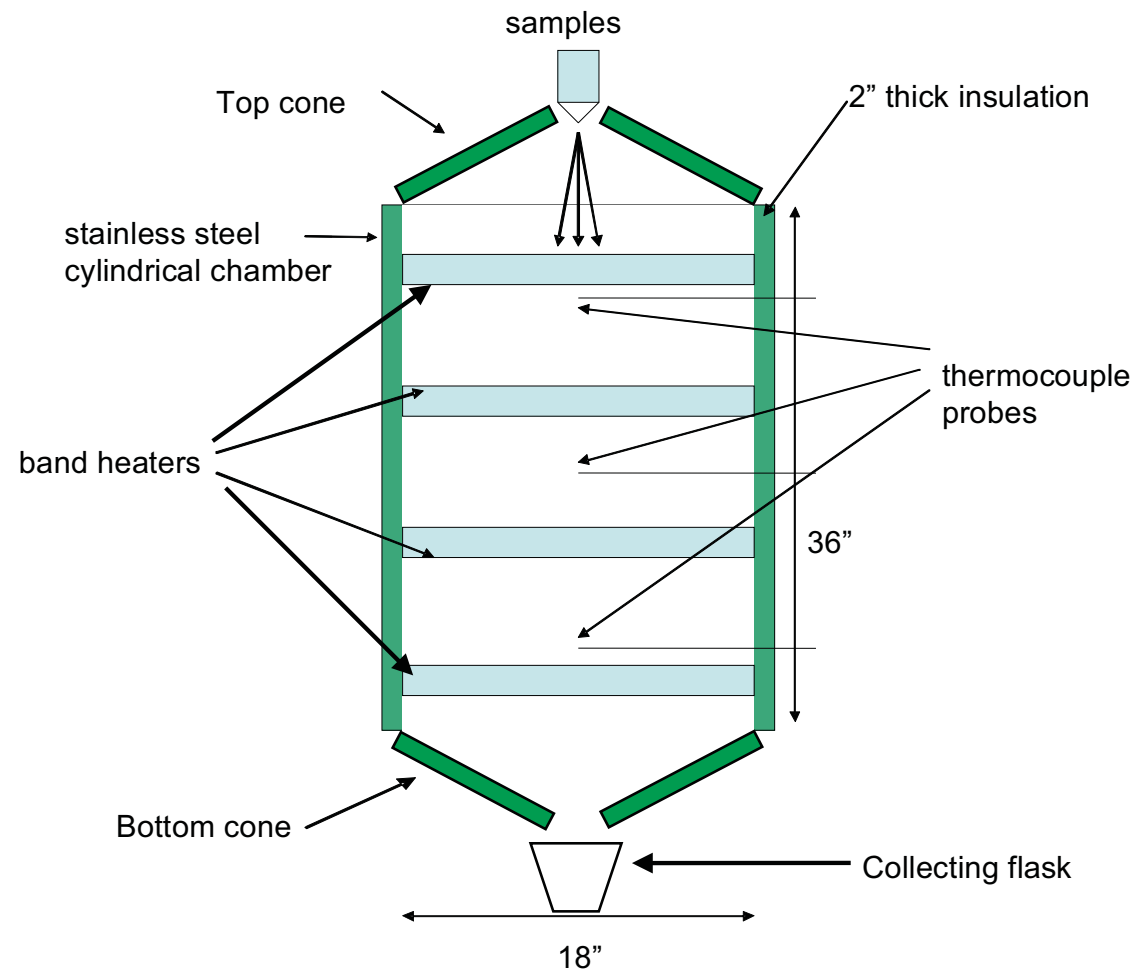

Fig. 107. Diagram of the temperature chamber used for the "fast heating profiles".

Table 17. Temperature measurements using LBO, MBO and CSO for samples dropped in temperature chamber (1-2 s).

\begin{tabular}{|ccc|}
\hline Chamber temperatures & Thermocouples & Material \\
\hline LBO & $197^{\circ} \mathrm{C}$ (top) & $20{ }^{\circ} \mathrm{C}$ \\
& $194^{\circ} \mathrm{C}$ (middle) & $206{ }^{\circ} \mathrm{C}$ \\
& $181{ }^{\circ} \mathrm{C}$ (bottom) & $206{ }^{\circ} \mathrm{C}$ \\
\hline MBO & $196{ }^{\circ} \mathrm{C}$ (top) & $180^{\circ} \mathrm{C}$ \\
& $198{ }^{\circ} \mathrm{C}$ (middle) & $200{ }^{\circ} \mathrm{C}$ \\
& $184{ }^{\circ} \mathrm{C}$ (bottom) & $168{ }^{\circ} \mathrm{C}$ \\
& & $201{ }^{\circ} \mathrm{C}$ \\
& & $197{ }^{\circ} \mathrm{C}$ \\
& & $210^{\circ} \mathrm{C}$ \\
& & $190^{\circ} \mathrm{C}$ \\
\hline CSO & - & $206{ }^{\circ} \mathrm{C}$ \\
\hline
\end{tabular}




\subsubsection{Nitrocellulose rapid combustion tests}

Due to the cost, time, impositions, and scarcity of explosive detonation testing using outside collaborators, we explored materials which combust rapidly without the destructive aspects-and attendant safety, regulatory, cost, and experimental requirements-of real high explosives. Although these deflagrations are an imperfect substitute for military-scale detonations, they are extremely beneficial because we can perform tests ourselves with minimal cost. Macroscale resistive temperature device (RTD) readings have shown a rapidly-heating, slowly-cooling temperature profile similar in shape to that seen in actual high explosive detonations.

Of the unregulated products tried thus far, the most promising is nitrocellulose paper or cotton treated with nitric acid (Fig. 108). This material burns rapidly and cleanly, can be molded into shapes to adjust the temperature and time of sample exposure, is readily available from theatrical effects retailers, and poses a minimal safety hazard in the lab. Its combustion temperature can be increased by mixing it with titanium particles, another child of the stage-pyrotechnics industry.

We have successfully partially depopulated TLD-100 powder embedded in a nitrocellulose plug and retrieved a temperature profile using the same analysis procedure as with detonation tests (Fig. 109). Experimentation with different configurations-placement, folding, additives, etc.-to tailor the combustion characteristics of flash paper and cotton has been carried out.

The simulation techniques and codebase used to recover temperature data from TL data have been subjected to ongoing refinement over the past year. This has yielded notable performance increases: it would have taken the better part of an hour in the middle of the project to achieve the precision levels of a thermal profile reconstruction that can now be completed in less than one minute.

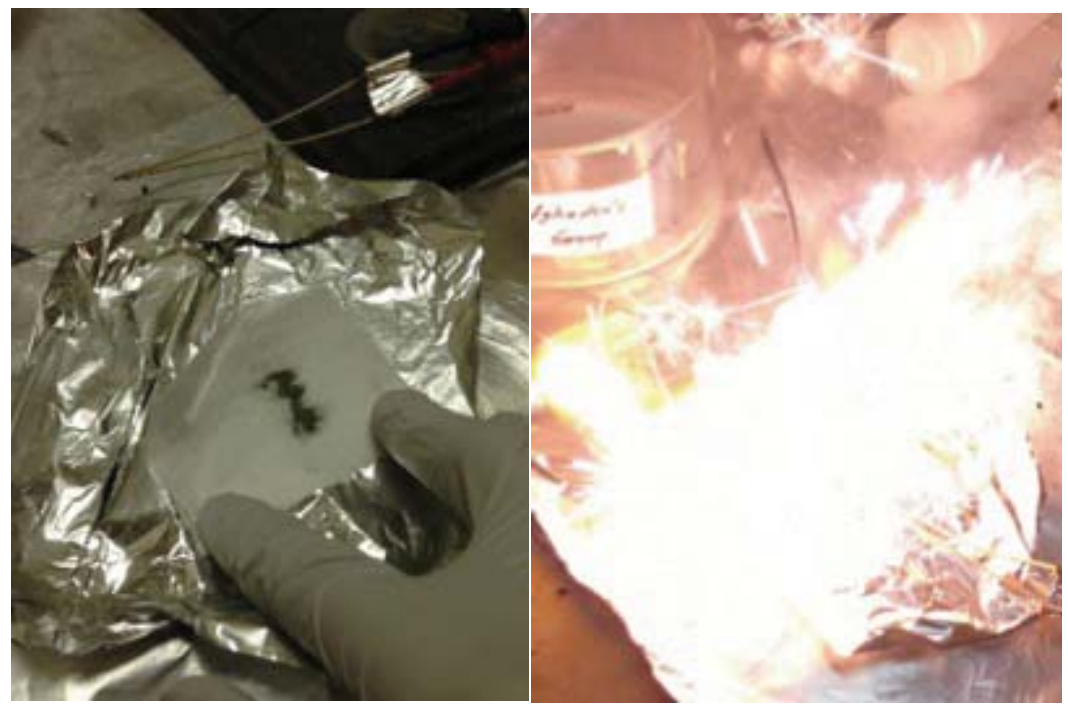

Fig. 108. A piece of flash paper with titanium powder being prepared (left) and in mid-burn (right). While not shown here, in runs testing TL materials, the TL powder is mixed into the titanium powder or placed wherever desired, and recovered from the container in which the burn takes place. Generally, very little unburned nitrocellulose or titanium powder remains. 


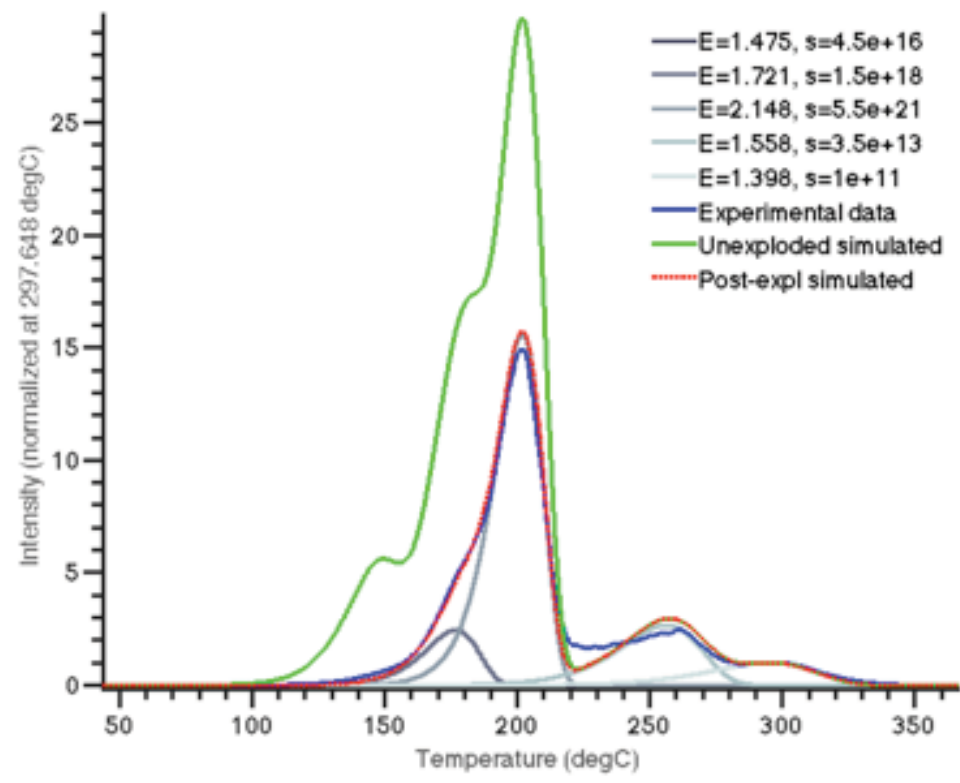

Fig. 109. Control, post-explosion (here post-combustion) observed, and simulated closest-match TL curves of TLD-100 burned in flash paper and Ti powder. The most likely scenario was calculated to have a $0.041 \mathrm{~s}$ linear heating ramp, a $284.1^{\circ} \mathrm{C}$ maximum temperature, and a $0.358 \mathrm{~s}$ cooling time, yielding a $14.9 \%$ average match error among the peaks. As a caveat, this run was a rather preliminary test and the TLD-100 first-order model was not initialized with the same rigor as usually used. 


\section{INVESTIGATION OF INFLUENCING FACTORS}

\subsection{Thin film development}

The investigation of pressure effects independent from the temperature experienced during detonations required us to develop thin film TL materials that could be subjected to various pressures in laboratory. This section describes the development of such thin films.

\subsubsection{Introduction}

The luminescent properties of rare-earth doped yttria are well known [55-59]. These materials show luminescence due to the trivalent lanthanide in the yttria host. In particular studies have been done on the cathodoluminescence [60], photoluminescence [61], and TL [62] of $\mathrm{Y}_{2} \mathrm{O}_{3}: \mathrm{Tb}$. The studies conclude that the luminescence comes from the interaction of the trivalent terbium ion with the yttria crystal, which introduces a luminescent center in the band gap of yttria.

Most TL thin films are grown using techniques such as spray pyrolysis [63] and sol-gel [64], methods that are not part of standard silicon integrated circuit processes. Conversely, the overwhelming majority of TL materials will not display TL when deposited in the same manner as a microelectronic film. In this work, we show that $\mathrm{Y}_{2} \mathrm{O}_{3}$ :Tb is a notable exception that deposits in a simple evaporation process and luminesces brightly after exposure to radiation and subsequent heating. Yttrium oxide is well known as a material that is deposited as a polycrystalline film [65].

The combination of strong luminescent and polycrystalline characteristics of $\mathrm{Y}_{2} \mathrm{O}_{3}: \mathrm{Tb}$ make it a good candidate for an integrated circuit TL thin film. And since this film can be deposited using e-beam evaporation [60] it can be used in standard microfabrication processes.

\subsubsection{Deposition process and measurements}

In this report, electron-beam evaporation is used to deposit a thin film of $\mathrm{Y}_{2} \mathrm{O}_{3}$ :Tb. Rare earth [55-62] and transition metals [66] impurities can be added to yttria to improve the luminescence. The dopant can be added during deposition by including small amounts of it in the host material of the electronbeam source. As the electron beam strikes the source, it will evaporate both the host material and impurity. With a subsequent annealing the polycrystalline grains can be grown and the impurities in the film activated.

Unlike the previously mentioned spray pyrolysis and sol-gel methods, this technique does not require careful mixing of the chemicals. The powders for the host material and impurity are blended and then compressed into pellets. The pellets are then ready for evaporation. $\mathrm{Y}_{2} \mathrm{O}_{3}: \mathrm{Tb}$ films were deposited on (100) silicon substrates. $\mathrm{Y}_{2} \mathrm{O}_{3}(99.99 \%)$ and $\mathrm{Tb}_{4} \mathrm{O}_{7}$ (99.9\%) powders were used as evaporation sources (Alfa-Aesar). The terbium oxide powder was mixed with the yttrium powder with a molar concentration of terbium in the mixture of $0.1 \%$. The resulting powder was a homogenous blend of the $\mathrm{Y}_{2} \mathrm{O}_{3}$ and $\mathrm{Tb}_{4} \mathrm{O}_{7}$ powders and was then compressed into pellets. The pellets were $1 \mathrm{~cm}$ in diameter and approximately $0.5 \mathrm{~cm}$ thick.

The pellets were placed in a graphite crucible liner for e-beam evaporation. The electron beam system used for evaporation was a Varian model number 3118. The voltage and current during evaporation were $4.19 \mathrm{kV}$ and $0.2 \mathrm{~A}$ respectively. A slow deposition rate of $2-3 \AA / \mathrm{s}$ was used to promote crystal growth. Since the source material was not solid, but rather consisted of pellets, the deposition rate fluctuated considerably. A quartz crystal monitor was used to keep track of the thickness of the film, which had a final thickness of $350 \mathrm{~nm}$. After the film was deposited, it was annealed using a Modular 
Pro RTP-600S for $30 \mathrm{~s}$ at a temperature of $1100{ }^{\circ} \mathrm{C}$ with a heating rate of $30{ }^{\circ} \mathrm{C} / \mathrm{s}$. The film was annealed under argon gas with a flow rate of $4 \mathrm{sccm}$, preceded by a 2 min argon purge. The silicon wafers with the $\mathrm{Y}_{2} \mathrm{O}_{3}: \mathrm{Tb}$ thin films were then cleaved into $2 \mathrm{~cm} \times 2 \mathrm{~cm}$ squares for measurement.

Three ionizing radiation sources (ultraviolet, $x$-ray, and gamma) were used to fill the traps of the $\mathrm{Y}_{2} \mathrm{O}_{3}: \mathrm{Tb}$. The UV source was a deuterium lamp with no filter; the $\mathrm{x}$-ray source was an XRAD-320 with a $2 \mathrm{~mm}$ Al filter; the gamma radiation source was a ${ }^{137} \mathrm{Cs}$ radioisotope. Measurements of TL intensity versus temperature were taken using a photomultiplier tube (PMT) Sens-Tech P25PC as a detector. The internal default heating rate for our hotplate (with a $115 \mathrm{Vrms}$ ) is $2.7^{\circ} \mathrm{C} / \mathrm{s}$, so this ramp rate was used to measure the TL curves. For some of the measurements a $550 \mathrm{~nm}$ cut-on short pass optical filter placed directly in front of the PMT was used. The filter greatly attenuated the black body signal allowing for the measurement of high temperature peaks above $350{ }^{\circ} \mathrm{C}$.

The emission spectra of the thin film were measured using a monochromator placed in front of the PMT and set to a series of wavelengths. Each wavelength was incremented $3 \mathrm{~nm}$ for 100 subsequent TL measurements untill the full range from 400 to $700 \mathrm{~nm}$ was covered. Additional scans were taken of the peak at $490 \mathrm{~nm}$ in $1 \mathrm{~nm}$ steps to increase resolution of the peak.

Bleaching affects were also measured. Bleaching occurs when the intensity of the TL (usually corresponding to emission at lower temperatures) decreases due to exposure to light after irradiation. The bleaching measurement was carried out by placing a monochromator in front of a tungstenhalogen lamp. In a similar manner to the emission spectra measurement, the set wavelength of the monochromator was incremented in $15 \mathrm{~nm}$ steps. The bleach testing was also done between 400 to $700 \mathrm{~nm}$.

\subsubsection{Data and analysis}

The TL curve measured $10 \mathrm{~s}$ after 15 min UV irradiation shows TL curve peaks at $140{ }^{\circ} \mathrm{C}$ and $230{ }^{\circ} \mathrm{C}$ (Fig. 110a). Longer irradiation times had no effect on the intensity of the TL curve. These peaks indicate that there are at least two traps in the band gap of yttria that are deep enough to be thermally stable at room temperature. These trapping centers could be due to defects in the film, terbium ion, or surface states. The terbium ion may act as both an electron and hole trap similar to $\mathrm{Dy}^{3+}$ in $\mathrm{YPO}_{4}$ [67]. After irradiation the electrons and holes get trapped at various terbium sites. Upon heating an electron can be excited to the conduction band where it is free to move. It then could combine with a hole trapped at a terbium site. Conversely this could happen with holes excited into the valence band and then recombining with a trapped electron. From the spectral emission peaks mentioned later the terbium ion is always acting as the recombination center, because the spectral peaks match the intraband transitions in the terbium ion [61].

$\mathrm{Y}_{2} \mathrm{O}_{3}: \mathrm{Tb}$ also had a TL curve after gamma and $\mathrm{x}$-ray (Fig. $110 \mathrm{~b}$ and c). Under these higher energy radiation sources a TL peak at $410{ }^{\circ} \mathrm{C}$ could be measured using the $550 \mathrm{~nm}$ short pass filter. The TL curves for gamma and $\mathrm{x}$-ray sources do show a peak at $170^{\circ} \mathrm{C}$ and $120^{\circ} \mathrm{C}$, respectively, indicating a sensitivity to these forms of ionizing radiation; the UV source, however, is much more accessible to our group; therefore, the TL curves used in the rest of this section are for UV irradiation. The UV source was used because it allowed for more rapid measurements. The x-ray and gamma sources require the sample to be moved after irradiation, whereas the UV irradiation can be done with the sample on the hot plate. 
(a)

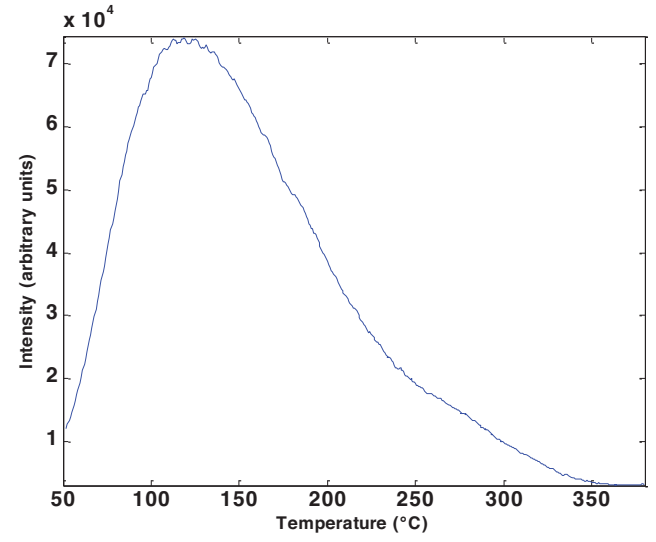

(b)

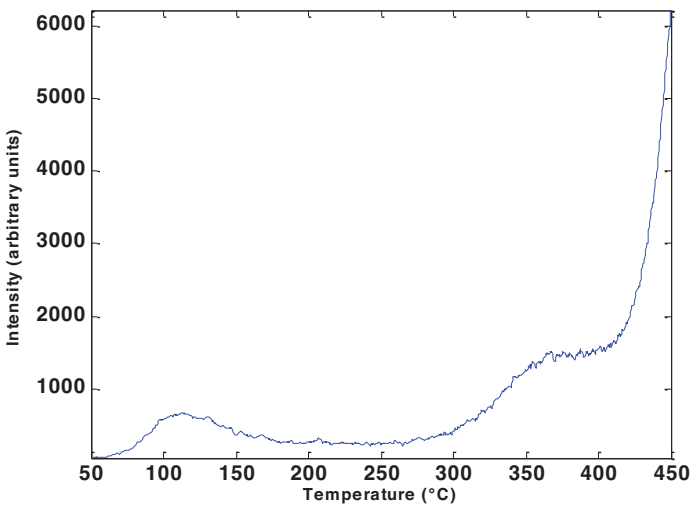

(c)

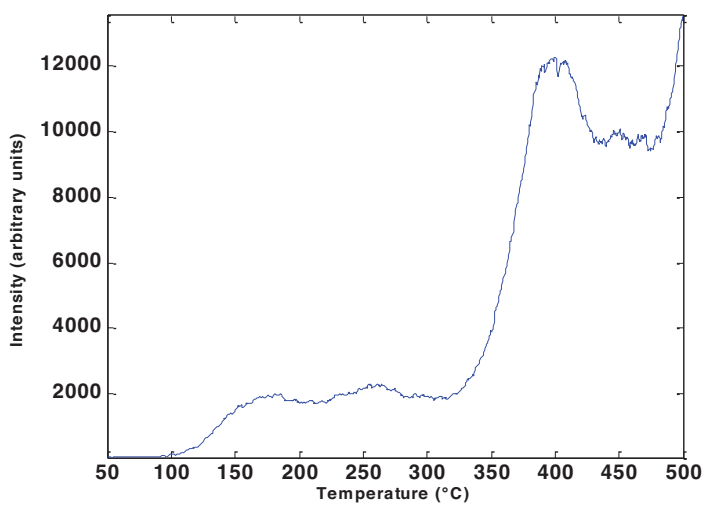

Fig. 110. TL curve for $\mathrm{Y}_{2} \mathrm{O}_{3}: \mathrm{Tb}$ (a) $10 \mathrm{~s}$ after UV irradiation. There are two peaks at $140^{\circ} \mathrm{C}$ and $230^{\circ} \mathrm{C}$. (b) 30 min after x-ray irradiation with peaks at $120^{\circ} \mathrm{C}$ and $360^{\circ} \mathrm{C}$. (c) 1 hour after gamma irradiation with peaks at $170^{\circ} \mathrm{C}, 260^{\circ} \mathrm{C}$, and $410^{\circ} \mathrm{C}$.

The emission spectrum shown in Fig. 111 matches well with prior results that describe $\mathrm{Y}_{2} \mathrm{O}_{3}: \mathrm{Tb}$ grown by other methods $[60,61]$. The two main peaks at $490 \mathrm{~nm}$ and $540 \mathrm{~nm}$ involve $4 \mathrm{f}-4 \mathrm{f}$ transitions of the $\mathrm{Tb}^{3+}$ ion [61]. The $490 \mathrm{~nm}$ and $540 \mathrm{~nm}$ peaks correspond to the ${ }^{5} \mathrm{D}_{4}$ to the ${ }^{7} \mathrm{~F}_{5}$ and ${ }^{7} \mathrm{~F}_{6}$ levels respectively. 
Normally intraband electric dipole transitions are forbidden. In the case of most lanthanides, however, the $4 \mathrm{f}-4 \mathrm{f}$ transition is the cause of the strong and narrow spectral lines. But since this transition occurs within the $4 \mathrm{f}$ energy band, it should be forbidden according to quantum mechanical selection rules. Specifically, the Laporte selection rules state that there must be a \pm 1 change in orbital and total orbital angular momentum, spin must be conserved and there must be a change in parity. In this case the parity rule is broken because it remains the same during the transition. The magnetic dipole interaction does not require a change in parity, but, because it is so weak, it cannot account for the intensity of the spectral lines seen from lanthanides. The Judd-Ofelt theory [68] states that if a higher order band such as the $5 d$ (even parity) band is mixed into the $4 f$ (odd parity) band than the parity selection rule is satisfied, allowing for an electric dipole transition to occur. There are characteristic peaks at $585 \mathrm{~nm}$ and $618 \mathrm{~nm}$ that do not appear or are difficult to attribute in Fig. 111. This is most likely because the signals are relatively too weak for the PMT to detect, given the intensity of the other peaks and the extremely small volume of material in the film. In Fig. 111 the peak at $585 \mathrm{~nm}$ is uncharacteristically sharp for this emission peak relative to others reported in the literature, and in Fig. 112 it exists for only a very small temperature range, which is also unusual. Although this could certainly be a contribution from the $585 \mathrm{~nm}$ TL emission, it is so weak relative to our noise level that we will ignore it.

Fig. 112 is a $3 \mathrm{D}$ rendering of the relationship between temperature and emission wavelength. One can separate the $490 \mathrm{~nm}$ and $540 \mathrm{~nm}$ emission peaks into two plots of TL versus temperature. When the two plots are compared, they appear almost identical. This indicates that the emission spectrum is not significantly temperature dependent and the recombination center is largely independent of the trap states.

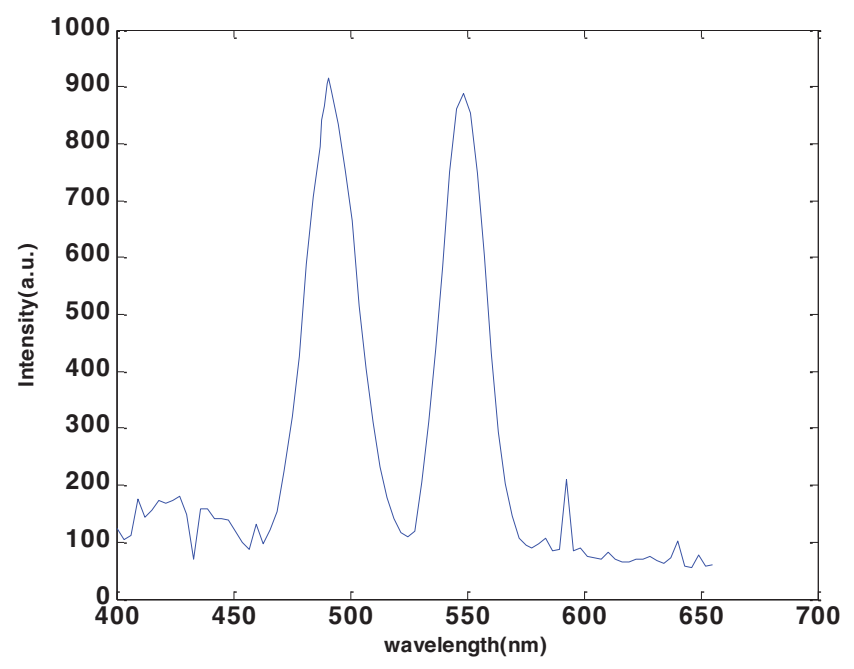

Fig. 111. Emission spectrum of $\mathrm{Y}_{2} \mathrm{O}_{3}: \mathrm{Tb}^{3+}$. The two main peaks at $490 \mathrm{~nm}$ and $540 \mathrm{~nm}$ correspond to the ${ }^{5} \mathrm{D}_{4}$ to the ${ }^{7} \mathrm{~F}_{5}$ and ${ }^{7} \mathrm{~F}_{6}$ levels respectively. 


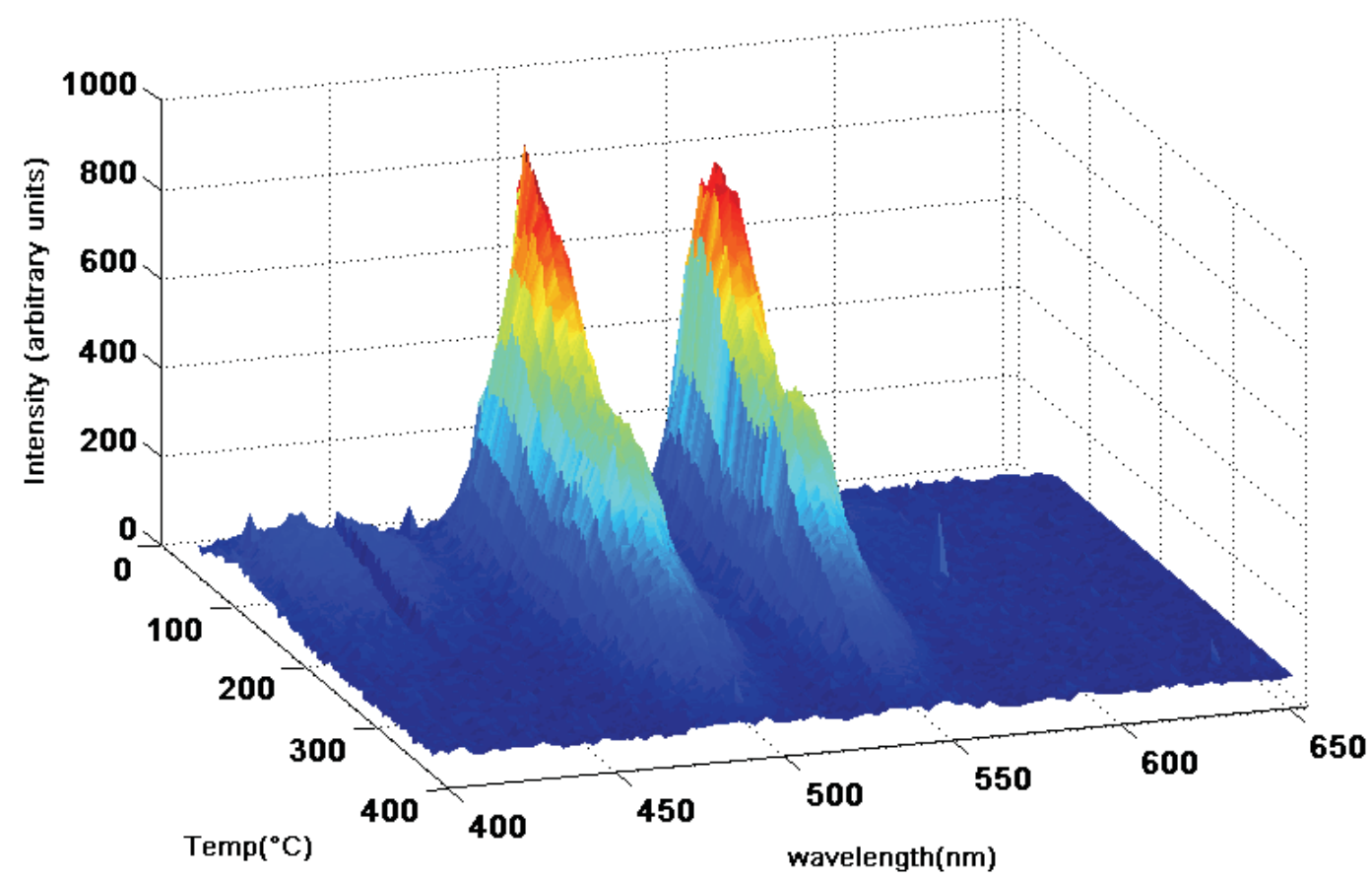

Fig. 112. 3D rendering showing that there is no temperature dependence on the emission spectra of $\mathrm{Y}_{2} \mathrm{O}_{3}: \mathrm{Tb}^{3+}$. The lack of temperature dependence can be seen from the similar shape of the two peaks.

There is a minimum annealing temperature required in order for the $\mathrm{Y}_{2} \mathrm{O}_{3}$ :Tb to become thermoluminescent. This requirement is closely related to the onset of crystallinity in the film as depicted in Fig. 113. The reflection planes [69] indicative of $\mathrm{Y}_{2} \mathrm{O}_{3}$ are also labeled in figure Fig. 113b.

X-ray diffraction was used to measure the crystallinity of the film after several different annealing temperatures. It can be seen from the XRD peak intensity that the film becomes more (poly)crystalline with increased annealing temperature. The thin film does not become thermoluminescent until a $900{ }^{\circ} \mathrm{C}$ anneal for $2 \mathrm{~min}$. Beyond this, the intensity of the TL increases with annealing temperature (Fig. 113a). This is probably due to an increase in crystal grain size and activation of the $\mathrm{Tb}^{3+}$ ion. The XRD data of Fig. 113b indicate that with higher annealing temperatures the peaks increase in intensity implying an increase in the (poly)crystallinity and grain size of the material. As the crystal grain size increases, more of the $\mathrm{Tb}^{3+}$ ions are incorporated into the cubic bixbyite [65] crystal structure.

(a) 


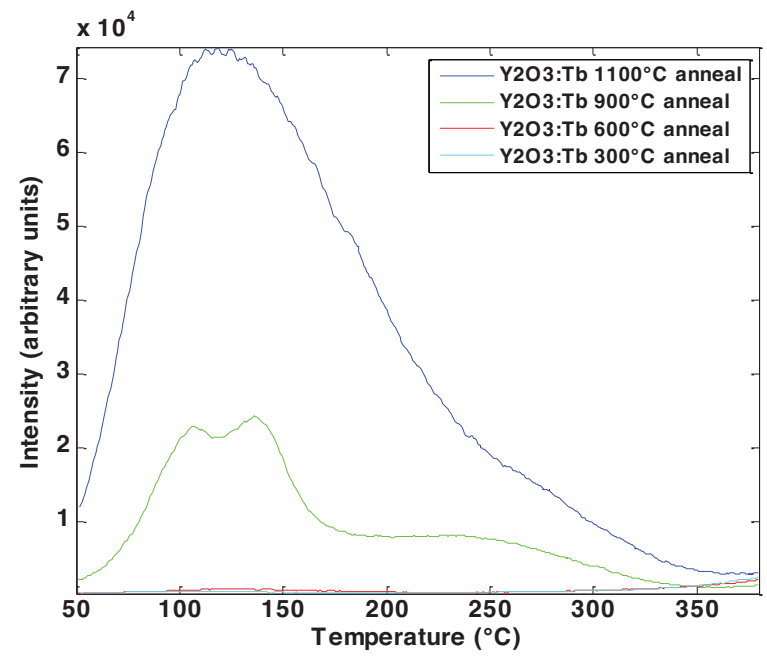

(b)

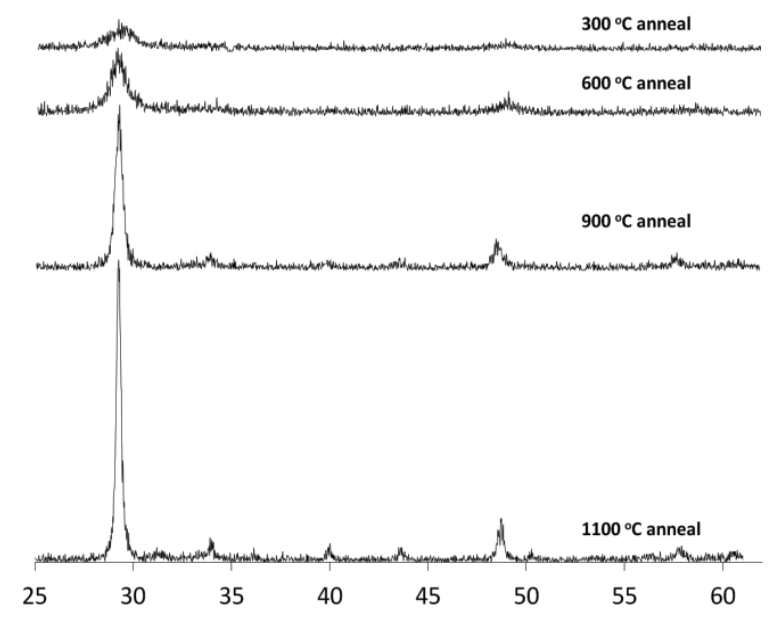

Fig. 113. (a) TL curves for different annealing temperatures. The two TL curves for the $600^{\circ} \mathrm{C}$ and $300^{\circ} \mathrm{C}$ are plotted but are very weak. (b) XRD measurements for different annealing temperatures. There is an obvious correlation between the intensity of the XRD peaks and intensity of the TL curves. The XRD peaks increase in intensity with increasing annealing temperature.

Rutherford backscattering spectrometry (RBS) was used to measure the impurity concentration of terbium in the yttria thin film. A graph of the RBS measurement is shown in Fig. 114. The concentration of the terbium was determined by calculating the area under the yttrium and terbium peaks. The scattering cross sections of the terbium and yttrium peaks are then used as a weighting factor on the area under the peaks. The ratio of the weighted areas will be the relative concentrations of atoms in the material. Since the XRD measurement indicates that the $\mathrm{Y}_{2} \mathrm{O}_{3}$ is at least partially crystalline, we have assumed a stoichiometric material and calculated the oxygen concentration accordingly from the yttrium concentration. The ratio of yttrium to terbium indicates a $1 \%$ concentration of terbium. This is 10 times more than the concentration in the source material, and is probably due to the higher vapor pressure of terbium oxide relative to yttrium oxide during the evaporation process. Which may be due to the slightly higher melting point of yttria being $2425^{\circ} \mathrm{C}$ and terbium oxide $2340{ }^{\circ} \mathrm{C}$. 


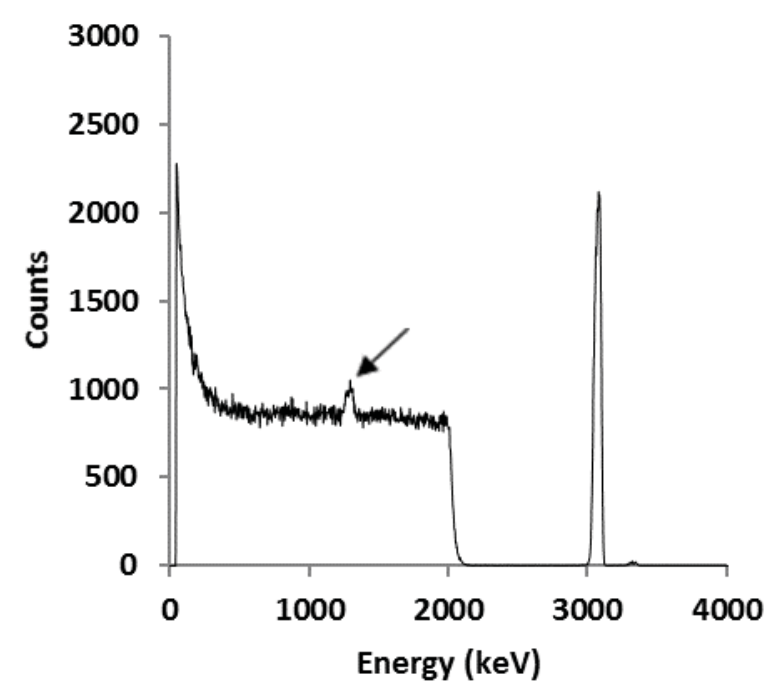

Fig. 114. The RBS data show the peaks for oxygen, yttrium and terbium. The large flat portion represents the $550 \mu \mathrm{m}$ thick silicon wafer.

Thermal fading has a significant effect on the $\mathrm{Y}_{2} \mathrm{O}_{3}$ :Tb. Fig. 115 shows an example of the thermal fading. The TL intensity peak fades by $75 \%$ after $10 \mathrm{~min}$ and $50 \%$ after $1 \mathrm{hr}$. For visible light with wavelengths shorter than $500 \mathrm{~nm}$, light will begin emptying traps that were filled during irradiation. This effect is weakly seen across the $500 \mathrm{~nm}$ to $700 \mathrm{~nm}$ range of visible light but is more apparent at shorter wavelengths.

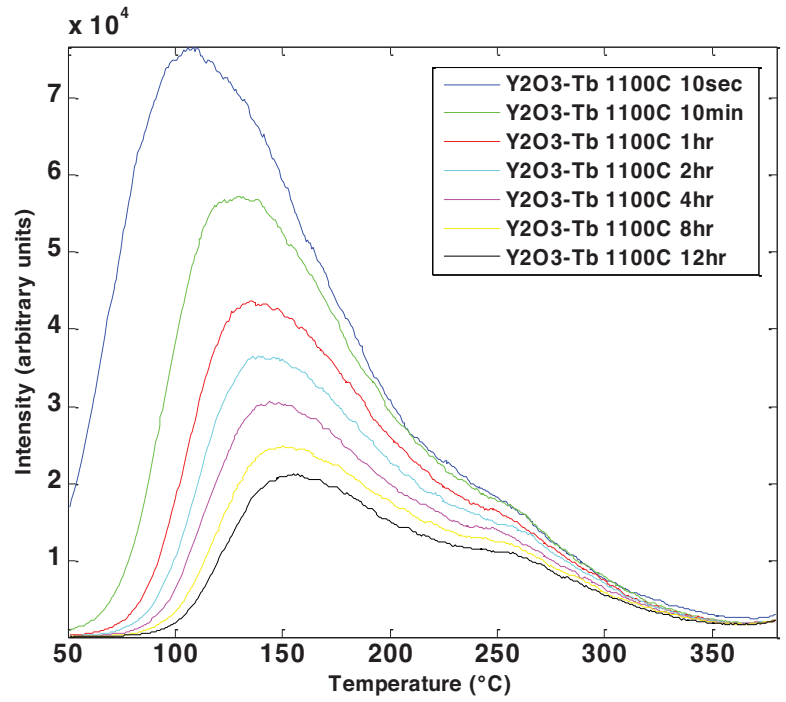

Fig. 115. There is significant thermal fading of the lower temperature TL curve peak, whereas the high temperature TL peaks show less fading.

Finally, the luminescent intensity per unit mass of $\mathrm{Y}_{2} \mathrm{O}_{3}: \mathrm{Tb}$ was compared to TLD-100 from Thermo Scientific. The thin film was $2 \mathrm{~cm} \times 2 \mathrm{~cm}$ and $350 \mathrm{~m}$ thick and the TLD-100 chip was $6.4 \mathrm{~mm} \times 6.4 \mathrm{~mm} \times$ 
$0.89 \mathrm{~mm}$. The mass and maximum intensities of the TL curves of the $\mathrm{Y}_{2} \mathrm{O}_{3}: T b$ and TLD-100 were $7.01 \times 10^{-4} \mathrm{~g}, 1160 \mathrm{au}, 9.64 \times 10^{-2} \mathrm{~g}$ and $5.84 \times 10^{6} \mathrm{au}$, respectively. The calculated TL intensity per unit mass for the $\mathrm{Y}_{2} \mathrm{O}_{3}: \mathrm{Tb}$ and TLD-100 is $1.65 \times 10^{6}$ and $60.8 \times 10^{8}$ respectively. The comparison can be seen in Fig. 116 between $\mathrm{Y}_{2} \mathrm{O}_{3}: \mathrm{Tb}$ and TLD-100 irradiated with $25 \mathrm{~Gy}$. The TL intensity per unit mass of the TLD-100 thin film was 37 times as intense as the $\mathrm{Y}_{2} \mathrm{O}_{3}:$ Tb.

(a)

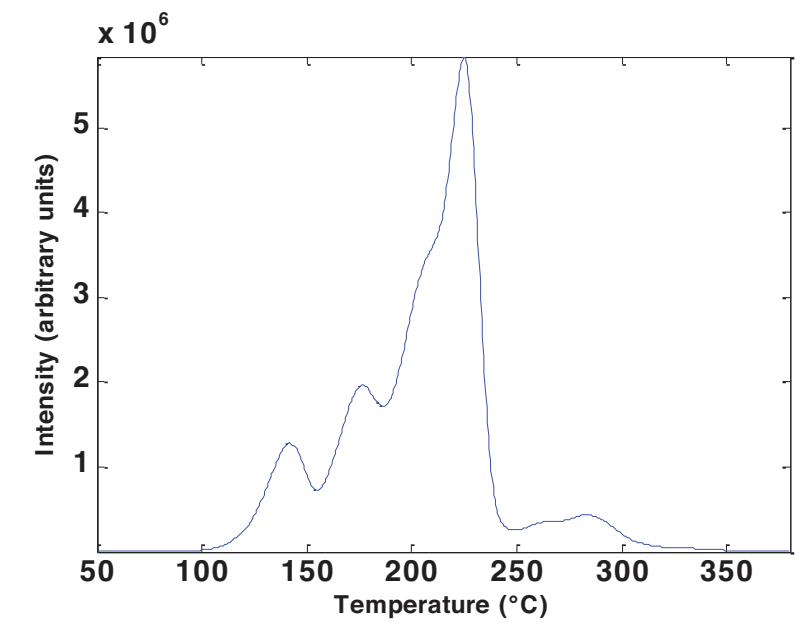

(b)

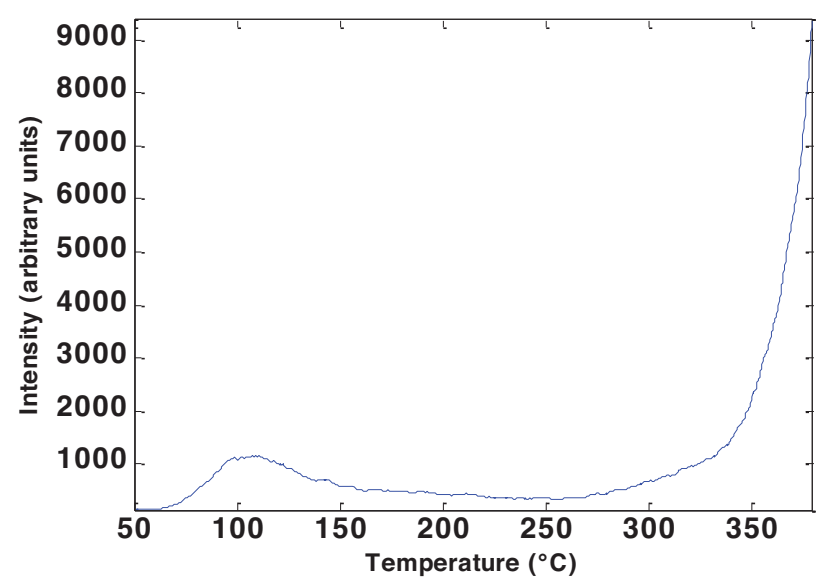

Fig. 116. A comparison between the intensities of the $\mathrm{Y}_{2} \mathrm{O}_{3}$ :Tb thin film and TLD-100. a) The TL curve for $\mathrm{Y}_{2} \mathrm{O}_{3}: \mathrm{Tb}$ after $25 \mathrm{~Gy}$ of x-ray irradiation. b) The TL curve of TLD-100 after 25 Gy of x-ray irradiation. The TL curve for the $\mathrm{Y}_{2} \mathrm{O}_{3}: \mathrm{Tb}$ is much weaker than that for TLD-100 but the mass of the $\mathrm{Y}_{2} \mathrm{O}_{3}$ :Tb thin film is much less than the TLD-100 chip. The luminescent intensity per unit mass was 37 times weaker for the $\mathrm{Y}_{2} \mathrm{O}_{3}: \mathrm{Tb}$.

\subsection{Pressure tests}

\subsubsection{Membrane stress}

This section describes work to ascertain the impact of mechanical stress on the luminescence of our TL materials. This subsection covers sudden mechanical stress on films. The next section covers impact effects. Regarding stress, we have seen none at MPa-scale pressures. The only changes in luminescence that we have seen have come under extensively repeated stressing using an electrostatic actuator, 
which is probably due to dielectric charging but could also be due to fatigue. Neither is seen in explosions.

We engineered a method to examine the effects of pressure - both sustained stress and sudden impact forces - on the TL of materials. The design of the actuator was refined to enable it to easily reach snapdown, where the membrane and the TL film it carries is driven to quickly impact the bottom electrode. Progress in fabrication methods has also improved yields and efficiency.

The actuator device is fabricated as two separate components, the membrane die and the bottom electrode die, and assembled for use. This approach greatly simplifies fabrication by eliminating a number of potential issues, such as the release of such a large membrane (usually at least $1 \mathrm{~cm}$ on a side) with limited etchant access to the sacrificial layer and the electrical isolation of electrodes, which must face each other across the actuator air gap. Non-permanent assembly also allows different TL samples to be swapped in and out, and parameters such as electrode distances to be adjusted, without requiring the entire fabrication process to be repeated each time; this latter benefit demonstrates its value on a regular basis when handling membranes a few hundreds of nanometers thick.

(a)

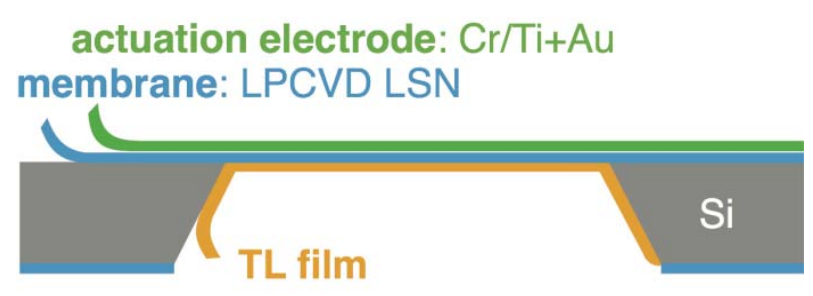

(b)

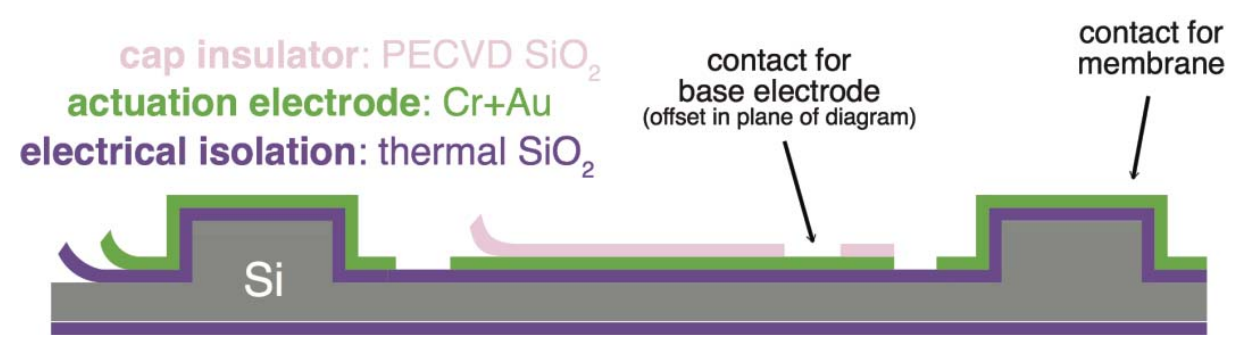

(c)

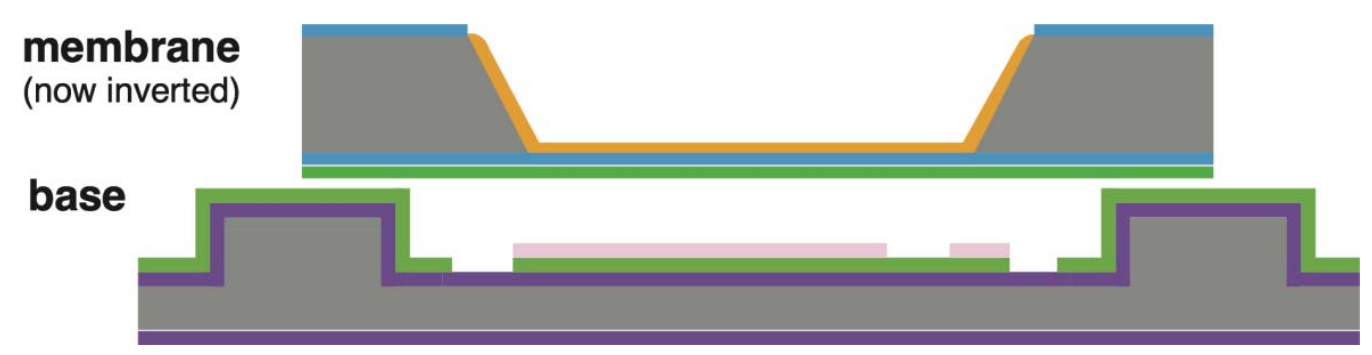

Fig. 117. Cross-sectional diagrams of the actuator components, and their assembly into the completed device: (a) the membrane die, a low-stress nitride membrane released from silicon by wet etch and coated on opposite sides with a metal 
layer and the TL film of interest; (b) the base die, with support pillars that have been Bosch-etched from Si, insulated with thermal oxide, and metallized; (c) the two dies in their assembled configuration. Note that the "peel-off" effect drawn in (a) and (b) is a bit of artistic license taken to clarify the different film layers; we have to date had no significant problems with film adhesion.

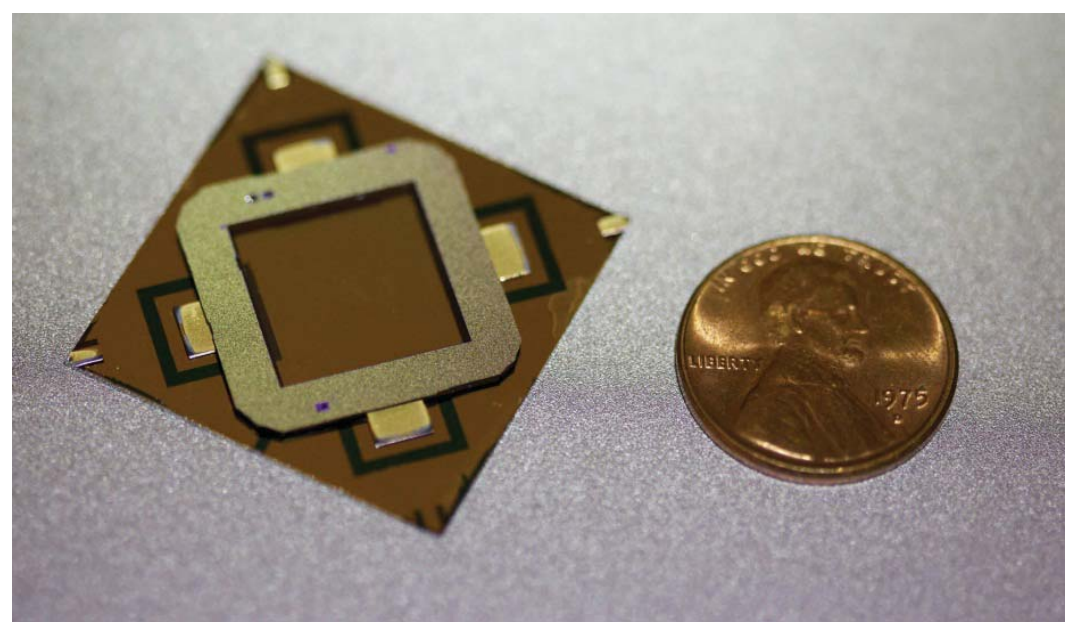

Fig. 118. An assembled actuator device. The pictured example is a slightly earlier-generation design, without an encapsulation layer on the base die.

By reducing the electrode separation - which also demonstrates again the benefits of separately fabricated base dies - and refining other fabrication steps, we are now able to realize controllable deflection up to the pull-in point using drive voltages of less than $100 \mathrm{~V}$.

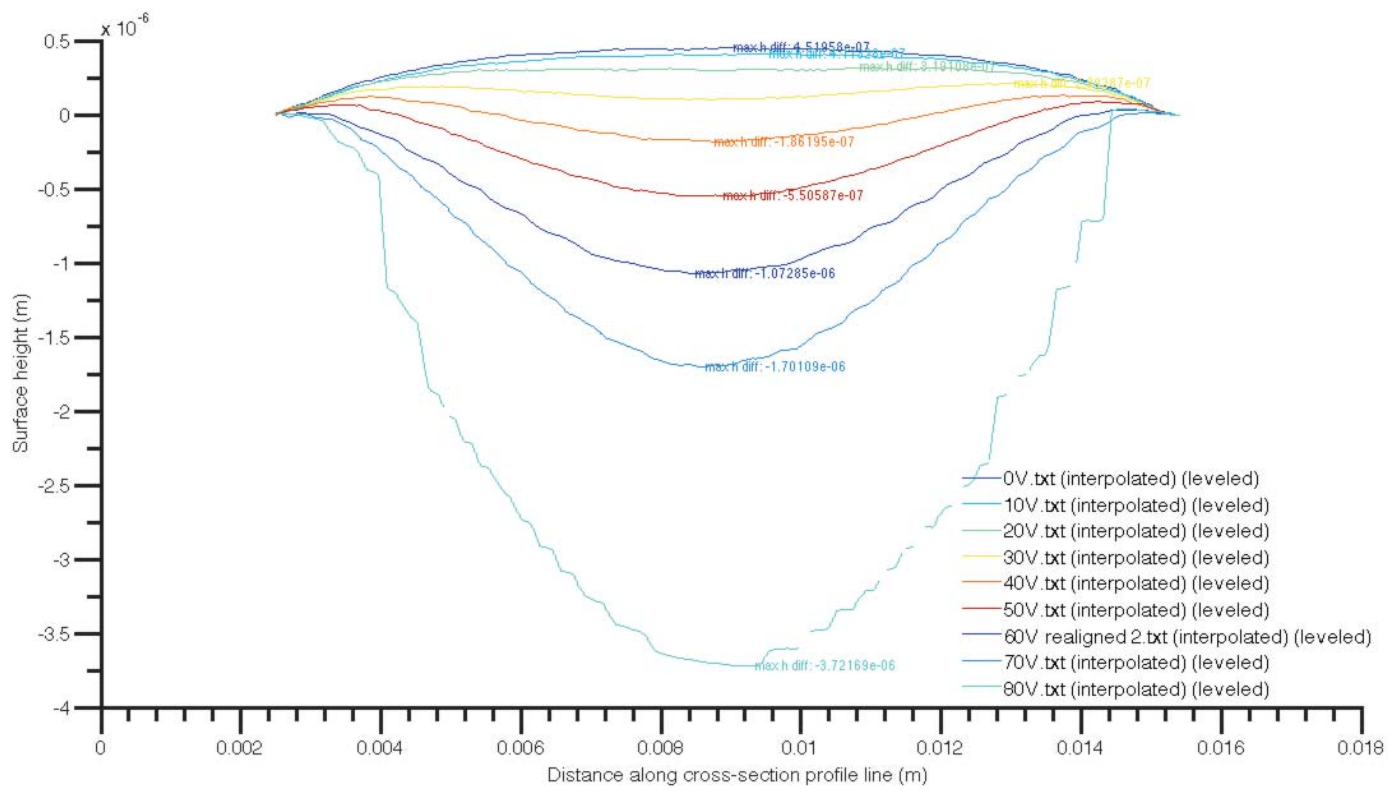

Fig. 119. The cross-sectional membrane height profile of an actuator membrane under drive voltages from 0 to $80 \mathrm{~V}$. Deflections of more than $4 \mu \mathrm{m}$ are reached at the membrane center. 
Interesting results have been observed when applying non-impact stress to our tested TL film, the ebeam evaporated terbium-doped yttrium oxide described elsewhere in this report. An experiment was run where the $\mathrm{Y}_{2} \mathrm{O}_{3}: \mathrm{Tb}$-coated membrane was irradiated to charge TL traps, alternatingly actuated with a given voltage and relaxed for $3 \mathrm{~s}$ each for one hundred cycles, and finally heated to collect a TL curve. From interferometric measurements of separate actuations, it is estimated that the application of $25 \mathrm{~V}$ produces a deflection at the membrane center of $0.15 \mu \mathrm{m}$; the actuation voltage was capped at $25 \mathrm{~V}$ to protect the films, which had not yet undergone testing to find their actuation and stress limits.

A decrease in overall TL curve intensity is seen with each increase in the actuation voltage (Fig. 120); the two intensity peaks at approximately $133{ }^{\circ} \mathrm{C}$ and $245{ }^{\circ} \mathrm{C}$ see a maximum decrease of $42 \%$ from $20 \mathrm{~V}$ to $25 \mathrm{~V}$. Some hysteresis does appear: returning to a $0 \mathrm{~V}$ actuation sees only a partial recovery in intensity, and applying an intermediate voltage less than the previously applied maximum causes no change from this value. The positions of the peaks do not appear to change, suggesting that the phenomenon does not come from alteration of first-order trap parameters.

(a)

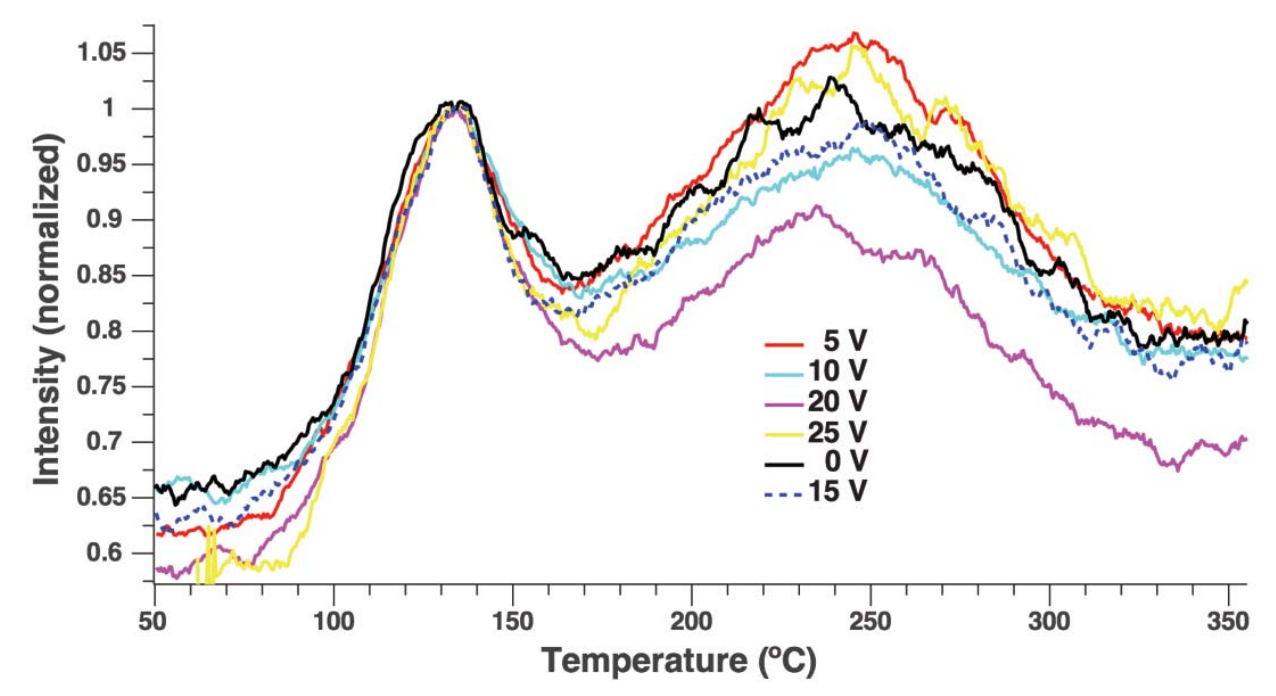

(b) 


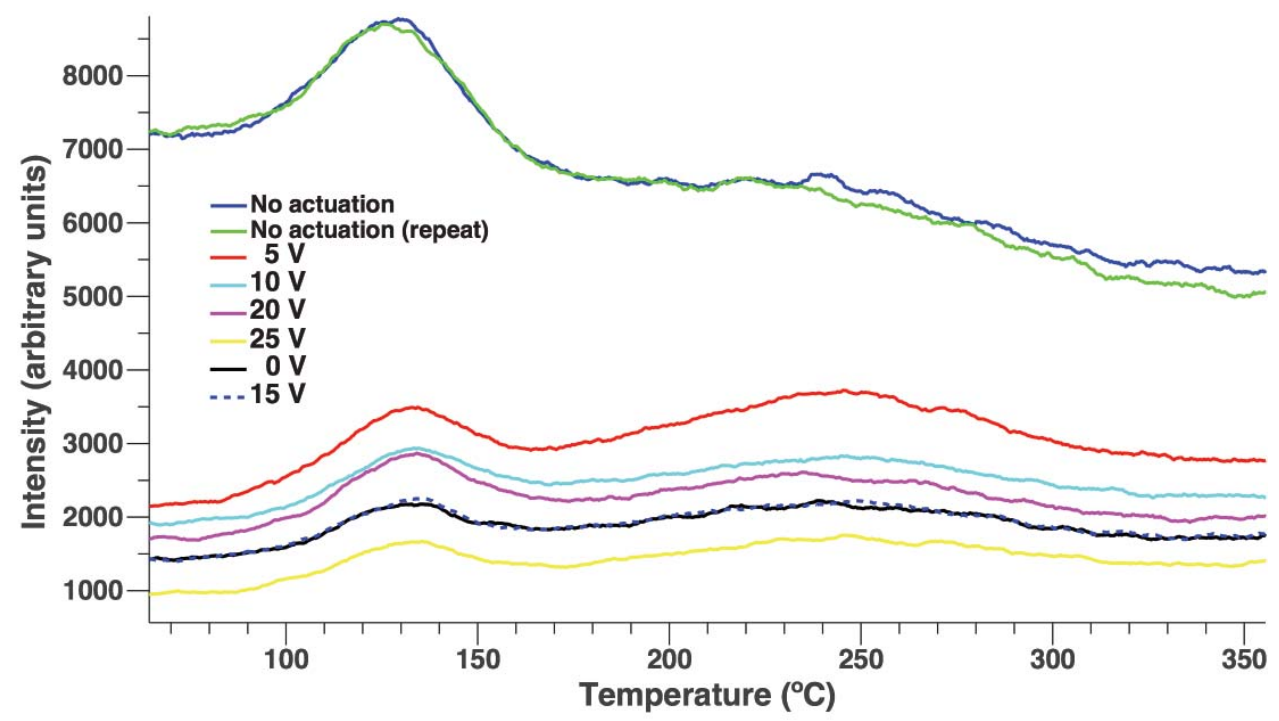

Fig. 120. Two representations-with (a) and without (b) normalization-of TL curves collected following repeated postirradiation actuation and relaxation cycles. The overall intensity of the curve sinks with increasing actuation voltage up to the maximum of $25 \mathrm{~V}$, then shows a partial recovery when a lower voltage is subsequently applied. Note that in both graphs, the legend lists the curves in the order in which they were run.

To rule out the intensity decrease being a simple case of the film aging, a series of TL curves with no actuation was run on a second membrane with an identically prepared film. An opposite trend of rising intensities with TL curve repetition is seen, suggesting that the effects of repetitive actuation may actually be larger than they appear (Fig. 121). There are, however, multiple possible explanations for the phenomenon, such as dielectric charging.

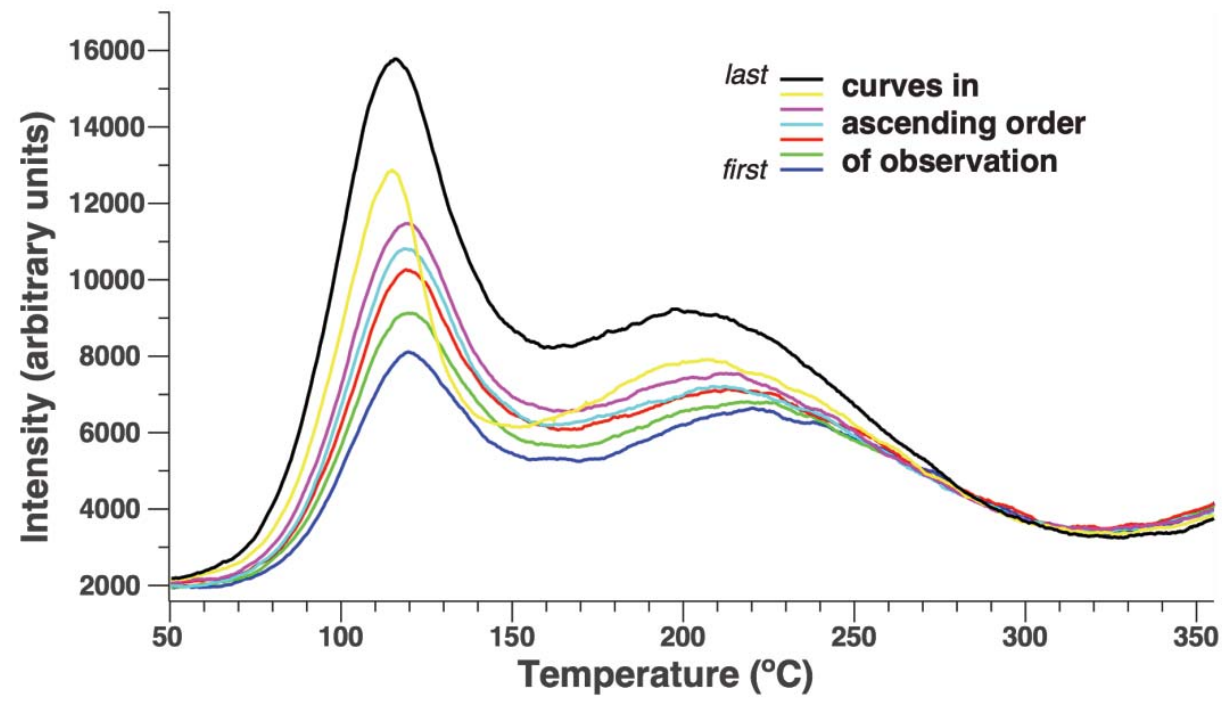

Fig. 121. A series of non-actuated TL curves (on an identically prepared film sample which had never been used for actuation experiments) collected to test for aging effects. The TL intensity grows over time and repeated use, which is the opposite trend of the actuation effects seen, and suggests that the change caused by actuation is actually stronger than it appears to counteract this aging effect. 


\subsubsection{Impact Triboluminescence}

Completing our studies of the effects of pressure and impact forces on TL materials, we have conducted experiments to test for pressure- and impact-induced luminescence. The membrane microdevices used to study changes in TL with strain can be optimized to study the effects of fast stress and impact forces with a few simple tweaks to the bottom die's fabrication parameters. The devices as used feature $10 \mathrm{~mm}$ square released membranes of $200 \mathrm{~nm}$ thick LPCVD low-stress nitride, metallized on the downward-facing side with $5+45 \mathrm{~nm}$ of sputtered Ti+Au and coated on top with the thin film of interest (Fig. 122). Pillars on the base die elevate the membrane $12 \mu \mathrm{m}$ above a gold bottom electrode, and provide probe contact points for the downward-facing membrane electrode.

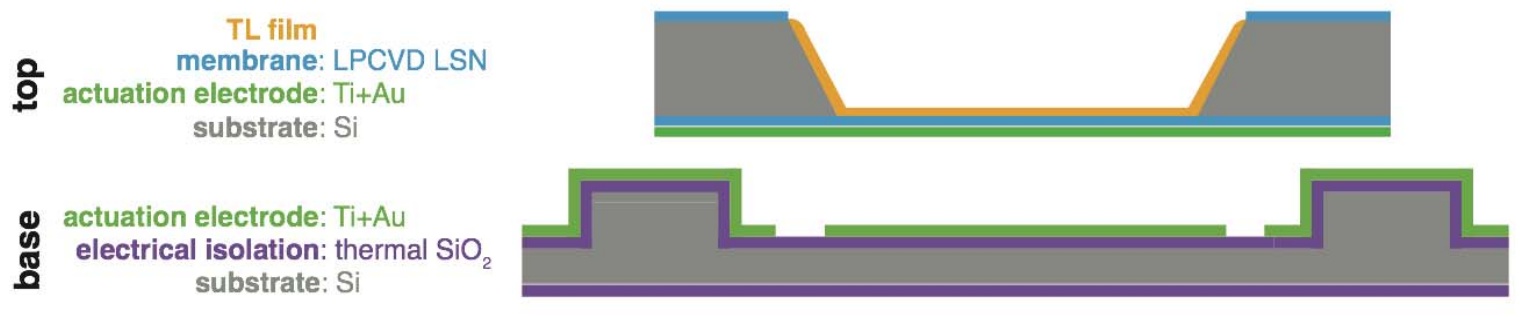

Fig. 122. Cross-sectional diagrams of the actuator as used for the results below. The two-die nature of the design shows its flexibility here: we can carry over verbatim the same top membrane dies used in previously published studies of strain effects on TL, but adjust the electrode spacing to optimize the device for fast impacts.

To profile the deflection of the membrane vs. time, we use a linear position-sensing detector (PSD) to measure the motion of a $675 \mathrm{~nm}$ collimated fiber-coupled diode laser incident at a $45^{\circ}$ angle upon the center of the membrane. The PSD is angled at a similar $45^{\circ}$ angle. Vertical deflection of the membrane causes lateral travel in the laser's point of incidence with the membrane, translating to a proportional motion of the reflected beam's intersection with the detector. The PSD outputs a voltage via a transimpedance amplifier, and this signal is digitized and recorded by a computer-linked oscilloscope.

The TL films under study are irradiated in situ with a 180-400 nm deuterium UV source. Sequences of voltage pulses are then applied while a photomultiplier tube, protected from stray light from the position-sensing laser by a $625 \mathrm{~nm}$ shortpass filter, observes the sample for induced luminescence. To verify that the PMT is operational and to check for barely-discernible signals, which may be drowned out by the position-sensing laser, one deflection pulse in every repeated chain is done with the laser deactivated. 


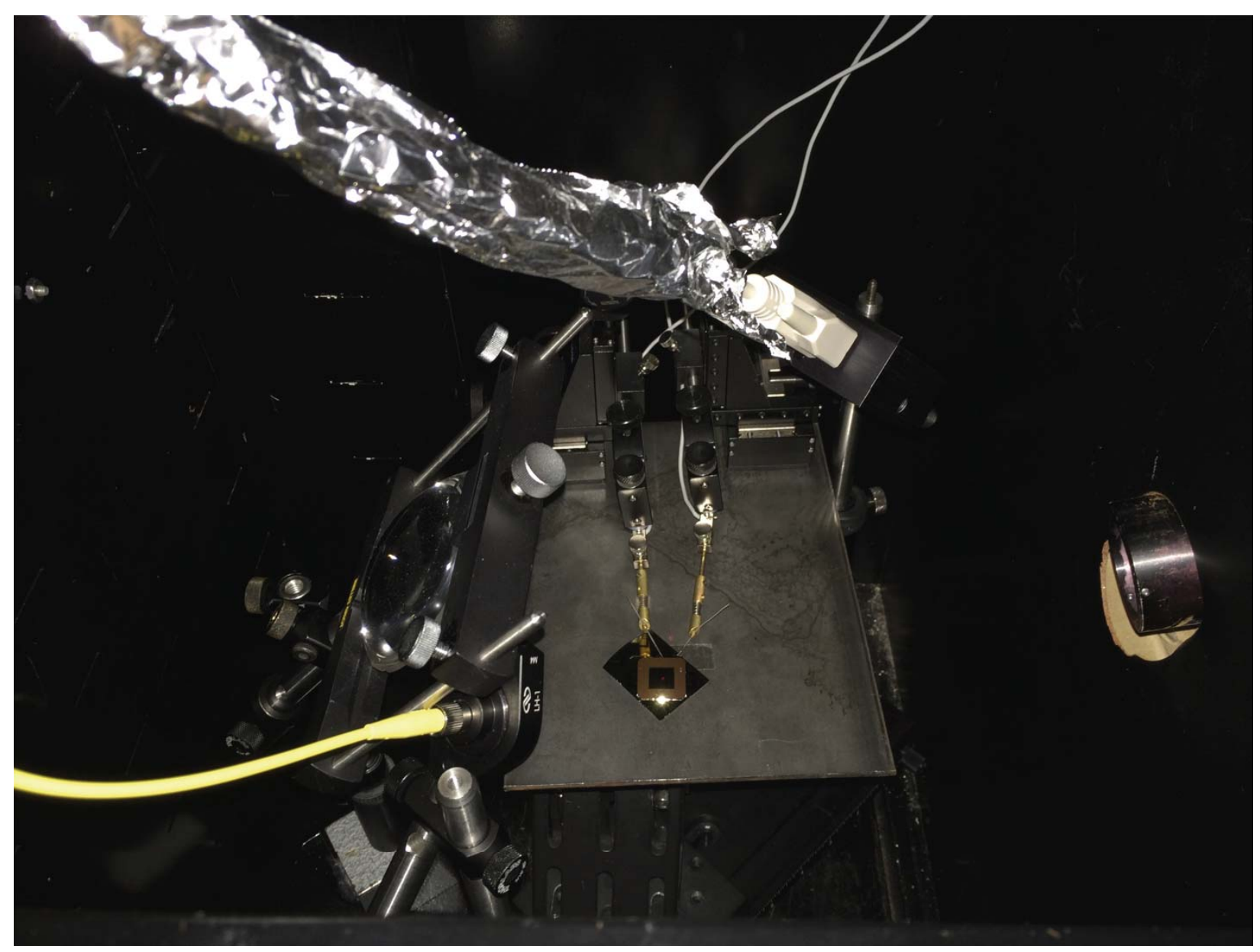

Fig. 123. Photograph of the actuator device, at lower center, and deflection measurement equipment in the experimental test chamber. The yellow cable at bottom right is the single-mode fiber carrying the $\mathbf{6 7 5} \mathbf{n m}$ laser for the position-sensing detector, which itself is the black box at the end of the tinfoil-wrapped cable. The large light-gathering lens can be seen just above the mount that holds the head of the optical fiber, and the output lens of the UV source makes an appearance at far right. The PMT sits outside the left edge of the photo.

Our experiments used a terbium-doped yttrium oxide $\left(\mathrm{Y}_{2} \mathrm{O}_{3}: \mathrm{Tb}\right)$ thin film, electron-beam evaporated onto the membranes at thicknesses of around $200 \mathrm{~nm}$ and annealed in argon at $900{ }^{\circ} \mathrm{C}$ for $30 \mathrm{~s}$. When irradiated with 180-400 $\mathrm{nm} \mathrm{UV}, \mathrm{Y}_{2} \mathrm{O}_{3}: \mathrm{Tb}$ shows two to three (depending on preparation) broad TL peaks between $100{ }^{\circ} \mathrm{C}$ and $300{ }^{\circ} \mathrm{C}$. These tend to become more pronounced over a new sample's first few TL curve readouts, probably due to annealing as the sample is heated to over $400{ }^{\circ} \mathrm{C}$, so membranes equipped with this film have several TL curves collected before being used for actuation. 

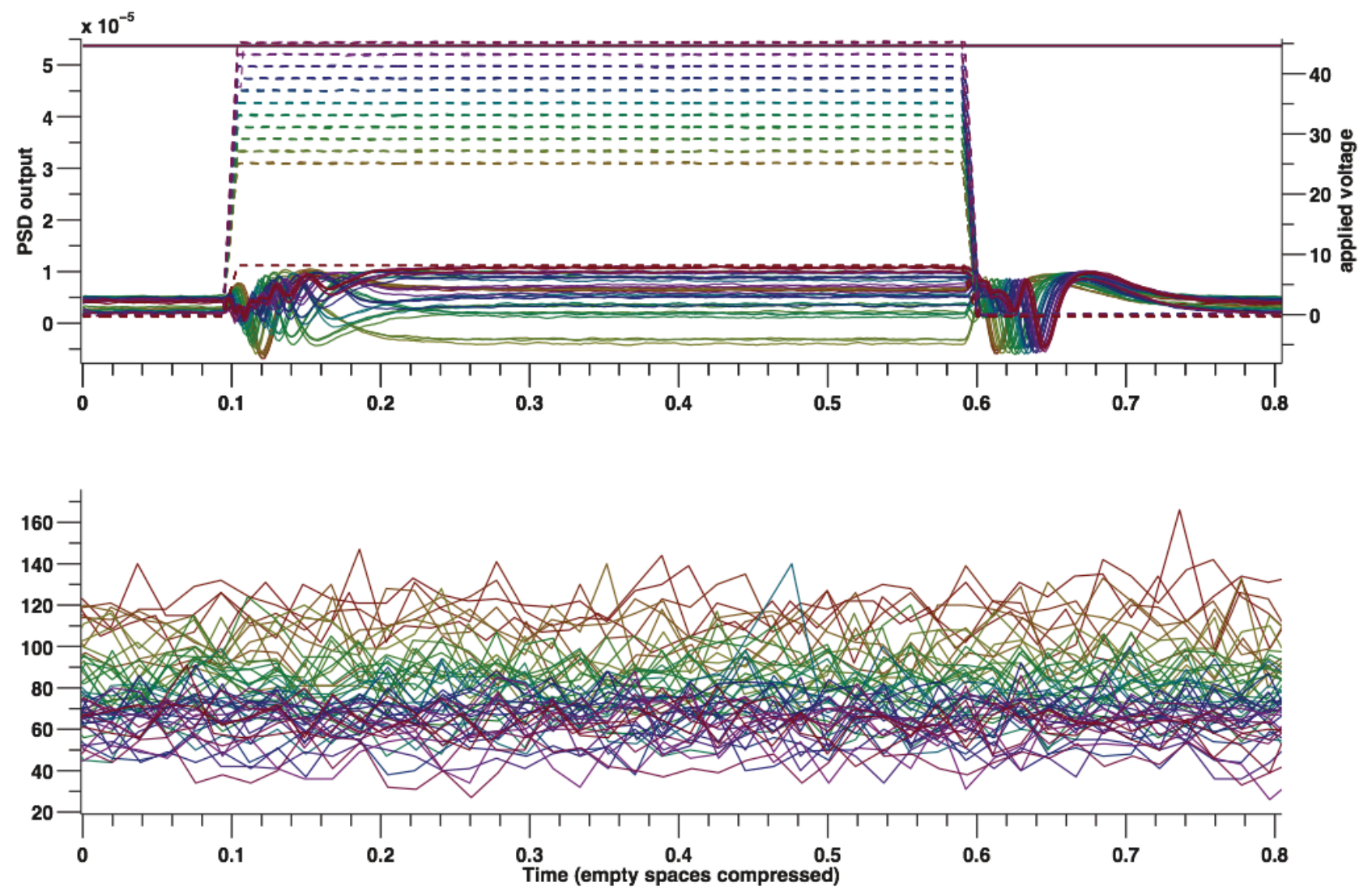

Fig. 124. (Top) The voltage-drawn with dashed lines and charted on the right-hand vertical axis-applied and measured to our membrane actuator device. The resulting output height data from the position-sensing detector (PSD) is shown in solid lines and charted on the left-hand vertical axis. Both series are plotted vs. a shared time scale, with shared colors indicating the voltage and PSD output series, which correspond with each other. All of the pulses shown above are $500 \mathrm{~ms}$ long. (Bottom) Recorded photomultiplier counts, representing observed light intensity, during the induced deflections above. The intensity data have not been averaged or filtered for single-point anomalies.

We drove the actuator conservatively, reaching about half the easily achievable voltage range, to protect our constrained sample quantities. (Although fabrication yields of the actuator membranes are now within a few percent of ideal, barring handling accidents, adding and annealing the $\mathrm{Y}_{2} \mathrm{O}_{3}: \mathrm{Tb}$ film often induces what is believed to be abrupt, explosive stress-induced ruptures.) PSD readings suggest that the actuator has achieved the full range of motion; this is supported by naked eye observations, which note clearly visible changes in the membrane surface appearance and interference fringes as voltage is applied. The response to applied voltage generally consists of a large and short-lived deflection downwards, as would be expected if the actuator reached pull-in to touch and short its electrodes together, and this pattern is repeated when the voltage is removed. The PSD data, however, also suggest that the membrane sometimes finds a stable elevation above its rest position for most of the duration of the voltage pulse. Although these behavior patterns are unintuitive, they are repeatable and seem consistent with increasing voltage. It is unclear whether this is an actual phenomenon, perhaps caused by bilayer behavior in the metallized membrane, or a result of our spatially-limited readout system, e.g. caused by the membrane center tilting during deflection. Experiments are being planned which seek to clarify this by consecutively sampling multiple points on the membrane surface, as well as varying the length of the pulses.

No clear strain- or impact-induced luminescence has been detected from this or any other of our films. 


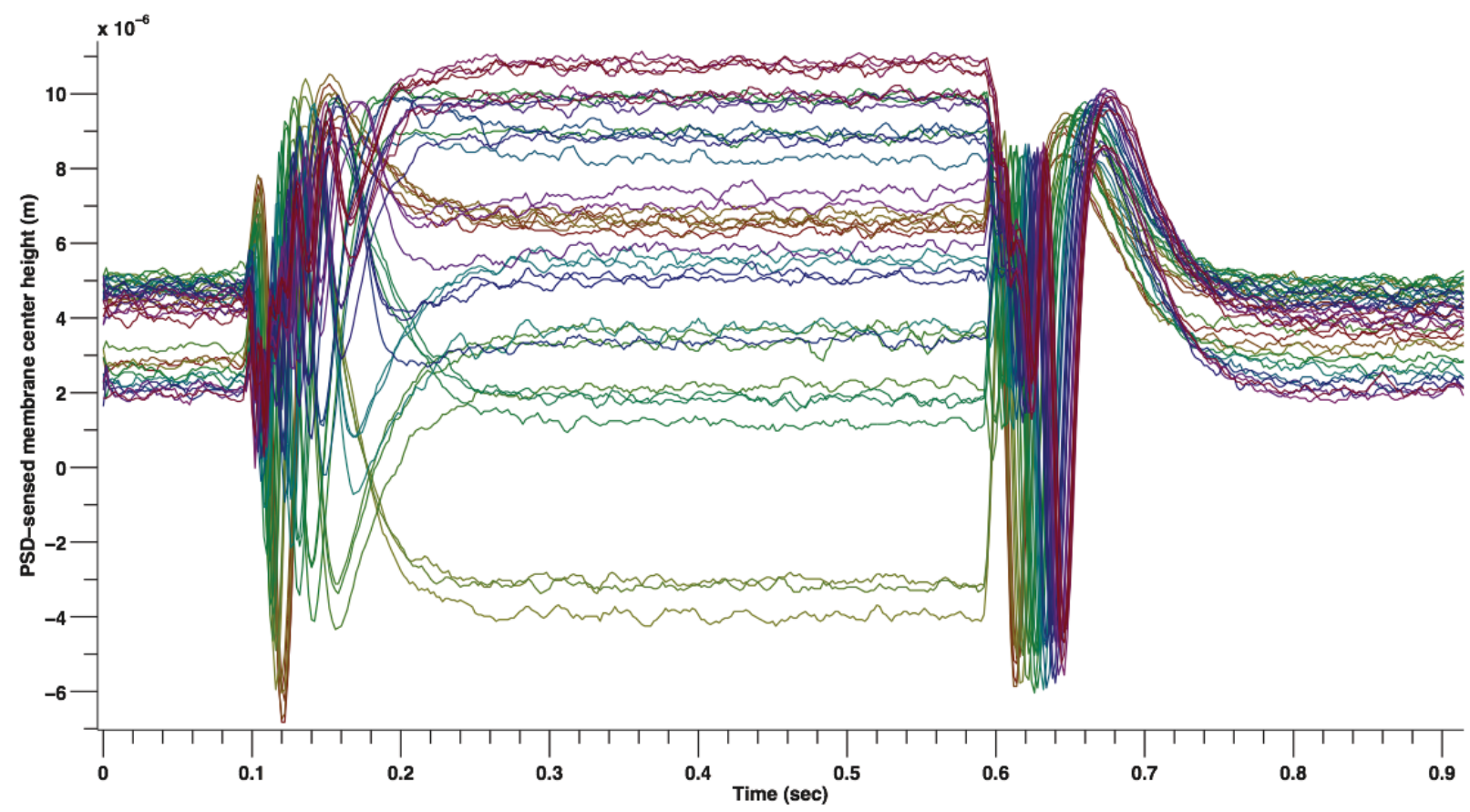

Fig. 125. The PSD-sensed membrane center height data seen in the previous figure, isolated and rescaled.

\subsection{Rare earth yttria sintered powder thin films}

In the past, when we made the source materials for electron beam evaporation, we would mix the host and dopant powder together and then compress the mixture into pellets. The pellets were then used for e-beam evaporation. We found $\mathrm{Y}_{2} \mathrm{O}_{3}: \mathrm{Tb}$ to be the only thin film that would luminesce strongly after deposition as a thin film. We decided to sinter the powders after mixture to increase the quality of the source material used for deposition. The hope was that this would diffuse the dopant material into the host material before deposition. The powder mixture is annealed at $1000^{\circ} \mathrm{C}$ for over $10 \mathrm{~h}$. This powder after sintering showed a TL curve. The TL curves are listed and compared to $350 \mathrm{~nm}$ thin films after deposition. The films are on 4" diameter p-type silicon wafers. The sintering appears to have helped bring out the TL of the material. Below are the TL curves for the powder and corresponding thin film.

Each of the above measurements were taken using a lens that increases the intensity by about an order of magnitude. All of these TL curves were too weak to make an accurate spectrum measurement, because the monochromator attenuates the TL signal too much for the PMT to detect anything above the noise level. The thin films have approximately 8-10 times the dopant concentration as the mixed powders. This is most likely due to the dopant evaporation more readily during deposition. This could explain why there is such a distinct difference between the powders and thin films. We are currently trying to modify the dopant concentration in evaporation source. In Fig. 126 the dopant concentration of the source is $0.1 \% \mathrm{~mol}$, but the thin films from this source have a concentration of about $1 \%$. 
(a) $\mathrm{Y}_{2} \mathrm{O}_{3}: \mathrm{Gd}$

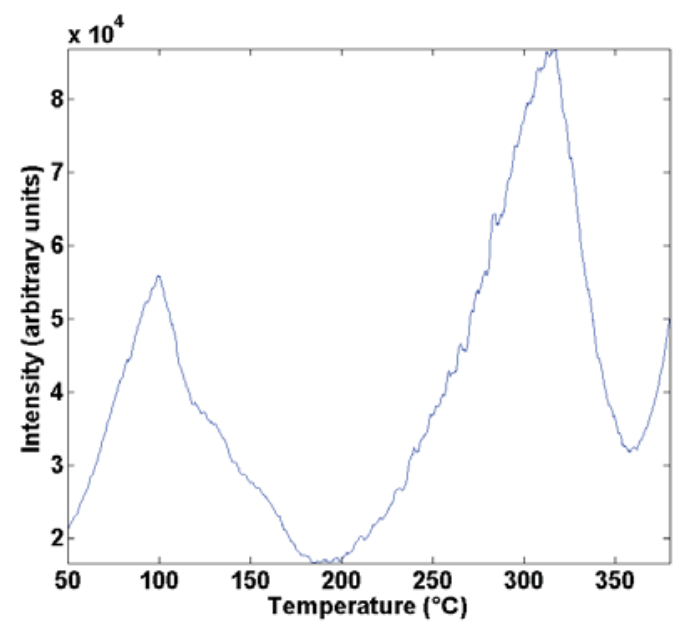

(c) $\mathrm{Y}_{2} \mathrm{O}_{3}: \mathrm{Yb}$

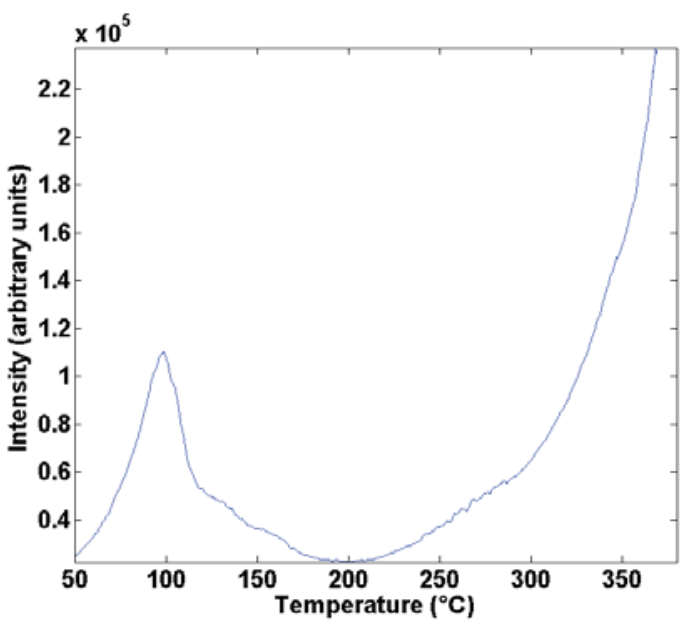

(e) $\mathrm{Y}_{2} \mathrm{O}_{3}: \mathrm{Dy}$

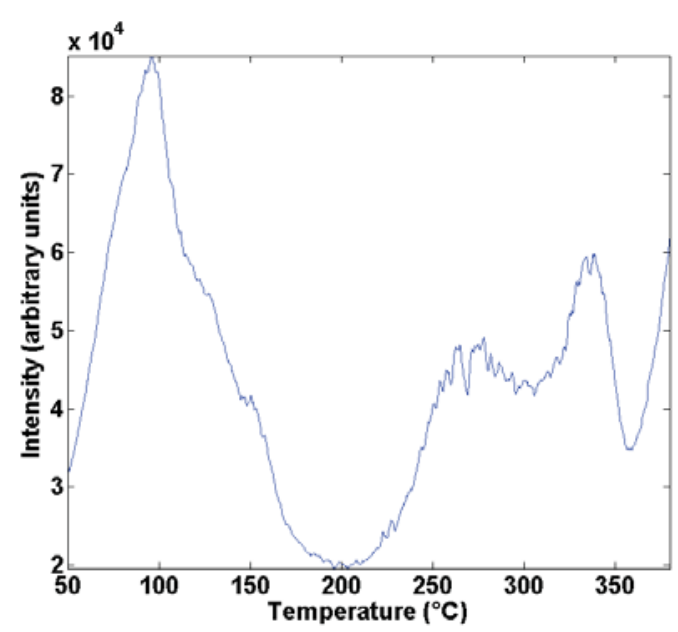

(b) $\mathrm{Y}_{2} \mathrm{O}_{3}$ :Gd thin

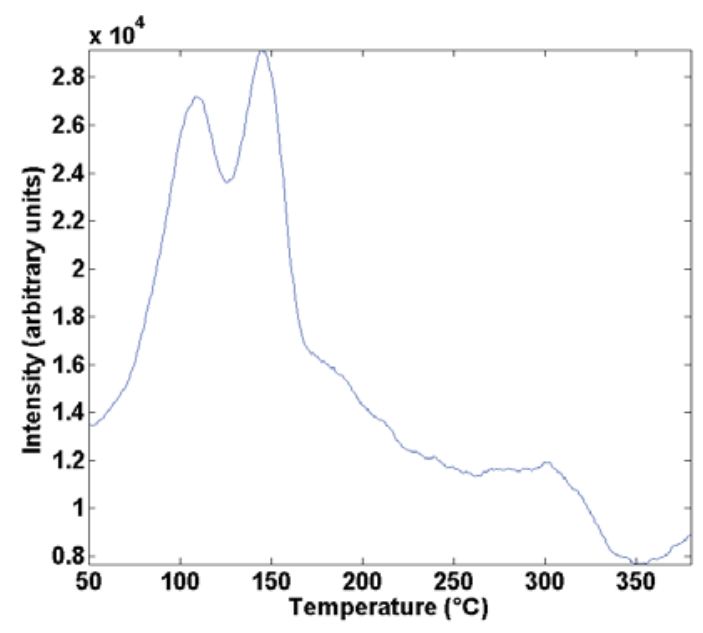

(d) $\mathrm{Y}_{2} \mathrm{O}_{3}: \mathrm{Yb}$ thin

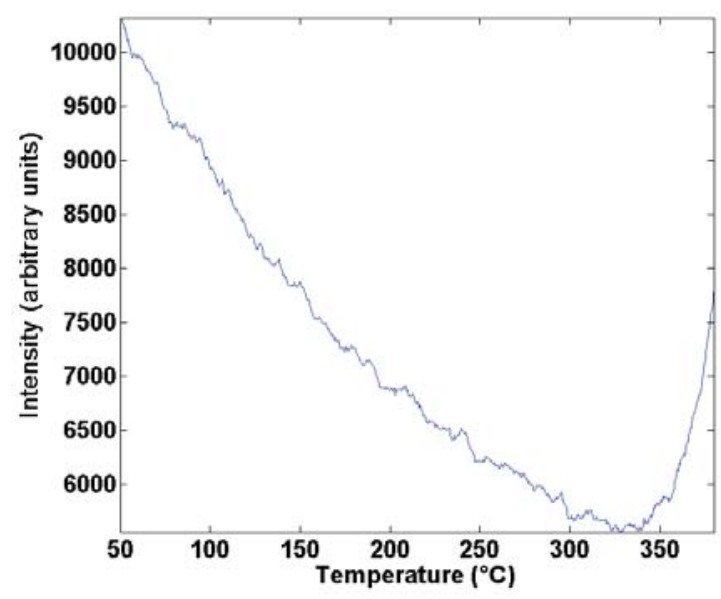

(f) $\mathrm{Y}_{2} \mathrm{O}_{3}$ :Dy thin

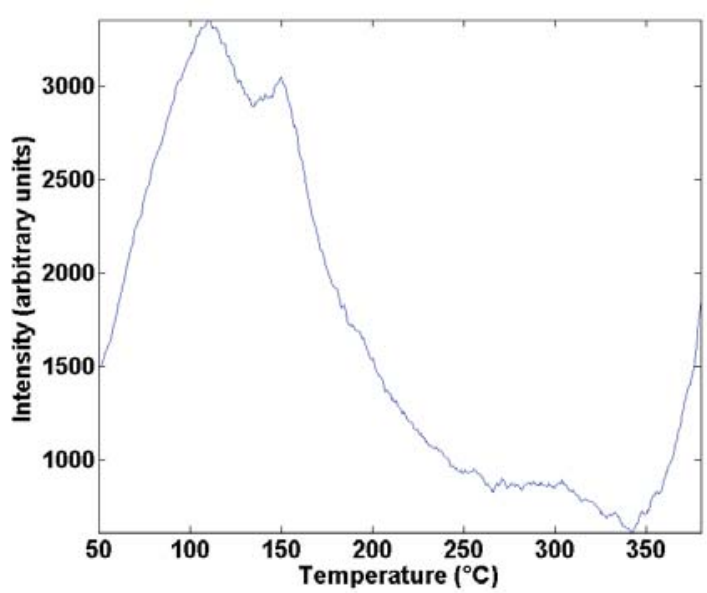


(g) $\mathrm{Y}_{2} \mathrm{O}_{3}:$ Er powder

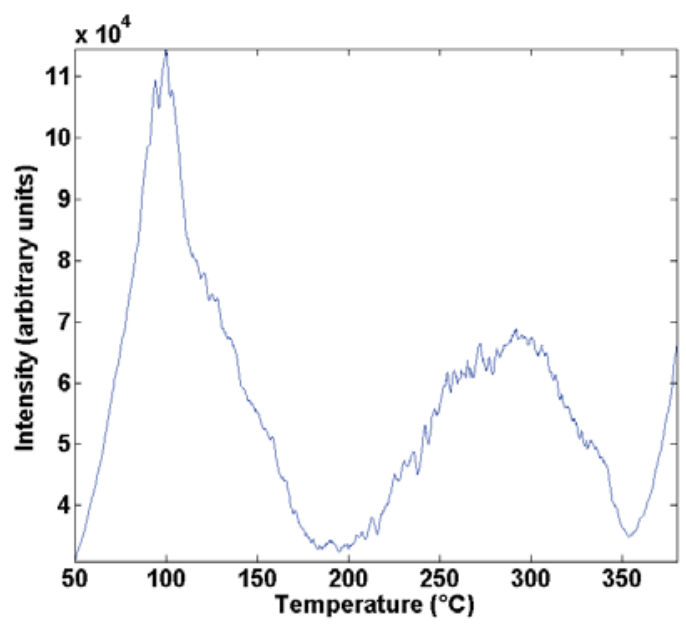

(h) $\mathrm{Y}_{2} \mathrm{O}_{3}$ :Er thin

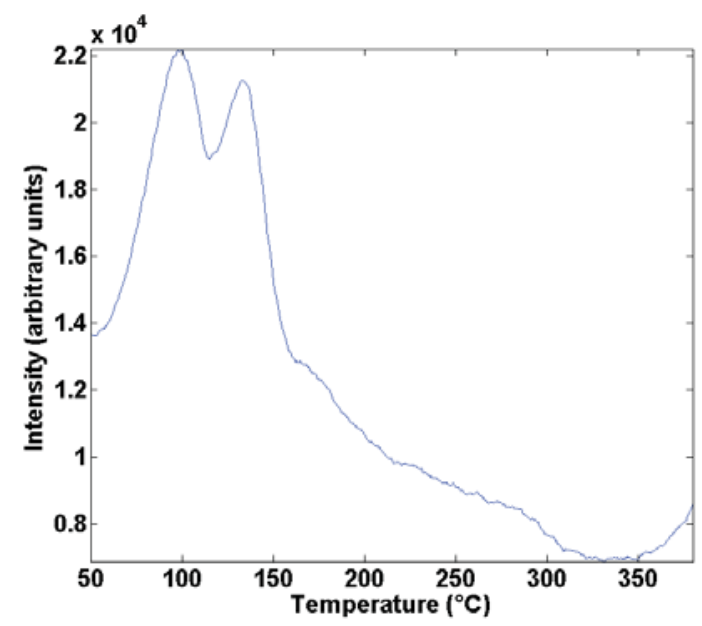

(i) $\mathrm{Y}_{2} \mathrm{O}_{3}$ :Eu thin film

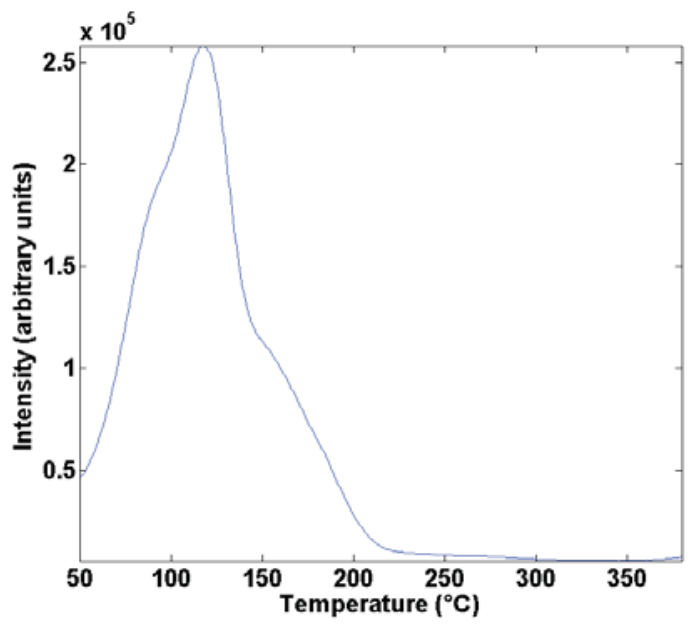

Fig. 126. TL curves of $\mathrm{Y}_{2} \mathrm{O}_{3}$ samples doped with different lanthanides.

\subsection{Effect of UV}

We also tested whether or not the TL peaks can be induced exposing the samples to UV light instead of ionizing radiation. Fig. 127 shows the TL curves obtained for LBO after beta irradiation or exposure to UV (deuterium lamp). The data show that the TL curves produced by UV or beta radiation are different. Beta irradiation seems to be more suitable because it produces peaks that are better defined and distributed over a wider temperature range.

A similar investigation was carried out for $\mathrm{MBO}$. Although the structure of the peaks is more similar after beta irradiation or UV exposure than LBO, the TL curves are still very different. In this case, whether or not to use beta rays or UV for the preparation of the sample depends on the convenience for the laboratory and on the specific protocol that was used during readout of the samples. 

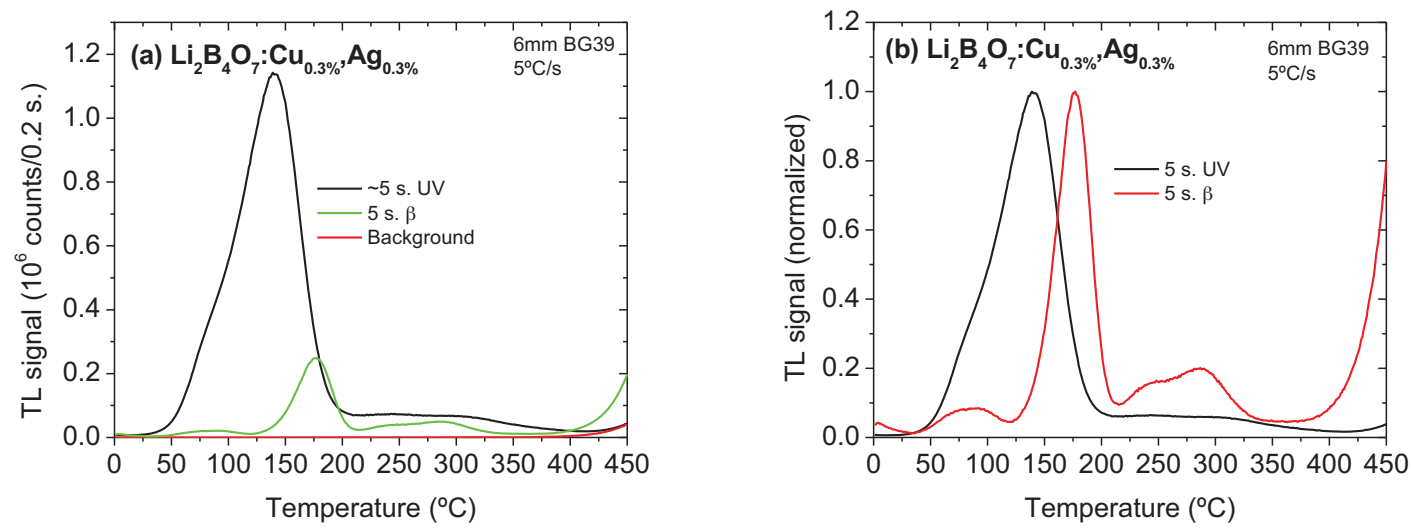

Fig. 127: (a) UV induced TL of LBO and (b) a comparison of normalized TL curves from beta and UV induced TL of LBO. Heating rate of $5^{\circ} \mathrm{C} / \mathrm{s}$ with TL readout to $450^{\circ} \mathrm{C}$. UV exposure was $5 \mathrm{~s}$ to a deuterium lamp and beta exposure was $5 \mathrm{~s}$ ( 0.5 Gy).
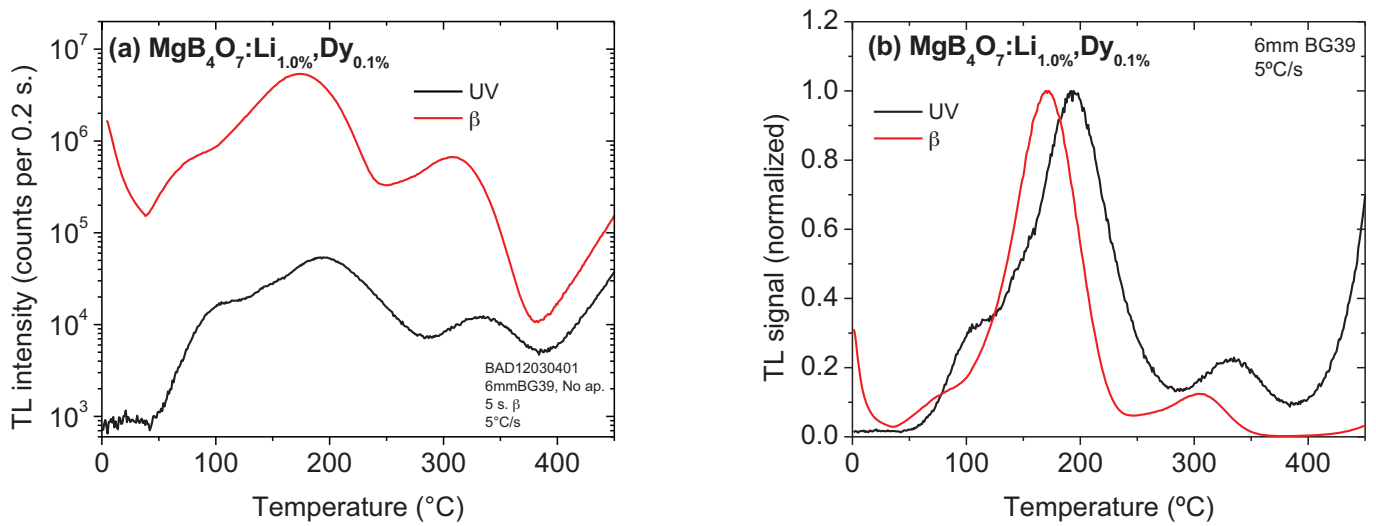

Fig. 128: (a) UV induced TL of MBO and (b) a comparison of normalized TL curves from beta and UV induced TL of MBO. Heating rate of $5^{\circ} \mathrm{C} / \mathrm{s}$ with TL readout to $450^{\circ} \mathrm{C}$. UV exposure was $5 \mathrm{~s}$ to a deuterium lamp and beta exposure was $5 \mathrm{~s}$ ( $0.5 \mathrm{~Gy})$.

\subsection{Influence of alcohol wash}

Since some of the temperature sensing experiments involves the use of alcohol to wash and collect the particles used for temperature sensing, we investigated the effect of alcohol on LBO, MBO and CSO, which are the samples currently used by OSU for temperature sensing. For the tests, $10 \mathrm{mg}$ of each samples (LBO, CSO and MBO) was irradiated with $500 \mathrm{~Gy}$. The irradiated sample was then placed in a $1.5 \mathrm{ml}$ vial with $\sim 1.4 \mathrm{ml}$ of ethanol and mixed to $5 \mathrm{~min}$. Then the vial was centrifuged and the ethanol was poured out; the remaining material was dried in a vacuum oven for $3 \mathrm{~h}$ without heating. The TL of the dried sample was measured and the sample was then re-irradiate with the same dose to record the regenerated TL curve.

Fig. 129 shows the TL curves of $\mathrm{LBO}, \mathrm{CSO}$ and $\mathrm{MBO}$ after the two TL readouts mentioned above. For the LBO, the TL curve shows the same feature before and after alcohol washing; for CSO, even the main TL peaks does not change, although there may be some change in the high temperature region $\left(>350^{\circ} \mathrm{C}\right.$ ). For MBO, the TL curves before and after alcohol washing are essential the same, except that there is an 
increase for the high temperature peak around $295{ }^{\circ} \mathrm{C}$. The phenomenon of sensitization previously discussed might explain this.
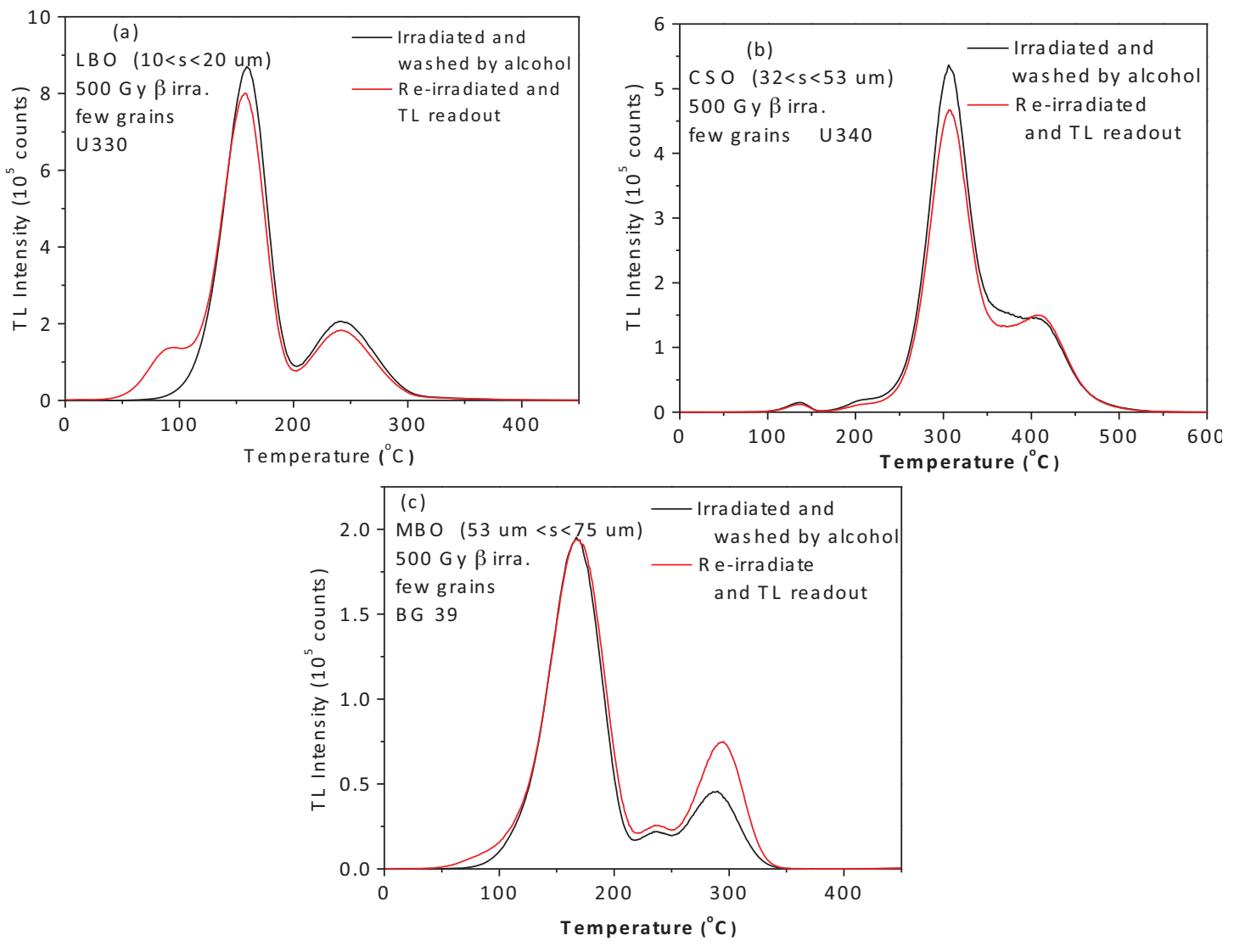

Fig. 129.TL curves of 500 Gy beta irradiated (a) LBO, (b) CSO and (c) MBO after alcohol washing (black line) and then reirradiated by 500 Gy $\beta$-ray and readout again (red line). The heating rate is $1^{\circ} \mathrm{C} / \mathrm{s}$. 


\section{DETONATION TESTS}

\subsection{NSWC (2011)}

Particles of LiF:Mg,Ti (TLD-100) were purchased from Thermo Scientific. After a standard annealing pretreatment of $400{ }^{\circ} \mathrm{C}$ for one hour, the particles were irradiated ( $220 \mathrm{~Gy}$ nominal dose) using a ${ }^{137} \mathrm{Cs}$ radioisotope source. This dose was chosen to produce strong luminescence for a small number of particles, since a large portion of the powder was expected to be scattered during the detonation tests.

With charge carrier traps filled by irradiation, the TLD-100 particles were exposed to the freefield detonation of a $20 \mathrm{~g}$ plastic-bonded explosive formulation containing cyclotetramethylenetetranitramine (HMX) and aluminum particles, with the center of the explosion located approximately $22 \mathrm{~cm}$ away, similar to that shows in Fig. 130. A conventional thermocouple was positioned at the same distance to provide a comparison temperature reading. The TLD-100 sample was suspended over a plastic collection cone, which contained a small amount of particles after detonation (Fig. 131).

For TL readout, the powder samples were placed in a shallow stainless steel crucible, the opening of which was covered with a thick fused quartz disc. A resistive heating element underneath the sample holder provided a linear temperature ramp of $0.77 \mathrm{~K} / \mathrm{s}$, as monitored via a platinum resistive temperature detector (RTD) mounted on the crucible surface. Luminescence intensity was measured using a Sens-Tech P25PC photomultiplier tube (PMT) interfaced to a BK 1823A frequency counter. The entire TL curve collection process is automated to be performed inside a light shielding chamber isolated in a darkened room; these measures reduce the background light intensity to under $0.3 \%$ of the maximum post-detonation PMT count rate. To provide a qualitative assessment for damage or surface contamination suffered in the explosion, the recovered particles were imaged with a JEOL 6610 scanning electron microscope (SEM).

The algorithm described in Section 6.2 was implemented in MATLAB to reconstruct the thermal history profile experienced by the TLD-100 particles. We assumed that the temperature profile experienced by the particles took the form of an instantaneous jump from ambient temperature to a maximum temperature $T_{\max }$, followed by an exponential decrease, as in simple convective cooling, back to the original ambient (Fig. 102). The time at which the temperature descends to $1{ }^{\circ} \mathrm{C}$ above the ambient was set as the cooling time $t_{\text {cooling. }}$. Comparisons with thermocouple measurements of the detonations bear this functional form out as a fairly good approximation, taking into account that the thermocouple dwarfs the microparticles in size and will respond much more slowly to rapid changes in temperature.

Under SEM imaging, the TLD-100 particles that had been exposed to explosive detonation displayed little to no significant change from the unexposed control sample (Fig. 132). A TL curve collection confirmed that the particle TL had indeed survived the explosion (Fig. 133). When normalized to the highest temperature intensity peaks, the three lowest-temperature easily distinguishable peak-like features (local inflection points) are seen to have decreased in intensity compared to the control sample. The experimental data were input to the reverse search algorithm, which was initiated with an initial $T_{\max }$ of 100 to $500{ }^{\circ} \mathrm{C}$ and $t_{\text {cooling }}$ of 0.1 to $10.1 \mathrm{~s}$. To eliminate the possibility of operator bias influencing the results, the program was executed with an intentional lack of knowledge of the thermocouple readings. The thermal heating profile converged upon, when reduced to a realistic order of precision for our experimental data, featured a maximum temperature of $240{ }^{\circ} \mathrm{C}$ and a cooling time of $0.4 \mathrm{~s}$. The simulated TLD-100 TL curve intensity resulting from this answer matches that of the experimental data to within $3 \%$ at all five comparison points (Fig. 134). This result is also compatible with the thermocouple readings of the same detonation. 


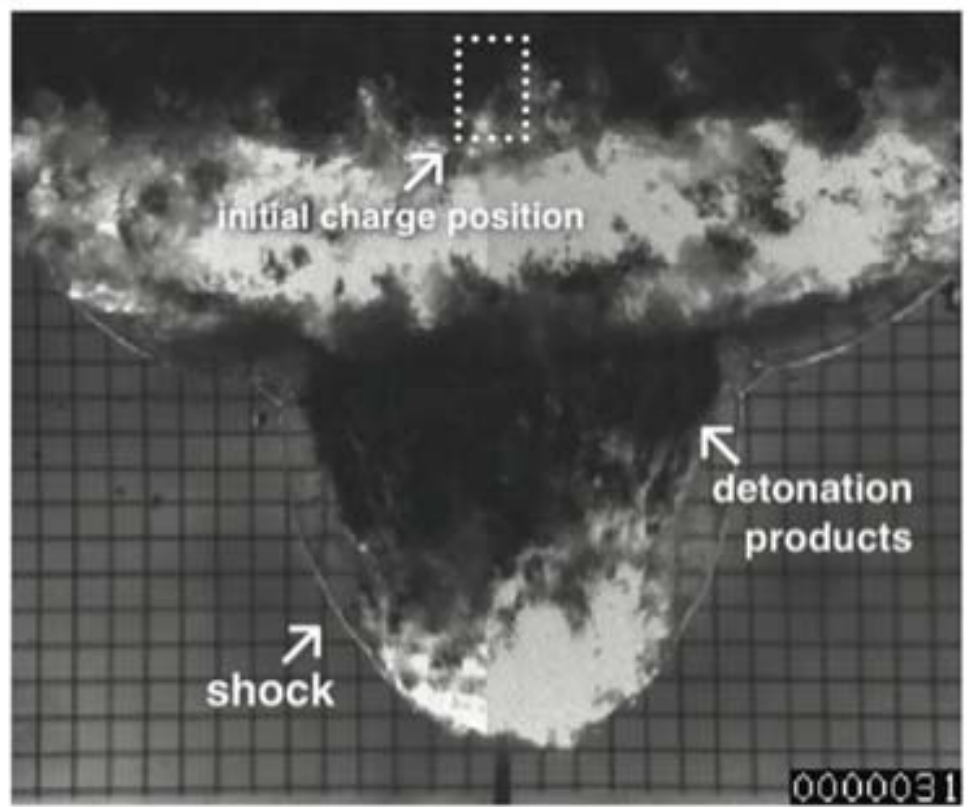

Fig. 130. High-speed photograph of the detonation of an aluminized explosive compound very similar to the one used here. The whitish areas of the fireball indicate aerobic aluminum combustion at temperatures of approximately $3000 \mathrm{~K}$
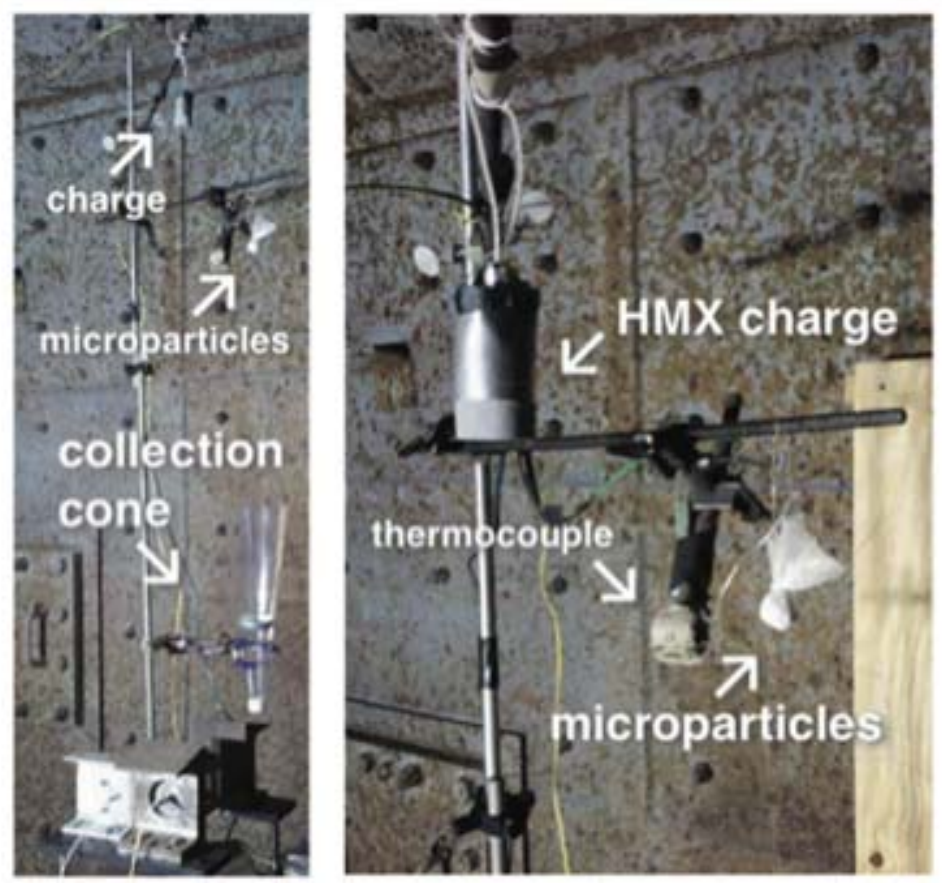

Fig. 131. Position of explosive, sample, and equipment for the detonation experiment. 


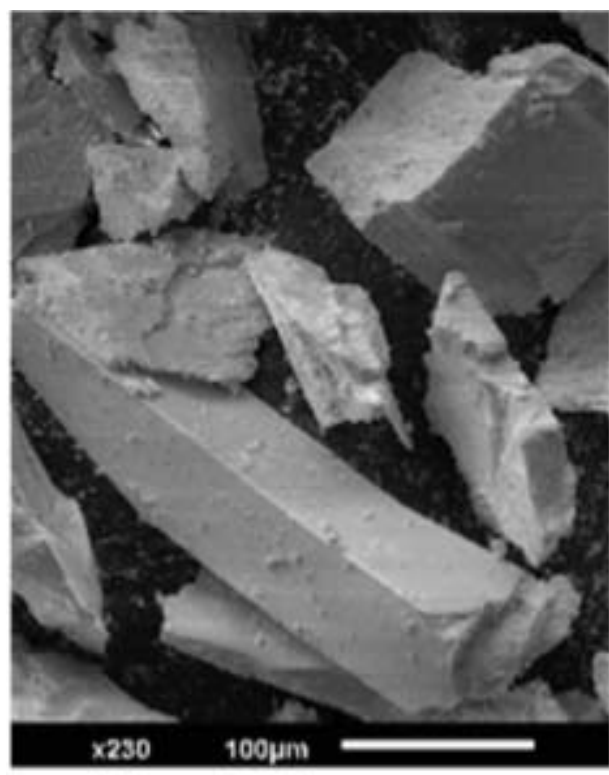

(a) Control

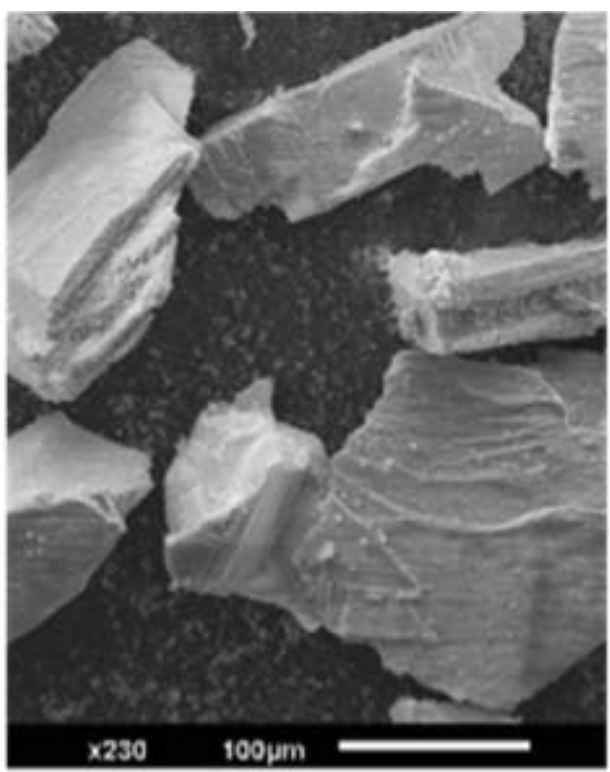

(b) Post-explosion

Fig. 132. SEM images of TLD-100 particles (a) before and (b) after exposure to high explosive detonation. Taken with an accelerating voltage of $5 \mathrm{kV}$; the dark substrate visible is carbon tape.

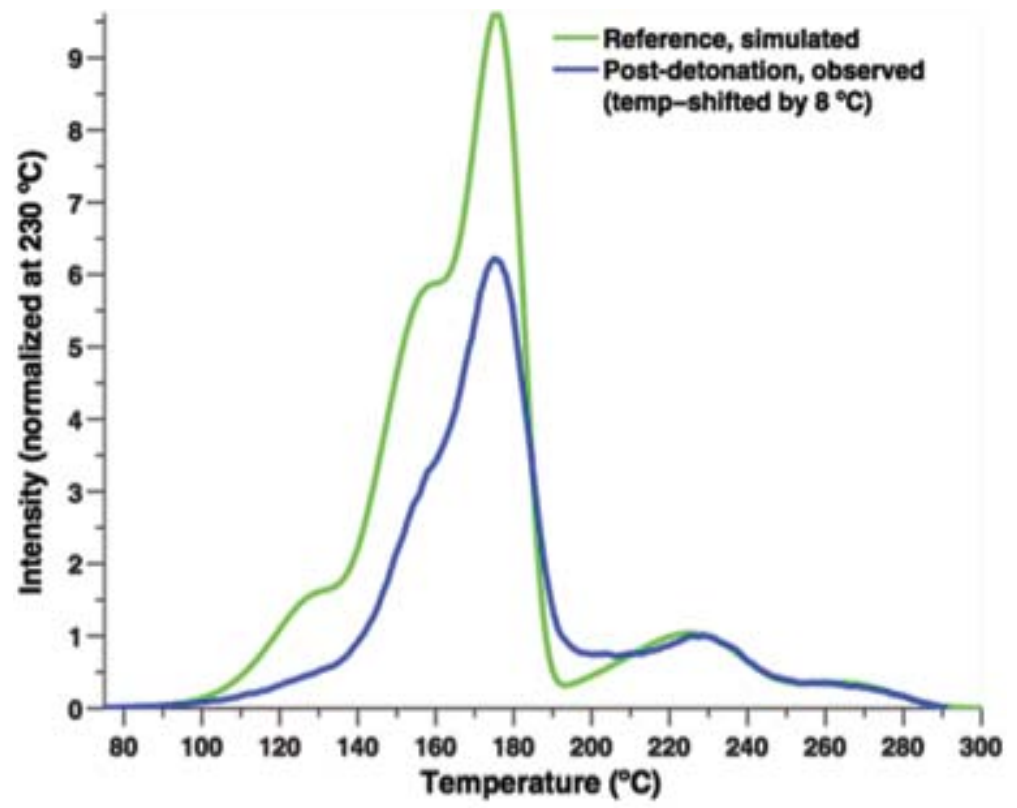

Fig. 133. TL curves of TLD-100 before and after exposure to high explosive detonation. The post-detonation curve has been shifted down in temperature by $8^{\circ} \mathrm{C}$ to better align with the control curve, a discrepancy most likely due to variations in thermal contact between the RTD, sample holder, and heating element. 


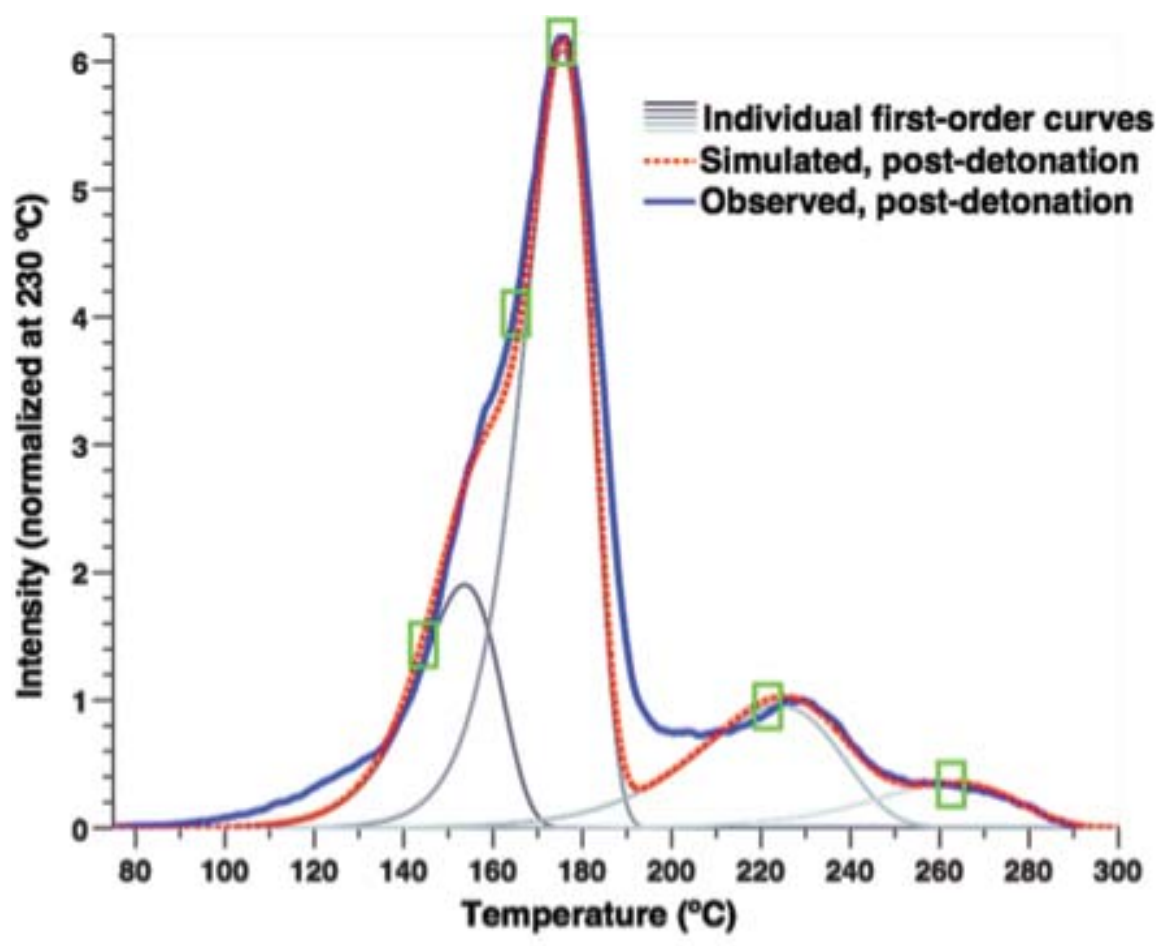

Fig. 134. The observed TL curve of post-detonation TLD-100 compared to the simulated curve of the proposed explosion temperature profile. Among the five comparison points used, observed and simulated curves differ by an average of $3 \%$.

A few other observations provided reassurance, although not confirmation, to the solution. Multiple runs of the thermal history recovery program were all in consensus, although occasionally other heating scenarios would be included; these were generally specified with less confidence and would likely be eliminated with further iterations or higher solution precision thresholds. Changes made to the initial search parameters, including slight modifications to the simulated TLD-100 trap parameters, did not prevent the program from converging upon the solution, although the time taken to do so was subject to variation. Thanks to the non-deterministic aspect introduced by the randomization of evaluation points, small fluctuations are seen in the number and exact values of the solutions produced from run to run. These were consistently within the targeted precision bounds of the simulation and minuscule compared to realistic precision limits, which are governed by our experimental TL curve collection and the manually-fitted first-order material model.

Although this brute-force search method of thermal history recovery is robustly adaptable to various materials and scenarios of increasing complexity, this comes at a price: the computational cost of such a brute-force approach scales as a power law with the number of independent temperature profile parameters. Fortunately, the individual TL curve simulations can be done completely independently of each other, implying that the computation time should scale inversely to the number of processor cores participating. Approximately $30 \mathrm{~min}$ of CPU time on an average desktop machine is already sufficient to attain an unrealistic level of precision with the current implementation, which features no parallelization or optimization except for a partially vectorized structure. Other solutions of a less computationally intensive nature are also likely to be possible. 


\subsection{NSWC (March 2012)}

\subsubsection{OSU results}

The detonation tests were carried out in the configuration shown in Fig. 135a. In each shot, two different materials were used ( $L B O$ and $M B O$ ) positioned in opposite sides of the charge.

A few grams of material were collected in each sample collector and sent back to OSU. From these, we read the surviving TL signal from several aliquots, each containing just a few grains, as shown in Fig. 135b. The readouts were carried out using the Ris $\varnothing$ TL/OSL reader. The signal from these grains was high enough to almost saturate the photomultiplier tube, which shows that the material had sufficient TL signal for the intended application.

The readouts were performed using the set of optical filters shown in Table 6, which means that we could separate the TL from the two materials, even though the samples were mixed during the detonation. So, for each sample we could measure the signal from LBO or from MBO separately.

\section{(a) Shots 2 and 4}

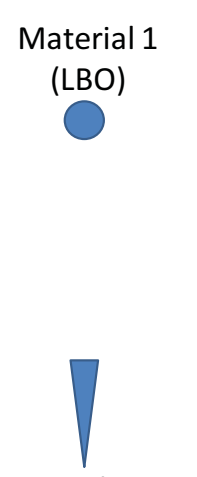

Sample 1

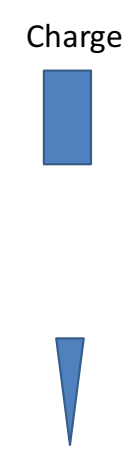

Sample 2

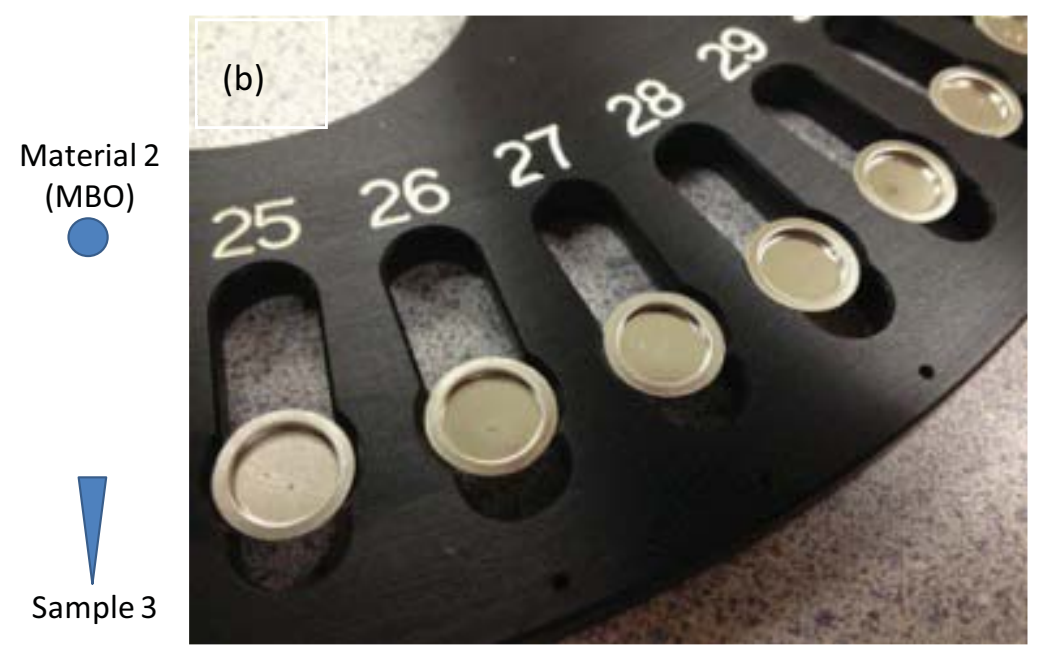

Fig. 135. (a) Configuration of the shots performed at NSWC Indian Head Division and the position of the materials; (b) few grains used for TL readout (out of several grams that were recovered).

\section{Results}

The most important results from these tests were that:

- The samples survived the experiment. Since these samples were recently developed and never tested before in actual conditions, we were not sure whether the experimental conditions (light exposure, humidity, chemicals, etc.) would affect the TL signal or not.

- The TL signal was sufficiently intense to allow us to use only a few grains in each readout (see Fig. 135b).

- The tests also showed that the TL was not affected by pressure or UV during the detonation.

Unfortunately, the samples were not subjected to sufficient heating to affect the TL curves.

Fig. 136 shows the TL from six different aliquots, each containing only a few grains of LBO. One can see that the TL is identical to the TL after laboratory irradiation, showing that the sample did not seem to 
be affected by the light exposure, humidity, chemicals present, pressure or UV in the experimental conditions of the detonation.

Fig. 137 shows similar data for MBO, where a difference in the TL peaks was observed. However, the same difference was observed in control samples (Fig. 138), which indicates that this was not caused by the temperature experienced by the particles.

Upon further investigation, we determined that this was caused by TL sensitivity changes due to repeated irradiation (i.e., accumulated dose). As the accumulated dose increases, the high TL temperature peak increases, as shown in Fig. 36b. This is a well-known phenomenon in TL [25, 34].

We found that this could be solved by either giving the sample a high dose prior to use or heating it to $600{ }^{\circ} \mathrm{C}$ during the TL readouts. The repeatability data shown in Fig. $32 \mathrm{~b}$ were obtained after we solved this problem, demonstrating the MBO samples can also be used for temperature sensing without the problems evidenced by detonation data discussed above (Fig. 137 and Fig. 138).
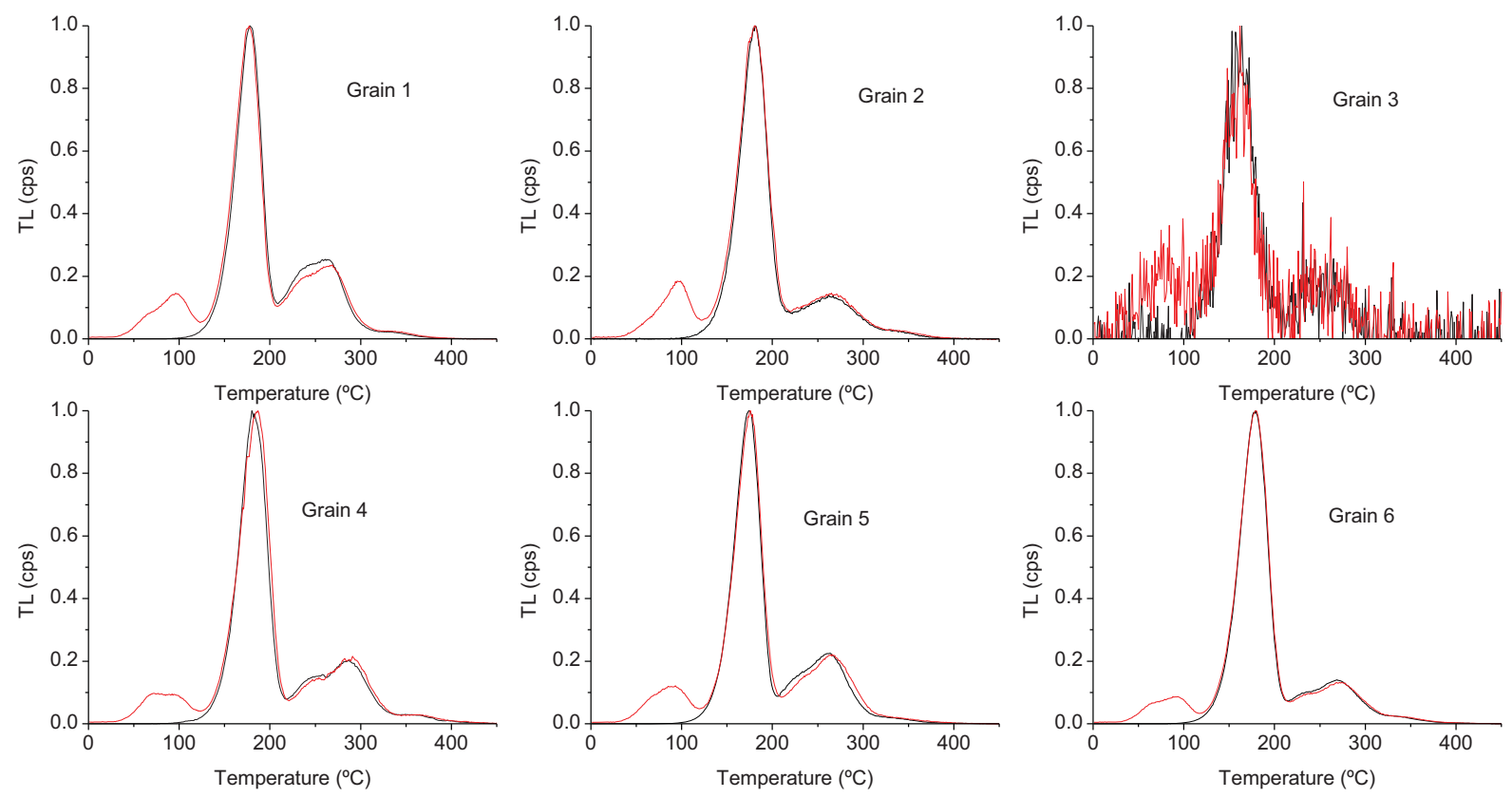

Fig. 136. Normalized TL from small number of grains of LBO after detonation (black) and after irradiation in laboratory (red). Except for the low temperature peaks that decay at room temperature, we did not observe significant differences in the TL curves. (Experimental conditions: $1^{\circ} \mathrm{C} / \mathrm{s}, 1000 \mathrm{~s}$ irradiation.) 

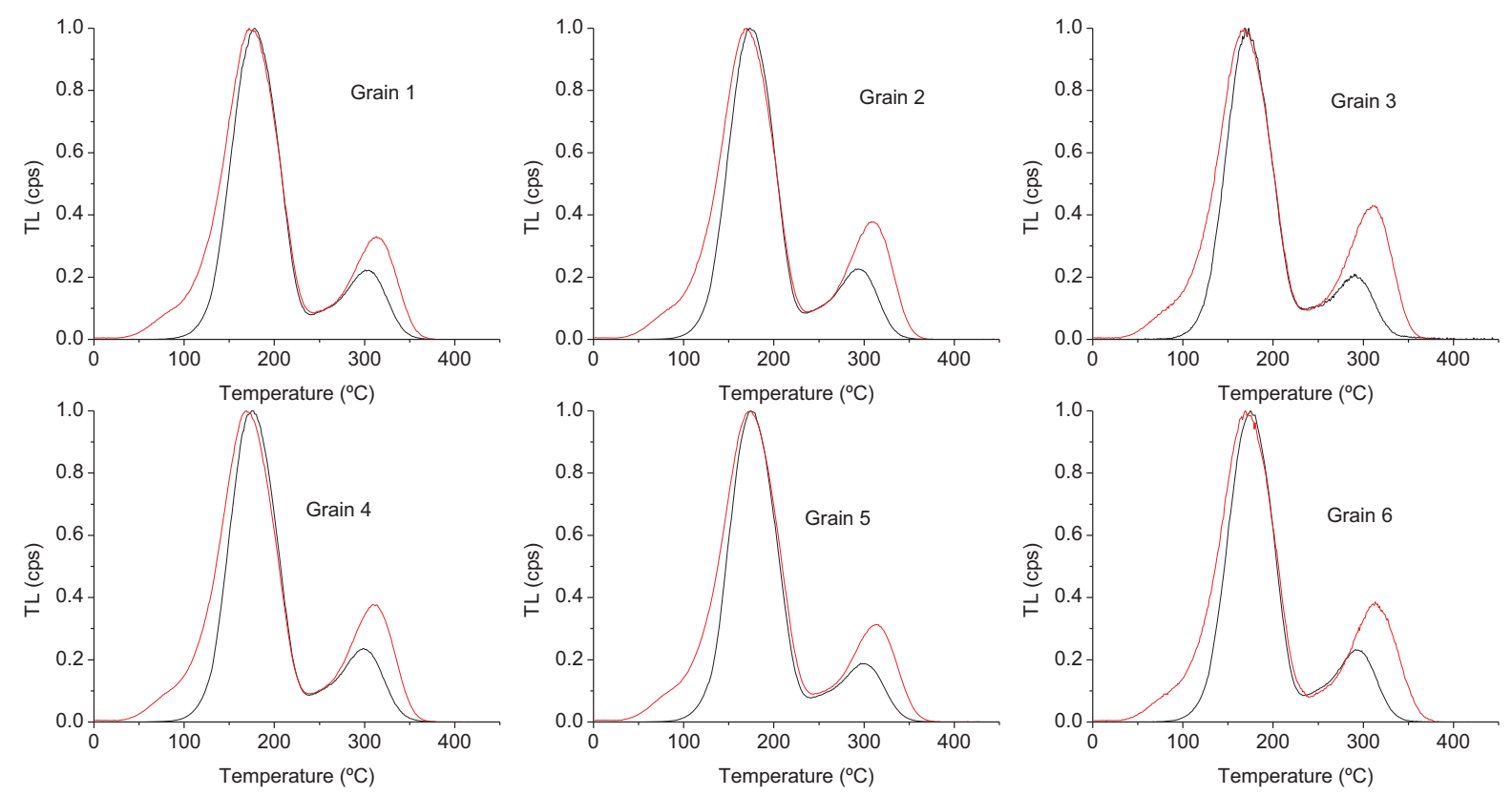

Fig. 137. Normalized TL from small number of grains of MBO after detonation (black) and after irradiation in laboratory (red). The difference observed was not due to the temperature, as similar differences were observed in control samples (see Fig. 138). This was caused by sensitivity changes that have now been solved (see text for more details). (Experimental conditions: $1^{\circ} \mathrm{C} / \mathrm{s}, 1000 \mathrm{~s}$ irradiation.)
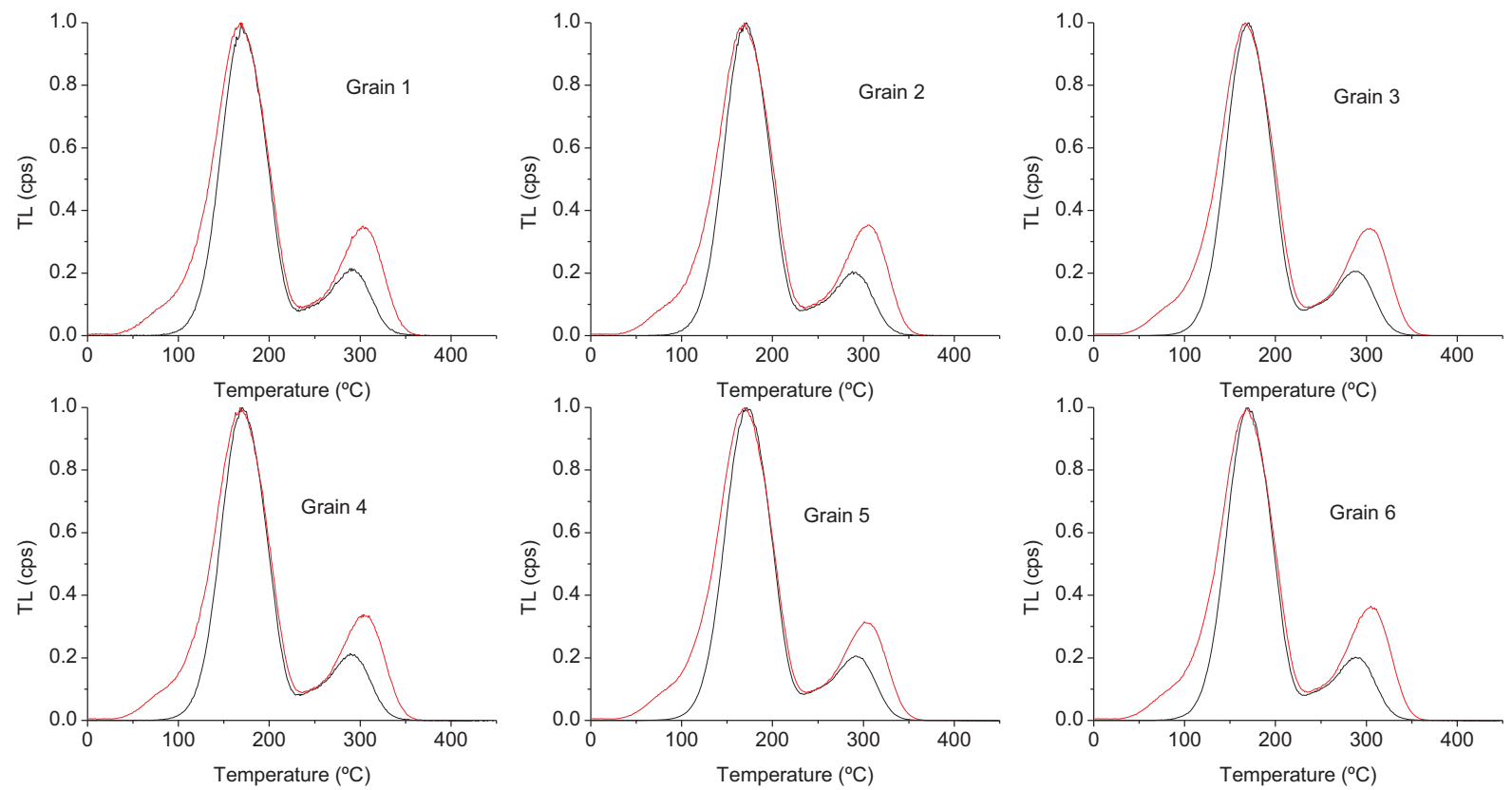

Fig. 138. Normalized TL from small number of grains of control samples of MBO as-received (black) or after irradiation in laboratory (red). The difference observed was caused by sensitivity changes that have now been solved (see text for more details). (Experimental conditions: $1^{\circ} \mathrm{C} / \mathrm{s}, 1000 \mathrm{~s}$ irradiation.) 


\subsubsection{UMN results}

We have collaborated with Dr. Jillian Horn of Indian Head Division, Naval Surface Warfare Center, to conduct tests of TL particles in detonations of high explosives. Following on previous successful tests with Dr. James M. Lightstone of Indian Head Division, whose three detonation samples yielded reconstructed temperature profiles which closely matched the luminescence signatures and agreed with thermocouple measurements, this new round of tests attempted to expand on both the range of detonations demonstrated and the materials used.

The common TL radiation dosimeter LiF:Mg,Ti, commercially known as TLD-100, is insensitive to photobleaching, well represented by first-order kinetics, and has been previously shown to retain $\mathrm{TL}$ properties through close proximity to high-explosive detonations.

To expand the range of temperatures precisely measurable by our technique, we added $\mathrm{CaF}_{2}: \mathrm{Dy}$, known as the dosimeter TLD-200. It has an order of magnitude more sensitivity to radiation dose than TLD-100, allowing it to produce higher intensities (although sensitivity is not a major factor in these detonation tests, where sufficient signal is expected with most TL materials. Far more important are the other luminescent peaks of $\mathrm{CaF}_{2}$ :Dy). Both materials were purchased from Thermo Fisher Scientific as sieved particles $75-176 \mu \mathrm{m}$ in size and irradiated with 200 or $300 \mathrm{~Gy}$ of gamma radiation from a ${ }^{167} \mathrm{Cs}$ source.

Post-detonation samples were collected by bins placed below the explosive charge, and the TL curves were collected from individual particles via microheater (Fig. 139). Unfortunately, the majority of samples measured showed insufficient depopulation to attribute to the explosive detonation. We believe that the amounts of material provided for each test was far too large. From the video provided by Indian Head (Fig. 140), this may have resulted in the bulk of the particles being propelled away from the explosion by the shock front before they had a chance to experience significant heating.

However, several samples did indicate trap depopulation of a sufficient magnitude to warrant examination (Fig. 141). For modeling to extract the thermal history from the TL curves, we used five monitoring points in the TL curve, which correspond roughly to the individual first-order peaks in our material TL curve model. These points were examined before (using a control sample) and after the explosion. There are several plausible regions, which neighbor each other but do not overlap. There were more than a dozen different shot samples, but to save space, only one representative example is shown here (Fig. 142). 

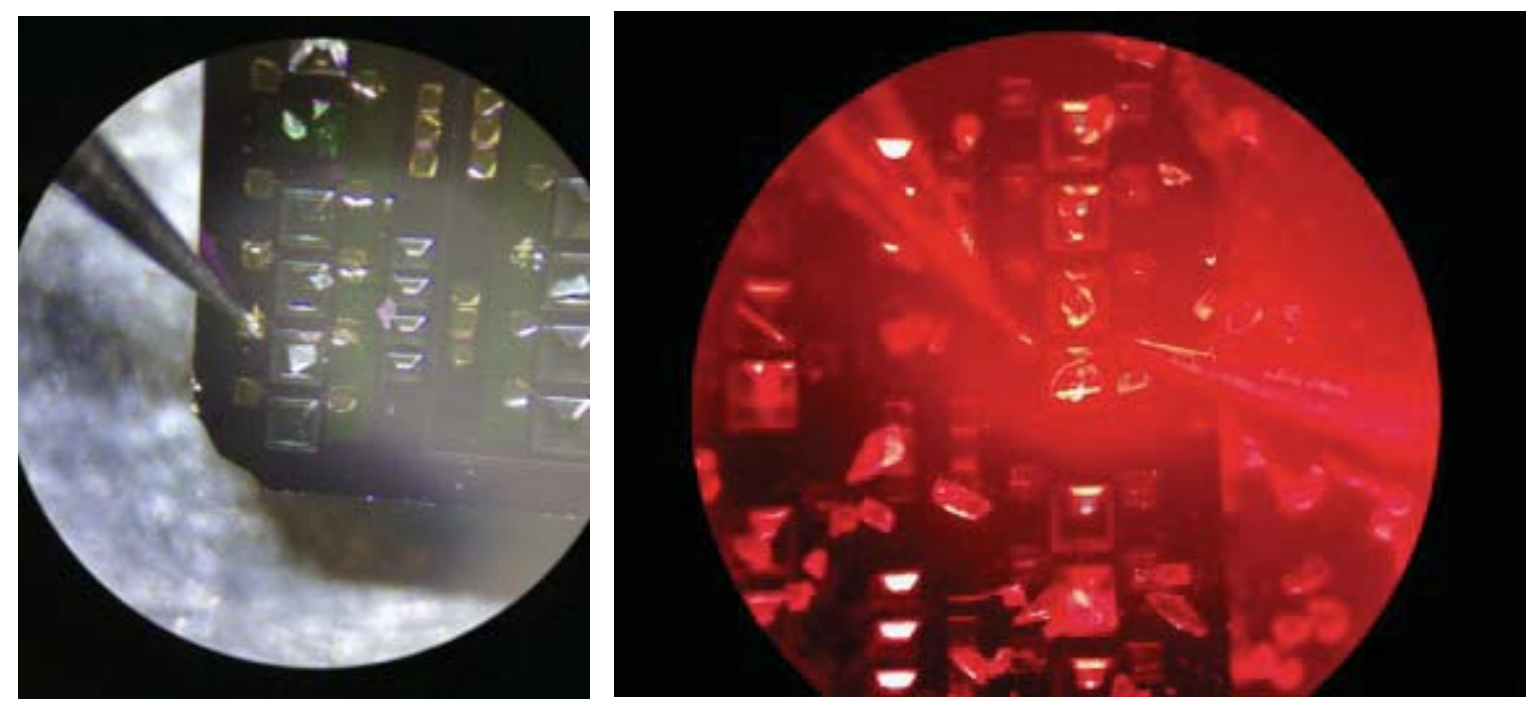

Fig. 139. Optical microscope images of returned post-detonation particles atop microheaters.

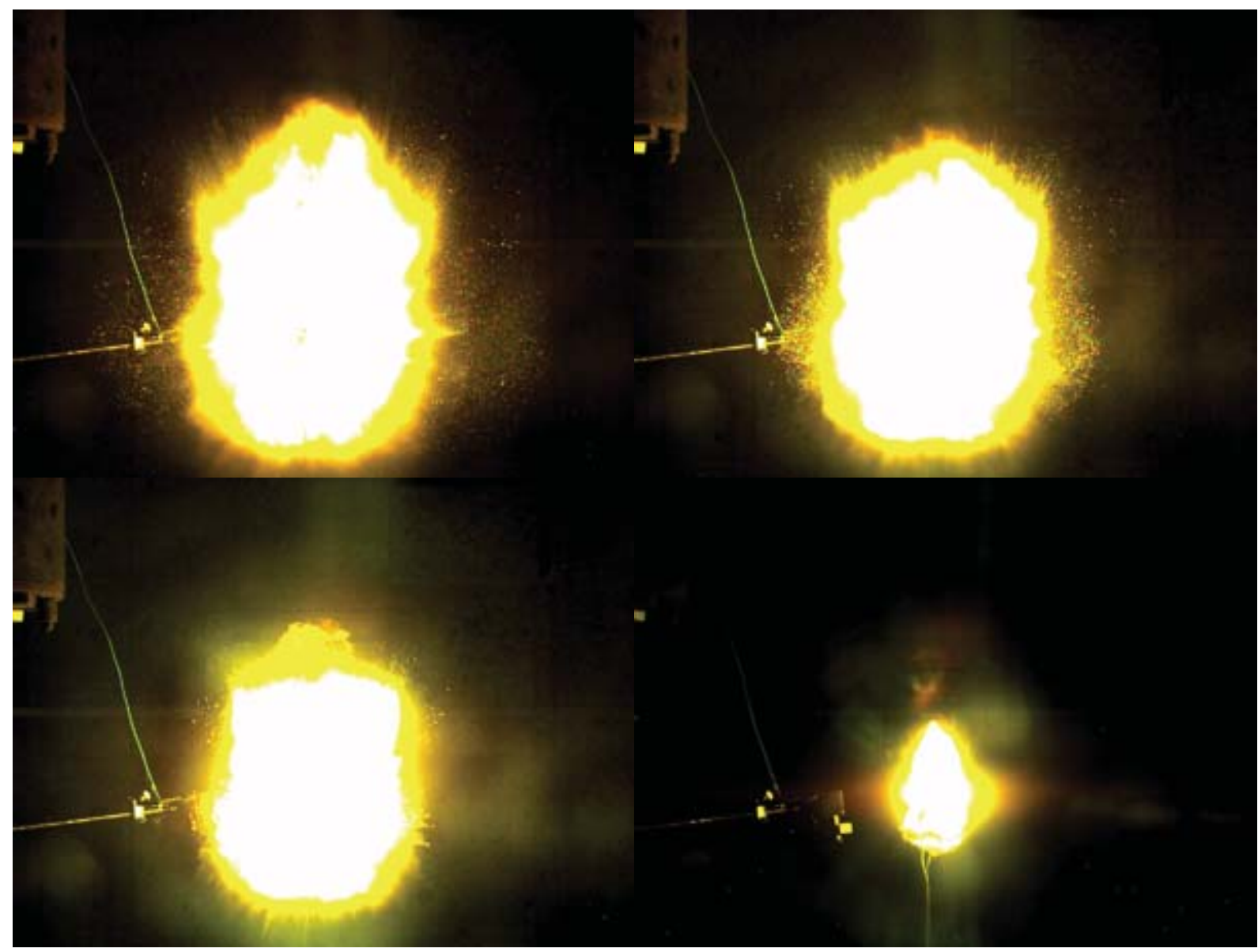

Fig. 140. Four frames taken from a high-speed video of one high explosive detonation test. The video was recorded at 22,006 frames per second. The particles seen near, and flying outwards from, the periphery of the fireball are likely particles or clumps of TL powder samples. Video courtesy Jillian Horn, Naval Surface Warfare Center, Indian Head Division. 


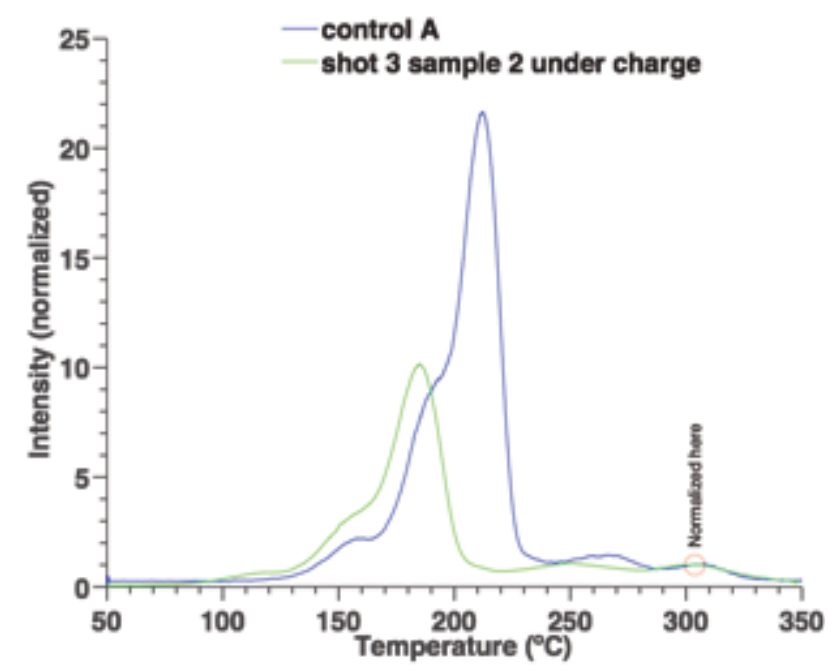

Fig. 141. Data for "shot 3 sample 2 under charge" compared to a TLD-100 control sample, showing significant depopulation. (The cause for the curve's shift in temperature is unknown, but is assumed to be due to differences in thermal contact with the microheater for the purposes of temperature recovery.)
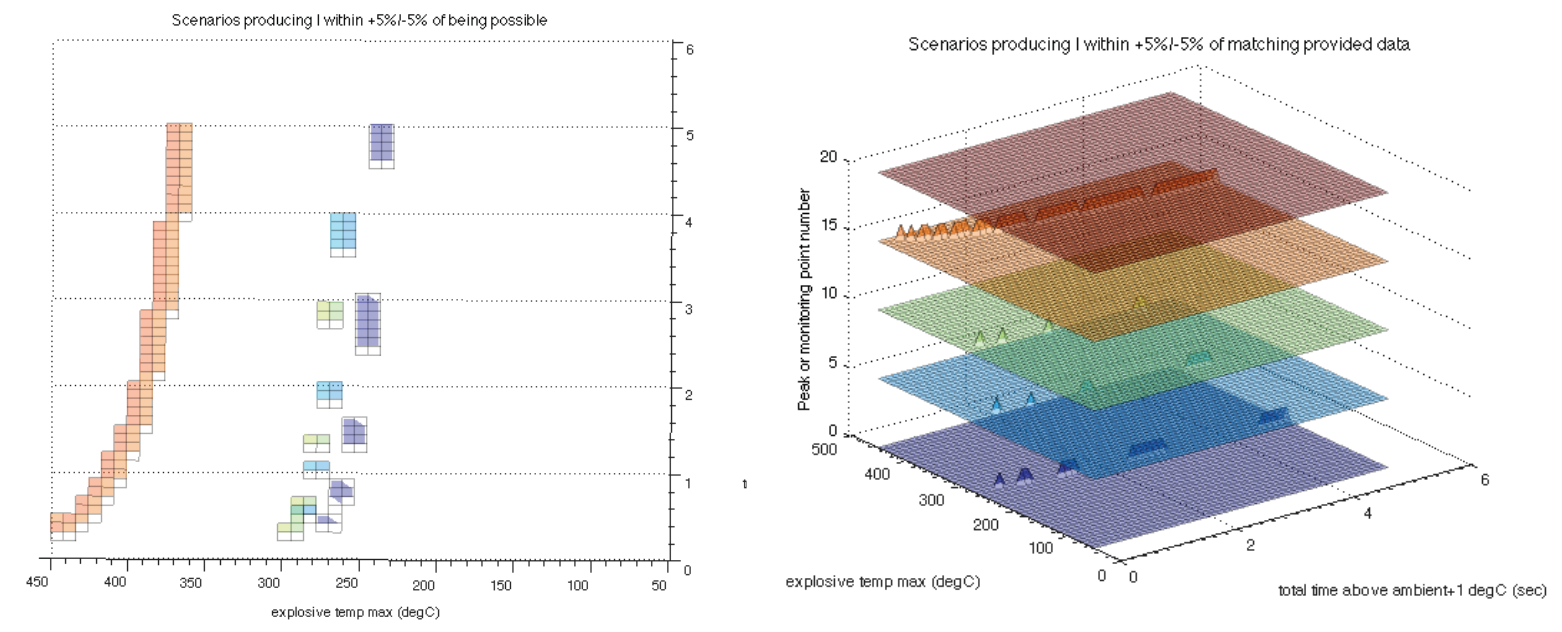

Fig. 142. Two views of the results of a temperature reconstruction simulation designed to allow dissenting peaks to produce a most likely result. The intensity of the experimental TL curve is sampled at several temperatures, chosen to correspond to the locations of the individual first-order TL curves comprising the combined curve; the modeled TL material is then subjected to simulated explosive heating to determine the range of heating parameters which result, at each monitoring point, in an intensity within a certain percent of that experimentally observed. This particular example for "shot 3 sample 2 under charge" shows that three monitoring points come very close to overlap in a certain $T_{\max } / t_{\text {cool }}$ range, but the fourth point insists on a higher temperature range. (Five monitoring points were used in this example, but the fifth is used for intensity normalization and thus cannot be compared.)

\section{Monitor samples}

TLD-100 is a light-insensitive material, well suited to thermal history sensing. However, TLD-200 is known to be vulnerable to photobleaching. To see what kind of effects photobleaching would have on data analysis, TLD-200 was included as one of the testing materials. To measure this potential source of error, we used separate powder samples, sealed in clear plastic vials, which were handled simultaneously with the detonation powder samples and thus should have been exposed to all incidental heat and light stimuli encountered during handling (Fig. 143). (Note that the actual detonation is, of course, excepted.) These undetonated parcels were dubbed "monitor" samples to 
differentiate them from our control batches, which were irradiated alongside but kept in our laboratory in light-tight storage instead of being shipped to Indian Head Division.

Although many of the samples showed no significant change in TL after detonation, they do illustrate the utility of the enhanced control accuracy provided by the monitor samples. In several cases, the post-detonation sample showed depopulation - or other change, if not a change attributable to our heating profile - when compared to the control sample, but was virtually identical to the monitor sample, saving us much consternation (Fig. 144).

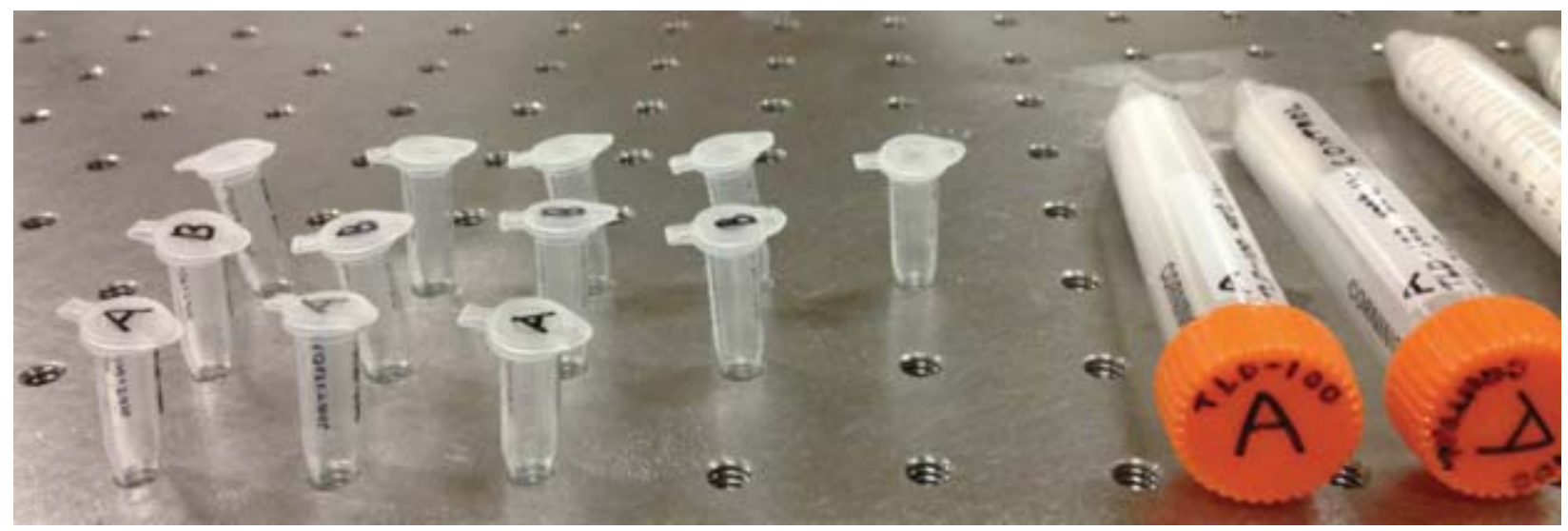

Fig. 143. The clear plastic vials housing monitor samples (on the left) next to the shipping vials of particles for detonation. Very small amounts of powder are required to provide plentiful single-particle readouts with microheaters, so the monitor samples in the tips of the vials are not visible in this photo. The monitor sample vials are not unsealed until they return for readout.
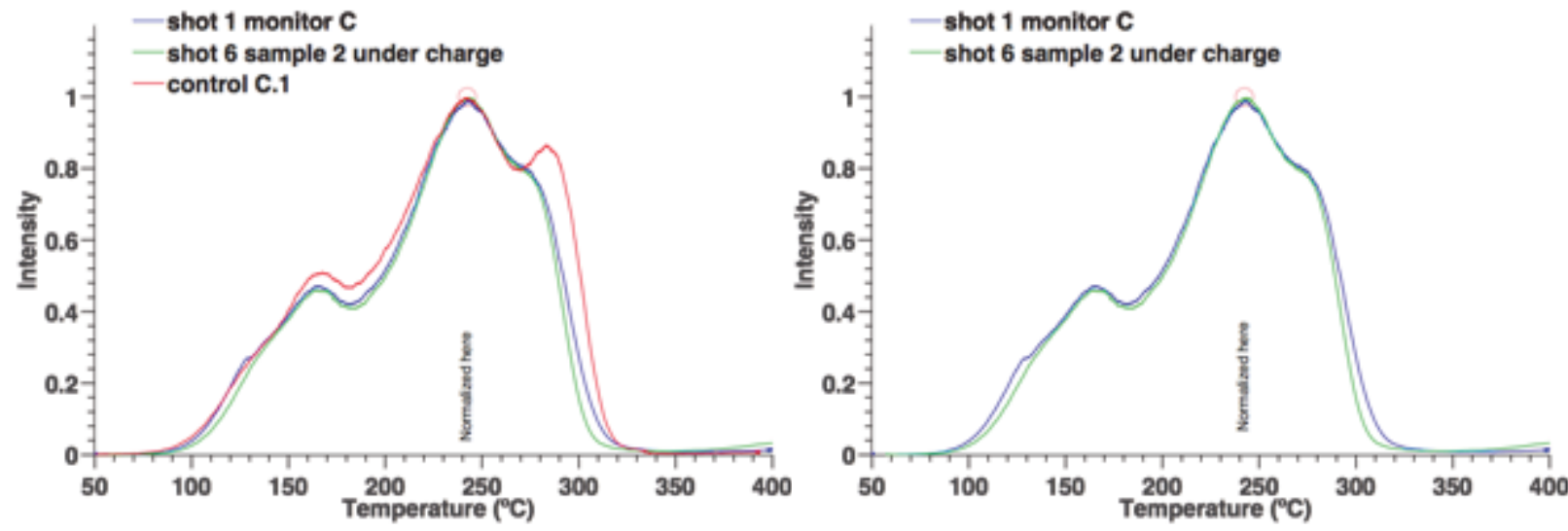

Fig. 144. The TL of post-detonation sample "shot 6 sample 2 under charge" appears nearly identical to the monitor sample "shot 1 monitor C", which comprises the same source material. (No monitor sample was available for shot 6.) The postdetonation and monitor samples appear markedly different from the unhandled "control C.1" sample, with differences including a high-temperature peak absent in both non-controls. This result outlines the usefulness of the monitor sample as a yardstick of incidental depopulation.

\subsection{NSWC (September 2012)}

Three materials developed at OSU $\left(\mathrm{Li}_{2} \mathrm{~B}_{4} \mathrm{O}_{7}: \mathrm{Ag}, \mathrm{Cu}, \mathrm{MgB}_{4} \mathrm{O}_{7}: \mathrm{Dy}\right.$, $\mathrm{Li}$ and $\left.\mathrm{CaSO}_{4}: \mathrm{Ce}, \mathrm{Tb}\right)$ were tested at NSWC in a total of six "shots" of different conditions. The results are detailed in a report presented to the DTRA and the NSWC. An overview of the results for each shot is shown in Table 18. 
Table 18. Overview of NSWC-IHD test results (Sep. 2012).

\begin{tabular}{|c|c|c|c|c|}
\hline & $\begin{array}{l}\text { Material } \\
\text { recovered }^{(a)}\end{array}$ & $\begin{array}{l}\text { TL } \\
\text { surviving } \\
\text { (\%) }\end{array}$ & $\begin{array}{l}\text { Temperature } \\
\text { estimated }\end{array}$ & Observations \\
\hline $\begin{array}{l}\text { Shot } 5 \\
\text { (LBO) }\end{array}$ & $0 \%$ & $7.9 \pm 15$ & N.A. & Not enough material was recovered. \\
\hline $\begin{array}{l}\text { Shot } 5 \\
\text { (MBO) }\end{array}$ & $0 \%$ & 0 & N.A. & Idem \\
\hline $\begin{array}{l}\text { Shot 5a } \\
\text { (LBO) }\end{array}$ & $47 \%$ & $1.4 \pm 2.4$ & $\begin{array}{l}>98 \% \text { heated } \\
\text { above capability }\end{array}$ & $\begin{array}{l}\text { A lot of material was recovered, but } \\
\text { almost no TL was observed, except for a } \\
\text { few grains. }\end{array}$ \\
\hline $\begin{array}{l}\text { Shot 5a } \\
\text { (MBO) }\end{array}$ & $27 \%$ & $0.1 \pm 0.4$ & $\begin{array}{l}>99 \% \text { heated } \\
\text { above capability }\end{array}$ & Idem \\
\hline $\begin{array}{l}\text { Shot } 6 \\
\text { (CSO) }\end{array}$ & $0 \%$ & Meaningless & N.A. & Not enough material was recovered. \\
\hline $\begin{array}{l}\text { Shot } 11 \\
\text { (LBO) }\end{array}$ & $0.6 \%$ & $0.9 \pm 1.2$ & $\begin{array}{l}>99 \% \text { heated } \\
\text { above capability }\end{array}$ & $\begin{array}{l}\text { Less than } 1 \% \text { of material was recovered, } \\
\text { but almost no TL was observed, except } \\
\text { for a few grains. }\end{array}$ \\
\hline $\begin{array}{l}\text { Shot } 11 \\
\text { (MBO) }\end{array}$ & $0.9 \%$ & 0 & $\begin{array}{l}100 \% \text { heated } \\
\text { above capability }\end{array}$ & $\begin{array}{l}\text { Less than } 1 \% \text { of material was recovered, } \\
\text { but no TL was observed. }\end{array}$ \\
\hline $\begin{array}{l}\text { Shot 11a } \\
\text { (LBO) }\end{array}$ & $58 \%$ & $5.9 \pm 0.2$ & $\begin{array}{l}477 \mathrm{~K}, 1.9 \mathrm{~h} \\
\text { (constant) }\end{array}$ & $\begin{array}{l}\text { A lot of material was recovered. Data } \\
\text { were analyzed using "E distribution TL } \\
\text { model" and "E-s distribution TL model". }\end{array}$ \\
\hline $\begin{array}{l}\text { Shot 11a } \\
\text { (MBO) }\end{array}$ & $22 \%$ & $13.3 \pm 1.4$ & $\begin{array}{l}480 \mathrm{~K}, \sim 2 \mathrm{~h} \text { (high } \\
\text { FOM) } \\
660 \mathrm{~K}, \sim 0.03 \mathrm{~s} \\
\text { (low FOM) }\end{array}$ & $\begin{array}{l}\text { A lot of material was recovered. Data } \\
\text { were analyzed using "E distribution TL } \\
\text { model" and "E-s distribution TL model". }\end{array}$ \\
\hline $\begin{array}{l}\text { Shot } 12 \\
\text { (CSO) }\end{array}$ & $0.5 \%$ & 0 & $\begin{array}{l}100 \% \text { heated } \\
\text { above capability }\end{array}$ & $\begin{array}{l}\text { Less than } 1 \% \text { of material was recovered, } \\
\text { but no TL was observed. }\end{array}$ \\
\hline
\end{tabular}

(a) Indicates amount of material recovered based on regenerated TL and confirmed by XRD.

(b) Indicates the fraction of TL observed in the sample divided by the total TL regenerated.

(c) Based on square heating pulse.

The main conclusions from these tests were:

a) The amount of material recovered varied from shot to shot. No material was recovered from shots 5 and 6 , less then $<1 \%$ was recovered from shots 11 and 12 , and $\sim 50 \%$ of material was recovered from shots 5 a and 11 a. Except for shots 5 and 6 , the other shots provided sufficient material for temperature determination.

b) Shots $5 a, 11$ and 12 were heated above the material capabilities. For LBO and MBO, the upper limit for a $0.1 \mathrm{~s}$ heating pulse was $\sim 700 \mathrm{~K}$. (We estimate that, for temperature below that, TL would have been observed.)

c) For shots 11a (LBO and MBO), we were able to extract temperatures using two models: a simple "E distribution TL model" (TL model with distribution of $E$ values and constant $s$ value) and a more sophisticated "E-s distribution TL model" (TL model with a distribution of both $E$ an 
$s$ values.) The temperature obtained was $\sim 480 \mathrm{~K}$ for $2 \mathrm{~h}$, which is consistent with report from the NSWC.

d) For the "E distribution TL model", temperature can be obtained if the heating period is estimated. For the "E-s distribution TL model", time and temperature could be determined, although the model needs refinement.

e) In shots in which most of the material was heated above the capabilities (e.g. shot 11), some grains showed TL consistent with no heating, indicating either incomplete heating during detonation or cross-contamination of the material during the experiments (i.e., irradiated grains ended up in heated material).

\subsection{NSWC 2013 tests}

The performance of different TL materials was tested as particle temperature sensors using the closed temperature detonation chamber at the Indian Head Explosive Ordnance Disposal Technology Division (NSWC-IHEODTD). TL materials tested included three developed at OSU, $\mathrm{Li}_{2} \mathrm{~B}_{4} \mathrm{O}_{7}: \mathrm{Cu}, \mathrm{Ag}$ (LBO), $\mathrm{MgB}_{4} \mathrm{O}_{7}: \mathrm{Dy}, \mathrm{Li}(\mathrm{MBO})$, and $\mathrm{CaSO}_{4}: \mathrm{Ce}, \mathrm{Tb}(\mathrm{CSO})$, and one commercial material used by UMN, LiF:Mg, $\mathrm{Ti}$ (TLD-100).

A total of 15 shots were performed at NSWC-IHEODTD using either a single or combination of different TL materials, and two different explosives: a high temperature plastic bonded explosive (HPBX) and a low temperature plastic bonded explosive (LPBX). The samples collected were returned to OSU and UMN for TL readout and analysis. TL measurements were performed at OSU and analyzed according to procedures tested previously in laboratory.

Complete reports on these experiments were presented by both OSU and UMN groups. Here we summarize the main findings.

\subsubsection{OSU results}

Fig. 145 shows examples of TL curves obtained for the various shorts for the materials produced at OSU: LBO, MBO and CSO. The data show clear modifications in the TL curves as a function of temperature exposure during the detonations.

These curves were analyzed using a method similar to the one used in previous laboratory tests and described elsewhere [10]. Based on these analyses, we obtained the data presented in Table 19.

LBO and MBO materials were able to measure temperatures for LPBX charges, whereas CSO was able to measure temperatures for both LPBX and HPBX charges. For LPBX charges, the temperatures determined were $\sim 550-670 \mathrm{~K}$, depending on the shot (excluding shot 7 , which used a smaller charge and resulted in heating only to $\sim 470 \mathrm{~K}$ ). For HPBX charges, the temperatures determined were $\sim 700-$ $780 \mathrm{~K}$, again depending on the shot.

The temperatures obtained were self-consistent and typically higher than the maximum thermocouple temperatures reported by NSWC-IHEODTD group, as expected. The analysis procedure was relatively robust, not strongly influenced by variations in the TL model, sample, shift of the TL curve in temperature, and timescale of the heating event. This indicates that TL may represent a reliable way of estimating the lowest temperature experienced by the particles.

Updated results have been published and can be found in Ref. [11]. 

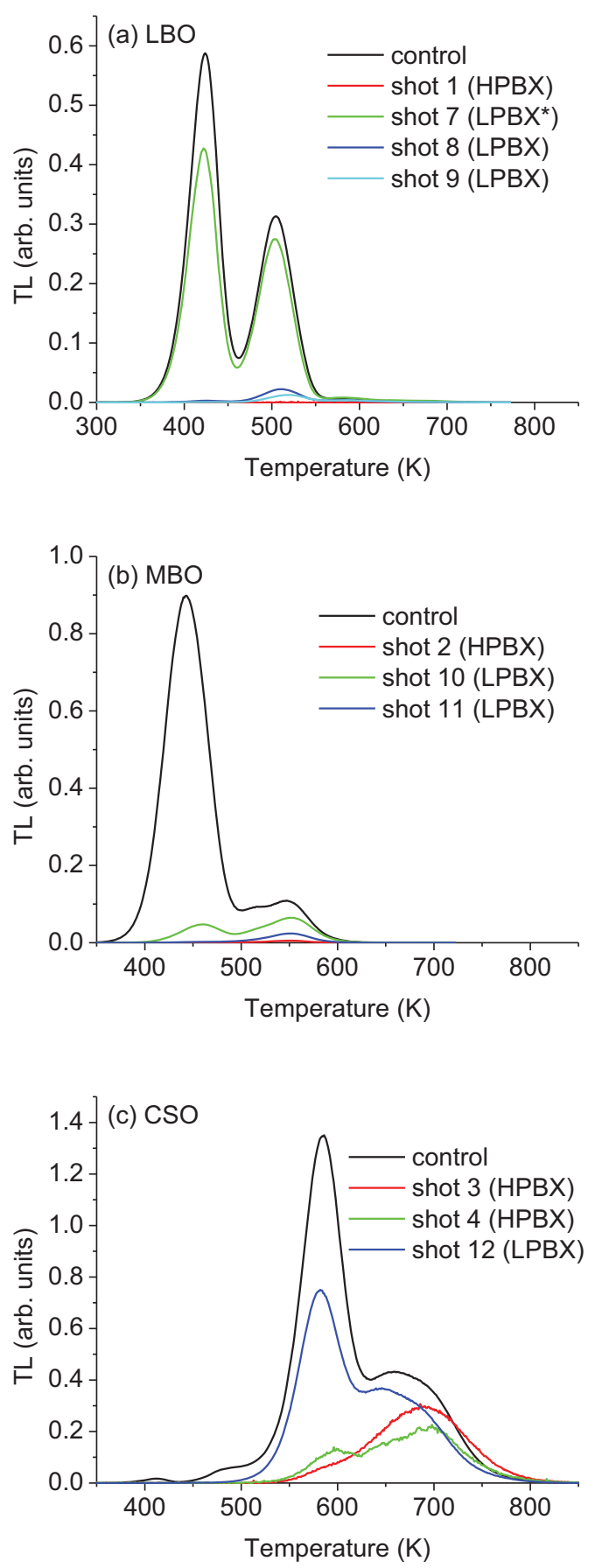

Fig. 145. TL curves obtained for the different shots. The curves are the average of five samples after normalization to the maximum TL of the regenerated curve. The * in shot 7 is to indicate that this shot was carried out with a smaller amount of explosive than shot 8 and 9.

Table 19. TL results assuming uniform heating by a pulse of $0.1 \mathrm{~s}$ of duration. The uncertainties are the experimental standard deviation of the data based on five TL curves obtained for each shot, therefore representing only the sample to sample variation.

\section{Shot Charge Material Temperature Residual TL Observations}




\begin{tabular}{|c|c|c|c|c|c|}
\hline & & & (K) & & \\
\hline 1 & HPBX & LBO & NA & $0 \%$ & Beyond material range \\
\hline 2 & HPBX & $\mathrm{MBO}$ & NA & $0 \%$ & Beyond material range \\
\hline 3 & HPBX & CSO & $723 \pm 12$ & $24 \%$ & \\
\hline 3 & HPBX & CSO sieved & $732 \pm 27$ & $15 \%$ & \\
\hline 4 & HPBX & CSO & $703 \pm 13$ & $20 \%$ & \\
\hline 4 & HPBX & CSO sieved & $717 \pm 21$ & $14 \%$ & \\
\hline 6 & HPBX & Mixed $(\mathrm{CSO})^{(\mathrm{a})}$ & $782 \pm 31$ & - & Contamination from LiF \\
\hline 7 & $\mathrm{LPBX}^{*}$ & LBO & $468 \pm 2$ & - & Contamination \\
\hline 8 & LPBX & $\operatorname{LBO}(53-75 \mu \mathrm{m})$ & $618 \pm 5$ & $4 \%$ & Evidence of partial heating \\
\hline 9 & LPBX & LBO $(<38 \mu \mathrm{m})$ & $626 \pm 1$ & $2 \%$ & Evidence of partial heating \\
\hline 10 & LPBX & $\mathrm{MBO}$ & $548 \pm 4$ & $12 \%$ & Evidence of partial heating \\
\hline 11 & LPBX & MBO & $607 \pm 4$ & $3 \%$ & Evidence of partial heating \\
\hline 12 & LPBX & $\mathrm{CSO}$ & $667 \pm 8$ & $59 \%$ & \\
\hline 13 & LPBX & Mixed $(\mathrm{CSO})^{(\mathrm{a})}$ & $663 \pm 5$ & $66 \%$ & \\
\hline
\end{tabular}

* lower explosive mass

(a) Read using Hoya U-340 filter to select CSO emission.

\subsubsection{UMN results}

In this section we discuss the results using LiF:Mg,Ti particles.

We once again prepared TL samples for testing by our collaborators at NSWC Indian Head Division, this time applying batch divisions by particle size. TL dosimetry material TLD-100 (LiF:Mg,Ti) from Thermo Scientific were filtered, with nylon CellMicroSieve mesh filters and IPA as a carrier liquid, into four size combinations: $<35 \mu \mathrm{m}, 35-80 \mu \mathrm{m},<80 \mu \mathrm{m}$, and 80-200 $\mu \mathrm{m}$. The samples were then dried with mild heat, given a standard sensitization anneal of $400{ }^{\circ} \mathrm{C}$ for 1 hour in air, and X-ray irradiated to $200 \mathrm{~Gy}$ before being detonated in three shots as determined by Indian Head personnel. Returned samples (e.g. Fig. 146) were sent to both UMN and OSU and read in both facilities to provide corroboration for temperature values.

Interestingly, the post-detonation material and debris produced varying TL strength from read sampling to sampling. "Shot 14 LPBX" and "Shot 15 LPBX" displayed two to three discernible peaks (Fig. 147). Unfortunately, shifts in the peak temperatures and unexpected changes in the TL curve shapes meant that agreement with the simulated models was off; the closest a standardized procedure (one that refrains from tuning the algorithm for each specific sample) could come were $49.1 \%$ and $65.4 \%$, respectively. This amount of agreement usually embodied two simulated peaks having their heights matched closely with their actual counterparts and a third peak significantly stronger or weaker than actual. "Shot 5 HPBX" proved the most depleted sample, with a maximum of two peaks appearing, and then only sporadically. 

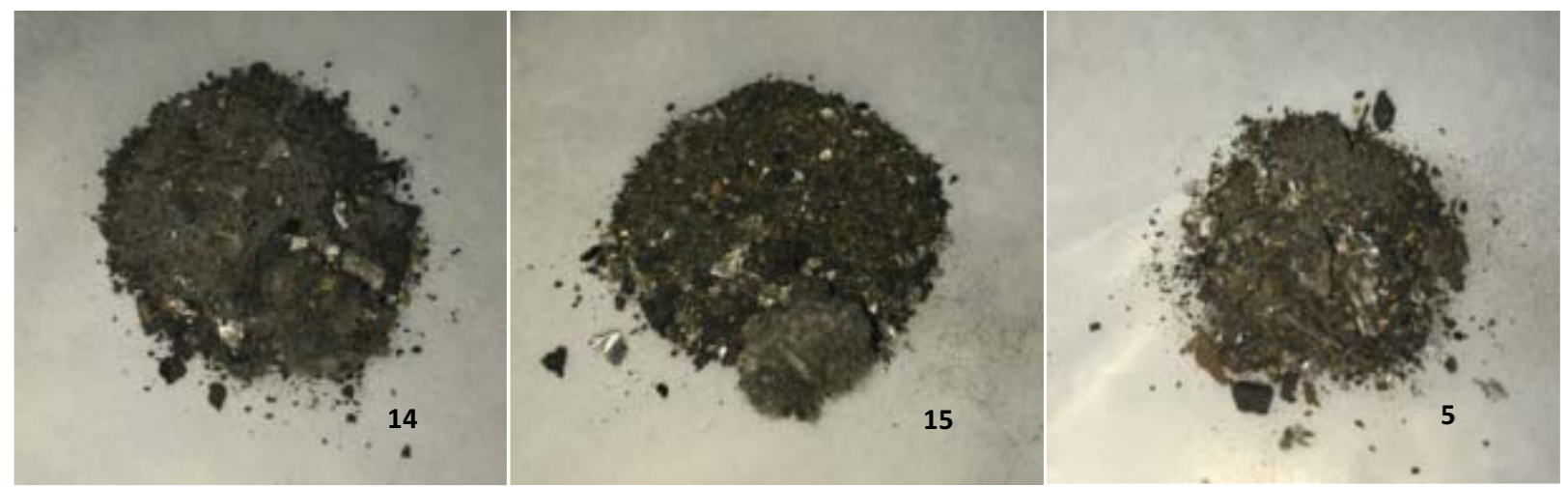

Fig. 146. Photographs of the post-detonation TLD-100 and debris returned from Indian Head. From left to right: "shot 14 LPBX", "shot 15 LPBX", and "shot 5 HPBX".

(a) Shot 14
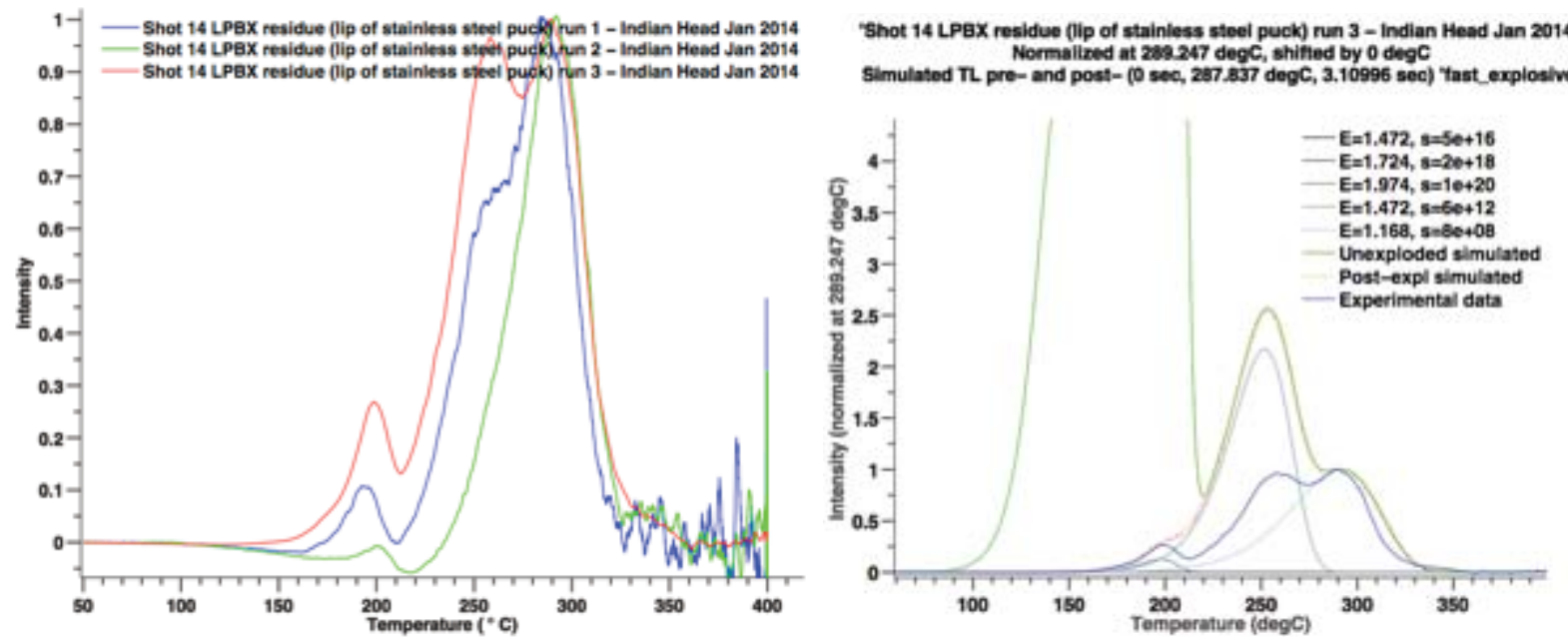

(b) Shot 15
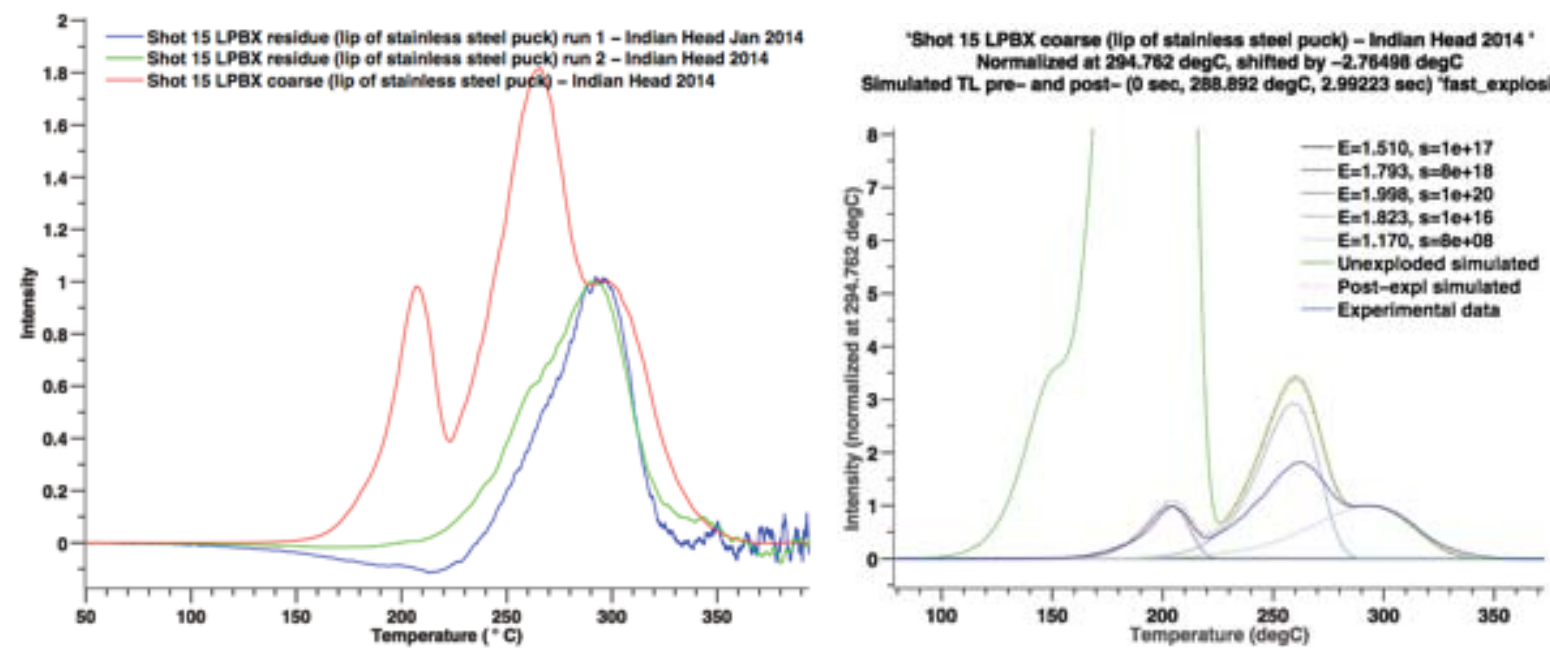

(c) Shot 5 (data courtesy OSU) 

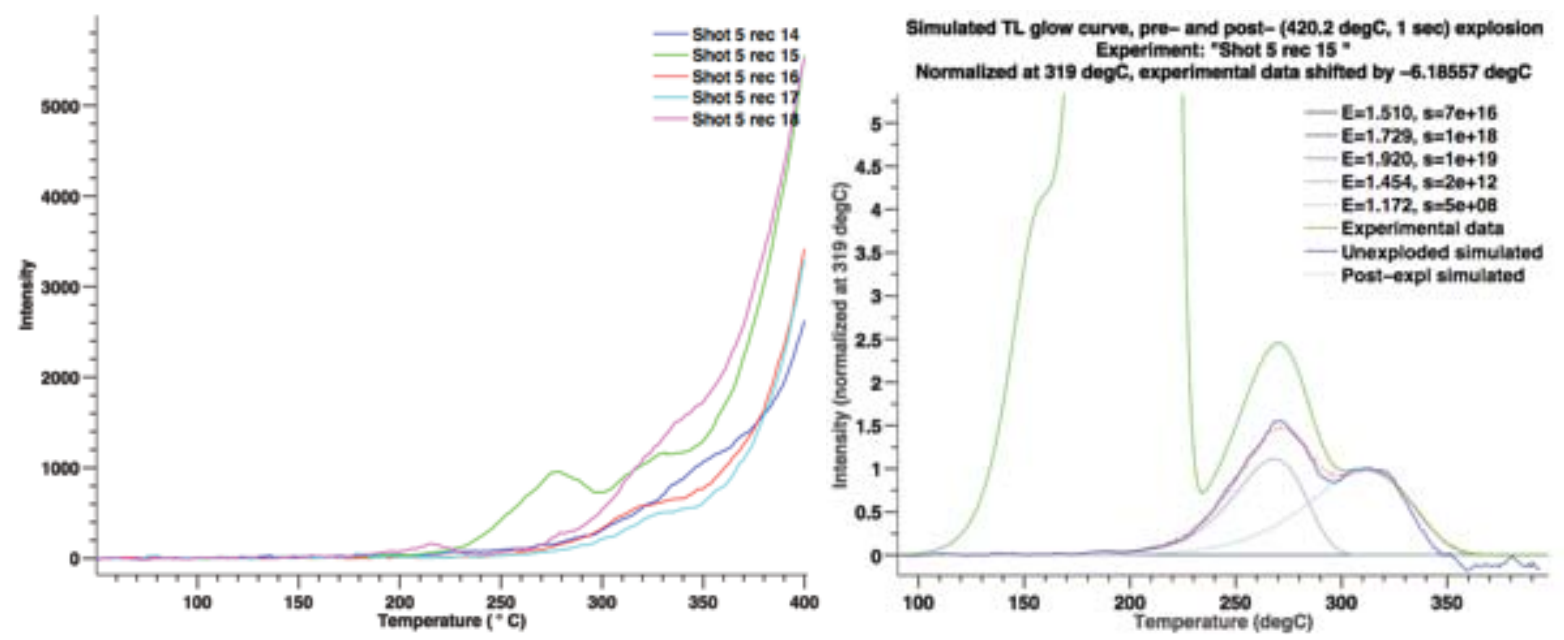

Fig. 147. TL data and simulated closest-match reconstructions for the three post-detonation samples.

This test was successful as all predicted temperatures and times are consistent with data obtained using thermocouples inside the blast chamber. One expects the microparticles to measure a temperature higher and more accurate than those of the thermocouples because the thermal masses of the thermocouples are high enough so that extremely rapid thermal excursions cannot be followed.

A summary of our results is shown in Table 20.

Table 20. Results obtained by UMN using LiF:Mg,Ti. The cooling time, $t_{\text {cool, }}$ is defined as the time it takes the sensor to cool from $T_{\max }$ to $\left[0.1\left(T_{\max }-T_{\text {ambient }}\right)+T_{\text {ambient }}\right]$.

\begin{tabular}{|llll|} 
Shot & Charge & $\boldsymbol{T}_{\max }$ & $\boldsymbol{t}_{\text {cool }}$ \\
\hline $\mathbf{5}$ & HPBX & $420{ }^{\circ} \mathrm{C}(693 \mathrm{~K})$ & $1.0 \mathrm{~s}$ \\
\hline $\mathbf{1 4}$ & LPBX & $303^{\circ} \mathrm{C}(576 \mathrm{~K})$ & $2.9 \mathrm{~s}$ \\
\hline $\mathbf{1 5}$ & LPBX & $310^{\circ} \mathrm{C}(583 \mathrm{~K})$ & $1.2 \mathrm{~s}$ \\
\hline
\end{tabular}

Further improvements were made to the process and simulation code for reconstructing thermal event histories from TL data. The basic concept remains the same: a first-order model of the control TLD-100 is subjected to the temperature profiles of various simulated detonations, and the resulting calculated TL curves compared to the actual post-detonation experimental TL curve data at five monitoring points (the highest-temperature one of which is used to normalize the others). The temperature profile of a high-explosive detonations seem to be represented fairly well by an instantaneous temperature increase followed by exponential cooling, which means that the thermal history is controlled by just two parameters, $t_{\text {cooling }}$ and $T_{\max }$. The temperature scenario most likely to have actually occurred is the one whose simulated TL curve most closely matches the actual data.

To make this rather brutish search more efficient, we use an iterative design, which progressively focuses in on the most likely scenarios of the previous go-around. Previous versions of this implementation have had to stick, more or less, to searching inside a rectangular grid of scenarios. However, the first-order TL model and this particular functional form of thermal profile result in a line of closest candidates that always curves from running parallel with the $T_{\max }$ axis to parallel with the $t_{\text {cooling }}$ axis; thus, a rectangle drawn to box it in has a lot of area coming nowhere close to that line, which translates to wasted computer time. Worse yet, the changing tilt of the line relative to a square 
grid means that the line can simply pass between even closely-spaced simulation points, and attempting to shift the points in such a variable way inside the box bogs down the simulation.

Here we used a completely restructured search code (Fig. 148), which finally enables a "freeform" sampling area. In the first iteration, a uniform grid is still used to roughly locate the "ridgeline" of the most-correct scenarios, but thereafter points are chosen in Gaussian-distributed spreads around the most correct points of the previous scenario. When combined with several overhauled TL helper functions, this approach boosts accuracy for a fraction of the processing time. The thresholds set by experimental error sources can generally be passed in $3 \mathrm{~s}$ with two heating parameters; moreover, it also allows the search technique to scale somewhat better, so adding a third variable to more accurately describe the shape of the thermal event does not push processing times into the hours range.
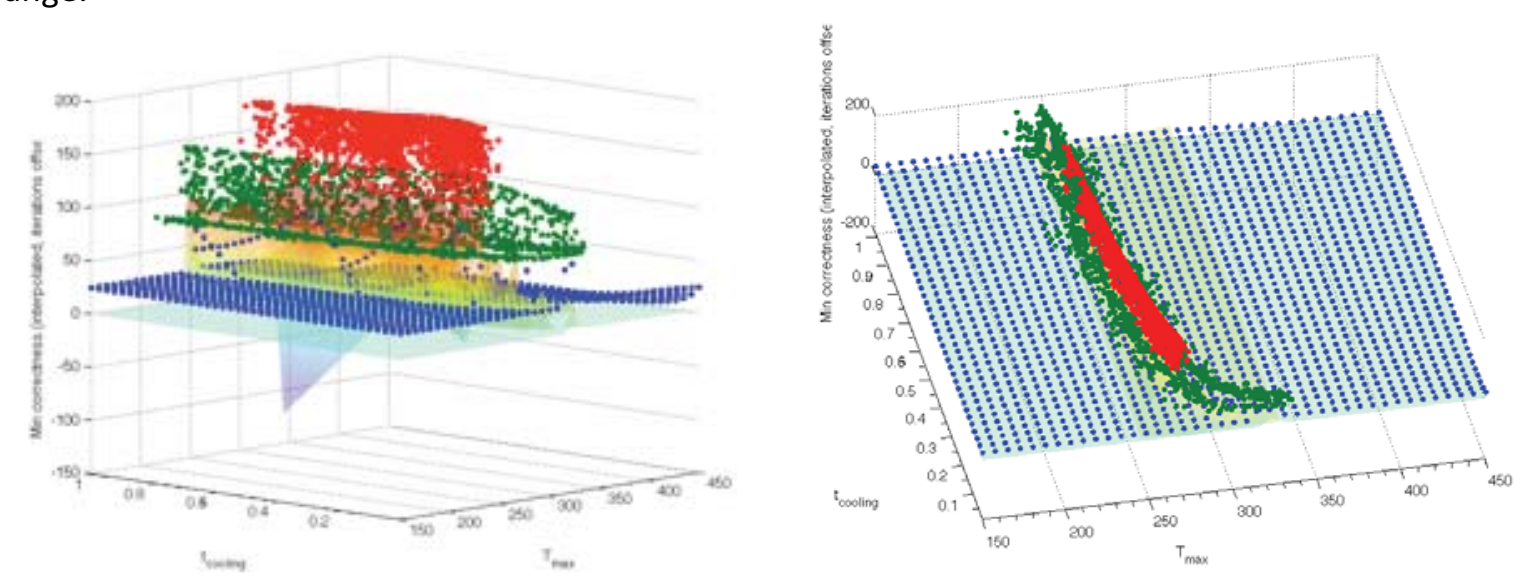

Fig. 148. Two angles of a plot illustrating the thermal history reconstruction process. The two variables required to describe each possible temperature scenario, cooling time $t_{\text {cooling }}$ and the maximum temperature $T_{\text {max }}$, form the horizontal plane; the vertical axis represents the minimum correctness (=1-\%error) among the five monitoring points between the actual postdetonation TL curve and the simulated result for that scenario. Colored dots represent points-thermal event scenarioswhich were simulated, with each iteration being assigned its own color. A vertical offset is also added to each iteration's results to visually separate them. Note that after the initial iteration's uniform grid, the simulated points hew very closely to the "ridgeline" of most correct scenarios, and quickly densify.

Since the TLD-100 prepared for this test was stratified by particle size, it was of great interest to see how the response to detonation might have differed. To this end, the two returned LPBX samples were filtered into six size ranges (Fig. 149) using the same mesh and IPA methods as in the initial preparation process (with the omission of heat-assisted drying). 

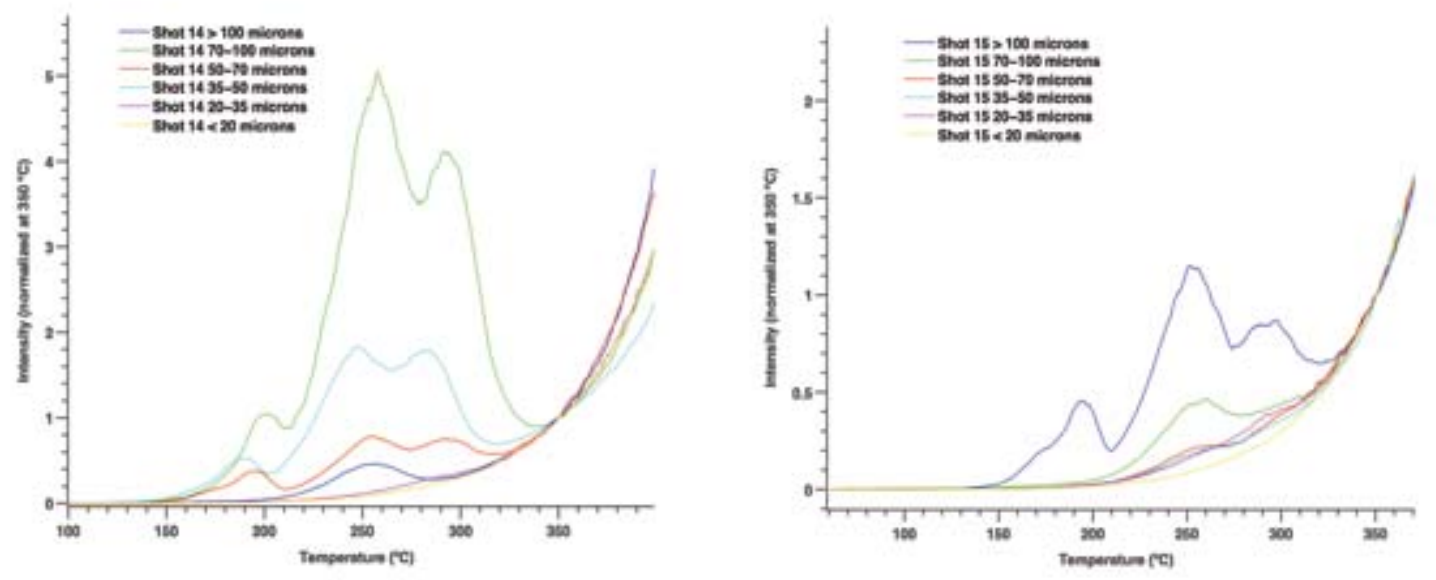

Fig. 149. TL data for shots 14 and 15, filtered by size.

Encouragingly, it is possible to identify which shot is which by matching the relative strengths of TL emission with the particle sizes that Indian Head reported having used; for example, particles $>100 \mu \mathrm{m}$ were included in shot 15 but not in shot 14, and thus dominate the TL emission in the former, whereas being questionably distinguishable as a partial TLD-100 curve in the latter. The TL curves also indicate that different sizes of particle experienced much different levels of TL carrier trap depopulation. This offers an explanation for the deviation of the unfiltered sample TL curves from our models. Tests with fresh size-filtered TLD-100 on rapidly-heating microheaters are planned to determine whether the differences are mostly due to the differences in thermal mass and response time, the size-dependency of their paths through the post-detonation environment, or a mixture of the two.

\subsection{DTRA Humble Gingko XIX (August 2014)}

Detonation tests were conducted at Kirkland AFB to determine the feasibility and performance of TL materials as temperature sensors in the Humble Gingko XIXB test facility. Particles tested include $\mathrm{MgB}_{4} \mathrm{O}_{7}: \mathrm{Dy}_{0.1 \%}, \mathrm{Li}_{1 \%}(\mathrm{MBO})$ and $\mathrm{CaSO}_{4}: \mathrm{Ce}_{0.2 \%}, \mathrm{~Tb}_{0.2 \%}$ (CSO), developed by OSU [4], and commercial $\mathrm{Al}_{2} \mathrm{O}_{3}: \mathrm{C}$. $\mathrm{Al}_{2} \mathrm{O}_{3}: \mathrm{C}$ was used to determine that $\mathrm{TL}$ material has been recovered. Light sensitivity prevents the use of $\mathrm{Al}_{2} \mathrm{O}_{3}: \mathrm{C}$ material for reliable temperature determination. OSU materials were sieved using a $75 \mu \mathrm{m}$ sieve size, whereas $\mathrm{Al}_{2} \mathrm{O}_{3}: \mathrm{C}$ has particle size of $<35 \mu \mathrm{m}$.

The Humble Gingko XIXB test facility is composed of two rooms, one with the explosive charge (source, SRC) and one connected to the source room (adjacent, ADJ). The samples were suspended in five unique positions and collected at one of 18 collection sites (9 SRC and 9 ADJ) following an explosion. For $\mathrm{MBO}$ and $\mathrm{CSO}$ the charge used was a $5 \mathrm{lb}$ Pentolite Bare Charge and for $\mathrm{Al}_{2} \mathrm{O}_{3}: \mathrm{C}$, an $8 \mathrm{lb} \mathrm{PBX} 109$ Steel Cased Charge. On site temperature measurements were provided by 4 thermocouples and 2 pyrometers.

Following collection of TL materials, samples were returned to UMN and OSU for analysis. A total of 44 sites and locations ( 15 for $\mathrm{MBO}, 14$ for $\mathrm{CSO}$, and 15 for $\mathrm{Al}_{2} \mathrm{O}_{3}: \mathrm{C}$ ) were received by OSU, but not all sites and locations were readout due to insufficient quantity of material. 


\subsubsection{UMN results}

\section{Sample preparation and readout}

Three types of TL samples were supplied for the Humble Gingko XIX tests. Of these, one-aluminum oxide, $\mathrm{Al}_{2} \mathrm{O}_{3}: \mathrm{C}$-was intended only for a basic material collection and minimal "survival" test; its trap distribution and sensitivity to UV light limits its usability for temperature reconstruction, but it is a relatively simple and hardy material to test for basic TL. The two materials meant for temperature reconstruction were $\mathrm{MgB}_{4} \mathrm{O}_{7}: \mathrm{Dy}, \mathrm{Li}(\mathrm{MBO})$ and $\mathrm{CaSO}_{4}: \mathrm{Ce}, \mathrm{Tb}(\mathrm{CSO})$. Both were synthesized by OSU and irradiated and packaged for testing by UMN.

For the tests, the samples were divided into five batches of $11 \mathrm{~g}$ each and placed at five sites within both detonation-affected rooms of the Gingko test structure. To ensure powder dispersal and elevate the sample above the floor before detonation, a lightweight open-top boat-made of a polystyrene weight boat, commercially-available fishing line, and a stainless steel lock washer-was used to hold each sample at its designated site (Fig. 150).

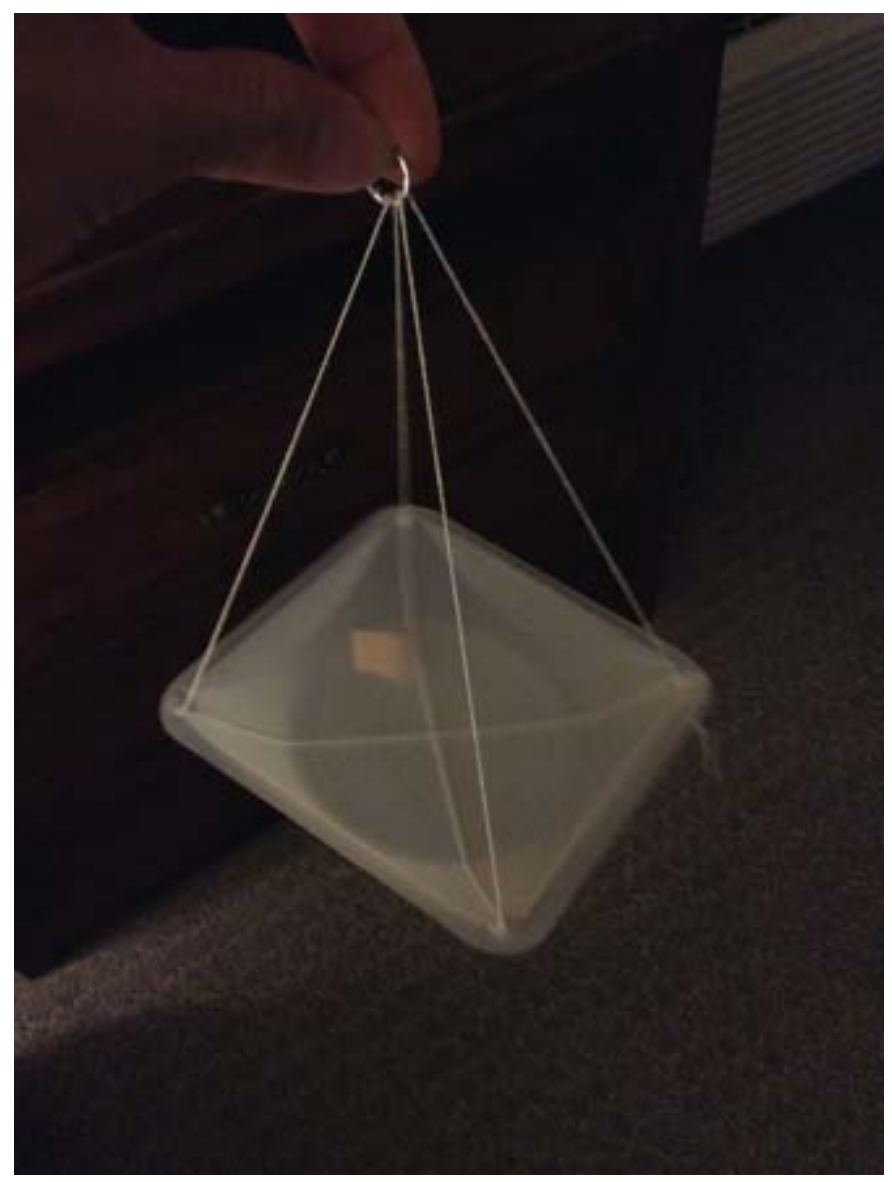

Fig. 150. A prototype sample boat. This "pre-production" example uses twine instead of fishing line, but the design is the same.

After detonation, powder was collected from 16 sites of interest (Fig. 151), pre-assigned and marked onto horizontal surfaces inside and around the Gingko structure. A natural-fiber 2" paintbrush (chosen primarily for its low static properties) and polystyrene weighboat were used by site safety personnel to sweep up powder and debris within each collection site. The collected samples were stored in plastic 
canisters and isolated along with the collection equipment in ziplock bags. Black light-opaque garbage bags were used to reduce the chances of photobleaching in the desert sun, and the collected samples were stored whenever possible in air-conditioned areas such as the testing site instrumentation vans. (Undetonated monitor samples were also used to measure for significant photobleaching and unintended thermal detrapping.) At the end of testing, all samples were returned to UMN and OSU in light-tight packaging by commercial parcel shipping.

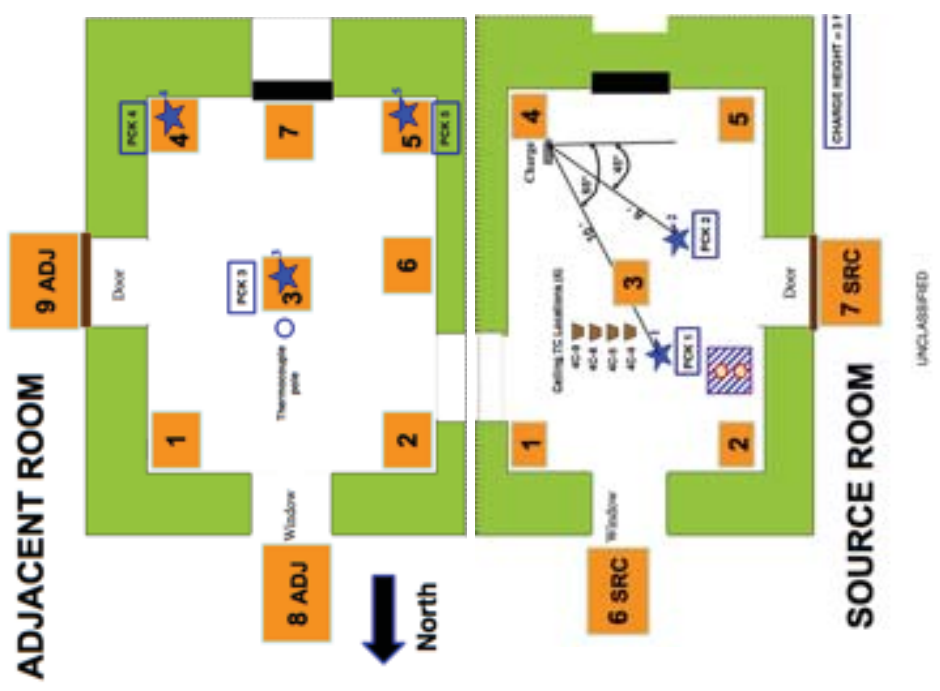

Fig. 151. The locations of the samples and collection sites within the two detonation-affected rooms of the Gingko test structure. After documentation provided by Howard Ross.

\section{Readout Procedure}

For primary temperature reconstructions, samples were read out using a resistive disc heater setup built and housed at UMN. Samples collected from Humble Gingko contained a lot of sand, small rocks, fibrous material, and other substances that one might expect to find in a structure open to the outdoor environment. Larger debris was removed by hand, and the remaining powder sifted using a tilted piece of aluminum foil to try and separate out the smallest visible particles. Although the samples recovered this way indubitably contain a very large amount of sand, our CSO and MBO materials are (presumably) the only substances recently irradiated with large doses of gamma radiation, and thus we have not noticed any spurious TL signals.

The TL was measured by a PMT interfaced to a frequency counter, with the count recorded multiple times per second by a computer. For CSO readout, we used a $550 \mathrm{~nm}$ optical shortpass filter before the PMT to reduce blackbody signal; for MBO readout, we used an optical bandpass filter, centered at 486 $\mathrm{nm}$ with $10 \mathrm{~nm}$ FWHM, to isolate the material's primary emission peak. The TL data was further processed after being recorded, with single data points of intensity 1.4 times greater than their immediate neighbors removed and a rolling 20-point $\left(\sim 1.5^{\circ} \mathrm{C}\right)$ average performed. A static background intensity level was averaged from the first ten data points recorded (after reaching $50^{\circ} \mathrm{C}$ ) and subtracted from the curve. A second TL measurement was performed on the now-depleted sample immediately after the first to obtain a record of the blackbody background; this second TL curve was processed similarly to the real TL data and subtracted from the latter.

A smaller copy of the TL readout system was built and transported (as checked airline luggage) to Albuquerque to provide rough material functionality checks in the field. It incorporates much of the 
same equipment as the full-sized system at UMN, but has a light-tight housing, which can be partially disassembled for portability (Fig. 152). Testing was successful, although time constraints precluded fullscale readout operations from proceeding en masse.

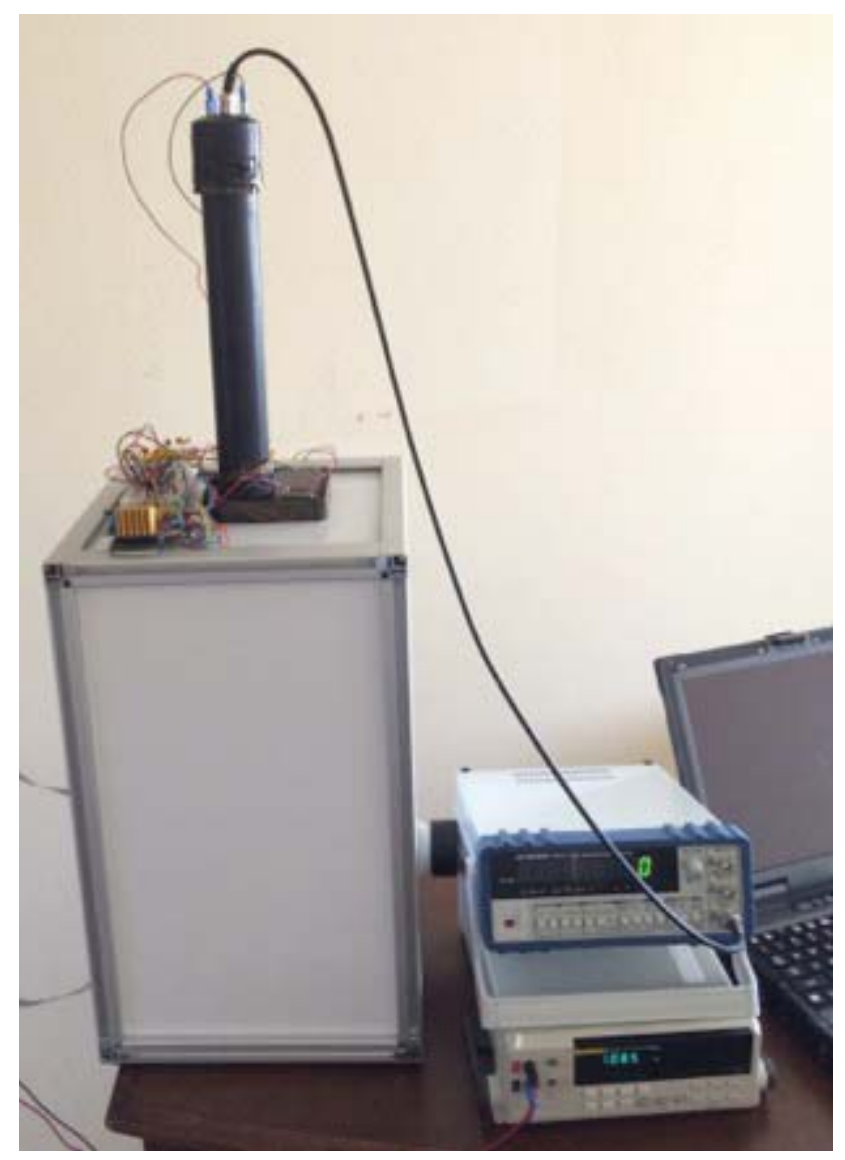

Fig. 152. Exterior of the portable TL readout system. From left to right: light-tight hotplate enclosure, with black tubular PMT mount and motorized shutter system on top; frequency counter and hotplate temperature sensor instruments; and data collection laptop.

\section{Reversal process}

Temperature reconstruction - or the "reversal" process for short-is done by an iterative search algorithm based on the simulation of multiple heating "scenarios", i.e. parameter variations of a functional thermal history form. This process is performed using third-generation simulation code developed for, and tested on, TLD-100, but is easily adapted to other materials and models.

The temperature profile experienced was assumed to be an essentially-instantaneous increase in temperature to a maximum $T_{\text {max }}$, followed by exponential cooling with time constant $\tau_{\text {cooling }}$. Both CSO and MBO materials were modeled as superpositions of multiple -7 and 5 , respectively - first-order TL traps, with parameters hand-fit to control readouts. MBO and CSO, however, have been studied for far less time than the decades of widespread examination and use enjoyed by TLD-100, and thus there is more uncertainty in the applicability of these fits. In particular, Prof. Yukihara and his team at OSU have proposed that CSO and MBO be modeled as a dense array of $\sim 30$ to 40 first-order traps $[10,11]$. Although our reconstruction code can accommodate this approach, the accuracy of the resulting model's behavior is not known to us. 


\section{Results and reversals: CSO}

Of the 16 CSO samples collected, all but four showed a healthy TL signal (Fig. 147). Of the remaining four, two from the adjacent room were either too heavily depopulated or lacked sufficient quantity of CSO material to give a significant signal strength; one each from the source and adjacent rooms showed insignificant TL signal, but as these areas were outside of the structure and samples consisted-as predicted beforehand-mostly of wood from the sacrificial plywood doors, it is likely that little CSO reached this collection site. Most of the eight samples with signal produced fits that, while gauged by the limited number of sampling points to be close, featured disagreement in the shapes of one or more peaks; agreement in four is good enough to trust that the maximum temperature has been approximated. The source room claimed three of these higher-confidence sites, with their maximum temperatures ranging from 380 to $490^{\circ} \mathrm{C}$ and cooling times estimated to be less than $10 \mathrm{~ms}$; the site in the adjacent room is expected to have reached only $270^{\circ} \mathrm{C}$ and cooled in $20 \mathrm{~ms}$. (As usual, the cooling time constant affects the TL less strongly than the maximum temperature reached.)

Fit and thus accuracy would likely be most effectively increased by improvements to the material TL model and in the temperature functional profile, e.g. factoring in any long-duration ( $>1 \mathrm{~s}$ ) heating of the surfaces on which the particles land post-detonation. Another possible factor in fit disagreements, and a source of concern, seen in CSO is that different samples taken from the same monitor tube show more disagreement than expected in peak height/ratio and position (Fig. 154). The cause is yet unclear.
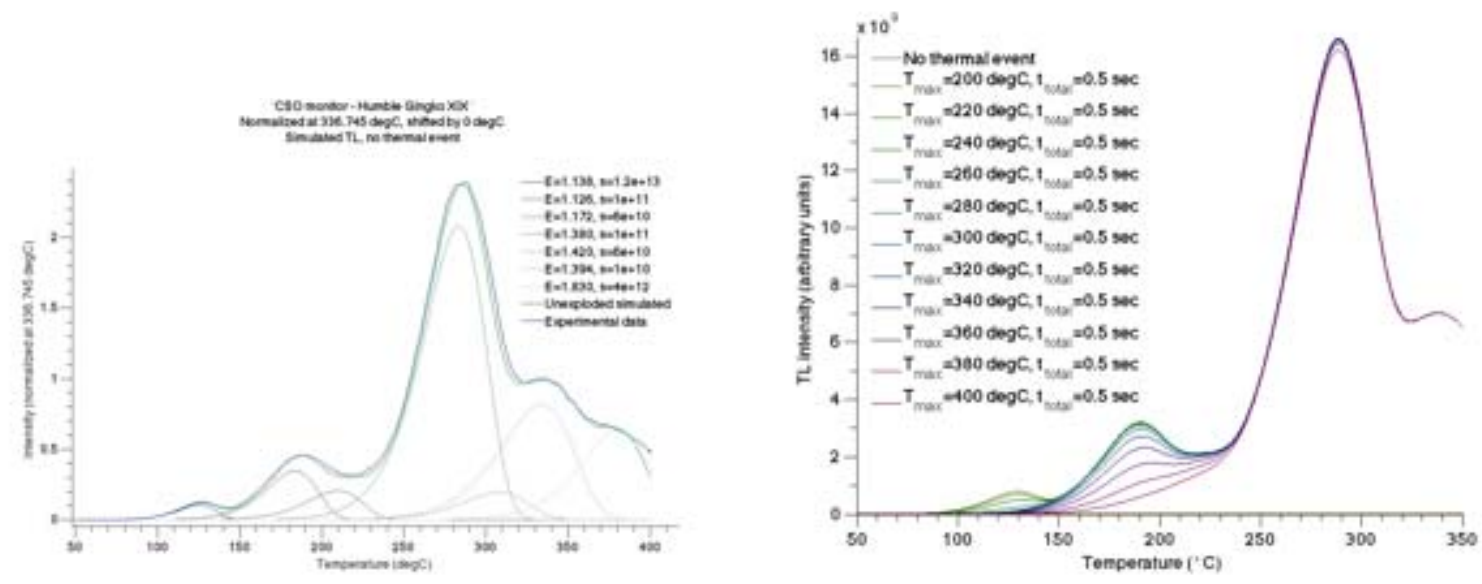

Fig. 153. (Left) TL curve for CSO monitor sample, compared with the hand-fit theoretical model. (Right) Simulated TL curves after a range of arbitrarily-chosen temperature events. 


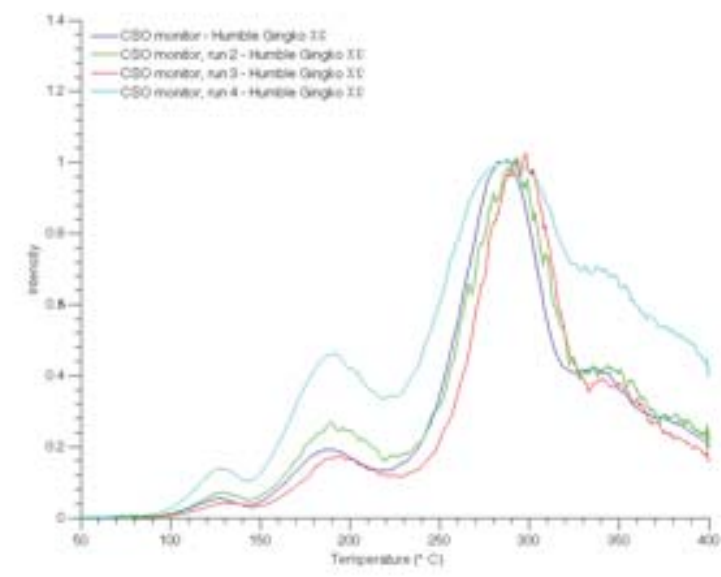

Fig. 154. Variation in different readouts of CSO monitor.
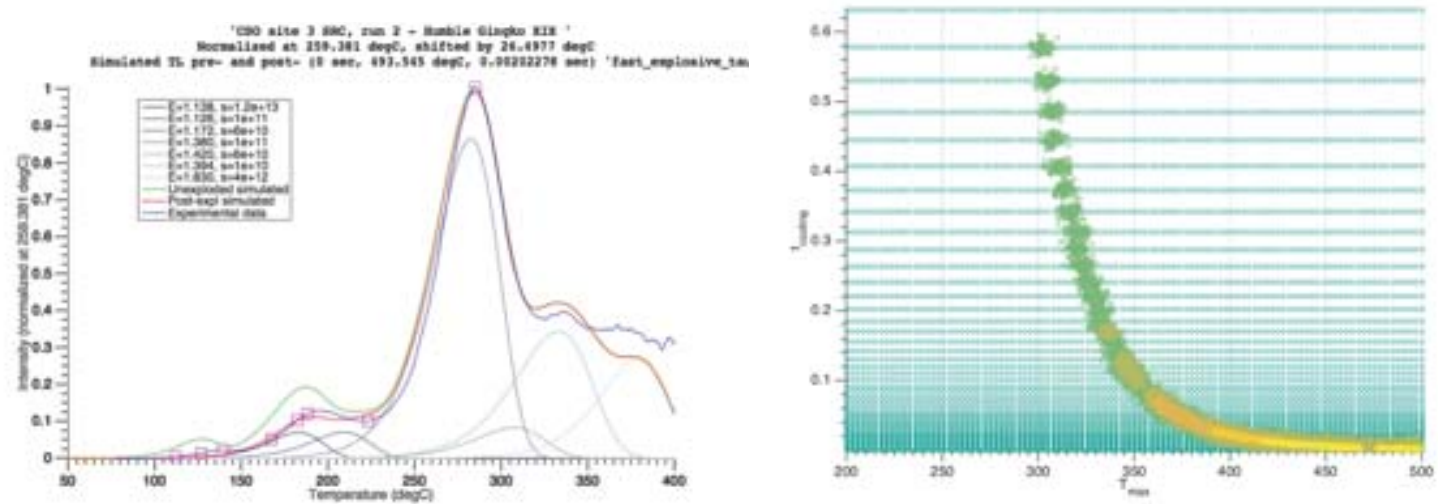

Fig. 155. (Left) A simulated temperature scenario's TL curve compared to the actual observed; note that the simulated curve expects more depopulation in the second peak from left than is actually observed. (Right) A plot of where heating parameters produced the closest match in simulated and actual TL curves. This example is CSO site 3 SRC. The time constant axis is shown in, and simulated using, a log scale.

\section{Results and reversals: $M B O$}

The MBO TL curve provides a broad high-temperature "shoulder", assumed to be composed of several higher-energy traps (Fig. 156). However, when normalized to the higher-temperature peaks, the lowertemperature peaks of several of the adjacent room MBO samples were found to be of greater relative intensity than they were in the monitor and model (Fig. 157). This could be explained by uniform heating, which affected the higher-temperature trap more than expected, or by misunderstood trap kinetics for the material. The simplest explanation, a monitor sample that had somehow been heated or modified drastically more than the detonated samples it accompanied, is ruled out by its close match to the self-held control stored at UMN.

The remainder of the adjacent room MBO samples showed negligible depopulation, suggesting that minimal heating was experienced in that room (Fig. 158). As seen in CSO, the collection sites outside of the adjacent and source rooms each showed no significant TL material present.

Samples from the source room showed behavior closer to the expected model. Maximum temperatures were clustered around $480-510^{\circ} \mathrm{C}$, with one outlier at $230^{\circ} \mathrm{C}$; cooling time constants were $1.5 \mathrm{~ms}$ and below, with the same curious outlier site at $0.64 \mathrm{~s}$. Again, improvements in the 
understanding of MBO's trap kinetics would effectively improve the areas of the fit that show less agreement, particularly given the seemingly aberrant responses of the adjacent room's samples.

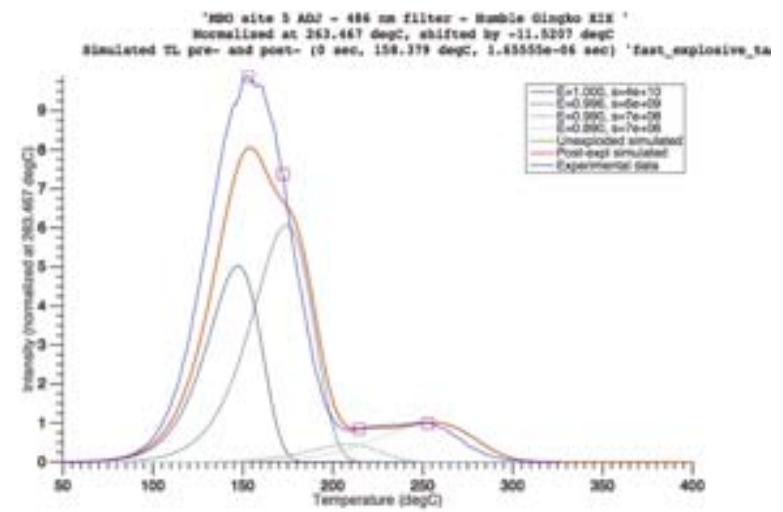

Fig. 156. MBO site $5 \mathrm{ADJ}$, displaying a normalized post-detonation main peak intensity that is much higher than the undetonated (simulated) control.

(a)

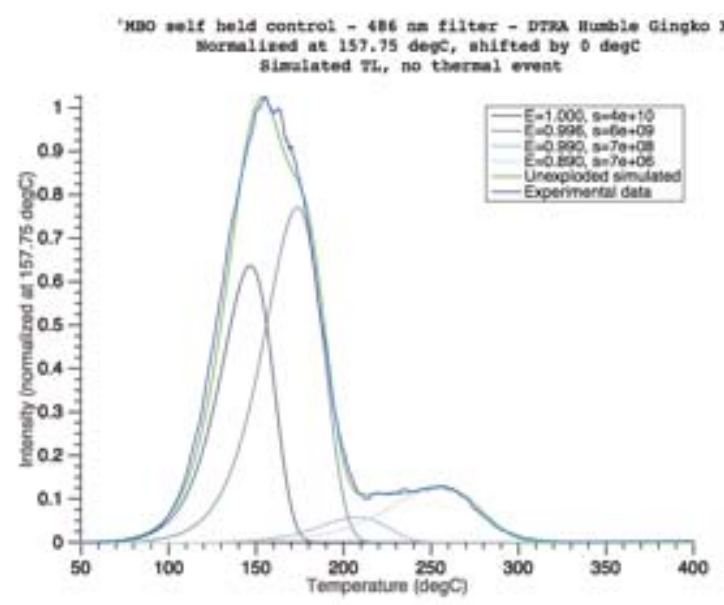

(b)

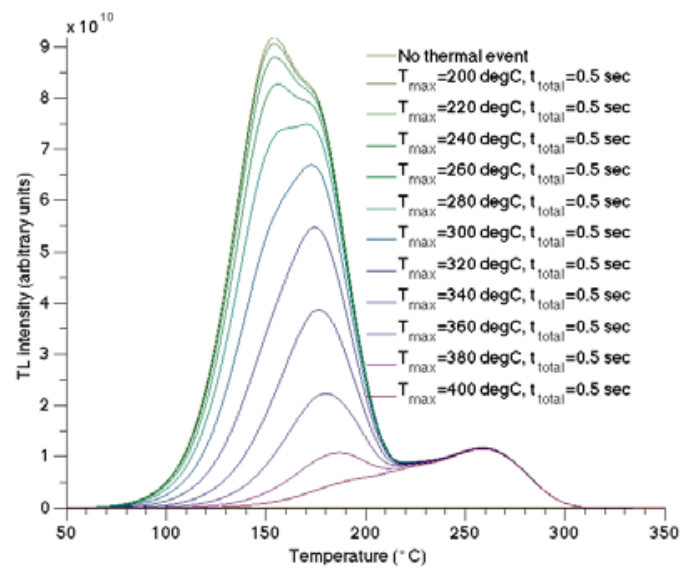

Fig. 157. (a) TL curve for MBO monitor sample, compared with the hand-fit theoretical model. (b) Simulated TL curves after a range of arbitrarily chosen temperature events. Note that the intensity of the high-temperature TL peak remains constant, whereas the main TL peak decays, as expected. 

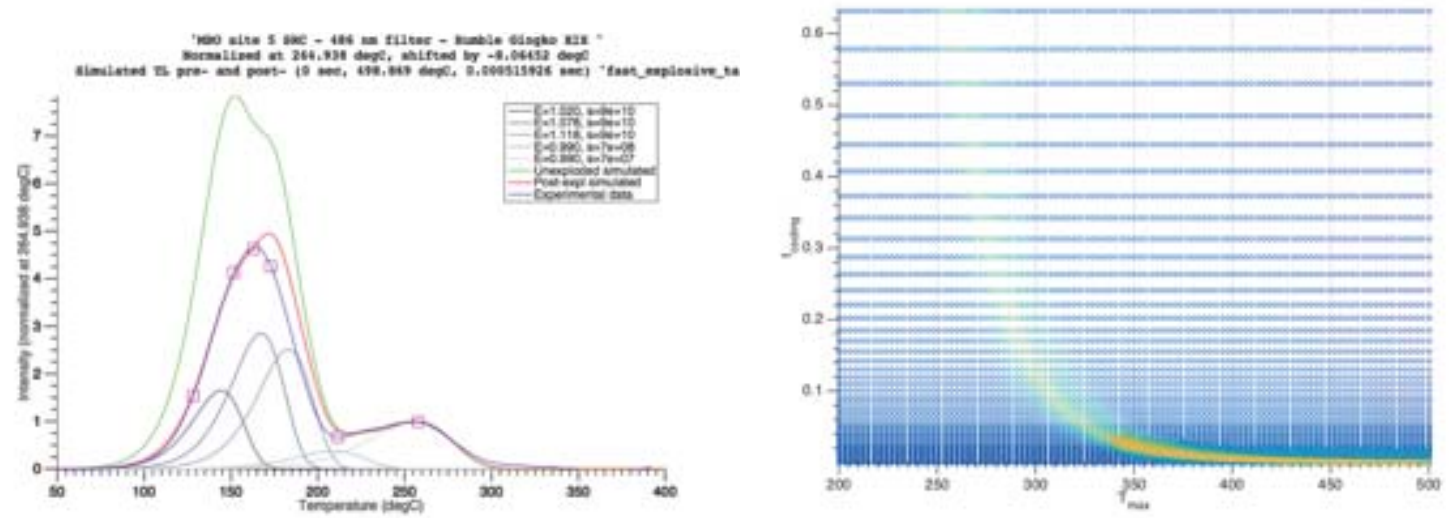

Fig. 158. (Left) A simulated temperature scenario's TL curve compared to the actual observed. The simulated scenario result departs the actual observed data primarily in the higher-temperature shoulder of the main peak. (Right) A plot of where heating parameters produced the closest match in simulated and actual TL curves. This example is MBO site 5 SRC.

\section{Summary Comments}

A luminescence intensity measurement was made on CSO control particles versus debris from the CSO test. The debris gave $0.038 \%$ as much signal as pure CSO. Even accounting for reduced TL due to temperature, the debris is overwhelmingly not TL particles; however, only tiny amounts of luminescence is necessary so we still have plenty of signal. Energy dispersive X-ray spectroscopy was applied to 15 MBO microparticles, selected by hand from debris because they looked like control particles. At most 9 particles were MBO as identified by EDS. 4 of the 15 were a calcium-rich compound, and 2 appeared to be a combination of MBO and Ca-rich.

Particle-by-particle studies, using micromachined heaters hundreds of $\mu \mathrm{m}$ in size, have been performed. One of the characteristics of open chambers that we noticed in the August 2014 Humble Gingko test was that individual particles were exposed to a huge range of temperatures. Some particles were completely bleached by extremely high temperatures, whereas others had minimal temperature exposure. Some example data for six tested particles taken from exactly the same site is as follows: Particle $4-280{ }^{\circ} \mathrm{C}$, Particle $9-396{ }^{\circ} \mathrm{C}$, Particle $12-440{ }^{\circ} \mathrm{C}$, Particle $15-344{ }^{\circ} \mathrm{C}$, Particle $18-156{ }^{\circ} \mathrm{C}$, and Particle $26-516^{\circ} \mathrm{C}$. It can be seen from even a small subset of measurements that many hundreds of degrees separate the temperature of the particles with the lowest temperature exposure and those of the highest. The number of possible temperature paths for particles that end up at the same spot is truly enormous. (Note that this characteristic is not seen in closed chamber tests, such as at Indian Head, where the particles show largely similar temperature exposures.) Since bleached particles do not contribute to TL, the remaining luminescence artificially selected lower temperature particles - ideal for agent defeat, but problematic for measuring average explosion temperatures.

Variations of the current simulation approach, using only the existing data detailed above, has been lightly explored. A temperature profile with a finite heating rate has been simulated, but did not produce a drastic jump in confidence; neither did a profile which holds the maximum temperature for some time before cooling begins. The limiting factor in the agreement of the simulated and observed data is currently believed to be the trap kinetics and parameters used for these materials, with which UMN was relatively unexperienced at the outset. 


\subsubsection{OSU results}

Received samples contained various non-TL materials including dirt, metal and wood. TL measurements and analysis were conducted for $\mathrm{MBO}, \mathrm{CSO}$, and $\mathrm{Al}_{2} \mathrm{O}_{3}: \mathrm{C}$ at OSU according to established methods [10, 11]. Representative experimental TL curves for each material are found in Fig. 159.
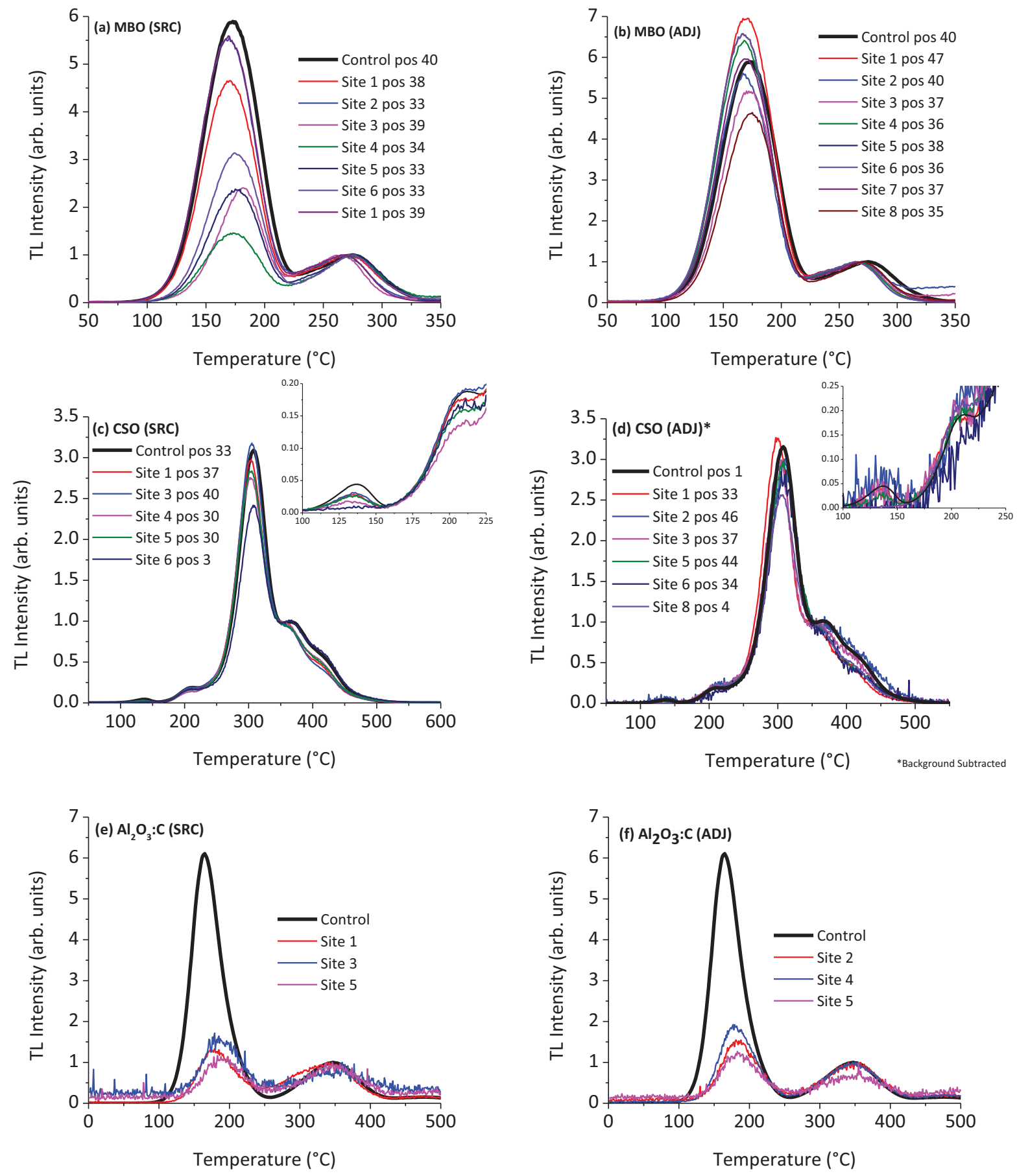

Fig. 159. "As-received" experimental TL curves from $\mathrm{MBO}, \mathrm{CSO}$, and $\mathrm{Al}_{2} \mathrm{O}_{3}: \mathrm{C}$. The first column shows representive curves from the source (SRC) placement and the second column shows representive curves from the adjecent (ADJ) placement. 
Temperature reconstruction was conducted for $\mathrm{MBO}$ and CSO using two heating profiles; constant heating ( $\tau=10 \mathrm{~ms}$ ) and exponential heating $(\tau=50 \mathrm{~ms})$ following previously established methods [10]. The range of applicability was established using the $10 \%$ - $90 \%$ residual TL area for MBO, or a physical interpretation of unheated TL peaks for CSO. The temperature reconstruction results are found in Table 21 and Table 22.

Analysis of MBO indicated that a majority of samples were heated below the material's applicable range ( $<515 \mathrm{~K}, 10 \mathrm{~ms}$ ) with SRC samples heated to temperatures between $515 \mathrm{~K}$ and $578 \mathrm{~K}$ (Table 21). Results for MBO ADJ sites indicate heating at or just above the $515 \mathrm{~K}$ limit indicating insufficient heating in most cases. In cases where temperatures were obtained for the exponential heating model the OSU results were within $\sim 50 \mathrm{~K}$ of those from UMN. CSO results indicate heating was insufficient for all sites except for SRC Site 6, which measured $700 \mathrm{~K}$ and $775 \mathrm{~K}$ for constant and exponential heating respectively. However UMN's analysis of this site returned a measurement of insufficient heating possibly indicating variability with in the sample sites themselves. Further analysis of CSO Site 6 SRC also indicates the possibility of heating to a distribution of temperatures.

The tests demonstrate that it is possible to recover sufficient material for TL analysis despite the large size of the chamber. Particles from a large fraction of sites were heated below the range of applicability for these materials indicating the need for a higher temperature charge and/or a decrease in particle size for future tests. It is also possible that the particles are "riding" the shock wave and are not being exposed to the peak temperatures.

When the reconstruction data from the particles are compared to data taken from thermocouples in the test chambers, UMN found that the particles do a good job of following the temperatures seen by an average of all of the thermocouples at time scales on the order of a few tenths of a second or longer, but do not follow individual thermocouples in any high temperature excursions seen by on the time scale of $50 \mathrm{~ms}$ or so. This is true even for small CSO particles, so it does not appear to be a time constant issue, but it may indicate that the TL emission is weighted towards brighter particles, corresponding to those that have followed trajectories in lower temperature portions of the blast. This would be the ideal case for agent defeat testing because one wishes to identify regions where infectious particles could have survived. If the low temperature trajectory hypothesis is true, then the distribution of temperatures seen by particles collected at any one particular spot will be large.

Table 21. Reconstructed temperatures for MBO samples. The analysis assumes either a constant heating profile with a $10 \mathrm{~ms}$ exposure time or an exponential decay with $50 \mathrm{~ms}$ time constant (for SRC samples only). Insufficient heating indicates temperatures $<515 \mathrm{~K}$ (or $<242{ }^{\circ} \mathrm{C}$ ).

\begin{tabular}{|llcccc|} 
Site & Aliquot & $\begin{array}{c}\Theta \\
\text { constant } \\
\tau=10 \mathrm{~ms}\end{array}$ & $\begin{array}{c}\Theta \\
\text { constant } \\
\tau=10 \mathrm{~ms}\end{array}$ & $\begin{array}{c}\Theta \\
\text { exp. decay } \\
\tau=50 \mathrm{~ms}\end{array}$ & $\begin{array}{c}\Theta \\
\text { exp. decay } \\
\tau=50 \mathrm{~ms}\end{array}$ \\
\hline SRC 1 & $31,33,34,35,37,39$ & $\begin{array}{c}\text { insufficient } \\
\text { insufficient }\end{array}$ & & \\
& 32 & $561 \mathrm{~K}$ & $288{ }^{\circ} \mathrm{C}$ & $581 \mathrm{~K}$ & $308{ }^{\circ} \mathrm{C}$ \\
& 36 & $517 \mathrm{~K}$ & $244^{\circ} \mathrm{C}$ & $533 \mathrm{~K}$ & $260^{\circ} \mathrm{C}$ \\
& 38 & $515 \mathrm{~K}$ & $242^{\circ} \mathrm{C}$ & $531 \mathrm{~K}$ & $258^{\circ} \mathrm{C}$ \\
\hline SRC 2 & $33,34,35$ & insufficient & insufficient & & \\
\hline SRC 3 & 41 & insufficient & insufficient & & \\
& 39 & $568 \mathrm{~K}$ & $295^{\circ} \mathrm{C}$ & $588 \mathrm{~K}$ & $315^{\circ} \mathrm{C}$ \\
& 40 & $547 \mathrm{~K}$ & $274{ }^{\circ} \mathrm{C}$ & $566 \mathrm{~K}$ & $293{ }^{\circ} \mathrm{C}$ \\
\hline SRC 4 & 33,35 & insufficient & insufficient & & \\
& 34 & $578 \mathrm{~K}$ & $305^{\circ} \mathrm{C}$ & $600 \mathrm{~K}$ & $327^{\circ} \mathrm{C}$ \\
\hline
\end{tabular}




\begin{tabular}{|c|c|c|c|c|c|}
\hline \multirow[t]{3}{*}{ SRC 5} & 33 & $563 \mathrm{~K}$ & $293^{\circ} \mathrm{C}$ & $585 \mathrm{~K}$ & $312{ }^{\circ} \mathrm{C}$ \\
\hline & 34 & $530 \mathrm{~K}$ & $257^{\circ} \mathrm{C}$ & $547 \mathrm{~K}$ & $274{ }^{\circ} \mathrm{C}$ \\
\hline & 35 & $536 \mathrm{~K}$ & $263^{\circ} \mathrm{C}$ & $554 \mathrm{~K}$ & $281^{\circ} \mathrm{C}$ \\
\hline \multirow[t]{3}{*}{ SRC 6} & 33 & $544 \mathrm{~K}$ & $271^{\circ} \mathrm{C}$ & $563 \mathrm{~K}$ & $290^{\circ} \mathrm{C}$ \\
\hline & 34 & $548 \mathrm{~K}$ & $275^{\circ} \mathrm{C}$ & $566 \mathrm{~K}$ & $293^{\circ} \mathrm{C}$ \\
\hline & 35 & $548 \mathrm{~K}$ & $275^{\circ} \mathrm{C}$ & $567 \mathrm{~K}$ & $294^{\circ} \mathrm{C}$ \\
\hline ADJ 1 & $43,44,45,46,47$ & insufficient & insufficient & & \\
\hline ADJ 2 & $40,41,42,43,44$ & insufficient & insufficient & & \\
\hline \multirow[t]{3}{*}{ ADJ 3} & 36 & insufficient & insufficient & & \\
\hline & 37 & $520 \mathrm{~K}$ & $247^{\circ} \mathrm{C}$ & & \\
\hline & 38 & $516 \mathrm{~K}$ & $243^{\circ} \mathrm{C}$ & & \\
\hline ADJ 4 & $36,37,38$ & insufficient & insufficient & & \\
\hline ADJ 5 & $36,37,38$ & insufficient & insufficient & & \\
\hline \multirow[t]{2}{*}{ ADJ 6} & 37,38 & insufficient & insufficient & & \\
\hline & 36 & $517 \mathrm{~K}$ & $244{ }^{\circ} \mathrm{C}$ & & \\
\hline ADJ 7 & $36,37,38$ & insufficient & insufficient & & \\
\hline \multirow[t]{2}{*}{ ADJ 8} & 33,35 & insufficient & insufficient & & \\
\hline & 34 & $515 \mathrm{~K}$ & $242{ }^{\circ} \mathrm{C}$ & & \\
\hline
\end{tabular}

Table 22. Reconstructed temperatures of CSO samples. The analysis assumes either a constant heating profile with a $10 \mathrm{~ms}$ exposure time or an exponential decay with $\mathbf{5 0} \mathrm{ms}$ time constant (for SRC samples only). Insufficient heating is indicated by $<571 \mathrm{~K}$ (or < $\left.298^{\circ} \mathrm{C}\right)$. “No TL" indicates TL curve with a distorted, null, or too weak signal.

\begin{tabular}{|c|c|c|c|c|c|}
\hline Site & Aliquot & $\begin{array}{c}\Theta \\
\text { constant } \\
\tau=10 \mathrm{~ms}\end{array}$ & $\begin{array}{c}\Theta \\
\text { constant } \\
\tau=10 \mathrm{~ms}\end{array}$ & $\begin{array}{c}\Theta \\
\text { exp. Decay } \\
\tau=50 \mathrm{~ms}\end{array}$ & $\begin{array}{c}\Theta \\
\text { exp. Decay } \\
\tau=50 \mathrm{~ms}\end{array}$ \\
\hline SRC 1 & $36,37,38$ & $<571 \mathrm{~K}$ & $<298{ }^{\circ} \mathrm{C}$ & & \\
\hline SRC 2 & $33,34,35$ (no TL) & & & & \\
\hline SRC 3 & $40,41,42$ & $<571 \mathrm{~K}$ & $<298{ }^{\circ} \mathrm{C}$ & & \\
\hline SRC 4 & $30,31,32$ & $<571 \mathrm{~K}$ & $<298{ }^{\circ} \mathrm{C}$ & & \\
\hline SRC 5 & $30,31,32$ & $<571 \mathrm{~K}$ & $<298{ }^{\circ} \mathrm{C}$ & & \\
\hline \multirow[t]{3}{*}{ SRC 6} & 1 & $704 \mathrm{~K}$ & $431{ }^{\circ} \mathrm{C}$ & $780 \mathrm{~K}$ & $507^{\circ} \mathrm{C}$ \\
\hline & 2 & $696 \mathrm{~K}$ & $423^{\circ} \mathrm{C}$ & $770 \mathrm{~K}$ & $497^{\circ} \mathrm{C}$ \\
\hline & 3 & $702 \mathrm{~K}$ & $429^{\circ} \mathrm{C}$ & $778 \mathrm{~K}$ & $505^{\circ} \mathrm{C}$ \\
\hline ADJ 1 & $44,45,46$ & $<571 \mathrm{~K}$ & $<298^{\circ} \mathrm{C}$ & & \\
\hline ADJ 2 & $36,37,38$ (no TL) & & & & \\
\hline ADJ 3 & $43,44,45$ & $<571 \mathrm{~K}$ & $<298{ }^{\circ} \mathrm{C}$ & & \\
\hline ADJ 4 & 33,35 & $<571 \mathrm{~K}$ & $<298{ }^{\circ} \mathrm{C}$ & & \\
\hline ADJ 5 & $33,34,35$ & $<571 \mathrm{~K}$ & $<298{ }^{\circ} \mathrm{C}$ & & \\
\hline ADJ 6 & 4,6 & $<571 \mathrm{~K}$ & $<298{ }^{\circ} \mathrm{C}$ & & \\
\hline ADJ 8 & $1,2,3$ & $<571 \mathrm{~K}$ & $<298{ }^{\circ} \mathrm{C}$ & & \\
\hline ADJ 9 & 4, 5, 6 (no TL) & & & & \\
\hline
\end{tabular}




\subsection{DTRA Humble Gingko XX-17 tests (August 2015)}

On August 27, 2015, the DTRA and University of Minnesota conducted a temperature measurement test of a 5 pound bare Pentolite charge in the Humble Gingko test chamber (Humble Gingko XX - 17) using CaSO4:Ce,Pr TLparticles. The particles were approximately 6-12 $\mu \mathrm{m}$ in size but there were many clustered groups in addition to individual particles. This report outlines the temperatures predicted by the TL particles.

One of the characteristics of open chambers that we noticed in the August 2014 Humble Gingko test was that individual particles were exposed to a huge range of temperatures. Some particles were completely bleached by extremely high temperatures, whereas others had minimal temperature exposure. (Note that this characteristic is not seen in closed chamber tests, such as at Indian Head, where the particles show largely similar temperature exposures.) Since bleached particles do not contribute to $\mathrm{TL}$, the remaining luminescence artificially selected lower temperature particles - ideal for agent defeat, but problematic for measuring average explosion temperatures.

To correct this flaw in the August 2015 tests, we also measured the absolute photon count from the debris samples, and then compared this to the photon count obtained when the same samples were re-irradiated. This ratio, referred to as the "TL curve area analysis" or "area analysis" corrects for bleached particles, and we were able to use this technique to correct artificially low temperature predictions using standard TL reconstruction. Note, however, that the temperatures experienced by individual particles (as measured in last year's tests) collected at any given site ranged from under $150{ }^{\circ} \mathrm{C}$ to more than several hundred ${ }^{\circ} \mathrm{C}$. There is no single temperature that can be quoted as the "explosion temperature". Each type of particle, TL or otherwise, will quote temperatures in its measurement range because that is the fraction of temperature pathways that the particles inherently select.

TL material was placed at 5 locations within the Humble Gingko Test Chamber. Each TL material source consisted of $3 \mathrm{~g}$ of $\mathrm{CaSO}_{4}$ : $\mathrm{Ce}$, $\mathrm{Pr}$ that had been irradiated with $240 \mathrm{~Gy}$ from a ${ }^{137} \mathrm{Cs}$ gamma source. The particles had been filtered with a $13 \mathrm{~mm}$ sieve but the particles tended to agglomerate after filtering, so there were many larger "particles" as well. See attached data in a following slide. After the detonation, debris was collected from a number of sites in and around the chamber. Depending on the amount of debris, between $2 \mathrm{~g}$ and $100 \mathrm{~g}$ was collected per site. Outside the chamber, much of this debris was unrelated debris such as wood chips, so the results herein are mainly concerned with debris collected from within the chamber. (Note, however, that external sites usually also showed TL.) All TL measurements were taken with a Hoya U340 optical filter.

As a final note, the time reconstruction of $\mathrm{CaSO}_{4}: \mathrm{Ce}, \mathrm{Pr}$ is not as precise as with $\mathrm{LiF}: \mathrm{Mg}, \mathrm{Ti}$, and in about half of the reconstructions, the time constant was set to $50 \mathrm{~ms}$. With LiF:Mg,Ti this would have been unnecessary, as shown in the data from the closed chamber Indian Head tests. Howard Ross of the DTRA has sent the DTRA thermocouple data from the test. The adjacent room thermocouple data and microparticles data match very closely. This represents a situation where the temperatures were primarily low enough that none of the particles were bleached; therefore, no further particle analysis was necessary. The source room temperatures, however, were high enough that the top end temperatures, $600{ }^{\circ} \mathrm{C}+$, bleached many particles. Therefore an area analysis was made to correct for this. Note that the high-temperature-corrected analysis matched the high temperature thermocouple measurements and the low-temperature distribution matched the low temperature thermocouple measurements, just as would be expected for properly operating particles. All of this data was presented before the thermocouple results were known! 
The results obtained are presented in Table 23 and comparison with thermocouple data are presented in Fig. 160. Examples of the temperature reconstructions are presented in Fig. 161.

Table 23. Humble Gingko XX results for $\mathrm{CaSO}_{4}: \mathrm{Ce}, \mathrm{Pr}$.

Site $\quad \mathrm{T}_{\max }\left({ }^{\circ} \mathrm{C}\right) \quad$ Time constant (ms)

SRC Site $1 \quad 372 \quad 50$

SRC Site $2 \quad 420 \quad 133$

SRC Site $3 \quad 436 \quad 50$

SRC Site $4 \quad 463 \quad 50$

SRC Site $5 \quad 455 \quad 50$

ADJ Site $1259 \quad 16$

ADJ Site $2193 \quad 249$

ADJ Site $3 \quad 211 \quad 353$

ADJ Site $4 \quad 219 \quad 178$

ADJ Site $5 \quad 250 \quad 51$

ADJ Site $6 \quad 236 \quad 108$




\section{Adjacent Room Measurements HUMBLE GINGKO XX}
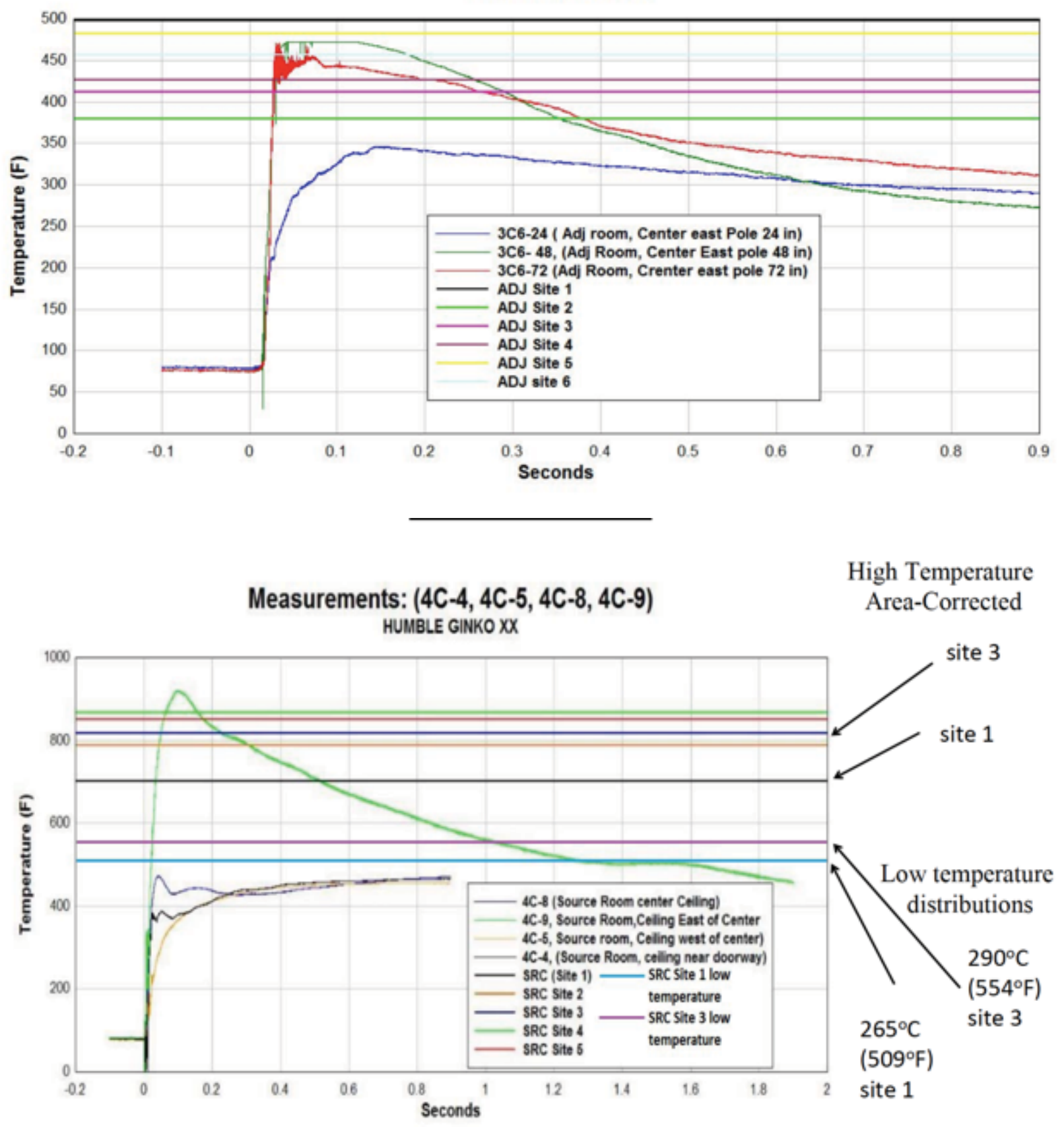

Fig. 160. Comparison of microparticle measurements with DTRA-acquired thermocouple data. 

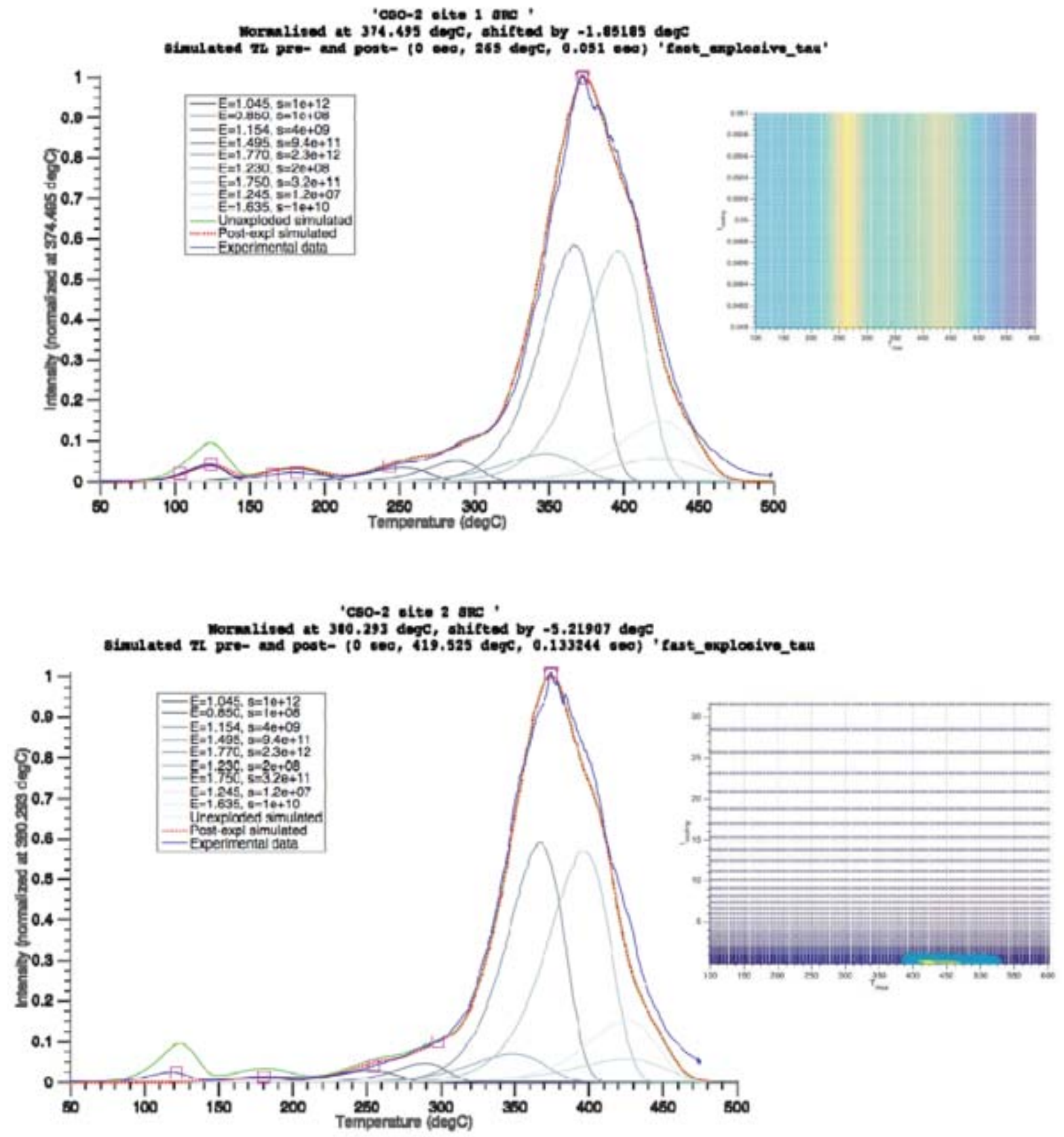

Fig. 161. Examples of temperature determination for the Humble Gingko XX tests.

\subsection{Shock-tube tests at University of Illinois Urbana-Champaign (Jan. 2013)}

\subsubsection{OSU results}

TL materials synthesized at OSU and commercial LiF:Mg,Ti were subjected to controlled detonation tests at University of Illinois Urbana-Champaign (UIUC) by Dr. Nick Glumac's group. OSU provided three 
types of TL materials to be tested: $\mathrm{Li}_{2} \mathrm{~B}_{4} \mathrm{O}_{7}: \mathrm{Cu}, \mathrm{Ag}(\mathrm{LBO}), \mathrm{MgB}_{4} \mathrm{O}_{7}: \mathrm{Li}, \mathrm{Dy}(\mathrm{MBO})$, and $\mathrm{CaSO}_{4}: \mathrm{Ce}, \mathrm{Tb}$ (CSO). Prior to the UIUC detonation tests, the materials were tested in our laboratory for repeatability, light sensitivity, signal stability, morphology, effect of grain size, and effect of alcohol. Before shipping, the samples were irradiated with a dose of $\sim 500 \mathrm{~Gy}$ (beta rays).

After the detonation tests, the samples were returned to OSU in alcohol solution. For the TL readouts, the samples were centrifuged and deposited in stainless steel cups for drying. After the first TL readout, the samples were re-irradiated with the same dose ( $500 \mathrm{~Gy}$ ) and the "regenerated TL" was read. This regenerated TL provided information on the amount of TL material recovered from the alcohol solution and deposited in the samples cups. This "regenerated TL" also provided information as to whether the TL from the material is consistent or not with exposure to a temperature profile.

Of the three tested materials, only MBO resulted in TL signal from "exposed" samples with sufficient intensity for analysis. LBO exhibited weak TL and in most cases the recorded TL curves were not reproducible from sample to sample. In the case of CSO, we obtained a regenerated signal, indicating that material was recovered. However, no meaningful TL signal was registered for the "exposed" samples.

In the case of MBO the TL curves showed considerable intensity and were further analyzed to determine the temperature using algorithms developed for these tests. The results, however, showed inconsistencies that we could not explain by the effect of temperature only. One hypothesis for the inconsistent results obtained is that the TL was affected by the washing procedure or by the storage in solvent. We had preliminary results indicating that the samples survive in alcohol for $24 \mathrm{~h}$, but discussion with Dr. Glumac clarified that the washing procedure involves a distilled water wash of the chamber.

Our results highlight the need to develop a methodology to recover the samples without altering the TL signal. A detailed report on these experiments was presented to DTRA.

\subsubsection{UMN results}

In January of 2013, we prepared TLD-100 powder samples with particle sizes of $10 \mu \mathrm{m}$ or less, filtered using a nylon membrane (CellMicroSieve) and with isopropyl alcohol (subsequently evaporated off) as a carrier, for tests performed by Prof. Nick Glumac of the UIUC. Four samples were returned, labeled as the annealed and unannealed results of two tests. None of these returned samples exhibited TL recognizable as TLD-100, even after gamma re-irradiation, indicating that handling induced some sort of chemical change in the particles. 


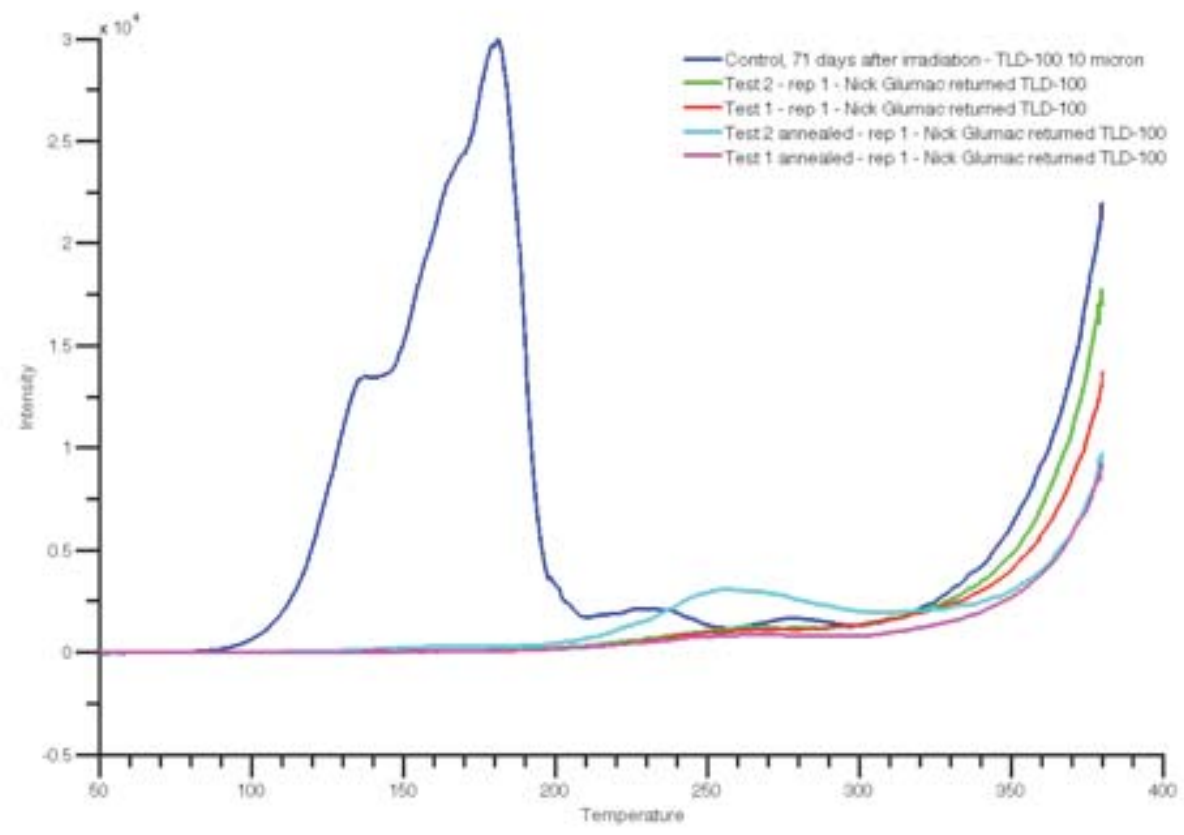

Fig. 162. TL curves for the four samples returned by Nick Glumac's group, compared to a self-held control sample. Although the samples may exhibit luminescence, it is not readily interpretable as any of the known TLD-100 peaks. Curves are plotted without normalization or temperature shift.

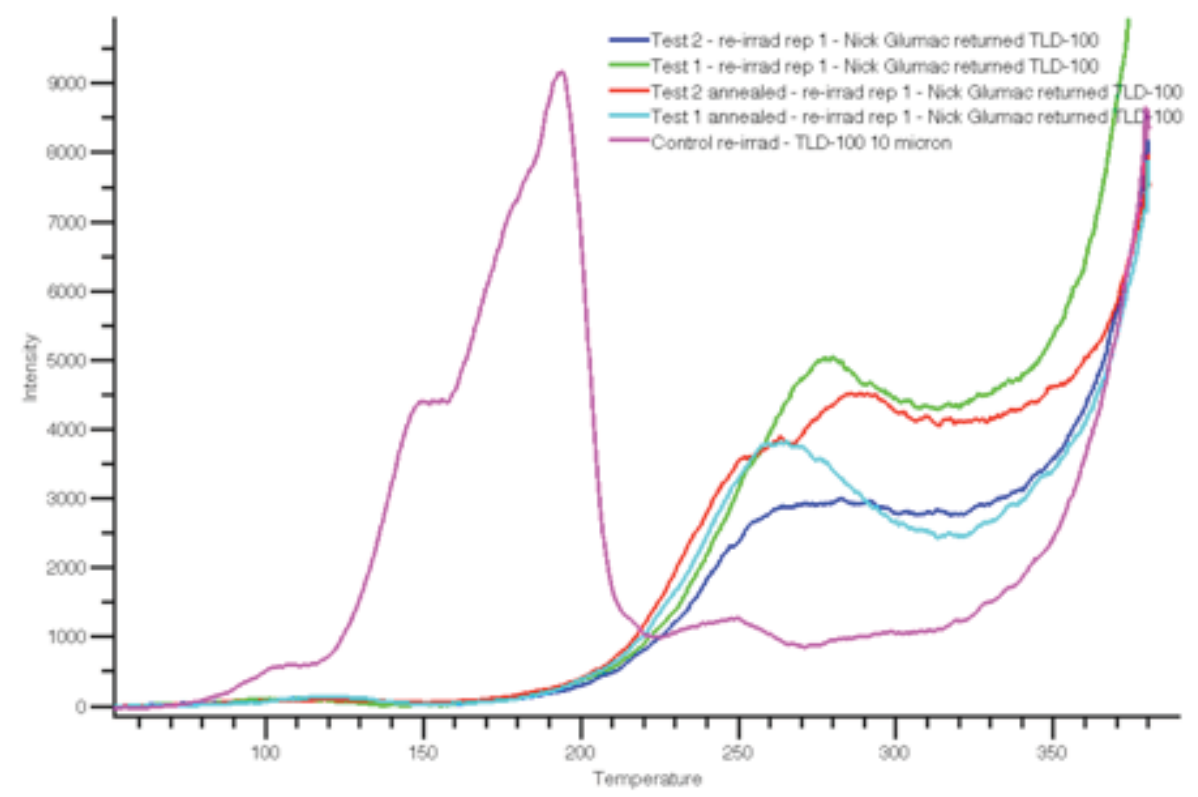

Fig. 163. TL curves for the four samples after re-irradiation. Luminescence has grown stronger, but it is now even less recognizable as TLD-100. Curves are plotted without normalization or temperature shift.

Energy dispersive spectroscopy (EDS) was performed as a rapid, low-precision composition test to rule out the possibility of our not having received the intended return samples. Fluorine-not the most common of contaminants-was readily detectable in all tested returned samples and a self-held control, but not in the SEM carbon tape or in debris of visibly different morphology found in the return 
vials, suggesting that the sample was indeed some form of the original samples we had prepared. Testing for Mg and Ti was inconclusive, as EDS is probably not sensitive enough technique to detect the expected concentrations of these dopants; Li cannot be detected using this instrument. (We were aware of these drawbacks before performing the analysis, but proceeded because the characterization adds very little overhead to the planned SEM imaging.)

(a) Control

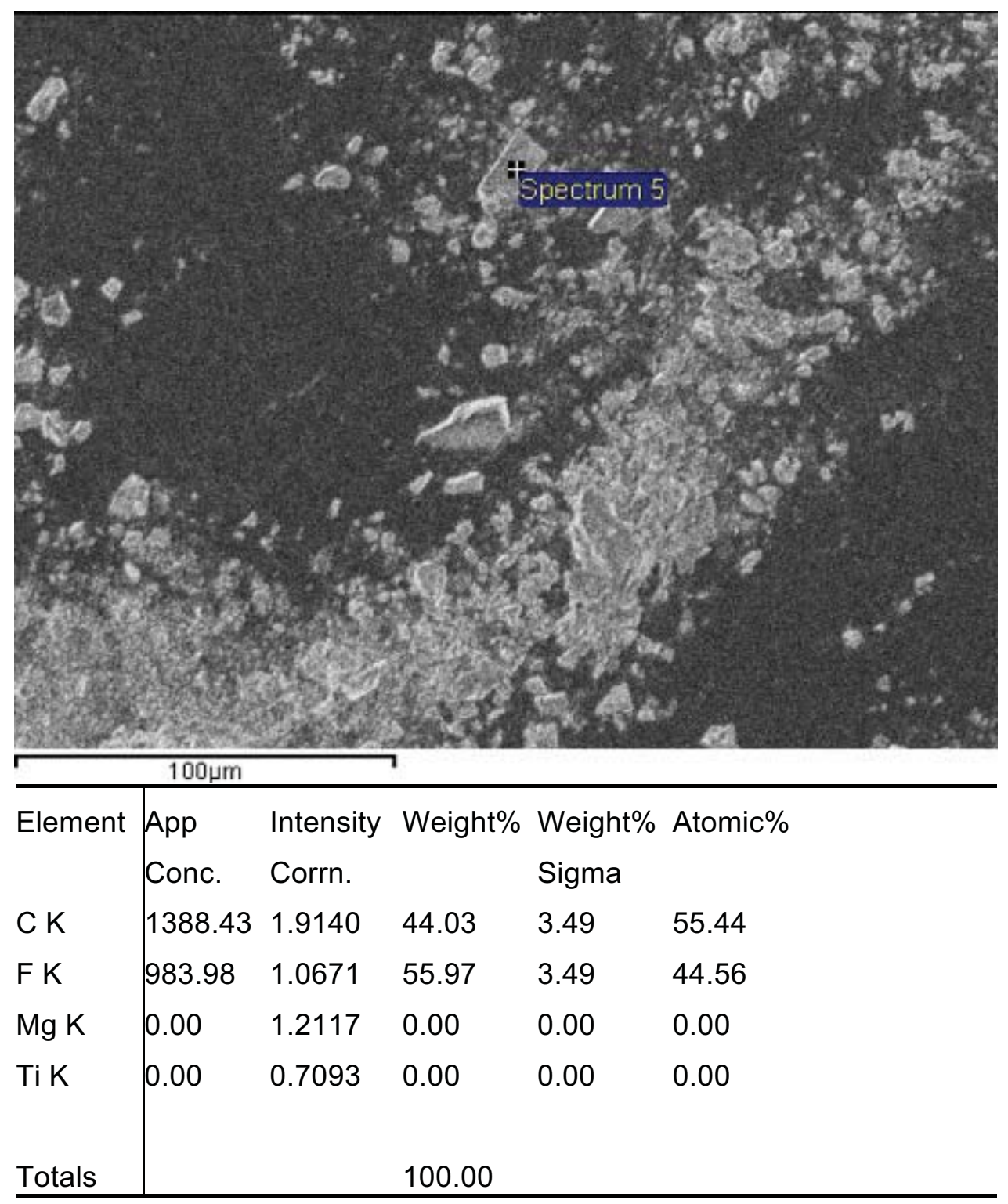

(b) "Test 1" 


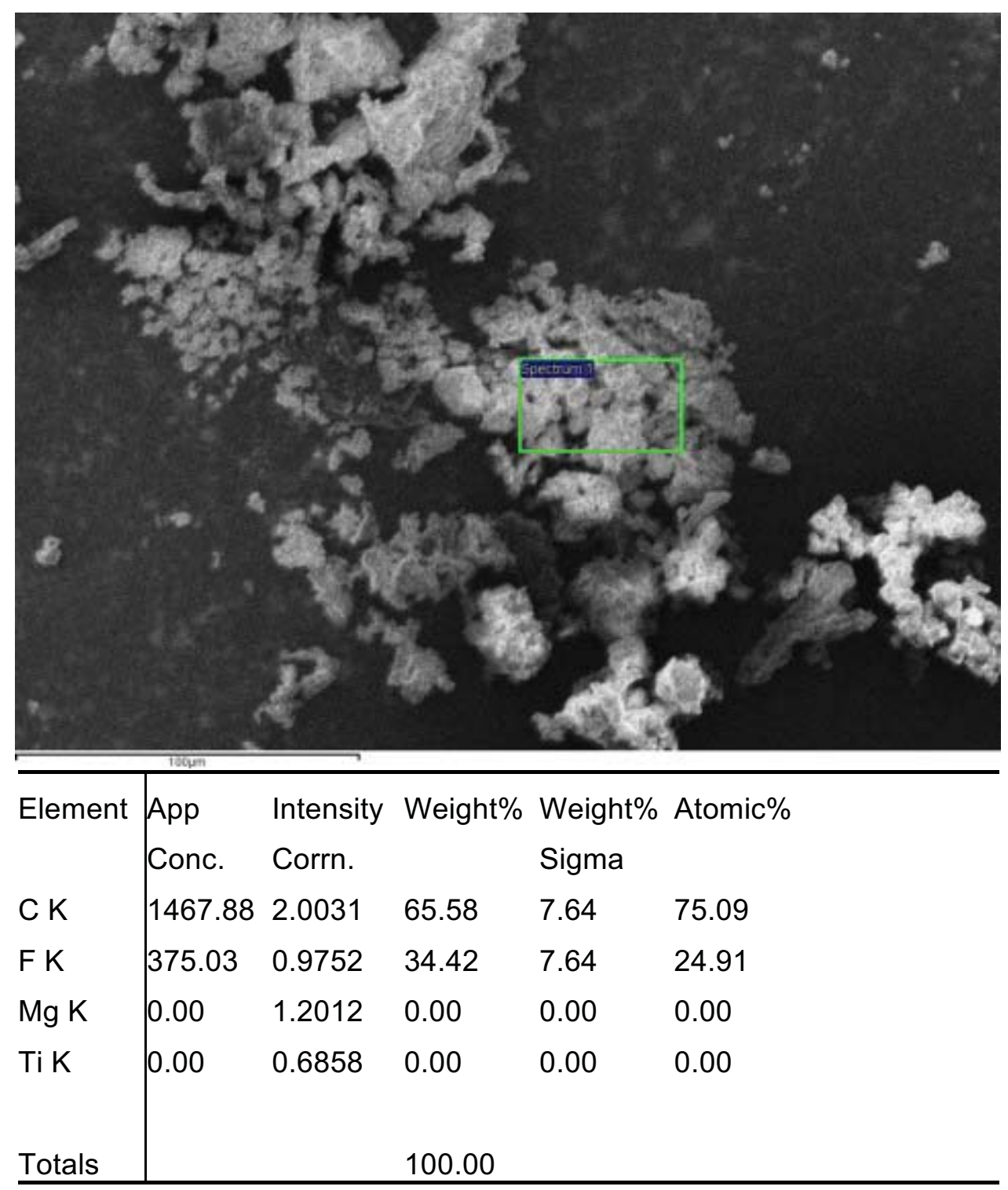

Fig. 164. SEM image, and EDS compositional analysis from the pictured portion of the sample, of a self-held control versus one of the returned samples. The dark background in both cases is carbon tape, tested negative for fluorine.

The samples were returned in an unidentified solvent, evaporated prior to TL curve collection. Control TLD-100 particles showed no unusual TL degradation or change when exposed to this solvent, even after over two months of immersion. Prior testing indicated that the luminescence of the TLD-100 is not affected by exposure to IPA. The samples were also exposed to water during the course of Prof. Glumac's experiment.

\subsection{Shock Tube tests at University of Illinois - Urbana Champaign (Oct. 2014)}

Shock tube tests were conducted at the University of Illinois at Urbana Champaign (UIUC) shock tube facility to investigate various material collection methods, as well as the performance of TL materials as particle temperatures sensors in a high temperature - short time scale ( $\sim 2-5 \mathrm{~ms}$ ) heating environment. Particles tested include three materials developed at OSU [4], $\mathrm{Li}_{2} \mathrm{~B}_{4} \mathrm{O}_{7}: \mathrm{Cu}_{0.4 \%}, \mathrm{Ag}_{0.1 \%}$ ( $(\mathrm{BOO})$, 
$\mathrm{MgB}_{4} \mathrm{O}_{7}: \mathrm{Dy}_{0.1 \%}, \mathrm{Li}_{1 \%}(\mathrm{MBO}), \mathrm{CaSO}_{4}: \mathrm{Ce}_{0.2 \%}, \mathrm{~Tb}_{0.2 \%}$ (CSO), and commercially available LiF:Mg,Ti (TLD-100) supplied by UMN. OSU materials were sieved using a $35 \mu \mathrm{m}$ mesh size prior to testing.

Shock tests took place a shock tube located at the UIUC Shock Tube Facility. Details of the shock tube testing environment and operational procedures are discussed elsewhere [70, 71]. On-site temperature measurements supplied by UIUC were obtained using a NASA Chemical Equilibrium with Applications (CEA) program, which uses the shock wave velocity as an input. The velocity is determined by knowledge of the distance traveled and time of arrival of the shock front as measured by internal pressure sensors.

A total of 11 shock tests were conducted at the UIUC Shock Tube Facility. Testing parameters are detailed below in Table 24. Two temperatures were investigated for each material; a low temperature of $\sim 650 \mathrm{~K}$ and high temperature of $\sim 850 \mathrm{~K}$. Temperatures were chosen such that for each material used at least one heating would fall within the range of application for each material as determined by pretest TL curve modeling.

Two collection methods were investigated; a bubbling/wash-down method and collection by adhesion to a cooled heat sink. The bubbling/wash down (henceforth referred to as wash down) involves bubbling posttest gas through water in collection beaker along with washing collected material down the sides of a dump tank. The second method employs a cooled (dry ice) heat sink to catch particles at end of the shock tube before they disperse in a large dump tank. The wash down method involves extended ( $24 \mathrm{~h}$ ) exposure to water and ethanol, whereas the heat sink does not.

OSU received samples from UIUC $\sim 2$ months following the test at UIUC. Material obtained through the wash down method was fibrous in nature, assumed to be the result of residual material left by Kimwipes during shock tube cleaning procedures. No visible TL material was present on the heat sinks, however rinsing the heat sinks with ethanol into a collection vial allowed for sufficient material to be obtained for TL measurement.

TL measurements and analysis was conducted at OSU according to established procedures $[10,11]$. TL models for TLD-100 were obtained by deconvolution into five $1^{\text {st }}$ order TL peaks using TL fit [72]. Representative experimental TL curves for all materials and shots tested can be found in Fig. 165.

Temperature reconstruction followed previous methods [10], using a constant heating profile with a time scale of $5 \mathrm{~ms}$. In the event of insufficient depletion of the TL curve a range of applicability was established using $10 \%-90 \%$ residual TL area for the TL materials. In the case of CSO a physical interpretation of the low temperature TL peaks was used. The temperature reconstruction results are found in Table 24. 

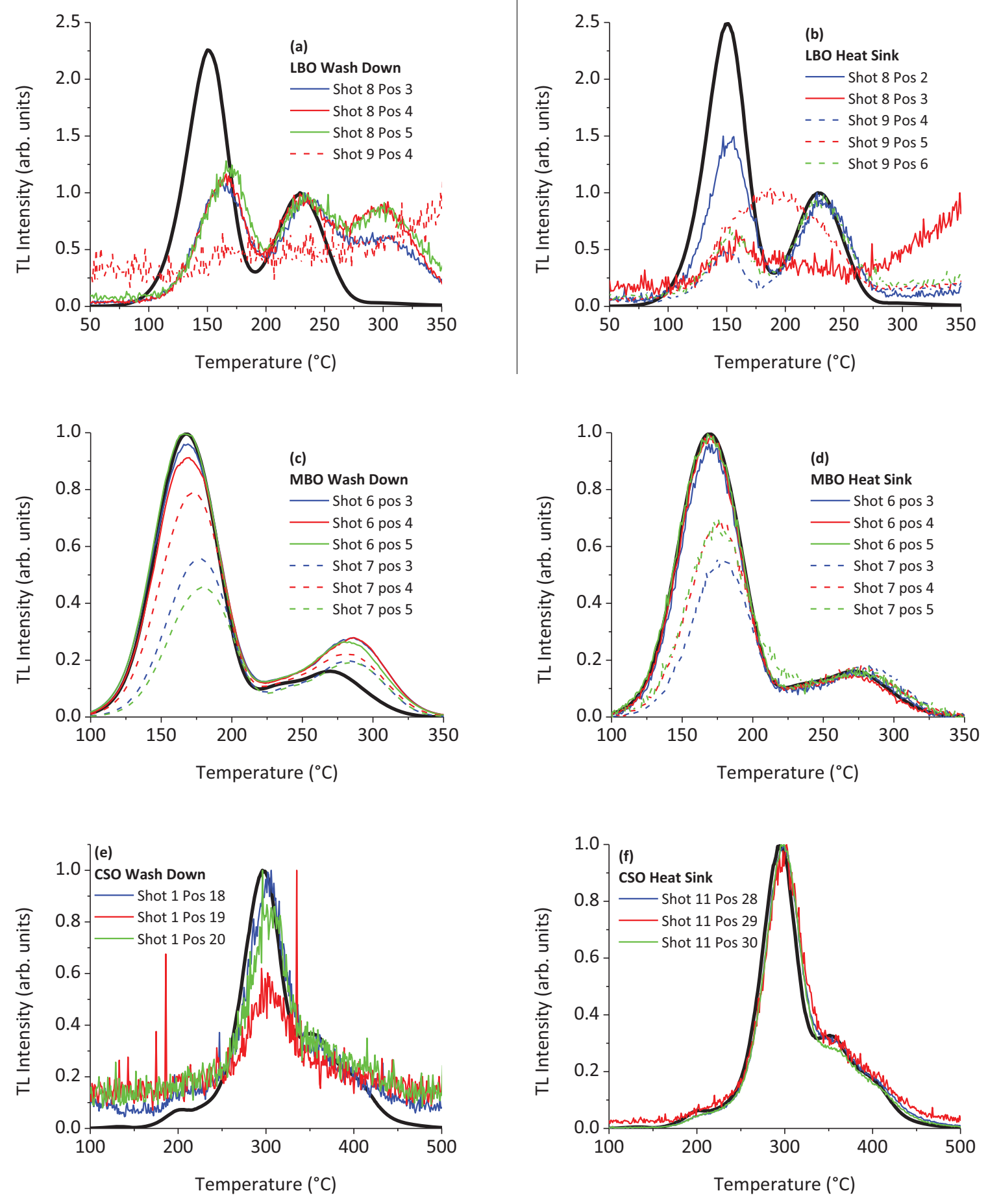

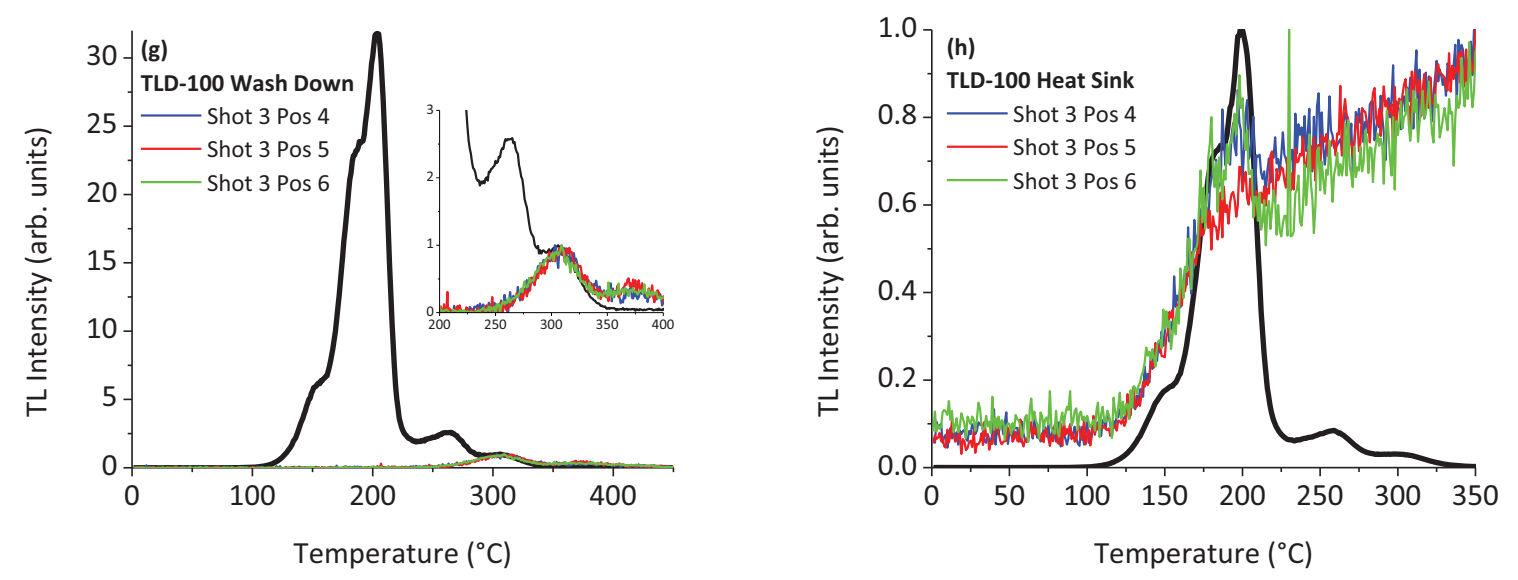

Fig. 165. "As-Received" experimental TL curves from materials tested at the UIUC shock tube facility for a low ( $\sim 50 \mathrm{~K}$ ) and high ( $\sim 850 \mathrm{~K}$ ) shock test. Curves on the left represent material collected by the wash down method, whereas curves on the right were obtained via a heat sink. Black curves represent control (unheated material) curves.

Table 24. Summary of testing parameters and heating results obtained from temperature reconstruction. Onsite temperature results were provided by UIUC and are listed as CEA (K).

\begin{tabular}{|llllll|}
\hline Shot \# & Material & Mass $(\mathbf{m g})$ & Wash Down (K) & Heat Sink (K) & CEA (K) \\
\hline $\mathbf{1}$ & CSO & 10 & Insufficient & Insufficient & 635 \\
\hline $\mathbf{2}$ & CSO & 10 & Insufficient & Insufficient & 850 \\
\hline $\mathbf{3}$ & TLD-100 & 10 & Beyond Range & N/A & 650 \\
\hline $\mathbf{4}$ & TLD-100 & 49 & N/A & N/A & 650 \\
\hline $\mathbf{5}$ & TLD-100 & 42 & N/A & N/A & 840 \\
\hline $\mathbf{6}$ & MBO & 35 & Insufficient & Insufficient & 650 \\
\hline $\mathbf{7}$ & MBO & 37 & $561 \pm 16$ & $560 \pm 7$ & 840 \\
\hline $\mathbf{8}$ & LBO & 29 & $545 \pm 2$ & 530 & 650 \\
\hline $\mathbf{9}$ & LBO & 35 & N/A & $565 \pm 4$ & 830 \\
\hline $\mathbf{1 0}$ & CSO & 25 & Insufficient & Insufficient & 630 \\
\hline $\mathbf{1 1}$ & CSO & 40 & Insufficient & $550 \mathrm{~K}<\Theta<630 \mathrm{~K}$ & 830 \\
\hline
\end{tabular}

The temperatures were consistent between collection methods. All OSU materials (MBO, LBO, and CSO) measured approximately the same temperature for the $~ 850 \mathrm{~K}$ heating, indicating a level of consistency between TL material temperature measurements, but temperatures obtained were consistently below those determined by the NASA CEA program.

Table 24 indicates that material was recovered in 8 of the 11 tests for both collection methods, but a temperature was not recovered in all cases. The majority of TL data was weak in intensity and collection of regenerated TL curves from test material failed to confirm the presence TL material (except MBO). Intensities of the as-received TL curves were similar between collection methods indicating that approximately the same amount of material was collected regardless of method. The heat sink method avoids prolonged exposure to water and ethanol as well as contamination from within the dump tank; however the delay in material return for analysis inadvertently kept materials in presence of a small amount of water for $\sim 2$ months. If future tests are to be repeated it is suggested 
that only the heat sink method be used, and material analyzed shortly after testing to minimized environmental effects.

The difference between temperatures obtained by TL materials and those found by the CEA program is of interest for future research. The consistency between temperatures obtained by OSU materials for the $~ 850 \mathrm{~K}$ tests seems to indicate the materials did measure the correct temperature for a small particle incident upon a shock wave. The discrepancy between the TL and CEA temperatures might indicate a slow thermal response time of TL material due to particle size, although previous calculations showed that, for a spherical CSO particle with a $100 \mu \mathrm{m}$ radius, the heating scale was $\sim 6 \mathrm{~ms}$. This calculation did not account for forced heating in a turbulent environment.

Furthermore it may be useful to understand how the material travels through the final $2 \mathrm{~m}$ of the shock tube with respect to the shock wave: do particles outpace the wave or do they sit behind in the high temperature region? Understanding these questions would be helpful in understanding how the TL materials behave in larger scale outdoor tests.

It is suggested that further testing at the UIUC shock tube facility concern itself with only the material obtained using the heat sink collection method. Though less material is collected from this method the reduction in exposure to elements is important to isolate effects on the TL curve and avoid contamination of regenerated TL curves. To overcome the weaker TL signal that results from the amount of material recovered from the heat sink it is suggested a higher dose (>500 Gy) is used during material preparation. Section 4.3.3 of this report discusses synthesis procedures conducted at the OSU laboratory that demonstrate that MBO and CSO can be obtained with particle sizes $<13 \mu \mathrm{m}$. One concern previously raised is that of the particle size reducing the TL material's ability to respond quickly ( $\sim \mathrm{ms})$ to a heating event. It is suggested that future studies investigate the effect of particle size using the new synthesis procedures developed at OSU. 


\section{OTHER DEVELOPMENTS}

\subsection{Development of low thermal emission heaters for high temperature TL analysis}

Micromachined heaters have applications in a wide variety of high temperature processes such as carbon nanotube growth [73], gas sensing [74-76], microfluidics [77] and measuring TL [15, 24, 78].

Unfortunately, traditional microheater designs are incompatible with measurements of low levels of light at high temperatures. Measuring faint signals of luminescence at elevated temperatures can be extraordinarily difficult because of the large amount of thermal background radiation emitted from heating elements. Typically, to mitigate background emission when monitoring material fluorescence, one must use a cutoff filter that blocks all but the shortest wavelengths, leaving only high energy UV fluorescence centers to be probed at very high temperatures, and even these are swamped when heating elements reach temperatures beyond several hundred ${ }^{\circ} \mathrm{C}$.

In this report, two methods are described to create microheaters that emit very little background radiation. The first method alters the heater design such that a large central region is composed solely of infrared transparent materials. Kirchoff's law, which states that the thermal emission of a material is exactly equal to its absorption, requires that the background emission be negligible. In theory, this central region could also be composed of highly reflective materials, but metals become more emissive at higher temperatures. The second method applies Kirchoff's law to a multilayer stack on the top and bottom of the microheater. The multilayer stack has a high reflectivity in the wavelength range of interest, therefore it can change the emissivity of the microheater/multilayer body in that spectral range [79].

The first method of using infrared-transparent materials in the center of a heater with an aperture to block the emission from the heating coils has the advantage of being able to block all wavelengths from the thermal radiation of the metal heating element. While in the second method the multilayer structure can only block a range of wavelengths depending on the thickness of the layers in the stack and the number of layers. One disadvantage to the first method is that it requires an aperture to be properly aligned with the center of the microheater and, since the center of the microheater does not have a heating element, there will be non-uniform heating from the center to edge of the heater. The multilayer microheater has uniform heating and does not require any careful alignment.

\subsubsection{Microheater design and fabrication}

The microheaters used in this study are composed of platinum and alumina, materials that have been used for other high temperature microstructures $[80,81]$. In Ref. [80] a Pt paste was deposited on an $\mathrm{Al}_{2} \mathrm{O}_{3}$ substrate and patterned using laser sintering. The method used in [81] was to deposit $\mathrm{Al}_{2} \mathrm{O}_{3}$ by reactive sputtering and then deposit Pt by DC magnetron sputtering. Both of these methods result in a microheater that operates without being released from the substrate. In the work presented here they consisted of $300 \mu \mathrm{m} \times 300 \mu \mathrm{m}$ and $500 \mu \mathrm{m} \times 500 \mu \mathrm{m}$ alumina platforms suspended by four legs with a platinum heating element on the platform. $\mathrm{Al}_{2} \mathrm{O}_{3}$ was chosen because of its high melting point and low thermal conductivity. Platinum was used as the heating element because of its relatively high melting point and linear temperature coefficient of resistance (TCR) over a wide range of temperatures. This design allowed the microheaters to be thermally isolated, so they could reach high temperatures with a small amount of current. The support leg length and cross sectional area were chosen to reduce the thermal conductance losses while maintaining mechanical stability. At very high temperatures, the power loss due to radiative emission can exceed the power thermally conducted to the substrate via the support legs. 
The fabrication process for the microheater begins by depositing $100 \mathrm{~nm}$ of $\mathrm{Al}_{2} \mathrm{O}_{3}$ onto a standard $\mathrm{p}$ type (100) 4 in. silicon wafer using atomic layer deposition (ALD). The heating element consisting of $5 \mathrm{~nm}$ of titanium and $50 \mathrm{~nm}$ of platinum is deposited using DC magnetron sputtering and patterned using a lift-off process. The $5 \mathrm{~nm}$ of titanium is to promote adhesion of the heating element to the $\mathrm{Al}_{2} \mathrm{O}_{3}$ film. The next layers are $10 \mathrm{~nm}$ of titanium and $200 \mathrm{~nm}$ of gold for the contact pads; these are also patterned using a lift-off process. The wafer is then encapsulated in $20 \mathrm{~nm}$ of $\mathrm{ALD} \mathrm{Al}_{2} \mathrm{O}_{3}$ to protect the devices from further processes and from the environment during testing. The platform and support legs are defined by etching through the $120 \mathrm{~nm}$ of $\mathrm{Al}_{2} \mathrm{O}_{3}$ by using a reactive ion etch (RIE) consisting of a $\mathrm{BCl}_{3}$ plasma. The same RIE process is used to etch through $20 \mathrm{~nm} \mathrm{Al} \mathrm{O}_{3}$ to make vias to the gold contact pads. The devices are released by doing a through etch of the wafer from the backside using the Bosch process. A summary of the process is shown in Fig. 166.
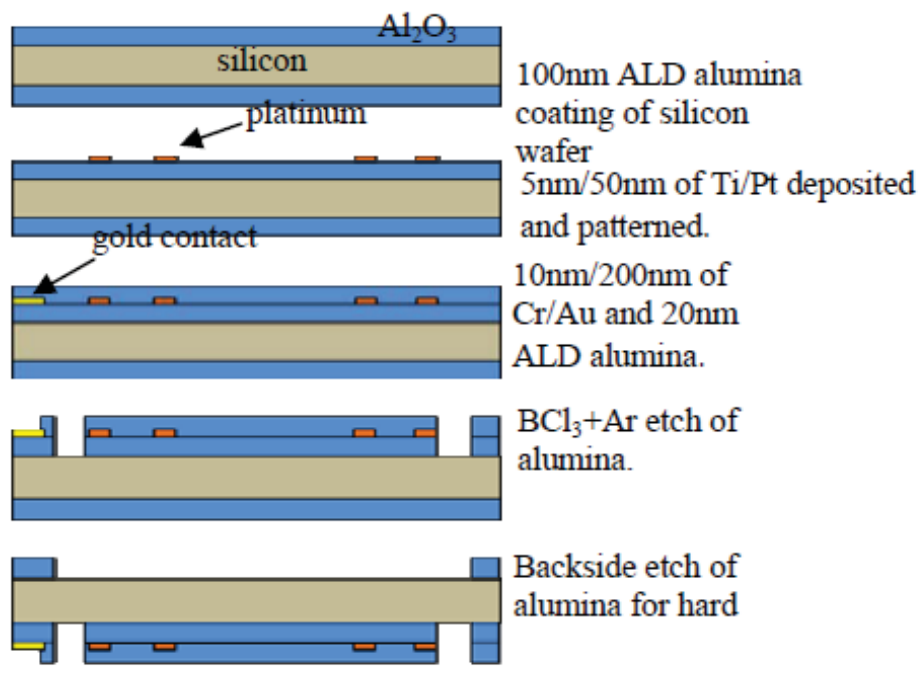

Backside etch of alumina for hard

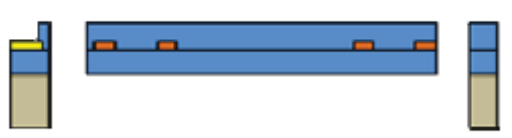

Bosch etch to release device.

Fig. 166. Infrared-transparent microheater process flow as described in the device fabrication section.

\subsubsection{Thermoluminescent materials}

TL was chosen to demonstrate the reduction in thermal emission because it is generally only weakly luminescent relative to current injection processes, such as LED emission. TL materials require thermal energy to excite charge carriers out of traps so that they can recombine with the oppositely charged carriers and emit photons. These characteristics make it useful to demonstrate how thermal emission affects the measurement of luminescence at high temperatures. TL materials often have deep level traps in their band gap, potentially occupied with either electrons or holes depending on the nature of the trap. Ionizing radiation such as UV light, X-rays, or gamma radiation is used to fill the deep level traps in the band gap. The traps of interest are thermally stable at room temperature but can be emptied at higher temperatures usually above $100{ }^{\circ} \mathrm{C}$. When a carrier leaves a trap, it can recombine and emit a photon. The TL intensity is measured by heating the material with a linearly increasing temperature and measuring the luminescence. 
We use two TL materials in this study. LiF:Ti,Mg (TLD-100) is a material that is used in the characterization of transparent microheaters using an aperture. LiF:Ti, Mg has a wide emission spectrum, which works well with the transparent microheaters because the aperture allows all wavelengths of emission to be measured while reducing thermal emission from the metal heating element. The TL material used for the microheaters with the multilayer intereference structure was $\mathrm{CaSO}_{4}: \mathrm{Ce}, \mathrm{Tb}$. The $\mathrm{CaSO}_{4}: \mathrm{Ce}, \mathrm{Tb}$ has sharp spectral emission peaks over a wavelength range where the multilayer structure can be designed to have lowered thermal emission. The spectral peak at $486 \mathrm{~nm}$ was chosen to for the design of the multilayer since it is the strongest emission peak.

\subsubsection{Multilayer design and fabrication}

The multilayer design was based on a distributed Bragg reflector (DBR). The DBR coating consisted of alternating $\lambda / 4$ layers with different indices of refraction, where $\lambda$ was the wavelength of light that

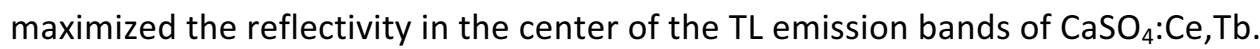

The reflectivity of a DBR was strongly dependent on the difference between the indices of refraction of the quarter-wave layers and the number of quarter-wave pairs. Ideally, one could use any number of pairs, but stress and processing issues made two pairs a good compromise between reflectivity and ease of fabrication. The two materials used were $\mathrm{Al}_{2} \mathrm{O}_{3}$ and $\mathrm{TiO}_{2}$ with refractive indexes of 1.78 and 2.62 at $490 \mathrm{~nm}$ respectively, measured using a spectroscopic ellipsometer. The spectroscopic ellipsometer measurement was done for wavelengths between $260 \mathrm{~nm}$ to $900 \mathrm{~nm}$. The $\mathrm{TiO}_{2}$ showed non-zero values for the imaginary part of its index of refraction. The peak reflectivity was chosen to be centered at a wavelength of $490 \mathrm{~nm}$, corresponding to $\mathrm{Al}_{2} \mathrm{O}_{3}$ and $\mathrm{TiO}_{2}$ thicknesses of $68.6 \mathrm{~nm}$ and $46.7 \mathrm{~nm}$ respectively. The $\mathrm{Al}_{2} \mathrm{O}_{3}$ and $\mathrm{TiO}_{2}$ were deposited onto the released microheaters by ALD. The ALD conformally coated both the top and bottom of the microheater, and, since the top of the microheater was already coated in $20 \mathrm{~nm}$ of $\mathrm{Al}_{2} \mathrm{O}_{3}$, a reduced thickness of $48.6 \mathrm{~nm}$ of $\mathrm{Al}_{2} \mathrm{O}_{3}$ was deposited as the first layer. The bottom of the microheater already had $100 \mathrm{~nm}$ of $\mathrm{Al}_{2} \mathrm{O}_{3}$ so the first layer of the DBR on the bottom was $80 \mathrm{~nm}$ thicker than a simple DBR design. Table 25 depicts the layer structure in the final device.

A simulation was done of the absorption using the transfer-matrix method [82] with indices of refraction extracted from data taken from a spectroscopic ellipsometer. Using Kirchoff's law of thermal radiation, the emissivity of the multilayer structure is known because the emissivity of a body is equal to its absorptivity. A plot of the emissivity of the multilayer structure with the platinum is shown in Fig. 167 , along with the emission spectra of $\mathrm{CaSO}_{4}: \mathrm{Ce}, \mathrm{Tb}$. The simulation shows that the emissivity is reduced from 0.47 to 0.08 roughly between $450-600 \mathrm{~nm}$, but this assumes a uniform platinum layer and does not account for the periodic structure of the serpentine resistor. According to this extremely crude model, the thermal emission intensity should be reduced by a factor of about 6 , but again, this ignores the non-emitting open areas and any diffractive or potential plasmonic effects. The DBR center wavelength of $490 \mathrm{~nm}$ was chosen because the simulation showed that it would decrease the emissivity of the microheater over a wavelength span containing the strongest emission peaks of the $\mathrm{CaSO}_{4}: \mathrm{Ce}, \mathrm{Tb}$. 

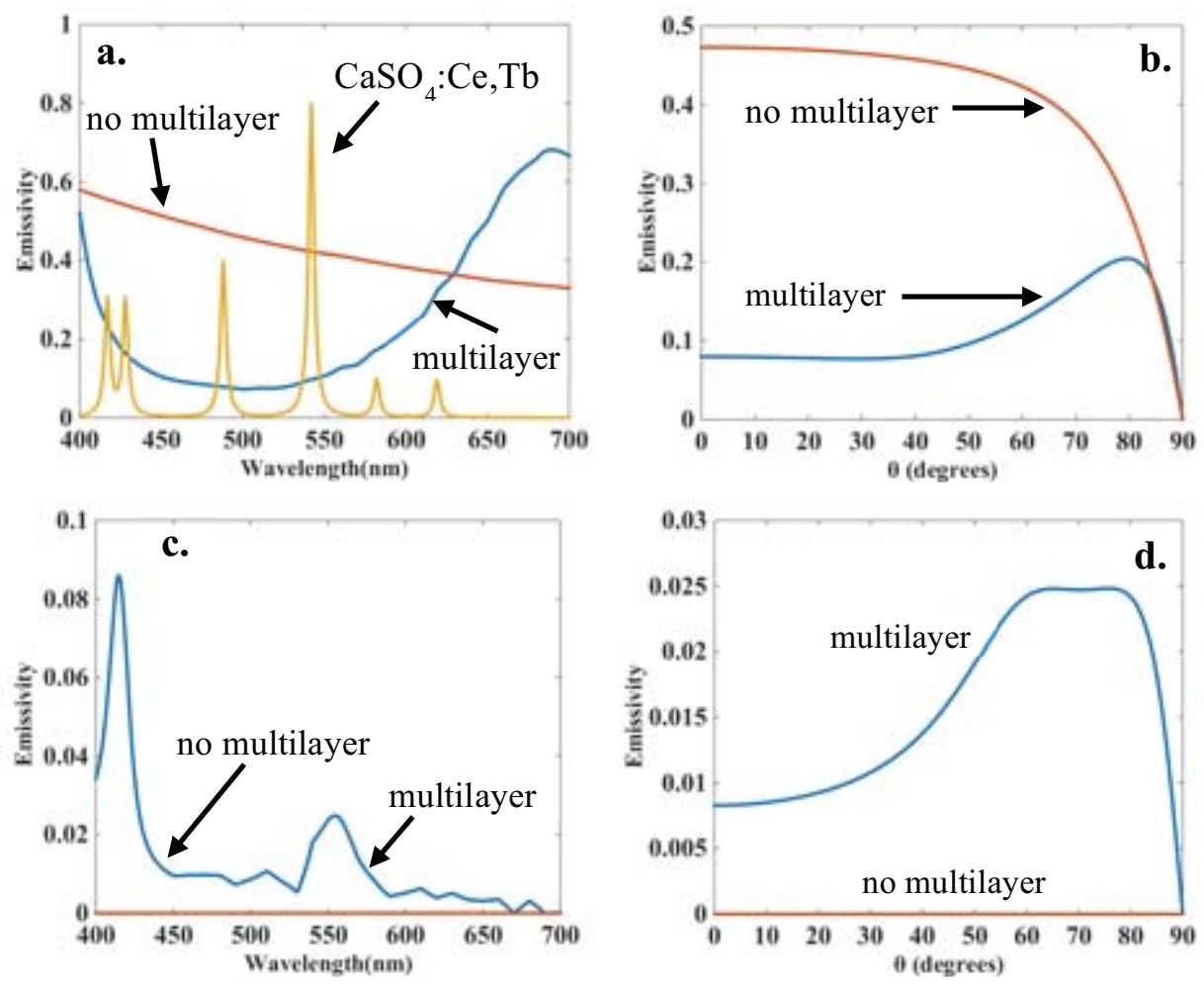

Fig. 167. Simulated emissivity of a microheater with and without a multilayer. a. The approximate emission spectra of $\mathrm{CaSO}_{4}: \mathrm{Ce}, \mathrm{Tb}$ is also shown and the main peaks overlap well with the region of reduced emissivity at an angle of 0 degrees. b. The emissivity at different angles from the microheater at a wavelength of $490 \mathrm{~nm}$. c. The emissivity of the multilayer structure between the platinum lines showing a very low emissivity compared to the metal at an angle of 0 degrees. $d$. The emissivity versus theta for the multilayer between the platinum lines at a wavelength of $490 \mathrm{~nm}$.

\subsubsection{Transparent heater experimental results}

A typical transparent microheater is shown in Fig. 168. The overall device is $500 \mu \mathrm{m} \times 500 \mu \mathrm{m}$ in area and the central transparent region without the heating element is $210 \mu \mathrm{m} \times 210 \mu \mathrm{m}$. The area of the microscope aperture is approximately $4.7 \times 10^{4} \mu \mathrm{m}^{2}$ and the total area of the microheater is $2.5 \times 10^{5} \mu \mathrm{m}^{2}$. The aperture covers about $19 \%$ of the microheater area.

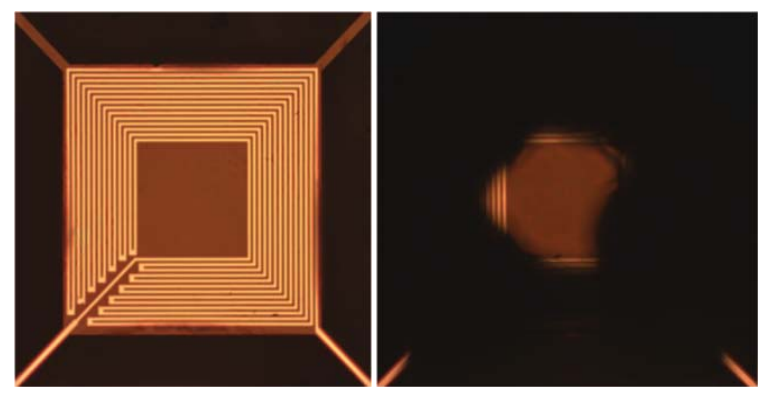

Fig. 168. Typical transparent microheater without aperture (left) with aperture (right). The central region is infraredtransparent. The overall microheater is $500 \times 500 \mu \mathrm{m}$ in area and the center square is $210 \times 210 \mu \mathrm{m}$. The area of the aperture is approximately $4.7 \times 10^{4}{\mu \mathrm{m}^{2}}^{2}$ and the total area of the microheater is $2.5 \times 10^{5} \mu \mathrm{m}^{2}$. The TCR of $2.3 \times 10^{-3} /{ }^{\circ} \mathrm{C}$ was measured by placing the microheater on a thermoelectric cooler/heater to control the temperature and then measuring the 
resistance in the temperature range of $15-60^{\circ} \mathrm{C}$. Current was applied to the microheater to raise its temperature from 50 $400^{\circ} \mathrm{C}$ where the temperature was measured using a thermal camera.

To test the thermal emission, the temperature of the heaters was gradually raised by applying current from a Keithley 2410 sourcemeter. The surface temperature was monitored using a Jenoptik IR-TCM 384 thermal camera with an areal density of 676 image pixels per microheater. The camera measurement had been previously calibrated using the microheater metal as a thermistor to verify accuracy. The microheater was placed on a thermoelectric cooler/heater to control the temperature, and the resistance was measured in the temperature range of $15-60{ }^{\circ} \mathrm{C}$. The resistance versus temperature measurement was then repeated by running current directly through the heating element of the microheater and measuring temperature using a thermal camera to measure temperature in a range of $50-400{ }^{\circ} \mathrm{C}$. The resistances measured during this process were calibrated so that measurements done with the thermoelectric and thermal camera corresponded in their overlap range. The thermal camera has a temperature uncertainty of $\pm 2 \%$. The TCR measured with the thermoelectric heating and the microheater heating was $2.3 \times 10^{-3} /{ }^{\circ} \mathrm{C}$. There was a significant temperature gradient across the microheater from center (lowest) to coil (highest), but we accounted for this using our thermal camera on identical "dummy" heaters with an absorbing region of Ti/Pt in the center. The Ti/Pt in the center was deposited and patterned along with the main heating element but was not electrically connected to the heating coils. The emissivity of the Ti/Pt in the center, however, was the same as the Ti/Pt that was being heated electrically. A comparison of the temperature between the heating element and center was performed with the thermal camera, calibrated to measure the temperature of the heating element. Therefore, when we indicate a temperature on the $x$-axis in Fig. 169 , that temperature was absolute within the limits of our measurement error. Data taken when the aperture was positioned over the center came from a higher current drive, to offset the microheater gradient, than data that was taken when the aperture was positioned over the heater coil. Fig. 169 shows the thermal emission versus temperature for three cases: the microheater emission without any aperture, the emission with the aperture placed over the platinum heater lines, and the aperture placed over the infrared-transparent central region. Note that the thermal emission through the aperture has been reduced by $90 \%$ in moving from the heating element to the infrared transparent region at the same temperature.

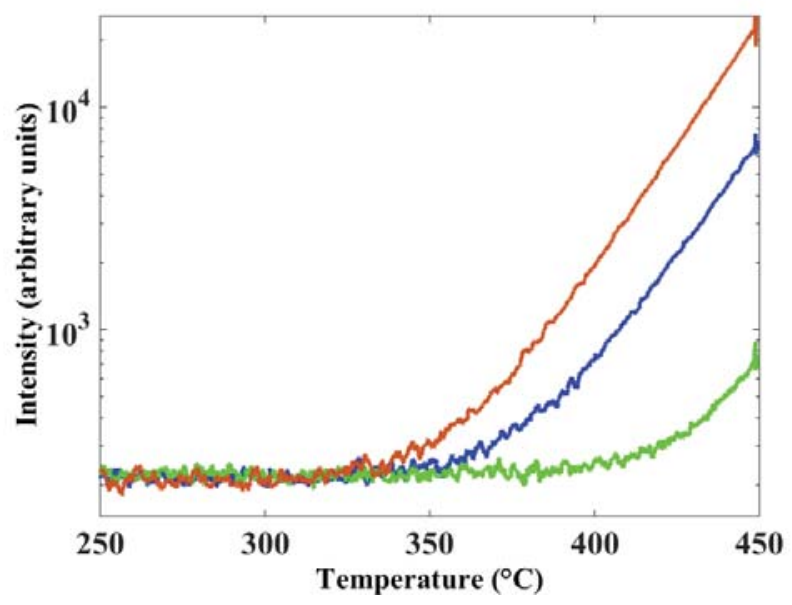

Fig. 169. Comparison of a thermal emission signal from a microheater with 1) no aperture, 2) an aperture offset so it is centered on the heating element, and 3 ) with aperture centered to the middle of the microheater. There is a $97 \%$ reduction 
between the thermal emission intensity with and without aperture. And a $90 \%$ reduction in intensity between the offset aperture on the heating element and the aperture centered on the infrared transparent region.

LiF:Ti,Mg (TLD-100), as discussed previously, is a TL material used as a characteristic low photon number emitter in the characterization of the transparent microheaters. LiF:Ti,Mg particles of $10-$ $20 \mu \mathrm{m}$ in size were distributed across the microheater surface. The TL intensity versus temperature of the LiF:Ti,Mg particles is shown in Error! Reference source not found.. The red curve shows emission from a heater and coils, whereas the green curve shows the emission of a transparent microheater using the aperture. The aperture was placed over the center of the microheater so that the TL of the LiF:Ti,Mg was detected, whereas the thermal emission from the heating element was blocked. At high temperatures the intensity of the thermal emission of the microheater without the aperture becomes comparable to the intensity of the LiF:Ti,Mg TL peak around $150^{\circ} \mathrm{C}$. For higher temperature $\mathrm{TL}$ measurements this thermal emission can obscure or overwhelm the signal making it impossible to take accurate measurements. But when the aperture is placed over the center of the microheater most of the thermal emission is blocked allowing for the possibility of measuring higher temperature TL peaks. All optical measurements were taken using a photomultiplier tube (PMT) because of the weak intensity of the luminescent or thermal emission signals.

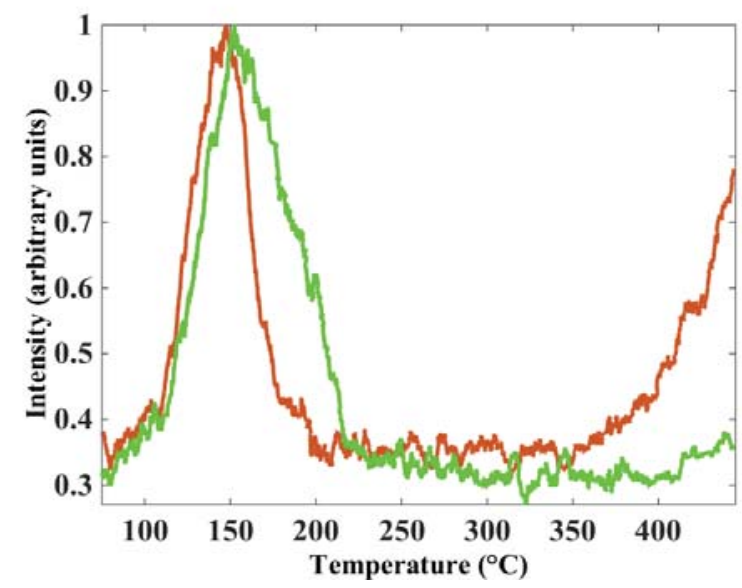

Fig. 170. Normalized LiF:Ti,Mg (TLD-100) TL intensity versus temperature glow curves of 10-20 $\mu \mathrm{m}$ diameter particles on the microheaters. The red curve shows emission from a heater and coils, whereas the green curve shows the emission of a transparent microheater using the aperture. A higher density of particles was placed on the microheater with the aperture to normalize the intensity of the TL emission.

\subsubsection{Multilayer method experimental results}

The microheaters used in the multilayer method are shown with and without the multilayer in Fig. 171. These microheaters have $300 \mu \mathrm{m} \times 300 \mu \mathrm{m}$ dimensions. The temperature dependence of the microheaters platinum resistors was measured before and after multilayer deposition to give TCRs of $2.3 \times 10^{-3} /{ }^{\circ} \mathrm{C}$ and $-4.4 \times 10^{-4} /{ }^{\circ} \mathrm{C}$ respectively. The transition from positive to negative was due to the impact of $\mathrm{TiO}_{2}$ on the resistance. It is inferred from our measurements that the $\mathrm{TiO}_{2}$ is somewhat electrically conductive, and it behaves in the same manner as semiconductors, which tend to have negative TCRs. The TCR was used to determine the drive voltages for the microheaters. The correlation between voltage and temperature was measured by increasing the voltage across the microheater and then measuring the current to determine the resistance, which is related to the temperature by using 
the TCR. A linear increase in voltage corresponds to a linear increase in temperature. The optical measurements were taken by placing the microheater in a dark chamber to reduce the ambient light. A source measurement unit (SMU) was used to source voltage and perform resistance measurements during a linear ramp in temperature, which is standard in TL analysis. A photomultiplier tube (PMT) was used to measure the light emissions from the multilayer structure and $\mathrm{CaSO}_{4}: \mathrm{Ce}, \mathrm{Tb}$. The counts were read by the frequency counter, which sent the data to a computer. The computer also received the resistance measurements from the SMU. The thermal emission intensity of the microheater with and without the multilayer was measured up to $600^{\circ} \mathrm{C}$ as shown in Fig. 172 . The multilayer reduced the thermal emission intensity by almost three orders of magnitude.

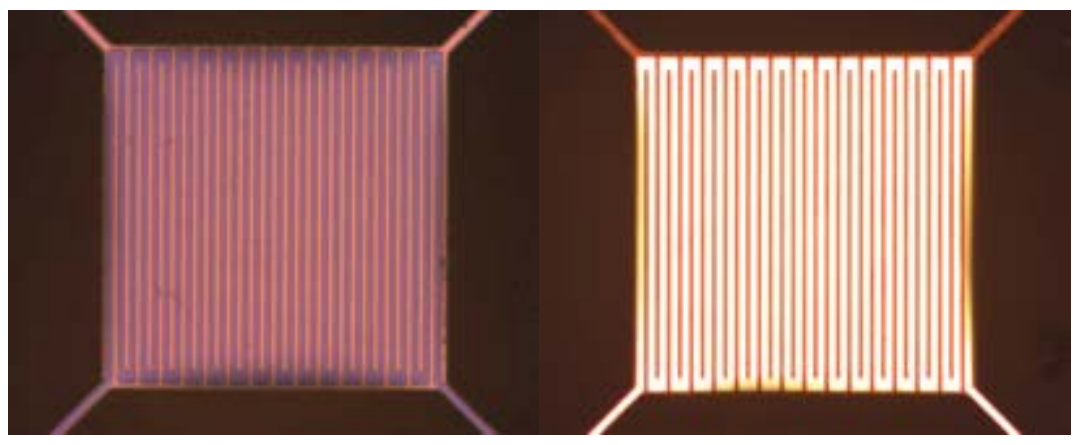

Fig. 171. Microheater without thermal emission reduction multilayer (left) and microheater with the multilayer (right). Both are $300 \mu \mathrm{m} \times 300 \mu \mathrm{m}$ platforms. The change in color is attributed to the change in reflectivity introduced by the multilayer. The TCRs before and after the multilayer deposition were $2.3 \times 10^{-3} /{ }^{\circ} \mathrm{C}$ and $-4.4 \times 10^{-4} /{ }^{\circ} \mathrm{C}$ respectively.

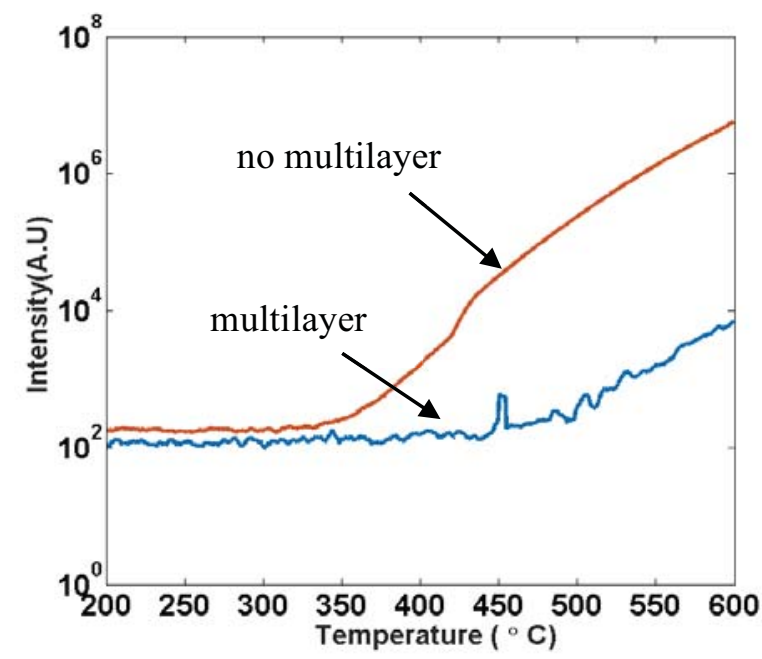

Fig. 172. Blackbody signals for microheaters with and without multilayer. The blackbody intensity at $600{ }^{\circ} \mathrm{C}$ was a factor of 800 less for the microheater with the multilayer.

To demonstrate the ability of the microheaters to block background radiation, microparticles of $\mathrm{CaSO}_{4}: \mathrm{Ce}$, Tb were deposited on a microheater. A few microparticles of $\mathrm{CaSO}_{4}: \mathrm{Ce}$, Tb would normally be very difficult to see in a standard heating system because of the heater thermal emission. Fig. 173 shows a SEM image of particles of $\mathrm{CaSO}_{4}: \mathrm{Ce}, \mathrm{Tb}$ on our microheater with particle size $<80 \mu \mathrm{m}$. Fig. 174 demonstrates the reduction in thermal emission intensity while measuring the luminescence of the $\mathrm{CaSO}_{4}: \mathrm{Ce}, \mathrm{Tb}$ microparticles. The PMT used for the optical measurements was only sensitive to wavelengths between 350 to $650 \mathrm{~nm}$. The thermal emission signal measured depended on a combination of the quantum efficiency of the PMT and the emissivity of the microheater with the 
multilayer. From Fig. 174 the PMT count at $420^{\circ} \mathrm{C}$ was 2474 photons from the particles and 153 photons from the thermal emission signal. The thermal emission signal for the microheater without the multilayer had a PMT count of 4386, which would have had a detrimental effect on the measurement. The efficiency of the interference structure increases even further at higher temperatures as the thermal emission power moves further towards shorter wavelengths.

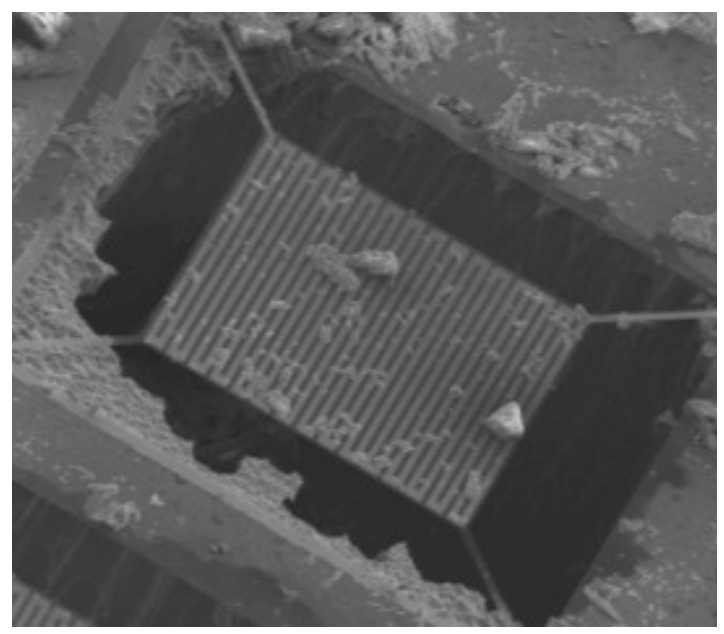

Fig. 173. SEM image of microheater with $\mathrm{CaSO}_{4}: \mathrm{Ce}, \mathrm{Tb}$ microparticles.

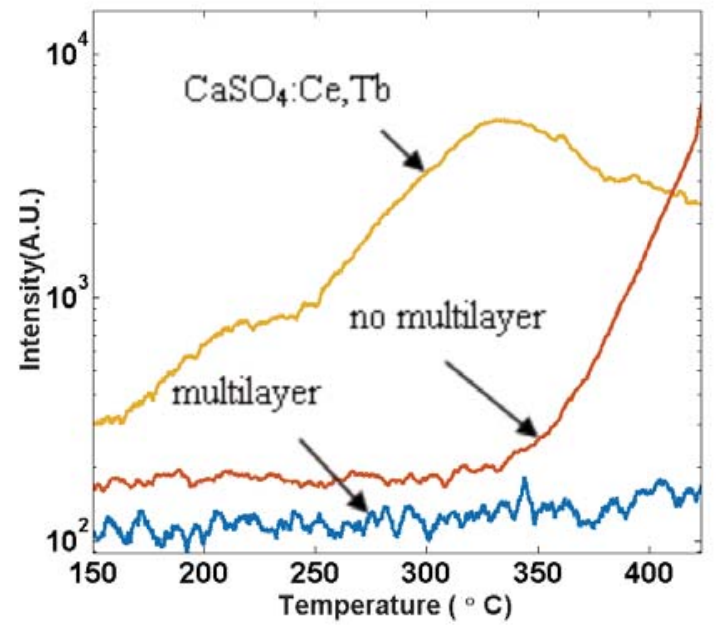

Fig. 174. TL of $\mathrm{CaSO}_{4}: \mathrm{Ce}$, Tb microparticles on a microheater with interference structure. The thermal emission from the microheater with no multilayer measured by the PMT is 4386 counts and the TL intensity was 2474 counts. This means the microheater thermal emission would have a detrimental effect on the measurement of the TL particle.

\subsubsection{Discussion}

It would be useful to discuss the relative advantages and disadvantages of the two methods used to reduce thermal emission during high temperature fluorescence measurements. Transparent heaters have low emissivity across extremely wide swaths of the infrared and visible spectrum. Their ultimate performance is limited by the intrinsic absorption of their constituent dielectric, but even this will be small in almost all cases because the transparent region can be made extremely thin. As one example, if a $10 \mathrm{~nm}$ layer were deposited by atomic layer deposition, the material would require an effective 
absorption coefficient of about 4.6 million $/ \mathrm{cm}^{-1}$ to have an emissivity of even $1 \%$. On the other hand, the central region is only a fraction of the overall area of the heater, so an aperture must be used to reject emission from the heater coils. This leads to an additional complication of non-uniform heating in the heater. The temperature of the coils will typically be hotter than the temperature of the transparent region; therefore, a calibration of the temperature of the central region relative to the coils must be performed at all temperature regions of interest using an external device such as a thermal camera or by calibrating relative to known temperature events such as characteristic TL peaks of known materials. An advantage of the transparent microheater method is relative ease of fabrication, since the transparent regions can be created in a standard microheaters process with no additional process steps.

The most fundamental disadvantage of the interference method is that interference structures must be optimized for specific wavelength ranges. These need not be continuous, but they are generally fixed by the fabrication process. Arbitrary tunability is extremely difficult for interference structures except for certain special cases such as Fabry-Perot resonances. In addition, the fabrication process is more difficult because incorporating interference structures is fabrication intensive. This applies whether the interference structure is a multilayer as in this work or a more advanced structure such as a photonic crystal. Advantages of the multilayer include the ability to use the entire heater without having to block emission from certain regions. The resulting optical collection system is then far simpler, and the amount of light that can be collected is significantly increased. While off-axis spectral performance can play a role, most collection optics use angular ranges that will shift interference performance a significant amount. In addition, as mentioned before, a photonic crystal could be used to block off light from any angle. If the spectral range of the weak emitter is limited, the efficiency of the interference structure in rejecting thermal emission over that range can be near $100 \%$.

\subsection{Thermal history of TL multilayers}

In this section, we suggest that TL multilayers can give supplementary information on the surface and deeper temperatures of the event. As a step towards this goal, we model the TL responses of a threelayer TL structure for the case where laser-induced heating on one TL layer diffuses into another TL layer, resulting in a nonuniform temperature distribution. To verify this simulation, a LiF:Mg, Ti and $\mathrm{CaF}_{2}$ :Dy bilayer sample is used. These bonded TL composites produced strong multiple peaks from their component materials whose individual contributions are distinguished in both the time and wavelength domains.

$\mathrm{CaF}_{2}$ :Dy was chosen because it tends to have different TL curve peaks as well as emission spectra from $\mathrm{LiF}: \mathrm{Mg}$,Ti. The drawback of using $\mathrm{CaF}_{2}:$ Dy is that it is roughly 16 times more sensitive than LiF:Mg,Ti. In other words, given the same dose, $\mathrm{CaF}_{2}$ :Dy emits at an intensity that is a factor of 16 higher than LiF:Mg,Ti. However, this drawback can be overcome by filtering the combined emission of the two materials and selecting a suitable emission wavelength where the intensities of the two materials are similar. A monochromator was used as a wavelength filter for this purpose. One can achieve similar results with other TL materials if they have TL peaks that occur at distinct temperatures.

Once we have extracted a set of trap parameters, we also measured the luminescent intensity versus wavelength for the $\mathrm{LiF}: \mathrm{Mg}, \mathrm{Ti}$ and $\mathrm{CaF}_{2}$ :Dy. A wavelength is selected where the intensities of the two materials are approximately the same and then the residual intensity ratio factor is included in our simulations. The results will be discussed in a subsequent section.

The $\mathrm{CaF}_{2}$ :Dy surface of the TL multilayer is coated with aluminum, and this serves as an absorption layer when the multilayer is heated by a Nd:YAG laser. The heat penetrates the multilayer over a period of 
time that depends on the multilayer thickness, multilayer diffusivity, and interlayer thermal contact conductances. This process empties some of the filled traps, which alters the TL that will be observed afterwards. A heat transfer simulation has been used to estimate the temperature distribution in the structure. This temperature as a function of time is fed into a TL simulation, derived in the earlier section, which estimates the trap depopulation and resulting TL curves. The heat transfer simulation assumes one-dimensional conduction, which is an accurate assumption because the thickness of the multilayer stack is very small compared to its lateral dimensions. The laser beam illumination is relatively uniform over the entire surface so lateral heat conduction can be ignored.

The LiF:Mg,Ti and $\mathrm{CaF}_{2}:$ Dy materials were purchased from Thermo Scientific. The material came in the form of $3.2 \mathrm{~mm} \times 3.2 \mathrm{~mm} \times 0.89 \mathrm{~mm}$ chips and were bonded with double-sided Kapton tape from DuPont. This Kapton tape can survive the high temperatures required for measurement.

A $400 \mathrm{~nm}$ thick light-absorbing aluminum layer was deposited on the surface of the $\mathrm{CaF}_{2}: \mathrm{Dy}_{\mathrm{b}}$ using electron beam evaporation. To fill the traps of the TL multilayer, it was irradiated with an X-RAD 320 dosage calibrated $X$-ray source. The source, with a beam hardening filter of $2 \mathrm{~mm}$ thick $\mathrm{Al}$, generates $2.7 \mathrm{~Gy} / \mathrm{min}$ at $320 \mathrm{kV}$ voltage and $12.5 \mathrm{~mA}$ current at a distance of $50 \mathrm{~cm}$. The LiF:Mg,Ti surface was turned toward the X-ray source to avoid potential attenuation of the $\mathrm{x}$-ray beam by the aluminum absorber on the $\mathrm{CaF}_{2}$. The radiation dose was set to be $700 \mathrm{cGy}$. It was observed that actual dose varied within $1 \mathrm{cG}$ of this value.

A beam from a Nd:YAG laser illuminated the aluminized surface of the TL multilayer (Fig. 175). The relative size of the composite has been exaggerated for better illustration, but the laser spot size was larger than the sample to ensure uniform heating. A 50:50 beam splitter divided the light so that the optical power could be simultaneously and independently measured by a silicon detector and optical power meter. Additional optics were used to focus the light. The laser was operated in continuous wave $(\mathrm{CW})$ mode, and the pulse duration was set by an external function generator. 


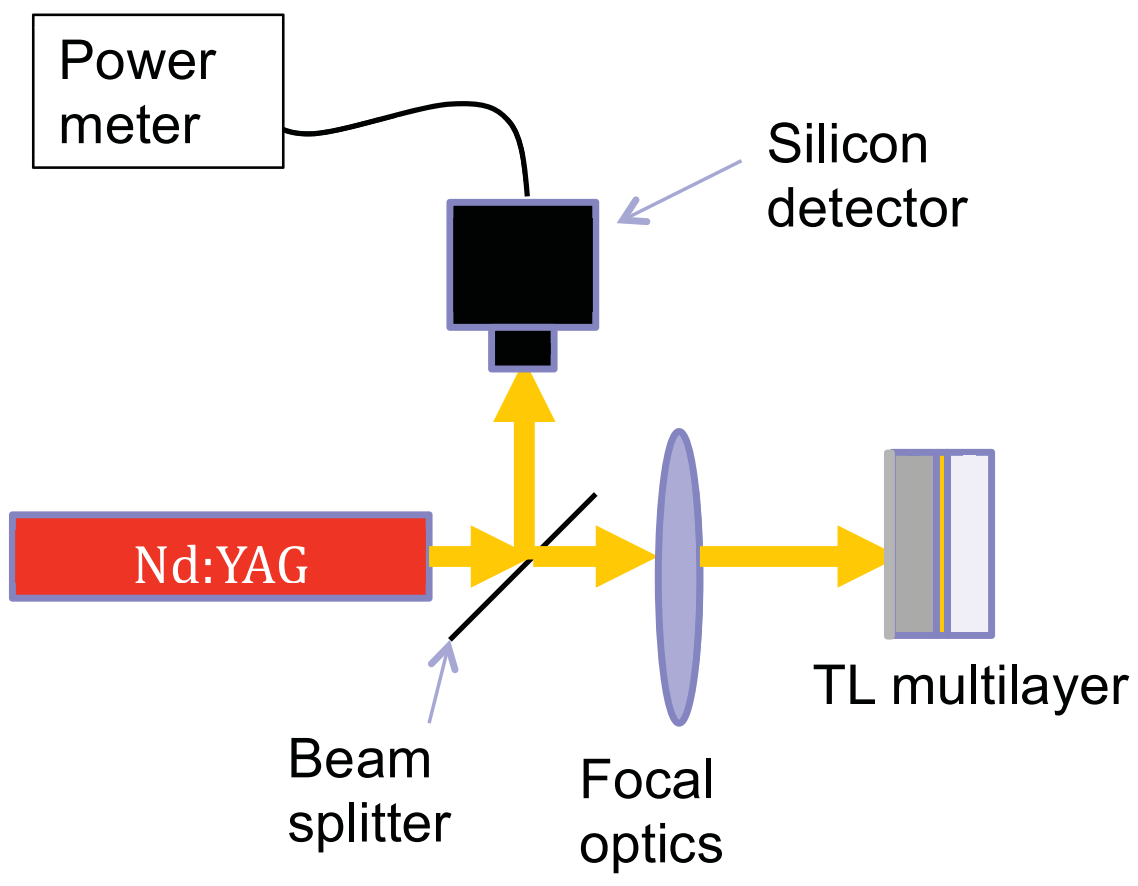

\section{$\Rightarrow$ Light path}

Fig. 175. Laser heating experiment set-up. The relative size of the TL multilayers has been exaggerated for better illustration but the laser spot size is bigger than the TL multilayers to ensure uniform heating across the surface.

The image below shows the experimental TL measurement system. The TL experiments were performed in a dark room and chamber $30 \mathrm{~min}$ after irradiation to ensure that effects due to thermal fading were consistent. The samples were carried in a black container to avoid optical fading effects.

A photomultiplier tube (PMT) was used to measure light output, and a monochromator was used to select the specific wavelength that balanced out the TL intensities of the LiF:Mg,Ti and $\mathrm{CaF}_{2}: \mathrm{Dy}$ materials. In this study, a wavelength of $460 \mathrm{~nm}$ with a $14 \mathrm{~nm}$ Full Width Half Maximum (FWHM) passband was chosen after several trials. To protect the Kapton tape, the samples were placed on a copper puck atop a hot plate to reduce the highest peak temperature from $400{ }^{\circ} \mathrm{C}$ to $350{ }^{\circ} \mathrm{C}$. 


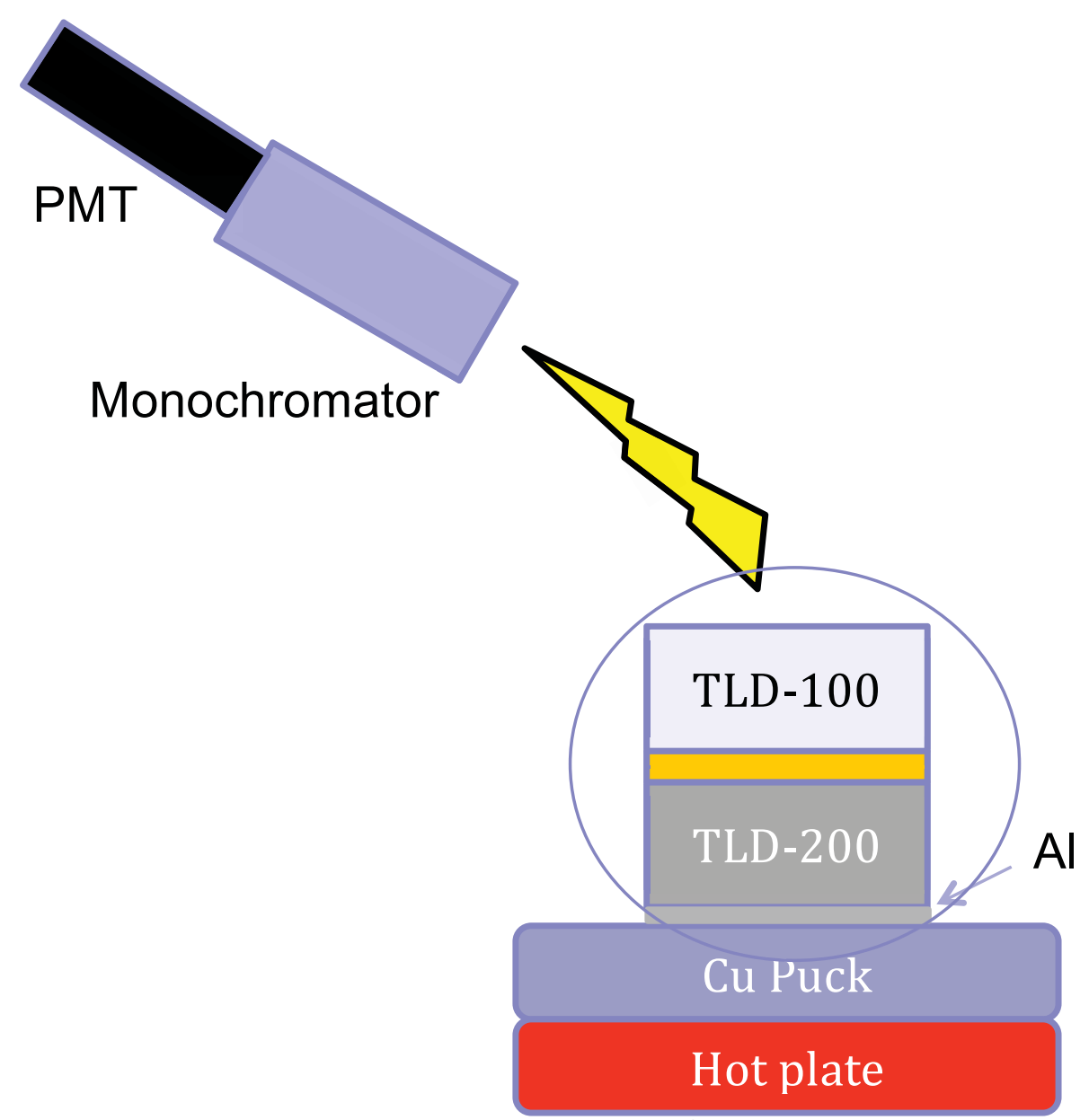

Fig. 176. Experimental set-up for TL test.

Below shows the typical TL curves of the multilayers. The peak at the $150{ }^{\circ} \mathrm{C}$ is associated with $\mathrm{CaF}_{2}: \mathrm{Dy}$ and the peak at $225^{\circ} \mathrm{C}$ with the LiF:Mg,Ti. The $150^{\circ} \mathrm{C}$ peak began to empty upon laser heating. The lower peaks of LiF:Mg,Ti depopulated, whereas the peak at $225^{\circ} \mathrm{C}$ still remained. These curves were normalized at $225^{\circ} \mathrm{C}$. The curves were also adjusted slightly to get stable peak temperature positions, because the peak temperatures drifted slightly due to variations in irradiation, heating rate, and thermal contact. The TL intensities also varied slightly from run-to-run due to these issues plus optical alignment. 


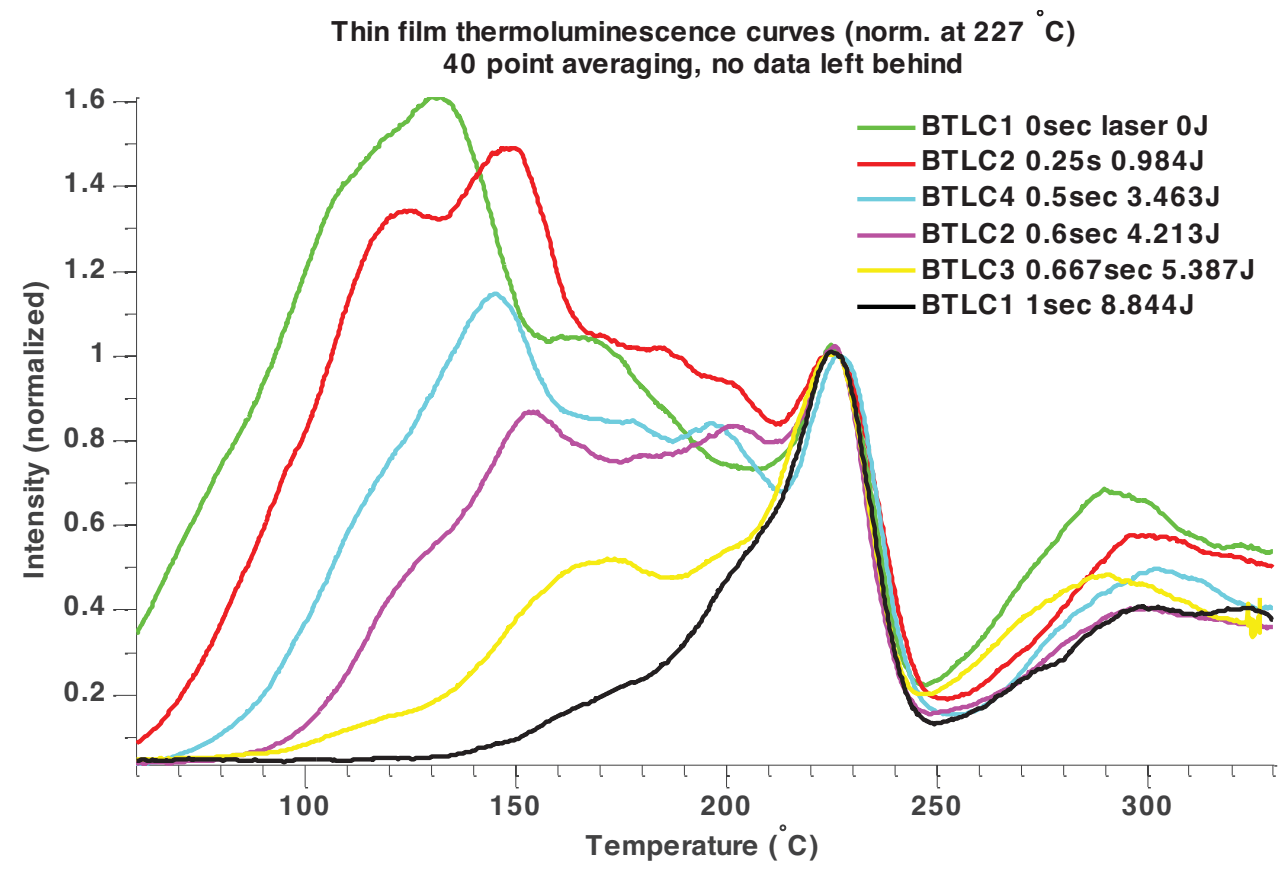

Fig. 177. Luminescence intensity curves for the TL multilayers with different laser heating conditions.

With the previous modeling, one can generate the simulation graphs. The simulation graphs are normalized in Fig. 178 to have the same maximum intensity as the experimental results. If the $150{ }^{\circ} \mathrm{C}$ peak is available, the simulation and experiment are set to the same intensity at $150{ }^{\circ} \mathrm{C}$ as depicted in Fig. 178(a)-(c), whereas the other temperature peaks might be off. For the $250 \mathrm{~ms}$ pulse, the peak of LiF:Mg, Ti at $225^{\circ} \mathrm{C}$ has a $6 \%$ intensity error between the simulation and experiment. If $150{ }^{\circ} \mathrm{C}$ peak is no longer available due to depopulation, the simulation is adjusted to have the same intensity at $225{ }^{\circ} \mathrm{C}$, as in Fig. 178(d)-(f). The location of the $\mathrm{CaF}_{2}$ :Dy peaks differed slightly from one experiment to another due to variations in the thermal contact between the samples and the Cu puck and in the hot plate heating rate; the curves are shifted in temperature to match the experimental control.

In this simulation, a thermal contact conductance was introduced between the aluminum and the $\mathrm{CaF}_{2}$ :Dy layer and was found to better match to experimental results than assuming an infinite interface conductivity. The aluminum was otherwise regarded as a perfect heat conductor that could absorb heat and dissipate it laterally. One dimensional heat transfer was assumed, neglecting convection and other heat transfer mechanisms. The Kapton thermal conductivity appeared to differ slightly from published values, since an increase in its simulated conductivity from 0.12 to 0.144 gave better matching results between simulation and experiment.

One can speculate that a multilayer system such as this could be used to measure the histories of very fast thermal events such as explosions. The advantage of a multilayer technique would be that the most rapid fluctuations could be measured using changes in the TL of the surface layers, whereas a slower thermal baseline would be measured in the deeper layers. In this work, the multilayers were one-dimensional, flat structures, suitable for devices where only one face of the structure was exposed to the temperature in question. Perhaps a more viable architecture for more general rapid thermal histories would be a core-shell(s) architecture where the layer thicknesses could be varied depending on the time constants of the events to be measured. 


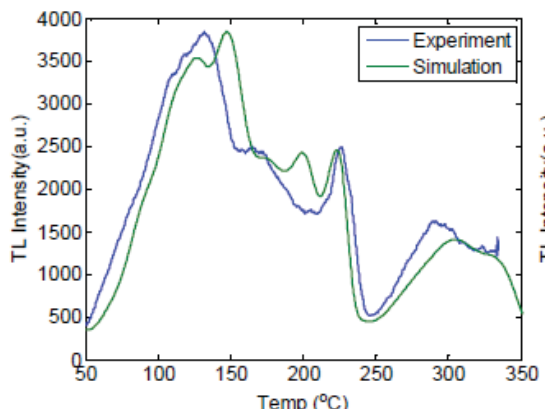

(a)

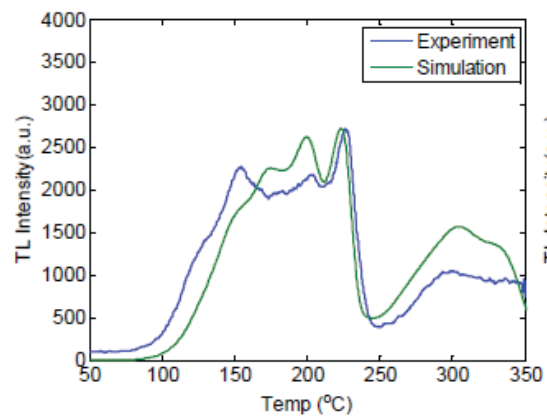

(d)

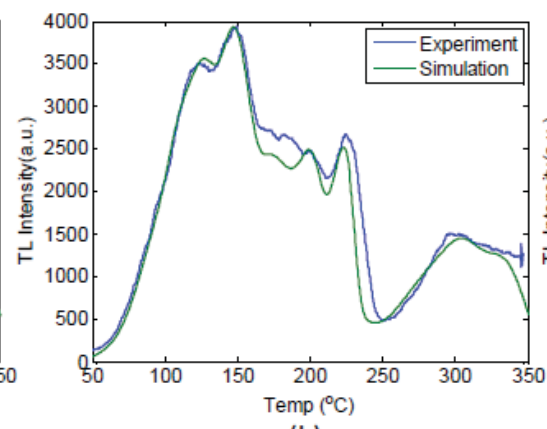

(b)

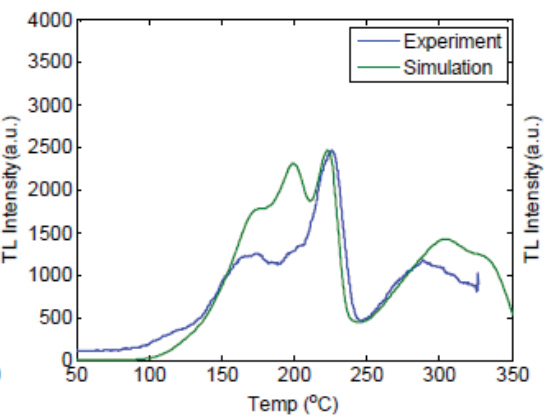

(e)

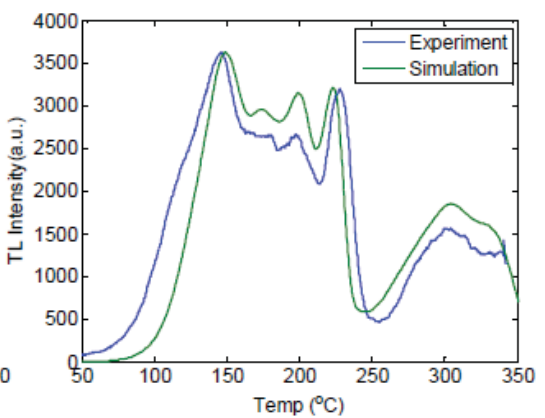

(c)

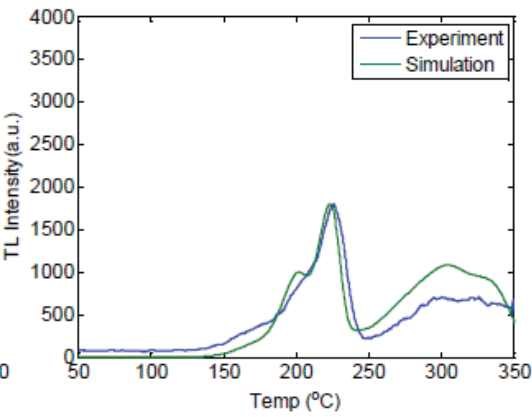

(f)

Fig. 178. Simulation results with experiment compared, in (a) for 0s, (b) $250 \mathrm{~ms}$, (c) $500 \mathrm{~ms}$, (d) $600 \mathrm{~ms}$, (e) $667 \mathrm{~ms}$ and (f) $1000 \mathrm{~ms}$.

\subsection{Thermally Assisted Optically Stimulated Luminescence of $\mathrm{Al}_{2} \mathrm{O}_{3}: \mathrm{C}$}

In this section we investigate the thermally assisted optically stimulated luminescence of $\mathrm{Al}_{2} \mathrm{O}_{3}: \mathrm{C}$ as an alternative for temperature measurements.

$\mathrm{Al}_{2} \mathrm{O}_{3}: \mathrm{C}$ would be a potential material for thermal sensing, if it was not for its light sensitivity and thermal quenching. $\mathrm{Al}_{2} \mathrm{O}_{3}: \mathrm{C}$ is known to be inert and have high melting temperature $\left(>2000{ }^{\circ} \mathrm{C}\right)$, high luminescence intensity, $\mathrm{TL}$ peaks over a wide range of temperatures, from $\sim 190{ }^{\circ} \mathrm{C}$ to $900{ }^{\circ} \mathrm{C}$ [83], and UV emission at $330 \mathrm{~nm}$ [84]. In addition, $\mathrm{Al}_{2} \mathrm{O}_{3}: \mathrm{C}$ is produced commercially in large quantities (>1.5 tons/year) by Landauer Inc. Nevertheless, light sensitivity decreases the intensity of the shallow peaks $\left(<400^{\circ} \mathrm{C}\right)$ and thermal quenching (reduction of luminescence with heating) prevents the measurement of high temperature peaks efficiently.

Therefore, in this study we investigated the possibility of using alternative approaches, such as thermally assisted OSL (TA-OSL) to estimate the deep trap population and use this material as a hightemperature temperature sensor.

Fig. 179 shows the $\mathrm{TL}$ curves of $\mathrm{Al}_{2} \mathrm{O}_{3}: \mathrm{C}$ after irradiation and pre-heating to increasing temperatures, showing the subsequent depletion of the TL peaks. This data confirms that there are TL peaks at temperatures $>600^{\circ} \mathrm{C}$, but their intensity is low because of thermal quenching. If these peaks could be assessed using TA-OSL, then they could be useful for temperature sensing. 


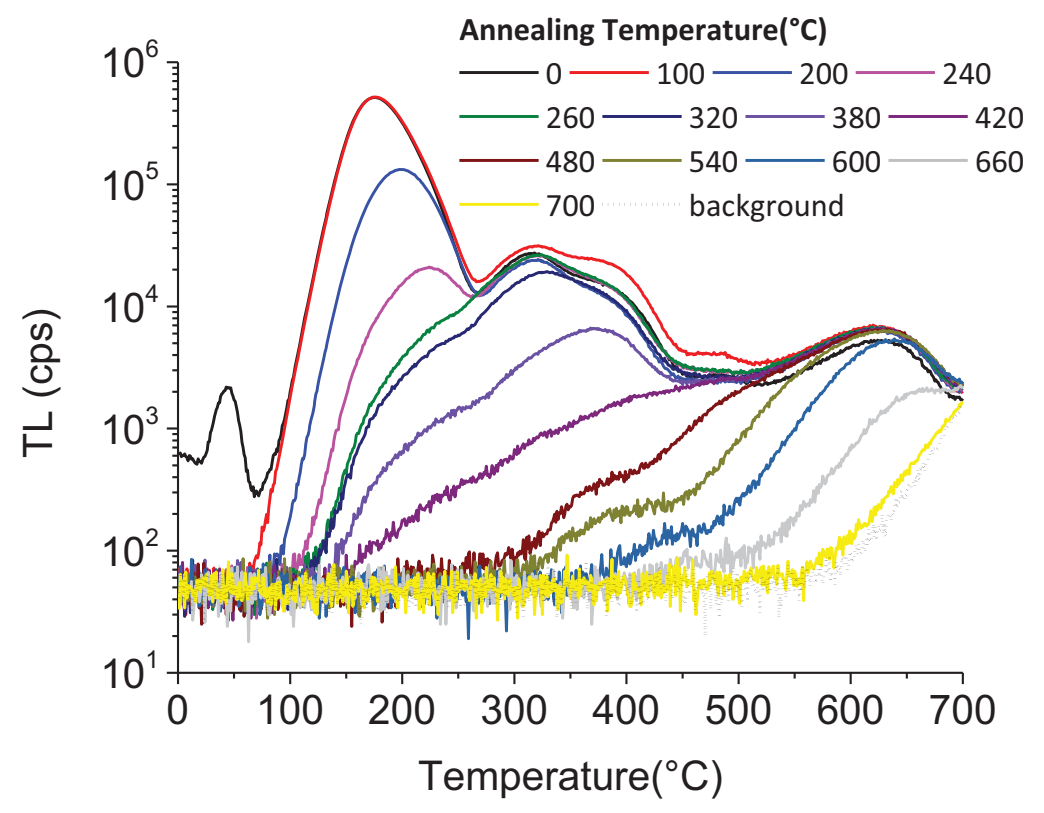

Fig. 179. $\mathrm{TL}$ curves of $\mathrm{Al}_{2} \mathrm{O}_{3}: \mathrm{C}$ after irradiation with $100 \mathrm{~Gy}$ and pre-heating to increasing temperatures at $1^{\circ} \mathrm{C} / \mathrm{s}$.

TA-OSL measurements consisted of stimulating the OSL signal at $150^{\circ} \mathrm{C}$. In our measurements this was carried out for $10 \mathrm{~s}$ and the total signal after background subtraction was obtained. Fig. 180 shows the TA-OSL signal as a function of heating in three different regimes. The data show a decreasing trend in total area with increases in final heating temperature as well as a decrease in area with an increase in heating rate as expected.

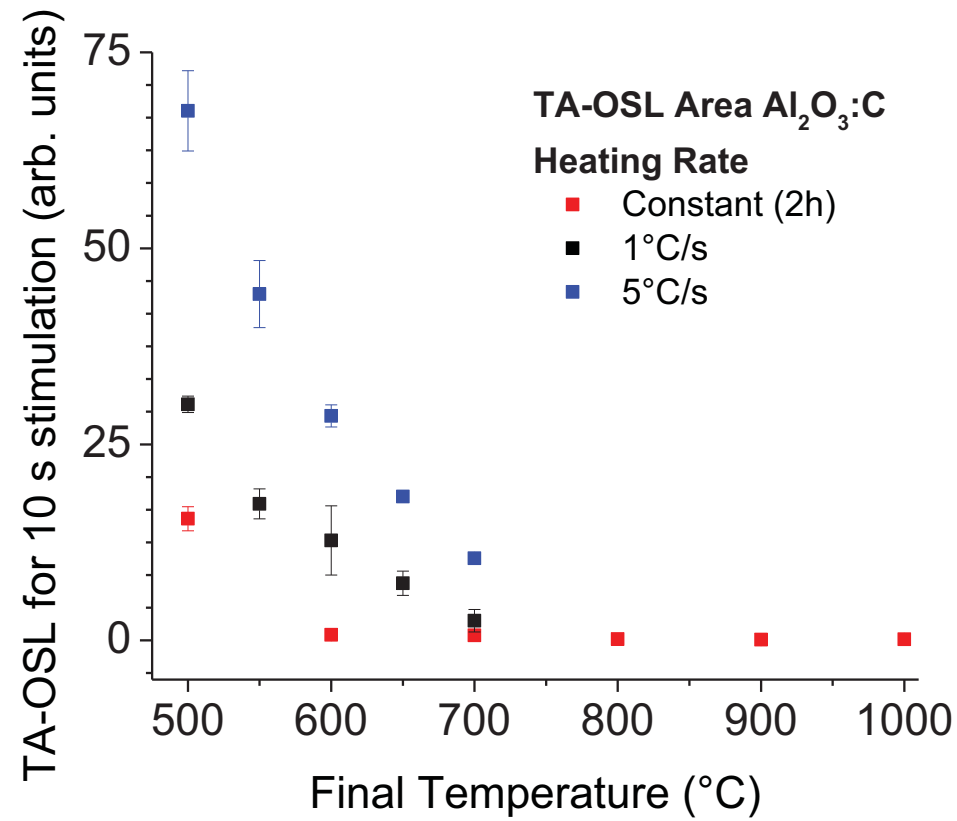

Fig. 180. Total luminescence counts during TA-OSL stimulation. Error calculated as one standard deviation.

We discovered, however, that even deep traps can be optically stimulated, causing a redistribution of trapped charges in different states. This changes the TA-OSL signal and confuses the interpretation of 
the data. The TL curves in Fig. 181 were obtained after irradiation of the sample and cleaning the shallow traps, so that only traps above $400{ }^{\circ} \mathrm{C}$ are observable (e.g. 0 s). Nevertheless, one can see that light exposure increases the intensity of the $\mathrm{TL}$ in shallow peaks (e.g., $250^{\circ} \mathrm{C}$ and $300{ }^{\circ} \mathrm{C}$ ), which can then give the impression that the samples were not heated to a temperature high enough.

Our conclusion is that this effect would be impossible to correct for in a real application environment, in which light exposure is unpredictable.

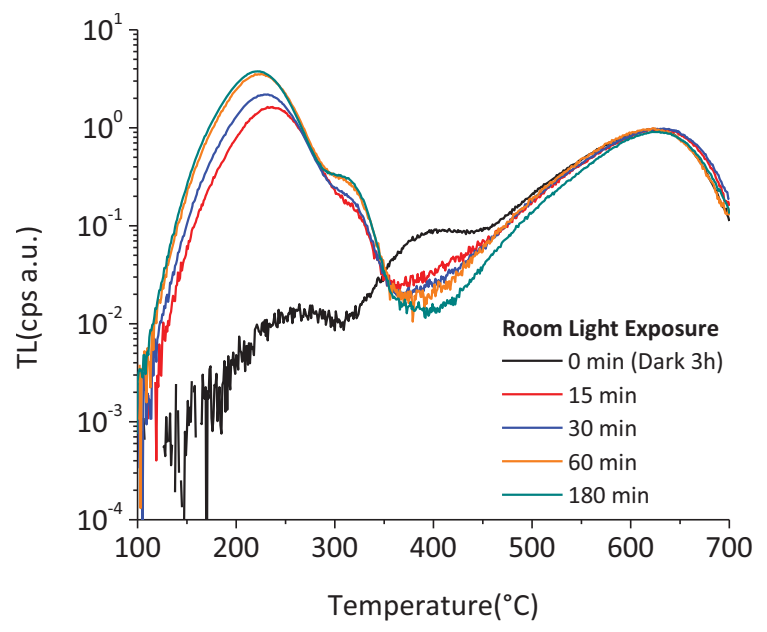

Fig. 181. Recovered TL signal of $\mathrm{Al}_{2} \mathrm{O}_{3}: \mathrm{C}$ following $400^{\circ} \mathrm{C} / 2 \mathrm{~h}$ and exposure to room light. The PTTL signal distorts the recovered $\mathrm{TL}$ signal, destroying information about heating history. 


\section{PUBLICATIONS AND PRESENTATIONS}

\subsection{Theses*}

1. Doull, B. A. 2013. Synthesis and Characterization of Luminescent Materials for Thermal Sensing and Proton Dosimetry. M. S. Thesis, Oklahoma State University.

*Other students have not yet completed their thesis by the time of this report.

\subsection{Book chapters}

1. Talghader, J.J. and Mah, M.L., 2012. Luminescent thermometry for sensing rapid thermal profiles in fires and explosions. In: Optical, Acoustic, Magnetic, and Mechanical Sensor Technologies, K. Iniewski (Ed.). CRC Press, 79-106.

2. Talghader , J. J. and Mah, M. L., 2013. Sensing Temperature inside Explosions, In: Smart Sensors for Industrial Applications, no. 16, K. Iniewski, Ed. CRC Press, 2013, pp. 259-272.

\subsection{Peer-reviewed publications}

1. Armstrong, P., Mah, M., Olson, K. D., and Talghader, J. J., 2015. Reduction of Thermal Emission Background in High Temperature Microheaters. Accepted for publication in the IEEE Journal of Microelectromechanical Systems, June 2015.

2. Armstrong, P., Mah, M., Kim, S., and Talghader, J. J. , 2014. Thermoluminescence of $\mathrm{Y}_{2} \mathrm{O}_{3}: \mathrm{Tb}^{3+}$ thin films deposited by electron beam evaporation. J. Lumin. 148, 225-229.

3. Doull, B.A., Oliveira, L.C., Wang, D.Y., Milliken, E.D. and Yukihara, E.G., 2014.

Thermoluminescent properties of lithium borate, magnesium borate and calcium sulfate developed for temperature sensing. J. Lumin. 146, 408-417.

4. Doull, B.A., Oliveira, L.C. and Yukihara, E.G., 2013. Effect of Annealing and Fuel Type on the Thermoluminescent Properties of $\mathrm{Li}_{2} \mathrm{~B}_{4} \mathrm{O}_{7}$ Synthesized by Solution Combustion Synthesis. Radiat. Meas. 56, 167-170.

5. Jacobsohn, L. G., Roy, A. L., McPherson, C. L., Kucera, C. J., Oliveira, L. C., Yukihara, E. G., and Ballato, J., 2013. Rare earth-doped nanocrystalline $\mathrm{MgF}_{2}$ : synthesis, luminescence and thermoluminescence. Opt. Mater. 35, 2461-2464.

6. Kim, S., Armstrong, P. R. , Mah, M. L., and Talghader, J. J., 2013. Depth-dependent temperature effects on thermoluminescence in multilayers. J. Appl. Phys. 114, 053519, 8 pages.

7. Mah, M.L., Armstrong, P.R., Kim, S.S., Carney, J.R., Lightstone, J.M. and Talghader, J.J., 2013. Sensing the thermal history of high-explosive detonations using thermoluminescent microparticles. IEEE Sens. J. 13, 1742-1747.

8. Mah, M.L., Manfred, M.E., Kim, S.S., Prokić, M., Yukihara, E.G. and Talghader, J.J., 2010. Measurement of rapid temperature profiles using thermoluminescent microparticles. IEEE Sens. J. 10, 311-315.

9. Milliken, E.D., Oliveira, L.C., Denis, G. and Yukihara, E.G., 2012. Testing a model-guided approach to the development of new thermoluminescent materials using YAG:Ln produced by solution combustion synthesis. J. Lumin. 132, 2495-2504. 
10. Orante-Barrón, V.R., Oliveira, L.C., Kelly, J.B., Milliken, E.D., Denis, G., Jacobsohn, L.G., Puckette, J. and Yukihara, E.G., 2011. Luminescence properties of MgO produced by Solution Combustion Synthesis and doped with lanthanides and Li. J. Lumin. 131, 1058-1065.

11. Wang, D., Doull, B.A., Oliveira, L.C. and Yukihara, E.G., 2013. Controlled synthesis of $\mathrm{Li}_{2} \mathrm{~B}_{4} \mathrm{O}_{7}: \mathrm{Cu}$ for temperature sensing. RSC Advances 3, 26127-26131.

12. Yukihara, E.G., Coleman, A.C., Bastani, S., Gustafson, T., Talghader, J.J., Daniels, A., Stamatis, D., Lightstone, J.M., Milby, C. and Svingala, F.R., 2015. Particle temperature measurements in closed chamber detonations using thermoluminescence from $\mathrm{Li}_{2} \mathrm{~B}_{4} \mathrm{O}_{7}: \mathrm{Ag}, \mathrm{Cu}, \mathrm{MgB}_{4} \mathrm{O}_{7}: \mathrm{Dy}, \mathrm{Li}$ and $\mathrm{CaSO}_{4}$ :Ce,Tb. J. Lumin. 165, 145-152.

13. Yukihara, E.G., Coleman, A.C. and Doull, B.A., 2014. Passive temperature sensing using thermoluminescence: laboratory tests using $\mathrm{Li}_{2} \mathrm{~B}_{4} \mathrm{O}_{7}: \mathrm{Cu}, \mathrm{Ag}, \mathrm{MgB}_{4} \mathrm{O}_{7}: \mathrm{Dy}, \mathrm{Li}$ and $\mathrm{CaSO}_{4}: \mathrm{Ce}, \mathrm{Tb} . \mathrm{J}$. Lumin. 146, 515-526.

14. Yukihara, E.G., Milliken, E.D. and Doull, B.A., 2014. Thermally stimulated and recombination processes in $\mathrm{MgB}_{4} \mathrm{O}_{7}$ investigated by systematic lanthanide doping. J. Lumin. 154, 251-259.

15. Yukihara, E.G., Milliken, E.D., Oliveira, L.C., Orante-Barrón, V.R., Jacobsohn, L.G. and Blair, M.W., 2013. Systematic development of new thermoluminescence and optically stimulated luminescence materials. J. Lumin. 133, 203-210.

16. Talghader, J. J., Mah, M. L.., Yukihara, E. G., and Coleman, A. C. Thermoluminescent microparticle thermal history sensors. Invited Review written for Microsystems and Nanoengineering, Nature Publishing Group, to be submitted in December 2015.

\subsection{Conference proceedings}

- P. Armstrong, M. Mah, and J. J. Talghader, "Microheater multilayer interference to reduce blackbody emission for low photon number luminescence measurement," Proceedings of the 2015 Transducers Conference, Anchorage, Alaska, June 21-25, 2015, pp. 924-927.

- P. R. Armstrong, A. K. Brown, K. D. Olson, and J. J. Talghader, "Microheater Controlled Part-PerMillion-Level Absorption Measurements Using Photothermal Common Path Interferometry," Proceedings of the IEEE Optical MEMS and Nanophotonics Conference, Jerusalem, pp. Tu4.3-1 4.3-2 August 2015.

- E. G. Yukihara. "Materials for temperature measurements: principles and state-of-the-art". Workshop on Time-Dependent Temperature Measurements in Energy Release Processes, October 10-11, Chicago, IL, USA (2012).

- J. J. Talghader. "Thermal History Sensing using Thermoluminescence: Measurement Concepts and Testing". Workshop on Time-Dependent Temperature Measurements in Energy Release Processes, October 10-11, Chicago, IL, USA (2012).

- B. Doull, L. C. Oliveira, and E. G. Yukihara. "Effect of annealing and fuel type on the thermoluminescent properties of Li2B4O7 synthesized by solution combustion synthesis". 8th International Conference on Luminescent Detectors and Transformers of Ionizing Radiation, Sep. 10-14, Halle (Saale), Germany (2012).

- M. L. Mah, P. R. Armstrong, J. J. Talghader, "Alteration by repeated electrostatic MEMS actuation of the thermoluminescence of thin films," 2013 IEEE Optical MEMS and Nanophotonics Conference, Kanazawa, Japan, August 18-22, 2013, pp. 55-56.

- P. R. Armstrong, M. L. Mah, L. Taylor, J. J. Talghader, "Reduced blackbody microheaters for measuring high temperature thermoluminescent glow curve peaks," 2014 IEEE International 
Conference on Optical MEMS and Nanophotonics, Glasgow, Scotland, August 2014, pp. 7-8, 2014.

\subsection{Presentations}

- E. G. Yukihara, J. J. Talghader“, A. Coleman, B. Doull, T. Gustafson, S. Bastani, M. L. Mah, P. R. Armstrong, "Particle Thermometry using Microparticle Thermoluminescence: Overview and Outlook,"', $15^{\text {th }}$ International Detonation Symposium, July 13-18, San Francisco, CA, (2014.). (Poster presentation by the student Adam Coleman.)

- J. J. Talghader and E. G. Yukihara, "Thermometry using Microparticle Thermoluminescence: Overview and Outlook," International Detonation Symposium, July 13-18, San Francisco, CA, 2014. (Oral presentation by the Co-PI Joey Talghader).

- E. G. Yukihara, D. Wang, B. A. Doull, L. C. Oliveira, B. Obryk, L. L. Campos, J. Lee, and J. J. Talghader. "Temperature sensing using thermoluminescence: Principles and State of the Art". 17th International Conference on Solid State Dosimetry, Sep 22 - 27, Recife, Brazil (2013)

- P. R. Armstrong, M. L. Mah, and J. J. Talghader, "The Accidental Sensor: 'Off-label' Uses for Thermoluminescent Radiation Dosimeters.", Medical Micro and Nano Systems session, Design of Medical Devices Conference, Minneapolis, April 9, 2013.

\subsection{Technology transfer presentations}

1. J. J. Talghader and E. G. Yukihara, "Agent Defeat Thermal History using Microparticle Thermoluminescence: Testing at Indian Head NSWC", DTRA, Ft. Belvoir, VA, April 22, 2014.

2. J. J. Talghader and E. G. Yukihara, "Agent Defeat Thermal History using Microparticle Thermoluminescence: Testing at Indian Head NSWC", DTRA, Albuquerque, NM, May 15, 2014 


\section{CONCLUSIONS}

The projected achieved the objective of creating and studying novel luminescence particles capable of sensing and retaining the time-temperature information to which they were exposed in their thermoluminescence ( $\mathrm{TL}$ ) curves. We investigated commercial candidate materials for use as temperature sensors, and performed a systematic study to synthesize and develop new materials by looking at various host/dopant combinations [2]. The study also included attempts to develop core/shell TL nanophosphors. Commercial and new TL materials developed were tested in various conditions (laboratory, closed-chamber detonation, open-chamber detonation, and shock-tube tests), demonstrating on the one hand the ability of the TL particles to accurate determine temperature (when comparisons with thermocouple data is feasible), and the complexity of the detonation environments (in which no reference is available for comparison, since the particles have unique properties and will experience different temperature excursions in comparison with fixed measured points such as thermocouples).

Specific findings of this project can be summarize as follow:

a) We found that specific host materials (e.g., $\mathrm{LiF}_{2} \mathrm{Li}_{2} \mathrm{~B}_{4} \mathrm{O}_{7}, \mathrm{MgB}_{4} \mathrm{O}_{7}, \mathrm{CaSO}_{4}$ ) are less light sensitive and can be used as the basis for development of temperature sensors [3,4]. With suitable dopants these materials can be made to emit in the UV region (e.g. by $\mathrm{Cu}^{+}$or $\mathrm{Ce}^{3+}$ doping), and $\mathrm{TL}$ peaks in a wide range of temperatures from $<100{ }^{\circ} \mathrm{C}$ up to $>600{ }^{\circ} \mathrm{C}$ (when using $1{ }^{\circ} \mathrm{C} / \mathrm{s}$ heating rate) can be introduced (e.g. using various lanthanides) $[5,6]$. The wide range of TL peaks that can be obtained has been demonstrated using $\mathrm{CaSO}_{4}$. We also demonstrate how the synthesis conditions (e.g. fuel type and annealing temperature) can be used to improve the luminescence properties [7, 8].

b) Commercial TL materials were identified (e.g. LiF, $\mathrm{CaF}_{2}: \mathrm{Tm}$ ) and new TL materials were developed (e.g. $\mathrm{Li}_{2} \mathrm{~B}_{4} \mathrm{O}_{7}, \mathrm{MgB}_{4} \mathrm{O}_{7}, \mathrm{CaSO}_{4}$ ). They were characterized for the structural and $\mathrm{TL}$ properties [4, 9], including a comprehensive analysis of the kinetic parameters. This provided the basic information to develop a multiparametric algorithm to extract the temperature profile from the TL curve data obtained from the particles.

c) Multiparametric algorithms for temperature recovery were developed at OSU and UMN. The OSU algorithm is based on the deconvolution of the TL from the samples to obtain a TL model, which can then be used together with experimental TL curves to estimate the temperature experience by the particles [10]. The UMN algorithm is based on the use of multiple points in the TL curve and a TL model for the materials to the same effect [9]. The results from both algorithms, developed completely independently, were comparable.

d) The materials mentioned above were also tested in laboratory (furnace and a temperature chamber) and showed perfect agreement with thermocouple data, except when particle aggregation prevented some of the grains from being heated uniformly [10].

e) Tests in close-chamber experiments also showed agreement with thermocouple data for various materials, while also highlighting the need to develop materials with a wider range of temperature applicability [11]. Materials with a narrow range of TL peaks also have a narrow range of applicability. Therefore, it is desirable to have materials with multiple TL peaks distributed over a wide range of temperatures.

f) Open chamber tests performed in collaboration with DTRA revealed the complexity of the temperature environment, with different particles resulting in different temperatures, probably because of the various possible paths during the detonation event. 
g) Thin TL films were developed to investigate the effect of pressure [12], but we did not observed any effect up to MPa pressures. Furthermore, because the samples are prepared with very high doses of ionizing radiation ( $k G y$ ), no effect of UV light in inducing or depleting the TL peaks was noticed during experiments.

h) The influence of particle size was investigated either by simulation of the response of core-shell nanophosphors, as well as experimentally by the use of TL materials with multilayers [13]. Materials for core-shell nanophosphors were also developed [14], but the properties were not sufficient for temperature sensing applications.

i) New microheaters with low thermal emission were also developed as part of this project with the objective of providing a method to measure TL peaks at high temperature, which has the potential to increase the range of applicability of the TL materials [15].

j) Shock tube tests at University of Illinois Urbana-Champaign (UIUC) showed that particles can be recovered using a cold heat sink. The temperatures obtained by different materials were consistent, but below the temperatures estimated by UIUC. The influence of particle size should be investigated in the future because of the very fast heating profiles.

Overall the project successfully demonstrated TL particles as thermal sensors and developed the fundamental materials science and data analysis procedures to apply it. Successful deployment of the technique in realistic conditions depends now on a close collaboration between researchers with expertise on the TL technique and TL materials, and researchers interested in employing the technique. The major obstacle seems to be not scientific or technical, but the need to bridge the gap between the two branches of investigation to identify and solve some of the practical issues related with sample collection and handling, choice of best materials for application, and strategies to obtain useful conclusions from the information provided by the particles, since the novelty of the technique also implies that there are no reference or "gold-standard" to compare the technique to in real applications. 


\section{REFERENCES}

[1] S. Wang, S. Westcott, W. Chen, J. Phys. Chem. B 106 (2002) 11203-11209.

[2] E. G. Yukihara, E. D. Milliken, L. C. Oliveira, V. R. Orante-Barrón, L. G. Jacobsohn, M. W. Blair, J. Lumin. 133 (2013) 203-210.

[3] J. J. Talghader, M. L. Mah, Luminescent thermometry for sensing rapid thermal profiles in fires and explosions, in Optical, Acoustic, Magnetic, and Mechanical Sensor Technologies, K. Iniewski, Ed.: CRC Press, 2012, pp. 79-106.

[4] B. A. Doull, L. C. Oliveira, D. Y. Wang, E. D. Milliken, E. G. Yukihara, J. Lumin. 146 (2014) 408417.

[5] E. D. Milliken, L. C. Oliveira, G. Denis, E. G. Yukihara, J. Lumin. 132 (2012) 2495-2504.

[6] E. G. Yukihara, E. D. Milliken, B. A. Doull, J. Lumin. 154 (2014) 251-259.

[7] B. A. Doull, L. C. Oliveira, E. G. Yukihara, Radiat. Meas. 56 (2013) 167-170.

[8] D. Wang, B. A. Doull, L. C. Oliveira, E. G. Yukihara, RSC Advances 3 (2013) 26127-26131.

[9] M. L. Mah, P. R. Armstrong, S. S. Kim, J. R. Carney, J. M. Lightstone, J. J. Talghader, IEEE Sens. J. 13 (2013) 1742-1747.

[10] E. G. Yukihara, A. C. Coleman, B. A. Doull, J. Lumin. 146 (2014) 515-526.

[11] E. G. Yukihara, A. C. Coleman, S. Bastani, T. Gustafson, J. J. Talghader, A. Daniels, D. Stamatis, J. M. Lightstone, C. Milby, F. R. Svingala, J. Lumin. 165 (2015) 145-152.

[12] P. R. Armstrong, M. L. Mah, S. S. Kim, J. J. Talghader, J. Lumin. 148 (2014) 225-229.

[13] S. S. Kim, P. R. Armstrong, M. L. Mah, J. J. Talghader, J. Appl. Phys. 114 (2013) 053519.

[14] L. G. Jacobsohn, A. L. Roy, C. L. McPherson, C. J. Kucera, L. C. Oliveira, E. G. Yukihara, J. Ballato, Opt. Mater. 35 (2013) 2461-2464.

[15] P. R. Armstrong, M. L. Mah, L. Taylor, J. J. Talghader, Reduced blackbody microheaters for measuring high temperature thermoluminescent glow curve peaks, in 2014 International Conference on Optical MEMS and Nanophotonics, Glasgow, 2014.

[16] J. J. Kingsley, K. C. Patil, Mater. Lett. 6 (1988) 427-432.

[17] L. A. Chick, L. R. Pederson, G. D. Maupin, J. L. Bates, L. E. Thomas, G. J. Exarhos, Mater. Lett. 10 (1990) 6-12.

[18] J. J. Kingsley, N. Manickam, K. C. Patil, Bull. Mater. Sci. 13 (1990) 179-189.

[19] L. E. Shea, J. McKittrick, O. A. Lopez, J. Am. Ceram. Soc. 79 (1996) 3257-3265.

[20] B. L. Cushing, V. L. Kolesnichenko, C. J. O'Connor, Chem. Rev. 104 (2004) 3893-3946.

[21] G. Hirata, N. Perea, M. Tejeda, J. A. Gonzalez-Ortega, J. McKittrick, Opt. Mater. 27 (2995) 13111315.

[22] J. R. DiMaio, C. Sabatier, B. Kokuoz, J. Ballato, PNAS 105 (2008) 1809-1813.

[23] M. W. Blair, L. G. Jacobsohn, B. L. Bennett, S. C. Tornga, E. G. Yukihara, E. A. McKigney, R. E. Muenchausen, Phys. Status Solidi A 206 (2009) 904-909.

[24] M. L. Mah, M. E. Manfred, S. S. Kim, M. Prokić, E. G. Yukihara, J. J. Talghader, IEEE Sens. J. 10 (2010) 311-315.

[25] S. W. S. McKeever, Thermoluminescence of Solids. Cambridge: Cambridge University Press (1985).

[26] K. C. Patil, S. T. Aruna, S. Ekambaram, Current Opinion in Solid State \& Materials Science 2 (1997) 158-165.

[27] L. G. Jacobsohn, K. B. Sprinkle, C. J. Kucera, T. L. James, S. A. Roberts, H. Qian, E. G. Yukihara, T. A. DeVol, J. Ballato, Opt. Mater. 33 (2010) 136-140.

[28] A. R. Lakshmanan, Phys. Status Solidi A 186 (2001) 153-166. 
[29] J. R. DiMaio, B. Kokuoz, T. L. James, T. Harkey, D. Monofsky, J. Ballato, Opt. Express 16 (2008) 11769-11775.

[30] C. J. Brinker, G. W. Sherer, Sol-Gel Science, the physics and chemistry of sol-gel processing. New York: Academic Press (1990).

[31] P. Dorenbos, Lanthanide level locations and its impact on phosphor performance, in Phosphor Handbook, W. M. Yen, S. Shionoya, and H. Yamamoto, Eds. Boca Raton, USA: CRC Press, 2007, pp. 139-154.

[32] V. R. Orante-Barrón, L. C. Oliveira, J. B. Kelly, E. D. Milliken, G. Denis, L. G. Jacobsohn, J. Puckette, E. G. Yukihara, J. Lumin. 131 (2011) 1058-1065.

[33] L. C. Oliveira, E. D. Milliken, E. G. Yukihara, J. Lumin. 133 (2013) 211-216.

[34] S. W. S. McKeever, M. Moscovitch, P. D. Townsend, Thermoluminescence Dosimetry Materials: Properties and Uses. Ashford: Nuclear Technology Publishing (1995).

[35] B. Obryk, P. Bilski, M. Budzanowski, M. Fuerstner, M. Glaser, C. J. Ilgner, P. Olko, A. Pajor, Z. Stuglik, IEEE Trans. Nucl. Sci 56 (2009) 3759-3763.

[36] L. G. Jacobsohn, M. W. Blair, S. C. Tornga, L. O. Brown, B. L. Bennett, R. E. Muenchausen, J. Appl. Phys. 104 (2008) 124303.

[37] Y. Fukuda, J. Radiat. Res. 43 (2002) S67-S69.

[38] Y. Fukuda, T. Niwa, Radiat. Prot. Dosim. 119 (2006) 153-156.

[39] B. Marczewska, P. Bilski, M. Budzanowski, P. Olko, V. Chernov, Radiat. Meas. 33 (2001) 571576.

[40] G. F. J. Garlick, A. F. Gibson, Proceedings of the Physical Society 60 (1948) 574.

[41] W. Hoogenstraaten, Philips Res. Rep 13 (1958) 515.

[42] R. Chen, S. A. A. Winer, Journal of Applied Physics 41 (1970) 5227-5232.

[43] R. Chen, S. W. S. McKeever, S. A. Durrani, Phys. Rev. B 24 (1981) 4931-4944.

[44] Y. S. Horowitz, D. Yossian, Radiat. Prot. Dosim. 60 (1995) 1-114.

[45] G. C. Taylor, E. Lilley, Journal of Physics D: Applied Physics 11 (1978) 567.

[46] P. Dorenbos, J. Lumin. 91 (2000) 155-176.

[47] P. Dorenbos, Phys. Rev. B 64 (2001) 125117 (12 pages).

[48] P. Dorenbos, J. Phys.: Condens. Matter 15 (2003) 8417-8434.

[49] P. Dorenbos, A. H. Krumpel, E. van der Kolk, P. Boutinaud, M. Bettinelli, E. Cavalli, Opt. Mater. 32 (2010) 1681-1685.

[50] J. T. Randall, M. H. F. Wilkins, Proc. Roy. Soc. A 184 (1945) 365-389.

[51] C. L. Lawson, R. J. Hanson, Solving Least Squares Problems. Englewoods Cliffs, New Jersey: Prentice-Hall, Inc. (1974).

[52] S. W. S. McKeever, Nucl. Instrum. Methods. Phys. Res. 175 (1980) 19-20.

[53] S. P. Kathuria, C. M. Sunta, J. Phys. D: Appl. Phys. 12 (1979) 1573-1587.

[54] E. Lilley, G. C. Taylor, J. Phys. D: Appl. Phys. 14 (1981) L13-L15.

[55] K. L. Choy, J. P. Feist, A. L. Heyes, B. Su, Journal of Materials Research 14 (1999) 3111-3114.

[56] V. Singh, V. K. Rai, I. Ledoux-Rak, S. Watanabe, T. K. G. Rao, J. F. D. Chubaci, L. Badie, F. Pelle, S. Ivanova, J. Phys. D: Appl. Phys. 42 (2009) 065104.

[57] M. A. Flores-Gonzales, G. Ledoux, S. Roux, K. Lebbou, P. Perriat, O. Tillement, J. Solid State Chem. 178 (2005) 989-997.

[58] P. A. Tanner, K. L. Wong, J. Phys. Chem. B 108 (2004) 136-142.

[59] P. J. R. Montes, M. E. G. Valerio, M. A. Macedo, F. Cunha, J. M. Sasaki, Microelectronics Journal 34 (2003) 557-559.

[60] D. Gozzi, A. Latini, G. Salviati, N. Armani, J. Appl. Phys. 99 (2006) 123524.

[61] J. C. Alonso, E. Haro-Poniatowski, R. Diamant, M. Fernández-Guasti, M. García, Thin Solid Films 303 (1997) 76-83. 
[62] N. Brahme, A. Gupta, D. P. Bisen, U. Kurrey, Recent Research in Science and Technology 4 (2012) 130-132.

[63] J. Hao, S. A. Studenikin, M. Cocivera, J. Lumin. 93 (2001) 313-319.

[64] A. J. Morales-Ramírez, A. García-Murillo, F. J. Carrillo-Romo, M. García-Hernández, J. MorenoPalmerin, R. Ruiz-Guerrero, International Journal of Molecular Sciences 12 (2011) 6240-6254.

[65] H. G. G. Long, D. R. Black, A. Feldman, E. N. Farabaugh, R. D. Spal, D. K. Tanaka, Z. Zhang, Thin Solid Films 217 (1992) 113-119.

[66] H. Kominami, C. Eguchi, M. Kottaisamy, Y. Nakanishi, Y. Hatanaka, Journal of Vacuum Science and Technology B 22 (2004) 1386.

[67] A. J. J. Bos, P. Dorenbos, A. Bessière, B. Viana, Radiat. Meas. 43 (2008) 222-226.

[68] M. P. Hehlen, M. G. Brikb, K. W. Krämerc, J. Lumin. 136 (2013) 221-239.

[69] T. Tsutsumi, Jpn. J. Appl. Phys. 9 (1970) 735-739.

[70] J. T. Brown, Comparison of Ignition Characteristics of Pure and Coated Aluminum Powder in a Shock Tube Facility, in Mechanical Engineering. vol. Master of Science University of Illinois at Urbana-Champaign: University of Illinois at Urbana-Champaign, 2007, p. 97.

[71] S. Guo, Turbulent interactions with normal shocks and their effects on aluminum particle burn time, in Mechanical Engineering. vol. Masters of Science University of Illinois at UrbanaChampaign: University of Illinois at Urbana-Champaign, 2015, p. 53.

[72] M. Puchalska, P. Bilski, Radiation Measurements 41 (2006) 659-664.

[73] J. Lu, T. Xu, J. Miao, Temperature Control of Microheaters for Localized Carbon Nanotube Synthesis, in 3rd International Nanoelectronics Conference (INEC), Hong Kong, 2010.

[74] O. Grudin, R. Marinescu, L. M. Landsberger, M. Kahrizi, G. Frolov, J. D. N. Cheeke, S. Chehab, M. Post, J. Tunney, X. Du, D. Yang, D. Segall, Journal of Vacuum Science and Technology A 20 (2002) 1100-1104.

[75] G.-S. Chung, J.-M. Jeong, Microelectronic Engineering 87 (2010) 2348-2352.

[76] H.-Y. Lee, S. Moon, S. J. Park, J. Lee, K.-H. Park, J. Kim, Electronics Letters 44 (2008) 1460 - 1461.

[77] M. Kim, R. Brukh, S. Kishore, S. Mitra, D. Misra, Sensors and Materials 18 (2004) 35-48.

[78] M. E. Manfred, N. T. Gabriel, E. G. Yukihara, J. J. Talghader, Radiat. Prot. Dosim. 139 (2010) 560564.

[79] X. He, Y. Li, L. Wang, Y. Sun, S. Zhang, Thin Solid Films 517 (2009) 5120-5129.

[80] Z. X. Cai, X. Y. Zeng, J. Duan, Thin Solid Films 519 (2011) 3893-3896.

[81] G.-S. Chung, S.-S. Noh, Sensors and Materials 10 (1998) 251-261.

[82] P. Yeh, Optical Waves in Layered Media. New York: Wiley (1988).

[83] E. G. Yukihara, V. H. Whitley, J. C. Polf, D. M. Klein, S. W. S. McKeever, A. E. Akselrod, M. S. Akselrod., Radiat. Meas. 37 (2003) 627-638.

[84] E. G. Yukihara, S. W. S. McKeever, Optically Stimulated Luminescence: Fundamentals and Applications. Chichester, West Sussex, UK: John Wiley \& Sons (2011). 


\title{
Appendix A: \\ Energy Distribution Determination
}

\author{
E.G. Yukihara, A.C. Coleman
}

Physics Department, Oklahoma State University, 145 Physical Sciences II, Stillwater, OK, 74078, USA

\section{- Summary}

This notebook is used to obtain a TL model for a given input curve, expressed as a superposition of 1st order TL curves, also know as Randall Wilkins Glow Curves (RW) under the assumption of a single frequency factor (in Log10). The user can choose the region of interest and the number of TL peaks to be used in the fitting procedure. The superposition of TL curves is output as a list of activation energies and weights (initial trap occupation). Two routines are implemented for fitting: Non-Negative Least Squares or Tikhonov. Cells with a light blue background provide information and comments, and cells in "light gray" require user input. The notebook ends with a "Figures" section which allows the user to view the results graphically and save the figures if desired.

\section{Output Files}

Edist.xls - Activation energy distribution (values and weights), used as input for "Temperature Determination" notebook.

TLcurves.xls - Data structure (list) with: Readout Temperature, Experimental TL data, Fitted TL curve, Fit Residuals, Individual Components.

Depletion.xls - Residual TL area for a temperature T, and time $\tau$. Format is column of T and column of residual TL area.

\section{- Version}

This notebook is version 1.1 and is written in Mathematica v. 10.1 (November $15^{\text {th }}, 2015$ )

\section{- Initialization}

Make sure the input file is located in the notebook directory. Execute the cell below to change the working directory to the notebook directory and define constants.

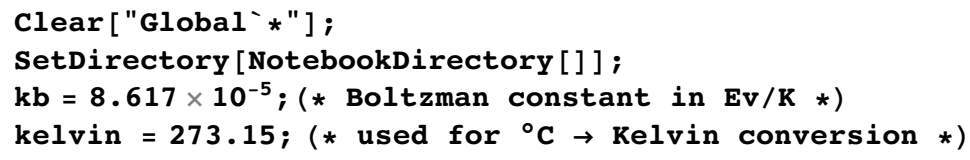

\section{Least Squares Routines \& TL Models}

\section{- Non-Negative Least Squares}

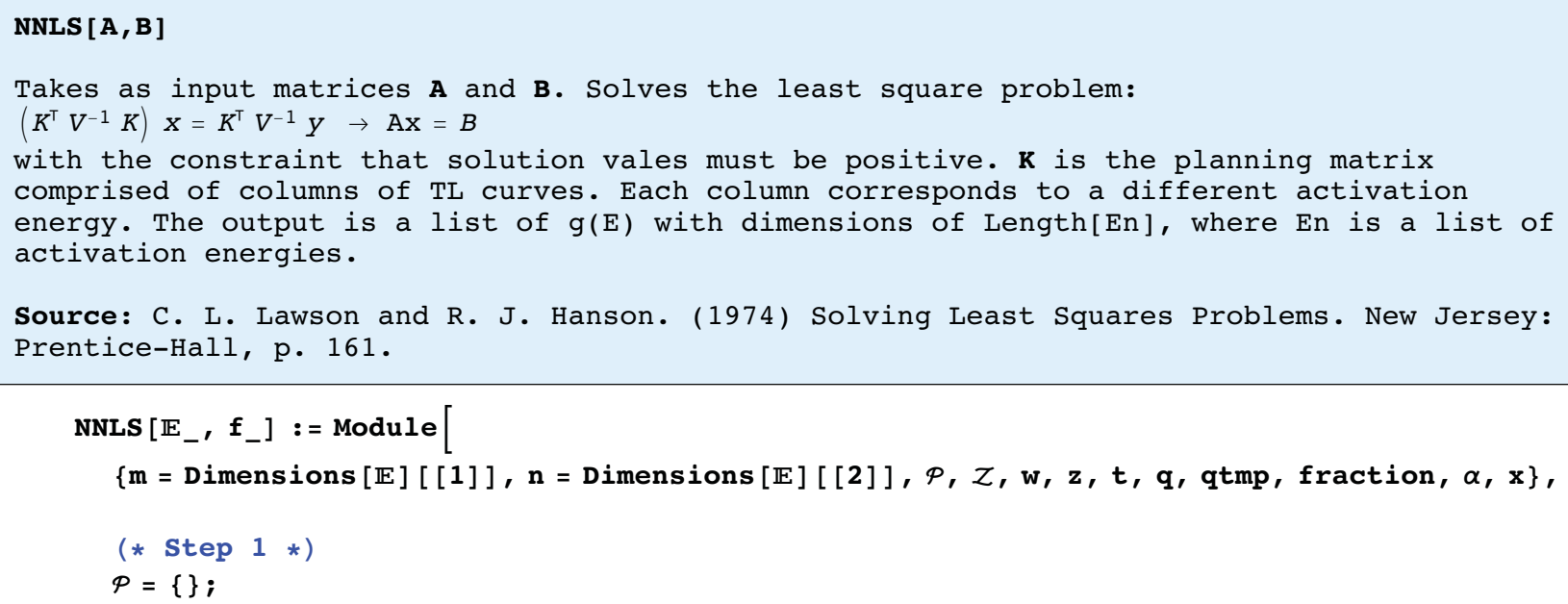


2 | E-dist determination.nb

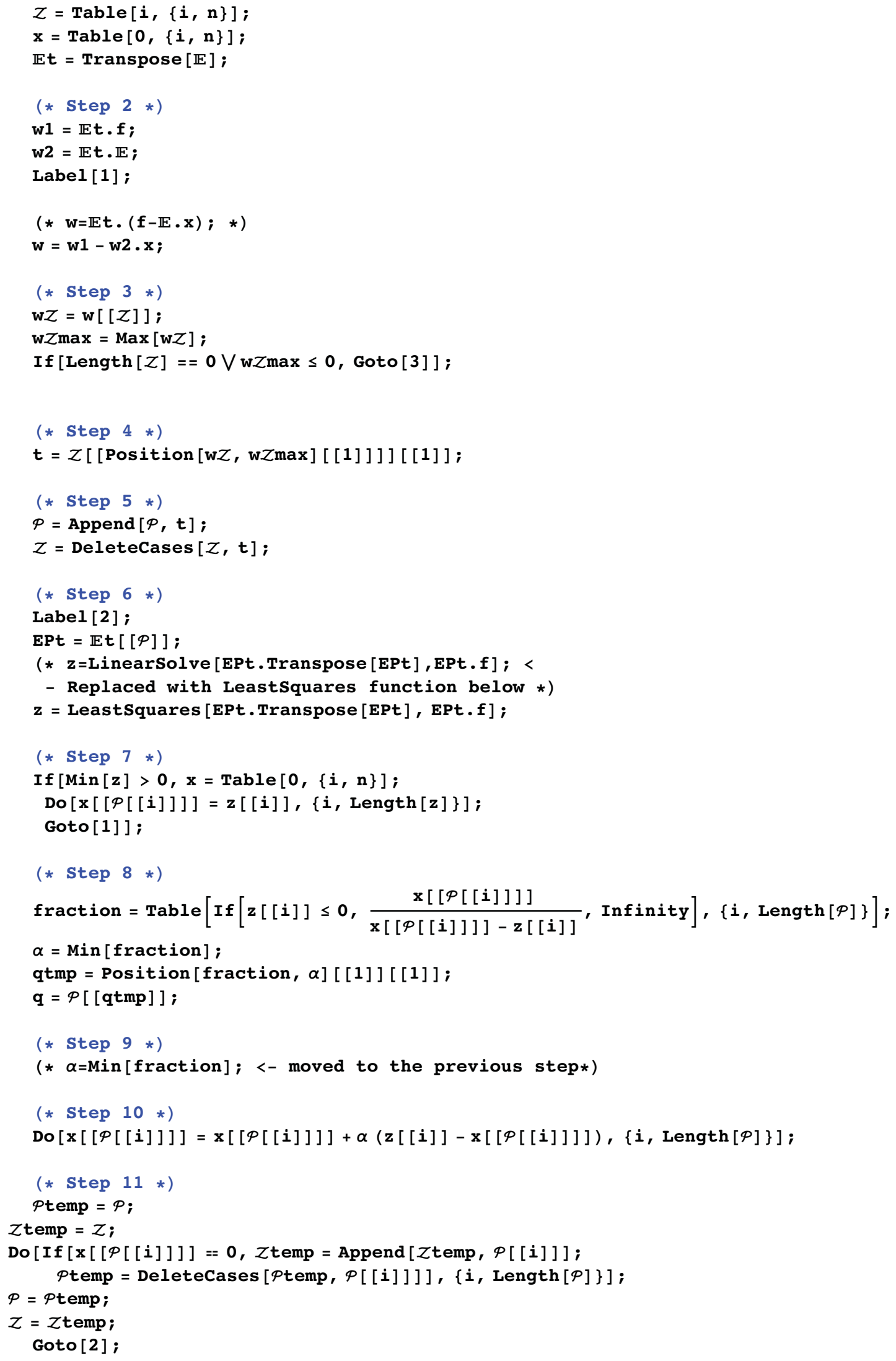




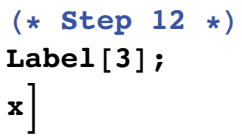

- Tikhonov Regularization Routines

Tikhonov [K, $\mathbf{f}, \mathrm{A}, \mathrm{B}$, tolerance, method $]$

Takes as input the matrices $\mathbf{K}, \mathbf{A}, \mathbf{B}$ as defined in NNLS Module. $\mathbf{f}$ corresponds to the intensities of the background removed TL control curve. This module solves the problem: $\left(K^{\top} V^{-1} K+\alpha^{2} S^{\top} S\right) x=K^{\top} V^{-1} y \rightarrow\left(\mathrm{A}+\alpha S^{\top} S\right) \mathrm{x}=\mathrm{B}$ where choice of $\mathrm{S}$ results in a constraint of minimizing amplitude (Method = 1 ) or a smoothing of the amplitudes (Method $=2$ ) of $\mathrm{x}$. Tolerance specifies how much the relative error can be increased from the minimum value possible. A tolerance of 0.1 ( $10 \%)$ is very reasonable. The routine will calculate the minimum relative error and then will increase it in $10 \%$ in order to find the corresponding a value. 


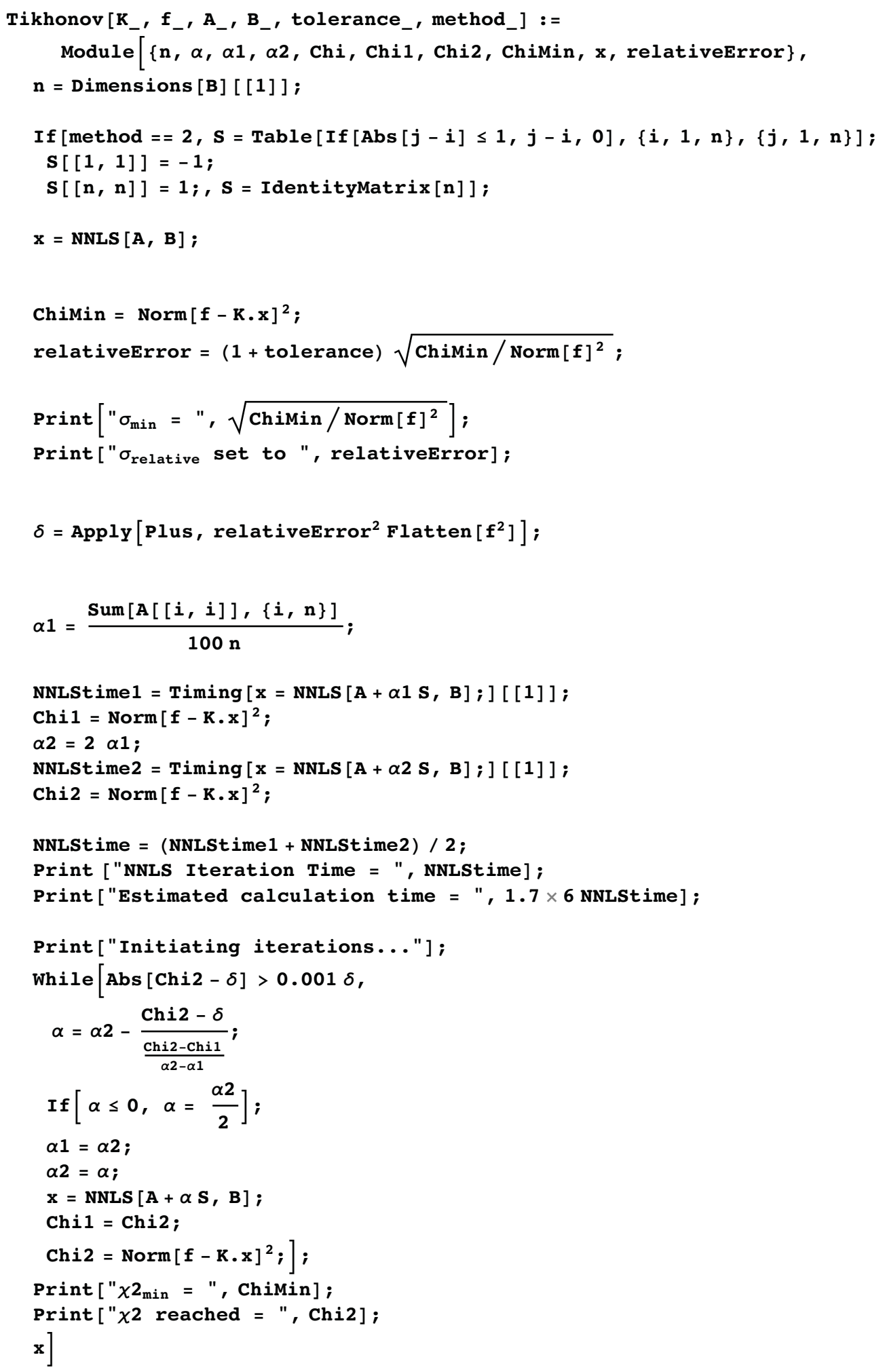

- TL Curve and Heating Profile 
in terms of energy $\mathbf{k T}$. The exponential integral in the RW model $\int e^{-E / \theta} \mathrm{d} \theta$ is approximated by

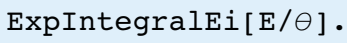

$\mathbf{f}[\mathrm{En}, \log s, \mathrm{~T}, \tau]$

Computes the depletion factor (between 0 . and 1.0 ) resulting from a constant heating profile of max temperature $\mathbf{T}$ (Kelvin) for a time period $\tau(\mathbf{s e c})$. First-order kinetics are assumed.

$\eta[\mathbf{T}, \tau]$ and $\mathbf{T L}[\mathbf{T}, \tau]$

Takes as input a max temperature $\mathbf{T}$, and a duration $\tau$. The function $\eta[$ ] generates a depletion factor between 0.0 and 1.0 , and the function TL[] applies the factor to solution TL curves.

$$
\begin{aligned}
& \left.\mathrm{RW}_{[\mathrm{En}}, \log _{-}, \mathrm{kT} \mathbf{T}_{-}\right]:=\operatorname{Chop}\left[10^{\operatorname{logs}} \operatorname{Exp}\left[-\frac{\mathrm{En}}{\mathrm{kT}}-10^{\operatorname{logs}}\left(e^{-\frac{\mathrm{En}}{\mathrm{kT}}} \frac{\mathrm{kT}}{\mathrm{kb}}+\frac{\text { En ExpIntegralEi}\left[-\frac{\mathrm{En}}{\mathrm{kT}}\right]}{\mathrm{kb}}\right)\right], 10^{-8}\right] \\
& f\left[E n_{-}, \log s_{-}, T_{-}, \tau_{-}\right]:=\operatorname{Chop}\left[\operatorname{Exp}\left[-10^{\operatorname{logs}} \operatorname{Exp}\left[-\frac{E n}{\mathrm{~kb} T}\right] \tau\right], 10^{-6}\right] \\
& \eta\left[\mathbf{T}_{-}, \tau_{-}\right]:=\mathbf{f}[\mathrm{En}, \mathbf{s}, \mathbf{T}, \tau] \\
& \mathrm{TL}\left[\bar{T}_{-}, \bar{\tau}_{-}\right]:=\mathrm{K} \cdot(\mathrm{n} 0 \eta[\mathrm{T}, \tau])
\end{aligned}
$$

\section{File Input}

Assumes file is type "*.xlsx" and is located in the same folder as notebook. Input file will have 4 columns: readout temperature, TL from exposed sample, TL from reference dose, TL from background.

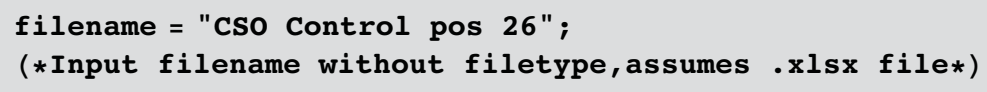

\section{Deconvolution}

\section{- Activation Energy Space}

User selects

- Log10[s] value (ex. $s=10^{14} \mathrm{~s}^{-1}$ becomes $\mathrm{s}=14$ )

- Range of data to be fit. Routine adds padding ( \pm ) to range. If fit is poor increase or

decrease padding and range as needed (See Figures Section)

- Number of equally spaced TL peaks in region of interest.

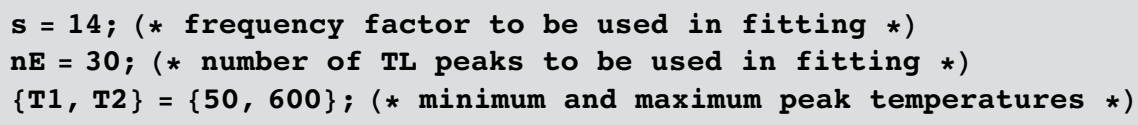

- The function $\mathcal{E}[\mathrm{T}]$ returns an activation energy the corresponding to a trap with a $\log 10$ frequency factor s, and a peak position $\mathrm{T}$. 


$$
\begin{aligned}
& \varepsilon\left[T_{-}\right]:=\operatorname{Quiet}\left[\varepsilon / . \operatorname{NSolve}\left[\varepsilon \operatorname{Exp}\left[\frac{\varepsilon}{\mathrm{kb}(T+k e l v i n)}\right]=10^{\mathrm{s}} \mathbf{k b}(T+273.15)^{2}, \varepsilon\right][[1]]\right] \\
& \{E n 1, \operatorname{En} 2\}=\{\varepsilon[\mathbf{T} 1+50], \varepsilon[\mathbf{T 2}-20]\} ; \\
& \text { (* Range of temperatures for the peaks to be fitted *) } \\
& \text { En }=\text { Range }\left[E n 1, E n 2, \frac{E n 2-E n 1}{n E-1}\right](* \text { range of energies *); }
\end{aligned}
$$

\section{- Deconvolution Routine}

User Selects

- Fitting method

- Uncertainty estimation

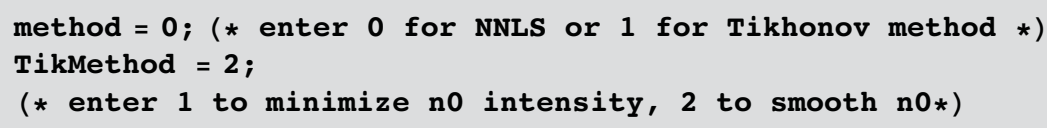

- Enter typical background (dark counts) that were subtracted from the curves (to estimate uncertainties associated with Poisson distribution):

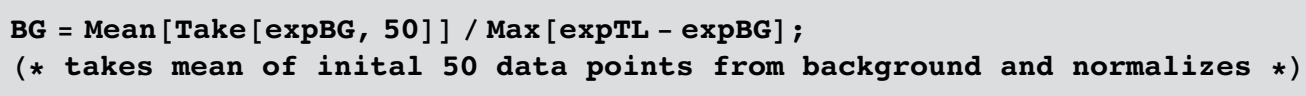

- First calculate the Planning matrix, $\mathbf{K}$, and its transpose $\mathbf{K}^{\mathrm{T}}$. Next calculate $\mathbf{V i n v}\left(V^{-1}\right)$ a diagonal matrix of the weights $\left(w_{i}=1 / \sigma_{i}^{2}\right)$ where the uncertainties are estimated to be Poisson from $\mathrm{y}$ and background. Finally calculate the matrices A and B for input for the least squares routines.

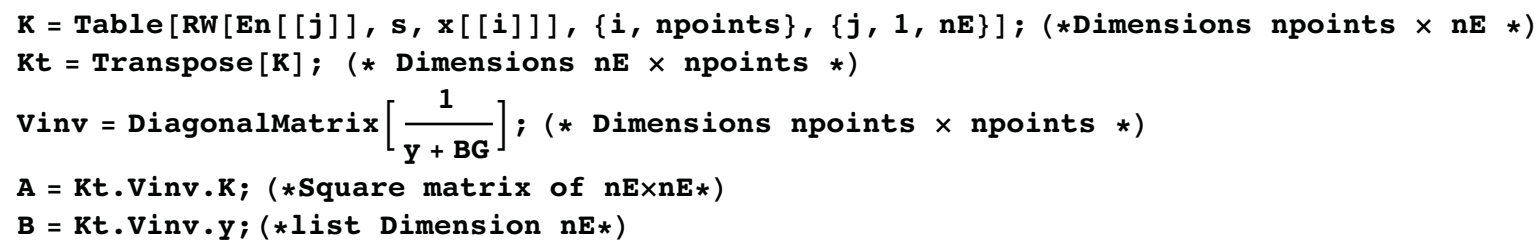

- Calculates E - Dist as a list $\mathbf{n} 0$ of length $\mathrm{nE}$.

If $[\operatorname{method}==0, \mathrm{n} 0=\operatorname{NNLS}[A, B], \mathrm{n} 0=\operatorname{Tikhonov}[\mathrm{K}, \mathrm{Y}, \mathrm{A}, \mathrm{B}, 0.001, \mathrm{TikMethod}]]$;

\section{Export}

Execute code below to export data sets. User Selects:

- Depletion timescale (in Log10)

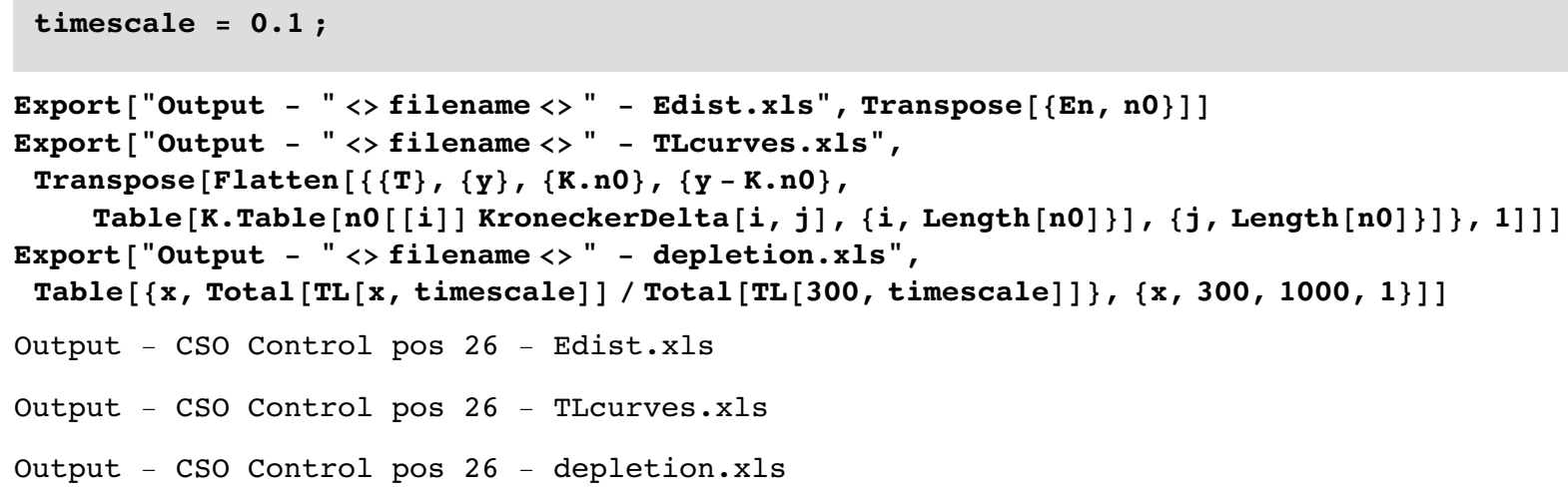




\section{Figures}
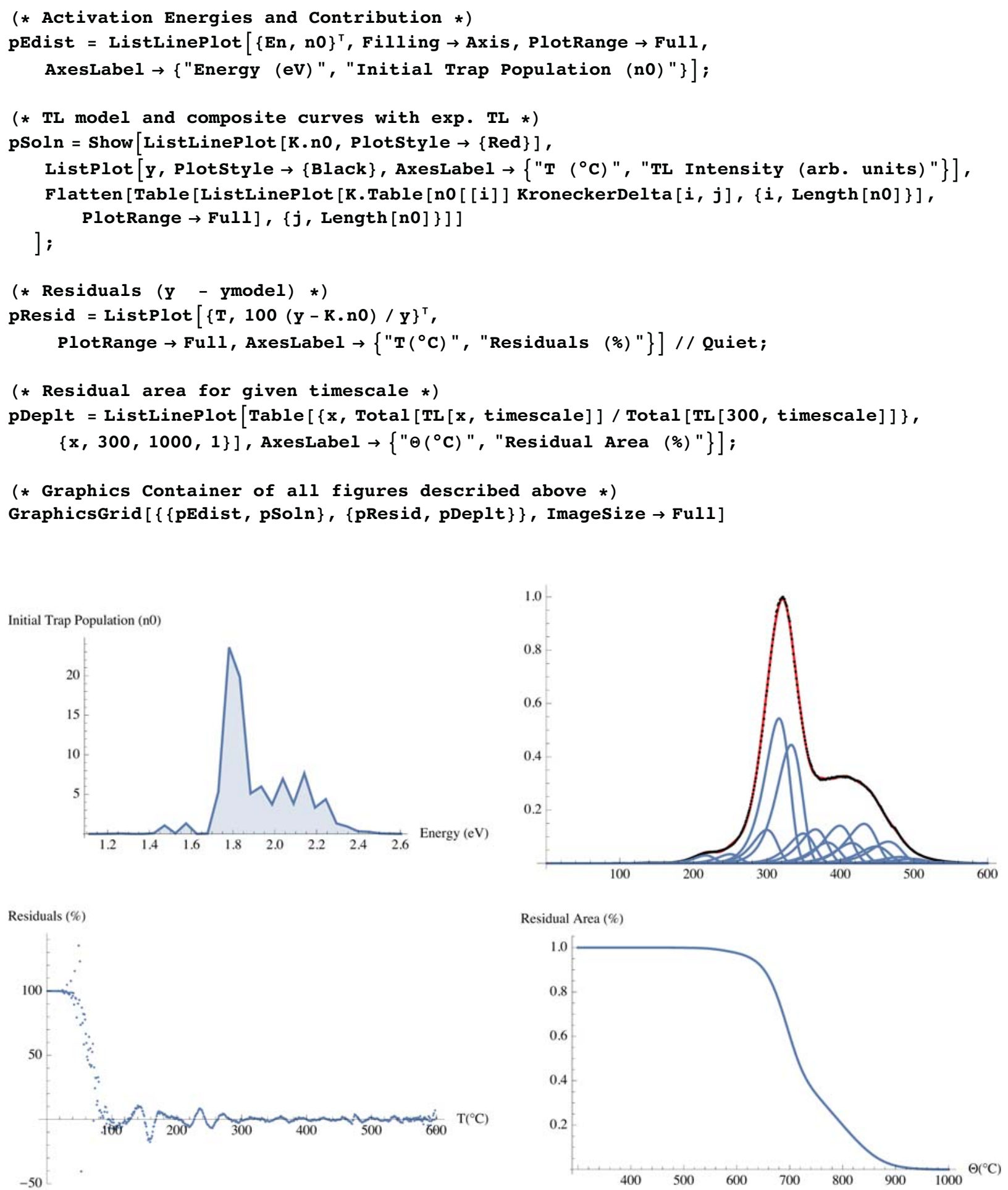

- $\quad$ File export of each individual figure. Execute cells of desired figures. Default export is *jpeg format.

Export ["Output - "<>filename <>" - edist.jpg", pEdist, ImageResolution $\rightarrow$ 600];

Export ["Output - "<>filename $<>"$ - solnTLcurves.jpg", pSoln, ImageResolution $\rightarrow$ 600]; 
8 E-dist determination.nb

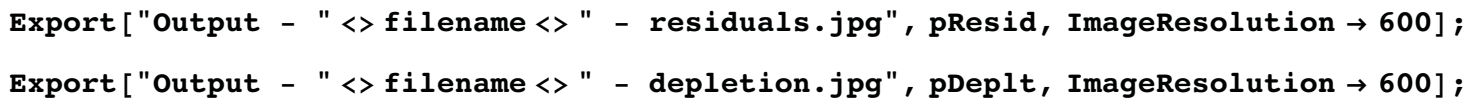




\title{
Appendix B: \\ Temperature Determination
}

\author{
E.G. Yukihara, A.C. Coleman
}

Physics Department, Oklahoma State University, 145 Physical Sciences II, Stillwater, OK, 74078, USA

\section{- Summary}

This notebook is used to obtain a temperature ("Tbest") that best explains a TL curve obtained from a heated sample. The algorithm loads an experimental TL curve and a TL model (Output from "E-Dist Determination" Notebook) obtained from a control sample. The model simulates various heated curves for a range of temperatures and heating times chosen by the user. The simulated curves are compared to the experimental TL curve to determine the heating profile that best matches the sample TL curve. Cells with a light blue background provide information and comments on a section, and cells in "light gray" require user input. The notebook ends with a "Figures" section which allows the user to view the results graphically and save the figures if desired.

\section{Output}

Files

Tbest.xls - Output file contains a value for Tbest $\left({ }^{\circ} \mathrm{C}\right)$ and shift $\Delta \mathrm{T}\left({ }^{\circ} \mathrm{C} / \mathrm{K}\right)$ for a user chosen timescale $\tau$. bestfit.csv - Output file contains readout temperature and 3 simulated TL curves for temperatures (Tbest, Tbest- $10^{\circ} \mathrm{C}, \mathrm{Tbest}+10^{\circ} \mathrm{C}$ ). profile.xls - Output file contains the calculated values for $\eta[\mathrm{T}, \tau]$, for a range of temperatures $\mathrm{T}$, and a single timescale $\tau$, chosen by the user.

\section{- Version}

This notebook is version 6, and is written in Mathematica v.10.1 (November $15^{\text {th }}, 2015$ )

\section{- Initialization}

Make sure the input file is located in the notebook directory. Execute the cell below to change the working directory to the notebook directory and define constants.

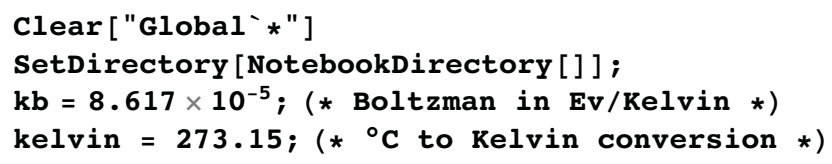

\section{TL and Pre-heating Model}

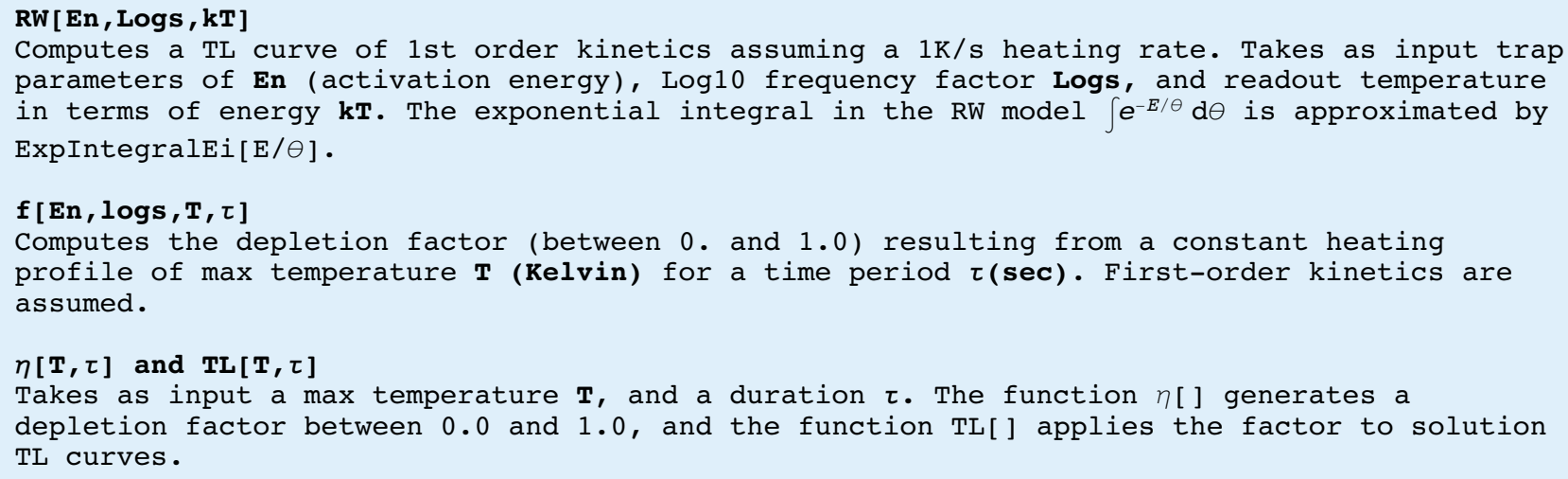




$$
\begin{aligned}
& \left.\mathrm{RW}_{[\mathrm{En}}, \log s_{-}, \mathrm{kT} \mathbf{T}_{-}\right]:=\operatorname{Chop}\left[10^{\operatorname{logs}} \operatorname{Exp}\left[-\frac{\mathrm{En}}{\mathrm{kT}}-10^{\operatorname{logs}}\left(\mathrm{e}^{-\frac{\mathrm{En}}{\mathrm{kT}}} \frac{\mathrm{kT}}{\mathrm{kb}}+\frac{\text { En ExpIntegralEi}\left[-\frac{\mathrm{En}}{\mathrm{kT}}\right]}{\mathrm{kb}}\right)\right], 10^{-8}\right] \\
& f\left[E n_{-}, \log s_{-}, T_{-}, \tau_{-}\right]:=\operatorname{Chop}\left[\operatorname{Exp}\left[-10^{\operatorname{logs}} \operatorname{Exp}\left[-\frac{E n}{\mathrm{~kb} T}\right] \tau\right], 10^{-6}\right] \\
& \eta\left[\mathbf{T}_{-}, \tau_{-}\right]:=\mathbf{f}[\mathrm{En}, \mathbf{s}, \mathbf{T}, \tau] \\
& \mathbf{T L}\left[\mathbf{T}_{-}, \tau_{-}\right]:=\mathrm{K} \cdot(\mathrm{n} 0 \eta[\mathrm{T}, \tau])
\end{aligned}
$$

\section{File Input}

\section{- Data Set}

Assumes file is type "*.xlsx" and is located in the same folder as notebook. Input file will have 4 columns: readout temperature, TL from exposed sample, TL from reference dose, TL from background.

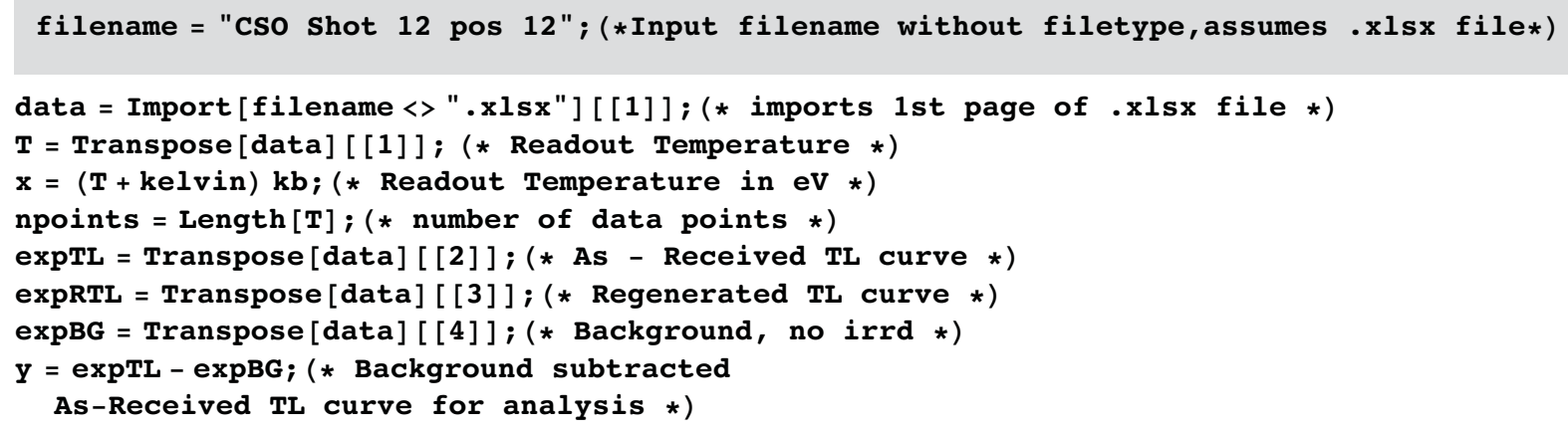

Assumes file is type "*.xls" and is located in the same folder as notebook. Input file will have 2 columns: Activation Energy and Initial Trap Population (Weighting).

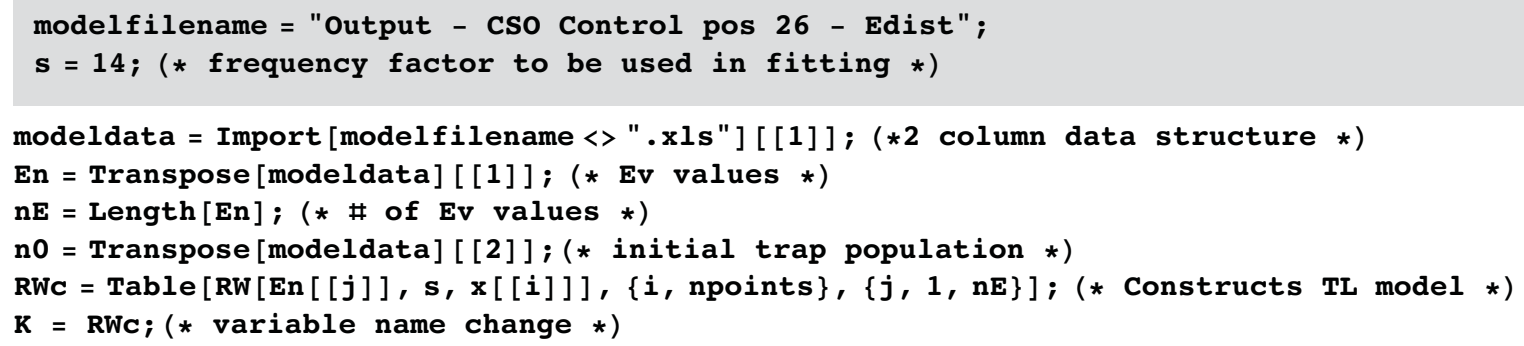

\section{Thermal Reconstruction}

Set of simulated TL curves are constructed for comparison to Exp. TL curves using a FOM of $\eta[T, \tau]=$ npoints $/\left|y-y_{\text {model }}\right|$. User must evaluate each subsection.

Solution Matrix: Calculates a 3D solution space, $1 / \eta[T, \tau]$, for a range of temperatures and timescales

Solution Profile: Calculates a 2D solution space, $1 / \eta[T, \tau 0]$, for a range of temperatures and a single timescale $\tau 0$.

User chooses

- Range of exposed temperatures in ${ }^{\circ} \mathrm{C}$

- Heating durations in Log10[sec]

- TL curve shift, accounts for exper. shift of TL curve. Interpret as $\pm \Delta$ Tmax. 
matrixx $=$ Range $[300,900,10] ;$ (* temperature in ${ }^{\circ} \mathrm{C}, \min , \max$, step *)

matrixy $=$ Range $[-3,2,0.2] ;\left(* \log _{10}\right.$ of duration $\tau$ in $s$, min, max, step $*$ )

$\Delta \operatorname{Tmax}=20$; (* Allowed tolerance in shift for TL curves *)

$\tau 0=0.1 ;$ (* Estimated timescale of event *)

\section{- Solution Matrix (range $\tau$ )}

- Create a multidimensional list of simulated TL curves over the range of temperatures and timescales.

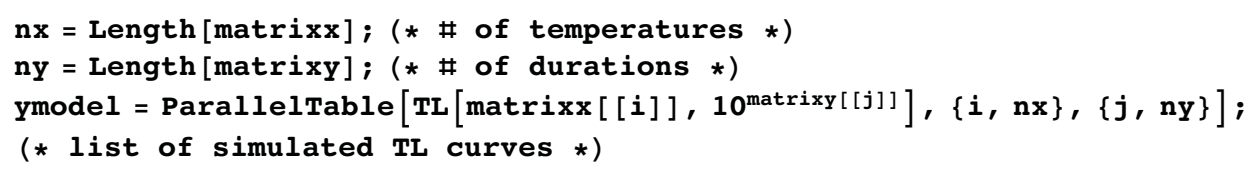

- Normalize simulated and sample TL curves.

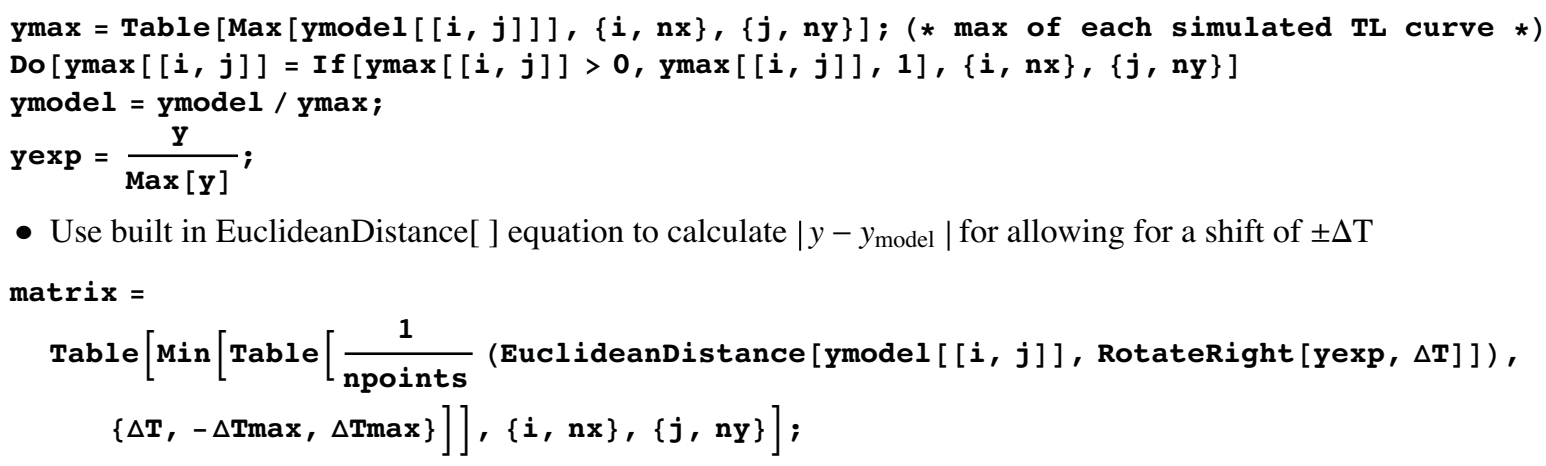

- Use built in EuclideanDistance[ ] equation to calculate $\left|y-y_{\text {model }}\right|$ for allowing for a shift of $\pm \Delta \mathrm{T}$

matrix $=$

\section{- Solution Line Profile (fixed $\tau$ )}

- Construct range of heating temperatures with $1^{\circ} \mathrm{C}$ step resolution.

- Simulate TL curves for each heating temperature for a hold time of $\tau 0$.

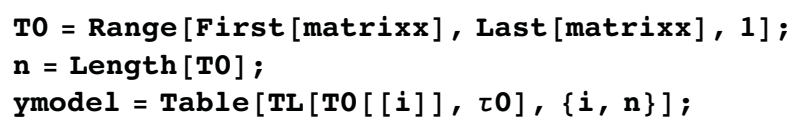

- Normalize simulated curves

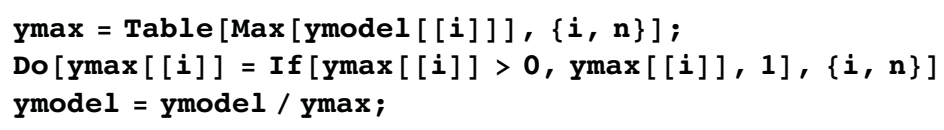

- Construct a $2 \mathrm{D}$ profile for $1 / \eta[\mathrm{T}, \tau 0]$

- Obtain the min value for $1 / \eta[\mathrm{T}, \tau 0]$, this is Tbest. This position also corresponds to the minimum shift $\Delta \mathrm{T}$ of the sample curve. 


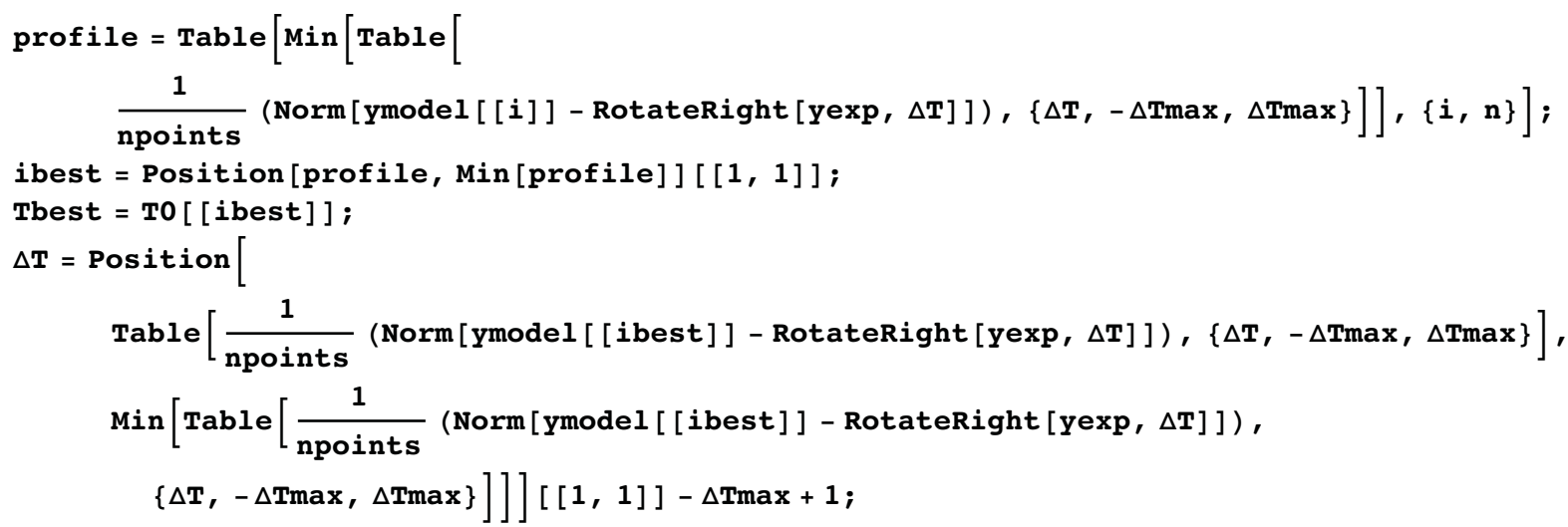

\section{Export Results}

Execute code below to export result data sets.

User Selects

- solution range, default is Tbest $\pm 10^{\circ} \mathrm{C}$

sol $=\{\{$ Tbest,$\tau 0\},\{$ Tbest $-10, \tau 0\},\{$ Tbest $+10, \tau 0\}\} ;$

plotdata $=$ Flatten $\left[\left\{\left\{\operatorname{RotateRight}\left[\frac{\text { yexp }}{\operatorname{Max}[y \exp ]}, \Delta \mathbf{T}\right]\right\}, \operatorname{Table}[\mathrm{TL}[\operatorname{sol}[[j, 1]]\right.\right.$, sol $[j j, 2]]] /$

$\operatorname{Max}[\operatorname{TL}[\operatorname{sol}[[1,1]], \operatorname{sol}[[1,2]]]],\{j, \operatorname{Length}[\operatorname{sol}]\}]\}, 1] ;$

Export ["Output - " <>filename <>" - bestfit.csv", Transpose[Prepend[plotdata, T] ]];

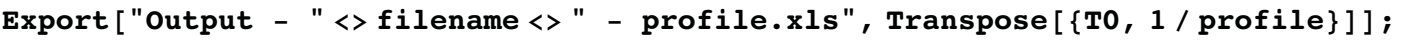

Export ["Output - "<>filename $<>"$ - Tbest.xls", \{Tbest, $\Delta$ T\}]; 


\section{Figures}

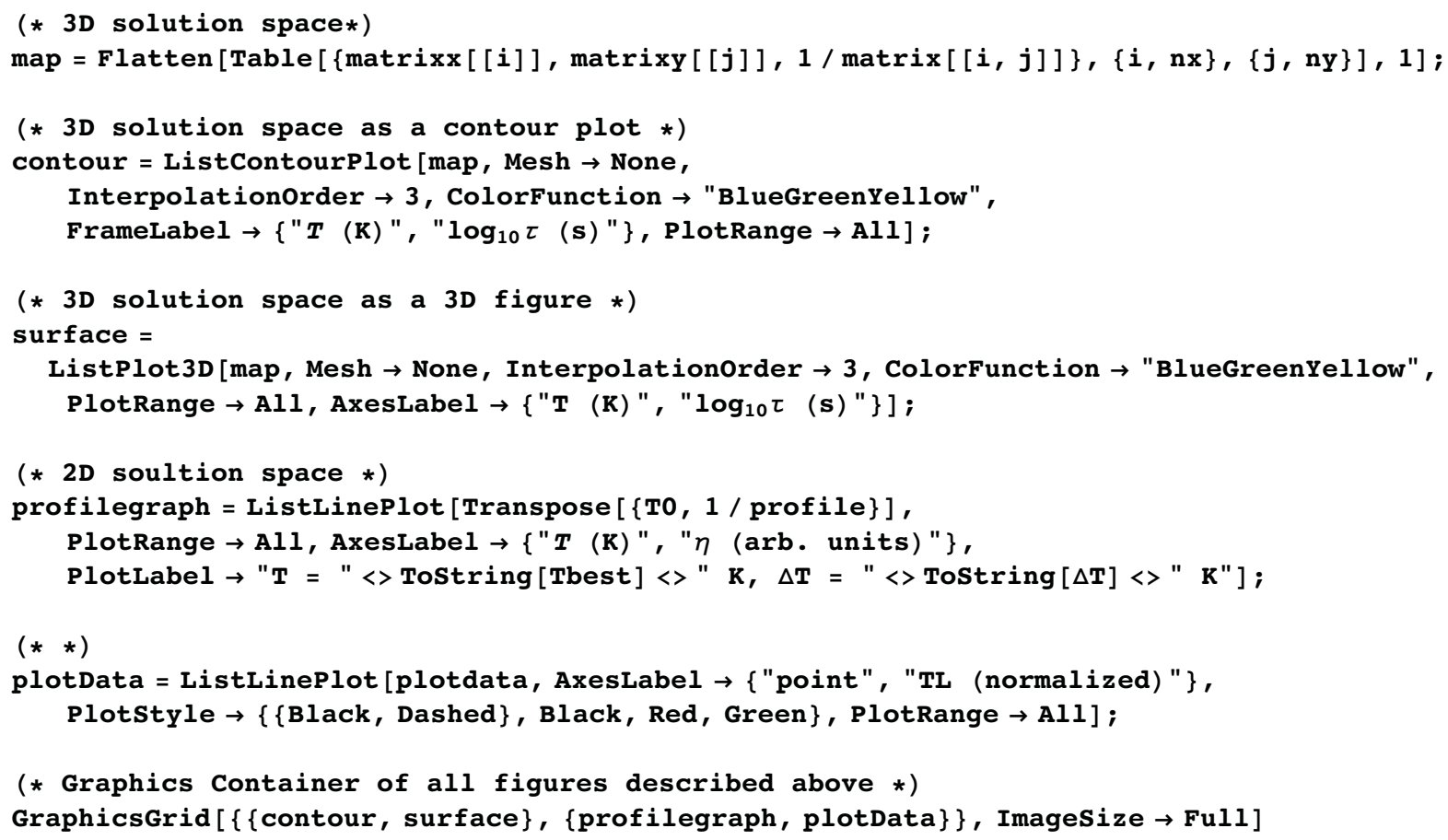



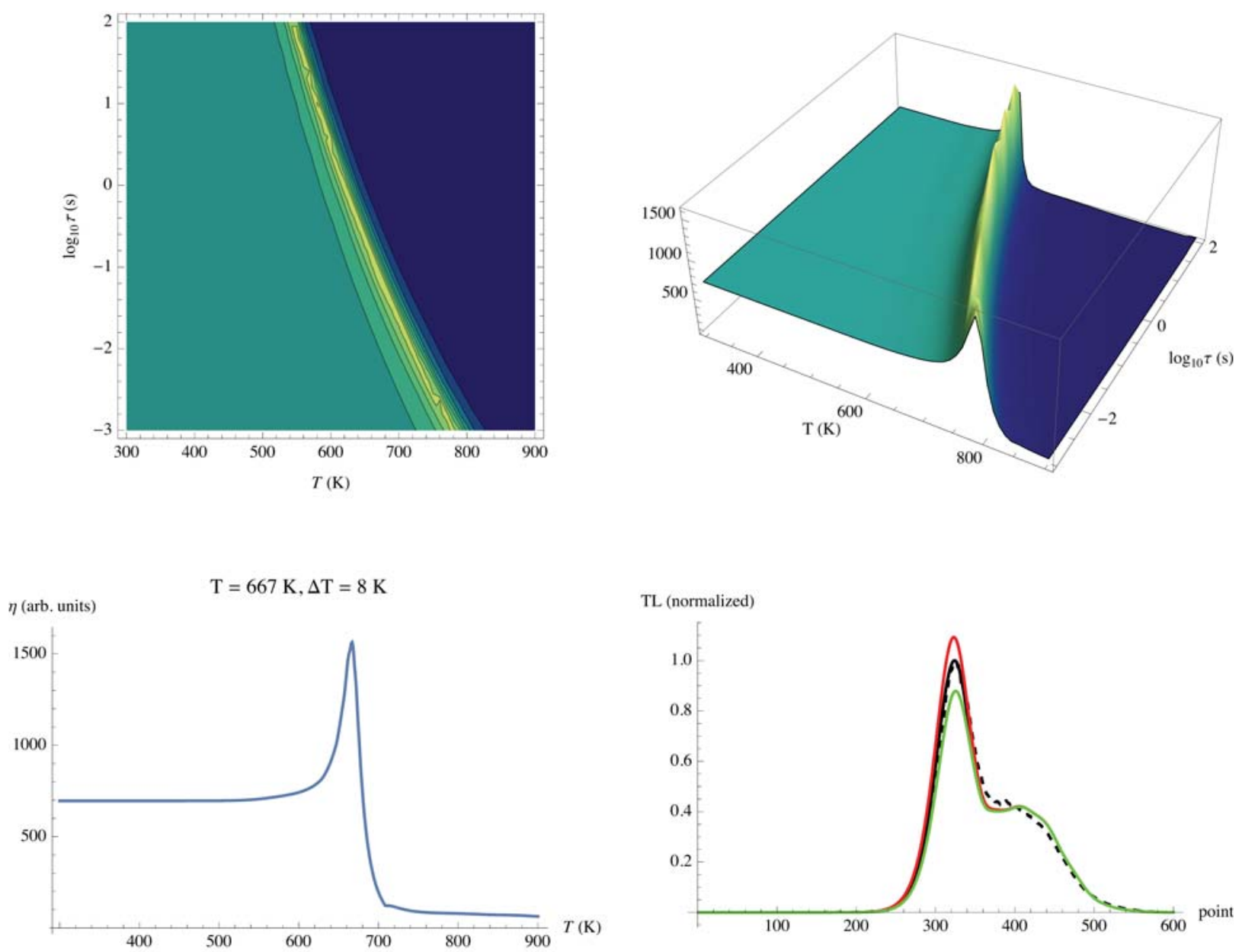

- File export of each individual figure. Execute cells of desired figures. Default export is *jpeg format.

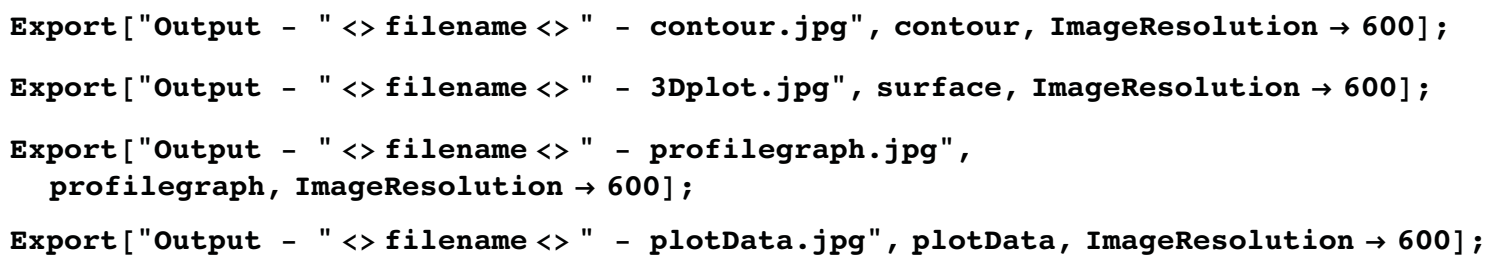




\section{DEPARTMENT OF DEFENSE}

DEFENSE THREAT REDUCTION

AGENCY

8725 JOHN J. KINGMAN ROAD

STOP 6201

FORT BELVOIR, VA 22060

ATTN: A. DALTON

DEFENSE TECHNICAL

INFORMATION CENTER

8725 JOHN J. KINGMAN ROAD,

SUITE 0944

FT. BELVOIR, VA 22060-6201

ATTN: DTIC/OCA

\section{DEPARTMENT OF DEFENSE}

CONTRACTORS

QUANTERION SOLUTIONS, INC.

1680 TEXAS STREET, SE

KIRTLAND AFB, NM 87117-5669

ATTN: DTRIAC 